CELLULOSE-BASED FIBERS FROM LIQUID CRYSTALLINE SOLUTIONS

by

Vipul Davé

Dissertation submitted to the Faculty of the

Virginia Polytechnic Institute and state University

in partial fulfillment of the requirements for the degree of

PhD

in

Materials Engineering Science

APPROVED :

bofgaum $q$ gheber

Alfred C. In e

Alfred C. Loos

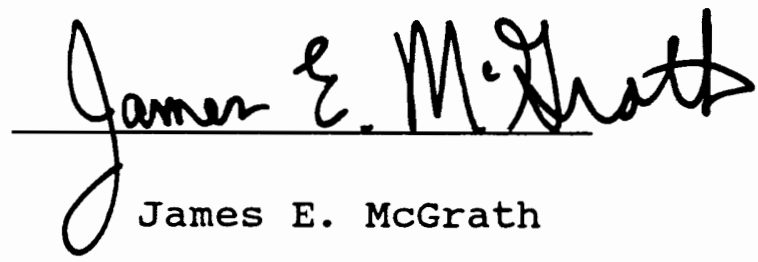

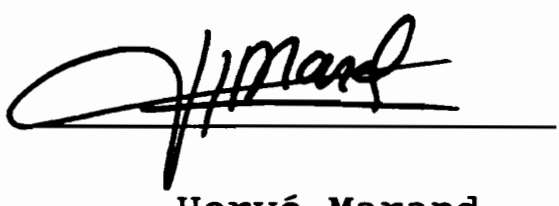

Hervé Maraud

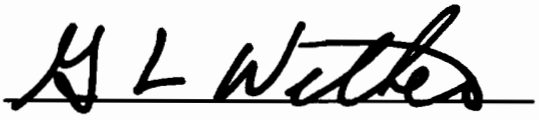

Garth L. Wilkes

February 1992

Blacksburg, Virginia 


\title{
CELLULOSE-BASED FIBERS FROM LIQUID CRYSTALLINE SOLUTIONS
}

\author{
by \\ Vipul Davé \\ Wolfgang G. Glasser, Chairman \\ Materials Engineering Science
}

(ABSTRACT)

Solutions of cellulose esters with different concentrations in dimethylacetamide (DMAC) and with different types of substituents were studied in relation to their liquid crystalline (LC) solution behavior. Classical LC behavior was revealed for all solutions. Critical polymer concentration $\left(V_{p}{ }^{c}\right)$ is highest for cellulose acetate $(C A)$ and lowest for cellulose acetate butyrate (CAB) with highest degree of butyration. This is opposite to the classical model by Flory which predicts an increase in $\mathrm{V}_{\mathrm{p}}{ }^{\mathrm{w}}$ with decreasing aspect ratio $(L / d)$.

Fibers were spun from isotropic and anisotropic DMAC solutions of cellulose esters by dry jet/wet spinning process. The mechanical properties, orientation, and crystallinity of the fibers increased as spinning progressed from the isotropic to the anisotropic solution state. High butyryl content enhances both overall solubility and the formation of LC solutions at lower concentration, but it results in lower fiber properties.

Unmodified cellulose (C) and cellulose hexanoate $(\mathrm{CH})$ 
also exhibited LC behavior. The $\mathrm{V}_{\mathrm{p}}{ }^{\mathrm{c}}$ value for $\mathrm{CH}$ was lower than that obtained for $C A B$ with maximum degree of butyration. This indicates that bulky substituents may lower $V_{p}^{c}$ values. The formation of high modulus (152 $\mathrm{g} / \mathrm{d})$ cellulose fibers from LC solutions is attributed to the air-gap that exists in the dry jet/wet spinning process.

Presence of lithium chloride (LiCl) in the LC solutions of $C A B$ exhibited ionic interactions. Mechano-sorptive creep behavior of the fibers spun from these solutions decreases in the presence of residual Licl salt.

Fibers from blends of $C A B$ and of $C$ with lignin (L) were spun from LC solution. Morphological investigations demonstrate that $\mathrm{CAB}$ and $\mathrm{L}$ formed intimately mixed blends whereas $C$ and $L$ were partially mixed. The mechanical properties of the fibers with $L$ increased due to good phase mixing of $\mathrm{CAB}$ and $\mathrm{L}$ molecules in the fiber matrix. 
To Dimple 


\section{PREFACE}

Besides the introduction, all the other chapters of this dissertation have been prepared as manuscripts which have been, or will be, submitted for publication. The terms like "we" and "our" refer to the authors of the manuscript. Each chapter has its own abstract, experimental section and literature citations. Apologies are made for this inconvenience. 


\section{ACKNOWLEDGEMENTS}

I sincerely thank Dr. Wolfgang G. Glasser for providing me the opportunity to pursue my Ph.D. under his guidance. I truly appreciate his supervision, support, and patience during the entire course of my research. I would like to extend my gratitude to my committee member, Dr. Garth L. Wilkes, for his invaluable help at every stage of my research. I would also like to thank the other members of my committee, Drs. A. C. Loos, H. Marand, and J. E. McGrath, for their interest and suggestions. Furthermore, thanks to Dr. Geza Ifju for his support and challenging racquetball.

Additionally, I am obliged to my colleagues in the laboratory for making the working environment very pleasant. I am thankful to Chip (NMR), Gamini (chemistry), Raj (lignin), Paul (DSC), Jody (MWD), Nancy (discussions), Laurence (humor), Klaus (computers), Willer (classical music), Bruno (stocks), Gil (party), and will (discussions) for all their help.

I would like to acknowledge the timely help by Dr. Phil Wapner (Southern Illinois University) and Harold Vandivort for constructing the fiber spinning apparatus.

I thank Dr. Dave Dillard, John Wang and Abaneshwar Prasad for their collaboration with my research activities. This association has been very productive. I wish to acknowledge the assistance provided by David Rodriguez on WAXS; Janet wimmer on instron; and Kathy on electron microscopy. My 
sincere thanks to sidhartha sen for being a good friend and for introducing me to the Bengali community in Blacksburg.

I wish to thank my family: my parents, Mr. and Mrs. B. M. Davé; my sister and brother-in-law, Aparna and Raju; my brother and sister-in-law, Parasar and Neetu; my best friend, Vivekanand; my mother-in-law, Mrs. P. J. Kusumgar; and my sister-in-law, Niki, for all their motivation, inspiration, and cooperation during my academic career.

I am deeply indebted to my wife, Dimple, as without her love and encouragement, none of this would have been possible. To her, I dedicate this dissertation. 


\section{Table of Contents}

1.0 Introduction . . . . . . . . . . . . . . . . . . . . 1

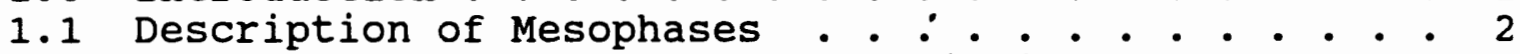

1.2 Nature, structure and Types of Liquid Crystals . . . 4

1.3 Molecular Theory of Liquid Crystals . . . . . . . . 11

1.3.1 Binary Lyotropic systems Consisting of

1.3.2 Mixtures of Rods and Random Coils

1.3.3 Semirigid Chains . . . . . . . . . 16

1.3.4 Intermolecular Interactions . . . . . . 18

1.4 Fiber Spinning Processes . . . . . . . . . . . . . 21

1.5 Fundamentals of Fiber Formation . . . . . . . . 25

1.5.1 Spinnability of Fluids . . . . . . . . 25

1.5.2 Fiber Spinning Variables . . . . . . . 28

1.5.3 Diffusion During the Coagulation Step

of Wet Spinning . . . . . . . . . . 30

1.6 Fiber spinning From Liquid Crystalline Solutions . . 40

1.6.1 Role of Liquid Crystalline State . . . . 40

1.6.2 Spinning from Nematic Solutions . . . . 43

1.6.3 Flow-induced Transitions to a Nematic

1.7 Research objectives $\bullet^{\cdot} \cdot \bullet^{-} \cdot \bullet^{-} \cdot \bullet^{-} \cdot \bullet^{-} \cdot \bullet^{-} 44$

1.8 References . . . . . . . . . . . . . . . . . . . 52

2.0 Cellulose-Based Fibers From Iiquid Crystalline Solutions I. Solution Properties of cellulose

Esters . . . . . . . . . . . . . . . 58

2.1 Abstract . . . . . . . . . . . . . . . 59

2.2 Introduction . . . . . . . . . . . . . . . . 59

2.3 Experimental . . . . . . . . . . . . . . . 62

2.3.1 Materials . . . . . . . . . . 62

2.3.2 Methods ................. 62

2.4 Results and Discussion . . . . . . . . . . . 63

2.5 Conclusions . . . . . . . . . . . . . . . . 77

2.6 Appendix A . . . . . . . . . . . . . . . 78

2.7 Literature Cited . . . . . . . . . . . . . 79

3.0 Cellulose-Based Fibers From Liquid Crystalline Solutions II. Processing and Morphology of

Acetate/Butyrate Esters . . . . . . . . . . 82

3.1 Abstract . . . . . . . . . . . . . . . . 83

3.2 Introduction . . . . . . . . . . . . . . . . . 84

3.3 Experimental ................... . 86

3.3.1 Materials .............. 86

3.3 .2 Methods . . . . . . . . . . . 86

3.4 Results and Discussion . . . . . . . . . . . . 92

3.4.1 Processing . . . . . . . . . . . . . 92

3.4.2 Mechanical Properties . . . . . . . 93

3.4.3 Morphology ............... 100 
3.5 Conclusions ................... 126

3.6 References . . . . . . . . . . . . . . 128

4.0 Cellulose-Based Fibers From Liquid Crystalline

solutions III. Processing and Morphology of

Cellulose and Cellulose Hexanoate Esters . • • . 132

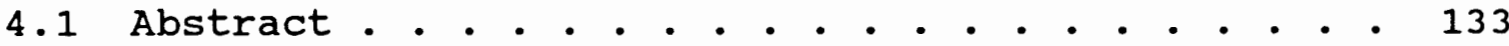

4.2 Introduction . . . . . . . . . . . . . . . . . 134

4.3 Experimental • . . . . . . . . . . . . . . . 139

4.3.1 Materials . . . . . . . . . . 139

4.3.2 Methods ............... 139

4.4 Results and Discussion . . . . . . . . . . . . 144

4.4.1 Dynamic Viscoelastic Properties . . . . 145

4.4.2 Polarized Optical Microscopy . . . . . 150

4.4.3 Theoretical Considerations for the Determination of Critical Volume

4.4.4 Processing .............. 158

4.4.5 Mechanical Properties ........ . 161

4.4.6 Morphology ............... 165

4.5 Conclusions . . . . . . . . . . . . . . . . . . 170

4.6 Appendix A . . . . . . . . . . . . . . . . . . . 171

4.7 References . . . . . . . . . . . . . . . . 173

5.0 Cellulose-Based Fibers From Liquid Crystalline Solutions IV. Effects of Iithium Chloride on Acetate/Butyrate Esters

5.1 Abstract . . . . . . . . . . . . . . . 179

5.2 Introduction . . . . . . . . . . . . . . . 180

5.3 Experimental . . . . . . . . . . . . . . 181

5.3.1 Materials ............... 181

5.3.2 Methods ................ 181

5.4 Results and Discussion . . . . . . . . . . . . . . 188

5.4.1 ${ }^{13} \mathrm{C}$ NMR Spectroscopy . . . . . . . . . . 188

5.4.2 Dynamic Viscoelastic Properties . . . 190

5.4.3 Polarized Optical Microscopy . . . . . 195

5.4.4 Processing and Mechanical Properties . . 195

5.4 .5 Morphology . . . . . . . . . . . 198

5.4.6 Moisture Absorption ......... . 201

5.4.7 Mechano-sorptive Creep Behavior . . . 201

5.5 Conclusions . • . . . . . . . . . . . . . . 212

5.6 References . . . . . . . . . . . . . . . 213

6.0 Cellulose-Based Fibers From Liquid Crystalline Solutions v. Processing and Morphology of Blends with Lignin . . . . . . . . . . . . . . . . 216

6.1 Abstract...................... . 217

6.2 Introduction..................... . 218

6.3 Experimental . . . . . . . . . . . . . . 220

6.3.1 Materials ............... 220 
6.3.2 Methods ................ 220

6.4 Results and Discussion . . . . . . . . . . . 225

6.4.1 Dynamic Viscoelastic Properties . . . . 226

6.4.2 Polarized Optical Microscopy . . . . 229

6.4.3 Processing . . . . . . . . . . . 234

6.4.4 Mechanical Properties . . . . . . . 234

6.4.5 Thermal Analysis...... . . . . . 236

6.4.6 Scanning Electron Microscopy . . . . . 238

6.4.7 Transmission Electron Microscopy . . . . 238

6.5 Conclusions . . . . . . . . . . . . . . . . . . 245

6.6 References . . . . . . . . . . . . . . . . . 246

7.0 Evidence For Cholesteric Morphology In Films of cellulose Acetate Butyrate BY Transmission

Electron Microscopy

7.1 Abstract..................... 250

7.2 Introduction . . . . . . . . . . . . . . . . . . 251

7.3 Experimental ................... 251

7.3.1 Materials ............... 252

7.3 .2 Methods ............... 252

7.4 Results and Discussion . . . . . . . . . . . . . 254

7.5 References . . . . . . . . . . . . . . 262

8.0 Molecular Organization of Lignin During

Carbonization . . . . . . . . . • . . . . 263

8.1 Abstract . . . . . . . . . . . . . . . . 264

8.2 Introduction . . . . . . . . . . . . . . . 265

8.3 Experimental . . . . . . . . . . . . . . . 270

8.3.1 Materials ............... 270

8.3.2 Methods . . . . . . . . . . 271

8.4 Results and Discussion . . . . . . . . . . . . 278

8.4.1 Lignin-like Model Compounds . . . . 278

8.4.2 Lignin................. 295

8.4.3 Pitch $(A-240)$............ 298

8.4.4 Blends of Pitch $(A-240)$ and Lignin. . . 301

8.4.5 Hydroxypropyl Lignin ......... . 303

8.4.6 Hydroxypropyl Lignin Pitch ...... . 305

8.4.7 Mechanical Properties .. . . . . . 308

8.4.8 Scanning Electron Microscopy ..... . 308

8.4.9 Transmission Electron Microscopy . . . . 308

8.5 Conclusions . . . . . . . • • • • . . . . 312

8.6 References . . . . . . . . . . . . . . . . . 314

9.0 Liquid Crystalline Phase Formation In Concentrated Tri-0- $\alpha$-Naphthylmethyl Cellulose solutions . . . 319

9.1 Abstract .. . . . . . . . . . . . . . 320

9.2 Introduction .................. 321

9.3 Experimental . . . . . . . . . . . . . 321

9.3.1 Materials ............. 321

9.3.2 Methods ............... 323

9.4 Results and Discussion . . . . . . . . . 325 
9.5 Conclusions . . . . . . . . . . . . . . 341

9.6 Appendix A................... 341

9.7 References.................. 343

10.0 Thermal Transitions of Cellulose Acetate.... . 345

10.1 Abstract . . . . . . . . . . . . . . . . 346

10.2 Introduction . . . . . . . . . . . . . . . 347

10.3 Experimental . . . . . . . . . . . . . 347

10.3.1 Materials ............. 347

10.3.2 Methods .............. . 347

10.4 Results and Discussion............. . 348

10.5 Conclusions . . . . . . . . . . . . . . 356

10.6 References . . . . . . . . . . . . . 357

11.0 Recommendations. . . . . . . . . . . . 358

12.0 Appendix . . . . . . . . . . . . . . . . . 360

A. Fiber Spinning Apparatus . . . . . . . . . . 361

B. Processing of Fibers ............. 367

vita . . . . . . . . . . . . . . 369 


\section{List of Illustrations}

\section{Chapter 1}

Fig. 1. Schematic diagram of the possible states of a liquid crystal forming material. . . . . . . 6

Fig. 2. Types of liquid crystal mesophase structures; (a) nematic (b) cholesteric (c) smectic. . . . 9

Fig. 3. (a) Rodlike particle oriented at an angle $\psi$ from the preferred axis $X-X$ and (b) its subdivision into $y$ sequences parallel to this axis as required for accommodation on the cubic lattice.

Fig. 4. Compositions in volume fraction of coexisting phases for rods of axial ratio $x=100$ subject to interaction denoted by parameter $\chi$. . . . 19

Fig. 5. Methods of solution spinning. . . . . . . . . 24

Fig. 6. Schematic representation of (a) cohesive

fracture of a steady-state liquid jet and (b) break-up of a liquid jet due to capillary wave mechanism. . . . . . . . . . . . 27

Fig. 7. Schematic representation of a fluid jet issuing from capillary. . . . . . . . . 29

Fig. 8. Schematic representation of coagulation and boundary movement. . . . . . . . . . . . 32

Fig. 9. Schematic representation of a non-uniform skin-core system formed in the process of wetspinning. Cross-hatched portion denotes rigid solidified layer. • . . . . . . . . . . 39

Fig. 10. Ensemble of molecules exhibiting maximum strength.

Fig. 11. Model representing the structures of melt-spun fibers of flexible polymers such as poly (ethylene terephthalate). . . . . . . . . 42

Fig. 12. The freely jointed chain; (a) the origin of the co-ordinate system is in the hydrodynamic center of the chain (b) the minimum energy of conformation. . . . . . . . . . . . . 445 


\section{Chapter 2}

Fig. 1. Dynamic viscosity vs. frequency for different concentration solution of cellulose esters in dimethylacetamide; (a) $\mathrm{CA}$ (b) $\mathrm{CAB}-1$ (c) $\mathrm{CAB}-2$

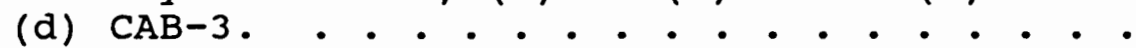

Fig. 2. Low-frequency limit (1 rad/sec) of dynamic viscosity vs. concentration for cellulose esters in dimethylacetamide. . . . . . . . 68

Fig. 3. Experimental and theoretical variation of critical volume fraction of cellulose esters with molecular weight of the repeating unit. $\quad 70$

Fig. 4. Dynamic elastic modulus vs. frequency for different concentration solutions of cellulose esters in dimethylacetamide; (a) $C A$ (b) $C A B-1$

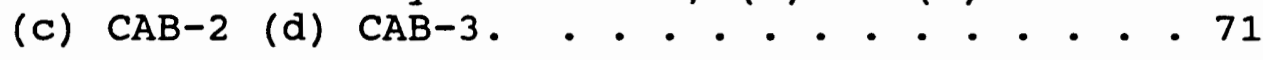

Fig. 5. Dynamic loss modulus vs. frequency for

different concentration solutions of cellulose esters in dimethylacetamide; (a) $C A$ (b) $C A B-1$

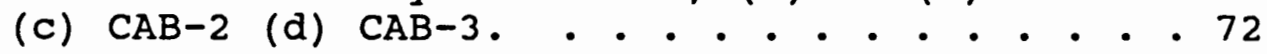

Fig. 6. Polarized optical micrographs of cellulose ester solutions in dimethylacetamide; (a) $\mathrm{CA}$, $50 \%(w / w)$ (b) $C A B-1,45 \%(w / w)$ (c) $C A B-1,50 \%$

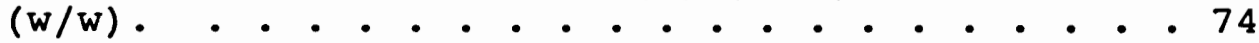

Fig. 7. Polarized optical micrographs of cellulose ester solutions in dimethylacetamide; (a) $C A B-2,40 \%(w / w)$ (b) $C A B-2,45 \%(w / w)$ (c) $C A B-3,30 \%(w / w)$ (d) $C A B-3,40 \%(w / w) . . .75$

Fig. 8. Structure-property map summarizing viscosity, morphology, and chemistry data for cellulose ester solutions in dimethylacetamide. . . . . 76

Chapter 3

Fig. 1 Modulus vs. polymer concentration of cellulose ester fibers spun at different take-up speeds;

(a) $\mathrm{CA}$ (b) $\mathrm{CAB}^{-1}$ (c) $\mathrm{CAB}-2$ (d) $\mathrm{CAB}-3$. . . . . 94

Fig. 2. Tenacity vs. polymer concentration of cellulose ester fibers spun at different take-up speeds; (a) $\mathrm{CA}$ (b) $\mathrm{CAB}-1$ (c) $\mathrm{CAB}-2$ (d) $\mathrm{CAB}-3$. . . . . . . . . . . . . 95 
Fig. 3. Modulus vs. degree of substitution (DS) of butyryl groups in cellulose ester fibers spun at different take-up speeds. . . . . . . .

Fig. 4. Minimum coagulation rate of cellulose ester fibers spun at different take-up speeds. . . 102

Fig. 5. SEM of the cross-section of cellulose ester fibers spun at different take-up speeds; (a) $\mathrm{CA}-35,8.5 \mathrm{~m} / \mathrm{min}$ (b) $\mathrm{CA}-35,16.6 \mathrm{~m} / \mathrm{min}$ (c) $\mathrm{CAB}-1-50,11 \mathrm{~m} / \mathrm{min}$ (d) $\mathrm{CAB}-1-50,21 \mathrm{~m} / \mathrm{min}$.

Fig. 6. SEM of the cross-section of cellulose ester fibers spun at $11 \mathrm{~m} / \mathrm{min}$; (a) $\mathrm{CAB}-1-35$ (b) $C A B-1-50$ (c) $C A B-2-35,7.5 \mathrm{~m} / \mathrm{min}$ (d) $C A B-2-50$ (e) $\mathrm{CAB}-3-25$ (f) $\mathrm{CAB}-3-50$. 106

Fig. 7. SEM of the surface of cellulose ester fibers spun at $11 \mathrm{~m} / \mathrm{min}$; (a) $C A-35$ (b) $C A B-1-35$ (c) $C A B-1-50$ (d) $C A B-2-50$ (e) $C A B-3-25$

Fig. 8. SEM of $\mathrm{CAB}-3$ fibers spun at $11 \mathrm{~m} / \mathrm{min}$; (a) CAB-3-25, circular cross-section (b) $C A B-3-50$, elliptical cross-section.

Fig. 9 .

(a) SEM of CAB-1-45 fiber cross-section schematic representation of 'skin-core' effect.

Fig. 10. WAXS pattern of cellulose ester fibers spun at $11 \mathrm{~m} / \mathrm{min}$;

(a) $C A-35$.

Fig. 10. WAXS pattern of cellulose ester fibers spun at $11 \mathrm{~m} / \mathrm{min}$; (b) $\mathrm{CAB}-1-35$

(c) $\mathrm{CAB}-1-50$. 114

Fig. 10. WAXS pattern of cellulose ester fibers spun at $11 \mathrm{~m} / \mathrm{min}$ (d) $\mathrm{CAB}-2-35,7.5 \mathrm{~m} / \mathrm{min}$ (e) $\mathrm{CAB}-2-50.115$

Fig. 10. WAXS pattern of cellulose ester fibers spun at $11 \mathrm{~m} / \mathrm{min}$ (f) $\mathrm{CAB}-3-25$ (g) $\mathrm{CAB}-3-50$. . . . . 116

Fig. 11. (a) DSC traces of CA (i) Powder (ii) Fibers, CA-35, $11 \mathrm{~m} / \mathrm{min}$.

Fig. 11. (b) DSC traces of $C A B-1$ (i) Powder (ii) Fibers, $C A B-1-35,11 \mathrm{~m} / \mathrm{min}$ (iii) Fibers,

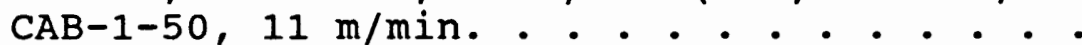
119 
Fig. 11 (c) DSC traces of $C A B-2$ (i) Powder (ii) Fibers CAB-2-35, $7.5 \mathrm{~m} / \mathrm{min}$ (iii) Fibers, CAB-2-50,

$11 \mathrm{~m} / \mathrm{min}$. . . . . . . . . . . . .

Fig. 11. (d) DSC traces of $C A B-3$ (i) Powder (ii) Fibers, $\mathrm{CAB}-3-25,11 \mathrm{~m} / \mathrm{min}$ (iii) $\mathrm{CAB}-3-50,11 \mathrm{~m} / \mathrm{min} .121$

Fig. 12. Heat of fusion vs polymer concentration of cellulose ester fibers spun at different take -up speeds (a) $C A$ (b) $C A B-1$ (c) $C A B-2$ (d) $\mathrm{CAB}-3$. . . . . . . . . . . . . . . .

\section{Chapter 4}

Fig. 1 Dynamic viscosity vs. concentration of cellulose solutions in DMAC/LiCl at different frequencies.

Fig. 2 Dynamic elastic modulus vs. concentration of cellulose solutions in DMAC/LiCl at different frequencies.

Fig. 3 Dynamic loss modulus vs. concentration of cellulose solutions in DMAC/LiCl at different frequencies. . . . . . . . . . . . . . .

Fig. 4 Dynamic viscosity vs. concentration of cellulose hexanoate solutions in DMAc at different frequencies.

Fig. 5 Dynamic elastic modulus vs. concentration of cellulose hexanoate solutions in DMAc at different frequencies.

Fig. 6 Dynamic loss modulus vs. concentration of cellulose hexanoate solutions in DMAC at different frequencies. . . . . . . . . . 153

Fig. 7 Polarized optical micrographs of cellulose solutions in DMAC/LiCl (a) $10 \%(\mathrm{w} / \mathrm{w})$ (b) $12 \%$

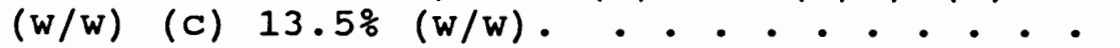
155

Fig. 8 Polarized optical micrographs of cellulose hexanoate solutions in DMAC (a) $25 \%(w / w)$ (b) $30 \%(w / w)$ (c) $35 \%(w / w)$ (d) $40 \%(w / w)$ 156

Fig. 9 Modulus vs polymer concentration of cellulose fibers spun at different take-up speeds for $\mathrm{V}_{\mathrm{o}}=21 \mathrm{~m} / \mathrm{min}$. 
Fig. 10 Modulus vs polymer concentration of cellulose hexanoate fibers spun at different take-up speeds for $V_{0}=42 \mathrm{~m} / \mathrm{min}$. . . . . . . . . 164

Fig. 11 SEM of the surface of cellulose fibers (a)

$6.5 \%(\mathrm{w} / \mathrm{w}), \mathrm{V}_{\mathrm{o}}=14 \mathrm{~m} / \mathrm{min}, \mathrm{V}_{\mathrm{L}}=21 \mathrm{~m} / \mathrm{min}$ (b)

$13.5 \%(\mathrm{w} / \mathrm{w}), \mathrm{V}_{\mathrm{o}}=21 \mathrm{~m} / \mathrm{min}, \mathrm{V}_{\mathrm{L}}=15 \mathrm{~m} / \mathrm{min}$.

Fig. 12 SEM of the surface of cellulose hexanoate fiber spun from $30 \%(\mathrm{w} / \mathrm{w})$ solution at $\mathrm{V}_{\mathrm{o}}=21 \mathrm{~m} / \mathrm{min}$ and $\mathrm{V}_{\mathrm{L}}=21 \mathrm{~m} / \mathrm{min}$.

Chapter 5

Fig. 1 (a) Diagram of the fiber creep test fixture.

(b) Relative humidity profile in the fiber

creep test. . . . . . . . . . . . . 186

Fig. $2{ }^{13} \mathrm{C}$ NMR spectra of CAB in DMAC (a) without

LiCl (b) with LiCl. . . . . . . . . . 189

Fig. 3 Dynamic viscosity vs frequency of $35 \%$ (w/w) and $50 \%$ (w/W) $C A B$ solutions without and with varying amount of LiCl. . . . . . . . . .

Fig. 4 Dynamic elastic modulus vs frequency of $35 \%$ $(w / w)$ and $50 \%(w / w)$ CAB solutions without and with varying amount of LiCl. . . . . . .

Fig. 5 Dynamic loss modulus vs frequency of $35 \%(\mathrm{w} / \mathrm{w})$ and $50 \%(\mathrm{w} / \mathrm{w}) \mathrm{CAB}$ solutions without and with varying amount of LiCl. . . . . . . .

Fig. 6 Polarized optical micrographs of $50 \%$ (w/w) CAB solutions (a) $0 \% \mathrm{LiCl}$ (b) $0.25 \% \mathrm{LiCl}$ (c) $1 \% \mathrm{LiCl}$. 196

Fig. 7 SEM of the surface of CAB fibers spun at 11 $\mathrm{m} / \mathrm{min}$ (a) $0 \mathrm{LiCl} \mu \mathrm{g} / \mathrm{g} \mathrm{(b)} 900 \mathrm{LiCl} \mu \mathrm{g} / \mathrm{g}$. . 199

Fig. 8 Weight change as a function of time of fibers exposed to air at $100 \%$ relative humidity. . . 200

Fig. 9 Creep of CAB-11 fiber. . . . . . . . . . 203

Fig. 10 Creep of $\mathrm{CAB}-21$ fiber. . . . . . . . . . 204

Fig. 11 Creep of CAB-11-S fiber. . . . . . . . . 205

Fig. 12 Creep of CAB-21-S fiber. . . . . . . . . 206 
Fig. 1 (a) Dynamic viscosity (b) dynamic elastic modulus and (c) dynamic loss modulus vs. concentration of $\mathrm{CAB} / \mathrm{L}$ solutions with varying amount of lignin content at different

Fig. 2 (a) Dynamic viscosity (b) dynamic elastic modulus and (c) dynamic loss modulus vs. frequency of $12 \%$ (w/w) cellulose solution and $12 \%(\mathrm{w} / \mathrm{W})$ cellulose solution containing $0.5 \%(\mathrm{w} / \mathrm{w})$ lignin. . . . . . . . . . . 228

Fig. 3 Polarized optical micrographs of $\mathrm{CAB} / \mathrm{L}$ solutions with varying amount of lignin; (a) $0 \%(w / w)$ (b) $4 \%(w / w)$ (c) $15 \%(w / w)$ (d) $20 \%$ $(w / w)$.

Fig. 4 Polarized optical micrographs of (a) $12 \%$ (w/w) cellulose solution and (b) $12 \%(\mathrm{w} / \mathrm{w})$ cellulose solution containing $0.5 \%(\mathrm{w} / \mathrm{w})$ lignin. . . 231

Fig. 5 (a) Modulus and (b) tenacity vs lignin content (wt. \%) for $\mathrm{CAB} / \mathrm{L}$ fibers at different take-up speeds. . . . . . . . . . . . . . . 235

Fig. 6 SEM of the surface of $C A B / L$ fibers with varying amount of lignin content (a) $15 \%(\mathrm{w} / \mathrm{w})$

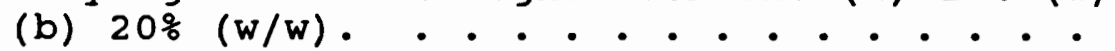

Fig. 7 TEM of the stained cross-section of (a) skin and (b) core of $\mathrm{CAB} / \mathrm{L}$ fibers containing $10 \%$ $(w / w)$ lignin.

Fig. 8 TEM of the stained cross-section of $\mathrm{C} / \mathrm{L}$ fibers containing $4 \%(\mathrm{w} / \mathrm{w})$ lignin. . . . . . . . 242

\section{Chapter 7}

Fig. 1 Polarized optical micrograph of CAB film containing $20 \%(\mathrm{w} / \mathrm{w})$ lignin. . . . . . . 255

Fig. 2 (a) TEM of an unstained cross-section of the $\mathrm{CAB}$ film containing $20 \%(\mathrm{w} / \mathrm{w})$ lignin. . . . 256

Fig. 2 (b) TEM of an unstained longitudinal-section of the $C A B$ film containing $20 \%$ (w/w) lignin.

Fig. 3 TEM of a stained cross-section of the CAB film containing $20 \%(\mathrm{w} / \mathrm{w})$ lignin. . . . . . . 261 
Fig. 1 Chemical structure of lignin-like model compounds (a) Biphenyl [5-5] (b) Biphenyl [5-5] (c) $\beta$-ether model (d) Phenyl coumaran $(\beta-5)$.

Fig. 2 Polarized optical micrographs of biphenyl [5-5] crystallized isothermally at $95^{\circ} \mathrm{C}$ and heated on hot-stage as a function of temperature (a) $95^{\circ} \mathrm{C}$ (b) $105^{\circ} \mathrm{C}$ (c) $113^{\circ} \mathrm{C}$ (d) $120^{\circ} \mathrm{C}$

Fig. 3 Heating and cooling DSC scans of biphenyl [5-5] (a) as-received sample (b) sample in (a) cooled from isotropic melt (c) sample in

(b) heated at $5^{\circ} \mathrm{C} / \mathrm{min} . . . . . . . .281$

Fig. 4 Polarized optical micrographs of biphenyl [5-5] crystallized isothermally at $98^{\circ} \mathrm{C}$ and heated on hot-stage as a function of temperature (a) $98^{\circ} \mathrm{C}$ (b) $118^{\circ} \mathrm{C}$ (c) $120^{\circ} \mathrm{C}$. . 284

Fig. 5 Heating and cooling DSC scans of biphenyl [5-5] (a) cooled at $2.5^{\circ} \mathrm{C} / \mathrm{min}$ from isotropic melt (b) sample in (a) heated at $5^{\circ} \mathrm{C} / \mathrm{min}$ (c) sample quenched from $135^{\circ} \mathrm{C}$ to room temperature and heated at $5^{\circ} \mathrm{C} / \mathrm{min}$.

Fig. 6 Polarized optical micrographs of $\beta$-ether model heated on hot-stage as a function of temperature (a) $102.5^{\circ} \mathrm{C}$ (b) $103.5^{\circ} \mathrm{C}$

Fig. 7 DSC scan of as-received $\beta$-ether model compound. 288

Fig. 8 DSC scans obtained on phenyl coumaran (a) asreceived sample (b) sample quenched from $140^{\circ} \mathrm{C}$ to room temperature and heated at $5^{\circ} \mathrm{C} / \mathrm{min}$ 290

Fig. 9 Polarized optical micrographs of phenyl coumaran crystallized isothermally as a function of temperature (a) $105^{\circ} \mathrm{C} / \mathrm{min}$ (b) $110^{\circ} \mathrm{C}$ (C) $130^{\circ} \mathrm{C}$ 291

Fig. 10 Polarized optical micrograph of lignin at $200^{\circ} \mathrm{C}$ when heated on hot-stage. . . . . . . 296

Fig. 11 Chemical structure of organosolv lignin. . . 297 
Fig. 12 Polarized optical micrographs of A-240 pitch when heated isothermally at $425^{\circ} \mathrm{C}$ on hot-stage as function of time (a) 1 hour (b) 2 hours. .

Fig. 13 Polarized optical micrographs of quenched A-240 pitch at room temperature after isothermal heat-treatment at $425^{\circ} \mathrm{C}$ with varying residence time (a) 1 hour (b) 2 hours. 300

Fig. 14 Polarized optical micrograph of the blend of A-240 pitch and lignin containing $25 \%$ (W/W) lignin after isothermal heating at $425^{\circ} \mathrm{C} / \mathrm{min}$ for 2 hours on hot-stage. . . . . . . .

Fig. 15 Schematic representation of hydroxypropyl (organosolv) lignin derivative $(n=1)$. . . 304

Fig. 16 Molecular weight distribution (a) HPL (b) hydrocracked HPL (c) heat-treated HPL. . . 306

Fig. $17{ }^{13} \mathrm{C}$ NMR spectra (a) HPL (b) hydrocracked HPL (c) heat-treated HPL. . . . . . . . 307

Fig. 18 SEM of the surface of fibers spun from heat-treated HPL (a) green fibers (b) carbon fibers. . . . . . . . . . . . 310

Fig. 19 TEM of the cross-section of carbon fibers from heat-treated HPL. . . . . . . . . . 311

\section{Chapter 9}

Fig. 1 (a) Proton NMR spectra of Tri-o- $\alpha$-naphthylmethyl Cellulose. . . . . 326

Fig. 1 (b) Two dimensional COSY (DQCOSY) spectra of Tri-0- $\alpha$-naphthylmethyl Cellulose. . . . . 327

Fig. $1 \quad$ (c) Chemical structure of Tri-0- $\alpha$-naphthylmethyl Cellulose. . . . . 328

Fig. $2 \quad{ }^{13} \mathrm{C}$ NMR spectra of Tri-0- $\alpha$-naphthylmethyl Cellulose. . . . . . . . . . . 330

Fig. 3 (a) Dynamic viscosity (b) dynamic elastic modulus and (c) dynamic loss modulus vs. concentration of tri-o- $\alpha$-naphthylmethyl cellulose solutions at different frequencies. 
Fig. 4 Polarized optical micrographs of tri-o- $\alpha-$ naphthylmethyl cellulose solutions (a) $40 \%$

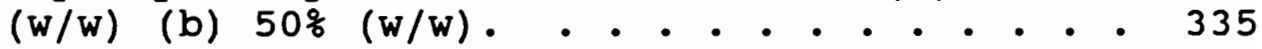

Fig. 5 Experimental and theoretical variation of critical volume fraction of cellulose derivatives with molecular weight of the repeating unit. . . . . . . . . . . 339

Chapter 10

Fig. 1 DSC trace of CA powder up to $125^{\circ} \mathrm{C} / \mathrm{min} . . .349$

Fig. 2 DSC trace of CA powder after being quenched

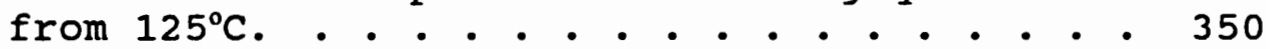

Fig. 3 FT-IR of CA film (a) undried (b) dried. . . 351

Fig. 4 DSC trace of CA powder after being quenched from $270^{\circ} \mathrm{C} / \mathrm{min}$ to $-60^{\circ} \mathrm{C} / \mathrm{min}$. . . . . . 353

Fig. 5 TGA trace of CA powder (a) undried (b) dried. 354

Fig. 6 Dynamic mechanical properties of CA (DS 2.4)

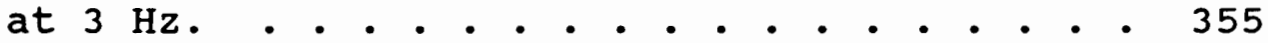

Appendix

Fig. 1 Design of spinning pack (a) top assembly b) bottom assembly............ . 362 Fig. 2 Design of single-hole spinnerette. . . . . 364 Fig. 3 Schematic representation of the take-up device.365 Fig. 4 Photograph of the fiber spinning apparatus. . 366 


\section{List of Tables}

\section{Chapter 2}

Table I.

Chemical and Molecular Characteristics

of Cellulose Esters. . . . . . . . . 64

Table II. Theoretical Values of Molecular

Parameters for Cellulose Esters. • . . . 73

Table III. Summary of Polarized Light Optical

Microscopy observations for Solutions

Containing 5-50\% (w/w) Cellulose Esters

In Dimethylacetamide. . . . . . . . 77

\section{Chapter 3}

Table I.

Spinning Conditions and Mechanical

Properties of Fibers.

Table II.

Glass Transition Temperature and Melting Parameters of the fibers determined by

\section{Chapter 4}

Table I.

Chemical and Molecular Characteristics of Carbanilated Derivatives of Cellulose and

Cellulose Hexanoates. . . . . . . . 143

Table II.

Theoretical values of Molecular

Parameters for Cellulose (C) and

Cellulose Hexanoate $(\mathrm{CH})$.

Table III. Spinning Conditions and Mechanical

Properties of Cellulose Fibers. . . . 159

Table IV. Spinning Conditions and Mechanical

Properties of Cellulose Hexanoate Fibers. 160

\section{Chapter 5}

Table I.

Spinning conditions and Mechanical

Properties of $\mathrm{CAB}$ Fibers containing

Lithium Chloride. . . . . . . . . 197

Table II.

Test Conditions for $C A B$ Fibers During

Creep Experiment. . . . . . . . . 202 
Table III.

Mechano-Sorptive Creep Results of CAB

Fibers. 208

Chapter 6

Table I.

Chemical and Molecular Characteristics of Cellulose Tricarbanilate, Cellulose Acetate Butyrate and Lignin. . . . . 224

Table II.

Spinning Conditions and Mechanical Properties of Fibers from blends of Cellulose Acetate Butyrate and Lignin. .

Table III. Spinning Conditions and Mechanical

Properties of Fibers from blends of

Cellulose and Lignin. . . . . . . .

Table IV.

Glass Transition temperature and melting parameter of fibers from blends of Cellulose Acetate Butyrate and Lignin

determined by DSC. . . . . . . . . 237

Chapter 8

Table I.

Chemical and Molecular Characteristics of Lignin, HPL, Hydrocracked HPL and Heattreated HPL. . . . . . . . . . .

Table II.

Typical Properties of Commercial Ashland

Table III. Summary of Liquid Crystalline Behavior of Lignin-like Model Compounds. . . . . 293

Table IV. Physical Properties of Carbon Fibers (CF) from Different Precursors. . . . . 309

\section{Chapter 9}

Table I.

Molecular, Chemical and Thermal

Characteristics of Tri-0- $\alpha-$ Naphthylmethyl Cellulose.

Table II.

Peak assignments of proton and carbon signals of tri-o- $\alpha$-naphthylmethyl cellulose.

Table III.

Theoretical Values of Molecular parameters for Cellulose Derivatives. 
1.0 INTRODUCTION. 


\section{$1.0 \quad$ INTRODUCTION}

Liquid crystalline polymers (LCP) have acquired importance in industry and academic research since 1970 (1). The interest in these materials was mainly due to the commercial development of exceptionally high modulus and high strength fibers. These materials have other useful properties which include excellent solvent resistance, low thermal expansion (2), low melt and solution viscosity, high use temperatures, and low permeability to many gases (3). Also, there is an increasing interest in the application of LCPs in blends with engineering thermoplastics and other resins as reinforcing agents (4).

This chapter deals with important concepts in the areas of liquid crystals (Sections 1.1-1.3), fiber spinning (Sections 1.4-1.5) and fiber spinning from liquid crystalline solutions (Section 1.6) which is followed by research objectives (section 1.7 ).

\subsection{Description of Mesophases}

Mesophase crystals (Greek, mesos = middle, phasis = appearance) may be in-between liquid and solid in macroscopic appearance. It is possible to divide all molecules into three major classes, small molecules with high aspect ratio, flexible macromolecules and rigid macromolecules. Mesophase materials are possible for all three classes of molecules. The three conventional states that form the limits within which 
the mesophases are formed are fully ordered crystal (long range, three dimensional order), amorphous solid (short and long range order) and isotropic liquid (no order).

The mesophase order is divided into three major types, namely: liquid crystals, plastic crystals and condis crystals (5). Definitions of the types of mesophase discussed here have been adapted from reference 5. The "positionally disordered" crystals or "orientationally ordered" liquids are widely known as liquid crystals. The molecules of liquid crystals always have a rigid, mesogenic group which is rod- or disc-like and causes a high activation energy to rotational reorientation.

The group of materials with a mesophase which shows "orientational disorder" but "positional order" and can be easily deformed are known as plastic crystals. The molecules of these crystals are compact and globular, and their reorientation is not opposed by a high activation barrier.

The third group of mesophase materials represents "condis crystals", which is a contraction of the term "conformationally disordered crystal". These crystals consist of flexible molecules which can undergo relatively easily hindered rotation to change conformation without losing positional or orientational order.

All three mesophases have some degree of long range order in common with the crystal and some degree of non-vibrational motion in common with the liquid. As a result, all mesophases must show just as liquids, a glass transition if 
crystallization to full order can be avoided on cooling. This is a key identifier for the mesomorphic state and is often overlooked (5). At temperatures below the respective glass transitions, there can be three further mesophases. These are, positionally disordered glasses (LC-glasses), orientationally disordered glasses (PC-glasses) and conformationally disordered glasses (CD-glasses). The terms LC-, PC- and CDglass stands for glass obtained by quenching a liquid crystal, plastic crystal and condis crystal, respectively. Only the liquid-crystal mesophases will be discussed in this chapter.

\subsection{Nature, Structure and Types of Liquid Crystals}

The term "liquid crystals" symbolizes the state of matter intermediate between the long-range and high degree of positional and orientational order of solid crystals and the long-range disorder of ordinary liquids. The phenomenon was first observed in 1888 by an Austrian botanist F. Reinitzer. On heating cholesteryl benzoate, he noticed the solid crystals melted to a turbid fluid which became a clear liquid at higher temperatures (6). In $1889,0 . \mathrm{z}$. Lehmann coined the term "Flussige Kristalle" (liquid crystal) to describe the physical state where a substance flows like a liquid but is optically anisotropic (7). Later the terms "crystalline liquids", "mesophases", and "mesomorphic phases" were embraced and are used interchangeably today.

In a crystalline solid, the molecules are arranged in a 
rigid lattice structure with three dimensional order. In contrast, the orientational and positional order in liquid crystals is shorter in range and the order is only one or two dimensional. A comparision of liquid crystals and isotropic liquids can be made too. The physical properties of isotropic liquids are same in all directions. Due to the presence of molecular ordering in liquid crystals, properties like viscosity are direction dependent on a microscopic scale (8). Liquid crystals are birefringent under both flow and quiescent conditions, whereas isotropic liquids exhibit birefringence only under flow conditions.

Liquid crystal forming materials may have five pure phases namely, fully ordered crystal, LC-glass, amorphous glass, liquid crystal and isotropic melt. Also, there may be four two phase materials: fully ordered crystal and LC-glass, fully ordered crystal and amorphous glass, fully ordered crystal and isotropic melt and fully ordered crystal and liquid crystal. The correlations between these phases are shown in Figure 1 (5).

There are two classes of liquid crystals, thermotropic liquid crystals and lyotropic liquid crystals. Thermotropic liquid crystals do not contain solvent and the transitions are governed by temperature, pressure and deformation. As the bulk polymer is heated, first order transition is observed from a solid to a liquid crystal (9). At higher temperatures, different transitions may occur and finally the material will 


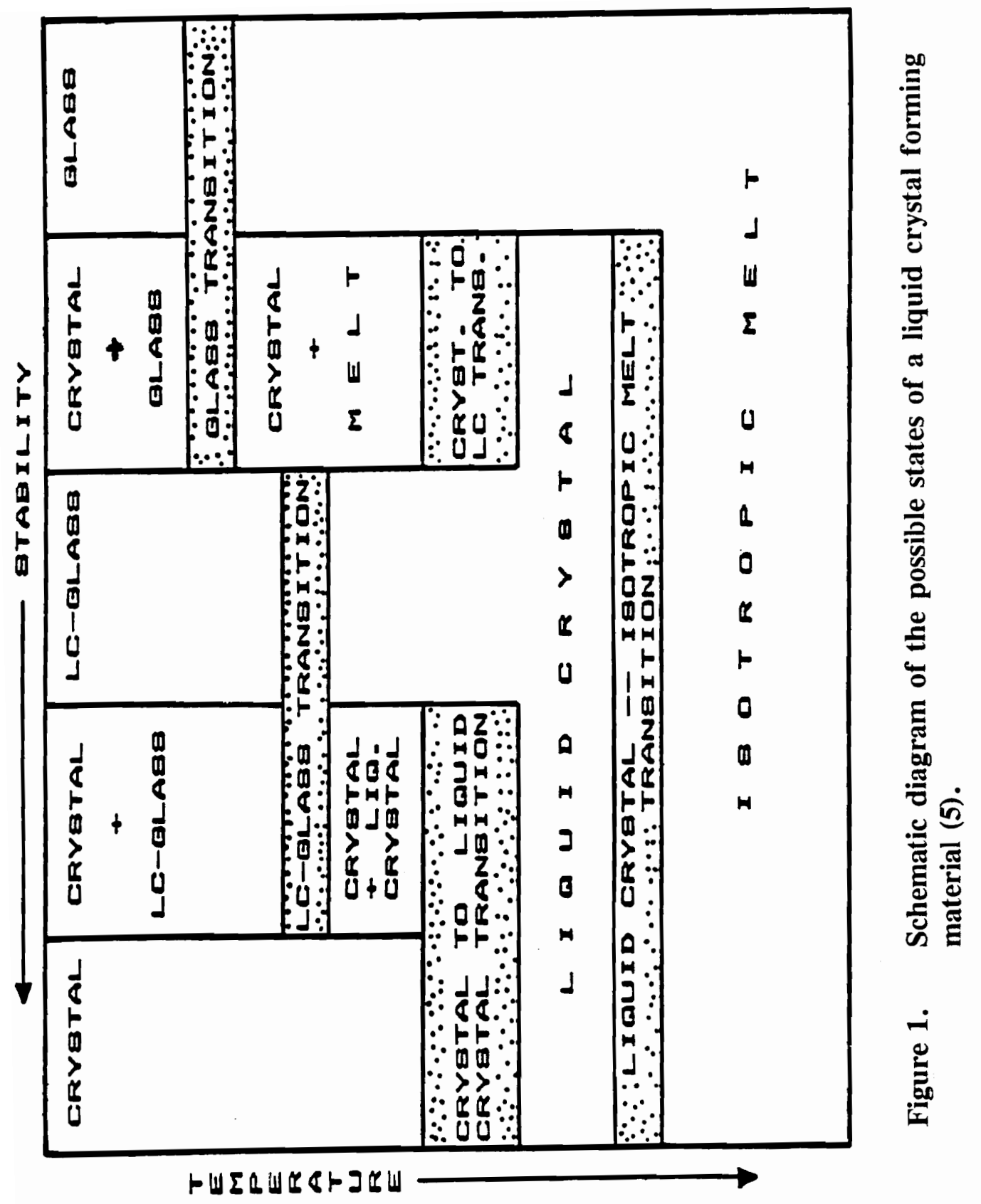


become isotropic or degrade depending on its chemical structure. In lyotropic liquid crystals a solvent or dispersing agent is present and the transitions are both concentration and temperature dependent. At low concentrations, the polymer solution is isotropic. As the concentration is raised the viscosity increases and reaches a maximum at a critical concentration, $V_{p}^{c}$, at which point the solution becomes birefringent and marks the commencement of liquid crystallinity. In the case of polymers, a two phase region (isotropic and liquid crystal phases) is routinely observed caused by polydispersity, impurities, or chain imperfections. As polymer concentration increases, the amount of material in the anisotropic phase increases, until the complete system becomes birefringent. At higher concentrations, the bulk polymer will precipitate out.

Molecular-level mesophase order can be achieved by a large number of different chemical structural units which are very stiff and have high aspect ratios (10). These two geometric conditions provide an ample justification for the formation of a thermodynamically stable anisotropic fluid (11). Such units are known as "mesogens". A summary of common molecular moieties or structures forming the liquid crystalline polymers can be found in reviews $(2,12-14)$. Based on molecular configuration and conformation, liquid crystalline polymers are usually divided into four groups 
(15). These groups are rigid rod-like molecules, molecules with rigid helical conformation, and block-like molecules with both mesogenic units and flexible spacer units in the main chain or with mesogenic units in the side groups but with a flexible chain backbone. Low molecular weight liquid crystals are usually composed of mesogenic groups, whereas the mesogenic units may be a part of the molecule in polymeric liquid crystals.

In addition to the distinction between being lyotropic and thermotropic, each of the four groups tends to form a particular mesophase structure, of which three have been well defined: nematic, cholesteric, and smectic. Figure 2 (15) illustrates schematically the arrangements of the mesogens in the three types of mesophases. The definitions of these phases are adapted from references 5, 9, 10, 15 and 16. The nematic phases (Greek, nema = thread) shows no long range order and correlation exists only in the direction of the mesogenic groups which results in one dimensional order. Their centers of gravity are randomly arranged. The molecules are greatly affected by external fields and forces due to the high mobility of the molecules in the nematic mesophase (10). Consequently, nematics are characterized by low viscosities.

A thermodynamically equivalent phase to the nematic phase is the cholesteric phase. It results if a mesogen is chiral or if a chiral molecule is dissolved in a nematic liquid crystal (5). The nematic structure of the cholesteric phase contains 

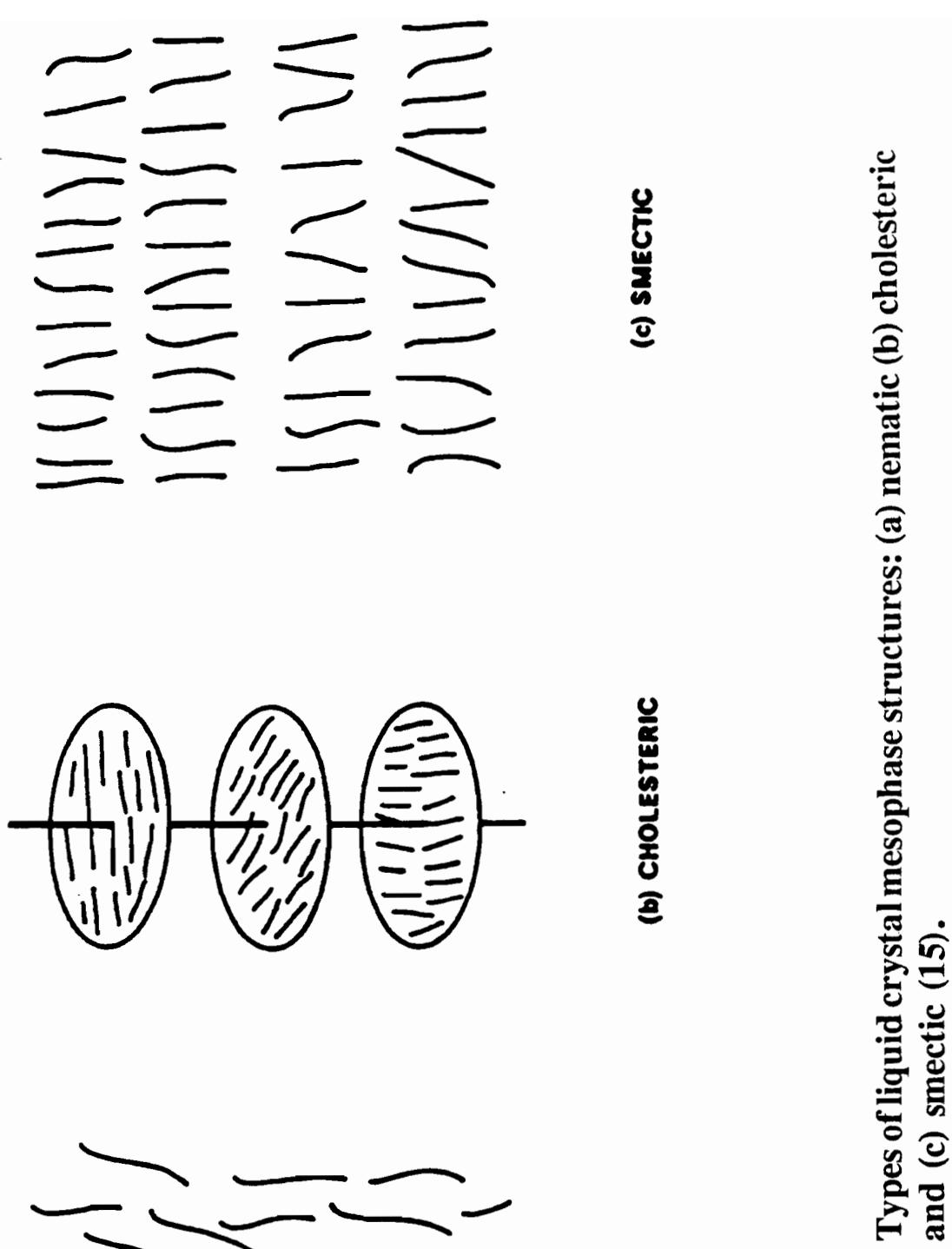

$\frac{0}{0}$
$\frac{0}{4}$
$\frac{0}{4}$
$\frac{0}{0}$
a

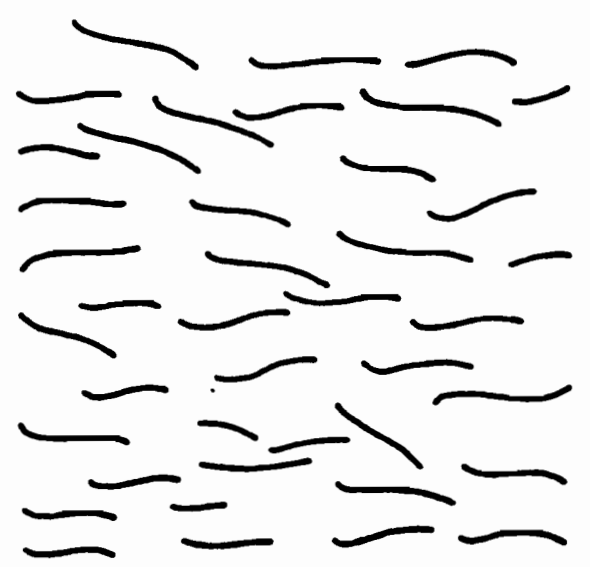

$\frac{0}{\frac{5}{5}}$

电 
a helical distortion of the orientation direction of longer periodicity than the molecular dimension (5). The chiral arrangement reduces the free energy and the molecules in each layer are twisted with respect to those above and below. Two such superhelicoidal structures are possible, one right-handed and one left-handed, but one is of lower energy than the other (16). The more highly ordered structure of the cholesteric mesophase results in a much higher viscosity relative to nematic.

The smectic phases show layered structures of the mesogens and exhibits the highest degree of order amongst the three mesophase types. The molecules are oriented parallel to one direction, while the centers of gravity of the molecules are arranged in distinct layers $(9,10)$. This two-dimensional order in a smectic mesophase restricts molecular motion and results in a viscosity much greater than that of nematic. As the degree of order increases in the smectic phases, it becomes difficult to distinguish them from fully ordered crystals.

Rod-like and helical molecules will tend to form nematic or cholesteric mesophases, either in solution or in bulk. The type of mesophase formed will depend on the geometrical symmetry of the rod or helical coil (10). Rigid molecules with uniaxial symmetry will be predisposed to form nematic mesophases, while non racemic mixtures of rod-like molecules or helical coils tend to form cholesteric mesophases (15). 
Poly ( $\gamma$-benzyl-L-glutamate) (PBLG) solutions form cholesteric mesophases whereas racemic mixture of PBLG and poly $(\gamma$-benzylD-glutamate) (PBDG) form nematic mesophases (17). Aromatic polyesters tend to form thermotropic liquid crystals whereas aromatic polyamides usually forms lyotropic liquid crystals as degradation occurs before melting. Many biological molecules like tobacco mosaic virus (18), ribonuclei acid (RNA), and deoxiribonucleic acid (DNA) (19) form rigid helical coils in bulk or in solvent.

Block-like molecules consist of alternating mesogenic units and flexible spacers or a flexible chain backbone with mesogenic units as side groups. The molecules with mesogenic units as side groups tend to form smectic type mesophase while those with mesogenic units along the backbone usually form all three types of mesophase structures depending on the type and size of the blocks (15).

\subsection{Molecular Theory of Liquid Crystals}

During the late $1930^{\prime}$ s, high molecular weight mesophases were first studied using the suspensions of tobacco mosaic virus (TMV). As the concentration of the TMV solution was increased, two phases were formed, one of which was birefringent (20). Phase separation occurred in suspensions containing only $1.8 \%$ needle-like TMV particles (21). In 1949, Onsager (22) presented a theoretical explanation for these observations for the first time. The theory is based on the 
analysis of the second virial coefficient for very long rodlike particles, and is especially applied to lyotropic systems. Elliot and Ambrose reported that a solution of PBLG in chloroform formed a birefringent phase as the concentration was increased (23). Encouraged by these observations, Isihara (24) and Flory (11) developed theoretical explanations for phase separation in polymer solutions. Flory's Lattice Model theory of liquids consisting of rigid and semi-rigid molecules has proved to be most generally useful in the treatment of liquid crystallinity in polymeric systems. Details of this theory will be discussed here and the versatility of this theory will be extended to different systems. The derivations of equations and fundamental concepts are adapted from reference 25 .

1.3.1 Binary Lyotropic systems Consisting of Hard Rod-like

\section{Particles and a Diluent}

The lattice theory deals with rod-like particles which do not have interactions with their neighbors other than the occurrence of short-range repulsions when the particles overlap.

a) Formulation of the Partition Function:

Conventional lattice methods can be employed for the treatment of a system of rigid, rod-like particles, or molecules, by the model shown in Figure 3 (25). The particle shown in Fig. $3 a$ is oriented at an angle $\psi$ with respect to the preferred axis of the surrounding domain. One of the principal 

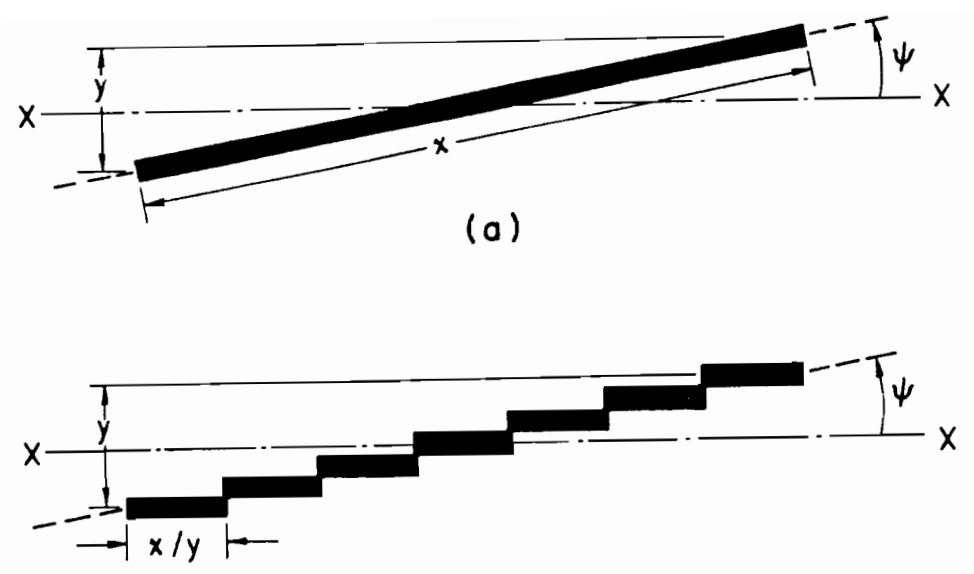

(b)

Figure 3. (a) Rodlike particle oriented at an angle $\psi$ from the preferred axis $\mathrm{X}-\mathrm{X}$ and (b) its subdivision into y sequences parallel to this axis as required for accommodation on the cubic lattice (25). 
axes of the cubic lattice is aligned with this axis. The particle comprises $\mathrm{x}$ isodiametric segments, each of a size that will occupy one cell of the lattice. It follows that $x$ so defined is also the axial (or aspect) ratio, $L / d$, of the particle. In order to accommodate the particle on the lattice when it is oriented as in Fig. $3 a$, it is to be subdivided into $y$ sequence of segments as represented in Fig. $3 b$, with each sequence parallel to the preferred axis. Parameter y serves as a measure of disorientation of the particle with respect to the domain axis (Fig. 3b). The analysis depends upon evaluation of the number $\nu_{j}$ of situations (i.e., appropriate sequence of empty lattice sites) accessible to molecule $j$ with orientation $y_{j}$ when $j-1$ molecules have been added previously to the space, or lattice, in which the mixture is confined. Reference 25 describes the complete formulation of the Partition Function.

A useful semi-empirical approximation for the critical volume fraction $v_{p}^{c}$ for incipience of "metastable order" that follows from the analysis is

$$
\begin{aligned}
\mathrm{V}_{\mathrm{p}}{ }^{\mathrm{s}} & \approx(8 / \mathrm{x})(1-2 / \mathrm{x}) \\
& \approx 8 / \mathrm{x} \text { for higher values of } \mathrm{x} .
\end{aligned}
$$

Equation 1 holds within $2 \%$ for $x>10$. This relation is widely used to represent the threshold volume fraction for appearance of a stable anisotropic phase, an assumption that appears to be well-justified. 
b) Biphasic Equilibrium:

The analysis of anisotropy in a system of rod-like particles ignores the possibility of separation of the system into two phases. Examination of the partition function shows that the critical point occurs in a range of composition that is metastable with respect to a system of two phases, one of them completely disordered and the other partially ordered but with retention of an appreciable degree of disorder.

The biphasic coexistence is treated by imposing the condition that the chemical potentials $\mu_{\mathrm{s}}$ (solvent) and $\mu_{\mathrm{p}}$ (polymer) \{obtained as partial derivatives of the free energy\} of the respective components in the two phases must be at equilibrium, i.e.,

$$
\left.\begin{array}{l}
\mu_{\mathrm{s}}=\mu_{\mathrm{s}}{ }^{\prime} \\
\mu_{\mathrm{p}}=\mu_{\mathrm{p}}{ }^{\prime}
\end{array}\right\}
$$

The prime denotes the anisotropic, or ordered phase. The calculated minimum axial ratio for stable nematic order in a neat liquid consisting of hard rods is 6.42 (26).

\subsubsection{Mixtures of Rods and Random Coils}

Application of the lattice scheme to a system consisting of rod-like molecules, random coils and a solvent is straightforward (27). The free energy of the nematic phase would be increased noticeably due to the presence of a significant proportion of the random coil. The latter component is predicted to be virtually excluded from this 
phase. This striking deduction from theory is solely from the "steric" requirements of the two kinds of macromolecules and no other interactions are required between them.

According to the theory, except within a limited range of composition, the two polymeric components may be considered as mutually incompatible. Some investigators have confirmed these predictions $(28,29)$. By rejecting the random coil, the nematic phase reveals a principal characteristic of crystals in general, and thus confirming the term "liquid crystal". The abundance of random coiled component in the coexisting isotropic phase increases the chemical potential of the rodlike component in that phase and concomitantly decreases the chemical potential of the diluent. Consequently, the concentration of the rod-like component in the anisotropic phase is caused to increase.

\subsubsection{Semirigid Chains}

Complete rod-like rigidity is not required to display liquid crystalline behavior. Semirigid or semiflexible chains may impart liquid crystallinity to their melts or solutions $(30,31)$. However, as flexibility increases, higher solution concentrations are required to achieve separation of the nematic phase from solution for the same molecular weight. It is important to address the issue of the effects of deviations from full rigidity on the isotropic-nematic transition.

At one extreme, the semi-rigid chains can be represented by wormlike model in which each unit deviates slightly from 
the direction of its predecessor. At the other extreme, there are chains in which the direction of the chain axis undergoes abrupt "bends" at occasional points along the chain. The units at which these sudden changes occur may differ chemically or structurally from the principal units of the chain (e.g., copolymer), or they may differ only in their conformation. Polymer chains that consist of alternating sequences of rigid and flexible units may exhibit liquid-crystallinity if the rigid sequences are sufficiently long $(32,33)$. It is difficult to expect that a single theory will comprehend all polymers of this kind. A simple model that gives insight into the tendency of a semirigid chain to induce formation of a nematic phase is the Kuhn chain which consists of bonds of appropriate length connected by flexible joints. This suggests that the theory of rigid rods may be adapted to semirigid chains by replacing the axial ratio $x$ by the axial ratio $x_{K}$ of the kuhn segment. $x_{K}$ can be calculated from the mean-square end-to-end length $\left\langle r^{2}\right\rangle_{0}$ of the unperturbed, random chain and its length $L$ at full extension. Therefore, these parameters can be calculated from the following relationships;

$$
\left.\begin{array}{l}
\mathrm{n}_{\mathrm{K}} \mathrm{l}_{\mathrm{K}}^{2}=\left\langle\mathrm{r}^{2}\right\rangle_{\mathrm{o}} \\
\mathrm{L}=\mathrm{n}_{\mathrm{K}} \mathrm{l}_{\mathrm{K}}
\end{array}\right\}
$$

$n_{K}$ is the number of segments in the model chain, and $l_{K}$ is the length of one of the segment. Also, $L=n l_{u}$, where $n$ and $l_{u}$ are the number of repeating units and the projected length of one 
of them on the axis of the extended chain, respectively. It follows that

$$
l_{\mathrm{K}}=\left\langle\mathrm{r}^{2}\right\rangle_{\mathrm{o}} / \mathrm{n} l_{\mathrm{u}}
$$

Cellulose and cellulose derivatives are characterized as semirigid polymers where flexibility arises from pseudorotation of the sugar ring, causing the $0-c$ and $c-0$ bonds to adopt transverse directions $(34,35)$. The application of Kuhn chain to cellulose derivatives is discussed in detail in chapter 2 .

\subsubsection{Intermolecular Interactions}

The lattice theory pertains to "hard" rods lacking interactions. The theoretical deductions originate from geometrical aspects of the molecules (i.e., aspect ratio) for rigid rods and kuhn segments for semirigid chains. The effects of isotropic and orientation-dependent intermolecular interactions between the molecules must be also assessed.

a) Isotropic Interactions:

Interactions of this type are included in the theory by the addition of the van Laar form to the free energy of mixing, with corresponding terms in the chemical potentials. For a binary mixture, the terms to be included for the solvent

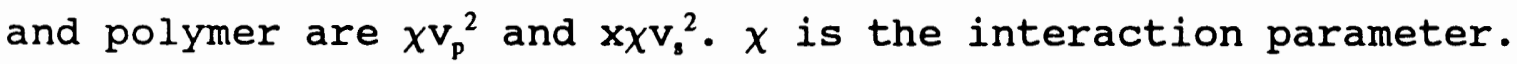
When $\chi<0$, the interaction between the solute molecules is repulsive. When $\chi>0$, the interaction between the solute segments is attractive, and the biphasic region widens. Fig. 


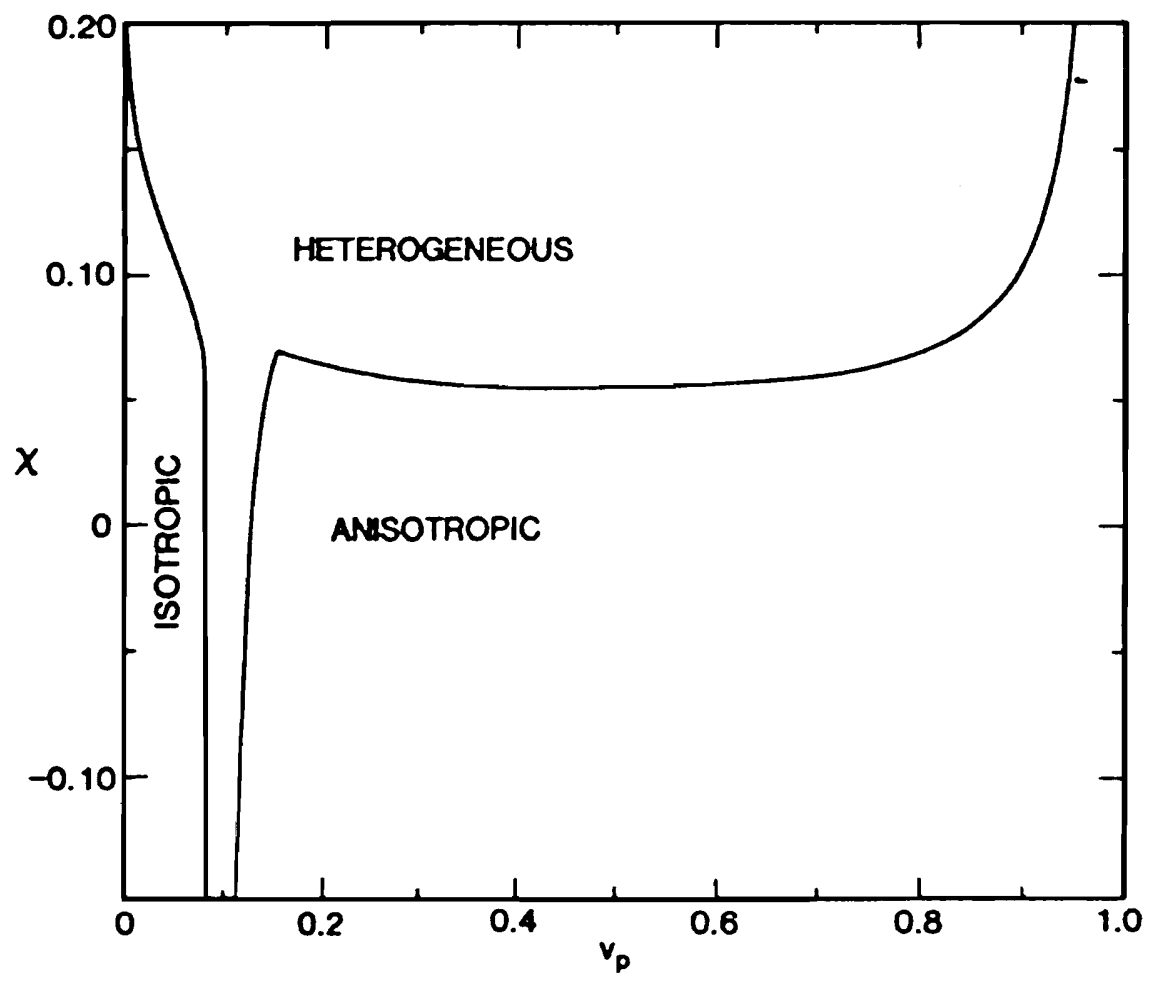

Figure 4. Compositions in volume fraction of coexisting phases for rods of axial ratio $x=100$ subject to interaction denoted by parameter $\chi(25)$. 
4 (25) shows a plot of $\chi$ as ordinate against the volume $v_{p}$ and $\mathrm{v}_{\mathrm{p}}{ }^{\prime}$ in the coexisting phases. The ordinate can be considered as an (inverse) measure of temperature. The narrow biphasic gap is slightly affected by the interactions when $\chi$ is negative. Above the critical point $(X=0.055)$, a shallow concave curve define the loci of coexisting anisotropic phases in addition to the isotropic and nematic phases of lower concentration within the narrow gap on the left. At $\chi=0.07$, the compositions of two of the phases, one from each of the respective pairs, reach the same value. Three phases coexist at this triple point. This phase diagram is well confirmed by experiments (36-38). The appearance of a concentrated, wellordered phase when $\chi$ exceeds a small positive value is due to attractive interactions between the rod-like particles.

b) Orientation-Dependent Interactions:

Polymer-polymer interactions are important in predicting the stability of the liquid-crystalline phases if the interactive forces are orientation-dependent. Certain groups (e.g., $-\mathrm{C} \equiv \mathrm{C}-, \quad-\mathrm{N}=\mathrm{N}-$, and $-\mathrm{C} \equiv \mathrm{N}$ ) in polymer chains can contribute to the stability of the LC state if their polarizabilities is higher along the bond axis than their perpendicular axis. These secondary interactions are calculated with respect to segmental contacts. The net effect of these interactions lowers the critical axial ratio needed for liquid-crystalline phase separation (25). These 
interactions play an important role in low molecular weight compounds and semiflexible long chain polymers in melt and concentrated solutions. The phase diagram developed (39) by calculations concerning interactions in lyotropic sytems is found similar to Fig. 4. The influence of orientationdependent, "soft" interactions on liquid-crystalline polymers have received little attention.

\subsection{Fiber Spinning Processes}

Commercial synthetic fibers are produced by either melt or solution spinning process. Melt spinning is the more rapid, efficient and most economical method of fiber formation. Its application is limited to those polymers which melt without degradation and are thermally stable in temperatures corresponding to high fluidity (e.g., polyamides, polyesters, polystyrene, polyolefins). The molten polymer is extruded through spinnerette(s) into a gaseous (or less often, liquid) medium where cooling and solidification of the materials occurs.

Spinning from solutions is applied to polymers which thermally degrade below their melting temperatures but which can be dissolved in high enough concentrations in lowmolecular liquids. The first polymers formed into fibers were cellulosics, and the first patents in this area involve solution spinning $(40,41)$. Presently, acrylics, aromatic polyamides and cellulosics are solution spun. When a polymer 
is soluble in a volatile solvent (e.g., acetone, tetrahydrofuran, ether, alcohols), dry-spinning procedure is applied. Dry-spinning of fibers from polymer solutions consists of extruding the spinning fluid into a vertical cell where jets of spinning solution leaving the spinnerette come into contact with a stream of hot gases (usually air). In the gas stream the solvent vaporizes, polymer concentration in the filament increases and the filaments solidify. Dry-spinning is widely used for the manufacture of fibers from cellulose acetate, polyvinyl chloride, polymer and copolymers of vinyl chloride, acrylonitrile, and others.

The other fundamental method of spinning from solutions is wet spinning. It is applied to polymers which do not melt and dissolve only in non-volatile or thermally unstable solvents. This procedure consists of the extrusion of polymer solution from a spinnerette "immersed" in the liquid bath containing low-molecular weight substances miscible with the solvent but not dissolving the polymer. In this "coagulation" bath the polymer is precipitated from the solution, and a solid "gel filament" containing considerable amounts of solvent and precipitating agent is formed. The filament is further washed to remove all the solvent. The surface speed of the take-up roller is lower than that of the roller used in melt or dry spinning due to the limitations imposed by hydrodynamic drag and liquid carryover. It is usually in the range of 5-40 m/min for an immersed jet (42). Wet spinning is 
the most complex spinning procedure. But this process provides the opportunity of obtaining fibers from materials which cannot be spun in other ways $\{$ e.g., cellulose (viscose rayon), proteins, aromatic polyamides, polyacrylonitrile and polyvinyl chloride\} •

An alternative arrangement is a short air gap between the spinnerette and coagulation bath (42). This process is known as "dry-jet" wet spinning or air-gap spinning. Its advantages over the normal immersed-jet process are higher maximum spinning speeds, elimination of spinnerette hole blockage by deposition of coagulated polymer and a stable temperature difference between spinnerette and coagulant. The "dry-jet" technique is used mainly in the production of highly oriented fibers from liquid-crystalline solutions of rigid-rod polymers such as para-linked aromatic polyamides [Kevlar aramid fibers]. The presence of an air-gap allows higher take-up speeds which result in higher orientation of nematic liquidcrystalline phase in the emerging filament before coagulation. The fibers produced in this way have higher tensile strength and modulus than the corresponding fibers from immersed-jet process. If this spinning method is used for a solution of a more flexible polymer (such as acrylic), it is likely to result in lower orientation as the air gap allows any orientation introduced during passage through the spinnerette hole to relax before coagulation. Fibers made by wet spinning have higher void content than those from other processes. With 


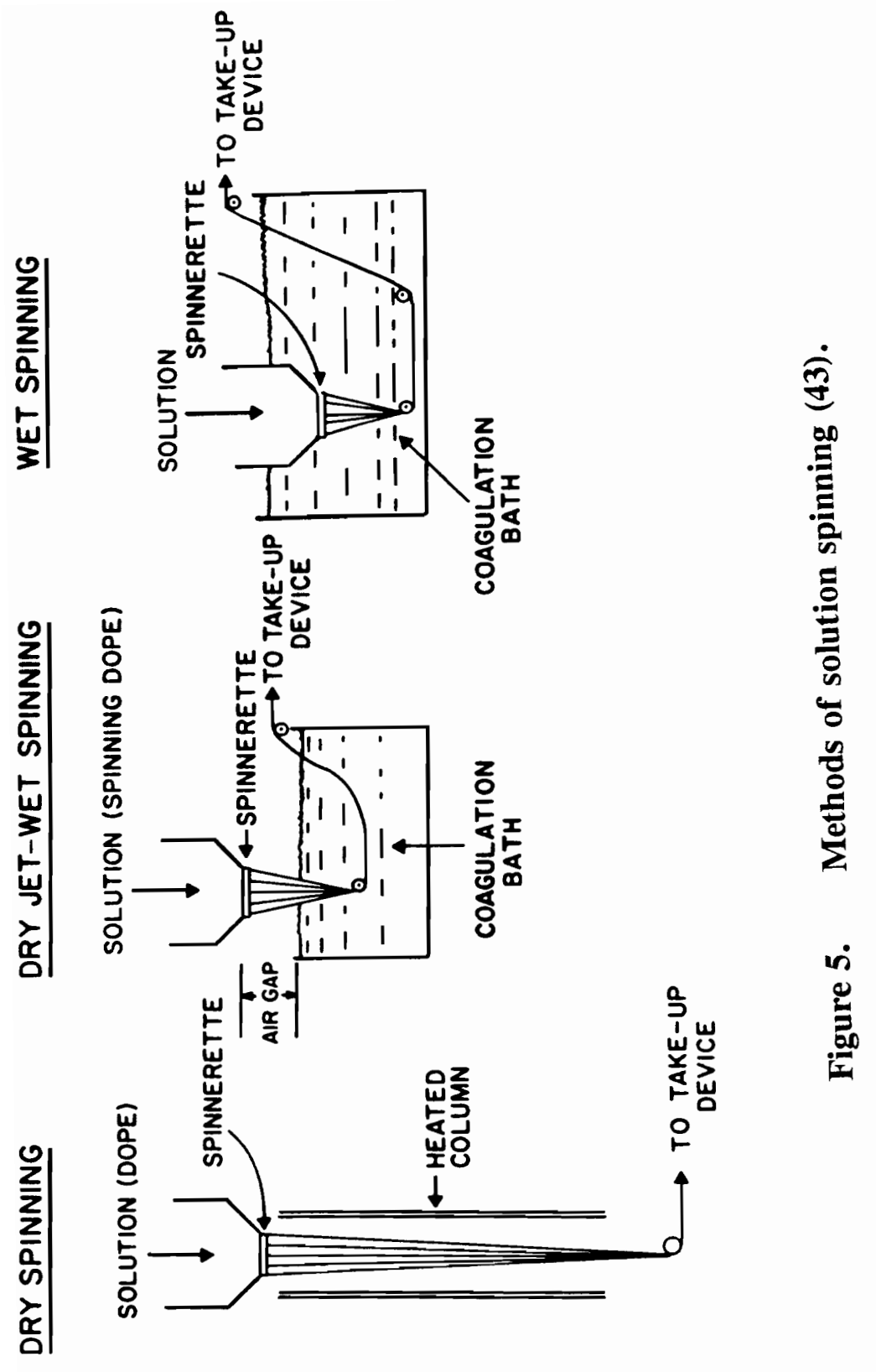


the application of dry-jet technique, fibers exhibit smooth surface and relatively nonporous structure which is characteristic of dry-spun fibers. The main disadvantage of dry-jet technique is that the holes in the spinnerette must be further apart than those in immersed-jet to prevent filament coalescence. This may lead to a net loss of production per spinning position. Fig. 5 is the schematic representation of the methods of solution spinning (43).

\subsection{Fundamentals of Fiber Formation}

In this section, the basic theories and principles of fiber formation will be discussed in the wet spinning process. Equations and concepts are adapted from reference 44.

\subsubsection{Spinnability of Fluids}

"Spinnable" usually means fiber-forming, i.e., suitable for manufacturing of fibers. The criterion of spinnability is defined as the fluid which is spinnable under given deformation conditions if steady-state, continuous elongation of the fluid jet proceeds without a break of any kind (44). There are two physical mechanisms of the breakage of fluid threads. The first process leading to the breakage of a fluid thread is a cohesive, brittle fracture occurring when the tensile stress in a viscoelastic jet exceeds some critical limit (tensile strength). Elasticity of the fluid plays an important role. An ideally viscous fluid would deform to an infinite extent and all the deformation energy is 
instantaneously dissipated. In a viscoelastic material a part of the deformation energy is stored, and on reaching some limiting value (resilience), results in a cohesive, brittle fracture. When a fluid jet is undergoing continuous elongation, the tensile stress, $p_{x x}$, changes along its axis as also does its tensile strength, $\mathrm{p}^{*}$, if the material characteristics are not constants. The deformation is possible up to the point $\mathrm{x}^{*}$ where the following condition is reached (Fig.6a);

$$
\left.\mathrm{p}^{*}(\mathrm{x})\right|_{\mathrm{x}=\mathrm{x}} *=\left.\mathrm{p}_{\mathrm{xx}}(\mathrm{x})\right|_{\mathrm{x}=\mathrm{x}} \text { * }
$$

Equation 5 determines the maximum thread length, $x^{*}$, controlled by the cohesive mechanism (44).

The other possible process of breaking fluid threads is associated with surface tension and "capillary waves" on the free surface of a liquid jet $(44,45)$. It can be shown that small, axisymmetrical distortions of the jet surface, $\delta_{0}$, will grow spontaneously leading to the break-up of the jet into drops (Equation 6, Fig. 6b) ;

$$
\delta(t)=\delta_{0} \exp (\mu t) \cos (2 \pi x / \lambda) ; \lambda>2 \pi R
$$

$R$ is the undistorted jet radius, $\lambda$ is the wavelength, and $\mu$ the growth factor. The general break-up condition for such a jet is given by;

$$
\left.\delta(x)\right|_{x=x} *=\left.R(x)\right|_{x=x} *
$$

Both the mechanisms described can act independently resulting in the breakage of the fluid thread. The former mechanism is 
(a)

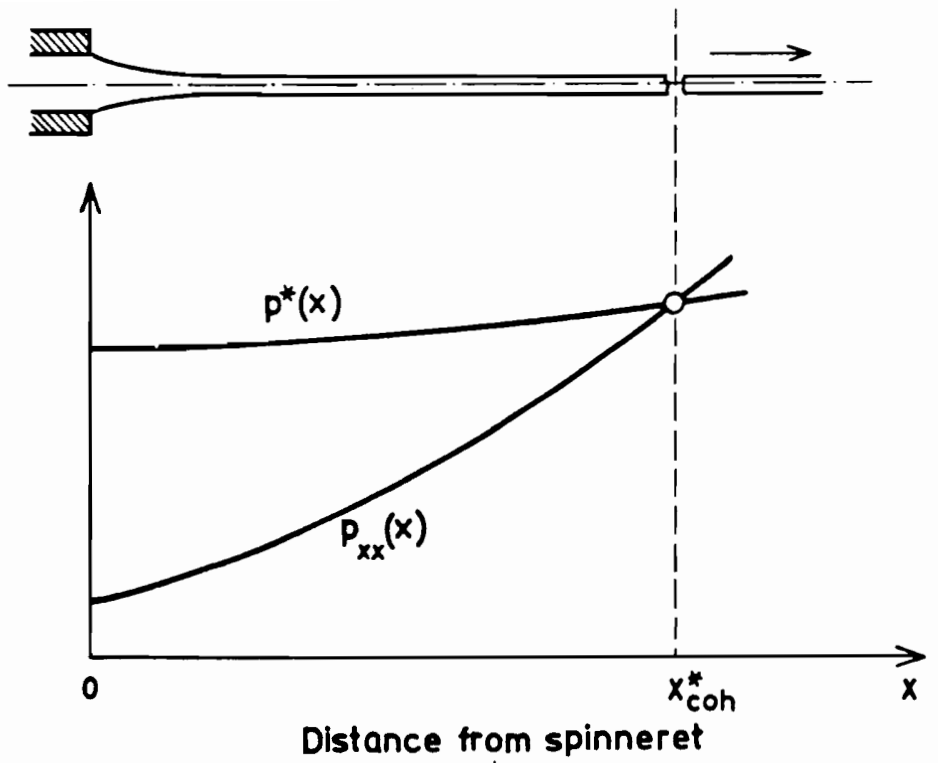

(b)

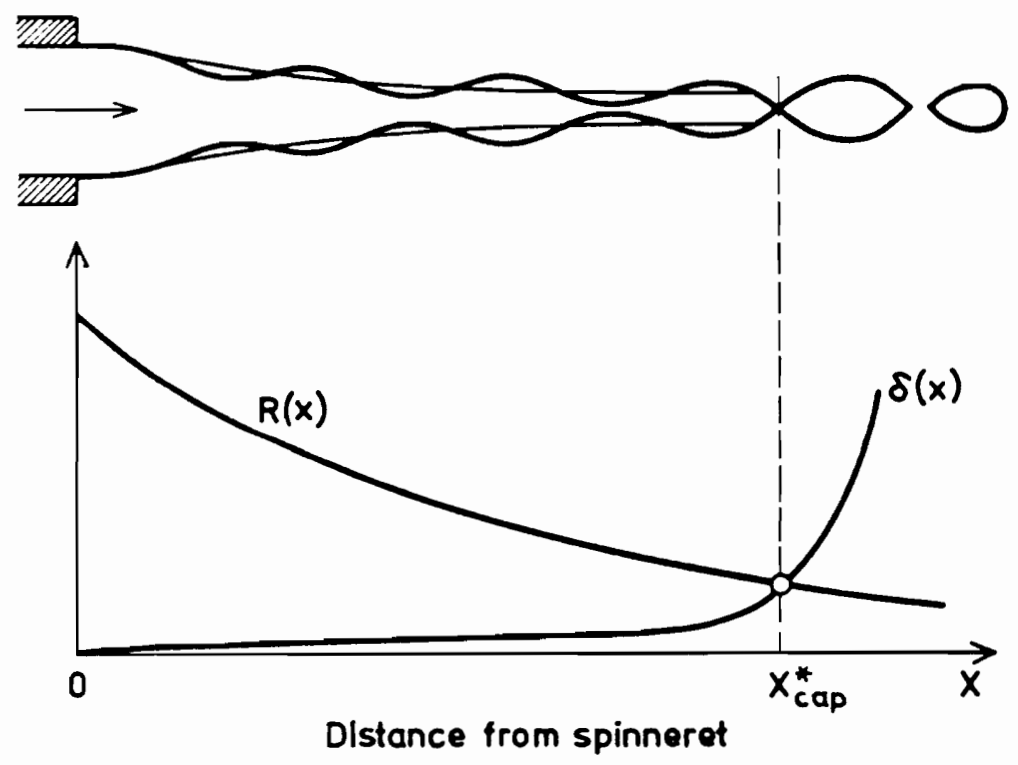

Figure 6. Schematic representation of (a) cohesive fracture of a steady-state liquid jet and (b) break-up of a liquid jet due to capillary wave mechanism (44). 
more important for wet-spinning.

\subsubsection{Fiber Spinning Variables}

The average velocity of the spinning solution in the spinnerette hole, $v_{0}$, is calculated from;

$$
\mathrm{V}_{\mathrm{o}}=\mathrm{Q} /(\pi / 4) \mathrm{D}^{2}
$$

where $Q$ is the flow rate per hole and $D$ is the hole diameter (46). The velocity, $v_{f}$, at which the filament emerges from the hole under conditions of free extrusion (i.e., no take-up) is smaller than $V_{0}$. This is because the elasticity of the spinning solution allows it to store energy originating from the shearing in the capillary and the deformation on entering the capillary, which is released on emerging from the hole. This free velocity, $v_{f}$, plays an important role in the problem of spinnability.

Consider a fluid to be forced through a hole of diameter $D$ at a flow rate $Q$. For elastic fluids, there is release of stored elastic energy. This causes the stream of fluid to broaden or swell to a diameter $D_{f}$, which is larger than the capillary diameter D. This phenomenon is "die swelling" and is also known as the Barus Effect (Fig. 7). This swelling causes the fluid jet or filament to slow down. The diameters and velocities are related by;

$$
\text { Die Swell Ratio }=D_{f} / D=\left(V_{o} / V_{f}\right)^{1 / 2}
$$

The die swelling phenomenon plays an important role with spin draw ratio in dry and wet spinning processes. The velocity of 


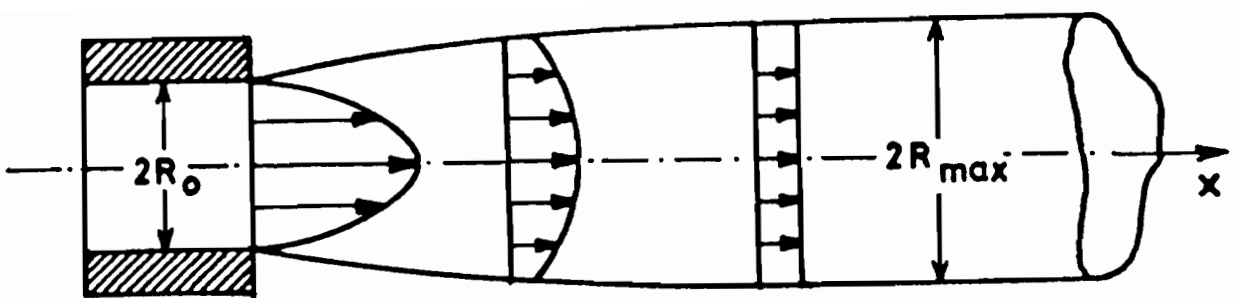

Figure 7. Schematic representation of a fluid jet issiung from capillary (44). 
the take-up roller is defined as $\mathrm{V}_{\mathrm{L}}$. The apparent draw ratio based on take-up and extrusion velocities is defined as,

$$
\text { (Draw Ratio) }_{\text {app }}=\mathrm{V}_{\mathrm{L}} / \mathrm{V}_{\mathrm{o}}
$$

This value, in some spinning conditions, is less than unity which would suggest "axial compression" of the jet and perpendicular orientation of the macromolecules.

If on the other hand, the draw ratio based on the free jet velocity $v_{f}$ corresponding to maximum die swell seems to be a more reasonable measure of deformation and is always greater than one (Equation 11).

Draw Ratio $=\mathrm{V}_{\mathrm{L}} / \mathrm{V}_{\mathrm{f}}$

This eliminates the "compression paradox" which does not make physical sense. L/D ratio of the capillary is an important die design variable for polymer extrusion. It has been shown that amount of die swelling decreases as the capillary is made longer (46).

The apparent shear rate at the capillary wall is given by $; \quad$.

$$
\gamma_{\mathrm{wa}}=4 Q / \pi(\mathrm{D} / 2)^{3}
$$

where $D$ is the hole diameter.

1.5.3 Diffusion During the Coagulation step of wet spinning

The most fundamental step in fiber formation from a polymer solution is solvent removal from the spinning fluid. The removal of solvent from the polymer solution must be 
accomplished by diffusional interchange with a nonsolvent bath. In this bath the polymer loses its solubility and is precipitated from solution as a consequence of the penetration of nonsolvent. This process causes the polymer to solidify and is termed "coagulation". This phenomenon has been analyzed by previous investigators for cellulose (47-49) and other polymer systems (50-53). An important feature of the coagulation process is that a distinct moving boundary is associated with the coagulation.

a) Coagulation and Boundary Movement:

A visible boundary separates the coagulating solution into two regions. One side of the boundary is a hard, elastic, coagulated polymer, while the other side is the soft, fluid solution. The boundary begins at the solution surface and moves inward with time until it reaches the center of the solution. The growth of this boundary seems to be related to the spinnability of polymer solutions (52) and structure of the wet-spun fibers (50). This boundary movement can be observed by a stereo microscope by immersing gelled rods of the polymer solution in a beaker of coagulating liquid for various lengths of time $(52,54-56)$. Fig. 8 shows this phenomenon schematically (55). $\xi$ is the depth of penetration. It is the radial distance between the outer circumference and the boundary and is a function of time. The boundary movement is based on Fick's diffusion law (57). The equation for the diffusion of a coagulant into the polymer solution for a 

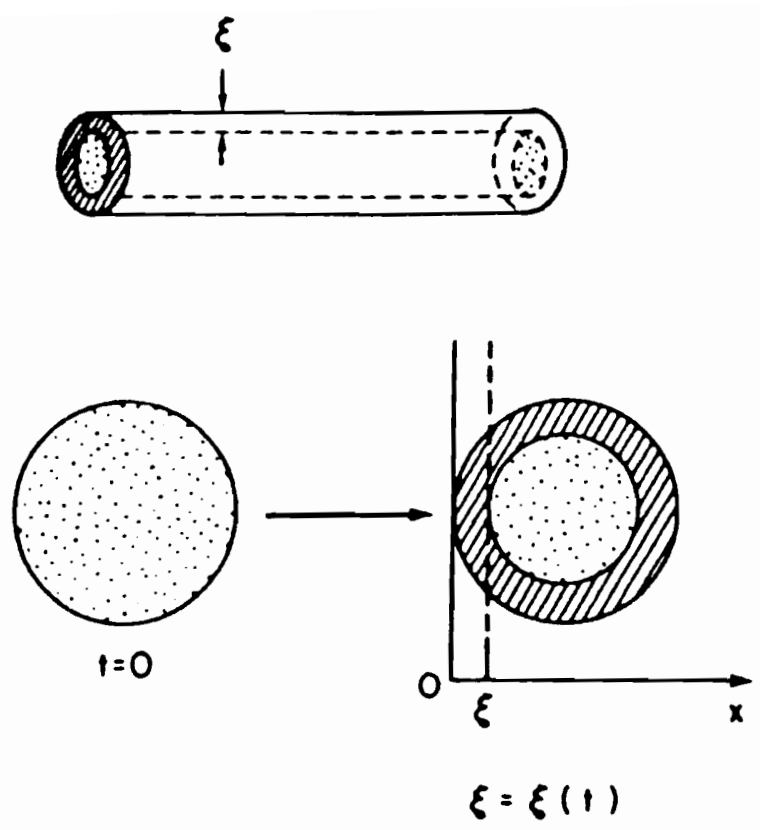

Figure 8. Schematic representation of coagulation and boundary movement (55). 
linear one-dimensional system is;

$$
\partial C_{n} / \partial t=D_{n}\left(\partial^{2} C_{n} / \partial x^{2}\right)
$$

where $C_{n}$ and $D_{n}$ are the concentration and the diffusion coefficients, respectively. References 58 and 59 describe the mathematical solution of this partial differential function. The results from the solution indicates that $\xi / \sqrt{ }$ should be a constant. $\xi / \sqrt{ }$ is known as "coagulation rate" and reflects the effects of coagulation variables on the coagulation of a polymer solution. At the coagulation boundary $(x=\xi)$, the concentration of coagulant reaches a critical value, $C_{n}{ }^{*}$, which causes the polymer to precipitate or crystallize from solution. Assuming that the diameter of the as-spun filament is same as the spinnerette hole, the relationship between the spinning conditions and the minimum coagulation rate $(\xi / V t)^{*}$ required for complete coagulation of the spinning solution in the coagulation bath is given by;

$$
(\xi / \sqrt{ })^{*}=0.7(D / 2)(<v>/ B)^{1 / 2}
$$

where $D$ is the diameter of the spinnerette hole, and $B$ and $\langle v\rangle$ are the immersion distance and the average velocity, respectively, of as-spun filament in the coagulation bath (56). In practice, the coagulant chosen in fiber spinning should provide sufficient coagulation rate to fully coagulate the as-spun filament within a specified time scale to avoid defects in the fully processed fiber. Equation 14 is definitely very valuable as it may be useful in predicting 
workable coagulants or coagulation conditions for a particular spinning system. This equation may become complicated by the effects of die-swell and stretching, as they relate to the diameter of as-spun filament.

The coagulation rate is affected by the molecular structure of the coagulant due to steric factors (56). The coagulation rate decreases at lower bath temperatures as the diffusion process is slow. This allows more time for adjustment to the internal stresses that cause expansion of the polymer network, and results in dense fiber with improved structure (54).

The effect of polymer concentration on the coagulation rate is controlled by two opposing factors (54). An increase in viscosity of the solution usually accompanies increasing polymer concentration and it is expected that the mobility of solvent and coagulant molecules will be reduced, leading to a decrease in coagulation rate. Another factor which must be considered is that higher concentration of polymer solution may increase the polymer intermolecular attractive force due to higher density of molecular chains which can increase the driving force for coagulation to occur. It seems that the polymer solution composition plays an important role in the coagulation process. At higher solids content, i.e., higher polymer concentration, less nonsolvent (coagulant) is required to diffuse into the solution to precipitate the polymer, and consequently, leads to a faster coagulation rate. Viscosity 
increase does not significantly affect the coagulation rate.

During the coagulation process, there are stresses in the filament which are transmitted across the boundary. Orientation of the molecules can take place by shear gradients established on the fluid side of the boundary. This orientation can be immobilized very quickly due to diffusion across the boundary $(60)$.

The key factor affecting the fiber structure and consequently fiber properties is governed by the composition of the coagulated layer. It seems that small correlation exists between the fiber properties and coagulation rate, as the coagulation rate reflects the growth rate of the coagulated layer of spinning thread during the process of fiber formation and does not control the composition of the coagulated layer. Mass transfer rate difference between coagulant and solvent is a dominating factor which controls the composition of the coagulated layer.

b) Coagulation and Mass Transfer Rate Difference:

In the polymer solution wet-spinning system, the solvent diffuses out of the solution, while coagulants diffuse in. The difference in the mass transfer rate between solvent and nonsolvent is the basic element influencing the composition of the coagulating polymer solution. The higher the rate difference, the more solidified the coagulating structure will be at a given time since more solvent is removed from the filaments compared with the amount of coagulants added to the 
filaments. Based on a mass balance of a coagulating polymer solution, the measurement of the weight change of a polymer solution as a function of time can provide the value of this rate difference. Normally, gelled model filaments are used as specimens in these measurements (54-56). Based on mass balance and Fick's laws of diffusion (57), the weight change measurements of the polymer solution with time can provide the value of the mass transfer rate difference $\Delta \mathrm{K}$. A workable coagulation system requires a mass transfer rate of solvent larger than that of coagulant, otherwise the polymer solution eventually becomes diluted with coagulant and dissipates into the coagulation bath.

The cross-sectional shape of wet-spun fibers varies with the type of coagulant due to the change in the mass transfer difference between solvent and coagulants; and deformability of the thin surface layer which forms on the filament at the very beginning of coagulation. When the mass transfer rate of the solvent is less than that of the coagulant $(\Delta K<0)$, the filament swells and a circular cross-section can be expected. When the solvent diffuses out of the filament at a higher rate than the absorption of coagulant $(\Delta K>0)$, the cross-sectional shape depends on the rigidity of the coagulated layer. With a soft, deformable surface layer the resulting shrinkage may lead to a circular cross-section. With a rigid surface layer, the collapse of the cross-section will lead to a noncircular, irregular shape. When the $\Delta \mathrm{K}$ value is very high, it can be 
expected that the surface layer has higher content of polymer, and therefore the rigidity tends to be higher. Therefore, rigidity gradient is the driving force which leads to a deviation from circularity of the cross-sectional shape. If $\Delta \mathrm{K}$ remains constant, then it will be expected that the rigidity gradient will increase with decreasing coagulation rate. Thus the ratio;

$$
\mathrm{G}_{\mathrm{r}}=\Delta \mathrm{K} /(\xi / \sqrt{ } \mathrm{t})
$$

plays an important role in determining the fiber crosssectional shape (56).

The initial modulus of the fibers increases with lower $\Delta \mathrm{K}$ values. This is due the fact that the as-spun filament tends to be more swollen and flexible, and consequently, providing higher stretching which contributes to higher degree of orientation of molecular chains.

Another important parameter associated with the composition of the coagulated polymer solution is the equilibrium swelling degree $\left[(\mathrm{SD})_{\mathrm{eq}}\right]$, which has been defined as the equilibrium weight of liquid per unit weight of polymer (54).

The presence of void structure in wet-spun fibers is a consequence of the coagulation process. The three characteristic void super-structures are hollow cores, radial fluted voids and relatively compacted structures. The key role in determining the void structure in the fibers is that of the 
solvent-coagulant interaction rather than the polymer itself. Interfacial instability between the miscible liquids leads to the formation of "fingers", which is primarily associated with the heats of solution $\left(\Delta \mathrm{H}_{\text {sol }}\right)$ of the liquids (61). A heat of solution of about 5.5 calories/gram of the solution or greater results in fingering. The fingering phenomenon is due to the interfacial turbulence observed at interfaces between low molecular weight liquids during mass transfer. These phenomena are generally called Marangoni or Thomson-Marangoni effects. Surface active agents can eliminate the interfacial turbulence. Solvent content in the bath is an important parameter which reduces the fingering phenomenon as the heat of solution decreases with increasing solvent content. Coagulation process is also retarded with increasing solvent content in the bath. It is a consequence of a dilution effect of solvent on the coagulant concentration in the bath (55). As the solvent content increases, the concentration of coagulant decreases in the bath, which in turn reduces the driving force for diffusion.

In all spinning processes, the structure and physical characteristics of the spinning line are differentiated from the cross-section of the fibers (44). In melt spinning it is due to the radial temperature gradient, in dry- and wetspinning it is due to the radial gradient of composition and phase structure of the partially solidified polymer solution. 


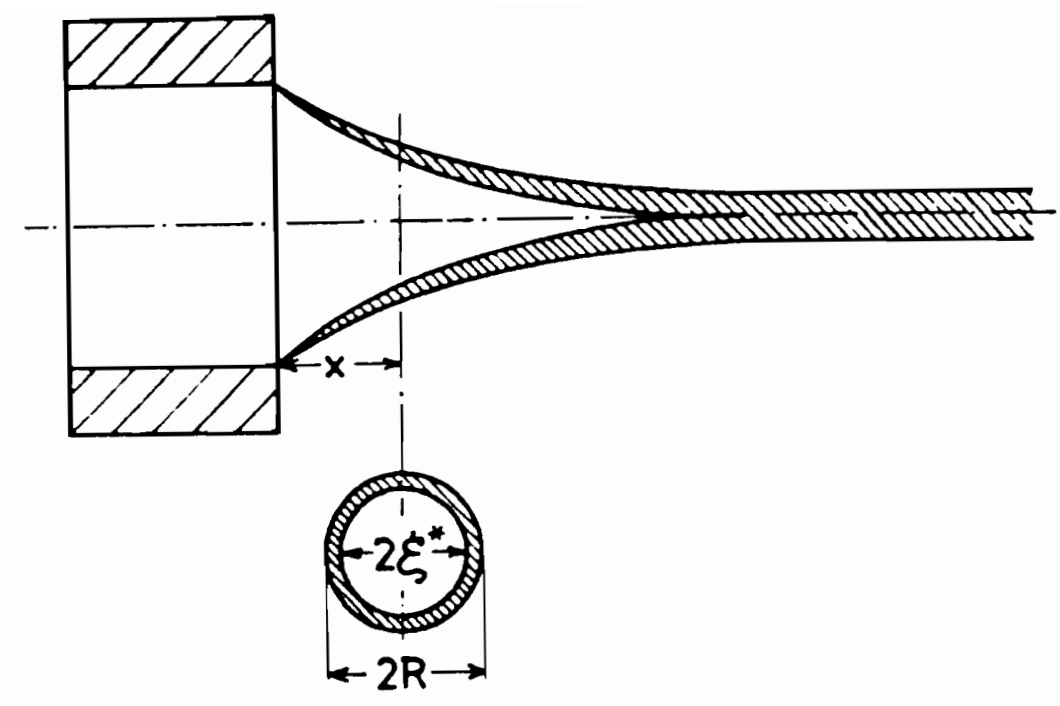

Figure 9. Schematic representation of a non-uniform skin-core system formed in the process of wet-spinning. Cross-hatched portion denotes rigid solidified layer (44). 
In melt and dry-spinning, radial distribution of polymer properties is continuous and the gradients are not large. However, it is different for wet-spinning. Solidification during coagulation is accompanied by formation of a rigid "skin" on the surface. The skin is separated by a distinct boundary from the fluid "core" (Fig. 9). This gives rise to the well known "Skin-Core Effect" and the resulting radial gradients of physical properties are extremely high.

\subsection{Fiber Spinning From Liquid-Crystalline Solutions}

A closely packed assembly of parallel extended polymer chains should exhibit the highest achievable specific strength. These unique characteristics of uniaxially oriented polymers are the basis for the technology of ultrahighstrength organic polymeric fibers (62). Some of the concepts in sections 1.6 .1 and 1.6 .2 are adapted from reference 62 .

\subsubsection{Role of Liquid Crystalline state}

Figure 10 represents an ensemble of molecules which exhibits maximum theoretical modulus and strength. This model resembles a nematic structure, except that in the case of high-strength fibers the distance between the chain ends is very large. On one extreme there are very rigid, insoluble, and infusible graphite fibers, and on the other, flexible low melting, soluble polyethylene fibers. Semi-rigid and rod-like polymers exhibiting thermotropic and/or lyotropic behavior fall in between these two extremes. 


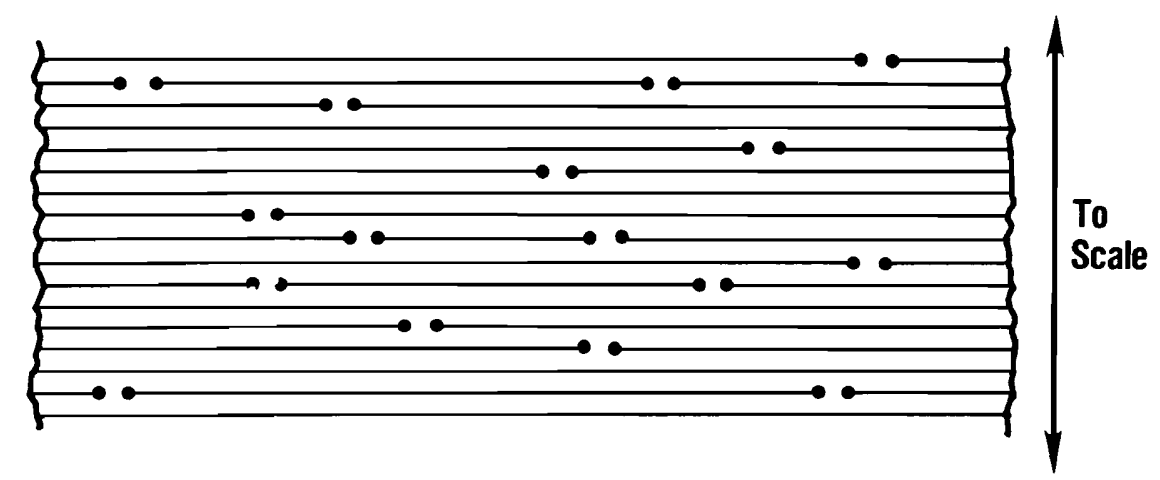

Reduced Scale -

Figure 10. Ensemble of molecules exhibiting maximum strength (62). 


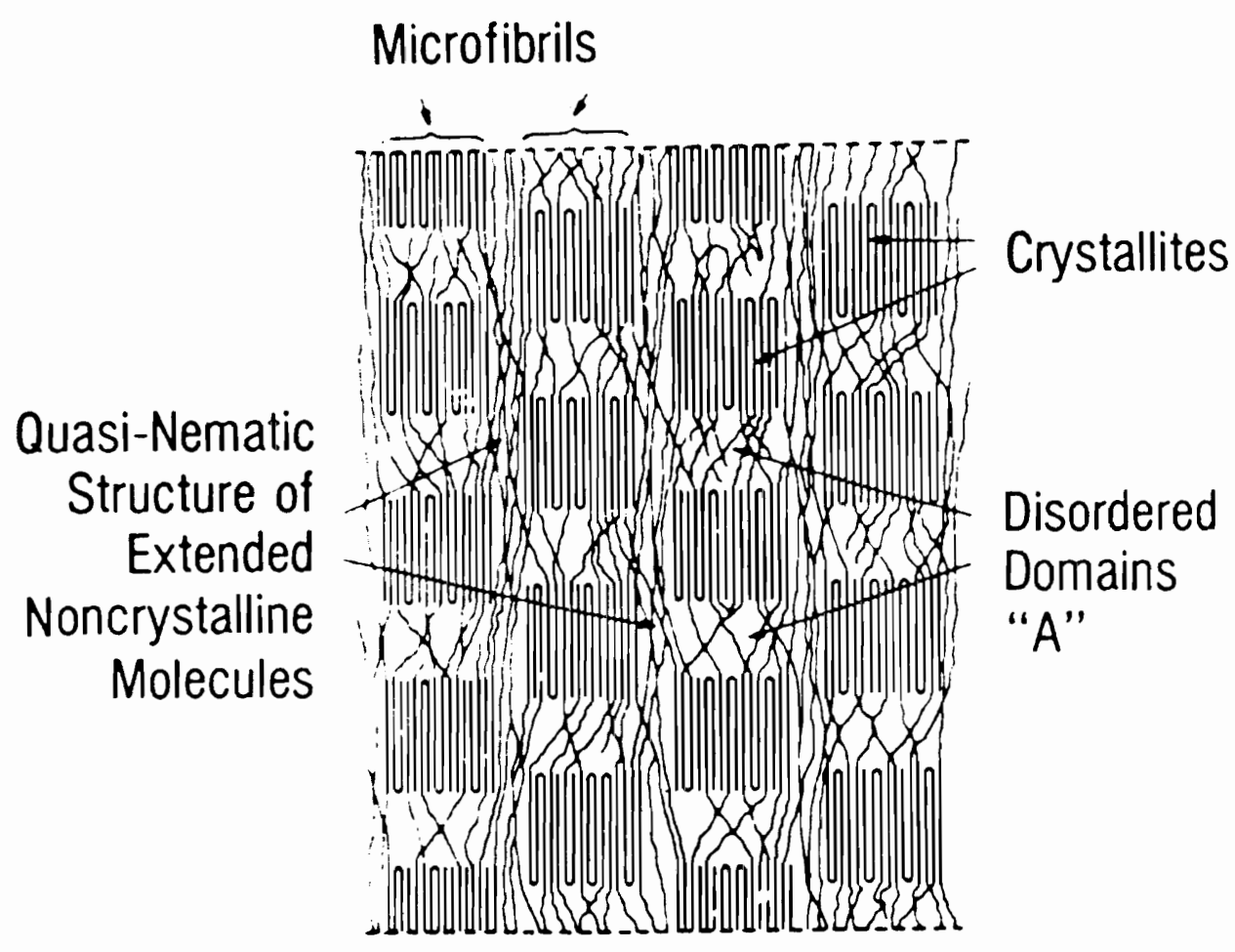

Figure 11. Model representing the structure of melt-spun fibers of flexible polymers such as poly (ethylene terephthalate) (62). 
A two-phase model which represents fibers melt-spun from flexible molecules is shown in Fig. 11. The problem of preparing fiber structures from flexible polymers close to the ideal model in Fig. 10 is that stretching is not enough to eliminate the microfibrillar two-phase structure and stacked chain folding, leading to high concentration of stress. However, it is possible to produce structures like Fig. 10 from polyethylene by solid state extrusion (63), drawing fibers from dilute solutions (64), and gel spinning (65). The chain-folds can be reduced by increasing the rigidity of the polymer molecule. This will lead the polymer to exhibit the "fold-free" liquid-crystalline behavior. But the increase in rigidity may reduce the solubility and increase the melting point of the polymer which limits processing from melt or solution. A great deal of research was required to design and synthesize polymers that are semirigid to a degree sufficient to give liquid crystalline behavior in melt (66) or in solution (67) at temperatures below their degradation.

\subsubsection{Spinning from Nematic Solutions}

Two general criteria that must be met to achieve high degree of orientation in as-spun fibers are that the polymer must be easily orientable by shear or elongational flow and this orientation must be preserved until the chain mobility in the fibers is frozen by the coagulation process. The first criterion is met by the existence of an anisotropic solution. The second criterion requires a long relaxation time, which 
must be of the same order or longer than the time needed to convert the spinning dope into a "solidified" filament in which the molecules are unable to undergo long-range molecular motion. Fiber from flexible polymers usually fail these criteria. The fibers produced from anisotropic solutions can, under specific conditions, approach the idealized structure of Fig. 10, and Kevlar-type aramid fibers represent the most important technological development based on the chemistry and physics of liquid crystalline high molecular weight polymers. Another important technological development which appears to hold promise is the spinning of cholesteric dopes of cellulose in aqueous solutions of a cyclic amine oxide, such as $\mathrm{N}-$ methylmorpholine $\mathrm{N}$-oxide, to yield strong fibers (68).

\subsubsection{Flow-Induced Transitions to a Nematic Phase}

since chain rigidity reduces polymer solubility, it is of technological interest to increase the chain flexibility and thereby expand the choice of solvents for solution spinning. But an increase in flexibility increases the critical polymer concentration at which isotropic-nematic transition occurs. Also, for more flexible polymers, there are occasion where the polymers crystallize from solution before reaching the critical polymer concentration. Recent theoretical and experimental developments predict that for semirigid polymers that do not form an anisotropic solution under quiescent conditions, an isotropic-nematic transition may be induced by shear or elongational flow (69-74). The basic theory of phase 

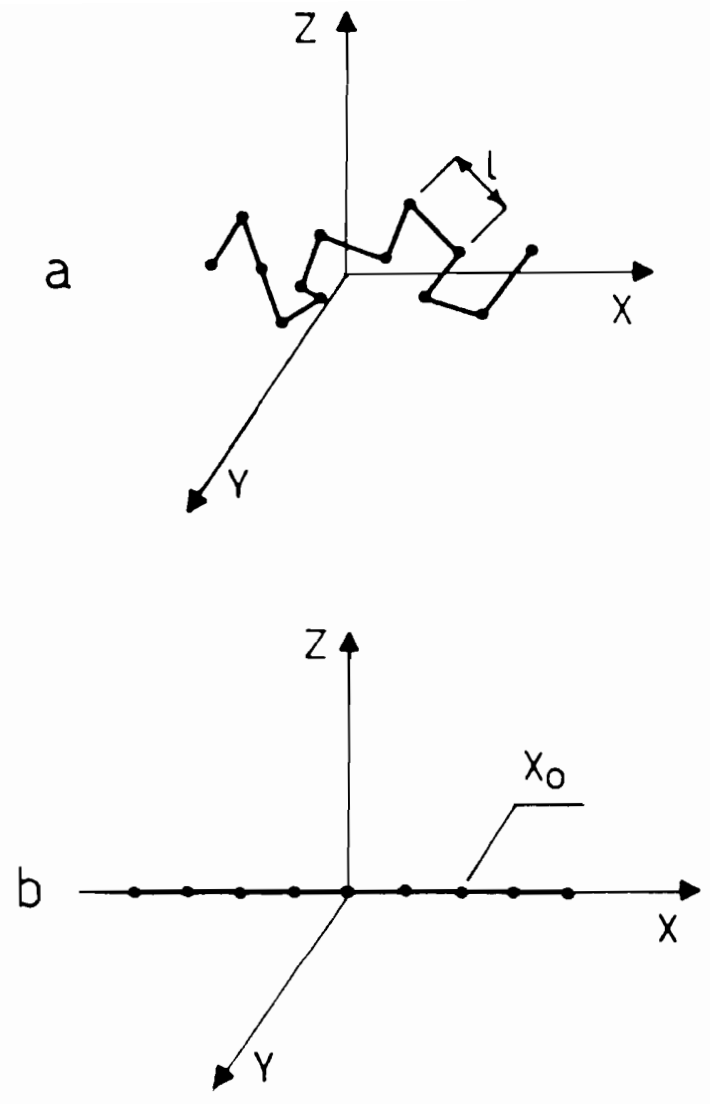

Figure 12. The freely jointed chain: (a) the origin of the co-ordinate system is in the hydrodynamic center of the chain; (b) the minimum energy of conformation (72). 
equilibria of rigid-rod molecules by flory is an equilibrium theory and does not include possible effects induced by a flow field. A brief discussion on the effect of flow field on phase transitions will be performed here. The discussions are adapted from reference nos. 72-74.

The flow effect is treated as a pseudo-equilibrium one in which the velocity field allows a potential. The model is applied to a general case of a freely jointed chain made of $\mathrm{N}$ equal links. This model chain includes the rod-like chain as a special case $(\mathrm{N}=1$ ) and can describe the semi-rigid (small $\mathrm{N}$ ) and the flexible (large $N$ ) cases (Fig. 12a). The free energy contribution due to flow is calculated and is added to the equilibrium free energy (72).

When a macromolecular solution undergoes flow, the distribution of macromolecular conformations and/or orientations will eventually reach a steady state which is different from the equilibrium. The friction force acting on a macromolecular segment is proportional to the relative velocity between the segment and the surrounding medium. To each conformation and orientation in space a potential energy can be associated just as if a force field were to act on the molecules, and the distribution will be a pseudo-equilibrium, i.e., Boltzmann distribution. When there is no flow, all conformations and orientations of the chain are energetically equivalent. When the elongational flow occurs, the chain assumes the minimum energy conformation state which is the 
fully stretched state oriented in the X-direction (Fig. 12b). Reference 72 describes the complete derivation of the energy contribution due to flow-field.

It has been shown that for a chain surrounded by an elongational flow the tension in the central zone is larger than that at the extremities due to the cumulative action of the friction along the chain (72).

$G$ is defined as the dimensionless stretching rate. In absence of a flow, $G=0$ and the anisotropic solutions at equilibrium depends only on the characteristic dimension of the rigid link, $x$, and is independent of overall molecular weight $\mathrm{N}$. When $\mathrm{G} \neq 0$, anisotropy is more easily achieved. The critical condition for anisotropy is given by;

$$
\left(G x^{3}\right)_{\text {crit }}=8 / 3 \mathrm{~N}
$$

Values of $G$ larger than $G_{c r i t}$ will generate anisotropic solutions even in the limit of dilute solutions. If $G$ is smaller than $G_{\text {crit }}$ sufficiently dilute solutions are isotropic. In the latter case anisotropy may set in, if $x$ is not too small, by increasing the concentration. The equilibrium between the isotropic and anisotropic phases is influenced by the applied flow field. The conditions in which the effect of the flow field could be essential in inducing anisotropy seems to be the semi-rigid chains having $x$ of the order of 10 .

During fiber spinning, elongational and shear flow exist which plays the role in deformation and orientation of 
molecules. Shear flow occurs through the tubing and spinnerette holes; and elongational flow occurs along the spinning line, or when ever there is a cross-sectional variation. The shear gradients at the spinnerette are very high which will greatly reduce the viscosity of the solution being spun. But such large variations of viscosity usually do not correspond to large variations of the orientation in the molecules. In case of isotropic solutions, the disorientation past the spinnerette hole induces only a modest orientation. In case of anisotropic solutions, flow field will not contribute to the orientation of the individual molecules and will merely orient the anisotropic domains along the continuous filament. Therefore, nematic phase is important only because it assures the "permanence" of orientation during coagulation even in the absence of flow fields.

A rather different situation occurs for polyterephthalamide of p-aminobenhydrazide $(x-500)$ which does not form anisotropic solution in dimethyl sulfoxide under quiescent condition. $x-500$ is characterized as a semi-rigid polymer and this condition seems to be sufficient to cause, under the influence of shear and elongational flow in the spin line, a transition from an isotropic to a nematic state (74). It seems that the shear gradient is large enough to induce fracture-type phenomena of the solution leading to anisotropic clusters. Although the rigidity of $x-500$ is insufficient to form nematic phase under quiescent conditions, it can be 
inferred that the system is probably very close to such a transition. Similar transitions are unlikely to occur in conventional flexible polymers due to the presence of many entanglements. 


\subsection{Research Objectives}

The first observation of a cholesteric liquid crystalline solution of cellulose derivative (hydroxypropyl cellulose) was made by Werbowyz and Gray (75) in 1976. Since this initial observation, the field has expanded and there are numerous reports of cellulose derivatives that form lyotropic Iiquid crystals (76-82). Investigators of cellulosic liquid crystals have two main motivations: to study mesophase formation primarily from a scientific viewpoint or a technological viewpoint. The primary focus of the latter has been on the potential of preparing high strength/high modulus regenerated fibers.

The main research objectives of the present study are to investigate both the scientific and technological aspects of lyotropic liquid-crystalline formation in cellulose and cellulose derivatives in direct solvent systems. This study is divided into four major sections which deal with:

1. Effects on the lyotropic liquid-crystalline behavior by different substituents on the cellulose backbone.

2. Structure-property relationships of the fibers spun from liquid-crystalline solutions of cellulose esters investigated in 1.

3. Effects of ionic interactions (lithium chloride) on the fibers spun from liquid-crystalline solutions of cellulose esters. 
4. Effects of an amorphous component (lignin) on the fibers spun from liquid-crystalline solutions of cellulose and cellulose esters. 


\subsection{References}

1. S. L. Kwolek, P. W. Morgan and J. R. Schaefgen, "Liquid Crystalline Polymers", in Encyclopedia of Poly. sci. and Engr., Vol. 9, 2nd Edition, John Wiley \& Sons, 1, 1987.

2. T. S. Chung, Polym. Eng. Sci., 26, 901, 1986.

3. J. S. Chiou and D. R. Paul, J. Poly. Sci. Poly. Phys. Ed., 25, 1699, 1987.

4. W. Brostow, T. S. Dziemianowicz, J. Romanski and W. Werber, Poly. Eng. Sci., 28(12), 785, 1988.

5. B. Wunderlich and J. Grebowicz, "Liquid Crystal Polymers II/III", Adv. in Poly. Sci. Ser, 60/61, 11984 .

6. F. Reinitzer, Monatsh., 9, 421, 1888 .

7. O. Lehmann, Phys. Chem., 4, 462, 1889.

8. M. Miesowicz, Nature, 158, 27, 1946.

9. P. G. Degennes, The Physics of Iiquid Crystals, Oxford Univ. Press (Clarendon), London and New York, 1974.

10. G. R. Luckhurst and G. W. Gray, The Molecular Physics of Liquid Crystals, Academic Press, London, 1979.

11. P. J. Flory, Proc. R. Soc. London, Ser. A, 234, 73, 1956.

12. G. W. Calundann and M. Jaffe, Proc. Robert A. Welch Found. Conf. Chem. Res., Res. Conf. XXVII, "Synthetic Polymers", 1982 .

13. Ch. K. Ober, J. I. Jin and R. W. Lenz, Adv. in Poly. Sci., 59, 103, 1984 .

14. C. Noel, Makromol. Chem. Makromol. Symp., 22, 95, 1988. 
15. T. Wilson, Ph.D. Dissertation, Virginia Polytechnic Institute and State University, VA, 1991.

16. A. DeVries, in Liquid crystals, The Fourth state of Matter, Chapter 1, F. S. Saeva, Ed., Marcel Dekker, New York, 1979 .

17. T. Asada, S. Onogi and H. Yanase, Poly. Eng. Sci., 24 (5), 355,1984 .

18. G. Oster, J. Gen. Physiology, 33, 445, 1950.

19. H. Aoki, J. L. White and J. F. Fellers, J. Appl. Poly. Sci., 23, 2293, 1979 .

20. F. C. Bawden and N. W. Pirie, Proc. R. Soc. London, Ser. B, $123,274,1937$.

21. J. D. Bernal and I. Fankuchen, J. Gen. Physiology, 25, $111,1941$.

22. L. Onsager, Ann. N.Y. Acad. Sci., 51, 627, 1949.

23. A. Elliot and E. J. Ambrose, Discuss. Faraday Soc., 9, 246,1950 .

24. A. Ishihara, J. Chem. Phys., 18, 1446, 1950.

25. P. J. Flory, Adv. in Poly. Sci., 59, 1, 1984.

26. P. J. Flory and G. Ronca, Mol. Cryst. Liq. Cryst., 54, 289,1979 .

27. P. J. Flory, Macromolecules, 11, 1138, 1978.

28. S. M. Aharoni, Polymer, 21, 21, 1980.

29. E. Bianchi, A. Ciferri and A. Tealdi, Macromolecules, 15, 1268,1982 . 
30. Iiquid Crystalline order in Polymers, A. Blumstein, Ed., Academic Press, New York, 1978.

31. Polymer Liquid Crystals, A. Ciferri, W. R. Krigbaum and R. B. Meyers, Eds., Academic Press, New York, 1982.

32. A. Ciferri, Chapter 3 of Ref. 31 .

33. A. Roviello and A. Sirigu, Makromol. Chem., 183, 895, 1982

34. D. A. Brant and K. D. Goebel, Macromolecules, 5, 536, 1972.

35. K. D. Goebel and C. E. Harvine and D. A. Brant, Applied Polymer Symp., 28, 671, 1976.

36. A. Nakajima, T. Hayashi and M. Ohmori, Biopolymers, 6, 973,1968 .

37. A. Nakajima, T. Hirai and T. Hayashi, Polym. Bull., 1, 143,1978 .

38. E. L. Wee and W. G. Miller, Liquid Crystals and ordered Fluids, Vol. 3, J. F. Johnson and R. S. Porter, Eds., Plenum Press, 371,1978 .

39. M. Warner and P. J. Flory, J. Chem. Phys., 73, 6327, 1980 .

40. H. de Chardonnet, U. S. Patent 531158, 1894 .

41. C. H. Stearn, British Patent 1020, 1898.

42. J. E. McIntyre and M. J. Denton, "Fibers", in Encyclopedia of Poly. Sci. and Eng., Vol.6, 2nd Edition, John Wiley \& Sons, 805, 1987. 
43. M. Jaffe and R. M. Jones, in High Technology Fibers, Part A, M. Lewin and J. Preston, Eds., International Fiber Sci. and Tech. Ser. No. 5, Marcel Dekker, 366, 1985.

44. A. Ziabicki, Fundamentals of Fiber Formation, John Wiley \& Sons, 15, 1976 .

45. Lord Rayleigh, Phil. Mag., 34, 145, 1892.

46. D. R. Paul, J. Appl. Poly. Sci., 12, $2273,1968$.

47. J. J. Hermans, J. Colloid Sci., 2, 387, 1947.

48. A. Takizawa, Sen-I Gakkaishi, 16, 839, 1960.

49. D. Vermaas, Textile Res. J., 32, 353, 1962.

50. V. Groebe, G. Mann and G. Duwe, Faserforsch. Textiltech., $17,142,1966$.

51. J. R. Booth, Appl. Poly. Symp., 6, 89, 1967.

52. D. R. Paul, J. App1. Poly. Sci., 12, 383, 1968.

53. A. Takizawa, Sen-I Gakkaishi, 17, 403, 1961.

54. J. J. Cho, S. H. Hudson and J. A. Cuculo, J. Poly. Sci.:Part B: Polym. Phys., 27, 1699, 1989.

55. C. K. Liu, J. A. Cuculo and B. Smith, J. Poly. Sci.:Part B: Poly. Phys., 27, 2493, 1989.

56. C. K. Liu, J. A. Cuculo and B. Smith, J. Poly. Sci.:Part B: Poly. Phys., 28, 449, 1990.

57. J. Frank, The Mathematics of Diffusion, Oxford University Press, London, 290, 1975.

58. G. S. Adair, Biochem. J., 14, 762, 1920.

59. R. H. Peters, Textile Chemistry, Vol. III, Elsevier, Oxford, 152, 1975. 
60. D. R. Paul, J.Appl. Poly. Sci,, 13, 817, 1969.

61. T. A. Hancock, J. L. White and J. E. Spruiell, Poly. Eng. Sci., $20(17), 1126,1980$.

62. D. C. Prevosek, Chapter 12 in Reference 31.

63. A. E. Zachariades, W. T. Mead and R. S. Porter in Ultra High Modulus Polymers, A. Ciferri and I. M. Ward, Eds., Appl. Sci., London, 77, 1979.

64. A. J. Pennings and K. E. Meihingen in Ultra High Modulus Polymers, A. Ciferri and I. M. Ward, Eds., London, 117, 1979.

65. P. Smith and P. J. Lemstra, Makromol. Chem., 180, 2983, 1979.

66. J. Preston in Ultra High Modulus Polymers, A. Ciferri and I. M. Ward, Eds., London, 155, 1979.

67. J. R. Schaefgen, T. I. Bair, J. W. Ballou, S. L. Kwolek, P. W. Morgan, M. Pavar and J. Zimmerman in Ultra High Modulus Polymers, A. Ciferri and I. M. Ward, Eds., London, 173, 1979 .

68. N. Franks and J. Varga, U. S. Patent 4,145,532, 1979.

69. G. Marrucci and A. Ciferri, Polym. Lett. Ed., 15, 643, 1977 .

70. B. Valenti and A. Ciferri, Polym. Lett. Ed., 16, 657, 1978

71. D. G. Baird, A. Ciferri, W. R. Krigbaum and F. Salaris, J. Poly. Sci.: Phys. Ed., 17, 1649, 1979. 
72. G. Marrucci and G. C. Sarti in Oltra High Modulus Polymers, A. Ciferri and I. M. Ward, Eds., London, 137, 1979 .

73. A. Ciferri and B. Valenti in Ultra High Modulus Polymers, A. Ciferri and I. M. Ward, Eds., London, 203, 1979.

74. B. Valenti, G. C. Alfonso, A. Ciferri and P. Giordani, J. Appl. Poly. Sci., 26, 3643, 1981.

75. R. S. Werbowyz and D. G. Gray, Mol. Cryst. Liq. Cryst., $34,97,1976$.

76. D. G. Gray, J. Appl. Polym. Sci., Appl. Polym. Symp., 37, 179,1983 .

77. R. D. Gilbert and P. A. Patton, Prog. in Poly. Sci., 9 $(2 / 3), 115,1983$.

78. V. G. Kulichikhin and L. K. Golova, Khim Drer, 3, 9, 1985 .

79. P. Sixou et al., Carbohydra. Polym., 2(4), 2381, 1982.

80. D. G. Gray, Poly. Sci. Technol. (Polymer Liquid Crystal), Plenum, 28, 369, 1985.

81. D. G. Gray, Faraday Discus. Chem. Soc., 79, 257, 1985.

82. R. D. Gilbert in ACs Symp. Ser. No. 433, Chapter 22, 259, 1990 . 
2.0 CELLULOSE-BASED FIBERS FROM LIQUID CRYSTALLINE SOLUTIONS. I. SOLUTION PROPERTIES OF CELLULOSE ESTERS. 


\title{
CELLULOSE-BASED FIBERS FROM \\ LIQUID CRYSTALLINE SOLUTIONS \\ I. SOLUTION PROPERTIES OF CELLULOSE ESTERS
}

\author{
Vipul Davé and Wolfgang G. Glasser \\ Department of Wood Science and Forest Products \\ Biobased Materials Center \\ Virginia Polytechnic Institute and State University \\ Blacksburg, Virginia, USA 24061
}

\begin{abstract}
Solutions of cellulose esters with different concentrations in dimethyl acetamide (DMAc) and with different types of substituents, but with nearly constant total degree of substitution and constant molecular weights, were studied in relation to their liquid crystalline solution behavior. Observations made by dynamic mechanical spectrometry and by cross-polarized optical microscopy revealed classical liquid crystalline behavior for all solutions. Critical polymer concentration levels $\left(V_{p}^{c}\right)$ were observed for all cellulose esters, and these were found to vary with substitution pattern. $V_{p}{ }^{c}$ is highest for cellulose acetate and lowest for the cellulose acetate butyrate with maximum degree of butyration. This is opposite to expected behavior based on the classical model by Flory, which predicts an increase in $V_{p}{ }^{c}$ with decreasing aspect ratio. Cellulose ester solutions are viscoelastic in nature.
\end{abstract}

Rigid rod-like polymers are recognized for their ability to form anisotropic liquid crystalline solutions $(1,2)$. For rod-like species, the classical Flory equation of:

$$
V_{p}^{c} \approx(8 / X)(1-2 / X)
$$

relates the critical volume fraction, $V_{p}{ }^{c}$, of the polymer in solution to the appearance of a stable anisotropic phase; where $X$ is the aspect ratio $(L / d)$ of the polymer; $L$ is the contour length; and 
$d$ is the average diameter of the polymer chain. Cellulose is a linear homopolymer of $\beta$-linked $1,4-$ anhydroglucose units. Liquid crystalline solution behavior has been observed with many cellulose and cellulose derivatives (3). Flory is credited with first commenting on the possibility of mesophase formation in cellulosic polymers (4). The most important parameter controlling the formation of a liquid crystalline phase appears to be chain stiffness for cellulose derivatives (5). The solution conformations of cellulosic polymers studied so far indicate that the cellulose backbone is characterized as a semi-rigid polymer (6). Like other semi-rigid polymers, cellulose and its derivatives are better represented by the Kratky-Porod worm-like chain model rather than the random flight model. For worm-like chains, persistence length $(q)$ is the measure of chain stiffness. It has been shown that the aspect ratio $(X)$ in Equation 1 is related to the persistence length $(q)$ through Equation $2(3)$.

$$
X=2 q / d
$$

Very long worm-like chains behave as random flight models and the persistence length can be related to the equivalent Kuhn segment length $\left(l_{k}\right)$ by Equation 3 .

$$
q=l_{k} / 2
$$

Thus, the stiffness of a worm-like chain may also be characterized by the equivalent Kuhn segment length. Hence, the aspect ratio $X$ in Equation 1 can be represented by Equation 4.

$$
\begin{gathered}
X_{k}=1_{k} / d \\
\text { where } 1_{k}=\left\langle r^{2}>_{\diamond} / n l u\right. \\
\text { and } d=\left(M u / N a_{\rho} l u\right)^{1 / 2}
\end{gathered}
$$

$M u$ is the molecular weight of the repeat unit, $\rho$ is the density of the polymer, $N a$ is the Avogadro number, and $l u$ is the length of the repeat unit projected on the molecular axis (7).

Since persistence length, Kuhn segment length, and diameter are known quantities for a given polymer, values of $V_{p}{ }^{c}$ can be both calculated and experimentally determined. There is, however, not 
an exact agreement between the experimental and theoretical values of $V_{p}{ }^{c}$. For cellulose derivatives, minor deviations between theoretical and experimental values of $V_{p}^{c}$ have been reported $(8,9)$. Attempts have been made to overcome these discrepancies by some appropriate modification of Flory's theory for specific solvents $(10,11)$. Values of $V_{p}{ }^{c}$ are influenced by solvent, temperature, molar mass and nature and degree of substitution mainly because these parameters affect chain conformation and stiffness $(5,12)$. The change of $V_{p}^{c}$ with solvent is primarily due to a change in the persistence length of the polymer chain in solution. Solvents which help in forming intra-chain hydrogen bonds between anhydroglucose units are expected to increase stiffness and hence reduce $V_{p}{ }^{c}$. Highly polar or acidic solvents generally favor mesophase separation at lower $V_{p}^{c}(13)$.

Persistence length decreases as temperature rises; and this influences $V_{p}^{c}$ as well (12).

It has been shown that values for $V_{p}^{e}$ increase with a decrease in molar mass of cellulose derivatives, and these observations are rationalized in terms of chain stiffness $(10,11,14)$.

Unmodified cellulose is soluble only in selected solvents and solvent mixtures at the high concentrations required for the formation of liquid crystalline solutions $(15,16)$. However, a myriad of cellulose derivatives can be formed due to the presence of three hydroxyl groups per anhydroglucose unit that may be available for modification. The presence of side chains; a high degree of substitution (DS); and a non-uniform pattern of substitution all increase the solubility of cellulose derivatives as crystallinity is repressed. The effective aspect ratio $(X$, Equation 1$)$ will also vary with changes in the chain diameter caused by substitution. Long flexible side chains may behave like an internal plasticizer for the cellulose backbone and aid in allowing some mobility to form thermotropic liquid crystals in the absence of a solvent (3).

Much of the current interest in polymeric liquid crystals can be attributed to the extraordinary physical properties that are obtained when solution or melt anisotropy are retained during the solidification process. Although flexible chain polymers orient during processing, retention of this orientation becomes difficult due to fast relaxation. Instead, entanglements seem to control the structure and properties of flexible chain polymers. Rigid polymers, by contrast, have properties 
that result from macroscopic structures which are the consequence of the rheological properties of polymeric liquid crystals (17). The viscosity of the anisotropic solutions (lyotropic liquid crystalline systems) increases with concentration and passes through a maximum with the onset of liquid crystalline order before it decreases with further increase in concentration (18). The liquid crystalline phase exhibits unique birefringence when viewed with the polarized light microscope under static conditions; and this phenomenon corresponds very closely to the concentration at which maximum viscosity is observed (19).

Commercially, polymeric liquid crystals are important because they can be processed into ultrahigh modulus fibers. There is always a good possibility to convert polymer liquid crystalline solutions into high performance fibers, and to date cellulose triacetate and Kevlar have shown this behavior. In order to understand the processing of liquid crystalline solutions, it is essential to characterize their rheological properties. The objective of the present study is to examine the rheological and morphological properties of liquid crystalline solutions of cellulose esters (cellulose acetate and cellulose acetate butyrate) in dimethyl acetamide (DMAc) in relation to the substitution pattern of butyrate and acetate substituents on the cellulose backbone.

\section{Experimental}

Materials. Cellulose acetate (CA) and cellulose acetate butyrates (CAB) were obtained from Eastman Kodak, Kingsport, Tennessee, as either commercial or experimental samples. The samples obtained were CA 394-60 (CA), CAB 171-15 (CAB-1), CAB 381-20 (CAB-2), and CAB 500-5 (CAB-3). Their average acetyl contents were 39.4, 29.5, 13.5, and $4.0 \mathrm{wt} . \%$, respectively; their butyryl contents were $0,17.1,38.1$, and $50.0 \mathrm{wt} . \%$, respectively; and their falling-ball viscosities were 60 (228 poise), 15 (57 poise), 20 (76 poise), and 5 (19 poise) seconds, respectively.

Reagent grade dimethyl acetamide (DMAc) was used as received.

Methods. Degree of substitution (DS) by acetate and butyrate groups on the cellulose backbone was determined using a JEOL Model GX-270 NMR spectrometer (operating at $270 \mathrm{MHz}$ for ${ }^{1} \mathrm{H}$ ) at $80^{\circ} \mathrm{C}(20,21)$. Deuterated dimethyl sulfoxide (DMSO) with a trace of trifluoroacetic acid served as 
the solvent.

The molecular parameters (molecular weight, Mark-Houwink-Sakurada constants, and intrinsic viscosity) of the cellulose esters were determined using gel permeation chromatography with a differential viscosity detector (Viscotek Model No. 100) and a differential refractive index (concentration) detector (Waters 410) in sequence. Solutions of the cellulose esters were prepared in HPLC-grade tetrahydrofuran (THF), and all the calculations were based on a universial calibration curve.

A complete range of solutions $[5-50 \%(w / w)]$ were prepared by mechanically mixing known weights of the cellulose esters with DMAc at ambient temperature. The solutions were allowed to equilibrate for 1-2 weeks prior to analysis. Extreme care was taken to treat the solutions similarly so that the relative aging effect is constant for all of them.

Viscosity measurement of the solutions below $20 \%(\mathrm{w} / \mathrm{w})$ concentration were made at $25^{\circ} \mathrm{C}$ using a Wells-Brookfield Cone/Plate Viscometer at different cone rotation speeds. A Rheometrics Mechanical Spectrometer (RMS 800) was used to determine the rheological properties of $20 \%$ (w/w) and higher concentrated solutions. The solutions were placed in a parallel-disk geometry. The dynamic mechanical properties were measured at $26^{\circ} \mathrm{C}$ using a strain amplitude of $25 \%$ of the value at which the respective sample showed viscoelasticity. The frequency ranged from 1.0 to 100.0 $\mathrm{rad} / \mathrm{sec}$.

Polarized light microscopy was performed with a Zeiss Axioplan Universal Microscope. Small portions of a cellulose solution were placed between microscope slide and cover slip, and this was examined for birefringence between the cross polarizers of the microscope at room temperature.

\section{Results and Discussion}

The chemical and molecular characteristics of the four cellulose ester derivatives employed in this study are summarized in Table I. The degree of substitution (DS) of acetyl (DS ${ }_{A C}$ ) and butyryl $\left(D_{B u}\right)$ was evaluated by proton NMR spectroscopy $(20,21)$. DS ${ }_{O H}$ was determined by difference [e.g., for $\left.\mathrm{CA} ; \mathrm{DS}_{O H}=3-2.44=0.56\right]$. The results (Table I) indicate that $\mathrm{DS}_{O H}$ is almost constant for all four cellulose ester derivatives with the exception of CA; that molecular weights differ only by a 
factor of 1.5 to 2; that the Mark-Houwink-Sakurada (MHS) constant is almost constant by classifying all four cellulose esters as semi-flexible polymer chains $(a \approx 0.90)$; and that intrinsic viscosity varies by less than a factor of 1.4. Although it is recognized that all factors, i.e., DS $O H$ (22), molecular weight $(10,11,14,22)$, chain flexibility $(6)$, and intrinsic viscosity $(10,11)$, influence liquid crystalline solution properties, the cellulose ester derivatives employed in this study differ mainly with regard to substitution pattern. No systematic study with respect to the effect of substitution pattern on liquid crystalline solution behavior of cellulose ester derivatives has been reported.

Table I. Chemical and Molecular Characteristics of Cellulose Esters.

\begin{tabular}{|c|c|c|c|c|}
\hline & CA & CAB-1 & $\mathrm{CAB}-2$ & CAB-3 \\
\hline $\mathrm{DS}_{A C^{1}}$ & 2.44 & 2.03 & 0.96 & 0.29 \\
\hline $\mathrm{DS}_{B u}{ }^{1}$ & 0 & 0.8 & 1.77 & 2.57 \\
\hline $\mathrm{DS}_{O H}{ }^{2}$ & 0.56 & 0.17 & 0.27 & 0.14 \\
\hline $\bar{M}_{n}\left(\times 10^{3}\right)$ & 41.5 & 66.8 & 60.0 & 52.6 \\
\hline $\bar{M}_{v}\left(\times 10^{3}\right)$ & 76.8 & 176.0 & 143.0 & 123.0 \\
\hline MHS - Constant (a) & 0.90 & 0.92 & 0.98 & 0.95 \\
\hline Intrinsic Viscosity $(\mathrm{dL} / \mathrm{g})$ & 1.88 & 1.63 & 2.07 & 1.51 \\
\hline
\end{tabular}

1 Determined by H-NMR spectroscopy.

${ }^{2}$ Determined by difference.

The relationship between dynamic shear viscosity, frequency or shear rate [applying the CoxMerz rule (23)] and concentration of the cellulose ester derivatives is illustrated in Figure 1. All four cellulose ester samples exhibit an increase in viscosity with increasing concentration up to a maximum before decreasing with further increase in concentration (i.e., after $V_{p}{ }^{c}$ ). Such an anomalous viscosity behavior is typical for liquid crystalline solutions, and this has been shown earlier for cellulose acetate $(22,24)$, ethyl cellulose (25), hydroxypropyl cellulose $(24,26)$, cellulose triacetate $(27)$, and cellulose acetate butyrate $(12,28)$. This change in viscosity is due to a change in the structure of the solutions. Baird has proposed an explanation that as the concentration rises, 


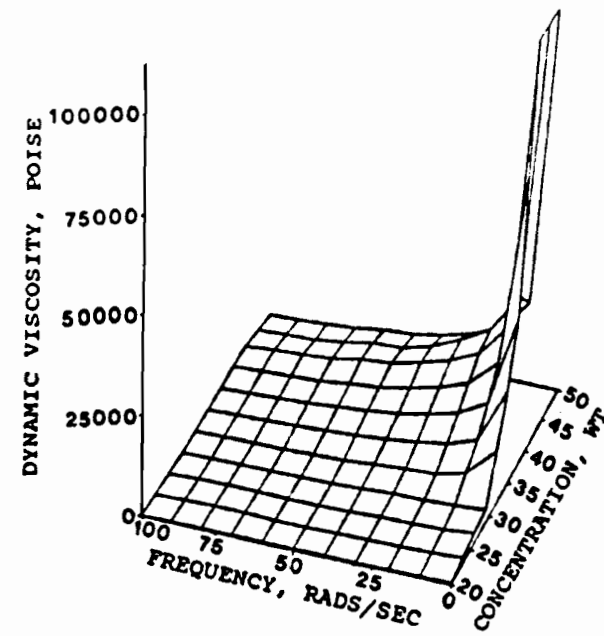

(a)

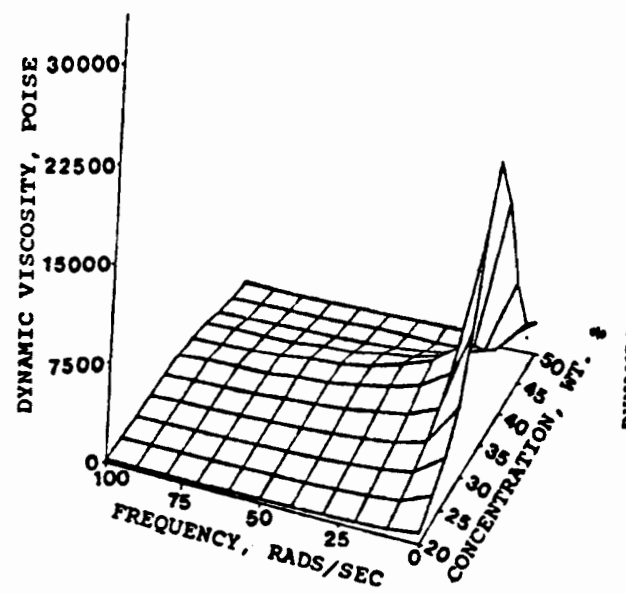

(c)

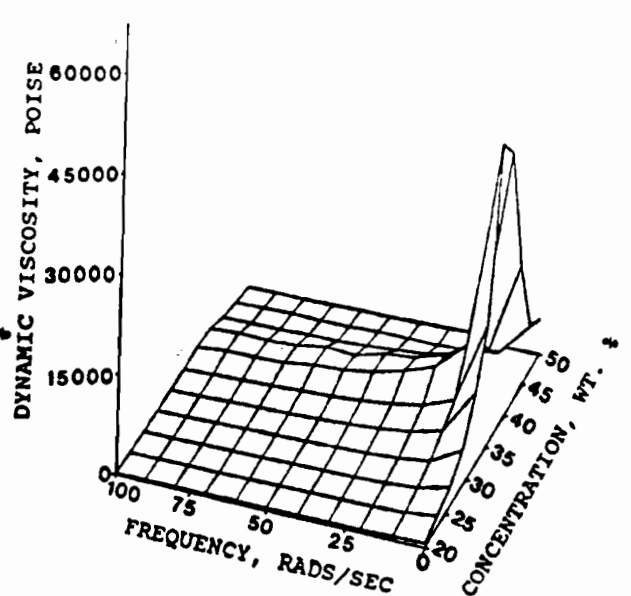

(b)

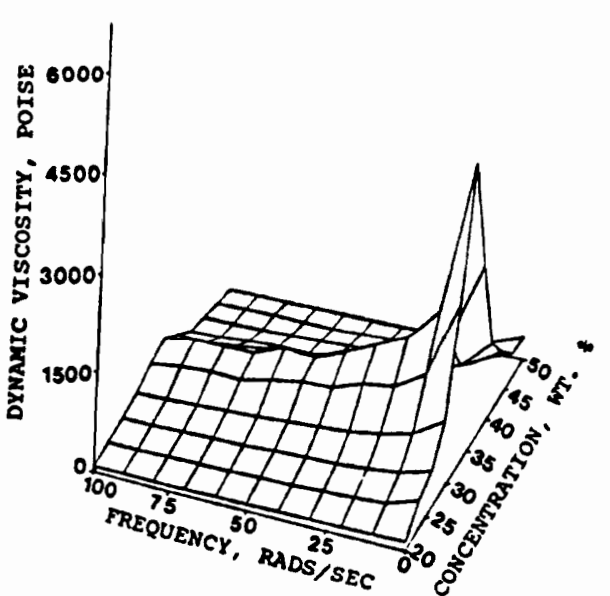

(d)

Figure 1. Dynamic viscosity versus frequency for different concentration solutions of cellulose esters in dimethylacetamide; (a) CA (b) CAB-1 (c) CAB-2 (d) CAB-3. 
the size and density of the ordered regions increases and a suspension of ordered clusters in an isotropic matrix is created (29). The energy dissipated by these clusters during flow is less than the total sum of the energy dissipated by each individual molecule; hence, the viscosity decreases with concentration. Another possible explanation for this anomalous viscosity behavior is that beyond $V_{p}{ }^{c}$ there may be a competition between ordering due to shear and ordering due to packing of the molecules in solution. The viscosity initially decreases with concentration at levels exceeding $V_{p}{ }^{c}$ as a consequence of shear ordering; and viscosity eventually increases again at higher concentrations as packing effects become important (24).

All four cellulose ester samples display a viscosity peak at constant concentration, irrespective of shear frequency. This indicates that unlike steady shear, dynamic shear is not responsible for driving the thermodynamic transition of the anisotropic phase to a different concentration (30). Previous investigators have made similar observations $(22,24,26,30)$. The overall magnitude of the dynamic shear viscosity peak decreases as the frequency increases. This behavior has been justified by several mechanisms. Hermans (31) suspected that there might be a competition between the ordering produced by shear and the ordering produced due to thermodynamic reasons. At high shear rates, the former dominates over the latter one. It is also possible that with increasing shear rates there is orientation of the rigid segments of macromolecular chains under flow giving rise to macroscopic texture (32). This ultimately causes the viscosity to decline. At high frequencies, the viscosity values are almost the same for all concentrations.

The drop in viscosity with increase in shear rate is known as "shear thinning" or "pseudoplastic behavior," and this phenomenon begins several decades of shear rate lower for an anisotropic solution than for an isotropic one of the same viscosity (33). The dependence of viscosity on shear rate for polymer liquid crystals has been divided into a three-region flow curve by Onogi and Asada (34). In region I, the apparent viscosity decreases rapidly with increasing shear rate. Region II is the plateau region sometimes called Newtonian region, and region III is the power-law shear thinning region. According to the three-region flow curve, all four cellulose esters seem to represent region I 
or region III behavior. Assuming region III is applicable for the cellulose esters, shear thinning is more pronounced for $\mathrm{CA}$ and reduces with high $\mathrm{DS}_{B u}$ (CAB's).

The rise in viscosity with polymer concentration is more pronounced for CA than for CAB's (Figure 1). The relationship between shear viscosity at $1 \mathrm{rad} / \mathrm{sec}$ frequency and concentration for the four cellulose esters (Figure 2) reveals that CA exhibits a greater increase in viscosity than CAB, and that the viscosity rise diminishes with butyryl content. Simultaneously, $V_{p}{ }^{c}$ shifts to lower concentrations as $\mathrm{DS}_{B u}$ rises (Figure 2). This shift in $V_{p}{ }^{c}$ cannot be accounted for by hydrogen bonds or molecular weight, as both of these parameters are almost constant for all four cellulose esters. Although it is not surprising that $V_{p}{ }^{e}$ is related to substituent size (and bulk), a decrease in $V_{p}^{c}$ with increase in substituent bulk is contrary to expectations. The experimental values of $V_{p}^{c}$ for $\mathrm{CA}$ and $\mathrm{CAB}-3$, i.e., 0.38 and 0.29 , are in reasonable agreement with those obtained by Dayan et al. [0.49] (22) and Bheda et al. [0.30] (12), respectively. The CAB used by Bheda et al. had unspecified characteristics in terms of its DS, and so it becomes difficult to correlate their CAB with the ones used in this study.

In order to verify the experimental values of $V_{p}{ }^{c}$, theoretical calculations of $V_{p}{ }^{c}$ are made using Equations 1-4 (see Appendix A). ${ }^{1}$ Diameter and molecular weight of the repeat unit increase from

1 The following direct quotation is taken from Flory (7): "Tanner and Berry (35) had obtained $\left\langle r^{2}\right\rangle_{\circ} / n=1080 \AA^{2}$ for secondary acetate (CA) of degree of substitution 2.45 dissolved in trifluoroacetic acid or in a mixture of methylene chloride and methanol. They found larger values of 1350 or greater for cellulose triacetate (CTA); Kamide, Miyazaki and Abe (38), on the other hand, report lower values of ca. 600 for CTA. The disparity may reflect the difficulties caused by aggregation of CTA. Hence, we adopt the same value for CTA as for CA, on the plausible grounds that they should be similar in this respect." Tanner and Berry had obtained relatively narrow range of values of $\left\langle r^{2}\right\rangle_{o} / n$ for cellulose hexanoate, cellulose nitrate and cellulose $N$-phenyl-carbamate in different solvent systems.

Light scattering measurements of some selected fractions of cellulose acetate butyrate $(29.5 \%$ acetyl and $17 \%$ butyryl by weight) in nitromethane and in ethyl acetate were carried out (39). The results were discarded because the apparent molecular weight varied from solvent to solvent; there were inconsistent changes of the second virial coefficient and root-mean-square end-to-end distance with the nature of solvent; and the curvilinear nature of the scattered light intensity envelops made extrapolation inaccurate. These problems could be due to the fact that the cellulose acetate butyrate chains are heterogeneous in composition (40-42).

It seems difficult to experimentally obtain coherent $\left\langle r^{2}\right\rangle_{0} / n$ values for cellulose acetate butyrates. Hence, it is reasonable to assume the same $\left\langle r^{2}\right\rangle_{0} / n$ (i.e., $1080 \AA^{2}$ ) value for the cellulose esters used in this study even though Tanner and Berry did not measure them. 


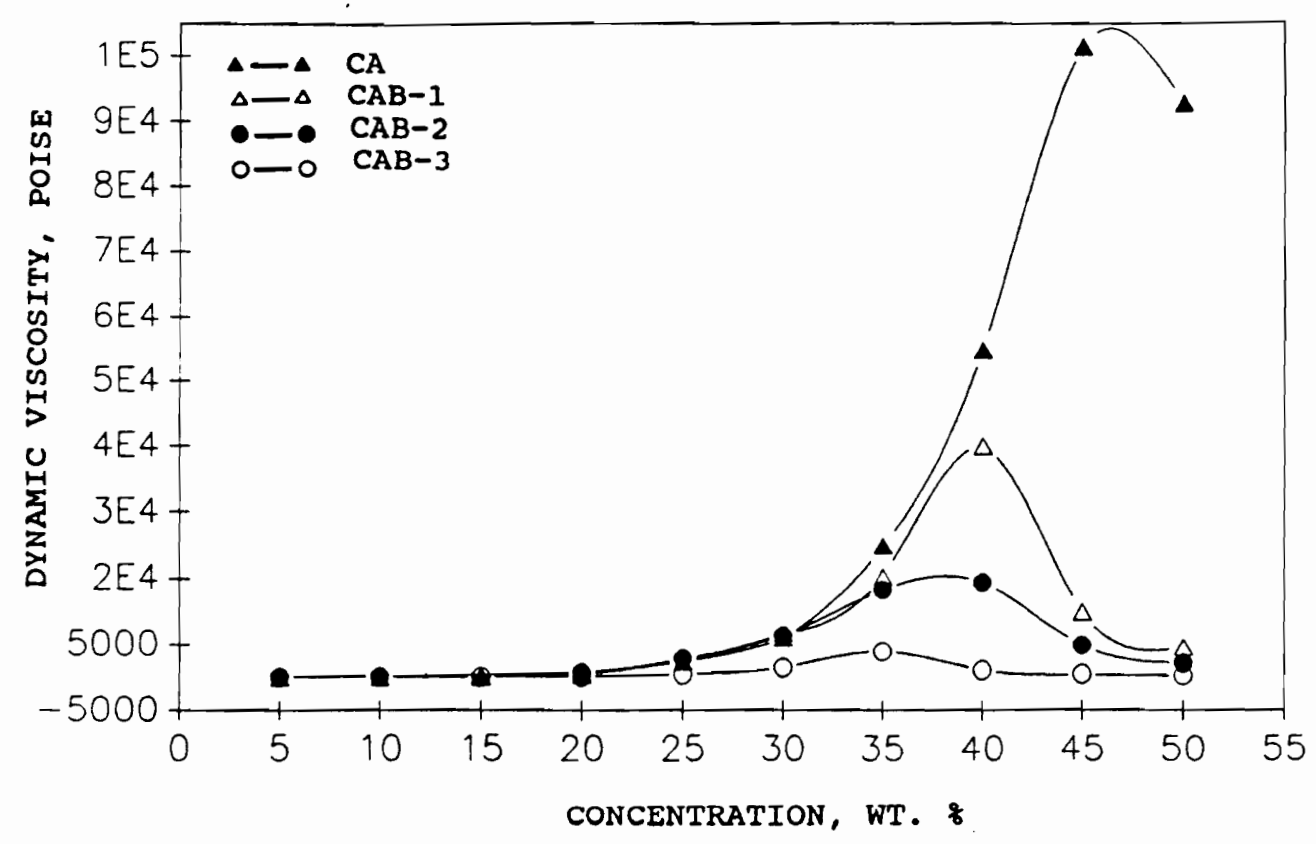

Figure 2. Low-frequency limit (1 rad/sec) of dynamic viscosity versus concentration for cellulose esters in dimethylacetamide. 
CA to CAB-3 (Table II). This observation is expected because the substituent bulk increases. The Kuhn segment length and persistence length of all the cellulose esters is constant. This implies that the chain stiffness does not change with an increase in substituent bulk and is in agreement with the unchanged value of MHS constant (a) for all cellulose esters. It can be inferred that the value of the axial ratio, $\mathrm{X}_{k}$, is affected only by $\mathrm{Mu}$. As $\mathrm{Mu}$ increases from 264.5 to $354.1, \mathrm{X}_{k}$ diminishes from 25.77 to 21.40 and simultaneously, the theoretical value of $V_{p}{ }^{c}$ increases from 0.29 to 0.34 . There is fairly good agreement between the absolute experimental and theoretical values of $V_{p}^{c}$ (Table II), but there is poor correlation in the trend of change in $V_{p}^{c}$ with $\mathrm{Mu}$ (Figure 3); i.e., $\left(\mathrm{dV}_{p}{ }^{c} / d M u\right)_{\text {expt }}<0$ and $\left(\mathrm{dV}_{p}{ }^{c} / d M u\right)_{T h}$. $>0$. Such an opposite trend is unexpected and conflicts with Flory's theory. In the derivation of Equation 1, the intermolecular and intramolecular interactions were not considered and the value of $V_{p} c$ is based only on geometrical aspects of the molecules. The orientation-dependent or anisotropic interactions considered by Flory and Ronca (36) may play an important role in concentrated solutions exhibiting liquid crystalline behavior. As $\mathrm{Mu}$ increases, there is crowding on the backbone which may lead to intermolecular and intramolecular interactions causing sufficient stiffening to form liquid-crystalline solutions at lower $V_{p}{ }^{c}$. The stiffness of the chains is much higher than predicted by the MHS equation. These reasons may explain the discrepancy in the experimental and theoretical values of $V_{p}{ }^{c}$. Based on these arguments, the shift to lower $V_{p}^{c}$ values with increasing substituent size may possibly be attributed to an increase in the chain stiffness caused by intermolecular and intramolecular interactions. This unexpected relationship has not yet been reported in the literature for cellulose esters. When the experimental $V_{p}{ }^{c}$ values are extrapolated to the molecular weight of anhydroglucose unit, i.e., 162 (Figure 3), $\left(V_{p}^{c}\right)_{\text {expt. }}$ is approximately 0.48 , and this is somewhat close to that predicted on theoretical grounds by Gilbert, i.e., $0.70(27)$.

The relationship of dynamic elastic modulus $\left(G^{\prime}\right)$ and dynamic loss modulus $\left(G^{\prime \prime}\right)$ at different frequency and concentration of the four cellulose ester solutions is summarized in Figures 4 and 5 , respectively. $G^{\prime}$ represents the amount of energy stored per cycle of deformation and $G^{\prime \prime}$ represents 


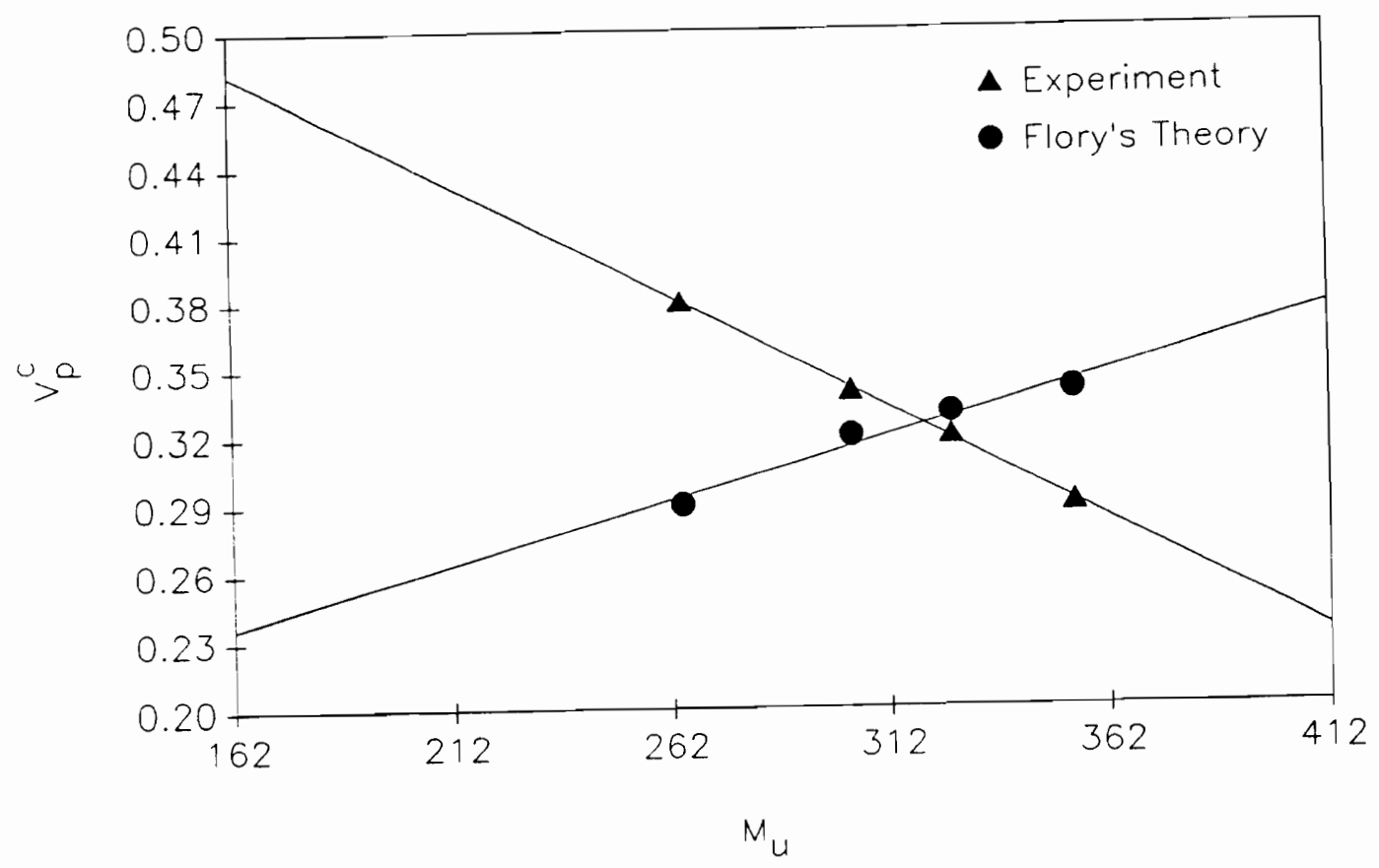

Figure 3. Experimental and theoretical variation of critical volume fraction of cellulose esters with molecular weight of the repeating unit. 


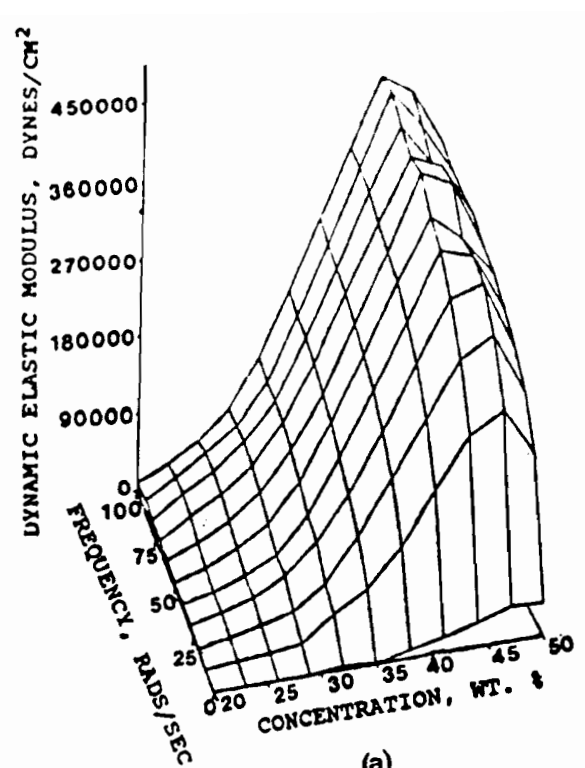

(a)

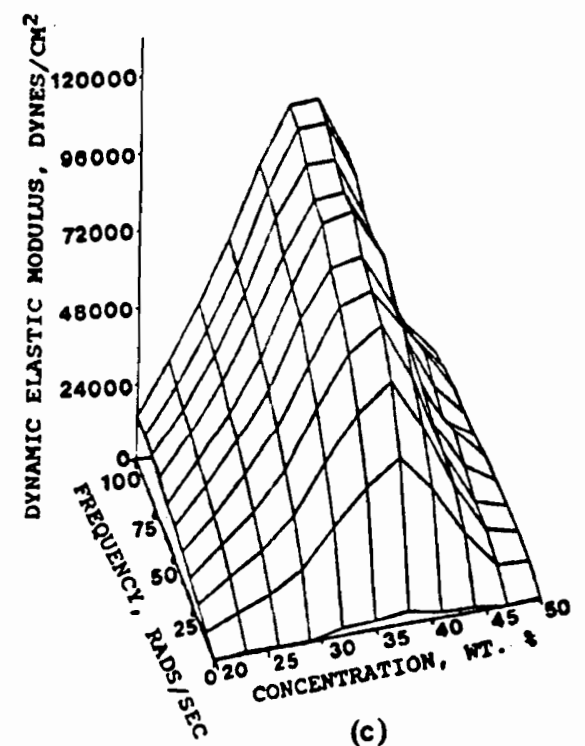

(c)

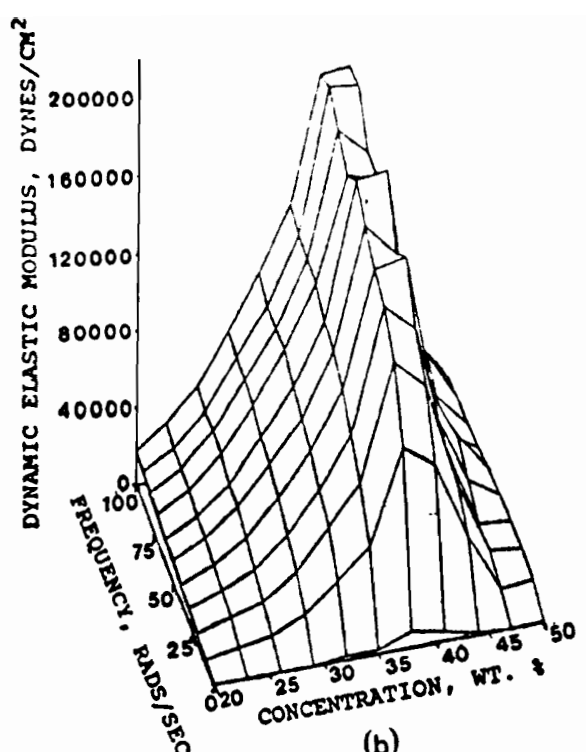

(b)

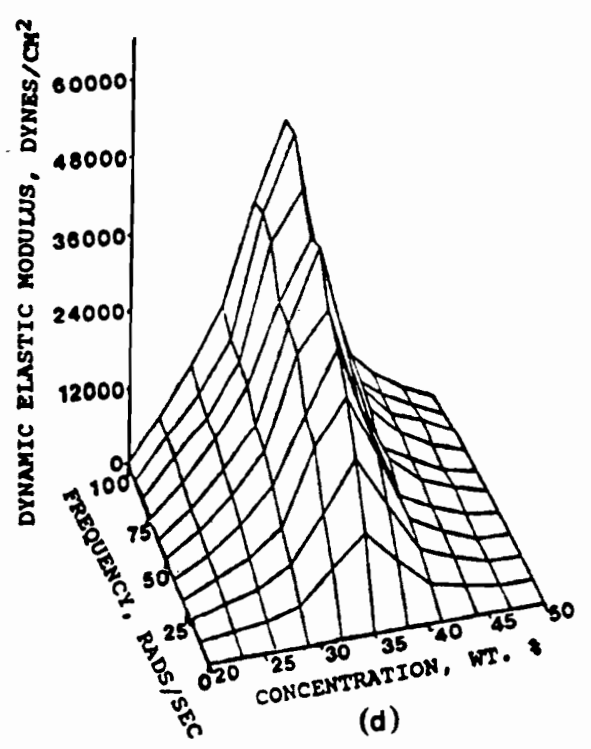

(d)

Figure 4. Dynamic elastic modulus versus frequency for different corcentration solutions of cellulose esters in dimethylacetamide; (a) CA (b) CAB-1 (c) CAB-2 (d) CAB-3. 


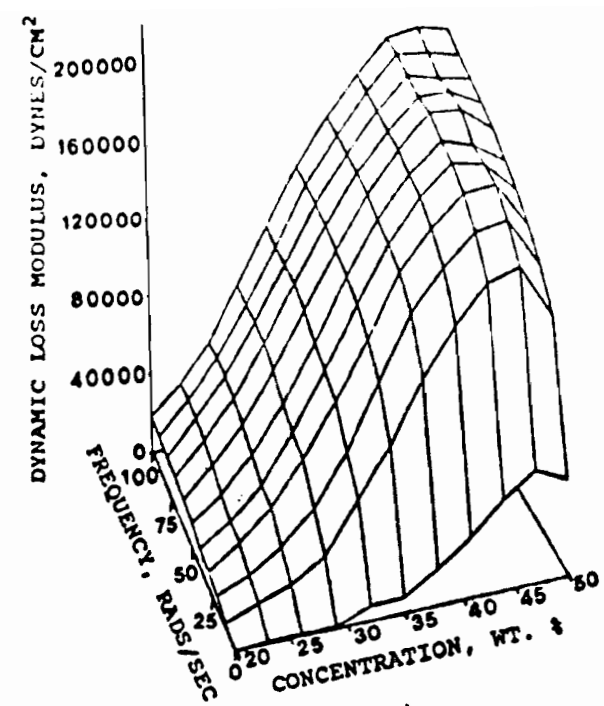

(a)

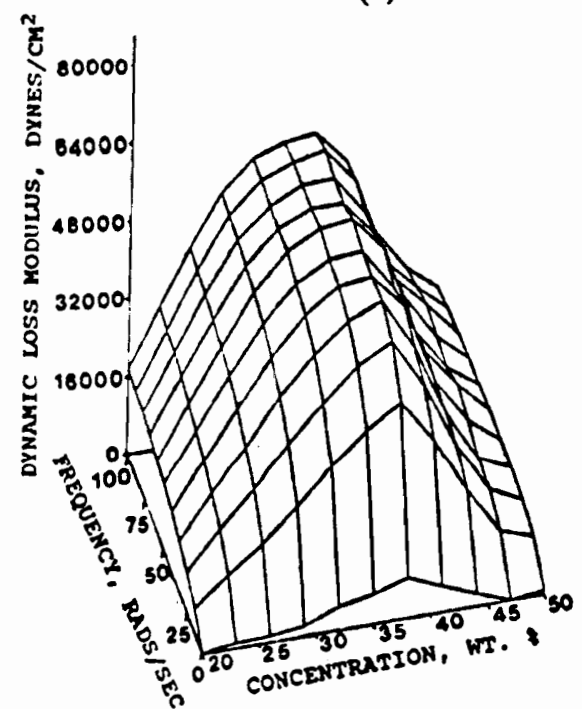

(c)

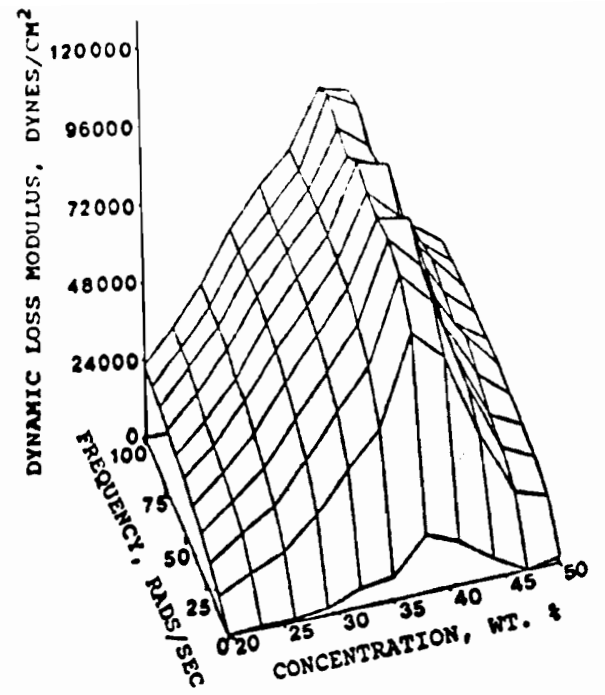

(b)

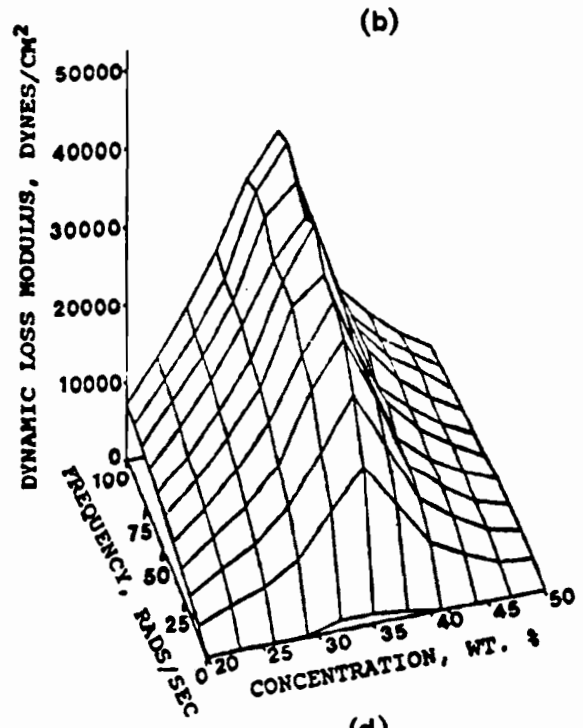

(d)

Figure 5. Dynamic loss modulus versus frequency for different concentration solutions of cellulose esters in dimethylacetamide; (a) $\mathrm{CA}$ (b) $\mathrm{CAB}-1$ (c) CAB-2 (d) CAB-3. 
Table II. Theoretical Values of Molecular Parameters for Cellulose Esters.

\begin{tabular}{lcccccccc}
\hline Cellulose & \multicolumn{3}{c}{$\mathrm{Mu}}$, & & & & Th. & Expt. \\
Esters & $\rho, \mathrm{g} / \mathrm{cm}^{3}$ & $\mathrm{~g} / \mathrm{mole}$ & $\mathrm{d}, \AA$ & $\mathrm{l}_{k}, \AA$ & $q, \AA$ & $\mathrm{X}_{k}$ & $V_{p}{ }^{c}$ & $V_{p}{ }^{c}$ \\
\hline $\mathrm{CA}$ & 1.3 & 264.5 & 8.06 & 207.7 & 103.85 & 25.77 & 0.29 & 0.38 \\
$\mathrm{CAB}-1$ & 1.2 & 303.3 & 8.98 & 207.7 & 103.85 & 23.13 & 0.32 & 0.34 \\
$\mathrm{CAB}-2$ & 1.2 & 326.2 & 9.32 & 207.7 & 103.85 & 22.29 & 0.33 & 0.32 \\
$\mathrm{CAB}-3$ & 1.2 & 354.1 & 9.71 & 207.7 & 103.85 & 21.40 & 0.34 & 0.29 \\
\hline
\end{tabular}

the energy loss per cycle of deformation. The feature of the concentration dependence of $\mathrm{G}^{\prime}$ and $\mathrm{G}^{\prime \prime}$ is the same as that seen for dynamic viscosity, i.e., after the solution becomes anisotropic $G^{\prime}$ and $\mathrm{G}^{\prime \prime}$ values decline. The solutions are seen to become more elastic with increasing concentration and even in the liquid crystalline phase its nature is that of a liquid rather than of a crystal. This is so because the solution will be highly elastic if the continuous liquid crystal structure is perfectly preserved (37). The liquid crystalline solutions are less elastic and some of the elastic properties can be due to regions of isotropic fluid dispersed in anisotropic regions (18). Similar to any viscoelastic material, $\mathrm{G}^{\prime}$ increases with increasing frequency. As the $\mathrm{DS}_{B u}$ increases, there is a gradual drop in $\mathrm{G}^{\prime}$ value and the solutions become less elastic.

$G^{\prime \prime}$ rises with increase in frequency. After reaching $V_{p}{ }^{c}, G^{\prime \prime}$ falls showing that damping characteristics of liquid crystalline solutions are lower than those of isotropic solutions. The rise of damping behavior with frequency is most pronounced with CA and least with CAB-3.

These results reveal that liquid crystalline state is fluid in nature and these systems are viscoelastic, i.e., they both store and dissipate energy during deformation.

During exposure to shear, such as during viscosity measurements, the phases are microscopically intermingled and they fail to reach a state of true equilibrium. In the absence of shear, phase equilibrium will be established, and the phases can be identified by cross-polarized light microscopy (19). Cross-polarization helps to distinguish isotropic (I), biphasic (B), and anisotropic (A) solution character empirically. Polarized light microscopy observations for solutions containing 5-50\% (w/w) 


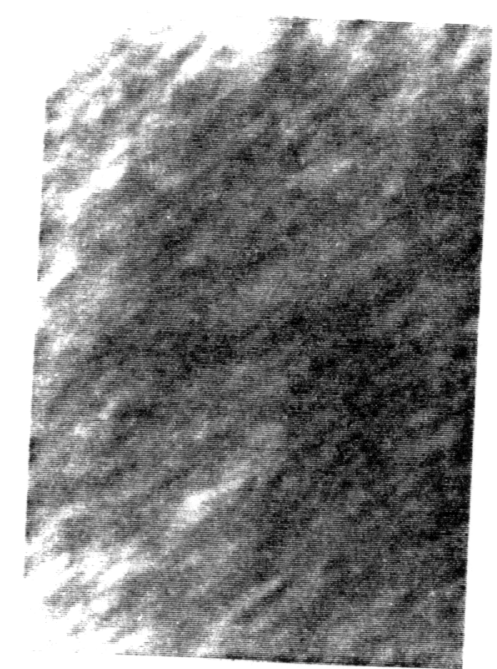

(a)

$50 \mu \mathrm{m}$

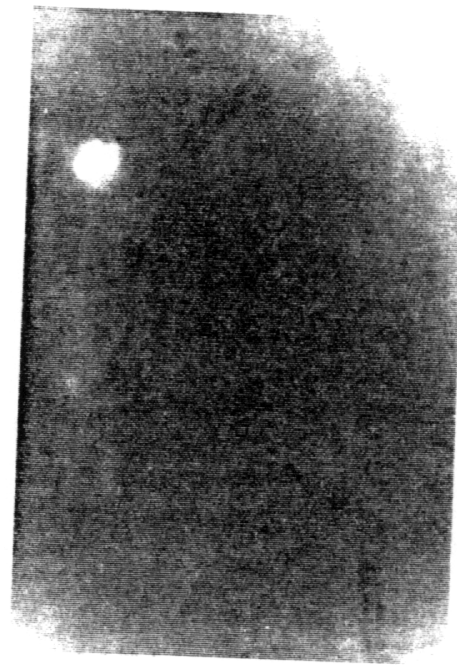

(b)

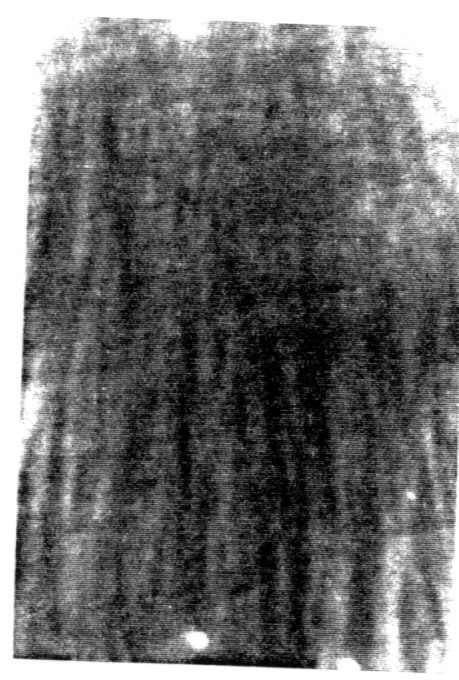

(C)

$50 \mu \mathrm{m}$

Figure 6. Polarized optical micrographs of cellulose ester solutions in dimethylacetamide; (a) CA, 50\% (w/w) conc. (b) CAB-1, 45\% (w/w) (c) $\mathrm{CAB}-1,50 \%(\mathrm{w} / \mathrm{w})$ conc. 


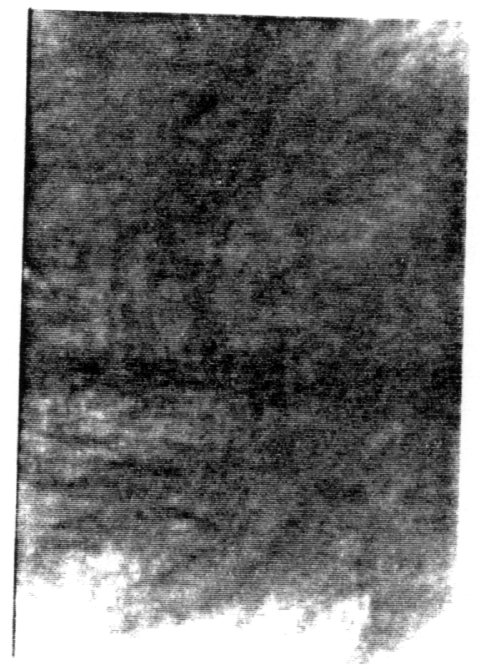

(a)

$50 \mu \mathrm{m}$

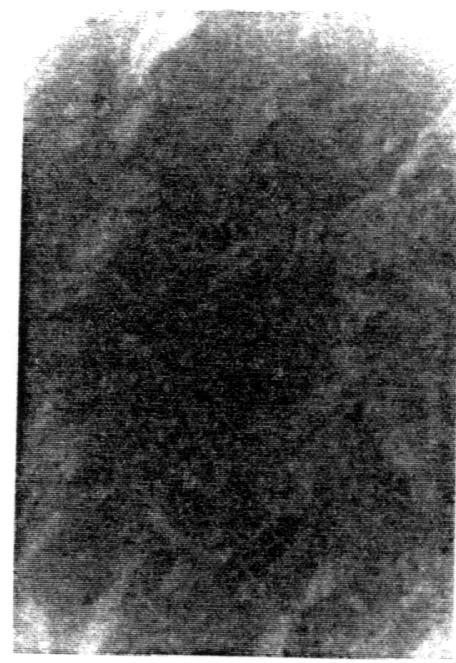

(C)

$50 \mu \mathrm{m}$

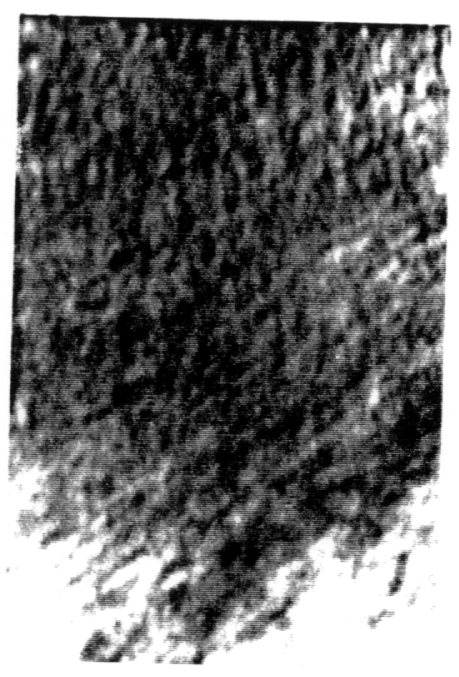

(b)

$50 \mu \mathrm{m}$

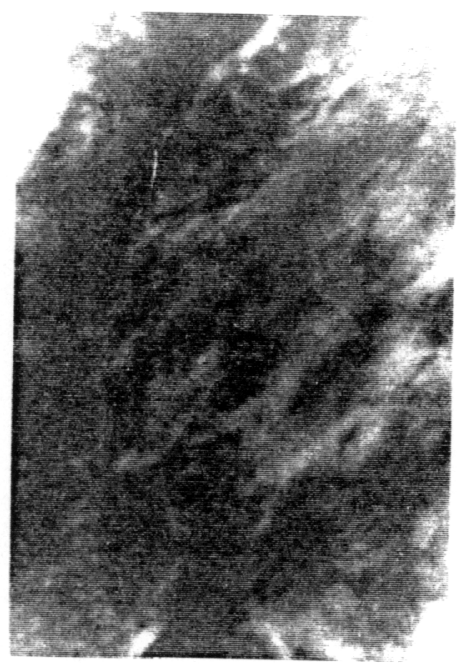

$50 \mu \mathrm{m}$

Figure 7. Polarized optical micrographs of cellulose ester solutions in dimethylacetamide; (a) CAB-2, 40\% (w/w) conc. (b) CAB-2, 45\% (w/w) conc. (c) $\mathrm{CAB}-3,30 \%(w / w)$ conc. (d) $\mathrm{CAB}-3,40 \%$ (w/w) conc. 


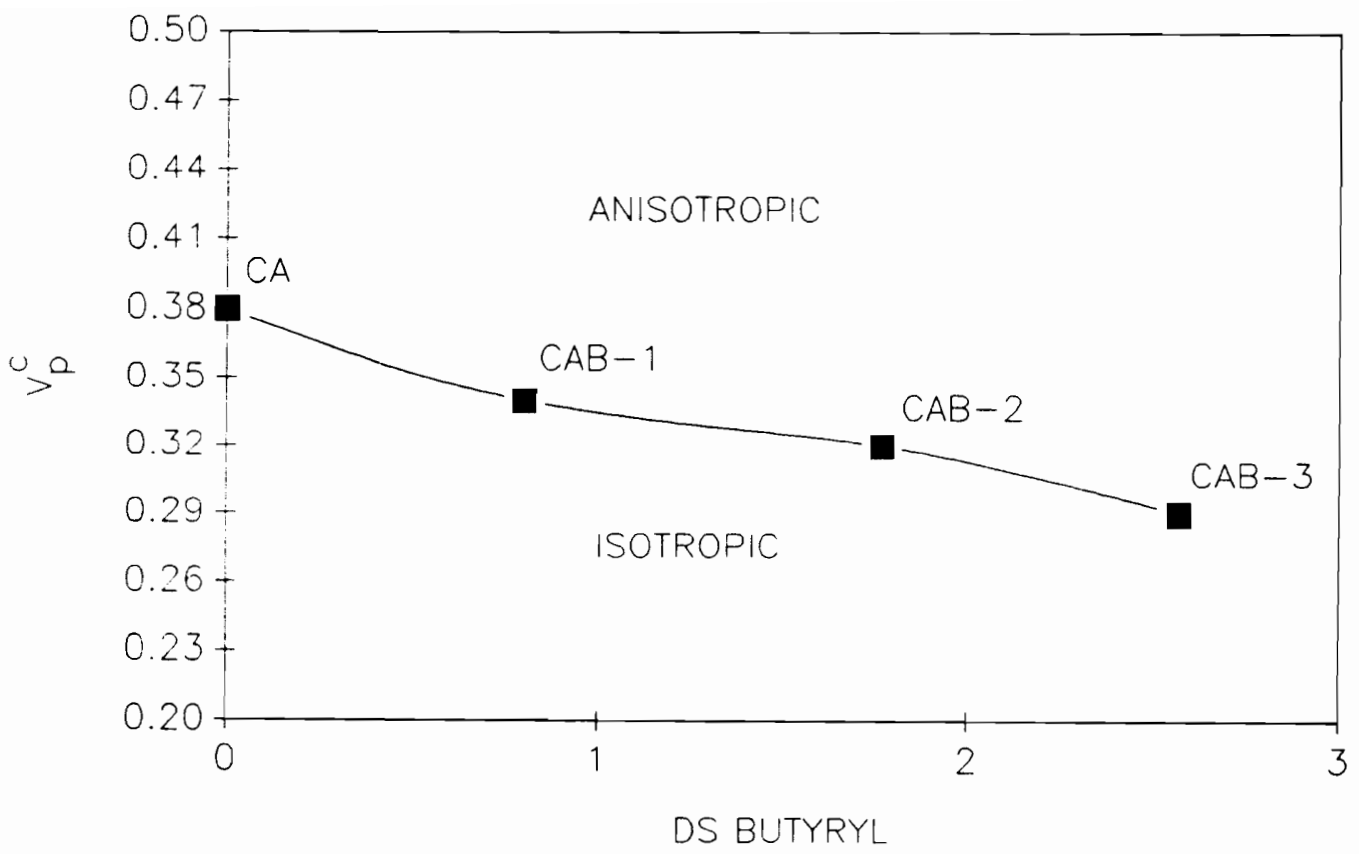

Figure 8. Structure-Property Map summarizing viscosity, morphology and chemistry data for cellulose ester solutions in dimethylacetamide. 
cellulose esters in DMAc are summarized in Table III. A distinctive band of biphasic behavior is recognized that is shifted to higher concentrations as butyryl content declines. The liquid crystalline phase appears at approximately the same concentration in this static experiment as the peak viscosity did in the dynamic test (Figure 2). A similar behavior had been observed for other lyotropic systems as well (19).

Table III. Summary of Polarized Light Microscopy Observations for Solutions Containing 5-50\% (w/w) Cellulose Esters in Dimethyl Acetamide.

\begin{tabular}{lcccccccccc}
\hline Sample & $5 \%$ & $10 \%$ & $15 \%$ & $20 \%$ & $25 \%$ & $30 \%$ & $35 \%$ & $40 \%$ & $45 \%$ & $50 \%$ \\
\hline CA & I & I & I & I & I & I & I & I & I & B \\
CAB-1 & I & I & I & I & I & I & I & I & B & A \\
CAB-2 & I & I & I & I & I & I & I & B & A & A \\
CAB-3 & I & I & I & I & I & I & B & B & A & A \\
\hline
\end{tabular}

Note: I, B, and A designate isotropic, biphasic, and anisotropic, respectively.

The photomicrographs of the four cellulose ester solutions illustrate the change in solution morphology in relation to concentration (Figures 6 and 7). Figure 6a shows that for CA, the solution becomes birefringent at approximately $50 \%$ concentration. The change from B to A for CAB-1 and CAB-2 is shown in Figures $6 \mathrm{~b}$ and $6 \mathrm{c}$ and Figures $7 \mathrm{a}$ and $7 \mathrm{~b}$, respectively. The transformation from isotropic to anisotropic phase for CAB-3 is seen in Figures $7 \mathrm{c}$ and $7 \mathrm{~d}$.

A structure-property map (Figure 8) is prepared which summarizes the information from rheology, morphology, and chemistry data for the cellulose ester solutions. $V_{p}^{c}$ represents the peak viscosity values from Figure 2. The map shows that as the $\mathrm{DS}_{B u}$ (chemistry) increases, the peak viscosity (rheology) falls and the solutions become anisotropic (morphology) at lower concentrations.

\section{Conclusion}

Cellulose acetate and cellulose acetate butyrate solutions in DMAc exhibit classical liquid crystalline solution behavior in agreement with earlier studies on cellulose derivatives. 
$V_{p}{ }^{c}$ is strongly related to the substitution pattern and it declines with increasing degree of butyration. This is contrary to the expected behavior since an increased substituent bulk (Mu) increases the diameter of the molecular chain and thereby increase the $V_{p}{ }^{c}$. This anomaly is explained with increased chain stiffness due to interchain and intrachain interaction caused by substituent crowding on the backbone. This implies that solution anisotropy can be reached at lower concentrations with cellulose esters with large and bulky substituents as compared to smaller ones. In spite of the anomaly, there is fairly good agreement between the absolute experimental and theoretical values of $V_{p}^{c}$ but not in the trend of $\mathrm{d} V_{p}^{c} / \mathrm{dMu}$.

There is a good correlation between dynamic viscosity measurements and the static crosspolarized light microscopy results.

The liquid crystalline state is fluid and the cellulose ester solutions are viscoelastic in nature.

\section{Acknowledgments}

Financial support for this study was provided by the NSF Science and Technology Center on High Performance Polymers and Adhesives of Virginia Tech, and by the Biobased Materials Center of the Center for Innovative Technology at Virginia Tech. The authors would like to thank Dr. H. Marand (Chemistry) and Dr. D. G. Baird (Chemical Engineering) for their help with polarized light microscopy and RMS, respectively. Helpful counsel by Dr. Rick Davis (Chemical Engineering) is acknowledged with gratitude.

\section{Appendix A}

1. Calculation of molecular weights of repeat units, $\mathrm{Mu}$ :

$\mathrm{CA} ; \quad \mathrm{Mu}=2.44 \times 42+162=264.5$

CAB-1; $\quad \mathrm{Mu}=2.03 \times 42+0.8 \times 70+162=303.30$

CAB-2; $\quad \mathrm{Mu}=0.96 \times 42+1.77 \times 70+162=326.2$

CAB-3; $\quad \mathrm{Mu}=0.29 \times 42+2.57 \times 70+162=354.1$

2. Calculation of chain diameter, $\mathrm{d}: \mathrm{d}=\left(\mathrm{Mu} / \mathrm{N}_{A} \rho / u\right)^{1 / 2}$ 
$l u=5.2 \AA(7) ; N_{A}=6.02 \times 10^{23}$ molecules $/$ mole.

$$
d_{C A}=\left(\frac{264.5}{6.02 \times 10^{23} \times 1.3 \times 10^{-24} \times 5.2}\right)^{1 / 2}=8.06 \AA
$$

3. Calculation of Kuhn segment length, $l_{k}: l_{k}=\left\langle r^{2}\right\rangle_{0} / n l u$;

From light-scattering [Tanner and Berry, (35)];

$<r^{2}>_{\circ} / n=1080 \AA^{2}$

A narrow range of values of $\left\langle r^{2}\right\rangle_{0} / n$ have been reported for diverse cellulose esters (35); therefore, $1080 \AA^{2}$ is assumed for all the cellulose esters in this study (see footnote in text).

$l_{k}=1080 / 5.2=207.70 \AA$

4. Calculation of persistence length, $q: q=l_{k} / 2=207.70 / 2=103.85 \AA$

5. Calculation of aspect ratio, $X_{k}: X_{k}=l_{k} / d_{C A}=\frac{207.70}{8.06}=25.77$

6. Calculation of critical concentration, $V_{p}{ }^{c}: V_{p}^{c}=\left(8 / X_{k}\right)\left(1-2 / X_{k}\right) ; V_{p}^{c} C_{A}=8 / 25.77(1-$ $2 / 25.77)=0.29$

\section{Literature Cited}

1. Papkov, S. P.; Kulichikhin, V. G.; Kalmkoya, V. D.; Malkin, A. Ya. J. Polym. Sci.: Polym. Phys. Ed. 1974, 12, 1753-1770.

2. Morgan, P. W. Macromolecules 1977, 10, 1381-1390.

3. Gray, D. G. J. Appl. Polym. Sci.: Appl. Polym. Symp. 1983, 37, 179-192.

4. Flory, P. J. Proc. R. Soc. London, Ser. A 1956, 234, 60.

5. Gray, D. G. Faraday Discuss. Chem. Soc. 1985, 79, 257-264.

6. Gilbert, R. D.; Patton, P. A. Prog. Polym. Sci. 1983, 9, 115-131.

7. Flory, P. J. Adv. in Polym. Sci. 1984, 59, 2-36.

8. Werbowyj, R. S.; Gray, D. G. Macromolecules 1980, 13(1), 69.

9. Werbowyj, R. S.; Gray, D. G. Mol. Cryst. Liq. Cryst. 1976, 34, 97. 
10. Conio, G.; Bianchi, E.; Ciferri, A.; Tealdi, A.; Aden, M. A. Macromolecules 1983, 16, 1264-1270.

11. Aden, M. A.; Bianchi, E.; Ciferri, A.; Conio, G.; Tealdi, A. Macromolecules 1984, 17, 2010-2015.

12. Bheda, J.; Fellers, J. F.; White, J. L. Colloid and Polymer Sci. 1980, 258, 1335-1342.

13. Aharoni, S. M. Mol. Cryst. Liq. Cryst. 1980, 56, 237.

14. Bhadani, S. N.; Tseng, S. L.; Gray, D. G. Makromol. Chem. 1983, 184, 1727-1740.

15. Chanzy, H.; Peguy, A.; Chaunis, S.; Monzie, P. J. Polym. Sci.: Polym. Phys. Ed. 1980, 18, 1137.

16. Patel, D. L.; Gilbert, R. D. J. Polym. Sci.: Polym. Phys. Ed. 1981, 19, 1231.

17. Baird, D. G. In Polymeric Liquid Crystals; Blumstein, A., Ed.; New York: Plenum Press, 1985; pp 119-143.

18. Baird, D. G. In Rheology; Astarita, G.; Marrucci, G.; Nicolais, L., Eds.; New York: Plenum Press, 1980; Vol. 3, pp 647-658.

19. Aharoni, S. M. Polymer 1980, 21, 1413-1422.

20. Buchanan, C. M.; Hyatt, J. A.; Lowman, D. W. Macromolecules 1987, 20, 2750-2754.

21. Buchanan, C. M.; Hyatt, J. A.; Lowman, D. W. Carbohydr. Res. 1988, 177, 228-234.

22. Dayan, S.; Maissa, P.; Vellutini, M. J.; Sixou, P. J. Polym. Sci.: Polym. Lett. Ed. 1982, 20, 33-43.

23. Bird, R. B.; Armstrong, R. C.; Hassager, O. In Dynamics of Polymeric Liquids; 1987; Vol. 1, p 150.

24. Dayan, S.; Gilli, J. M.; Sixou, P. J. Appl. Polym. Sci. 1983, 28, 1527-1534.

25. Suto, S. J. Polym. Sci.: Polym. Phys. Ed. 1984, 22, 637-646.

26. Navard, P.; Haudin, J. M. J. Polym. Sci.: Polym. Phys. Ed. 1986, 35, 189-201.

27. Hong, Y. K.; Hawkinson, D. E.; Kohout, E.; Garrard, A.; Fornes, R. E.; Gilbert, R. D. In Polymer Association Structure; El-Nokaly, M. A., Ed.; ACS Symp. Ser. 384, 1989; Ch. 12, pp 184-203.

28. Bheda, J.; Fellers, J. G.; White, J. L. J. Appl. Polym. Sci. 1981, 26, 3955-3961.

29. Baird, D. G. J. Rheology 1980, 24(4), 465-482. 
30. Kiss, G.; Porter, R. S. J. Polym. Sci.: Polym. Symp. \#65 1978, 193-211.

31. Hermans, J. J. Colloid Sci. 1962, 17, 638.

32. Kiss, G.; Porter, R. S. J. Polym. Sci.: Polym. Phys. Ed. 1980, 18, 361.

33. Wissbrun, K. F. J. Rheology 1981, 25(6), 619-662.

34. Onogi, S.; Asada, T. In Rheology; Astarita, G.; Marrucci, G.; Nicolais, L., Eds.; New York: Plenum Press, 1980; Vol. I, pp 127-147.

35. Tanner, D. W.; Berry, G. C. J. Polym. Sci.: Polym. Phys. Ed. 1974, 12, 941.

36. Flory, P. J.; Ronca, G. Mol. Cryst. Liq. Cryst. 1979, 54, 311.

37. Iizuka, E. Mol. Cryst. Liq. Cryst. 1974, 25, 287-298.

38. Kamide, K.; Miyazaki, Y.; Abe, T. Polym. J. 1979, 11, 523.

39. Vyas, N. G.; Shashikant, S.; Patel, C. K.; Patel, R. D. J. Polym. Sci.: Polym. Phys. Ed. 1979, $17,2021$.

40. Goodlett, V. W.; Dougherty, J. T.; Patton, H. W. J. Polym. Sci. 1971, A-1, 9, 155.

41. Vasilév, B. V.; Grishin, E. P.; Zhegalova, N. N.; Malinine, L. P.; Pogosov, Yu. L. Vysokomol. Soedin. 1974, Ser. A, 16, 136.

42. Baker, W. O. Ind. Eng. Chem. 1945, 37, 246. 
3.0 CELLULOSE-BASED FIBERS FROM LIQUID CRYSTALLINE SOLUTIONS.

II. PROCESSING AND MORPHOLOGY OF ACETATE/BUTYRATE ESTERS. 


\title{
CELLULOSE-BASED FIBERS FROM LIQUID CRYSTALLINE SOLUTIONS.
}

\author{
II. Processing and Morphology of Acetate/Butyrate Esters.
}

\begin{abstract}
Fibers were spun from isotropic and anisotropic dimethylacetamide solutions of cellulose esters. Take-up speeds of the dry jet/wet spinning process varied. Water served as coagulant. The mechanical properties of the fibers increased as spinning progressed from the isotropic to the anisotropic solution state. A trade-off was noted for butyryl to acetyl ratio, solubility and fiber properties. Whereas high butyryl content enhances both overall solubility and the formation of liquid crystalline solutions at lower concentration, it results in lower fiber modulus and strength. Morphology of the fibers depended on the coagulation rate which was influenced by the concentration of the spinning solution. The level of orientation and crystallinity of the fibers increased when they were spun from liquid crystalline solutions. This study demonstrates that fiber spinning from liquid crystalline solutions is the only method by which to obtain solid state ordered materials from cellulose acetate /butyrate esters.
\end{abstract}




\section{INTRODUCTION}

Panar and wilcox were the first to identify that cellulose acetate butyrate ( $C A B)$, cellulose acetate ( $C A)$, cellulose triacetate (CTA) and other cellulose derivatives form liquid crystalline solutions in various solvents (1). Bheda et al. (2) confirmed this behavior for CAB by structural investigations. Thermal analysis, quiescent polarized optical microscopy, and rheological investigations (3) demonstrated that $C A B$ formed an isotropic, viscoelastic melt similar to flexible chain polymers like polystyrene.

The liquid crystalline behavior of $C A$ was studied in thirty solvents by Aharoni $(4,5)$ and the effects of molecular weight and degree of substitution on this behavior was investigated by Dayan et al. (6). NMR (7) and DSC investigations (8) for anisotropic solutions of $C A$ in trifluoroacetic acid have been reported. Detailed analysis of the mesophase formation of $C A$ in dimethylacetamide was done by Ciferri et al. (9).

Patel and Gilbert (10-12) showed that CTA formed mesophases in mixtures of trifluoroacetic acid (TFA) and chlorinated alkanes. Extensive studies of this system were reported by several investigators (13-15). Thermal behavior of these liquid crystalline solutions in TFA have also been reported (16-19). Sixou et al. (20) showed that circular dichroism of cholesteric CTA solutions in trifluoroacetic acid 
relied on the molecular weight of CTA. Navard et al. (19) studied gel formation and liquid crystallinity in trifluoroacetic acid-water solutions of CTA. Bheda et al. (2) showed that CTA formed mesophase also in dichloroacetic acid. Fiber spinning from liquid crystalline solutions of $C A B$, CA, CTA and other cellulose derivatives was patented in 1976 (1). A preliminary study (21) on wet spinning was extended to anisotropic solutions of $\mathrm{CAB}$ in dimethylacetamide and CTA in trifluoroacetic acid. CTA fibers were also wet spun from liquid crystalline solutions of trifluoroacetic acid-methylene chloride $(13,22)$ and trifluoroacetic acid-water (22). Wet spinning from acetic acid-water (23) and dry spinning from acetone (24) of CA fibers from isotropic solutions have also been reported. In addition to cellulose esters, fiber spinning studies were also carried out for liquid crystalline melts (25) and solutions (26) of hydroxypropyl cellulose.

No systematic study has been done on fiber spinning from liquid crystalline solutions of cellulose esters in relation to substitution pattern of acetate and butyrate substituents on the cellulose backbone. In our earlier paper in this series, we have reported the liquid crystalline solution properties of cellulose acetate and different cellulose acetate butyrates (27). Critical volume fraction $\left(V_{p}^{c}\right)$ was observed for all the cellulose esters, and these were found to vary with substitution pattern. $V_{p}^{c}$ was highest for cellulose 
acetate and lowest for cellulose acetate butyrate with maximum degree of butyration. The main objective of this study is to establish structure-property relationships of the fibers spun from isotropic and anisotropic solutions of the cellulose esters (cellulose acetate and cellulose acetate butyrates).

\section{EXPERIMENTAL}

\section{Materials.}

Cellulose acetate (CA) and cellulose acetate butyrate (CAB) were obtained from Eastman Kodak, Kingsport, TN as either commercial or experimental samples. The samples obtained were $C A$ 394-60 (CA), CAB 171-15 (CAB-1), CAB 381-20 $(C A B-2)$ and $C A B$ 500-5 ( $C A B-3)$. Their average acetyl contents were $39.4,29.5,13.5$ and 4.0 weight respectively; and their average butyryl contents were $0,17.1,38.1$ and 50.0 weight $\%$ respectively. The chemical and molecular characteristics of the cellulose esters were described in our previous publication (27).

Reagent grade dimethylacetamide (DMAC) was used as received.

II. Methods.

1. Preparation of Concentrated Fiber Spinning Dopes:

Concentrated stock solutions were prepared by mechanically mixing weighed amounts of cellulose esters and DMAC at room temperature. Polymer concentration of stock solution, $C_{p}$, given as grams of cellulose ester per 100 grams 
of binary (cellulose ester + DMAC) solution, varied between $25 \%$ to $50 \%$ (Table I). The solutions were allowed to equilibrate for 2-3 weeks prior to fiber spinning. The critical concentration, $C_{p}{ }^{\prime}$, at which the liquid crystalline phase appears was determined by rheological measurements supported by optical microscopy (27).

2. Fiber Spinning:

(a) Design of Fiber Spinning Apparatus.

Continuous fibers were processed using the dry jet/wet spinning method which Blades (28) introduced for liquidcrystalline solutions of p-linked aromatic polyamides. The spinning apparatus consisted of (i) Solution Delivery System, (ii) Spinning Pack Assembly, (iii) Capillary Spinnerette, (iv) Coagulation Bath, and (v) Take-up Device. The delivery system comprised of a programmable infusion and withdrawal syringe pump (Harvard Apparatus). The pump was capable of delivering the solutions at a constant steady flow rate via a plunger. The cylindrical spinning pack assembly functioned as solution reservoir. The combination of the pump and the cylindrical pack assembly operated similar to a piston-cylinder unit. The volume of the reservoir was approximately $100 \mathrm{~cm}^{3}$. The capillary spinnerette had a single hole with capillary diameter of $0.3 \mathrm{~mm}$, capillary length of $1.2 \mathrm{~mm}$ (i.e., L/D Ratio $=4$ ) and entrance angle of $60^{\circ}$. The spinnerette was fitted at the bottom of the spinning pack assembly. The 
dimensions of the coagulation bath were 1 meter (length) $x$ 0.15 meter (breadth) $\times 0.11$ meter (depth). Water was used as the coagulant at approximately $30^{\circ} \mathrm{C}$. Take-up device had interchangeable spool tubes on which the wet-spun fibers were wound up as they emerged from the coagulation bath. The take-up speed of the spool tubes could be varied to produce fibers at different draw ratio.

(b) Fiber Spinning:

Concentrated solutions were transferred into the cylindrical reservoir. They were extruded through the singlehole spinnerette by the pump into the coagulation bath at a steady flow rate of $1.0 \mathrm{~cm}^{3} / \mathrm{min}$. The air gap between the spinnerette and the water surface was approximately 1 inch. The fibers were wound on the spools at various take-up speeds, $\mathrm{V}_{\mathrm{L}}$. To assure complete removal of solvent from the fibers, the fiber-wound spools were immersed in beakers containing water for 8 hours. The spools were allowed to air-dry for 48 hours, and then vacuum oven-dried for 24 hours at approximately $50^{\circ} \mathrm{C}$. No post-spinning treatments (thermal or mechanical) were performed.

3. Fiber Diameter:

Fiber diameter (D) was determined by a Nikon UM-2 Measurescope equipped with a Quadra-Chek III attachment. The diameter was generally constant along an extended length of fiber. The reported $D$ value in Table $I$ is the average of three 
to four measurements along the fiber length.

4. Mechanical Properties:

The mechanical properties of the fibers were determined on Instron 1130 Test Instrument following the procedures from ASTM D 3822. Tests were conducted at room temperature and $67 \%$ relative humidity on single fiber with 1 inch gauge length at strain rate of 0.2 inches/min. Load - elongation curve and linear density (mass per unit length) were used to determine the mechanical properties of the fibers. Linear density varied between 95 and 380 denier. The values of initial modulus (E), breaking tenacity $\left(\sigma_{b}\right)$, breaking toughness (BT) and elongation at break $\left(\epsilon_{\mathrm{b}}\right)$ represented averages of 6-10 measurements. 5. Scanning Electron Microscopy:

The fibers were observed on a JEOL JSM-35C scanning electron microscope with an accelerating voltage of $15 \mathrm{kV}$. Fracture surfaces were formed in liquid nitrogen. The fibers were mounted on aluminum specimen mounts (EMSL) with an angle of $45^{\circ}$. To avoid electron charging effects, the fibers were coated at the bottom by Ladd Silver conducting Paint and were then sputter coated by pure gold for 1 minute (thickness $~ 9$ $n m)$ in a SPI sputter coater.

6. Wide-Angle X-Ray Scattering:

The wide angle $x$-ray patterns were taken on a table-top Phillips PW 1720 x-ray generator model PW 1170, set at $40 \mathrm{kV}$ and 20 milli-amperes, equipped with a standard vacuum sealed 
Warhus photographic pinhole camera. A bundle of 6-8 fibers were used for each pattern and the exposure time was 20 hours. The x-ray beam was perpendicular to fiber surface. Calculations of d-spacing were based on Bragg's law ( $\mathrm{n} \lambda=2 \mathrm{~d}$ $\sin \theta)$, where $\mathrm{n}$ is a positive integer, $\lambda$ is the wavelength of radiation ( $\lambda=1.54 \AA$ ), and $2 \theta$ is the scattering angle.

7. Differential Scanning Calorimetry:

The thermal analysis of the fibers was determined on a DuPont $912 \mathrm{DSC}$ at a heating rate of $10^{\circ} \mathrm{C} / \mathrm{min}$ under nitrogen atmosphere from $30^{\circ} \mathrm{C}$ to $280^{\circ} \mathrm{C}$. After the first heating run, the samples were quenched to $30^{\circ} \mathrm{C}$ and re-heated to $280^{\circ} \mathrm{C}$ to observe the glass transition temperature of the material. During the sample preparation, the fibers were cut into small pieces and were placed very carefully into the DSC pans. The pans were then sealed cautiously. The weight of the samples was low and varied between 3 to $6 \mathrm{mgs}$. as it was difficult to fill the pan completely with short light-weight fibers.

8. Fiber Shrinkage Experiment:

Single fibers, 3 inches long and spun at same take-up speeds from isotropic and anisotropic solutions of $C A B-1, C A B-$ 2 and $C A B-3$ were heated in a vacuum oven above the respective glass transition temperatures for 30 minutes. The length of the fibers was again measured after cooling them to room temperature. 
Table I. Spinning Conditions and Mechanical Properties of Fibers.

\begin{tabular}{|c|c|c|c|c|c|c|c|c|}
\hline $\begin{array}{c}C_{p} \\
(w / w z)\end{array}$ & Phase ${ }^{\text {l) }}$ & $\begin{array}{c}\mathrm{V}_{\mathrm{L}}^{2)} \\
\mathrm{m} / \mathrm{min}\end{array}$ & $\begin{array}{l}\text { Draw } \\
\text { Ratio }\end{array}$ & $\begin{array}{l}D \\
\mu \mathrm{m}\end{array}$ & $\begin{array}{c}E \\
\left.g / d^{4}\right)\end{array}$ & $\begin{array}{c}\sigma_{b} \\
g / d\end{array}$ & $\begin{array}{l}\epsilon_{b} \\
q\end{array}$ & $\begin{array}{l}B T^{5)} \\
g / d\end{array}$ \\
\hline$C A-35$ & $I$ & 8.5 & 0.60 & 213 & 25.6 & 1.0 & 41.2 & 0.40 \\
\hline$C A-35$ & $I$ & 11.0 & 0.80 & 193 & 27.7 & 1.1 & 52.6 & 0.50 \\
\hline$C A-35$ & $I$ & 13.6 & 0.97 & 187 & 27.6 & 1.0 & 46.0 & 0.30 \\
\hline $\mathrm{CA}-35$ & $I$ & 15.0 & 1.04 & 173 & 35.1 & 1.2 & 44.8 & 0.50 \\
\hline$C A-35$ & $I$ & 16.6 & 1.18 & 167 & 23.3 & 0.9 & 46.2 & 0.30 \\
\hline$C A B-1-35$ & $I$ & 11.0 & 0.80 & 276 & 19.4 & 0.4 & 8.2 & 0.02 \\
\hline$C A B-1-40$ & $I$ & 11.0 & 0.80 & 315 & 20.7 & 0.5 & 9.3 & 0.03 \\
\hline $\mathrm{CAB}-1-40$ & $I$ & 13.6 & 0.97 & 275 & 23.0 & 0.6 & 10.4 & 0.03 \\
\hline$C A B-1-45$ & $\mathrm{~B}$ & 11.0 & 0.80 & 229 & 24.8 & 1.5 & 21.2 & 0.20 \\
\hline$C A B-1-45$ & B & 15.0 & 1.07 & 191 & 35.6 & 1.9 & 18.0 & 0.22 \\
\hline $\mathrm{CAB}-1-45$ & B & 21.0 & 1.50 & 160 & 48.0 & 2.4 & 19.6 & 0.27 \\
\hline$C A B-1-50$ & $A$ & 11.0 & 0.80 & 178 & 65.2 & 2.9 & 13.0 & 0.27 \\
\hline$C A B-1-50$ & $A$ & 15.0 & 1.07 & 169 & 76.4 & 3.3 & 12.7 & 0.27 \\
\hline$C A B-1-50$ & $\mathrm{~A}$ & 21.0 & 1.50 & 152 & 122 & 3.8 & 10.2 & 0.27 \\
\hline$C A B-2-35$ & $I$ & 7.5 & 0.50 & 378 & 13.8 & 0.3 & 21.0 & 0.06 \\
\hline$C A B-2-40$ & $\mathrm{~B}$ & 11.0 & 0.80 & 252 & 10.3 & 0.5 & 34.3 & 0.13 \\
\hline$C A B-2-45$ & A & 11.0 & 0.80 & 223 & 19.7 & 0.7 & 21.6 & 0.13 \\
\hline$C A B-2-45$ & A & 15.0 & 1.07 & 183 & 22.0 & 0.8 & 17.8 & 0.11 \\
\hline $\mathrm{CAB}-2-45$ & $A$ & 21.0 & 1.50 & 146 & 24.0 & 0.9 & 17.6 & 0.10 \\
\hline$C A B-2-50$ & A & 11.0 & 0.80 & 247 & 21.5 & 0.7 & 14.7 & 0.08 \\
\hline $\mathrm{CAB}-2-50$ & $A$ & 15.0 & 1.07 & 201 & 29.3 & 1.0 & 21.3 & 0.14 \\
\hline $\mathrm{CAB}-2-50$ & A & 21.0 & 1.50 & 199 & 38.4 & 1.1 & 15.2 & 0.11 \\
\hline$C A B-3-25$ & $I$ & 11.0 & 0.80 & 283 & 4.6 & 0.1 & 10.2 & 0.01 \\
\hline$C A B-3-25$ & I & 15.0 & 1.07 & 222 & 9.6 & 0.3 & 17.4 & 0.04 \\
\hline$C A B-3-25$ & I & 21.0 & 1.50 & 204 & 11.0 & 0.4 & 21.6 & 0.05 \\
\hline$C A B-3-40$ & B & 11.0 & 0.80 & 214 & 10.5 & 0.4 & 16.0 & 0.05 \\
\hline$C A B-3-40$ & B & 15.0 & 1.07 & 156 & 12.2 & 0.6 & 21.3 & 0.08 \\
\hline$C A B-3-40$ & $\mathbf{B}$ & 21.0 & 1.50 & 155 & 22.6 & 0.8 & 11.0 & 0.06 \\
\hline$C A B-3-50$ & A & 11.0 & 0.80 & 220 & 14.0 & 0.5 & 24.4 & 0.09 \\
\hline$C A B-3-50$ & A & 15.0 & 1.07 & 216 & 15.0 & 0.6 & 27.7 & 0.12 \\
\hline$C A B-3-50$ & A & 21.0 & 1.50 & 190 & 16.0 & 0.7 & 26.3 & 0.12 \\
\hline
\end{tabular}

1) I, B, and A designate isotropic, biphasic and anisotropic phases respectively.

2) $V_{L}$ represents the velocity of the take-up roller. $V_{0}$ represents the velocity of the spinning solution in the spinnerette hole and was kept constant at $14 \mathrm{~m} / \mathrm{min}$ for all the fibers.

3) Draw ratio $=V_{L} / V_{0}$.

4) $1 \mathrm{GPa} \approx 8.5 \mathrm{~g} / \mathrm{d}$.

5) $\mathrm{BT}=$ Breaking Toughness. 


\section{RESULTS AND DISCUSSION}

I. Processing:

Table I shows the spinning conditions and mechanical properties of the as-spun fibers. The first column represents the types and concentrations of the cellulose ester solutions from which the fibers were spun. The velocity of the spinning solution in the spinnerette hole, $V_{0}$, was $14 \mathrm{~m} / \mathrm{min}$ and was kept constant for all the fibers. $V_{0}$ was calculated based on the solution flow rate $\left(1.0 \mathrm{~cm}^{3} / \mathrm{min}\right)$ controlled by the pump and the spinnerette hole diameter $(300 \mu \mathrm{m})$. The selection of relatively large spinnerette diameter (compared to industrial standards) was done on purpose to avoid difficulties that may be encountered during spinning from a small hole. The shear rate at the capillary wall for all the solutions was approximately $6300 \mathrm{~s}^{-1}$ for the given $\mathrm{V}_{0}$ and hole diameter. The take-up speed, $V_{L}$, varied between $7.5-21.0 \mathrm{~m} / \mathrm{min}$.

Since all the solutions were elastic (27), there was release of stored energy which resulted in die-swelling of the solutions and seemed to have slowed down the filament velocity as it emerged from the die. At low take-up speeds, draw ratio (i.e., $V_{L} / V_{0}$ ) was less than 1 (Table $I$ ) as it was calculated based on $V_{0}$ instead of the reduced filament velocity. Therefore, the draw ratio values of less than unity do not suggest axial compression of the jet. The diameter of the fibers, spun at the same draw ratio, from isotropic solutions 
was somewhat larger than those from anisotropic solutions (Table I). This maybe an indication of more die-swelling in case of isotropic solutions than anisotropic solutions due to shorter relaxation times. Die swelling for anisotropic solutions is unexpected as they usually have long relaxation times (29-31). However, die swelling had been observed for poly (p-phenylene terephthalamide) during fiber spinning with an air gap using photographic techniques (32) . High L/D (i.e., 4 ) of the die capillary seemed to have reduced die swelling (33) and fiber breakage.

There was a marked difference in the spinnability of isotropic and anisotropic cellulose ester solutions. It was difficult to spin fibers from isotropic solutions at high take-up speeds whereas the anisotropic solutions could be wet spun over a wide range of conditions. Liquid crystalline solutions greatly improved the spinnability, and this is in agreement with some earlier studies (21).

II. Mechanical Properties:

Figures 1 and 2 represent the modulus and the tenacity variations with spinning dope concentrations on going through the isotropic - anisotropic transition at $c_{p}$ ' of the cellulose ester fibers spun at different take-up speeds. CA fibers were spun only from isotropic solution (35\%), as at higher concentrations the pump was unable to extrude the solutions due to extremely high viscosities. Figures 1 and 2 clearly 
(a)

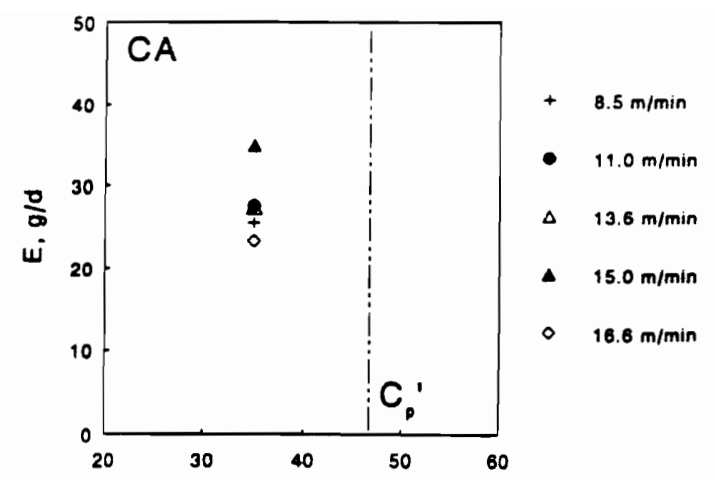

(b)

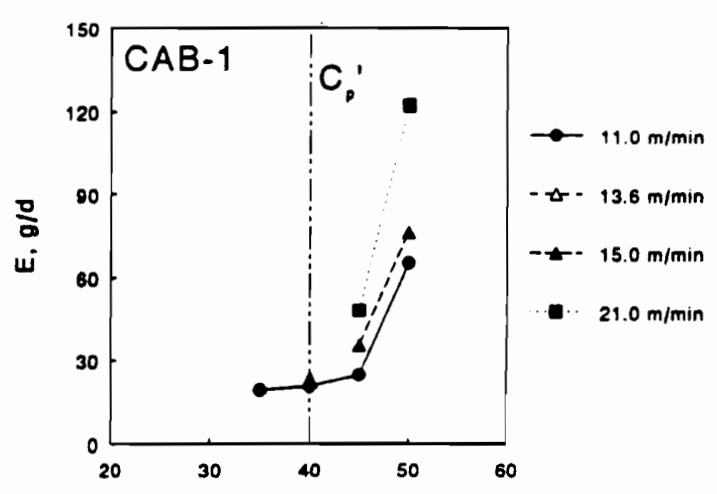

(c)

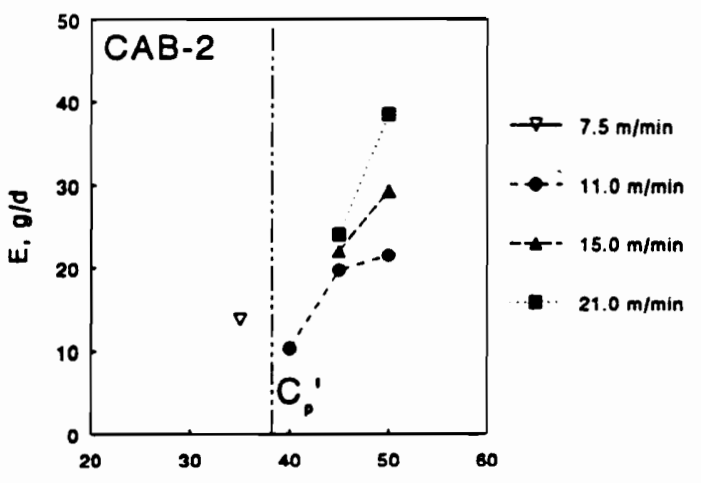

(d)

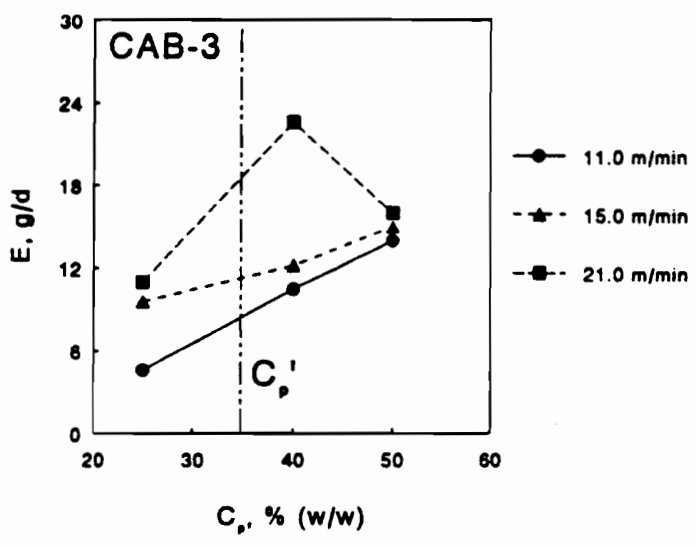

Figure 1. Modulus versus polymer concentration of cellulose ester fibers spun at different take-up speeds; (a) CA (b) CAB-1 (c) CAB-2 (d) CAB-3. 
(a)

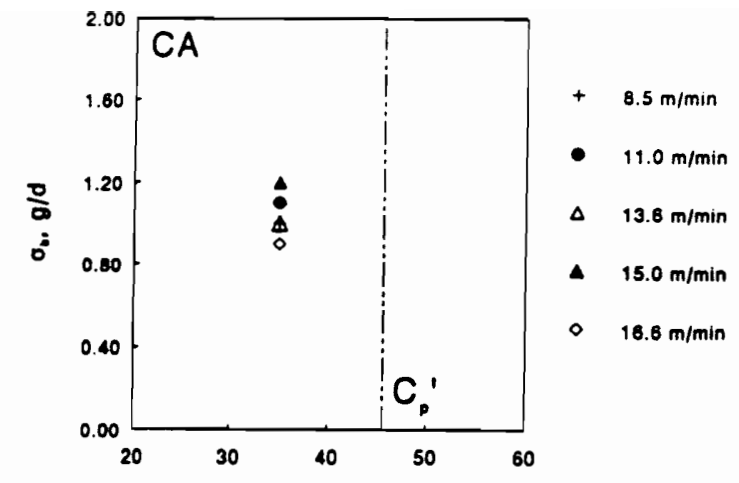

(b)

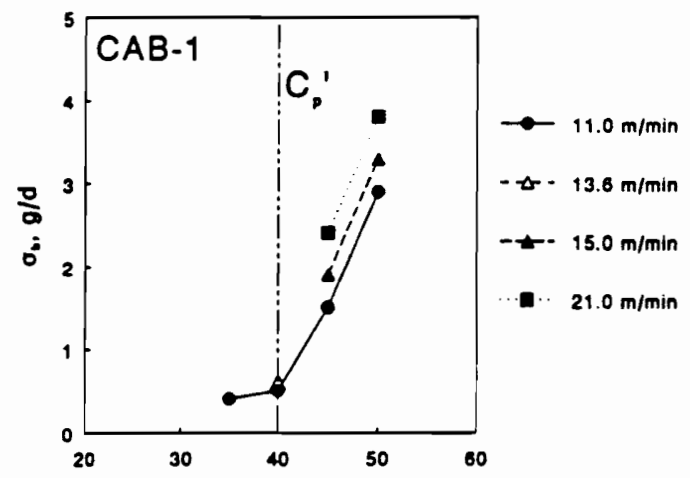

(c)

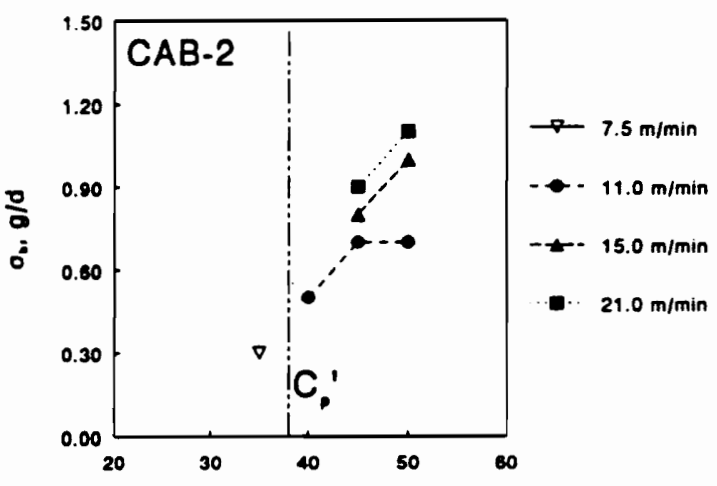

(d)

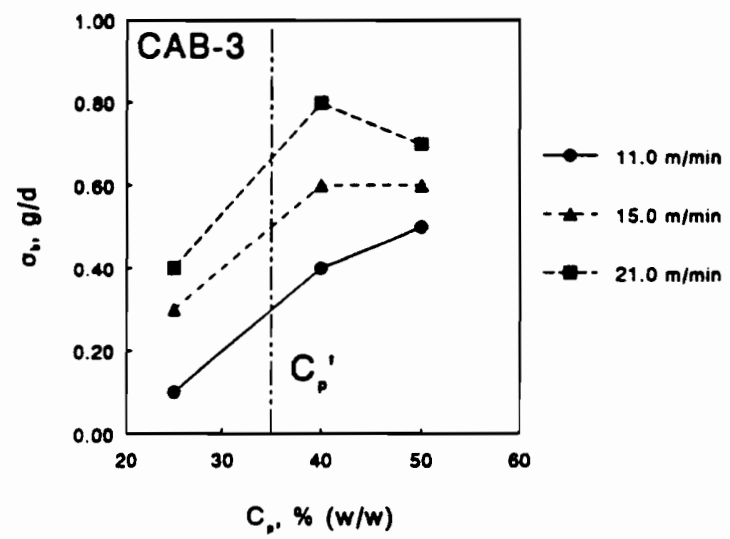

Figure 2. Tenacity versus polymer concentration of cellulose ester fibers spun at different take-up speeds; (a) CA (b) CAB-1 (c) CAB-2 (d) CAB-3. 
illustrate that the modulus and tenacity values "jump" for the fibers spun from anisotropic solutions. Similar observations were also made for poly (p-benzamide) and cellulose fibers (34, 35). Since the isotropic-anisotropic transition was bridged by a narrow biphasic gap, the discontinuity is not very sharp. As modulus is a direct measure of orientation (36), fibers from anisotropic solutions should be more oriented than those from isotropic solutions. Increase in the modulus and tenacity values of fibers from isotropic and anisotropic solutions was further enhanced by the increase in the take-up speed (i.e., draw ratio). These results establish the direct connection between the improved mechanical properties and the appearance of mesophase solution morphology. As soon as the mesophase is encountered, there is improved fluid orientation which leads to high modulus fibers. The properties maximize at higher concentrations as the mesophase is fully developed, i.e., completely anisotropic. This suggests a strong relationship between fiber properties and nature of the spinning solution.

On comparing the mechanical properties of all the fibers from isotropic solutions, CA fibers possess the highest modulus and tenacity. CA fibers were also stiffer and stronger than most $C A B-2$ fibers and all $C A B-3$ fibers spun from anisotropic solutions. This indicates that $C A$, if spun from anisotropic solution, could have the potential of producing ultrahigh modulus fibers. The maximum values of modulus and tenacity, $35.1 \mathrm{~g} / \mathrm{d}$ and $1.2 \mathrm{~g} / \mathrm{d}$ respectively, obtained in this 
study for CA are higher than those reported in the literature [i.e., $12.5 \mathrm{~g} / \mathrm{d}$ and $0.86 \mathrm{~g} / \mathrm{d}$ respectively] (23). CA fibers were also tougher ( $\mathrm{BT}=0.5 \mathrm{~g} / \mathrm{d}$ ) than the other cellulose ester fibers studied here.

The modulus and tenacity of $C A B-1$ fibers spun from anisotropic solution surpass all the fibers investigated in this study. The increase in the values is significantly higher in $C A B-1$ than the other cellulose esters. The modulus and tenacity values (i.e., 122 and $3.8 \mathrm{~g} / \mathrm{d}$ respectively, Table I) of CAB-1 fibers are comparable to those reported for CTA [i.e., 164 and $4.1 \mathrm{~g} / \mathrm{d}$, respectively] (13). It seems that the mechanical properties of $C A B-1$ fibers can be further improved if they are spun from higher concentrations and at higher take-up speeds.

CAB-2 fibers spun from liquid crystalline solutions have higher modulus and tenacity in this study, $38.4 \mathrm{~g} / \mathrm{d}$ and 1.1 g/d respectively, than those reported in the literature [i.e., $19.55 \mathrm{~g} / \mathrm{d}$ and $0.94 \mathrm{~g} / \mathrm{d}$ respectively] (21).

CAB-3 fibers are the weakest despite $C A B-3$ forming liquid crystalline solutions at a lower concentration than the other cellulose esters (i.e., $C_{p}^{\prime}=35 \%$ ). Fiber properties reach a plateau at higher solution concentration, and this does not improve greatly at high take-up speeds. These fibers were easiest to spin as viscosity of the concentrated solutions was comparatively low. $\mathrm{CAB}-3$ can be of interest for exploratory 
research as it forms anisotropic solutions at low concentration (35\%), and these are easy to process. Breaking toughness and elongation at break of all the cellulose ester fibers improved when spun from liquid crystalline solution.

Based on optical microscopy and small angle light scattering studies with liquid crystalline solutions of cellulose derivatives, Bheda et al. (2) had concluded that cellulose triacetate solutions (in dichloroacetic acid) had a better defined structure than CAB solutions (in DMAC). During a preliminary study of fiber spinning from liquid crystalline solutions, it was evident that fibers from cellulose triacetate had better mechanical properties than those from $C A B$ (21). Therefore, it appears that the degree of structural perfection in the liquid crystalline state is determined primarily by the chemical structural regularity of the polymer. The cellulose ester derivatives employed in this study differ mainly with regard to the substitution pattern. Due to better chemical structural regularity, CAB-1 seemed to have developed a well-defined liquid crystalline structure than $C A B-3$. This feature must have led to improved mechanical properties of fibers with lower butyryl content. Fig. 3 shows that the modulus of the cellulose ester fibers gradually deteriorates with increase in butyryl content. However, it was shown earlier that liquid crystalline solutions can be reached at lower concentrations with increasing butyryl content (27). Consequently, there is a trade-off as a high butyryl content 


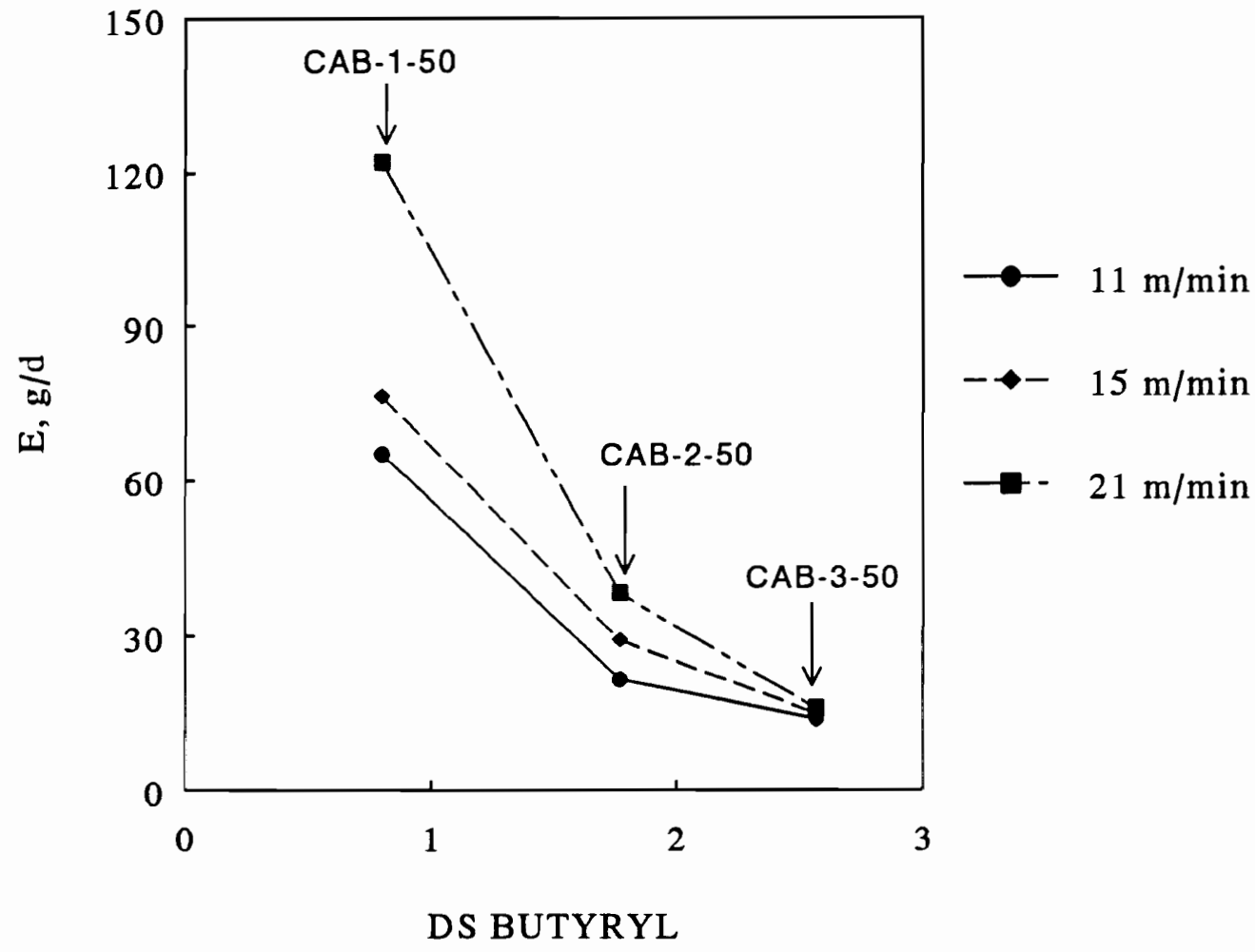

Figure 3. Modulus versus degree of substitution (DS) of butyryl groups in cellulose ester fibers spun at different take-up speeds. 
has a negative effect on fiber performance, but it has a positive effect on the formation of a liquid crystalline solution state. Therefore, CAB-1 (with lowest butyryl content) produces the best cellulose ester fibers as it displays the lowest performance reduction while exhibiting the greatest positive effect on the formation of liquid crystalline solutions. From this systematic study, it can be concluded that the mechanical properties of the fibers are closely related to the physical structure developed in the liquid crystalline solution state which in turn relies on the chemical structure of the polymer. This investigation also reveals the role of the mesophase in fiber spinning, and it establishes a structure-property type relationship for liquid crystalline cellulose ester derivatives.

III. Morphology:

a. Coagulation Effects.

(i) Effect of Take-up Speeds:

The most fundamental step in fiber formation from a polymer solution is solvent removal from the spinning fluid by diffusional interchange with a non-solvent bath. This process causes the polymer to solidify; it is termed coagulation. $\xi$ is the depth of penetration of the non-solvent in the polymer solution during coagulation as a function of time ( $t)$. The coagulation rate, defined as $\xi / V t$, exhibits the effects of coagulation variables on the coagulation of a polymer solution (37-39). Assuming that the diameter of the as-spun filament is 
the same as the spinnerette hole, the relationship between the spinning conditions and the minimum coagulation rate $(\xi / V t)^{*}$ required for complete coagulation of the spinning solution in the coagulation bath is given by,

$$
(\xi / \sqrt{ } t)^{*}=0.7(D / 2)(\langle V\rangle / B)^{1 / 2}
$$

where $D$ is the diameter of the spinnerette hole, and $B$ and $\langle v\rangle$ are the immersion distance and the average velocity, respectively, of as-spun filament in the coagulation bath (38). This equation may become complicated by the effects of die-swell and stretching, as they relate to the diameter of as-spun filament.

Model experiments were performed by Paul (40) on gelled solutions of acrylic polymer in dimethylacetamide to determine the coagulation rates of solvent and non-solvent (water) during coagulation. It was found that the coagulation rate for DMAc-water system at $30^{\circ} \mathrm{C}$ was $4.2 \times 10^{-4} \mathrm{~m} / \sqrt{\mathrm{min}}$. The key role in determining the fiber structure during the process of coagulation is solely dependent on the interfacial interaction between the solvent and the coagulant with the polymer playing a neutral role (41). Based on this knowledge, the coagulation rate determined for acrylic polymers can be applied in this study as a similar solvent-coagulant system was employed here. Minimum coagulation rate, $(\xi / V t)^{*}$, required for complete coagulation of the cellulose ester spinning solutions in the coagulation bath was determined by Eq. 1. The diameter of the 


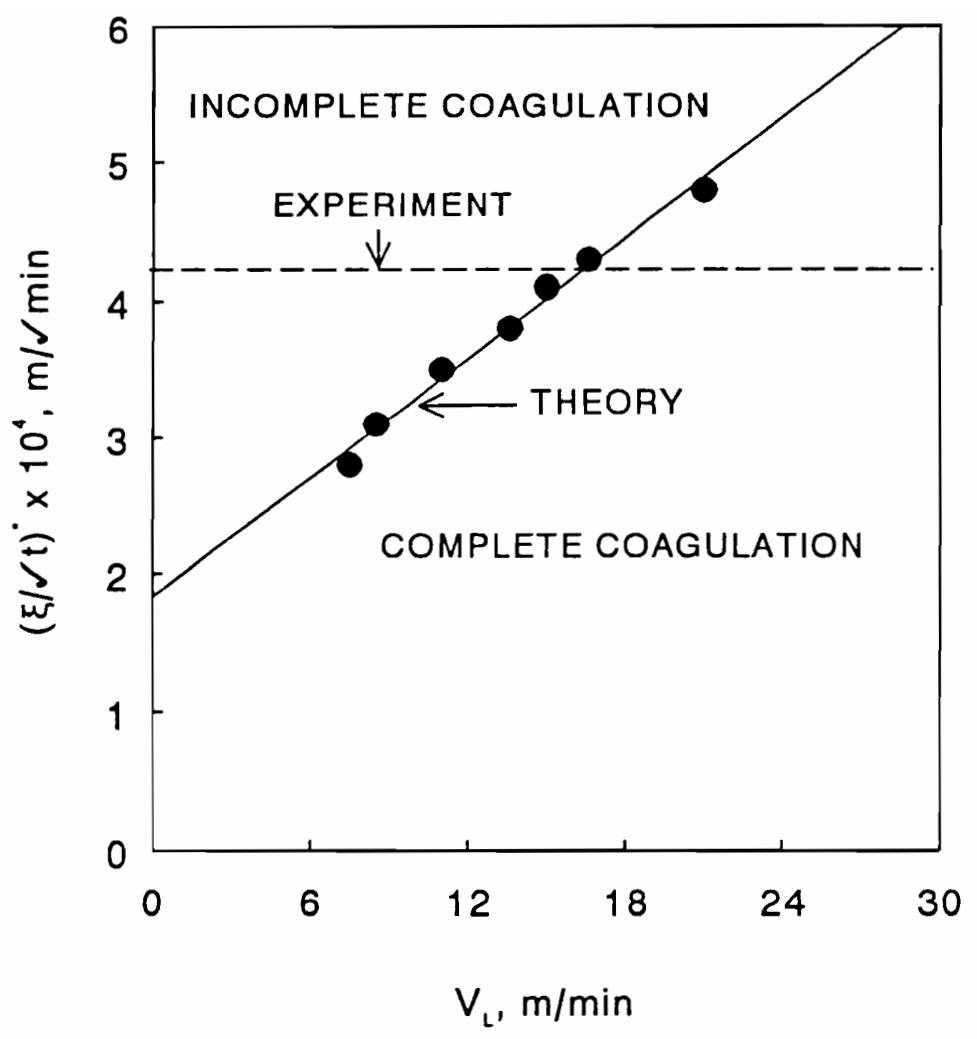

Figure 4. Minimum coagulation rate of cellulose ester fibers spun at different take-up speeds. 
spinnerette was $300 \mu \mathrm{m}$, immersion distance of the as-spun filaments was 1 meter, and the average velocity, $V_{L}$, varied from 7.5 to $21 \mathrm{~m} / \mathrm{min}$. The calculation is based on the assumption that the diameter of the as-spun filament is the same as the spinnerette hole. Figure 4 shows the variation of minimum coagulation rates required for complete coagulation of the cellulose ester fibers spun at different take-up speeds. Minimum coagulation rate required for complete coagulation increased with increase in the spinning speed. This is due to the reduced residence time of the as-spun filament in the coagulation bath at higher speeds. Since the experimental value was higher than the requirements (except for the 21 $\mathrm{m} / \mathrm{min}$ samples), complete coagulation was assured qualitatively. The effects of spinning speeds on coagulation rates were verified especially by SEM.

Figure 5 shows the scanning electron micrographs of the cross-section of the fibers spun from isotropic and anisotropic solutions at different take-up speeds. Figs. 5 (a) and (b) showed no holes or hollow cores in the center of the fibers which are signs of complete coagulation. Similar observations were made for fibers spun from $21 \mathrm{~m} / \mathrm{min}$ [Fig. 5(d)] which confirms complete coagulation even though the computation of Fig. 4 suggests borderline coagulation. Although, the coagulation requirement for the fibers spun at $21 \mathrm{~m} / \mathrm{min}$ was somewhat higher than the experimental value, it 

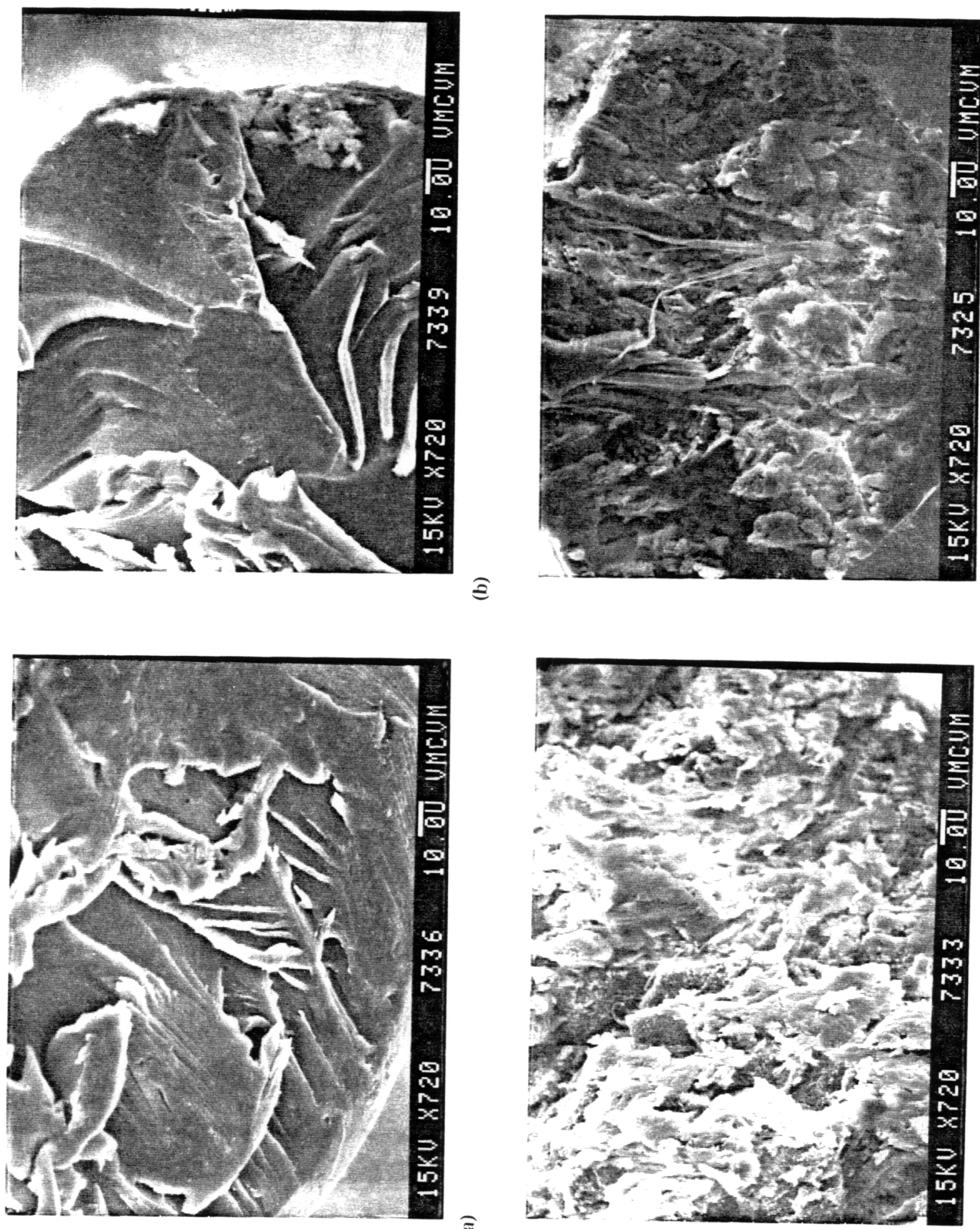

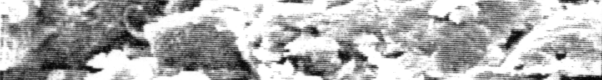

อ

¿

记

ช

을

를

¿

o in

$\approx \infty \frac{1}{\circ}$

至

造

它已

이

0

용

Е छ

- क

을 을

돈

on

¿

ฮบ

ธอ อ

范

¿

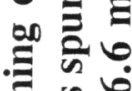

อ

ฮู่

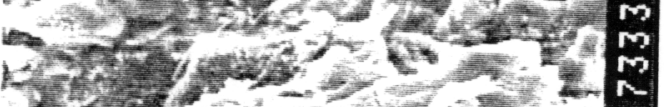

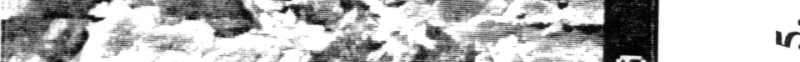

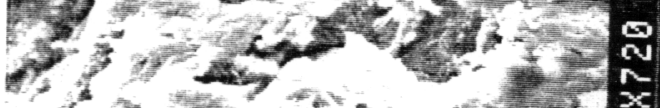

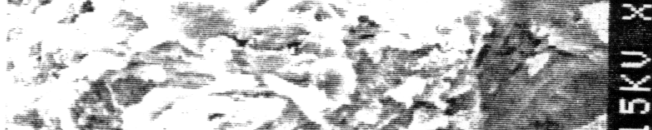

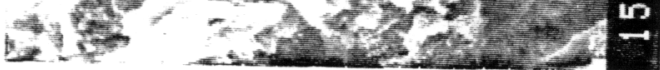


seems to be within experimental error based on SEM results. There was almost no difference in the structure of the fiber cross-sections at different speeds from similar solution phases. These results clearly demonstrate that, due to complete coagulation, high spinning speeds for different solution phases (i.e., isotropic and anisotropic) were not responsible for any defects incorporated in the fiber crosssection.

\section{(ii) Effect of Concentration:}

The effect of concentration on the cross-sectional structure of the fibers is shown in Figure 6. Fibers spun from low concentrations were uniform and open as compared to the fibers from high concentrations. This interesting morphological feature was detected in all the cellulose ester fibers. At higher polymer concentrations, less coagulant was required to diffuse into the solution to precipitate the polymer, and this, consequently, led to a faster coagulation rate. This does not allow enough time for the adjustment to the internal stresses that had caused expansion of the polymer network and made the structure rough. Following the same reasoning, at low concentrations the coagulation rates were relatively slow and thereby led to a uniform structure. The fibers from high concentrations seemed to be denser than those from low concentrations. High concentration solutions were in the liquid crystalline phase with molecules aligned in one direction. Upon coagulation at faster rates, these molecules 

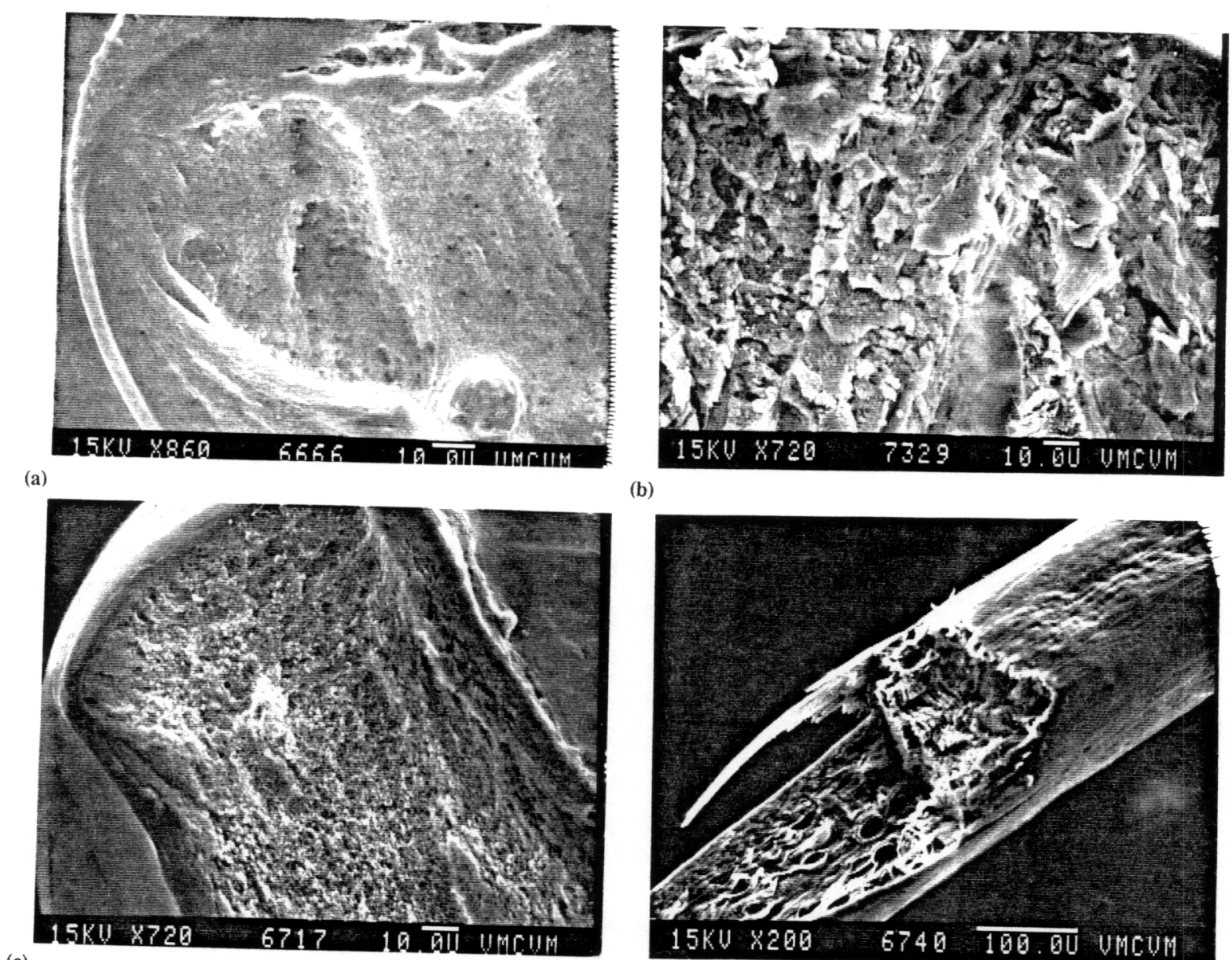

(b)

(c)

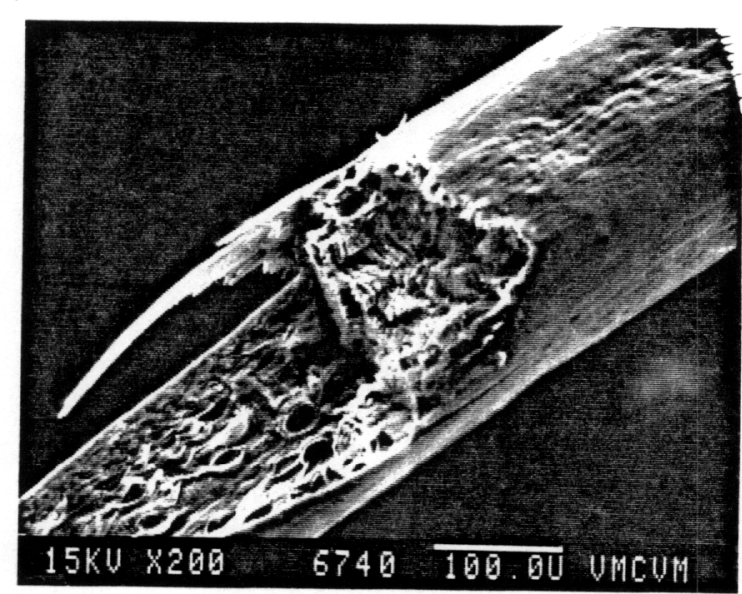

(d)

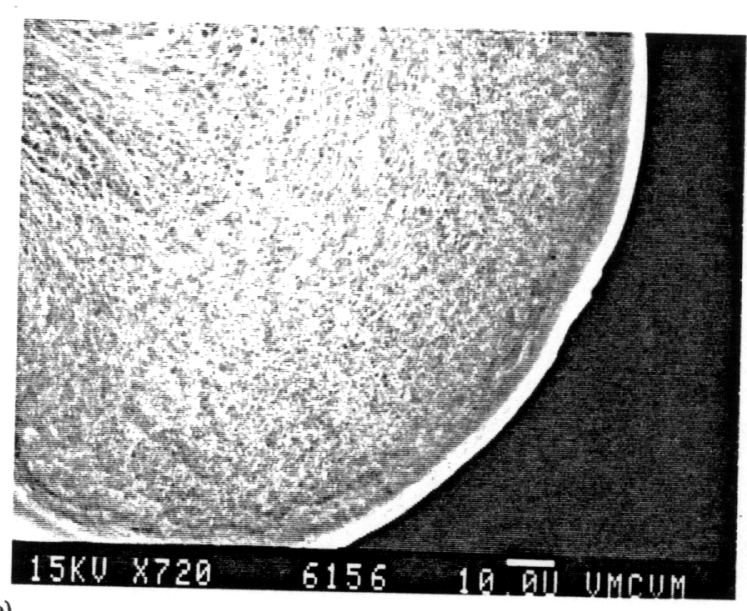

(e)

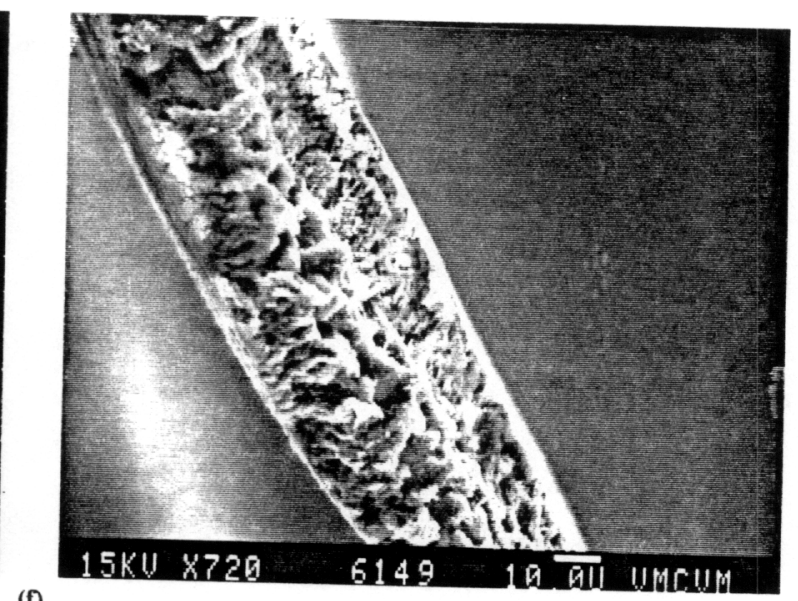

Figure 6. Scanning electron micrographs of the cross-section of cellulose ester fibers spun at $11 \mathrm{~m} / \mathrm{min}$; (a) CAB-1-35 (b) CAB-1-50 (c) CAB-2-35, 7.5 m/min (d) CAB-2-50 (e) CAB-3-25 (f) CAB-3-50. 
did not get the opportunity to relax which resulted in a dense structure.

Figure 7 show the micrographs of the surfaces of the fibers spun from low and high concentration spinning solutions. Fibers from low concentrations had smooth surface morphology whereas the fibers from high concentrations were coarse. However, the surface morphology varied with the type of cellulose ester. $\mathrm{CAB}-1$ fibers had a fibrillar ring structure at regular intervals, $\mathrm{CAB}-2$ fibers appeared to have alternating sections of dented and flat surfaces; and $\mathrm{CAB}-3$ fibers had a very porous and open structure. The surface morphology of the fibers spun from high concentrations seemed to follow a trend which implied an increase in irregularity and roughness with increase in butyryl content. This difference in structure can be qualitatively related to the increase in the coagulation rate with the increase in the hydrophobic non-polar alkyl chains as the butyryl content increases. This makes $C A B-3$ more porous in relation to $C A B-1$. These results suggest that the fiber morphology was dependent on chemical structure of the polymer; on concentration; and the phase of the spinning solution.

(iii) Effect of Mass Transfer Rate:

Figure 8 shows the SEM of the CAB-3 fibers from isotropic and anisotropic solutions. The fibers from isotropic solution were circular in cross-section whereas the fibers from anisotropic solutions were elliptical. 


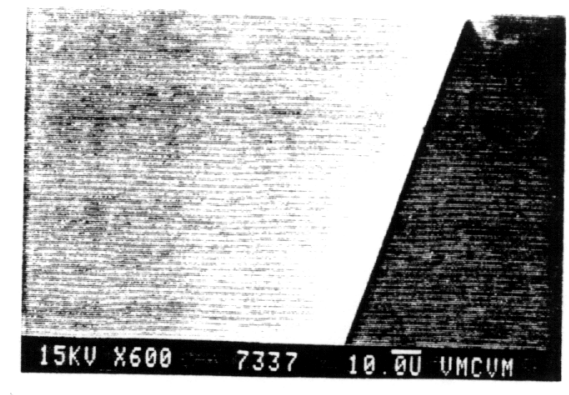

(a)
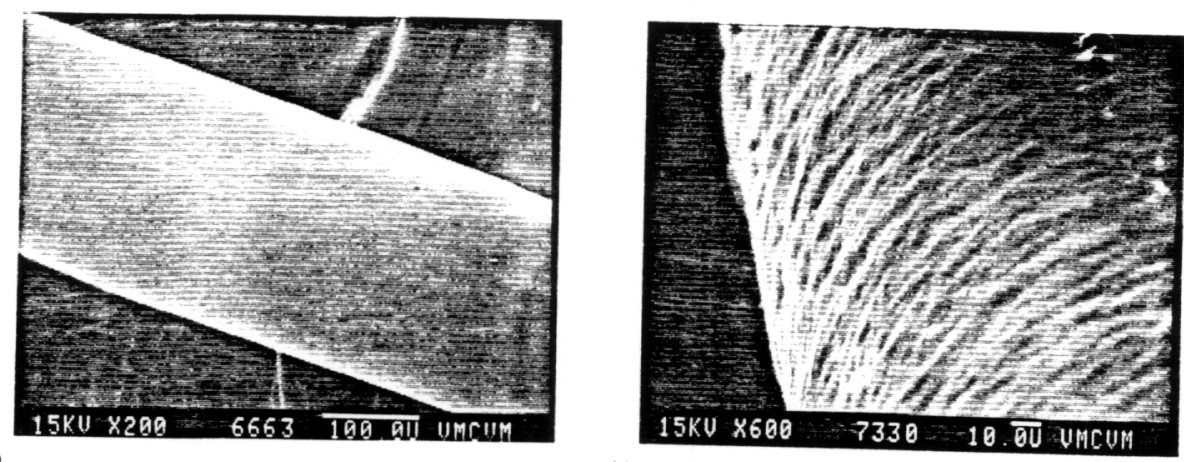

(b)

(c)
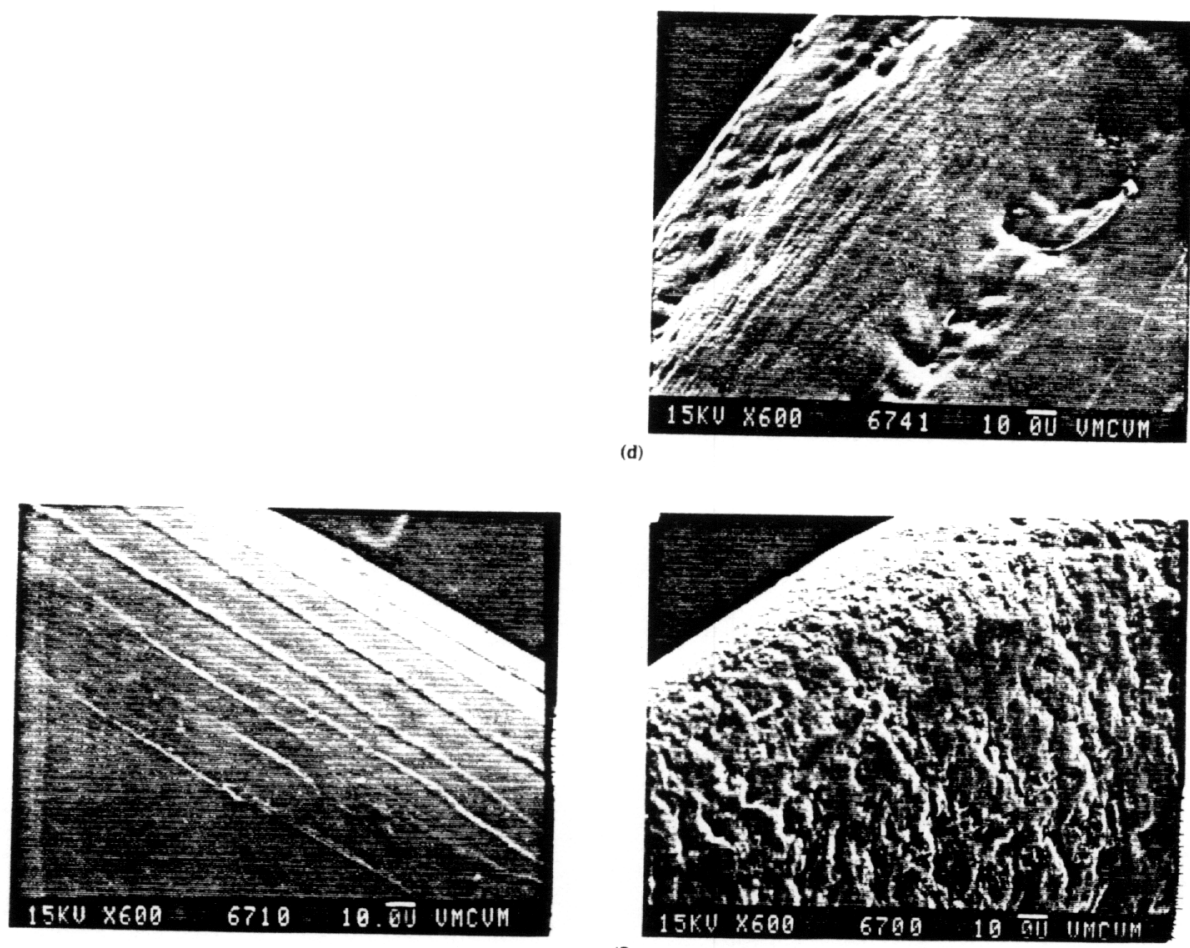

(f)

Figure 7. Scanning electron micrographs of the surface of cellulose ester fibers spun at $11 \mathrm{~m} / \mathrm{min}$; (a) CA-35 (b) CAB-1-35 (c) CAB-1-50

(d) CAB-2-50 (e) CAB-3-25 (f) CAB-3-50. 
(a)

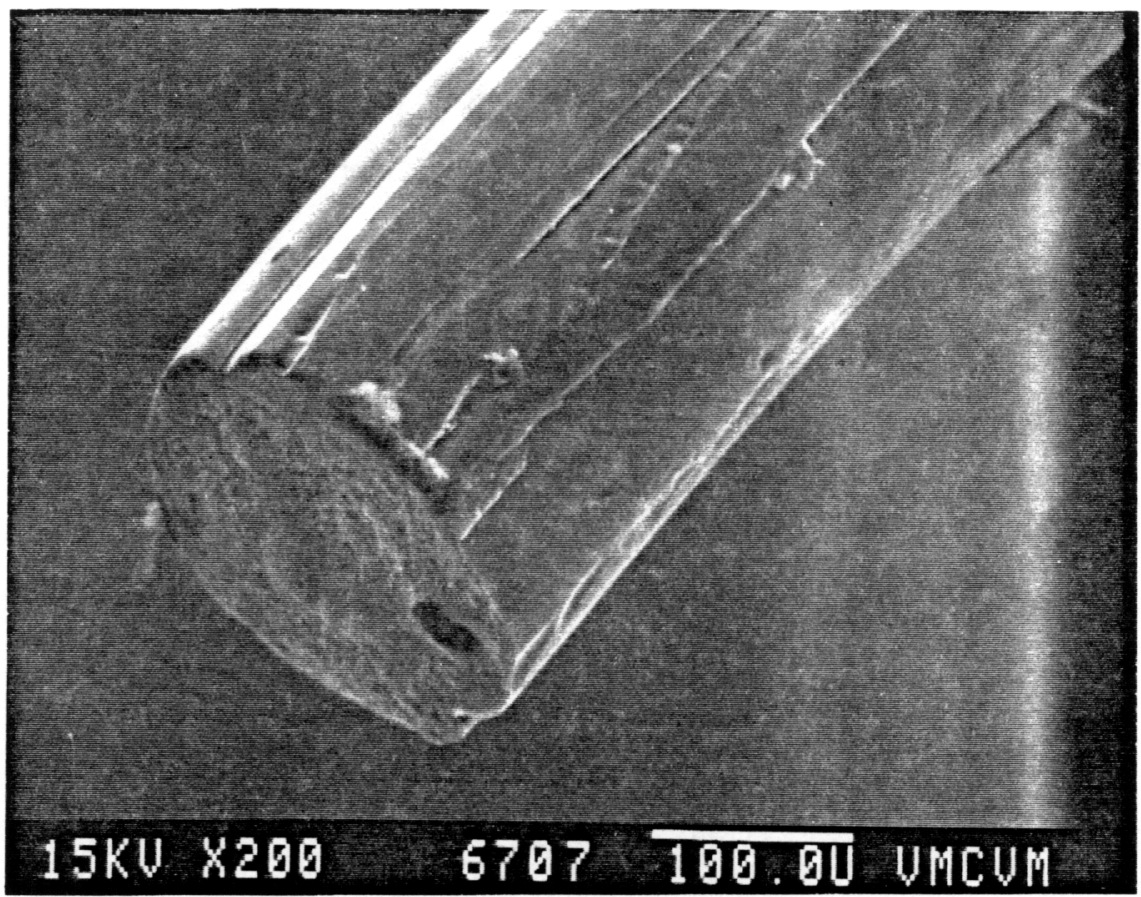

(b)

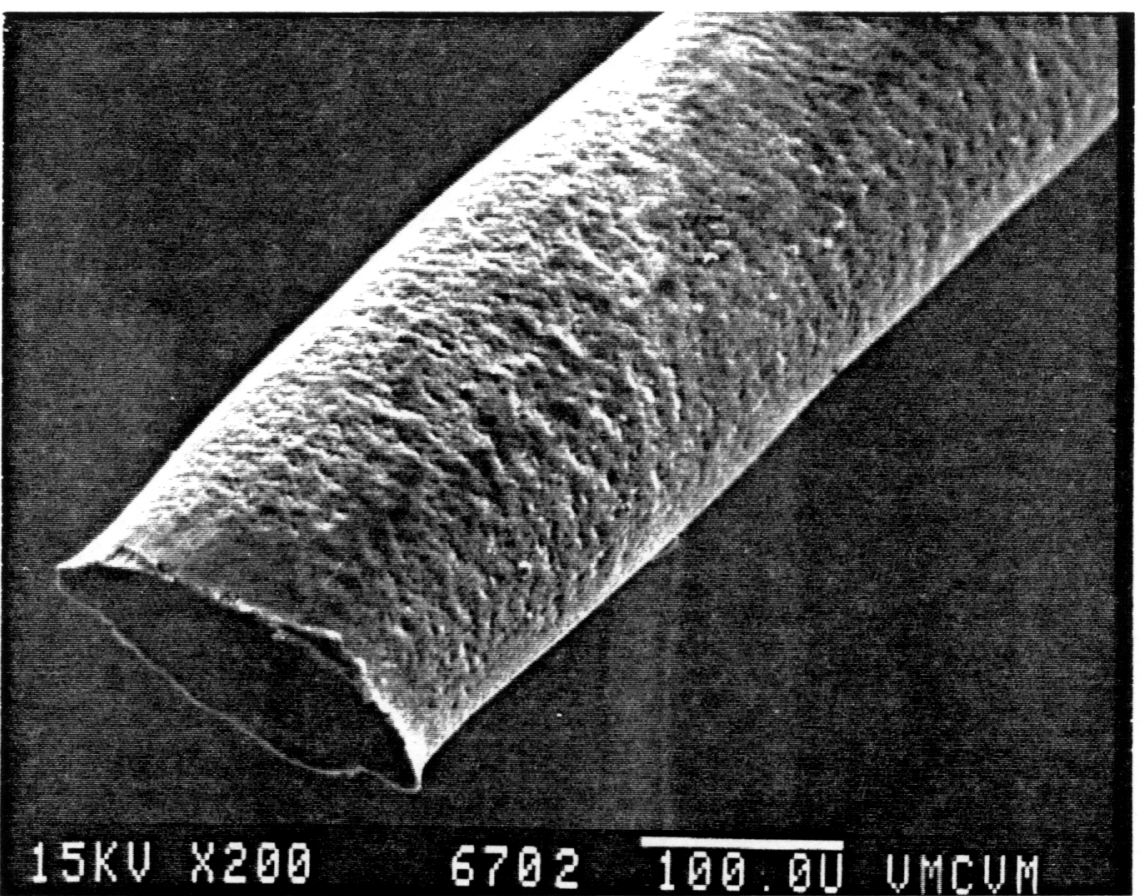

Figure 8. Scanning electron micrographs of CAB-3 fibers spun at $11 \mathrm{~m} / \mathrm{min}$; (a) CAB-3-25, circular cross-section (b) CAB-3-50, elliptical cross-section. 
In the polymer solution wet-spinning system, the solvent diffuses out of the solution, while coagulants diffuse in. The difference in the mass transfer rate between solvent and nonsolvent is an important element that influences the composition of the coagulating polymer solution. The crosssectional shape of wet-spun fibers varies with the type of coagulant due to the change in the mass transfer difference, $\Delta \mathrm{K}$, between solvent and coagulants; and deformability of the thin surface layer which forms on the filament at the initiation of coagulation (39). When the mass transfer rate of the solvent is less than that of the coagulant, the filament swells and a circular cross-section is formed. But when the solvent diffuses out of the filament at a higher rate than the absorption of coagulant, the cross-sectional shape depends on the rigidity of the coagulated layer. With a rigid surface layer, the cross-section will collapse and lead to a noncircular, irregular shape.

This implies that DMAC diffused out of the CAB filaments from high concentrations at a higher rate than the absorption of water and made the surface layer very rigid which collapsed the cross-section into an elliptical shape. Due to this collapse of the fiber cross-section during coagulation, the opportunity to stretch the molecules diminished. Therefore, the increase in modulus of $\mathrm{CAB}-3$ fibers with increase in concentration was not outstanding. 
(a)

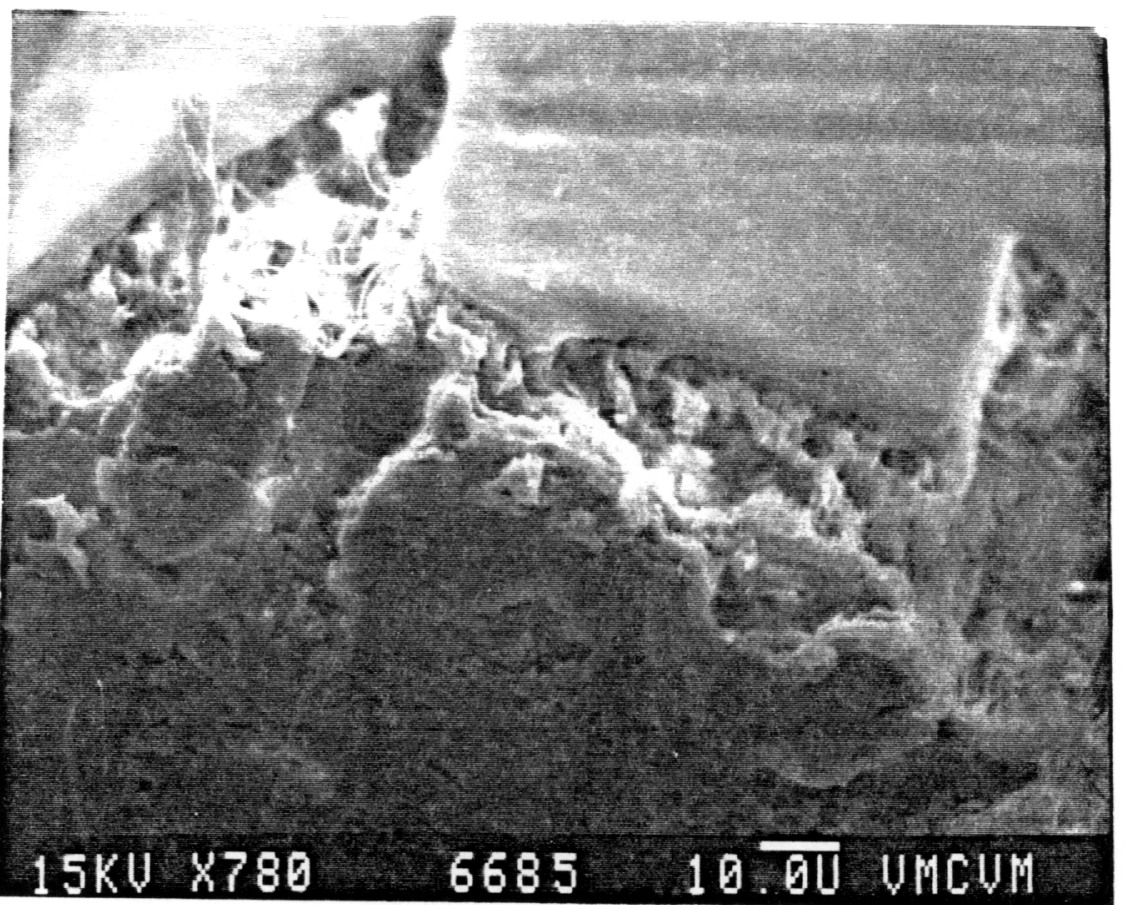

(b)

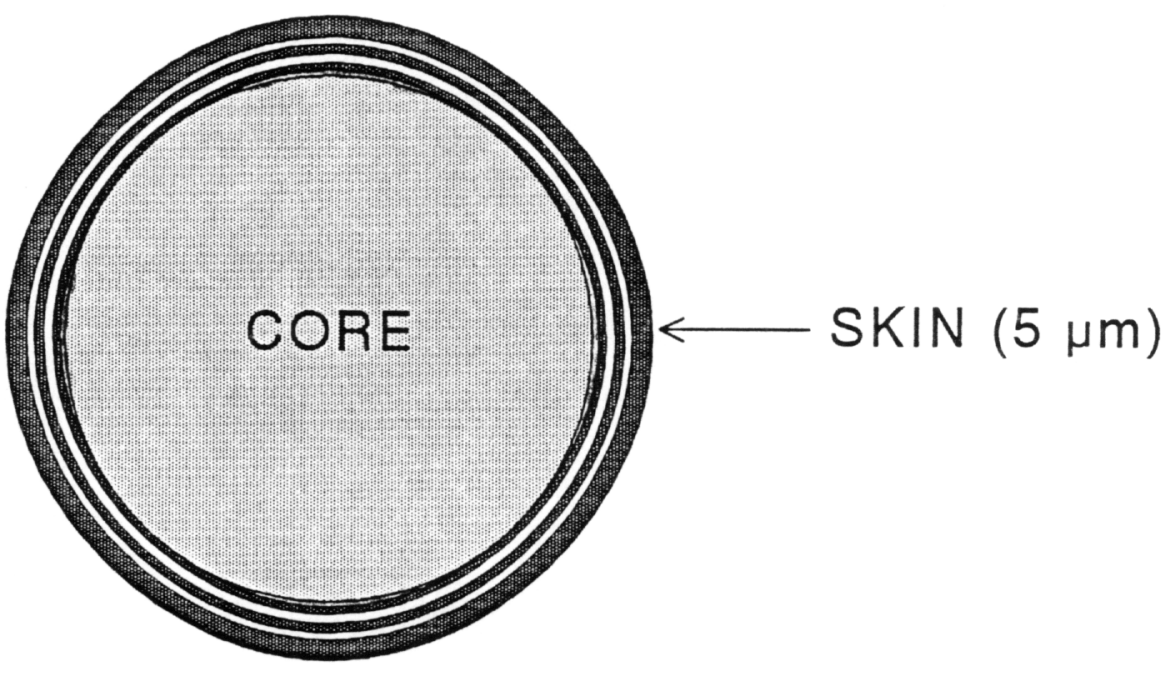

Figure 9. (a) Scanning electron micrograph of CAB-1-45 fiber cross-section (b) schematic representation of 'skin-core' effect. 


\section{(iv) Skin-Core Effect:}

Figure 9a shows the skin-core effect for $C A B-1$ fiber, where the rigid skin was separated from the flexible core. Thickness of the skin is approximately $5 \mu \mathrm{m}$.

During coagulation in wet spinning, there is always a radial gradient of composition and phase structure of the partially solidified polymer solution. Solidification during coagulation is accompanied by formation of a "skin" on the surface which is separated by a distinct boundary from the "core" (see Fig. 9b) (42). This "skin-core" morphology was observed in all the cellulose ester fibers.

b. Orientation and Crystallinity Effects.

(i) Wide Angle X-ray scattering (WAXS):

Figure 10 shows the WAXS patterns of the cellulose ester fibers spun at the same draw ratio from isotropic and anisotropic solutions. WAXs patterns were taken of fibers to qualitatively determine the level of orientation. Amongst the fibers spun from isotropic solutions, CA fibers showed [Fig. 10 (a) ] a diffuse crystalline ring ( $d "$ spacing $=2.13 \AA$ ) as compared to a completely amorphous ring for $\mathrm{CAB}-1, \mathrm{CAB}-2$ and CAB-3 fibers [Figs. 10 (b), 10 (d) and 10 (f) respectively]. This showed that $C A$ fibers were relatively more oriented than the other fibers from isotropic solutions, and this was the best evidence to explain its superior mechanical properties. Figures 10 (c), 10 (e) and 10 (g) show the WAXs patterns of the fibers spun from anisotropic solutions of $C A B-1, C A B-2$ and 


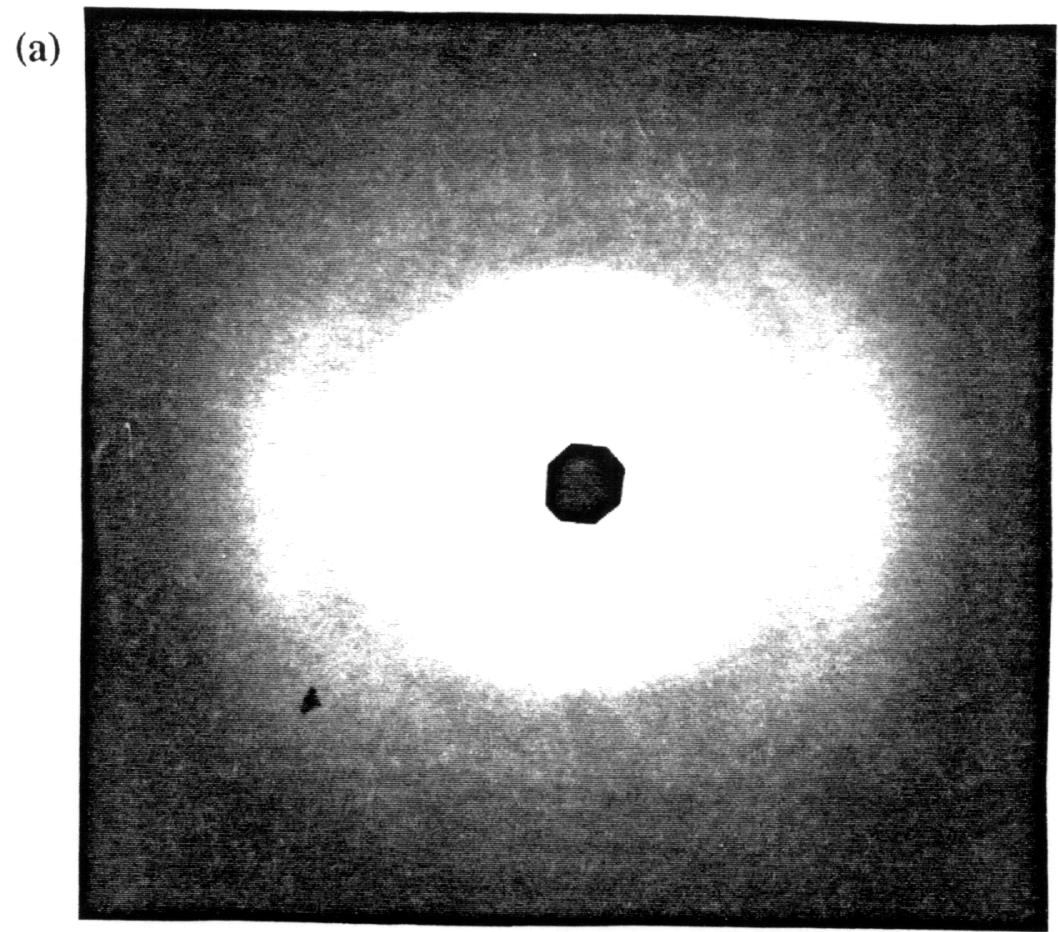

Figure 10. WAXS pattern of cellulose ester fibers spun at $11 \mathrm{~m} / \mathrm{min}$; (a) CA-35. 
(b)

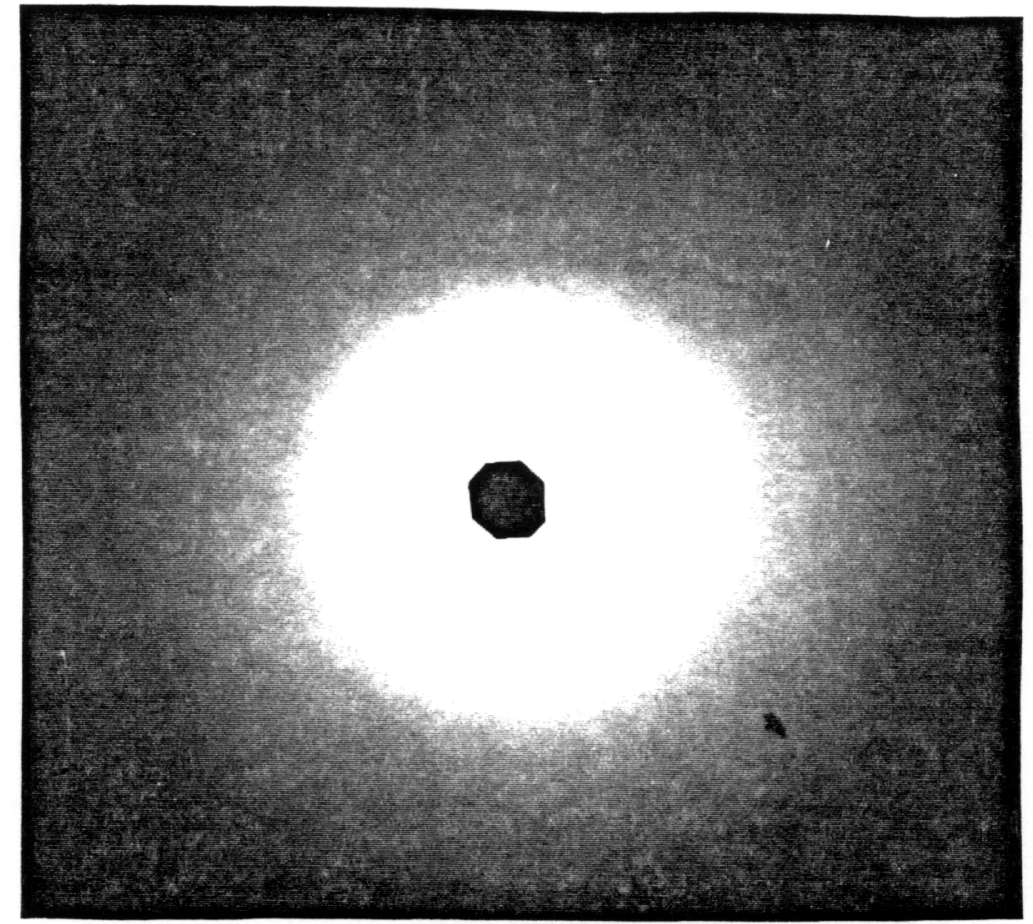

(c)

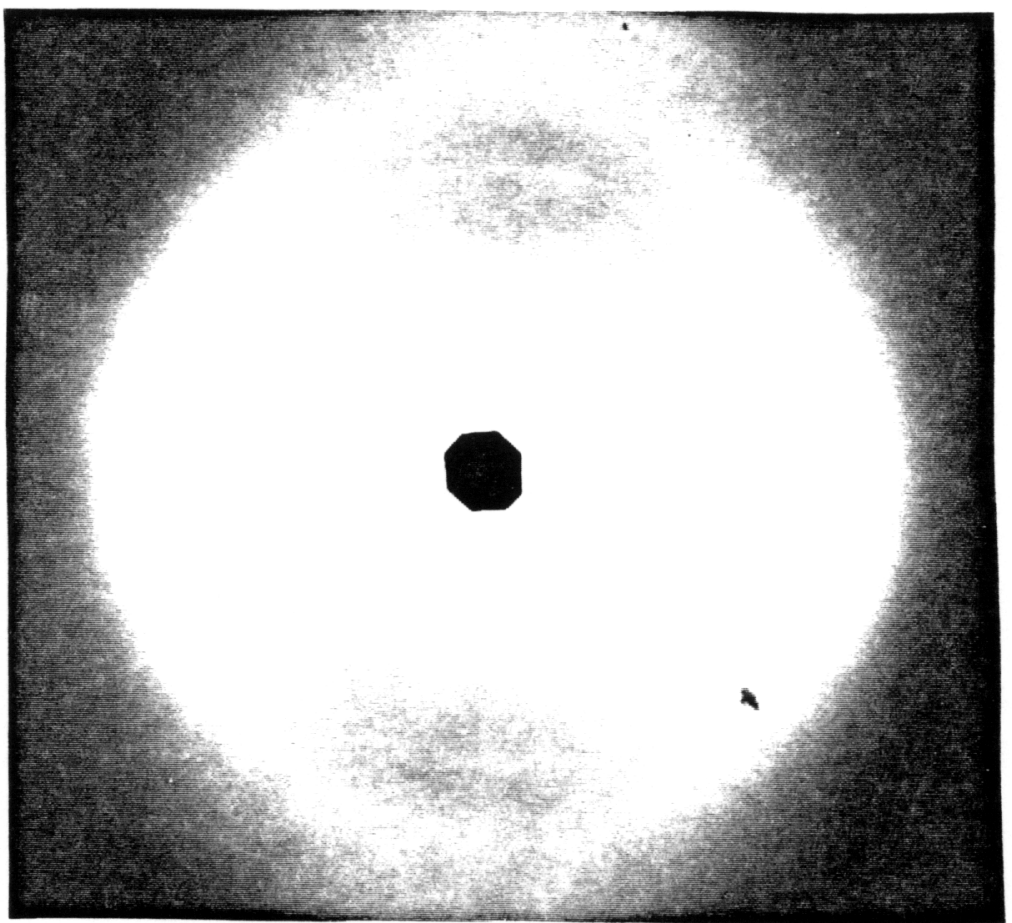

Figure 10. WAXS patterns of cellulose ester fibers spun at $11 \mathrm{~m} / \mathrm{min}$; (b) CAB-1-35 (c) CAB-1-50. 
(d)

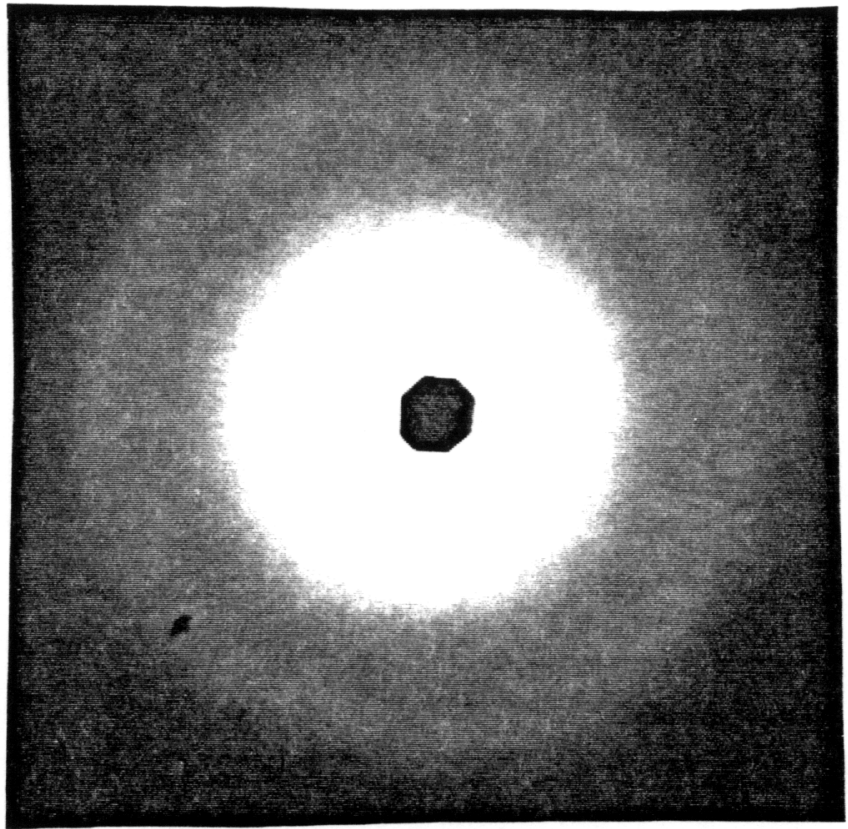

(e)

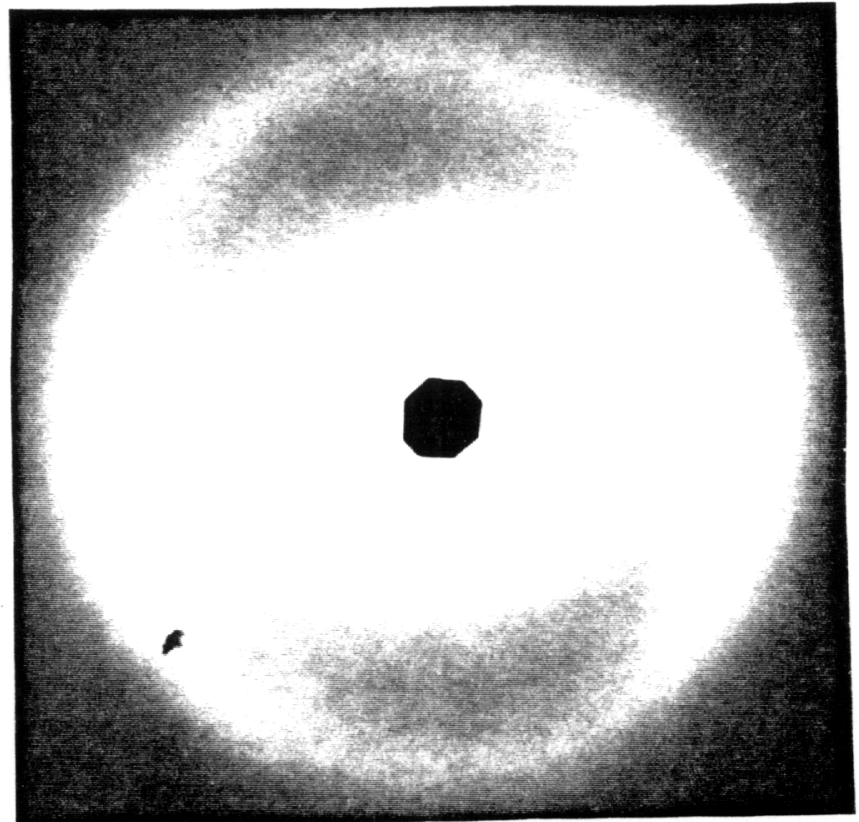

Figure 10. WAXS patterns of cellulose ester fibers spun at $11 \mathrm{~m} / \mathrm{min}$; (d) CAB-2-35, $7.5 \mathrm{~m} / \mathrm{min}$ (e) CAB-2-50. 
(f)

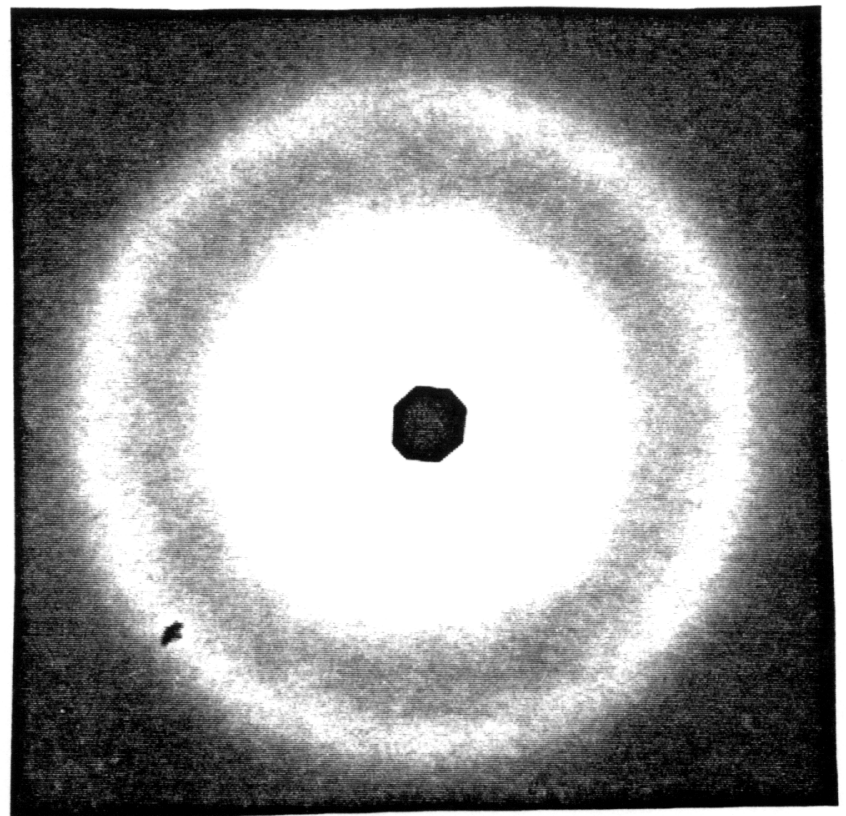

(g)

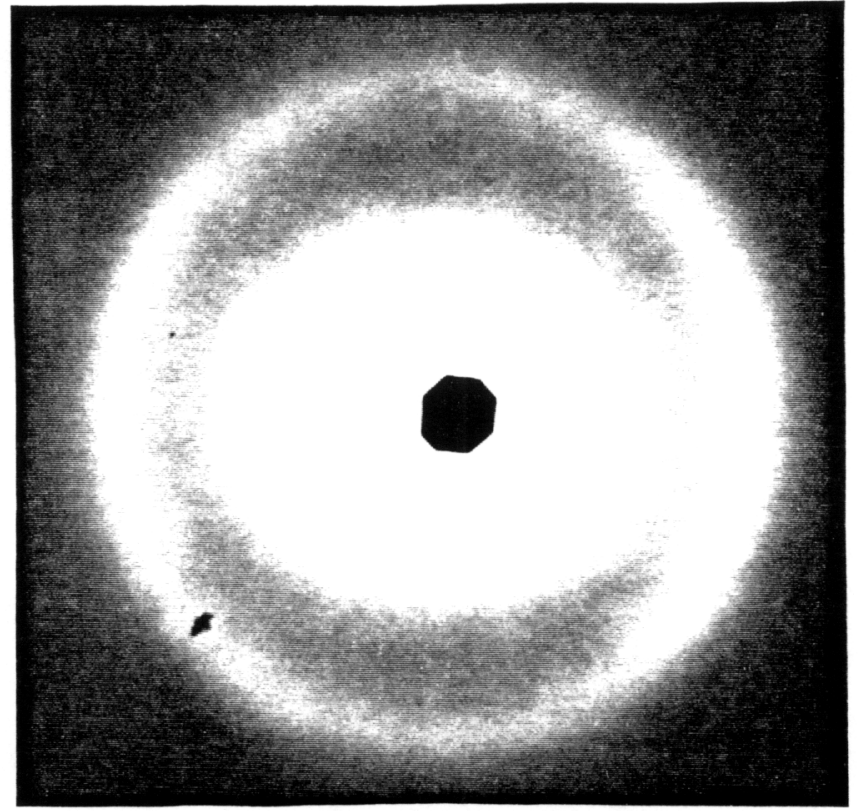

Figure 10. WAXS patterns of cellulose ester fibers spun at $11 \mathrm{~m} / \mathrm{min}$;

(f) CAB-3-25 (g) CAB-3-50. 
CAB-3 respectively $\mathrm{CAB}-1$ and $\mathrm{CAB}-2$ fibers exhibited some degree of molecular orientation and showed dependence on the azimuthal angle along the equatorial direction. CAB-3 fibers did not show noticeable molecular orientation. CAB-1 displayed sharp rings at " $d$ " spacing of $2.13 \AA$. CAB-2 and $C A B-3$ had somewhat diffuse rings at " $d$ " spacings of $2.24 \AA$ and $2.55 \AA$, respectively. The "d" spacing increased with the substituent size on the cellulose backbone. No improvement in orientation was obtained with increase in draw ratio. These wAXs patterns clearly illustrate the improvement in molecular orientation of the fibers spun from liquid crystalline solutions. The degree of orientation was highest for $\mathrm{CAB}-1$ and least for $\mathrm{CAB}-3$ fibers and correlated very well with the mechanical properties. Increase in butyryl content reduces the molecular orientation of the cellulose ester fibers due to reduction in the structural regularity in the liquid crystalline state. Once again there is a trade off as high butyryl content has a negative effect on fiber performance but forms liquid crystalline solution at a lower concentration.

(ii) Differential Scanning Calorimetry (DSC):

Figures 11 (a) - (d) are the DSC traces of powder, fibers from isotropic solutions and fibers from anisotropic solutions of $C A, C A B-1, C A B-2$ and $C A B-3$ respectively. $A$ broad endothermic transition was observed for $C A$ powder and fibers. The transition migrated between $70^{\circ} \mathrm{C}$ and $80^{\circ} \mathrm{C}$, and was also 


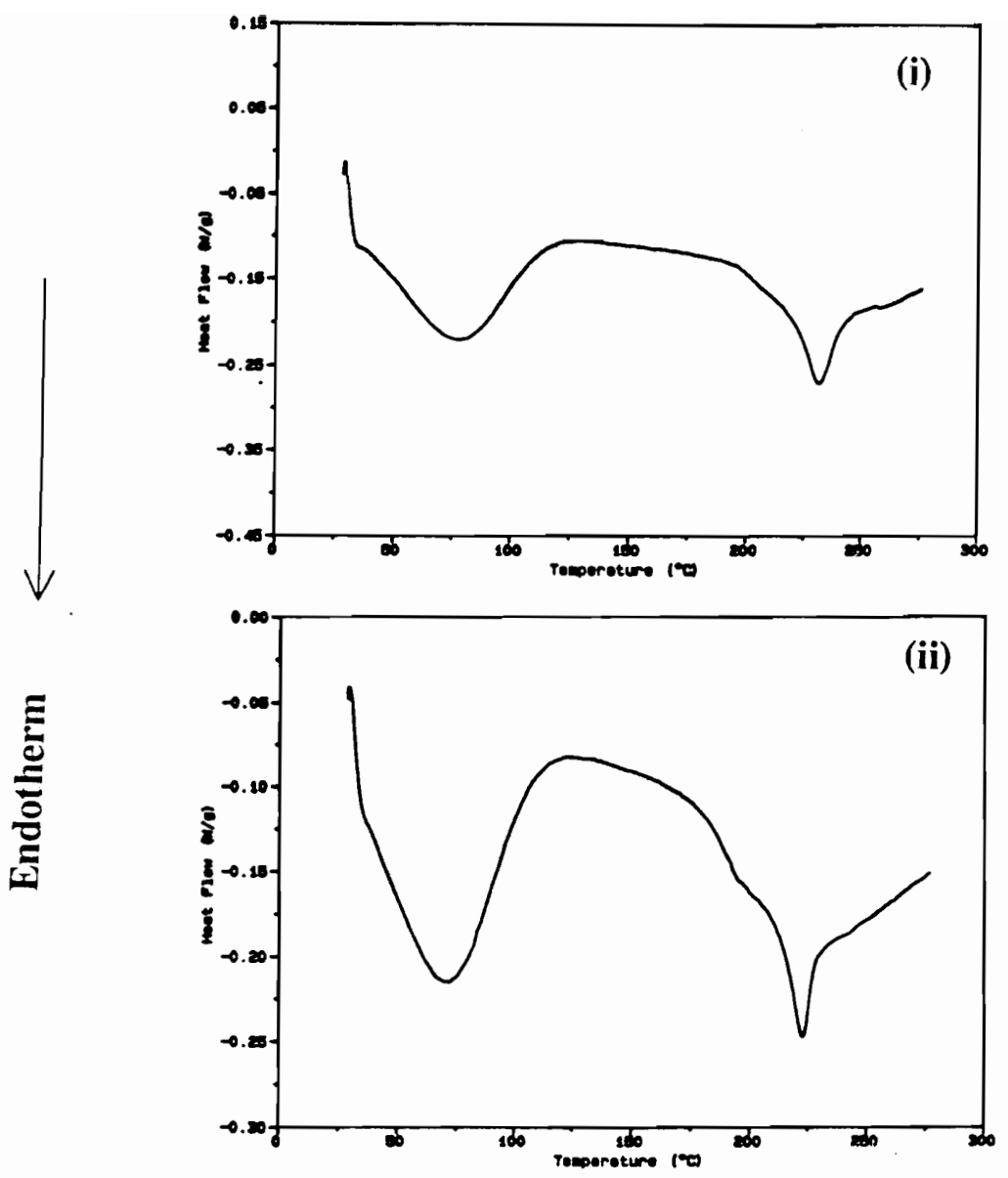

Figure 11. (a) DSC traces of CA; (i) Powder (ii) Fibers, CA-35, $11 \mathrm{~m} / \mathrm{min}$. 

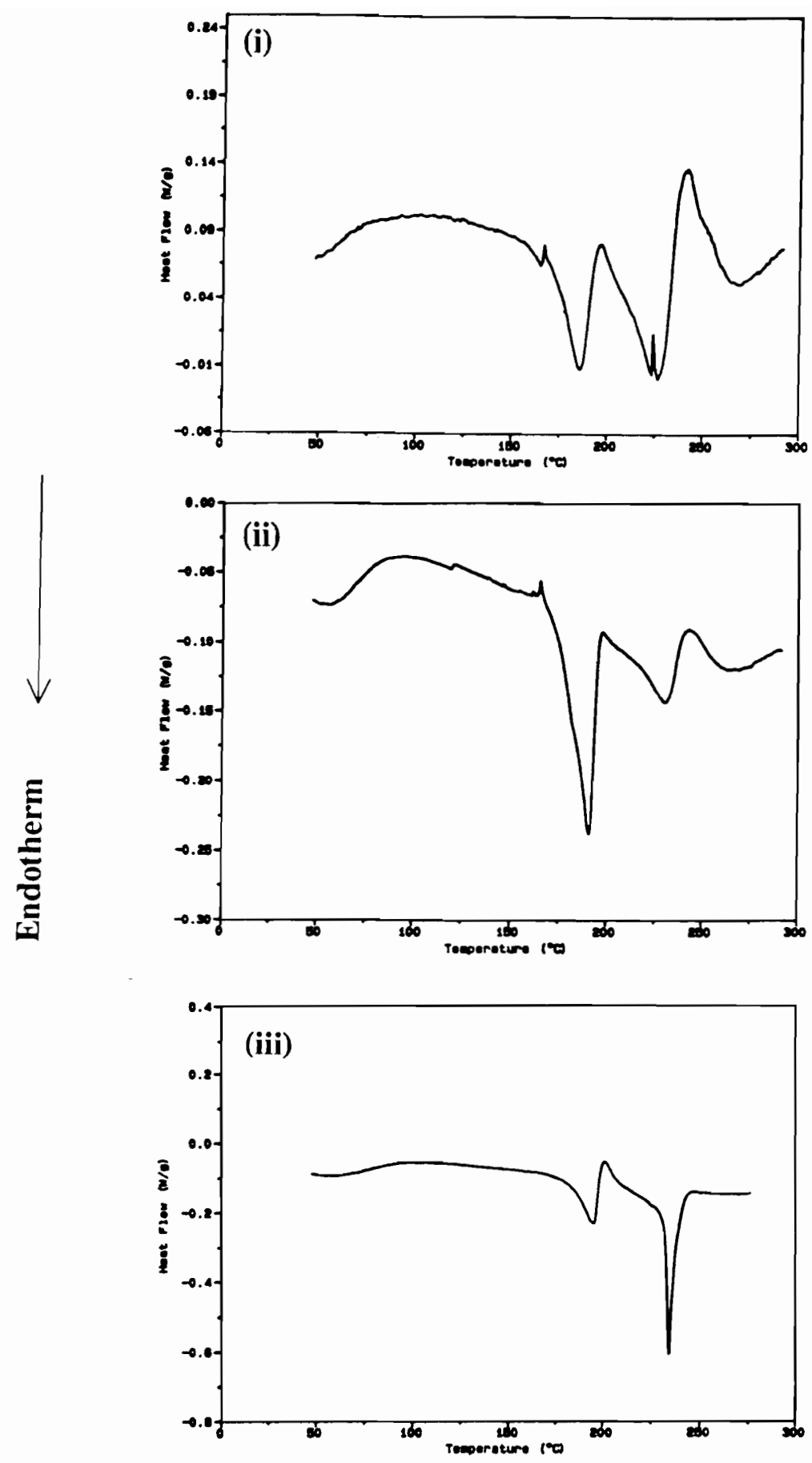

Figure 11. (b) DSC traces of CAB-1; (i) Powder (ii) Fibers, CAB-1-35, $11 \mathrm{~m} / \mathrm{min}$ (iii) Fibers, CAB-1-50, $11 \mathrm{~m} / \mathrm{min}$. 

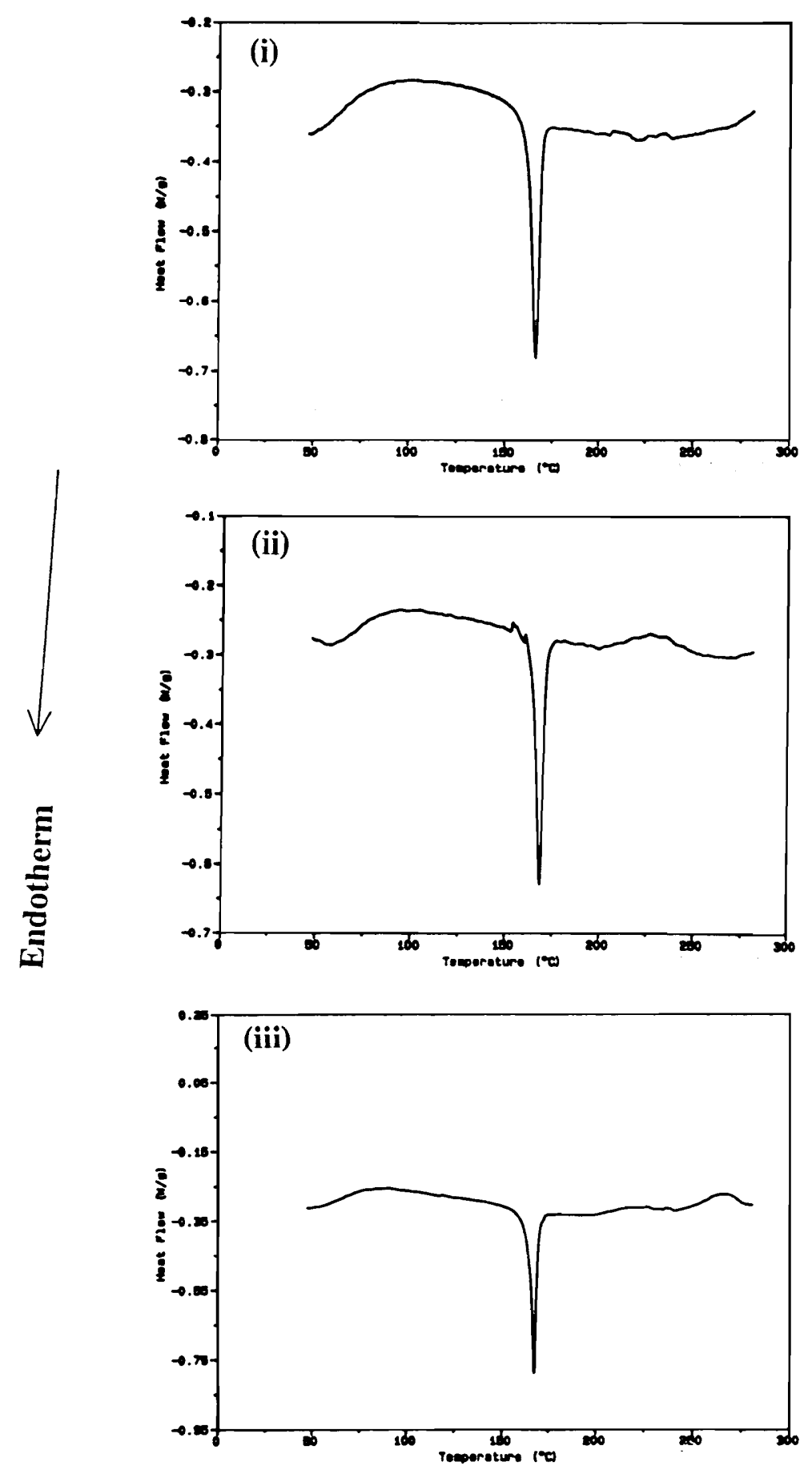

Figure 11. (c) DSC traces of CAB-2; (i) Powder (ii) Fibers, CAB-2-35, $7.5 \mathrm{~m} / \mathrm{min}$ (iii) Fibers, CAB-2-50, $11 \mathrm{~m} / \mathrm{min}$. 

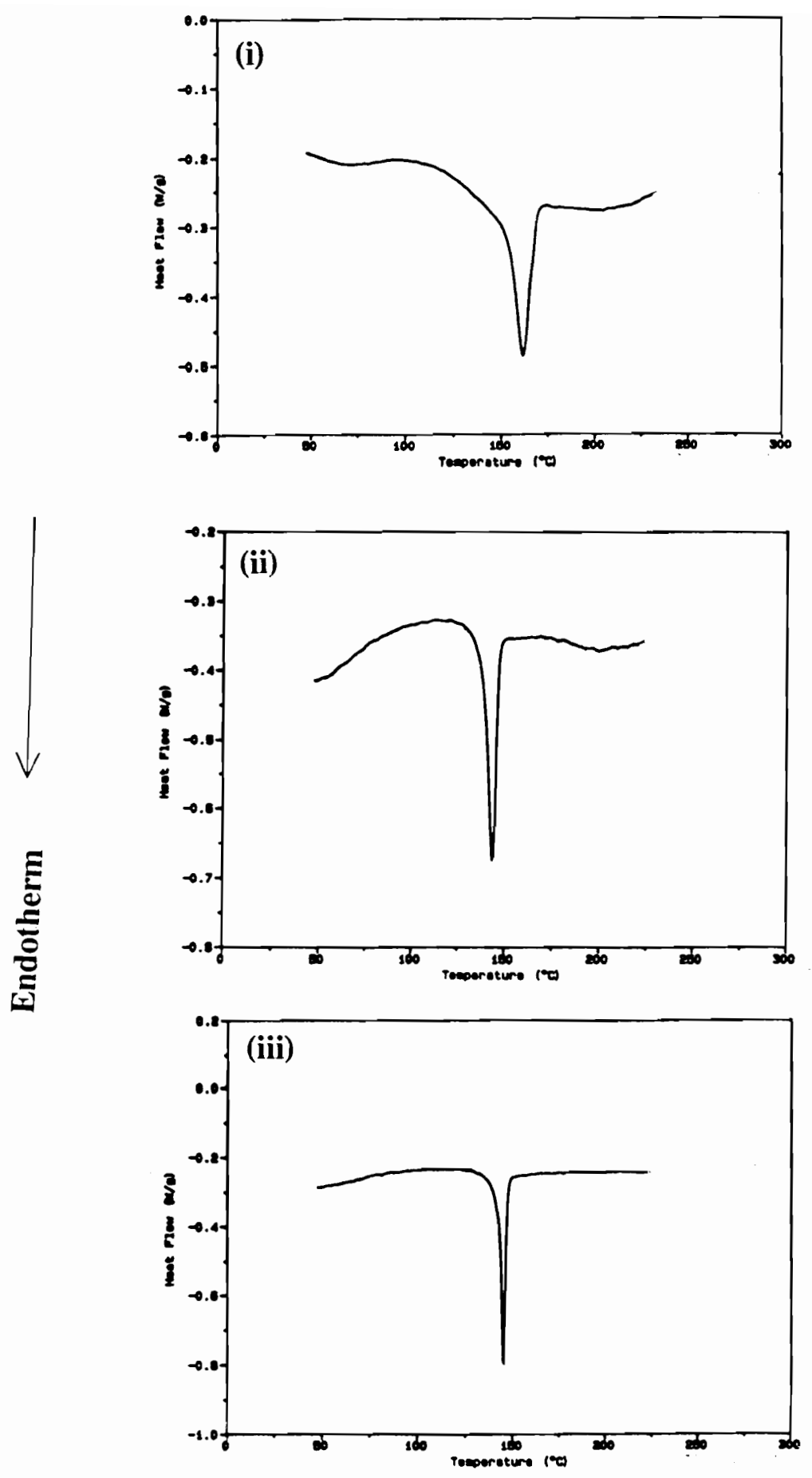

Figure 11. (d) DSC traces of CAB-3; (i) Powder (ii) Fibers, CAB-3-25, $11 \mathrm{~m} / \mathrm{min}$ (iii) Fibers, CAB-3-50, $11 \mathrm{~m} / \mathrm{min}$. 
observed by previous investigators (43). It was concluded, after a detailed analysis (44), that presence of water caused the occurrence of this transition. The melting transition and the heat of fusion of the $C A$ fibers was lower than the $C A$ powder.

Two melting transitions were observed for $C A B-1$ powder. The transitions at $186^{\circ} \mathrm{C}$ and $227^{\circ} \mathrm{C}$ were for the butyrate and acetate blocks, respectively. Such transitions for cellulose acetate butyrate have been observed in the past (45). CAB-1 is prepared at Eastman Kodak by simultaneous addition of butyric acid and acetic anhydride to the solution of activated cellulose. Sulfuric acid serves as the catalyst for the reaction. Mixed anhydride is formed as the reaction proceeds. In the presence of sulfuric acid, the rate of esterification of cellulose with acetic anhydride is faster than butyric anhydride (46). This difference in reactivity may lead to the formation of acetate and butyrate blocks along the cellulose backbone. Besides the DSC results, there are no other evidences in our study to support the presence of these blocks. CAB-1 fibers from the isotropic solutions exhibited a sharp melting transition of the butyrate blocks. The melting behavior reverses for the fibers spun from anisotropic solutions, where it was observed that the acetate blocks displayed an extremely sharp melting transition. The "d" spacing values by WAXS of the $C A$ and $C A B-1$ fibers are 
identical. This means that in $\mathrm{CAB}-1$, the acetate blocks are responsible for the molecular orientation whereas the butyrate blocks enhance the solubility of the ester derivative. A sharp melting transition of this nature showed that the crystals were very well organized and that the fibers from anisotropic solutions were highly crystalline.

$\mathrm{CAB}-2$ and $\mathrm{CAB}-3$ powders had only one melting transition at $166^{\circ} \mathrm{C}$ and $162^{\circ} \mathrm{C}$, respectively and represents the melting of butyrate blocks. The transitions of $\mathrm{CAB}-2$ and $\mathrm{CAB}-3$ fibers from anisotropic solutions were relatively sharper than those from isotropic solutions. Crystallinity of the fibers does not improve significantly when they are spun from anisotropic solutions. Table II summarizes the thermal transitions of the cellulose ester powders and fibers.

When the fibers were quenched from the melt, they were completely glassy as no melting transitions were observed. This means the only route to obtain a solid state ordered material from cellulose acetate butyrate is by spinning fibers from liquid crystalline solutions.

Figure 12 shows the change in heat of fusion of the fibers spun from isotropic and anisotropic solutions at different take-up speeds. Heat of fusion (i.e. crystallinity) of the fibers from liquid crystalline solutions was undoubtedly higher than those from isotropic solutions.

Fiber shrinkage experiments were carried out to get 
Table II. Glass transition temperature $\left(T_{g}\right)$ and melting parameters $\left(\mathrm{T}_{\mathrm{m}}, \Delta \mathrm{H}\right)$ of the fibers determined by DSC.

\begin{tabular}{|c|c|c|c|c|c|c|c|}
\hline $\begin{array}{c}C_{p} \\
(w / w q)\end{array}$ & $\begin{array}{c}\mathrm{V}_{\mathrm{L}} \\
\mathrm{m} / \mathrm{min}\end{array}$ & ${ }^{\circ} \mathrm{C}^{\mathrm{T}_{8}}$ & $\begin{array}{l}\mathrm{T}_{\mathrm{ml}} \\
{ }^{\circ} \mathrm{C}\end{array}$ & $\begin{array}{l}\Delta \mathrm{H}_{1} \\
\mathrm{~J} / \mathrm{g}\end{array}$ & $\begin{array}{l}\mathrm{T}_{\mathrm{m} 2} \\
{ }^{\circ} \mathrm{C} \\
\end{array}$ & $\begin{array}{l}\Delta \mathrm{H}_{2} \\
\mathrm{~J} / \mathrm{g}\end{array}$ & $\begin{array}{l}\Delta \mathrm{H}_{\mathrm{T}} \\
\mathrm{J} / \mathrm{g}\end{array}$ \\
\hline $\begin{array}{c}\text { CA } \\
\text { Powder }\end{array}$ & ---- & 191 & -- & ---- & 232 & 9.05 & 9.05 \\
\hline$C A-35$ & 8.5 & 190 & --- & ---- & 221 & 2.79 & 2.79 \\
\hline$C A-35$ & 11.0 & 188 & --- & ---- & 223 & 3.15 & 3.15 \\
\hline$C A-35$ & 13.6 & 190 & --- & ---- & 223 & 3.11 & 3.11 \\
\hline$C A-35$ & 15.0 & 188 & --- & ---- & 222 & 2.60 & 2.60 \\
\hline$C A-35$ & 16.6 & 189 & --- & ---- & 220 & 3.00 & 3.00 \\
\hline $\begin{array}{l}\text { CAB-1 } \\
\text { Powder }\end{array}$ & ---- & 146 & 186 & 7.03 & 227 & 12.75 & 19.78 \\
\hline$C A B-1-35$ & 11.0 & 150 & 191 & 11.95 & 231 & 6.45 & 18.40 \\
\hline$C A B-1-40$ & 11.0 & 153 & 194 & 10.80 & 236 & 12.19 & 22.99 \\
\hline$C A B-1-40$ & 13.6 & 153 & 192 & 10.50 & 236 & 15.41 & 25.91 \\
\hline$C A B-1-45$ & 11.0 & 153 & 194 & 2.85 & 235 & 20.15 & 23.00 \\
\hline$C A B-1-45$ & 15.0 & 153 & 195 & 6.46 & 235 & 17.08 & 23.54 \\
\hline$C A B-1-45$ & 21.0 & 153 & 193 & 3.51 & 235 & 16.74 & 20.25 \\
\hline$C A B-1-50$ & 11.0 & 153 & 196 & 8.63 & 234 & 17.03 & 25.66 \\
\hline$C A B-1-50$ & 15.0 & 153 & 194 & 7.80 & 234 & 16.20 & 24.00 \\
\hline$C A B-1-50$ & 21.0 & 153 & 194 & 8.65 & 234 & 16.50 & 25.15 \\
\hline $\begin{array}{l}\text { CAB-2 } \\
\text { Powder }\end{array}$ & $-\cdots$ & 130 & 166 & 11.46 & --- & ---- & 11.46 \\
\hline$C A B-2-35$ & 7.5 & 132 & 169 & 11.05 & $-\cdots$ & ----- & 11.05 \\
\hline $\mathrm{CAB}-2-40$ & 11.0 & 130 & 169 & 10.76 & --- & ---- & 10.76 \\
\hline$C A B-2-45$ & 11.0 & 130 & 166 & 11.69 & $-\cdots$ & --- & 11.69 \\
\hline$C A B-2-45$ & 15.0 & 127 & 167 & 11.00 & --- & $-\cdots-$ & 11.00 \\
\hline$C A B-2-45$ & 21.0 & 127 & 167 & 10.02 & ---- & ---- & 10.02 \\
\hline$C A B-2-50$ & 11.0 & 132 & 168 & 11.88 & ---- & --- & 11.88 \\
\hline$C A B-2-50$ & 15.0 & 130 & 168 & 12.33 & ----- & ----- & 12.33 \\
\hline $\mathrm{CAB}-2-50$ & 21.0 & 128 & 168 & 11.18 & ---- & ---- & 11.18 \\
\hline $\begin{array}{l}\text { CAB-3 } \\
\text { Powder }\end{array}$ & ---- & 97 & 162 & 13.67 & ----- & $-\infty--$ & 13.67 \\
\hline$C A B-3-25$ & 11.0 & 101 & 144 & $12 \cdot 32$ & $---\infty$ & ---- & 12.32 \\
\hline$C A B-3-25$ & 15.0 & 105 & 144 & 10.68 & ---- & ---- & 10.68 \\
\hline $\mathrm{CAB}-3-25$ & 21.0 & 105 & 144 & 12.06 & ----- & ----- & 12.06 \\
\hline $\mathrm{CAB}-3-40$ & 11.0 & 103 & 148 & 13.23 & ---- & ---- & 13.23 \\
\hline$C A B-3-40$ & 15.0 & 102 & 147 & 12.91 & ---- & ---- & 12.91 \\
\hline$C A B-3-40$ & 21.0 & 102 & 147 & 13.09 & $---\cdots$ & ---- & 13.09 \\
\hline$C A B-3-50$ & 11.0 & 101 & 145 & 13.00 & ----- & $-\cdots$ & 13.00 \\
\hline$C A B-3-50$ & 15.0 & 107 & 145 & 12.80 & $=--$ & ----- & 12.80 \\
\hline$C A B-3-50$ & 21.0 & 105 & 145 & 12.50 & ----- & ---- & 12.50 \\
\hline
\end{tabular}


(a)

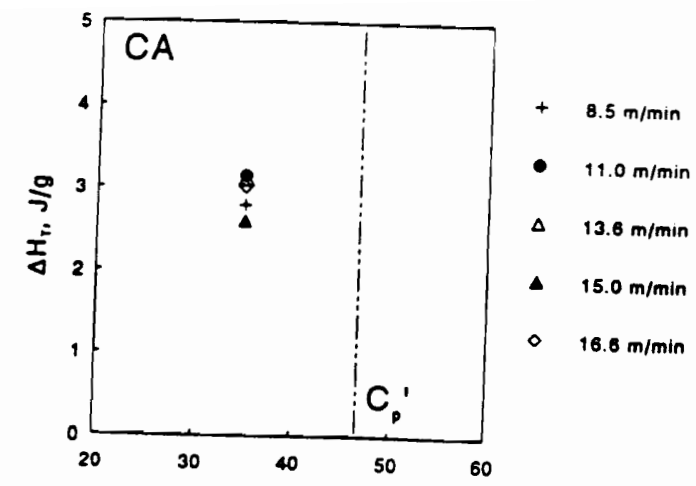

(b)

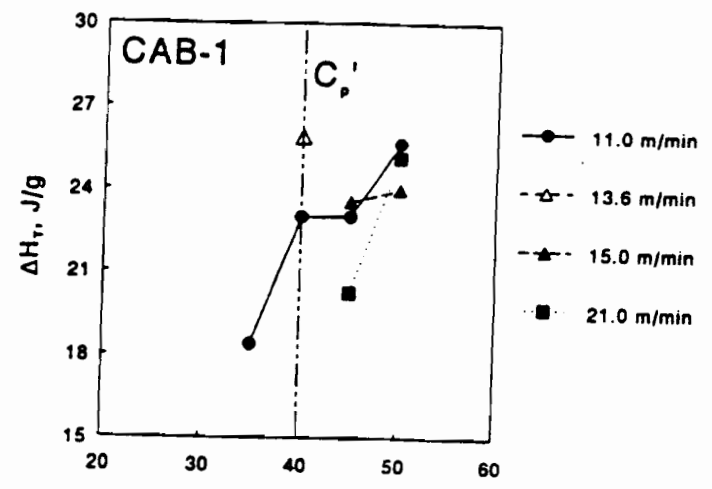

(c)

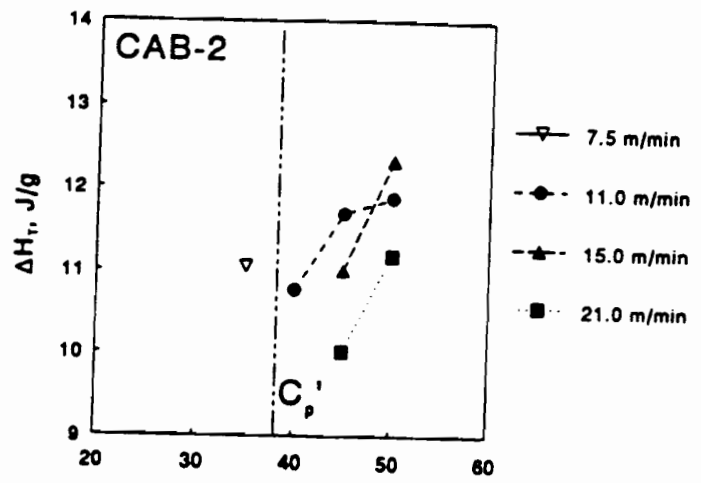

(d)

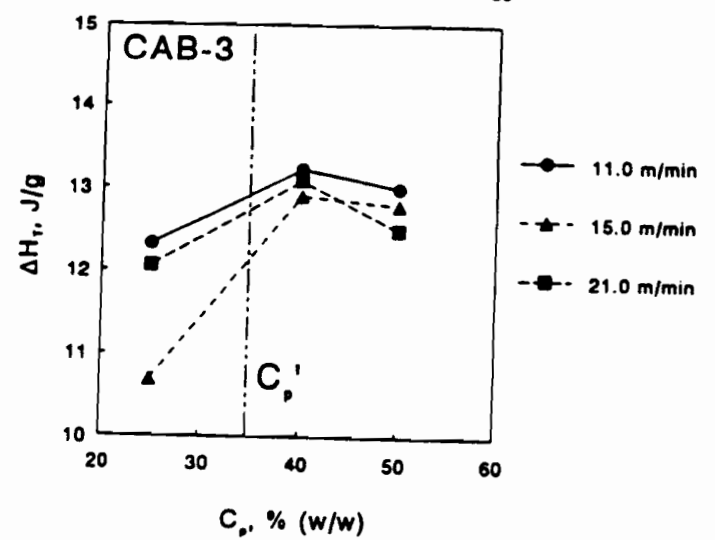

Figure 12. Heat of fusion versus polymer concentration of cellulose ester fibers spun at different take-up speeds; (a) CA (b) CAB-1 (c) CAB-2 (d) CAB-3. 
further insight about the morphology of the fibers. $C A B-1$ fibers spun from $35 \%(\mathrm{w} / \mathrm{w})$ and $50 \%(\mathrm{w} / \mathrm{w})$ solutions at $11 \mathrm{~m} / \mathrm{min}$ were heated in the vacuum oven at $160^{\circ} \mathrm{C}$ for the shrinkage experiment. After cooling to room temperature, the fibers from isotropic solution [35\% (w/w)] were kinked and shrunk by $14 \%$ whereas the fibers from anisotropic solutions [50\% (w/w)] retained its shape and shrunk by only $0.26 \%$. The experiment demonstrated that the amorphous regions in the fibers from isotropic solutions relaxed when heated above the glass transition temperature and caused the fibers to shrink. However, the fibers from liquid crystalline solutions were highly crystalline and retained its length and shape. This simple experiment qualitatively confirms the quantitative results obtained by DSC (i.e., heat of fusion) that fibers from anisotropic solutions are more crystalline than those from isotropic solutions.

\section{CONCLUSIONS}

1. Modulus and tenacity values increase for the fibers spun from liquid crystalline solutions of cellulose esters and this "jump" was further enhanced by increasing the draw ratio.

2. Mechanical properties of the cellulose ester fibers gradually deteriorated with increase in butyryl content.

3. All the fibers were completely coagulated during fiber spinning at different take-up speeds. 
4. Fibers spun from low concentration isotropic solutions had uniform and open cross-sectional structure and a smooth surface morphology. But the fibers from high concentration liquid crystalline solutions had nonuniform and dense cross-sectional structure and a coarse surface morphology which varied with the type of cellulose ester derivative.

5. "Skin-Core" morphology was observed in all the fibers.

6. The fibers from liquid crystalline solutions were oriented and highly crystalline as compared to the fibers from isotropic solutions. No improvement was observed in orientation with increase in draw ratio. 


\section{REFERENCES}

1. M. Panar and O. B. Wilcox, Belg. Pat. Appl. 656359, 1976; Fr. Pat. Appl. 2340344, 1977; Fed. Rep. of Germany Pat. Appl. 2705382,1977 .

2. J. Bheda, J. F. Fellers and J. L. White, colloid Polym. Sci., 258, 1335, 1980.

3. S. Suto, J. L. White and J. F. Fellers, Rheol. Acta, 21, $62,1982$.

4. S. M. Aharoni, Mol. Cryst. Liq. Cryst. (Letters), 56, $237,1980$.

5. S. M. Aharoni, J. Macromol. Sci. - Phy., B 21(2), 287, 1982 .

6. S. Dayan, P. Maissa, M. J. Vellutini and P. Sixou, J. Polym. Sci. Polym. Lett. Ed., 20, 33, 1982.

7. S. Dayan, F. Fried, J. M. Gilli and P. Sixou, J. Appl. Polym. Sci.: Appl. Polym. Symp., 37, 193, 1983.

8. P. Navard, J. M. Haudin, S. Dayan and P. Sixou, J. Appl. Polym. Sci.: Appl. Polym. Symp., 37, 211, 1083.

9. E. Bianchi, A. Ciferri, G. Conio, L. Lanzavecchia and M. Terbojevich, Macromolecules, $19(3), 630,1986$.

10. D. L. Patel and R. D. Gilbert, J. Polym. Sci.: Polym. Phys. Ed., 19, 1449, 1981.

11. D. L. Patel and R. D. Gilbert, J. Polym. Sci.: Polym. Phys. Ed., 20, 1019, 1982 . 
12. D. L. Patel and R. D. Gilbert, J. Polym. Sci.: Polym. Phys. Ed., 21, 1079, 1983.

13. Y. K. Hong, D. E. Hawkinson, E. Kohout, A. Garrard, R. E. Fornes and R. D. Gilbert, in Polymer Association structures, ACS Symposium Series 384 , A. M. El-Nokaly, Ed. $184,1989$.

14. V. V. Myasoedova, O. V. Alekseeva and G. A. Krestov, Zh. Prikl. Khim., (Leningrad), 60 (10), 2526, 1987.

15. G. A. Krestov, V. V. Myasoedova, O. V. Alekseeva and S. Y. Belov, Dokl. Akad. Nauk SSSR, 293 (1), 174, 1987.

16. P. Navard and J. Haudin, Br. Polym. J., 12 (4), 174, 1980.

17. P. Navard, J. M. Haudin, S. Dayan and P. Sixou, J. Polym. Sci.: Polym. Lett. Ed., 19 (8), 379, 1981.

18. P. Navard and J. M. Haudin, Calorim. Anal. Therm., 14, 207,1983

19. G. H. Meeten and P. Navard, Polymer, 23, 1727, 1982.

20. P. Sixou, J. Lematre, A. Ten Bosch, J. M. Gilli and S. Dayan, Mol. Cryst. Liq. Cryst., 91 (3-4), 277, 1983.

21. J. Bheda, J. F. Fellers and J. L. White, J. Appl. Polym. Sci., 26, 3955, 1981 .

22. J. P. O'Brien, U. S. Patent 4464323; 4501886, 1984.

23. J. Ferguson and K. M. Ibrahim, Polymer, 10 (2), 135, 1969.

24. P. D. Griswold and J. A. Cuculo, J. Appl. Polym. Sci., $18,2887,1974$ 
25. K. Shimamura, J. L. White and J. F. Fellers, J. Appl. Polym. Sci., 26, 2165, 1981 .

26. M. Horio, S. Ishikawa and K. Oda, J. Appl. Polym. Sci.: Appl. Polym. Symp., 41 (U.S. - Japan Semin. Polym. Liq. (cryst.), 269, 1983 .

27. V. Davé and W. G. Glasser, in viscoelasticity of Biomaterials, ACS Symp. Series, W. G..Glasser and H. Hatakeyama, Eds., (In press).

28. H. Blades, U. S. Patent 3767756,1972 .

29. A. Ciferri, "Developments in Oriented Polymers-2", I. M. Ward, Ed., Elsevier Applied Science Publishers, Ltd., 1986 .

30. G. Marrucci, IUPAC Symposium on Non Crystalline order in Polymers, Naples, Italy, May, 1985.

31. G. Marrucci, IX International Congress of Rheology, B. Mena, A. Garcia Rejon and C. Range Naisale, Ed., University National Aut. de Mexico, Acapulco, 441, 1984 .

32. M. Jaffe and R. Sidney Jones in "High Technology Fibers", Part A, M. Lewin and J. Preston, Eds., Handbook of Fiber science and Technology: Vol. III, Marcel Dekker, New York, 349, 1985 .

33. D. R. Paul, J. Appl. Polym. Sci,, 12, 2273, 1968.

34. G. Conio, R. Bruzzone, A. Ciferri, E. Bianchi and A. Tealdi, Polym. J., 19 (6), 757, 1987.

35. E. Bianchi, A. Ciferri, G. Conio and A. Tealdi, J. Polym. Sci.: Part B: Polym. Phys., 27, 1477, 1989. 
36. M. G. Northholt and J. J. van Aartsen, Europhysics Conf. Abstr., 10, 79, April, 1976.

37. J. J. Cho, S. H. Hudson and J. A. Cuculo, J. Polym. Sci.: Part B: Polym. Phys., 27, 1699, 1989.

38. C. K. Liu, J. A. Cuculo and B. Smith, J. Polym. Sci.: Part B: Polym. Phys., 27, 2493, 1989.

39. C. K. Liu, J. A. Cuculo and B. Smith, J. Polym. Sci.: Part B: Polym. Phys., 28, 449, 1990.

40. D. R. Paul, J. Appl. Polym. Sci., 12, 383, 1968.

41. T. A. Hancock, J. L. White and J. E. Spruiell, Polym. Sci. Engr., 20 (17), 1126, 1980.

42. A. Ziabicki, "Fundamentals of Fiber Formation", John Wiley \& Sons, Ltd., 1976.

43. H. C. Trivedi, K. C. Patel and R. D. Patel, J. Macromol. Sci.-Chem., A $19(1), 85,1983$.

44. M. Scandola and G. Ceccorulli, Polymer, 26, 1953, 1985. 45. V. F. Uryash, I. B. Rabinovich and A. N. Mochalov and T. B. Khlyustova, Thermochimicia Acta, 93, 409, 1985.

46. C. J. Malm, L. J. Tanghe, H. M. Herzog and M. H. Stewart, Ind. Eng. Chem., 50 (7), 1061, 1958 . 
4.0 CELLULOSE-BASED FIBERS FROM LIQUID CRYSTALLINE SOLUTIONS.

III. PROCESSING AND MORPHOLOGY OF CELLULOSE AND CELLULOSE HEXANOATE ESTERS. 


\title{
CELLULOSE-BASED FIBERS FROM LIQUID CRYSTALLINE SOLUTIONS.
}

III. Processing and Morphology of Cellulose and Cellulose Hexanoate Esters.

\begin{abstract}
Cellulose and cellulose hexanoate ester exhibited liquid crystalline behavior in dimethylacetamide/lithium chloride and dimethylacetamide, respectively. The experimentally observed critical volume fraction $\left(V_{p}{ }^{c}\right)$ of cellulose was lower than predicted by Flory's theory, whereas the experimental and theoretical values of $\mathrm{V}_{\mathrm{p}}^{\mathrm{c}}$ were within $70 \%$ of prediction for cellulose hexanoate. The $\mathrm{V}_{\mathrm{p}}^{\mathrm{c}}$ value obtained for cellulose hexanoate was lower than that previously reported for cellulose acetate butyrate with maximum degree of butyration $(C A B-3)$. This indicates that bulky substituents may lower $v_{p}{ }^{c}$ values. Fibers were spun from isotropic and anisotropic solutions of cellulose and cellulose hexanoate by dry jet/wet spinning method. There was an increase in mechanical properties through the isotropic to anisotropic transition with moduli reaching $152 \mathrm{~g} / \mathrm{d}(20.8 \mathrm{GPa})$ for cellulose fibers. The formation of cellulose fibers with high modulus at large extrusion rates and large take-up speeds (draw ratio) is explained with molecular organization prior to coagulation. This unexpected enhancement is attributed to the air-gap that exists in the dry jet/wet spinning process. similar improvements were not observed for cellulose hexanoate fibers.
\end{abstract}


This is explained with incomplete development of liquid crystalline structure at the solution concentrations from which the fibers were spun.

\section{INTRODUCTION}

Cellulosic fibers (rayon) have been manufactured by the viscose process for almost a century. However, slow extrusion speeds, high pollution, high energy consumption and high investment costs have made the process un-competitive. Research in the area of liquid crystal formation of cellulose in new solvent systems has been primarily driven by the aspiration to develop new non-polluting processes to produce regenerated cellulose fibers. Since cellulose is the most abundant renewable organic raw material, it can replace petrochemicals. Due to its complex morphology of crystalline regions and hydrogen bonding, cellulose is difficult to dissolve in common solvents. Indirect solvent systems such as nitrogen tetroxide/dimethyl formamide or dimethyl sulfoxide $(1,2)$, paraformaldehyde /dimethyl sulfoxide (3), chloral/dimethyl formamide/pyridine (4) and urea/sodium hydroxide (5) form cellulose derivatives during the dissolution of cellulose, at least temporarily. Direct solvent systems may form a complex with cellulose but no new covalent bonds are formed, and cellulose maintains its original chemical structure. Dissolution process, by-product control and solvent recovery make direct solvent systems superior to 
their indirect counterparts for fiber spinning. A detailed review was published on different solvent systems of unmodified cellulose ( 6 ). Only a brief summary of liquid crystalline studies of cellulose solutions in direct solvent systems is presented here.

Graenacher and Sallman (7) first discovered that a family of tertiary amine oxides is capable of dissolving cellulose. This was rediscovered by Johnson thirty years later (8). The detailed mechanism of dissolution and fiber spinning of cellulose from this solvent system was reported by investigators at American Enka (9-14). The physical properties of these fibers were comparable to rayon fibers.

Chanzy and Peguy (15) were the first to report the formation of fibers from lyotropic mesophase of cellulose in the mixture of $\mathrm{N}$-methyl-morpholine $\mathrm{N}$-oxide (MMNO) and water. The anisotropy depended on temperature of the solution $\left(>90^{\circ} \mathrm{C}\right)$, concentration of cellulose $[>20 \%(\mathrm{w} / \mathrm{w})]$, mole ratio of water/anhydrous MMNO $(<1)$, and degree of polymerization of the dissolved cellulose. Structural and molecular characterization of MMNO in its anhydrous and monohydrate states was undertaken to investigate the basic geometry and conformation of MMNO and the nature of its interactions with water molecules $(16-18)$. Detailed investigations have been carried out on limiting viscosity number-molecular weight relationship (19), rheology $(20,21)$, swelling (22), 
crystallization (23), phase behavior (24), and fiber spinning (25-27) of cellulose in MMNO. Modulus and tenacity of dry jet/ wet spun fibers were $21 \mathrm{GPa}$ and $0.5 \mathrm{GPa}$, respectively, from cellulose with DP $=600(27)$. These fibers are in the process of getting commercialized under the trade-name of Tencel by Courtaulds. MMNO was also utilized to prepare solutions from numerous polysaccharides (except chitin); and most of them formed anisotropic solutions (28).

The highest cellulose concentrations achieved for this solvent system were $55 \%(w / w)$ for microcrystalline cellulose (DP 35) and 30-35\% (w/w) for cellulose with DP $600-1000$. It is recommended that a stabilizer such as n-propyl gallate be added to the solution to reduce cellulose degradation. High processing temperatures appear to be the limitation for spinning fibers from this system.

Patel and Gilbert showed lyotropic mesophase formation of cellulose in mixtures of trifluoroacetic acid (TFA) and chlorinated alkanes at room temperature (29). The mesophase was identified to be cholesteric, and this is expected from the chirality of cellulose. An anisotropic phase was observed in $4 \%(\mathrm{w} / \mathrm{w})$ solutions of whatman cellulose in TFA $-\mathrm{CH}_{2} \mathrm{Cl}_{2} \quad(70: 30$ $\mathrm{v} / \mathrm{v})(30)$. Degradation of cellulose occurred in $\mathrm{TFA}-\mathrm{CH}_{2} \mathrm{Cl}_{2}$, presumably due to the attack of TFA at the glycosidic linkages and the rate of degradation decreased with decreasing TFA $/ \mathrm{CH}_{2} \mathrm{Cl}_{2}$ ratio (30). There are no reports of fiber spinning 
from this solvent system. A number of Russian workers have studied the cellulose-TFA and cellulose-TFA-ClCH $\mathrm{CH}_{2} \mathrm{Cl}$ systems (31-33) and have prepared solutions up to $20 \%(\mathrm{w} / \mathrm{w})$ concentrations. Dissolution occurred without cellulose modification or oxidative degradation with little or no trifluroacetylation of cellulose $(30,31)$.

Although first reported by Scherer in 1931 (34), the ammonia/ammonium thiocyanate solvent system for cellulose was systematically studied only recently by Hudson and Cuculo (3537). This solvent system is relatively non-toxic; it causes little or no degradation of cellulose; and the dissolution procedure is simple and safe. A detailed coagulation study for this solvent system was done in order to understand the fiber forming ability of cellulose under various conditions (38-40) . A cellulose with DP 210 formed a cholesteric phase at $3.5 \%$ $(\mathrm{w} / \mathrm{v})$ and a nematic phase at $8-16 \%(\mathrm{w} / \mathrm{v})$ at $25^{\circ} \mathrm{C}$ in a solvent containing $75.5 \%(\mathrm{w} / \mathrm{w})$ ammonium thiocyanate and $24.5 \%(\mathrm{w} / \mathrm{w})$ ammonia (41). This type of lyotropic system makes it possible to selectively produce cellulose mesophases in either cholesteric or nematic phase. Fibers spun from nematic cellulose solutions by the dry jet/wet spinning method had modulus and tenacity values of $44 \mathrm{~g} / \mathrm{d}$ and $0.98 \mathrm{~g} / \mathrm{d}$ (DP 210 ); and of $167 \mathrm{~g} / \mathrm{d}$ and $3.4 \mathrm{~g} / \mathrm{d}$ (DP 765), respectively (41). McCormick showed (42) that dimethylacetamide/lithium chloride is a good solvent system for cellulose, and he and 
his associates demonstrated the utility of this system for synthesizing various cellulose derivatives $(42,43)$. Solution studies of cellulose, and the formation of cholesteric mesophase order were reported in this system by Mccormick $(44,45)$ followed by Ciferri (46). Turbak also studied the dissolution process and was first to describe fiber spinning of cellulose in this solvent system (47). A critical aspect of this system is the necessity of activating the cellulose prior to dissolution. Maximum cellulose concentration achieved was $16 \%(w / w)$ with DP of 550 (47). Licl-DMAC does not degrade or react with cellulose (48), but it does form a complex with the hydroxyl groups on the cellulose backbone which results in its dissolution $(48,49)$. A pure anisotropic phase was not observed due to solubility limits. This prevents attainment of complete anisotropy. Bianchi et al. (50) reported on wet spun fibers from isotropic and anisotropic solutions of cellulose (DP 290) in LiCl (7.8\%)/DMAC. Wet spinning involved the processing of solutions without an air gap. The fiber properties increased through the isotropic-anisotropic transition with modulus and tenacity values reaching $161 \mathrm{~g} / \mathrm{d}(22 \mathrm{GPa})$ and $2.5 \mathrm{~g} / \mathrm{d}$ $(0.35 \mathrm{GPa})$, respectively.

Previously (51) we established that solution anisotropy can be reached at increasingly lower concentrations if cellulose esters possess substituent groups that are increasingly large and bulky. This study is to further examine a cellulose derivative with an unusually large substituent 
(i.e., hexanoate) so as to test whether this principle applies in general. The objectives of this study are to investigate the rheological and morphological properties of liquid crystalline solutions of cellulose and cellulose hexanoate; and to establish structure-property relationships of fibers spun from isotropic and anisotropic solutions of cellulose and cellulose hexanoate by dry jet/wet spinning process.

\section{EXPERIMENTAL}

\section{Materials.}

Whatman CF-11 cellulose powder was used as received. Cellulose hexanoate was prepared in our laboratory by the acylation of Whatman cellulose in homogenous phase (3\% DMAC/LiCl-9\%) with hexanoic anhydride using N,Ndicyclohexylcarbodiimide [DCC] and 4-pyrrolidinopyridine [PP] as catalyst $(52)$.

Reagent grade dimethylacetamide and lithium chloride was used as received.

\section{Methods.}

1. Determination of Molecular Parameters:

Carbanilate derivatives of cellulose and cellulose hexanoate were prepared (53) to make solutions in HPLC-grade tetrahydrofuran (THF). The molecular parameters like molecular weight, degree of polymerization (DP), intrinsic viscosity and Mark-Houwink-Sakurda constants were determined using gel permeation chromatography with a differential viscosity 
detector (Viscotek Model No. 100) and a differential refractive index (concentration) detector (Waters 410) in sequence. All the calculations were based on a universal calibration curve.

2. Preparation of Solutions:

Solutions of cellulose were prepared in DMAC/LiCl following the method described by Turbak (47) with which complete dissolution was better achieved than with the method reported by McCormick (54). Weighed amount of DMAC was added to weighed amount of cellulose which was soaked in DMAc for 3 days. The mixture was heated to reflux temperature (approximately $165^{\circ} \mathrm{C}$ ) for 20-30 minutes while being mechanically stirred under nitrogen atmosphere. The mixture was allowed to cool to about $100^{\circ} \mathrm{C}$, and a weighed amount of lithium chloride was added under steady stirring. The mixture was stirred for about 30 minutes at $80^{\circ} \mathrm{C}$ and was allowed to gradually cool to room temperature. Stirring was continued for about 20 hours at room temperature. The cooled solution was slightly brown. Polymer concentration, $c_{p}$, given as grams of cellulose per 100 grams of ternary solution (cellulose, DMAc and $\operatorname{LiCl}$ ), varied between 6.5 and $13.5 \%$. Salt concentration, $\mathrm{C}_{\mathrm{s}}$, given as grams of LiCl per 100 grams of binary solution (DMAC and LiCl) was kept at $7.8 \%$ for all solutions.

A complete range of solutions [5-40\% (w/w) ] were prepared by mechanically mixing known weights of cellulose hexanoate 
with DMAC at ambient temperature. LiCl was not required for these solutions.

The solutions were allowed to equilibrate for 2 weeks prior to analysis. Extreme care was taken to treat the solutions similarly so that relative aging effect was constant for all of them.

3. Viscosity Measurements:

Viscosity measurements of the solutions below $6.5 \%$ (w/w) and $15 \%(w / w)$ concentration, for cellulose and cellulose hexanoate, respectively, were made at $25^{\circ} \mathrm{C}$ using a wellsBrookfield Cone/Plate Viscometer at different cone rotation speeds. A Rheometrics Mechanical Spectrometer (RMS 800) was used to determine the rheological properties of $15 \%(\mathrm{w} / \mathrm{w})$ and higher concentrated solutions of cellulose hexanoate and all the other cellulose solutions. The solutions were placed in a parallel-disk geometry. The dynamic mechanical properties were measured at $26^{\circ} \mathrm{C}$ using a strain amplitude of $25 \%$ of the value at which the respective sample showed viscoelasticity. The frequency ranged from 0.1 to $100.0 \mathrm{rad} / \mathrm{sec}$.

4. Polarized Optical Microscopy:

Polarized optical microscopy was performed with a Zeiss Axioplan Universal Microscope. Small amounts of cellulose and cellulose hexanoate solutions were placed between microscope slide and cover slip, and these were examined for birefringence between the cross polarizers of the microscope 
at room temperature.

5. Fiber Spinning:

Continuous fibers were processed from a single hole with capillary diameter of $0.3 \mathrm{~mm}$ and using dry jet/wet spinning method as described in our earlier publication (55). The flow rate of the solutions was varied between 0.4 and $3 \mathrm{~cm}^{3} / \mathrm{min}$ by the extrusion pump. The fibers were wound on the spools at take-up speeds varying from $11-46 \mathrm{~m} / \mathrm{min}$. The air-gap was about 1 inch. Water was used as the coagulant at approximately $30^{\circ} \mathrm{C}$. The fiber-wound spools were immersed in beakers containing water for 8 hours and were allowed to air-dry for about 8-10 hours. The fibers were further vacuum-dried for 24 hours at approximately $50^{\circ} \mathrm{C}$. No post-spinning treatments (thermal or mechanical) were performed.

6. Fiber Diameter:

Fiber diameter (D) was determined by a Nikon UM-2 Measurescope equipped with a Quadra-Chek III attachment. The diameter was generally constant along an extended length of the fiber. The reported $D$ value is the average of three to four measurements along the fiber length.

7. Mechanical Properties:

The mechanical properties of the fibers were determined on an Instron 1130 Test Instrument following the procedure of ASTM standard D3822. Tests were conducted at room temperature and $67 \%$ relative humidity with 1 inch gauge length at strain 
Table I. Chemical and Molecular Characteristics of Carbanilated Derivatives of Cellulose and Cellulose Hexanoate.

\begin{tabular}{|c|c|c|}
\hline & $\begin{array}{c}\text { Cellulose } \\
\text { Tricarbanilate }\end{array}$ & $\begin{array}{l}\text { Carbanilated } \\
\text { Cellulose Hexanoate }\end{array}$ \\
\hline $\mathrm{DS}_{\mathrm{CAR}}$ & 3.0 & 2.31 \\
\hline $\mathrm{DS}_{\mathrm{HEx}}{ }^{1)}$ & 0 & 0.69 \\
\hline$\left\langle M_{n}\right\rangle\left(\times 10^{5}\right)$ & 1.1 & 0.95 \\
\hline$\left\langle\mathrm{M}_{\mathrm{w}}\right\rangle\left(\times 10^{5}\right)$ & 1.8 & 1.4 \\
\hline$D P_{n}$ & 212 & 190 \\
\hline MHS - constant (a) & 0.83 & 0.86 \\
\hline $\begin{array}{c}\text { Intrinsic Viscosity } \\
(\mathrm{dL} / \mathrm{g})\end{array}$ & 1.34 & 1.05 \\
\hline $\log K$ & -4.19 & -4.36 \\
\hline
\end{tabular}

1) Determined by H-NMR spectroscopy. 
rate of 0.2 inches/min. Single fibers were used for cellulose and cellulose hexanoate during testing. Linear density varied between 48 and 270 denier for cellulose hexanoate fibers and between 36 and 180 denier for cellulose fibers. The values of initial modulus $(E)$, breaking tenacity $\left(\sigma_{b}\right)$, breaking toughness (BT) and elongation at break $\left(\varepsilon_{b}\right)$ were the average of 6-10 measurements.

8. Scanning Electron Microscopy:

The fibers were examined on a JEOL JSM-35C scanning electron microscope with an accelerating voltage of $15 \mathrm{kV}$. Fracture surfaces were formed in liquid nitrogen. The fibers were mounted on aluminum specimen mounts (EMSL) with an angle of $45^{\circ}$. The fibers were coated at the bottom by Ladd silver Conducting Paint and then sputter coated by pure gold for 1 minute (thickness about $9 \mathrm{~nm}$ ) in a SPI Sputter coater.

\section{RESULTS AND DISCUSSION}

Table I summarizes the chemical and molecular characteristics of the carbanilated derivatives of cellulose and cellulose hexanoate employed in this study. The degree of substitution (DS) of hexanoic groups ( $\left.\mathrm{DS}_{\mathrm{HEX}}\right)$ was 0.69 as evaluated by proton NMR spectroscopy (52). Unsubstituted hydroxyl groups $\left(D_{\mathrm{OH}}=2.31\right)$ in cellulose hexanoate were derivatized by the carbanilate groups to prepare solutions in THF for molecular weight determination. The $\mathrm{DP}_{\mathrm{n}}$ of cellulose and cellulose hexanoate was 212 and 190, respectively. It 
should be noted that the MHS constant ' $a$ ' was lower than expected for unsubstituted cellulose (i.e., 0.83). This is due to the bulky carbanilate groups.

I. Dynamic Viscoelastic Properties:

(a) Cellulose.

The relationship between dynamic shear viscosity and concentration of cellulose solutions at different shear rates or frequencies [applying the Cox-Merz rule (56)] is demonstrated in Figure 1 . The dynamic viscosity decreases and then increases at higher concentrations. The drop in viscosity after $7 \%(\mathrm{w} / \mathrm{w})$ concentration is due to the onset of liquid crystalline behavior of the cellulose solutions. $7 \%(\mathrm{w} / \mathrm{w})$ concentration is considered as the critical concentration value $\left(C_{p}^{\prime}\right)$ beyond which cellulose solutions exhibit liquid crystalline behavior in DMAC/LiCl. This value corresponds to $V_{p}^{c}=0.044$. This drop in viscosity was not observed for cellulose solutions in DMAc/LiCl by McCormick (44) or in TFA$\mathrm{CH}_{2} \mathrm{Cl}_{2}$ by $\mathrm{Gilbert}(30)$; it was, however, reported for cellulose solutions in MMNO as a function of temperature (20). To our knowledge, the observation of a critical concentration value by rheological measurements with cellulose solutions in DMAC/Licl has never been reported before. The drop in viscosity is observed at constant concentration. This indicates that dynamic shear does not drive the thermodynamic transition of the anisotropic phase to a different 


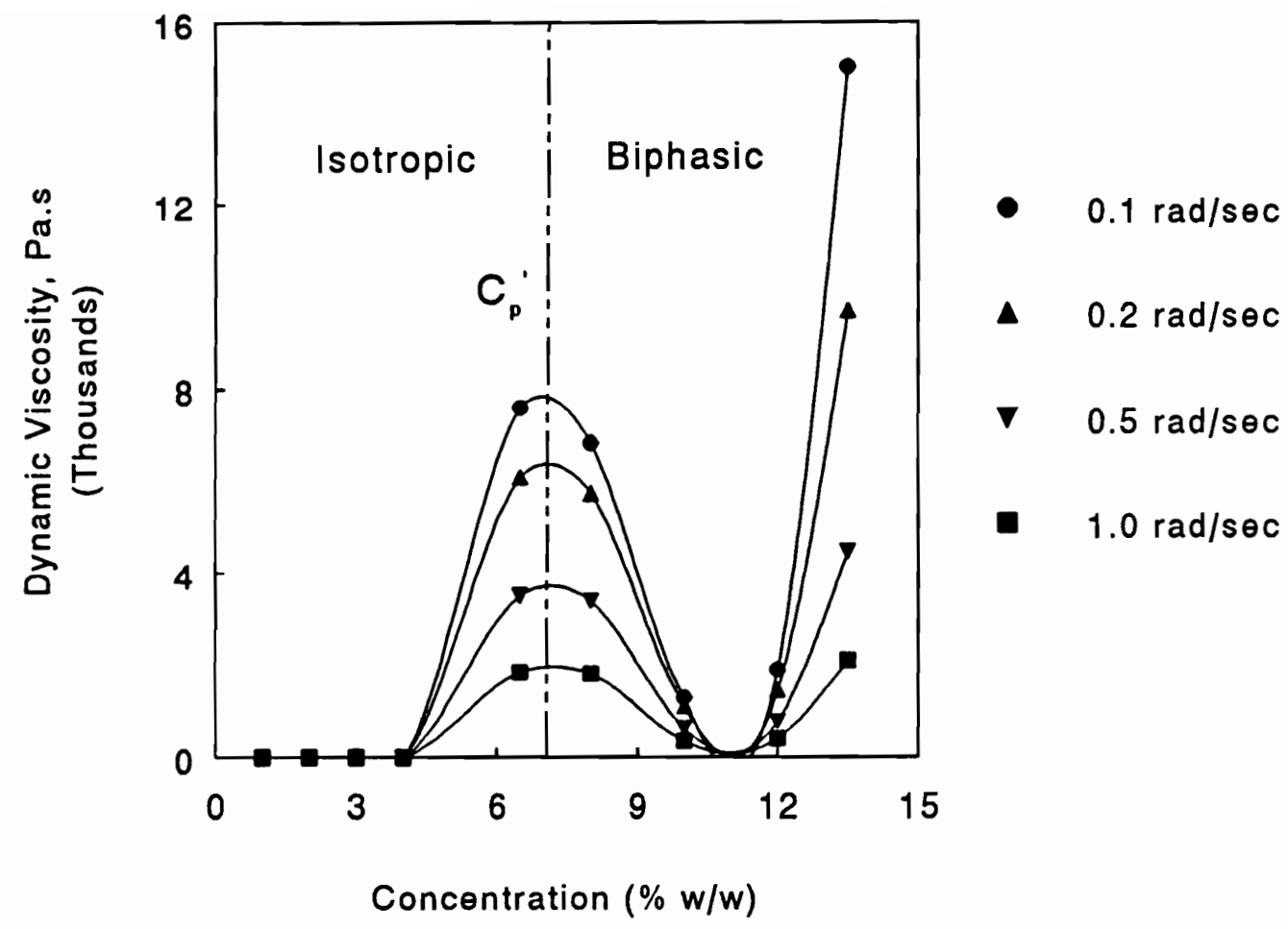

Figure 1. Dynamic viscosity versus concentration of cellulose solutions in $\mathrm{DMAc} / \mathrm{LiCl}$ at different frequencies. 
concentration.

At higher concentrations [i.e., above $12 \%$ (w/w)], the viscosity is seen to increase again. A similar rise in viscosity for cellulose solutions was universally observed by previous investigators $(30,44)$. Based on the phase diagram of cellulose in DMAC/LiCl, Ciferri (46) showed that the cholesteric mesophase forms in the proximity of the limit of solubility. Therefore, the formation of a liquid crystalline phase represents a metastable condition that is closely associated with crystallization (49). Consequently, the viscosity increases again at higher solution concentration. The viscosity of the solutions decreases with an increase in shear rate, and this confirms shear thinning behavior.

The relationship of dynamic elastic modulus (G') and dynamic loss modulus (G'') and concentration at different frequencies is shown in Figures 2 and 3 , respectively. The feature of the concentration dependence of $G^{\prime}$ and $G^{\prime \prime}$ is identical to that seen for the dynamic viscosity, i.e., after the solution becomes liquid crystalline, G' and G' decrease before increasing again at higher concentration. Similar to any viscoelastic material, $G^{\prime}$ increases with increasing frequency. G'' rises with frequency and does not fall even though the solutions are liquid crystalline. This shows that the damping characteristics of liquid crystalline solutions are higher than those of isotropic solutions. These results reveal that liquid crystalline cellulose solutions are 


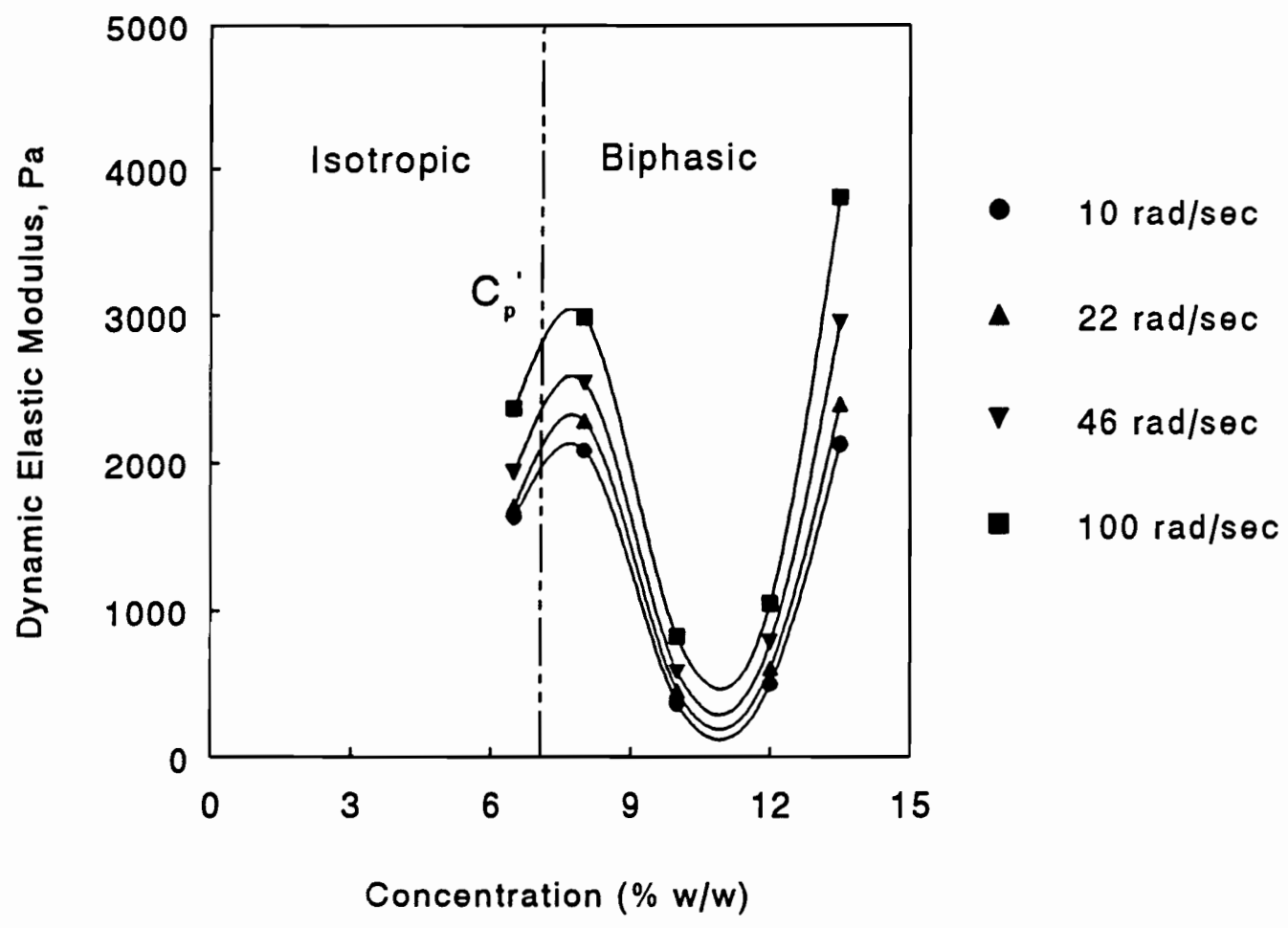

Figure 2. Dynamic elastic modulus versus concentration of cellulose solutions in $\mathrm{DMAc} / \mathrm{LiCl}$ at different frequencies. 


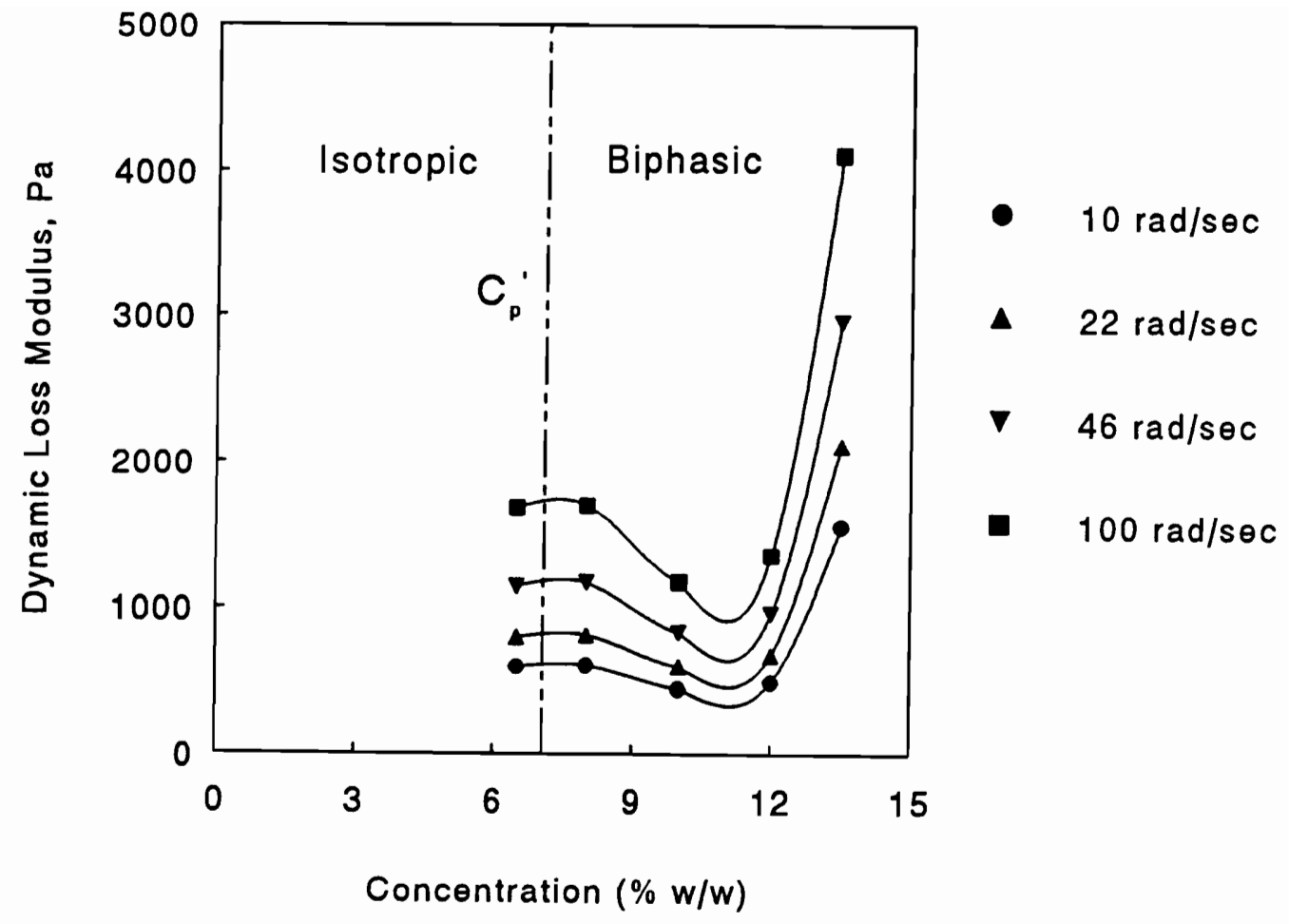

Figure 3. Dynamic loss modulus versus concentration of cellulose solutions in $\mathrm{DMAc} / \mathrm{LiCl}$ at different frequencies. 
viscoelastic in nature.

(b) Cellulose Hexanoate.

The relationship between dynamic shear viscosity and concentration of cellulose hexanoate solutions at different frequencies is shown in Figure 4. The viscosity increases with concentration and decreases with frequency. The expected decrease in viscosity at some critical concentration is not observed, and this is in contrast to typical polymer liquid crystalline solutions.

The relationships between dynamic elastic modulus $\left(G^{\prime}\right)$ and dynamic loss modulus $\left(G^{\prime}{ }^{\prime}\right)$ and concentration at different frequencies are shown in Figs. 5 and 6 , respectively. The characteristics of the concentration dependence of $\mathrm{G}^{\prime}$ and $\mathrm{G}^{\prime \prime}$ are the same as those observed for the dynamic viscosity. Similar trends were observed for cellulose solutions at higher concentrations (i.e., above 12\%).

II. Polarized Optical Microscopy:

a) Cellulose.

Figure 7 (a) - (c) shows the photomicrographs of $10 \%$ $(\mathrm{w} / \mathrm{w}), 12 \%(\mathrm{w} / \mathrm{w})$ and $13.5 \%(\mathrm{w} / \mathrm{w})$ cellulose solutions, respectively. An increase in birefringence was observed as the solution concentration increased. Solutions below $10 \%$ (W/W) were isotropic. By contrast, the onset of liquid crystalline behavior by rheological observations was at $7 \%$ (w/w). Lyotropic mesophases of cellulose in DMAC/LiCl were reported to occur (by microscopy) at $11 \%$ (w/w) (DP 288) and $10 \%$ (w/w) 


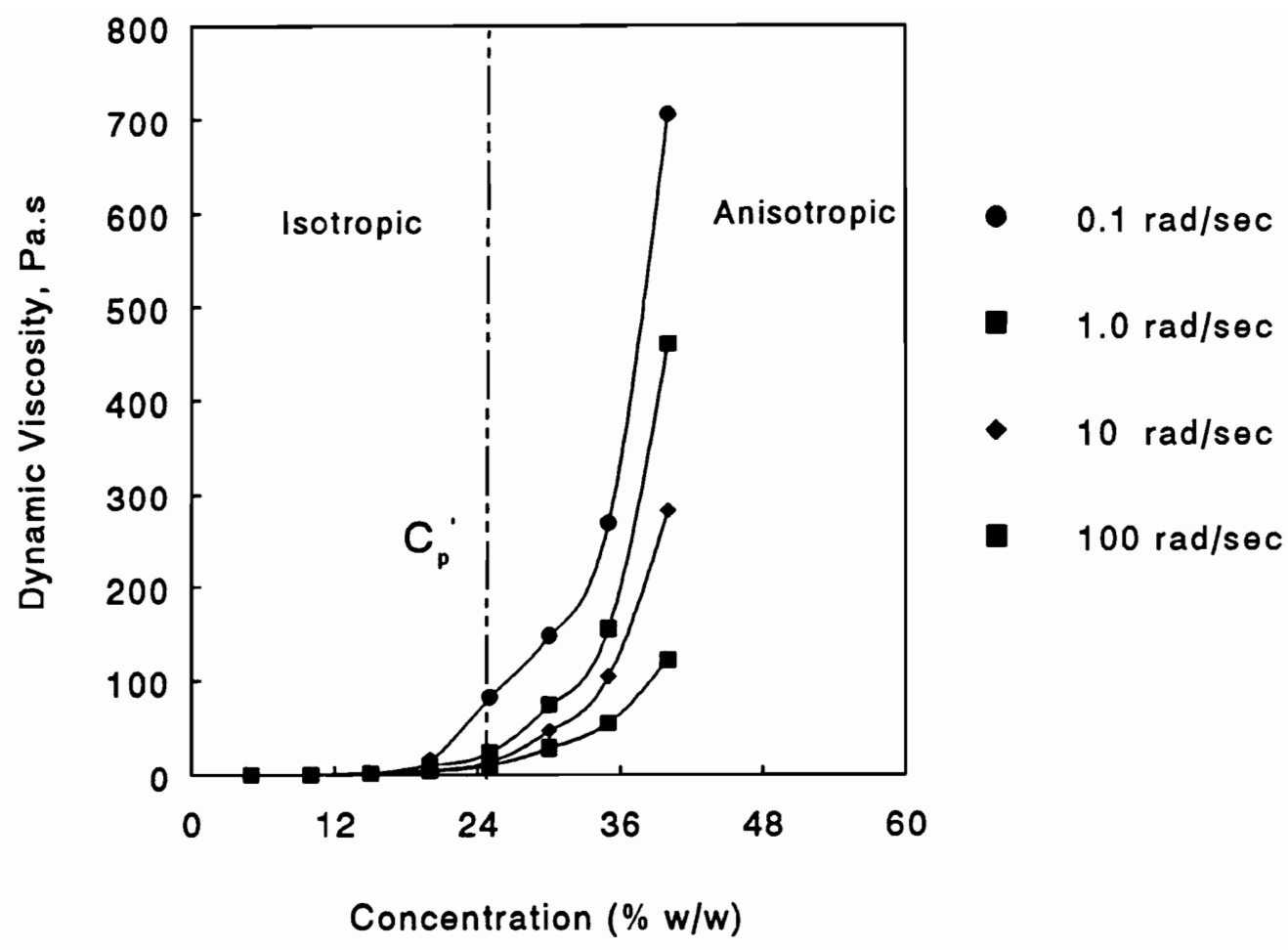

Figure 4. Dynamic viscosity versus concentration of cellulose hexanoate solutions in DMAc at different frequencies. 


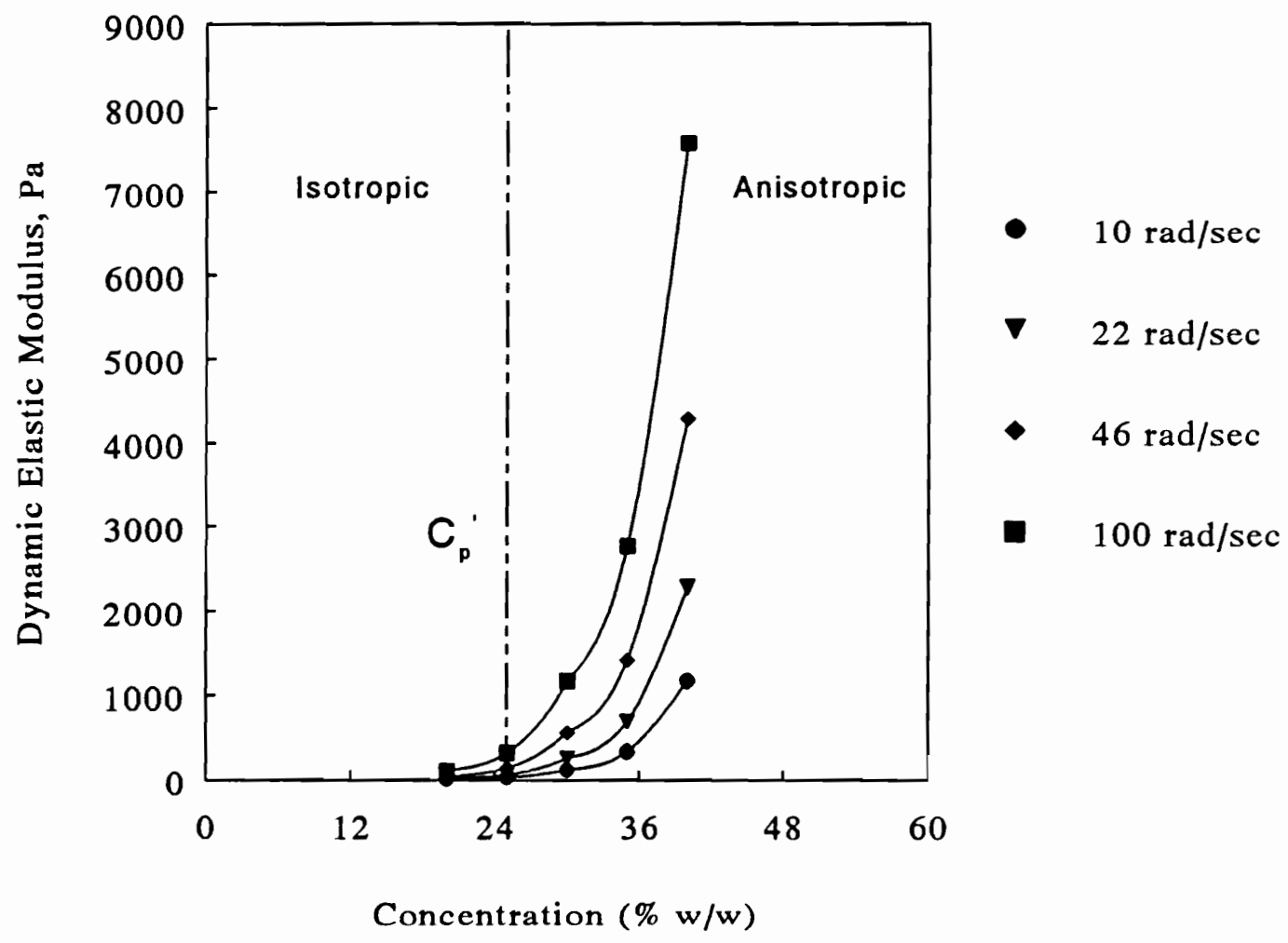

Figure 5. Dynamic elastic modulus versus concentration of cellulose hexanoate solutions in DMAc at different frequencies. 


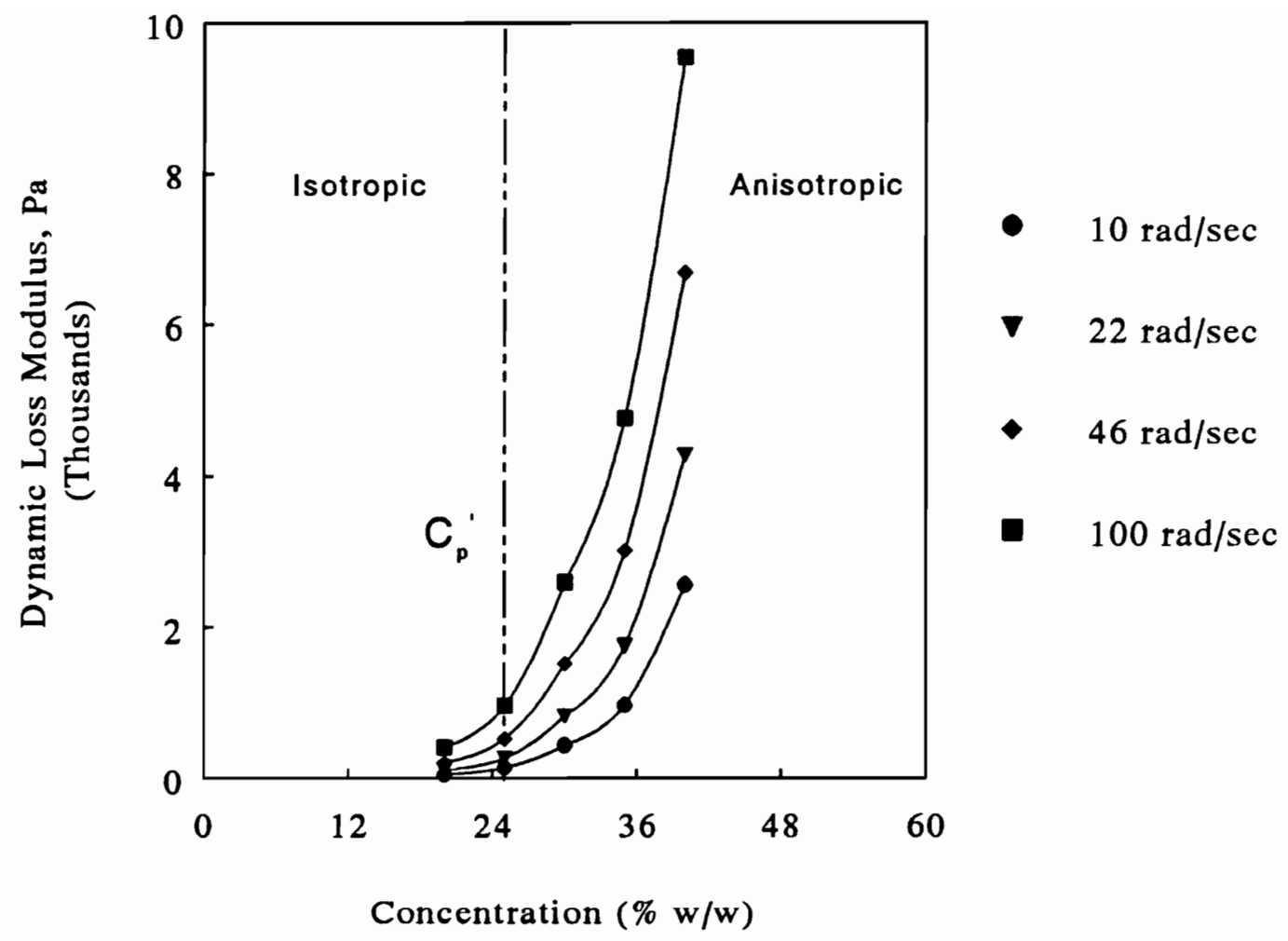

Figure 6. Dynamic loss modulus versus concentration of cellulose hexanoate solutions in DMAc at different frequencies. 
concentrations by Ciferri (46) and Mccormick (44), respectively. These values are in overall agreement, and rheological measurements must be suspected to be more sensitive to the onset of molecular organization than optical microscopy in this particular case.

b) Cellulose Hexanoate.

Figures 8 (a) - (d) show the photomicrographs of 25\% $(w / w), 30 \%(w / w), 35 \%(w / w)$ and $40 \%(w / w)$ cellulose hexanoate solutions, respectively. The onset of liquid crystallinity is detected at approximately $25 \%(\mathrm{w} / \mathrm{w})$. The solution becomes anisotropic at $40 \%(\mathrm{w} / \mathrm{w})$ concentration. Since the viscosity measurements (Figs. 4-6) failed to reveal typical liquid crystalline solution behavior, $c_{p}^{\prime}$ is in this case defined as the value determined by optical microscopy, i.e., $25 \%$ (w/w). This value corresponds to $\mathrm{V}_{\mathrm{p}}^{\mathrm{c}}=0.16$. The lyotropic mesophase formation of cellulose hexanoate has not been reported before. This study reveals that cellulose (derivative) solutions must be observed by optical microscopy in addition to rheology for detecting behavior typical of liquid crystallinity.

III. Theoretical Considerations for the Determination of Critical Volume Fractions $\left(\mathrm{V}_{\mathrm{p}}^{\mathrm{c}}\right.$ :

a) Cellulose.

Table II shows the theoretical values of molecular parameters for cellulose and cellulose hexanoate. (Please see the appendix for the calculations). Flory's lattice theory 


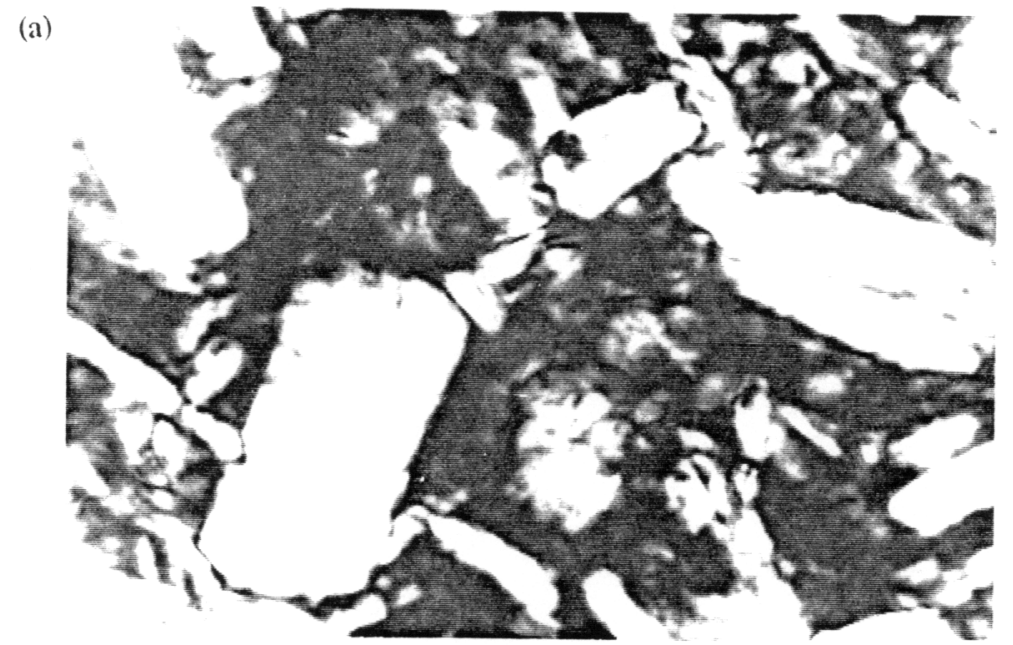

(b)

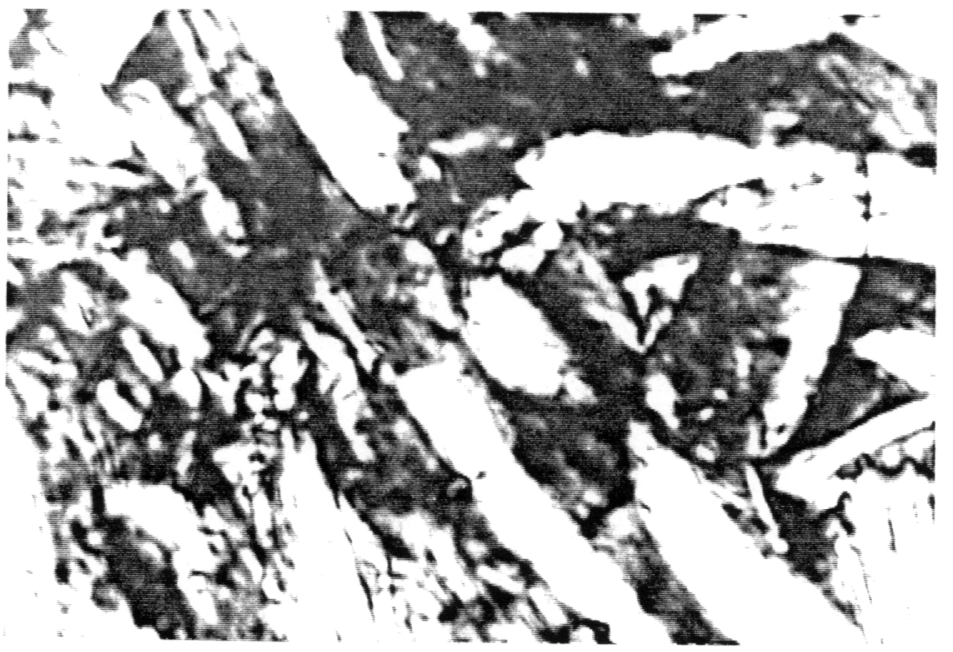

(c)

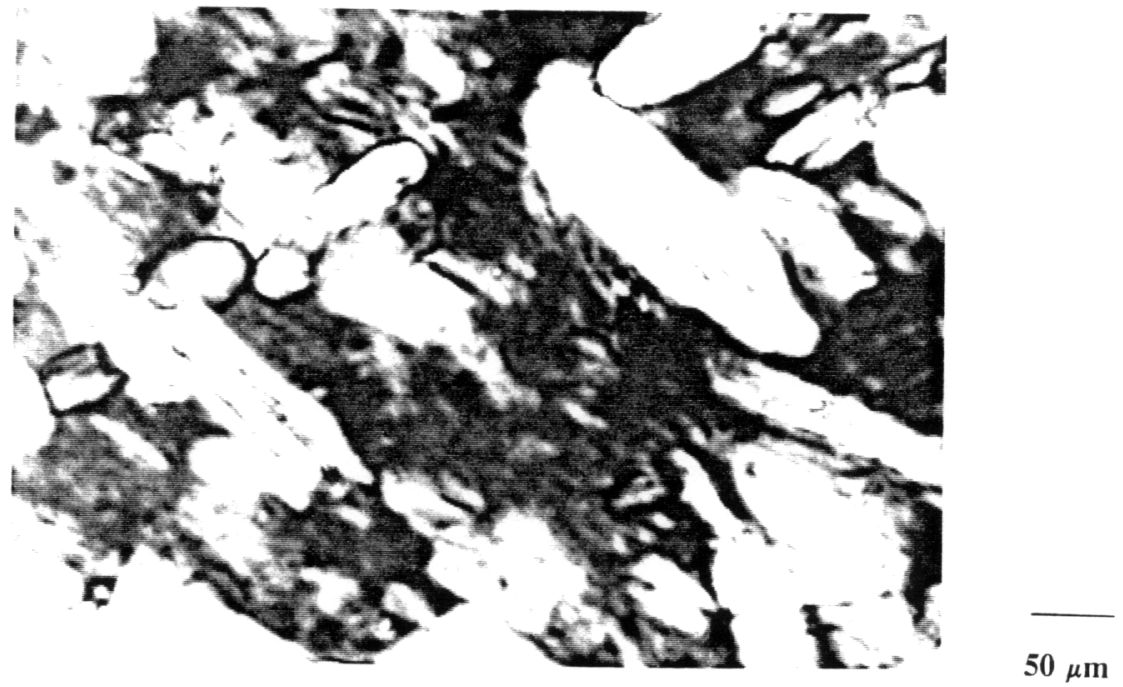

Figure 7. Polarized optical micrographs of cellulose solutions in $\mathrm{DMAc} / \mathrm{LiCl}$; (a) $10 \%(w / w)$ conc. (b) $12 \%(w / w)$ conc. (c) $13.5 \%(w / w)$ conc. 

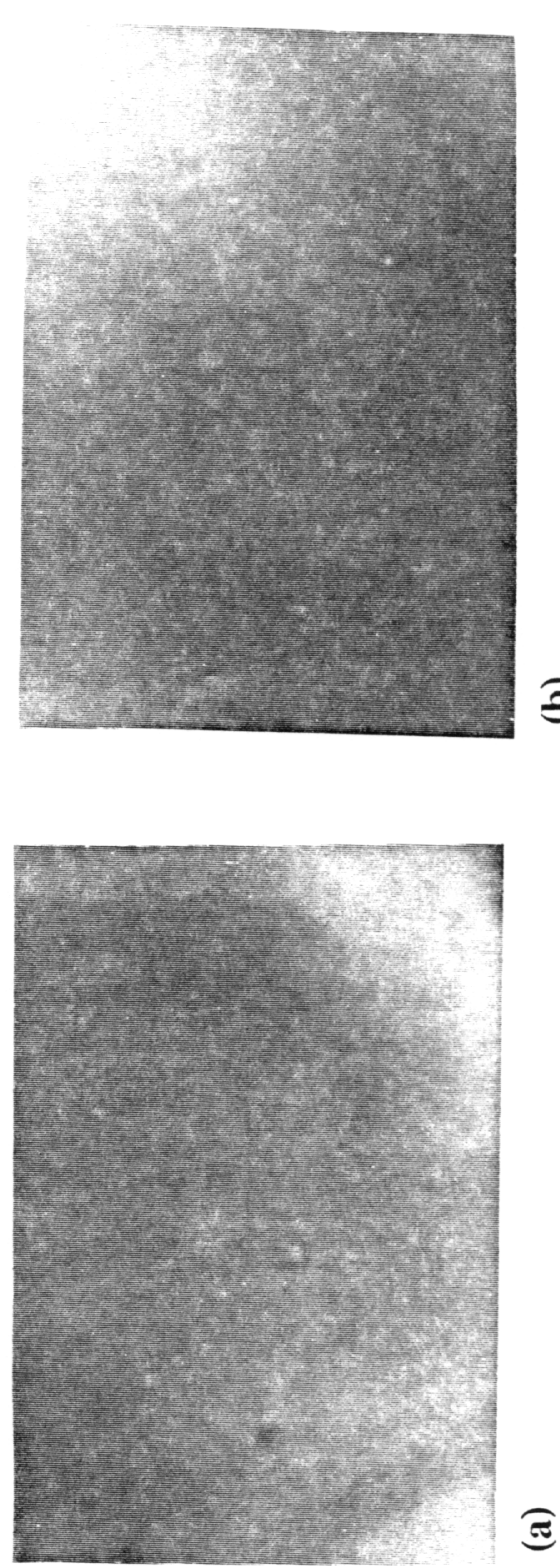
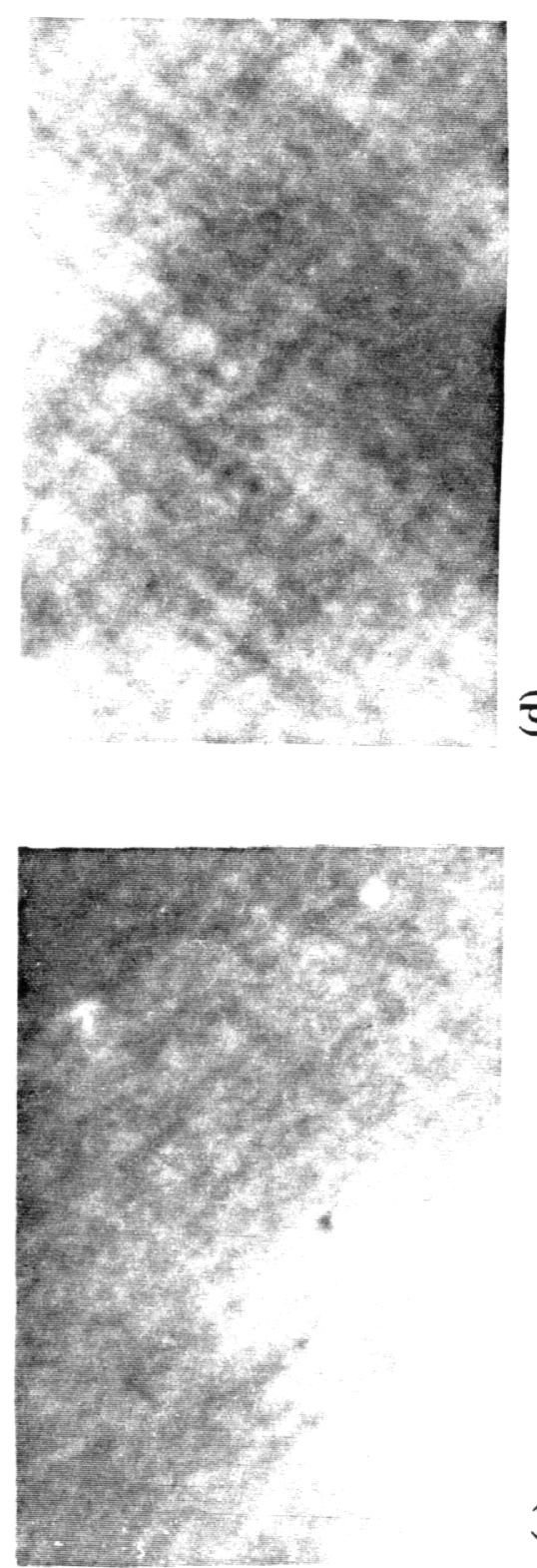

.

空

อ อ

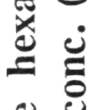

을

¿

$\Xi$

言高量

色送

รี

층 的

- है

- ن웡

농

e

$\stackrel{\infty}{0}$ 
Table II. Theoretical Values of Molecular Parameters for Cellulose (C) and Cellulose Hexanoate ( $\mathrm{CH})$.

\begin{tabular}{||l|l|l|l|l|l|l|l|l||}
\hline & $\begin{array}{c}\rho \\
g / \mathrm{cm}^{3}\end{array}$ & $\begin{array}{c}\mathrm{M}_{u} \\
\mathrm{gm} / \mathrm{mole}\end{array}$ & $\mathrm{d}, \AA$ & $I_{\mathrm{K}} \AA$ & $\mathrm{q}, \AA$ & \multicolumn{1}{|c|}{$\mathrm{X}_{\mathrm{K}}$} & $\begin{array}{l}\text { Th. } \\
\mathrm{V}_{\mathrm{p}}^{\mathrm{c}}\end{array}$ & $\begin{array}{l}\text { Expt. } \\
\mathrm{V}_{\mathrm{p}}^{\mathrm{c}}\end{array}$ \\
\hline $\mathrm{C}$ & 1.6 & 162 & 5.7 & 250 & 125 & 43.86 & 0.17 & $0.04^{1)}$ \\
\hline $\mathrm{CH}$ & 1.6 & 229.62 & 6.8 & 207.7 & 103.85 & 30.5 & 0.24 & $0.16^{2)}$ \\
\hline
\end{tabular}

1) Based on viscosity measurements.

2) Based on polarized optical microscopy observations. 
overestimates the $\mathrm{V}_{\mathrm{p}}^{\mathrm{c}}$ - value of cellulose in DMAC/Licl as observed by the deviation between the kuhn model and experimental data. Such deviations were observed by other investigators in other solvents $(30,41)$, as well as in DMAC/Licl $(44,46)$. The cellulose molecule seems to be stiffened by complexation and association with DMAC/Licl $(48,49)$. Presently there is a lack of careful studies which would allow theoretical treatments of cellulose in ordered phases.

b) Cellulose Hexanoate.

The absolute experimental and theoretical values of $\mathrm{V}_{\mathrm{p}}^{\mathrm{c}}$ for cellulose hexanoate are in reasonable agreement (Table II). The unsubstituted hydroxyl groups ( $\left.\mathrm{DS}_{\mathrm{OH}}=2.31\right)$ may form hydrogen bonds and stiffen the backbone. The bulky substituents $\left(M_{u}=229.62\right)$ on the backbone will reduce the hydrogen bonding to a certain extent, but they would contribute to an increase in intermolecular interactions. These two factors may influence the $V_{p}^{c}$ in cellulose hexanoate. IV. Processing:

Tables III and IV show the spinning conditions and mechanical properties of the cellulose and cellulose hexanoate fibers. The first column represents the concentration of the solutions from which the fibers were spun. The extrusion rates of the spinning solution, $V_{0}$, were 14,21 and $42 \mathrm{~m} / \mathrm{min}$. The values of shear rates to the corresponding $V_{0}$ values were 
Table III. Spinning Conditions and Mechanical Properties of Cellulose Fibers.

\begin{tabular}{|c|c|c|c|c|c|c|c|c|c|}
\hline$\underset{(w / w z)}{C_{p}}$ & $\mathrm{Ph}$ & $\begin{array}{c}v_{0} \\
m / m i n\end{array}$ & $\begin{array}{c}\mathrm{V}_{\mathrm{L}} \\
\mathrm{m} / \mathrm{min}\end{array}$ & $\begin{array}{l}\text { Draw1) } \\
\text { Ratio }\end{array}$ & $\begin{array}{l}\mathrm{D} \\
\mu \mathrm{m}\end{array}$ & $\begin{array}{l}E \\
g / d^{2)}\end{array}$ & $\begin{array}{l}\sigma_{\mathrm{b}} \\
\mathrm{g} / \mathrm{d}\end{array}$ & $\begin{array}{l}\varepsilon_{b} \\
\frac{g}{8} \\
\end{array}$ & $\begin{array}{l}\mathrm{BT} \\
\mathrm{g} / \mathrm{d}\end{array}$ \\
\hline$c-6.5$ & $I$ & 14 & 11 & 0.80 & 107 & 23.9 & .58 & 1.0 & .02 \\
\hline$c-6.5$ & $I$ & 14 & 15 & 1.07 & 95 & 34.7 & .56 & 1.1 & .02 \\
\hline$c-6.5$ & $I$ & 14 & 21 & 1.5 & 98 & 79.2 & .75 & 1.7 & .05 \\
\hline$c-6.5$ & $I$ & 21 & 11 & 0.52 & 144 & 33.8 & .57 & 1.5 & .02 \\
\hline$c-6.5$ & $I$ & 21 & 21 & 1.0 & 137 & 53.9 & .68 & 1.0 & .02 \\
\hline$c-6.5$ & I & 21 & 30 & 1.43 & 126 & 58.1 & .97 & 1.8 & .04 \\
\hline$c-6.5$ & I & 21 & 46 & 2.2 & 50 & 34.4 & 1.1 & 1.5 & .07 \\
\hline$c-8.0$ & $I$ & 14 & 11 & 0.8 & 128 & 24.9 & .62 & 0.7 & .01 \\
\hline$C-8.0$ & $I$ & 14 & 15 & 1.07 & 89 & 50.2 & .73 & 0.4 & .01 \\
\hline$c-8.0$ & I & 14 & 21 & 1.5 & 88 & 71.0 & .96 & 1.0 & .05 \\
\hline$c-8.0$ & I & 14 & 30 & 2.14 & 81 & 60.0 & .93 & 5.8 & .11 \\
\hline$c-8.0$ & $I$ & 21 & 11 & 0.52 & 161 & 67.7 & .88 & 0.8 & .03 \\
\hline$C-8.0$ & I & 21 & 21 & 1.0 & 91 & 35.6 & .55 & 0.2 & .02 \\
\hline$c-8.0$ & I & 21 & 30 & 1.43 & 86 & 40.9 & .61 & 1.5 & .02 \\
\hline$c-10.0$ & $B$ & 14 & 11 & 0.8 & 139 & 108.2 & .74 & 1.6 & .01 \\
\hline$c-10.0$ & B & 14 & 21 & 1.5 & 100 & 101.2 & .65 & 1.0 & .01 \\
\hline$C-10.0$ & B & 14 & 30 & 2.14 & 91 & 112.8 & 1.1 & 1.0 & .01 \\
\hline$c-10.0$ & B & 21 & 21 & 1.0 & 110 & 70.6 & .71 & 2.8 & .03 \\
\hline$c-10.0$ & B & 21 & 30 & 1.43 & 105 & 115.3 & .63 & 2.0 & .02 \\
\hline $\mathrm{C}-12.0$ & B & 21 & 15 & 0.72 & 144 & 105.1 & .61 & 1.5 & .01 \\
\hline$C-12.0$ & B & 21 & 21 & 1.0 & 115 & 110.5 & .42 & 2.0 & .02 \\
\hline$c-12.0$ & B & 21 & 30 & 1.43 & 103 & 144.1 & 1.1 & 2.0 & .02 \\
\hline$c-13.5$ & B & 14 & 11 & 0.8 & 180 & 101.5 & .73 & 2.4 & .02 \\
\hline$c-13.5$ & B & 14 & 15 & 1.07 & 145 & 119.4 & .82 & 1.5 & .01 \\
\hline$c-13.5$ & B & 21 & 15 & 0.72 & 171 & 115.2 & 1.1 & 2.9 & .02 \\
\hline$C-13.5$ & B & 21 & 21 & 1.0 & 151 & 120.6 & .82 & 2.9 & .02 \\
\hline$c-13.5$ & B & 21 & 25 & 1.2 & 85 & 151.9 & .85 & 2.2 & .02 \\
\hline
\end{tabular}

1) Draw Ratio $=V_{L} / V_{0}$.

2) $1 \mathrm{GPa} \approx 7.3 \mathrm{~g} / \mathrm{d}$. 
Table IV. Spinning Conditions and Mechanical Properties of Cellulose Hexanoate Fibers.

\begin{tabular}{||c|c|c|c|c|l|l|l|l|l||}
\hline $\begin{array}{c}\mathrm{C}_{\mathrm{p}} \\
(\mathrm{w} / \mathrm{wg})\end{array}$ & $\mathrm{Ph} .^{1)}$ & $\begin{array}{c}\mathrm{V}_{\mathrm{o}} \\
\mathrm{m} / \mathrm{min}\end{array}$ & $\begin{array}{c}\mathrm{V}_{\mathrm{L}} \\
\mathrm{m} / \mathrm{min}\end{array}$ & $\begin{array}{l}\text { Draw } \\
\text { Ratio }\end{array}$ & $\begin{array}{l}\mathrm{D} \\
\mu \mathrm{m}\end{array}$ & $\begin{array}{l}\mathrm{E} \\
\mathrm{g} / \mathrm{d}^{3)}\end{array}$ & $\begin{array}{l}\sigma_{\mathrm{b}} \\
\mathrm{g} / \mathrm{d}\end{array}$ & $\begin{array}{l}\varepsilon_{\mathrm{b}} \\
\mathrm{g}\end{array}$ & $\begin{array}{l}\left.\mathrm{BT}^{4}\right) \\
\mathrm{g} / \mathrm{d}\end{array}$ \\
\hline $\mathrm{CH}-20$ & $\mathrm{I}$ & 42 & 15 & 0.36 & 197 & 22.7 & 0.4 & 6 & .01 \\
\hline $\mathrm{CH}-20$ & $\mathrm{I}$ & 42 & 21 & 0.50 & 173 & 19.4 & 0.5 & 16 & .07 \\
\hline $\mathrm{CH}-20$ & $\mathrm{I}$ & 42 & 35 & 0.80 & 151 & 16.8 & 0.4 & 16 & .05 \\
\hline $\mathrm{CH}-30$ & $\mathrm{~B}$ & 21 & 11 & 0.52 & 185 & 15.2 & 0.5 & 26 & .11 \\
\hline $\mathrm{CH}-30$ & $\mathrm{~B}$ & 21 & 21 & 1.0 & 150 & 17.8 & 0.7 & 25 & .13 \\
\hline $\mathrm{CH}-30$ & $\mathrm{~B}$ & 42 & 21 & 0.52 & 190 & 18.9 & 0.5 & 30 & .13 \\
\hline $\mathrm{CH}-30$ & $\mathrm{~B}$ & 42 & 35 & 0.83 & 176 & 22.0 & 0.5 & 25 & .10 \\
\hline
\end{tabular}

1) Ph. represents different phases. I and $B$ designate isotropic and biphasic phases.

2) Draw Ratio $=V_{L} / V_{0}$.

3) $1 \mathrm{GPa} \approx 7.3 \mathrm{~g} / \mathrm{d}$.

4) $\mathrm{BT}=$ Breaking Toughness. 
6220,9330 and $18660 \mathrm{~s}^{-1}$ respectively. The take-up speed, $\mathrm{V}_{\mathrm{L}}$, varied between $11-46 \mathrm{~m} / \mathrm{min}$.

A draw ratio of less than 1 reflects die swelling due to the release of stored energy as the solutions were elastic. The draw ratio values are expected to be higher than reported if the velocity of the freely extruded filaments were used instead of $V_{0}$. Die swelling was larger for isotropic solutions than liquid crystalline solutions. A detailed study on die swelling for cellulose solutions was reported by Bianchi et al. $(50)$.

Cellulose solutions were wet spun under a wide range of conditions in comparison to cellulose hexanoate and other cellulose esters like cellulose acetate and cellulose acetate butyrate studied earlier (55). There was no marked difference in the spinnability of isotropic and liquid crystalline cellulose and cellulose hexanoate solutions.

V. Mechanical Properties:

a) Cellulose.

Figure 9 represents the modulus variation with spinning dope concentration of cellulose fibers spun at different takeup speeds (only data for $\mathrm{v}_{\mathrm{o}}=21 \mathrm{~m} / \mathrm{min}$ are shown). The modulus values increase for the fibers spun from anisotropic solutions. The modulus values were also higher when the takeup speed was increased. Extrusion rate does not seem to affect the fiber properties as high modulus values, i.e., $119.4 \mathrm{~g} / \mathrm{d}$ 


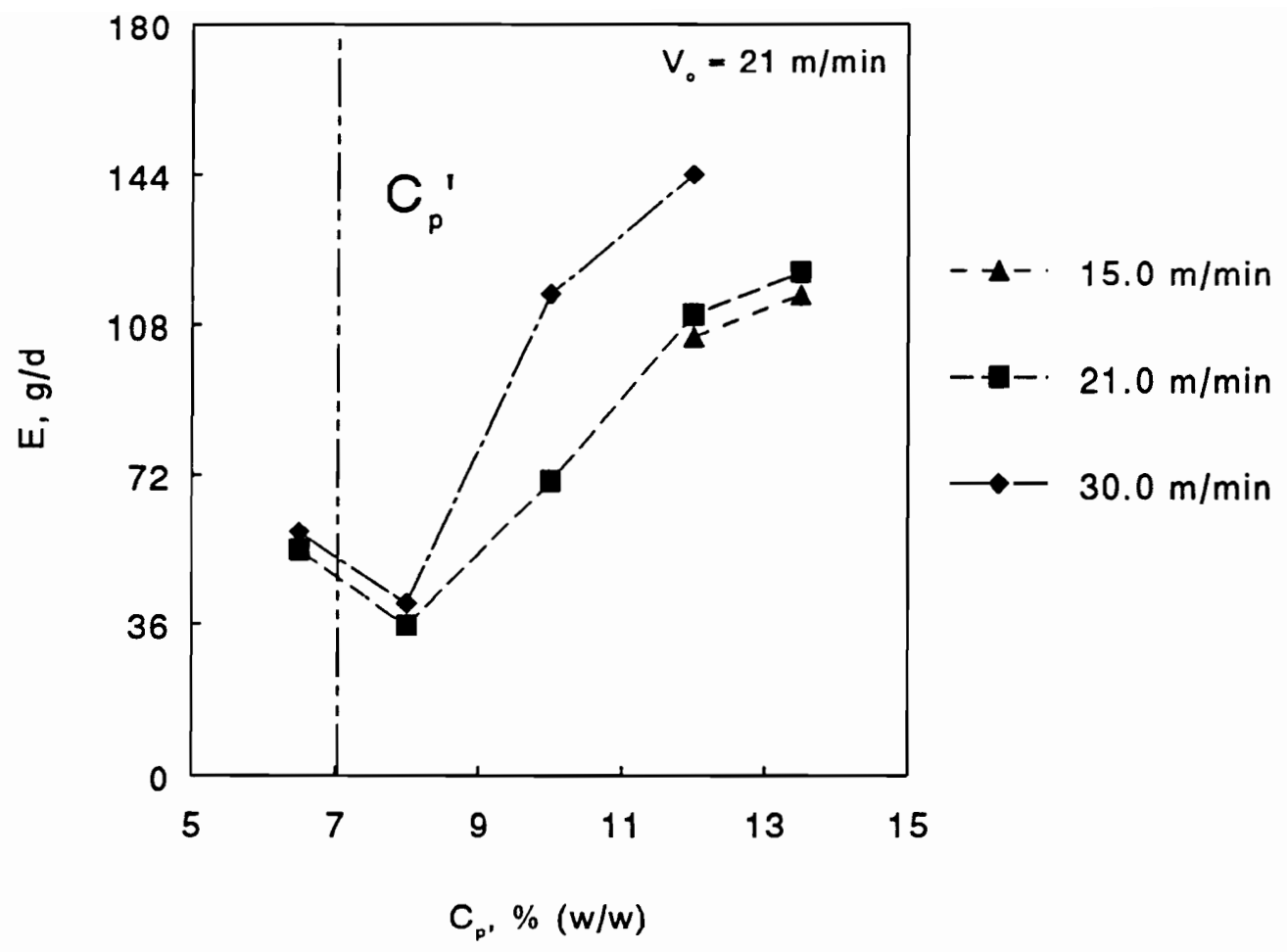

Figure 9. Modulus versus polymer concentration of cellulose fibers spun at different take-up speeds for $V_{0}=21 \mathrm{~m} / \mathrm{min}$. 
and $115.2 \mathrm{~g} / \mathrm{d}$, were obtained from $\mathrm{V}_{\mathrm{o}}$ values of $14 \mathrm{~m} / \mathrm{min}$ and 21 $\mathrm{m} / \mathrm{min}$, respectively, at take-up speeds of $15 \mathrm{~m} / \mathrm{min}$. The "dry jet" spinning method allows higher take-up speeds resulting in higher fiber orientation. The weakening effect caused by high extrusion rates observed by Bianchi (55) was eliminated in our study by spinning the fibers at high take-up speeds. Highest modulus and tenacity values obtained in this study were 151.9 $\mathrm{g} / \mathrm{d}(20.8 \mathrm{GPa})$ and $1.1 \mathrm{~g} / \mathrm{d}(0.15 \mathrm{GPa})$ respectively, for the cellulose fibers. These values are comparable $(27,55)$ and superior (41) to the cellulose fibers obtained by other investigators. Unlike cellulose derivatives, the solubility limit of cellulose does not allow cellulose solutions to become completely anisotropic. The biphasic liquid crystalline solution state restricts cellulose to reach its maximum mechanical properties. The strength (tenacity) values do not show great improvements at different take-up speeds and different liquid crystalline contents of the solutions. The fiber properties can be further improved by using high molecular weight cellulose and longer maturation and nucleation times of the solutions.

b) Cellulose Hexanoate.

Figure 10 shows the variation of modulus of cellulose hexanoate fibers on going through the isotropic-anisotropic transition at different take-up speeds for $V_{0}=42 \mathrm{~m} / \mathrm{min}$. Modulus values do not increase, as expected, possibly due to 


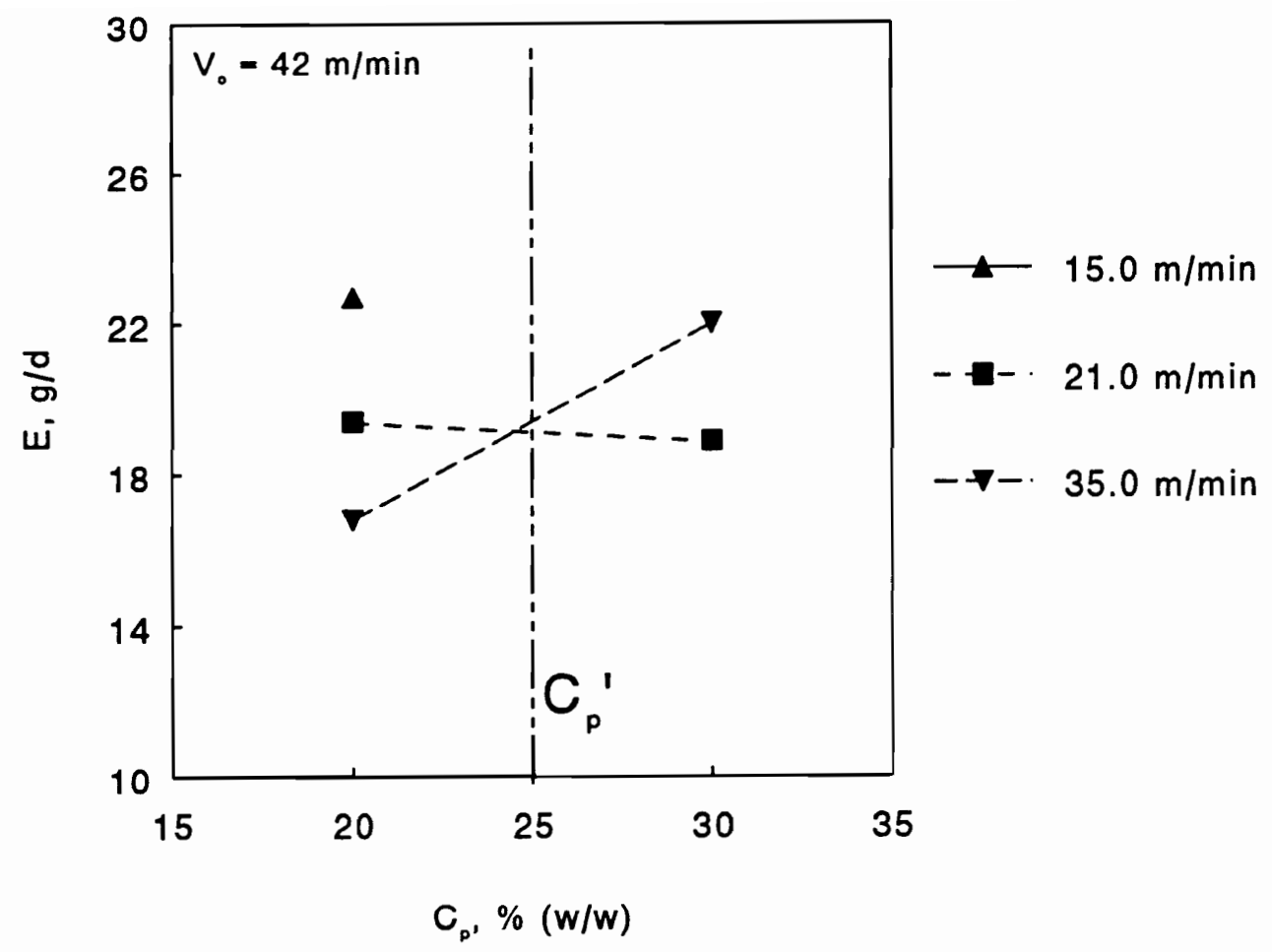

Figure 10. Modulus versus polymer concentration of cellulose hexanoate fibers spun at different take-up speeds for $V_{0}=42 \mathrm{~m} / \mathrm{min}$. 
the coexistence of an isotropic phase in the biphasic liquid crystalline solution. It should be noted that cellulose hexanoate fibers were not spun from completely anisotropic solution concentration, i.e., $40 \%(\mathrm{w} / \mathrm{w})$. However, the modulus value improved for the fibers spun at take-up speeds of 35 $\mathrm{m} / \mathrm{min}$. But the take-up speeds were probably not high enough to compensate for the weakening effect caused by the high extrusion rate (i.e., $42 \mathrm{~m} / \mathrm{min}$ ). No one has yet reported fibers from cellulose hexanoate.

VI. Morphology:

Cellulose fibers were slightly tacky immediately after they emerged from the coagulation bath at all take-up speeds. This could be due to complexation and association of DMAC/LiCl with cellulose which may have influenced the coagulation rate. (i) Effect of Concentration.

a) Cellulose.

Figure 11 shows the scanning electron micrographs of the surfaces of fibers spun from low (6.5\%) and high (13.5\%) concentration. The fibers from low concentration had smooth surfaces whereas the fibers from high concentration were rough. Fig. 11 (b) is the micrograph of a fiber bundle. At low concentrations, more coagulant was needed to diffuse into the solution to precipitate the polymer, and consequently, this led to a slower coagulation rate. This resulted in a uniform structure. The coagulation rate of higher concentration solutions was faster as less coagulant was required to 


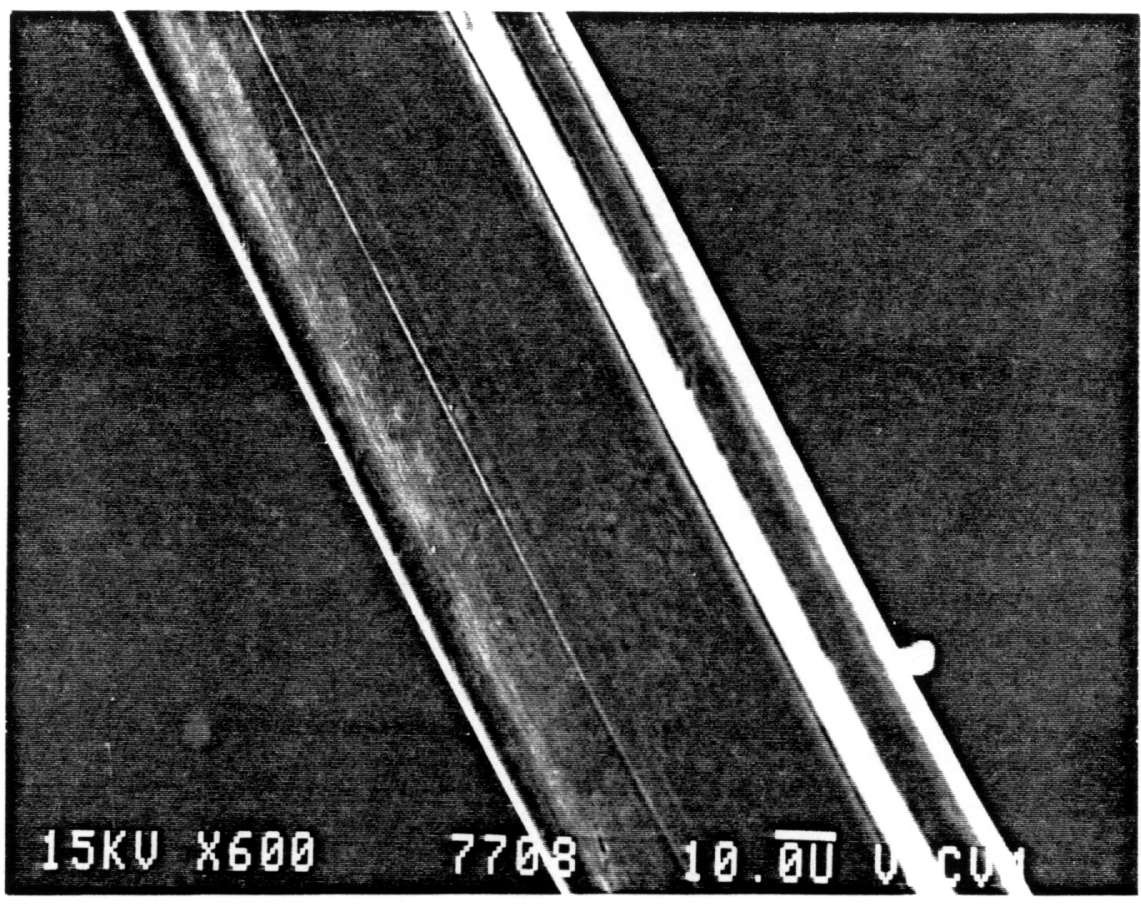

(a)

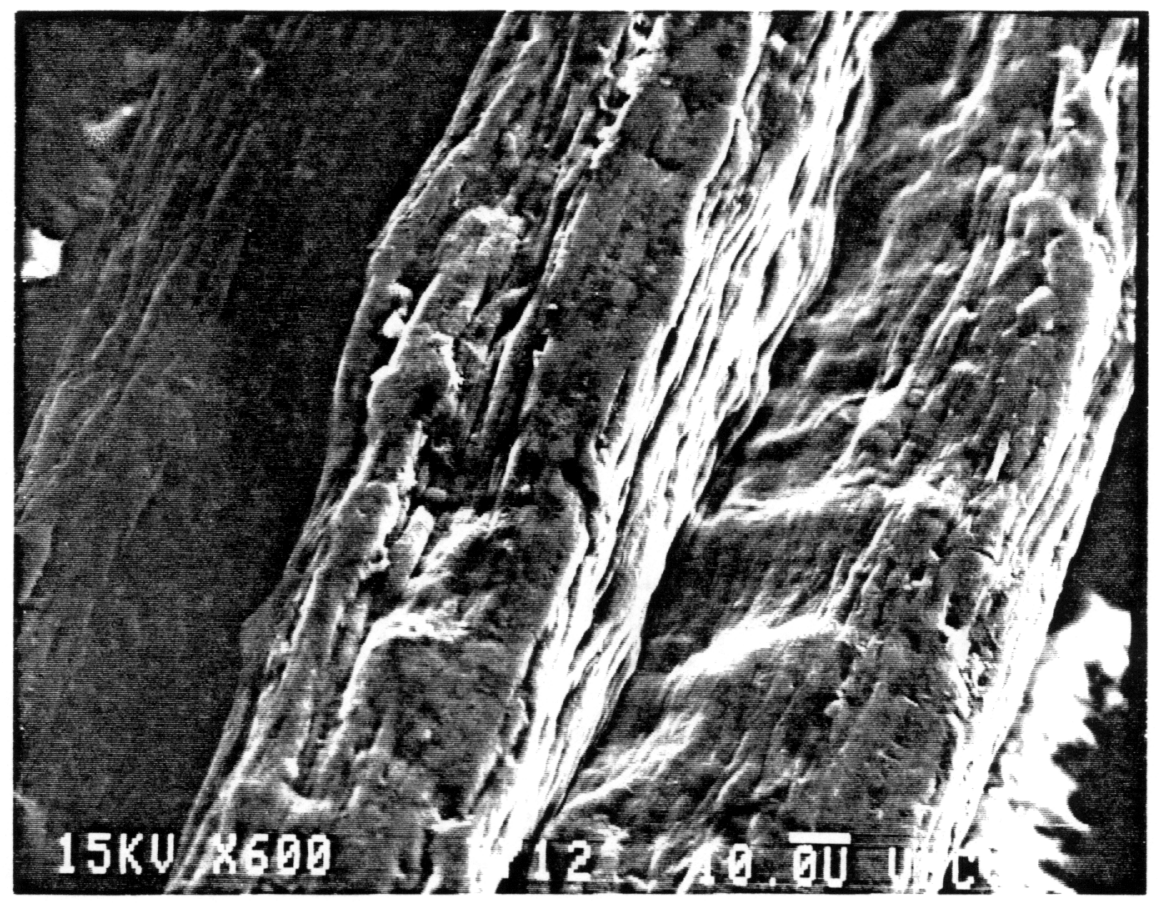

(b)

Figure 11. Scanning electron micrographs of the surface of cellulose fibers; (a) $6.5 \%(\mathrm{w} / \mathrm{w}), \mathrm{V}_{0}=14 \mathrm{~m} / \mathrm{min}, \mathrm{V}_{\mathrm{L}}=21 \mathrm{~m} / \mathrm{min}$ (b) $13.5 \%(\mathrm{w} / \mathrm{w}), \mathrm{V}_{0}=21$ $\mathrm{m} / \mathrm{min}, \mathrm{V}_{\mathrm{L}}=15 \mathrm{~m} / \mathrm{min}$. 
precipitate the polymer. This resulted in a rougher structure. b) Cellulose Hexanoate.

Figure 12 shows the surface morphology of cellulose hexanoate fibers spun from $30 \%(\mathrm{w} / \mathrm{w})$ solution. (The surface structure of fibers from $20 \%$ (w/W) solution was similar to that of $30 \%(\mathrm{w} / \mathrm{w})$ solution.) The smooth and uniform structure indicates slow coagulation due to relatively low concentration. Concentration effects on morphology maybe observed for fibers spun from higher concentrations.

The cellulose hexanoate examined in this study is expected to (a) retain the properties of cellulose due to its low degree of substitution $\left(\mathrm{DS}_{\mathrm{OH}}=0.69\right)$, and $(b)$ to exhibit behavior resembling cellulose ester derivatives due to its improved solubility. Derivatization seems to have reduced the hydrogen bonding to some extent as $\mathrm{LiCl}$ was not required to dissolve cellulose hexanoate. This is evident as the viscosity of cellulose hexanoate solutions was lower than that of cellulose solutions. However, in contrast to cellulose and cellulose derivatives, the viscosity of cellulose hexanoate solutions did not drop after the onset of liquid crystallinity. This difference in viscosity behavior may be explained by the presence of interactions and associations between the long side-chain substituents of hexanoic acid groups. The extent of these interactions is expected to be relatively lower for derivatives with shorter side chains like 


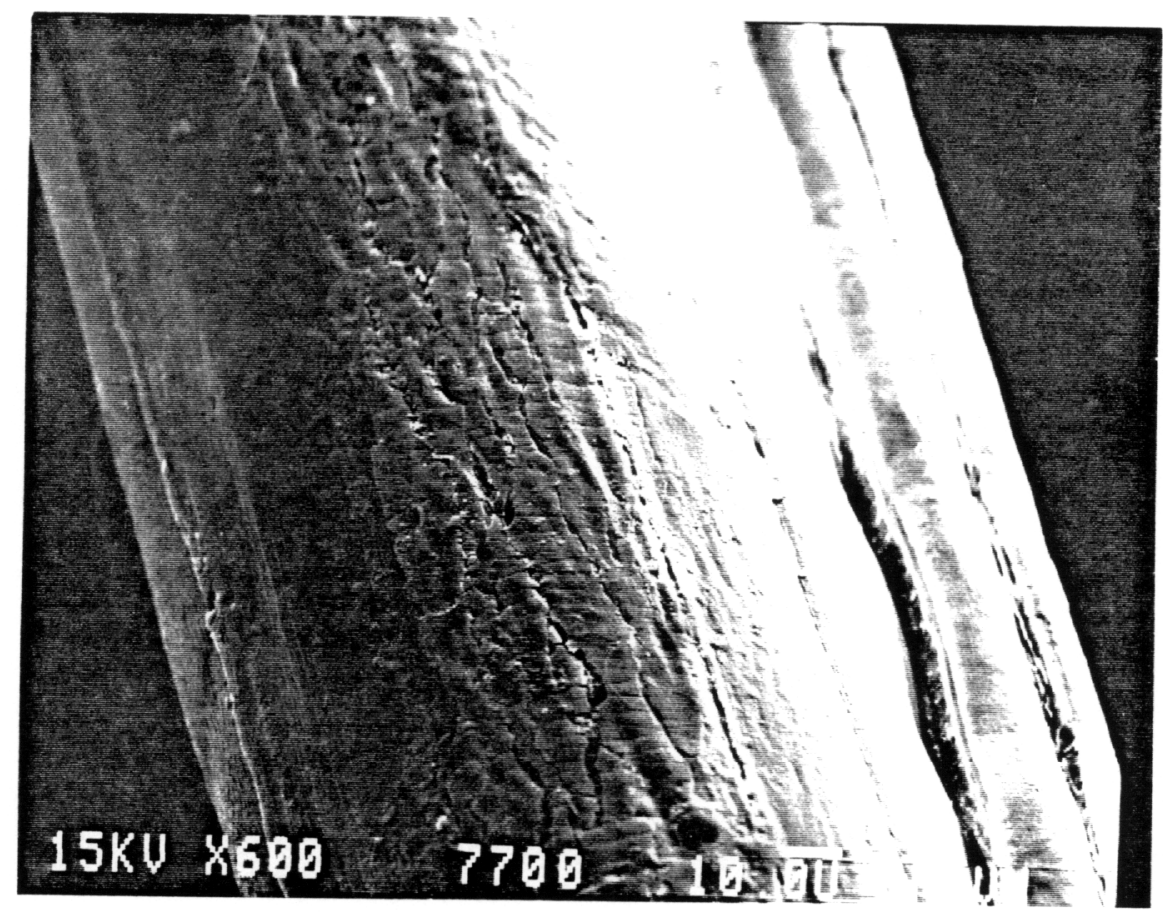

Figure 12. Scanning electron micrograph of the surface of cellulose hexanoate fiber spun from $30 \%(w / w)$ solution at $V_{0}=21 \mathrm{~m} / \mathrm{min}$ and $V_{L}=21$ $\mathrm{m} / \mathrm{min}$. 
cellulose acetate $(C A)$ and cellulose acetate butyrate (CAB). In case of cellulose, $C A$ and $C A B$, at critical concentration the viscosity drops as the liquid crystalline order seems to overcome the association between side-chains. However, in case of cellulose hexanoate, it seems that extensive side-chain association dominate the liquid crystalline order and inhibit the drop in viscosity. In fact, these side-chain interactions and associations may also be responsible for lowering the critical concentration at which liquid crystalline solutions form for the cellulose esters. The viscosity behavior at high solution concentration of cellulose hexanoate and cellulose is analogous. Unlike cellulose, cellulose hexanoate is highly soluble, and it presumably is not in a metastable condition. similar to other cellulose derivatives, at high concentrations packing effects of the cellulose hexanoate molecules may be responsible for the continuing rise in viscosity with concentration (57).

The experimental value of $V_{p}^{c}(0.16)$ of cellulose hexanoate was lower than the cellulose ester with maximum degree of butyration, $C A B-3$, studied earlier (51). The decrease in $V_{p}^{c}$ could be due to the bulky hexanoic acid groups which raise intermolecular interaction. However, the comparison may not be justified as cellulose hexanoate has a low DS which provides ample opportunity for hydrogen bonding. our prediction that solution anisotropy can be reached at 
lower concentrations in cellulose esters with large and bulky substituents seems correct, however, further investigation is required to deconvolute the conflicting effects of substituent size and hydrogen bonding.

The highest modulus and tenacity for cellulose hexanoate fibers achieved in this study is $22.7 \mathrm{~g} / \mathrm{d}$ and $0.7 \mathrm{~g} / \mathrm{d}$, respectively. These values are higher than those obtained from all other fibers produced from isotropic solutions, and they are higher than those of some of the fibers produced from anisotropic solutions of cellulose esters (i.e., cellulose acetate butyrate) reported earlier (55). This could be due to the presence of hydrogen bonding in cellulose hexanoate. However, these properties are lower than those of cellulose fibers. Therefore, the properties of cellulose hexanoate fibers are in-between those of unmodified cellulose and those of completely derivatized cellulose (ester) fibers.

\section{CONCLUSIONS}

1. Cellulose and cellulose hexanoate solutions were liquid crystalline above $7 \%(\mathrm{w} / \mathrm{w})$ and $25 \%(\mathrm{w} / \mathrm{w})$ concentrations, respectively. Cellulose formed biphasic solutions at all concentrations due to limited solubility, whereas cellulose hexanoate became completely anisotropic (see Fig. 8) at higher concentrations. The viscosity of cellulose solutions resembles typical liquid crystalline behavior. However, the viscosity of cellulose hexanoate 
solutions did not decrease at the onset of liquid crystalline behavior, possibly because of extensive sidechain interactions.

2. The experimental value of the critical volume fraction, $V_{p}{ }^{c}$, of cellulose hexanoate $(0.16)$ was lower than that of cellulose esters with maximum degree of butyration, CAB3, (i.e., 0.29) studied earlier.

3. The modulus of the cellulose fibers increased when spun from liquid crystalline solutions. Similar observations were not evident for cellulose hexanoate fibers. This may be explained with the weakening effect of the high extrusion rates applied, and of the incomplete development of the liquid crystalline structure at low concentrations.

4. Cellulose fibers spun from low concentration isotropic solutions had a smooth surface morphology, whereas the fibers from high concentration liquid crystalline solutions had a coarse surface structure.

\section{APPENDIX A}

1. Calculation of molecular weights of repeat units, $\mathbf{M}_{\mathbf{u}}$ : Cellulose;

$$
\begin{aligned}
& \mathrm{M}_{u}=162 \\
& \mathrm{M}_{u}=162+98 \times 0.69=229.62
\end{aligned}
$$

Cellulose Hexanoate;

2. Calculation of chain diameter, $d: d=\left(M_{u} / N_{A} \rho l_{u}\right)^{1 / 2}$

$I_{u}=5.2 \AA, N_{A}=6.02 \times 10^{23}$ molecules $/$ mole.

Cellulose; 
$d=\left(162 / 6.02 \times 10^{23} \times 1.6 \times 10^{-24} \times 5.2\right)^{1 / 2}=5.7 \AA$

Cellulose Hexanoate;

$d=\left(229.62 / 6.02 \times 10^{23} \times 1.6 \times 10^{-24} \times 5.2\right)^{1 / 2}=6.8 \AA$

3. Calculation of Kuhn segment length, $l_{k}: l_{k}=\left\langle r^{2}\right\rangle_{0} / n l_{u}$ i

From light scattering [Tanner and Berry, (58)];

$\left\langle\mathrm{r}^{2}\right\rangle_{\mathrm{o}} / \mathrm{n}=1080 \AA^{2}$;

A narrow range of values of $\left\langle r^{2}\right\rangle_{0} / n$ have been reported for diverse cellulose esters (58); therefore $1080 \AA^{2}$ is assumed for cellulose hexanoate in this study.

$l_{k}=1080 / 5.2=207.7 \AA$

4. Calculation of persistence length, $q: q=l_{k} / 2$

Cellulose;

$q=125 \AA(49)$

Cellulose Hexanoate;

$q=207.7 / 2=103.85 \AA$

5. Calculation of aspect ratio, $x_{k}=2 q / d$
Cellulose;
$X_{k}=2 \times 125 / 5.7=43.86$
Cellulose Hexanoate;
$X_{k}=2 \times 103.85 / 6.8=30.5$

6. Calculation of critical concentration, $V_{p}^{c}$;

Cellulose;

Cellulose Hexanoate;
$V_{p}^{c}=8 / 43.86(1-2 / 43.86)=0.17$

$V_{p}^{c}=8 / 30.5(1-2 / 30.5)=0.24$ 


\section{REFERENCES}

1. D. M. MacDonald, in Solvent Spun Rayon, Modified Cellulose Fibers and Derivatives, A. F. Turbak, Ed., ACS Symp. Ser. \# 58, 25-39, 1977.

2. R. B. Hammer and A. F. Turbak, in Solvent Spun Rayon, Modified Cellulose Fibers and Derivatives, A. F. Turbak, Ed., ACS Symp. Ser. \#58, 44-51, 1977.

3. D. C. Johnson, M. D. Nicholson and F. C. Haigh, Appl. Polym. Symp., 28, 931, 1976.

4. K. Kamide, K. Okajima, T. Matsui and S. Manabe, Polym. J. (Tokyo), 12, 521, 1980 .

5. O. Turunen, L. Mandell, V. Eklund, K. Ekman and J. Huttunen, US Patent 4486585, 1984.

6. S. M. Hudson and J. A. Cuculo, J. Macromol. Sci.-Rev. Macromol. Chem., C18 (1), 1-82, 1980.

7. C. Graenacher and R. Sallman, US Patent 2179181, 1939.

8. D. L. Johnson, US Patent 3447939, 1969.

9. N. E. Franks and J. K. Varga, US Patent 4145532, 1979.

10. C. C. McCorsley and J. K. Varga, US Patent 4142913, 1979.

11. C. C. McCorsley, US Patent $4144080,1979$.

12. C. C. McCorsley and J. K. Varga, US Patent 4211574, 1980.

13. R. N. Armstrong, C. C. McCorsley and J. K. Varga, Proc. of Fifth Int. Tappi Pulp Conf., Vienna, 100, 1980.

14. C. C. Mccorsley, US Patent 4246221, 1981. 
15. H. Chanzy, A. Peguy, S. Chaunis and P. Monzie, J. Polym. Sci.: Polym. Phys. Ed., 18, 1137-1144, 1980.

16. E. Maia, A. Peguy and S. Perez, Acta. Cryst., B37, 1858$1862,1981$.

17. E. Maia and S. Perez, Acta. Cryst., B 38, 849-852, 1982 .

18. H. Chanzy, E. Maia and S. Perez, Acta. Cryst., B 38, 852855,1982 .

19. K. Okamura, M. Fujita and T. Umezawa, Bulletin of the Kyoto University Forests, 53 (11), 254, 1981.

20. P. Navard and J. M. Haudin, Br. Polym. J., 12, 174-178, 1980 .

21. P. Navard and J. M. Haudin, Polym. Proc. Engr,, 3 (3), $291-301,1985$

22. H. Chanzy, P. Noe, M. Paillet and P. Smith, J. Appl. Polym. Sci., 37, 239-259, 1983.

23. H. Chanzy, M. Dube and R. H. Marchessault, J. Polym. Sci.: Polym. Lett. Ed., 17, 219-226, 1979.

24. H. Chanzy, S. Nawrot, A. Peguy, P. Smith and J. Chevalier, J. Polym. Sci.: Polym. Phys. Ed., 20, 19091924,1982 .

25. H. Chanzy, A. Peguy, S. Chaunis and P. Monzie, Proc. of Fifth Int. Tappi Dissl. Pulp Conf,, Vienna, 105-108, 1980 .

26. H. Chanzy, S. Nawrot, S. Perez and P. Smith, Proc. of Int. Tappi Dissl. Pulp Conf., Atlanta, 127-132, 1983. 
27. H. Chanzy, M. Paillet and R. Hagege, Polymer, 30 (3), $400-405,1990$.

28. H. Chanzy, B. Chumpitazi and A. Peguy, Carbohyd. Polym., $2,35-42,1982$.

29. D. L. Patel and R. D. Gilbert, J. Polym. Sci.: Polym. Phys. Ed., 19, 1231-1236, 1981.

30. Y. K. Hong, D. E. Hawkinson, E. Kohout, A. Garrard, R. E. Fornes and R. D. Gilbert, in Polymer Association structures, ACS Symp. Ser. \# 384, M. A. El-Nokaly, Ed., 184-203, 1989.

31. V. V. Myasoedova, O. A. Adamova and G. A. Krestov, Vysokomol. Soedin, Ser. B, 26 (3), 215, 1984 .

32. S. P. Papkov, Y. Y. Belousov and V. E. Kulichikhin, Khim. Volokna, 3, 8, 1983 .

33. V. V. Myasoedova, O. V. Alekseeva and G. A. Krestov, Zh. Prikl. Khim. (Leningrad), 60 (10), 2526, 1987.

34. P. Scherer, J. Am. Chem. Soc., 53, 4009, 1931.

35. S. M. Hudson and J. A. Cuculo, J. Polym. Sci.: Polym. Chem. Ed., 18, 3469-3481, 1980 .

36. J. A. Cuculo and S. M. Hudson, US Patent $4367191,1983$.

37. S. M. Hudson, J. A. Cuculo and L. C. Wadsworth, J. Polym. Sci.: Polym. Chem. Ed., 21, 651, 1983.

38. C. K. Liu, J. A. Cuculo and B. Smith, J. Polym. Sci.: Part B: Polym. Phys., 27, 2493-2511, 1989.

39. J. J. Cho, S. M. Hudson and J.A. Cuculo, J. Polym. Sci.: Part B: Polym. Phys., 27, 1699-1719, 1989. 
40. C. K. Liu, J. A. Cuculo and B. Smith, J. Polym. Sci.: Part B: Polym. Phys., 28, 449-465, 1990.

41. K. S. Yang, M. H. Theil and J. A. Cuculo, in Polymer Association structures, ACS Symp. Ser. \# 384, M. A. ElNokaly, Ed., 156-183, 1989.

42. C. L. McCormick, US Patent 4278790, 1981.

43. C. L. McCormick and P. A. Callais, Polymer, 28, 23172323,1987 .

44. C. L. McCormick, P. A. Callais and B. H. Hutchinson, Jr., Macromolecules, 18 (12), 2394-2401, 1985.

45. C. L. McCormick, P. A. Callais and B. H. Hutchinson, Jr., Polym. Prepr. (ACS Div. Polym. Chem.), 24 (2), $271,1983$.

46. G. Conio, P. Corazza, E. Bianchi, A. Tealdi and A. Ciferri, J. Polym. Sci.: Polym. Lett. Ed., 22, 273-277, 1984 .

47. A. F. Turbak, A. Kafraw, F. Synder and A. Auerback, US Patents 4302252 (1981) and 4352770 (1982).

48. M. Terbojevich, A. Cosani, G. Conio, A. Ciferri and E. Bianchi, Macromolecules, 18, 640-646, 1985.

49. E. Bianchi, A. Ciferri, G. Conio, A. Cosani and M. Terbojevich, Macromolecules, 18, 646-650, 1985.

50. E. Bianchi, A. Ciferri, G. Conio, A. Cosani and A. Tealdi, J. Polym. Sci.: Part B: Polym. Phys., 27, 1477$1484,1989$. 
51. V. Davé and W. G. Glasser, in viscoelasticity of Biomaterials, ACS Symp. Ser., W. G. Glasser and H. Hatakeyama, Eds. (In Press).

52. G. Samaranayake and W. G. Glasser (In Preparation).

53. R. Evans and A. F. A. Wallis, Fourth Int. Symp. on Wood and Pulp. Chem., Paris, 1, 201-205, 1987.

54. C. L. McCormick and T. S. Chen, Macromolecular solutions, R. B. Seymour and G. A. Stahl, Eds., Pergamon Press, New York, 1982 .

55. V. Davé, W. G. Glasser and G. L. Wilkes (To Be Published).

56. R. B. Bird, R. C. Armstrong and O. Hassager, in Dynamics of Polymeric Liquids, Vol. 1, 150, 1987.

57. S. Dayan, J. M. Gilli, and P. Sixou, J. Appl. Polym. Sci., $28,1527,1983$.

58. W. D. Tanner and G. C. Berry, J. Polym. Sci.: Polym. Phys. Ed., 12, 941, 1974. 
5.0 CELLULOSE-BASED FIBERS FROM LIQUID CRYSTALLINE SOLUTIONS. IV. EFFECTS OF LITHIUM CHLORIDE ON ACETATE/BUTYRATE ESTERS. 


\title{
CELLULOSE-BASED FIBERS FROM LIQUID CRYSTALLINE SOLUTIONS.
}

IV. Effects of Lithium Chloride on Acetate/Butyrate Esters.

\begin{abstract}
Cellulose acetate butyrate fibers were prepared by spinning from liquid crystalline solutions containing lithium chloride. Ionic interactions were observed between carbonyl oxygen and lithium cation by ${ }^{13} \mathrm{C}$ NMR spectroscopy. This result is supported by rheological experiments with $C A B$ solutions containing LiCl. Mechano-sorptive creep behavior decreases in the presence of residual Licl salt, possibly due to ion induced electrostatic interactions between the carbonyl oxygen of the acetyl and butyryl substituents and the unsubstituted hydroxyl groups on the cellulose backbone. The combination of liquid crystalline behavior and ionic interactions has produced a better understanding of the molecular interaction in fibers, and this should be employed for producing improved fibers for various applications.
\end{abstract}




\section{INTRODUCTION}

By spinning cellulose-based fibers from liquid crystalline solutions, we have already demonstrated a route for improving the mechanical properties of cellulose ester fibers (1). During the preparation of rayon fibers, improvements in the mechanical properties are achieved by adding of salts (and other chemicals) to the viscose coagulation solution (2).

Mechano-sorptive creep phenomena as recently reviewed

(3) has been observed in our earlier studies on commercial aramid fibers like Kevlar which are spun from liquid crystalline solutions, and which have hydrogen bonds between their crystallites (4). It has been hypothesized that the observed increase in creep under cyclic moisture conditions is due to the presence of residual salt molecules in the Kevlar ${ }^{\circledR}$ fibers. Therefore, creep measurements under cyclic moisture conditions could be a good method to understand the interaction between salt additives and the polymer backbone.

The objective of the present study was to study the effect of the presence of salt on the behavior of cellulose esters in liquid crystalline solutions and in fibers by rheology and optical microscopy; by ${ }^{13} \mathrm{C}$ NMR spectroscopy; and by mechano-sorptive creep. 


\section{EXPERIMENTAL}

I. Materials.

Cellulose acetate butyrate (CAB 171-15) was obtained from Eastman Kodak, Kingsport, TN. The average acetyl and butyryl content was 29.5 and 17.1 weight $\%$, respectively. The degree of substitution (DS) of acetyl $\left(D^{A C}=2.03\right)$ and butyryl (DS $=0.8)$ was evaluated by proton NMR spectroscopy $(5,6) \cdot$ DS $_{\mathrm{OH}}$ was determined by difference $\left[D S_{\mathrm{OH}}=3-\{2.03+0.8\}=0.17\right]$. Number average molecular weight $\left(\left\langle M_{n}\right\rangle\right)$, number average degree of polymerization $\left(\left\langle D P_{n}\right\rangle\right)$ and intrinsic viscosity (I.V.) were $66,800 \mathrm{gm} / \mathrm{mole}, 220$ and $1.63 \mathrm{dL} / \mathrm{gm}$, respectively. Detailed molecular characteristics of the cellulose ester were described in our previous publication (7).

six salt additives were selected for this study: ammonium chloride $\left(\mathrm{NH}_{4} \mathrm{Cl}\right)$, ammonium sulphate $\left[\left(\mathrm{NH}_{4}\right)_{2} \mathrm{SO}_{4}\right]$, sodium sulphate $\left(\mathrm{Na}_{2} \mathrm{SO}_{4}\right)$, lithium sulphate $\left(\mathrm{Li}_{2} \mathrm{SO}_{4}\right)$, lithium chloride (LiCl) and calcium chloride $\left(\mathrm{CaCl}_{2}\right)$.

Reagent grade dimethylacetamide (DMAC) was used as received. Deuterated DMAC was obtained from Schweizerhall, Inc., South Plainfield, N.J.

II. Methods.

1. Preparation of Solution:

Weighed amounts of the above mentioned salts and DMAC were mixed and heated to about $100^{\circ} \mathrm{C}$ with constant stirring for 1 hour followed by cooling to room temperature. Amongst 
the salt additives used, only lithium chloride dissolved in DMAC. Concentrated stock solutions were prepared by mechanically mixing weighed amounts of dried cellulose acetate butyrate $(\mathrm{CAB})$ to the DMAC solutions containing lithium chloride at room temperature. The concentrations of polymer and lithium chloride in the stock solutions were defined as grams of cellulose ester and lithium chloride per 100 grams of the ternary (cellulose ester + DMAC + LiCl) solution, respectively. The solutions were allowed to equilibrate for 23 weeks prior to analysis and fiber spinning.

2. Rheological Measurements:

A Rheometrics Mechanical Spectrometer (RMS 800) was used to determine the rheological properties of the concentrated solutions of $C A B$ containing lithium chloride. The solutions were placed in a parallel-disk geometry. The dynamic mechanical properties were measured at $26^{\circ} \mathrm{C}$ using a strain amplitude of $25 \%$ of the value at which the respective sample showed viscoelasticity. $1 \%$ strain amplitude was used for $50 \%$ (w/w) $C A B$ solution containing $1 \%(w / w)$ lithium chloride. The frequency ranged from 0.1 to $100.0 \mathrm{rad} / \mathrm{sec}$.

3. Polarized Optical Microscopy:

Polarized optical microscopy was performed with a zeiss Axioplan Universal Microscope. Small portions of the solutions were placed between microscope slide and cover slip, and these were examined for birefringence between the cross polarizers 
of the microscope at room temperature.

4. ${ }^{13} \mathrm{C}$ NMR Spectroscopy:

The ${ }^{13} \mathrm{C}$ NMR spectra of $\mathrm{CAB} /$ deuterated DMAC $[100 \mathrm{mgs} / 2.5$ $\mathrm{mls}$ ] and $\mathrm{CAB} / \mathrm{LiCl} /$ deuterated DMAC [100 $\mathrm{mgs} / 120 \mathrm{mgs} / 2.5 \mathrm{mls}$ ] solutions were recorded on a Varian 400 FT-NMR spectrometer operated at $100 \mathrm{MHz}$ and a spectral width of $25,000 \mathrm{~Hz}$. The observed spectra for the $\mathrm{CAB}$ solutions without and with $\mathrm{LiCl}$ were obtained after 3456 and 2304 repetitions, respectively, at $80^{\circ} \mathrm{C}$ in a $5 \mathrm{~mm}$ NMR tube.

5. Fiber Spinning:

Continuous fibers were processed using the dry jet/wet spinning method which Blades (8) introduced for liquidcrystalline solutions of p-linked aromatic polyamides. The spinning apparatus consisted of (i) Solution Delivery System, (ii) Spinning Pack Assembly, (iii) Capillary Spinnerette, (iv) Coagulation Bath, and (v) Take-up Device. The delivery system was comprised of a programmable infusion and withdrawal syringe pump. The capillary spinnerette had a single hole with capillary diameter of $300 \mu \mathrm{m}$, capillary length of $1.2 \mathrm{~mm}$ (i.e., L/D Ratio $=4$ ) and entrance angle of $60^{\circ}$. The flow rate of $1.0 \mathrm{~cm}^{3} / \mathrm{min}$ was kept constant for all the solutions. The velocity in the spinnerette hole, Vo, was $14 \mathrm{~m} / \mathrm{min}$ and the shear rate at the capillary wall was approximately $6220 \mathrm{~s}^{-1}$ for all solutions. The solutions were coagulated in a 1 meter long coagulating water bath at room temperature. The air-gap 
between the spinnerette and the water surface was approximately $25.4 \mathrm{~mm}$. The fibers were wound on to the spools at take-up speeds, $V_{L}$, varying from $11-21 \mathrm{~m} / \mathrm{min}$. It was not possible to spin fibers from $50 \%$ ( $w / w)$ CAB containing $1 \%(w / w)$ lithium chloride solution as the pump was unable to extrude the solution due to extremely high viscosity. The fiber-wound spools were immersed in beakers containing water for 8 hours to remove the residual solvent from the fibers. The spools were allowed to air-dry for 48 hours and then vacuum ovendried for 24 hours at $50^{\circ} \mathrm{C}$. No post-spinning treatments (thermal or mechanical) were performed.

6. Fiber Diameter:

Fiber diameter (D) was determined by a Nikon UM-2 Measurescope equipped with a Quadra-Chek III attachment. The reported $D$ value is the average of three to four measurements along the fiber length.

7. Elemental Analysis:

The analysis of Licl in the fibers was carried out by Galbraith Laboratories, Inc. and is expressed as micrograms of Licl per gram of fiber $(\mu \mathrm{g} / \mathrm{g})$.

8. Mechanical Properties:

The mechanical properties (modulus and strength) of the fibers were determined on Miniature Materials Tester (Polymer Laboratories). Tests were conducted on single fibers at $50^{\circ} \mathrm{C}$ with $25.4 \mathrm{~mm}$ gauge length at a strain rate of $0.25 \mathrm{~mm} / \mathrm{min}$. The 
values represent an average of 5-7 measurements. Load and deformation data were transferred to a personal computer where they were recalculated and replotted as stress vs. strain, load vs. deformation or modulus vs. strain curves. The calculation of modulus and strength was based on the crosssectional area of the fibers.

9. Scanning Electron Microscopy:

The fibers were observed on a JEOL JSM-35C scanning electron microscope with an accelerating voltage of $15 \mathrm{kV}$. Fracture surfaces were formed in liquid nitrogen. The fibers were mounted on aluminum specimen mounts (EMSL) with an angle of $45^{\circ}$. The fibers were coated at the bottom by Ladd silver Conducting Paint and were then sputter coated by pure gold for 1 minute in a SPI sputter coater. 10. Mechano-sorptive Creep Experiment:

The creep tests were conducted on a specially designed fixture as shown in Fig. 1 (a) (9). The fixture is composed of three systems: the central loading system, the environment generating system, and the control and data acquisition system. A single fiber is encapsulated in a glass tube to maintain an appropriate environment. Fig. 1 (b) is the humidity profile used in the tests. Before the start of each test, a fiber was conditioned in the glass tube with a relative humidity level of $95 \%$ and $50^{\circ} \mathrm{C}$ for a sufficiently long time (12-14 hours) for the fiber to reach its equilibrium 


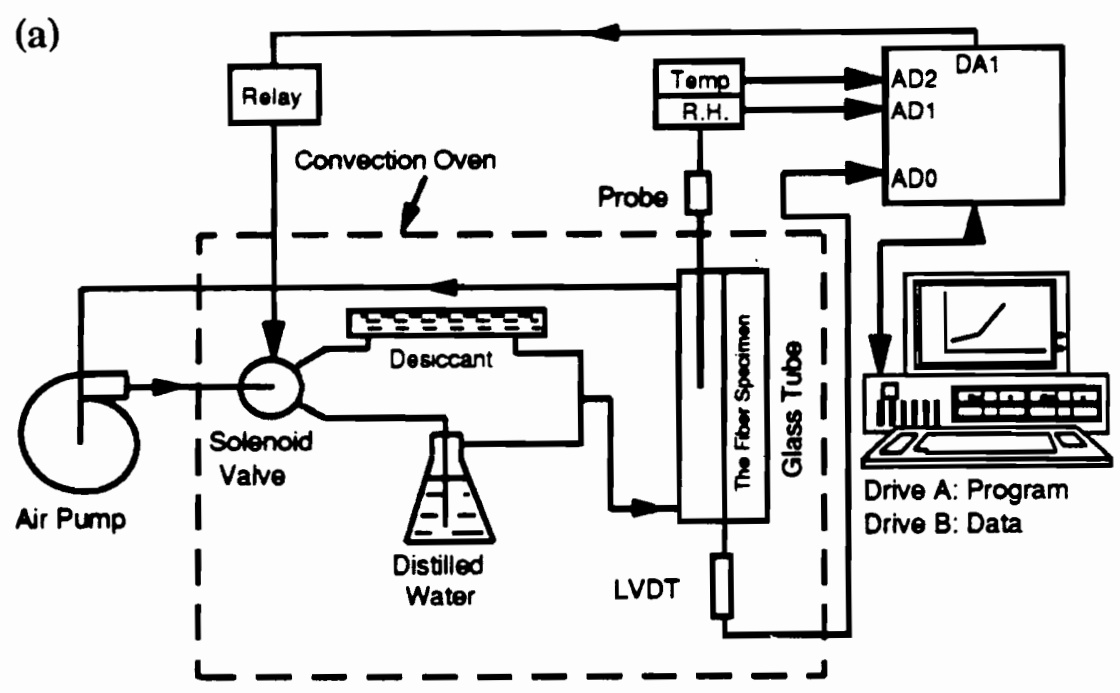

(b)

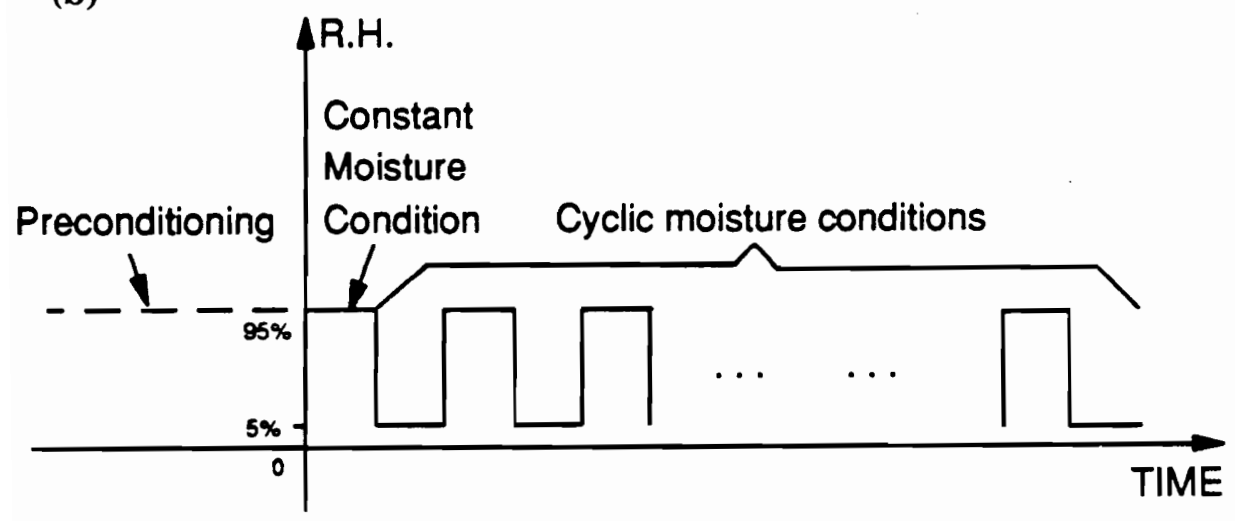

Figure 1. (a) Diagram of the fiber creep test fixture.

(b) Relative humidity profile in the fiber creep test. 
moisture content. After the mositure equilibrium was established, a constant stress was applied to the fiber and the test was started with the same relative humudity and temperature as in the preconditioning stage so that during the first two-hour period the moisture content in the fiber was constant. After the first two-hours, the humidity levels were cycled between 5 and $95 \%$ to observe the mechano-sorptive creep behavior. In this way, the creep rates at constant moisture content and cyclic moisture variations could be compared on the same fiber to minimize data scattering due to fiber variability.

Load is applied to the fiber by cementing a loadings train onto one end of the fiber. The displacement of the fiber is measured by a linear variable differential transducer (LVDT). The temperature was maintained at $50^{\circ} \mathrm{C}$ by accommodating the fixture in a conventional oven. Creep displacement, relative humidity and temperature in the glass tube were collected by a personal computer.

11. Moisture Absorption Experiment:

Fibers were dried at $65^{\circ} \mathrm{C}$ for 60 hours in a vacuum oven before testing. Weighed amounts of dried fiber bundles were placed in open wide-mouthed bottles which were in turn positioned on a frame above the water level in a temperature controlled water bath. Care was taken to use fiber bundles with almost equal weight. The temperature of the bath was 
maintained at $50^{\circ} \mathrm{C}$. The relative humidity was approximately $100 \%$ as the water bath was covered throughout the experiment. The weight of the fibers was measured at regular intervals. The experiment was discontinued after 15 hours due to excessive condensation of moisture on the fiber surface. The reported weight change data represent averages of two sets of fiber bundles.

\section{RESULTS AND DISCUSSION}

1. ${ }^{13} \mathrm{C}$ NMR Spectroscopy:

Figure 2 show the ${ }^{13} \mathrm{C}$ NMR spectra of the carbonyl region of $C A B$ in DMAC without and with LiCl, respectively. A downfield shift is observed for all the carbons.

Detailed investigations have been conducted by ${ }^{13} \mathrm{C}$ NMR spectroscopy on the interaction of solutions of cellulose/LiCl/DMAC (10-12). For DMAC/LiCl solutions, there is a downfield (deshielding) shift for all carbon atoms of the amide in DMAC upon addition of LiCl with the carbonyl carbons exhibiting the maximum change. This is due to direct interaction of the $\mathrm{Li}$ cation with the carbonyl oxygen of DMAc. This causes a general decrease in electron density of the entire amide molecule particularly at the carbonyl carbons and to a lesser extent at each of the other carbon atoms (10). It has also been shown earlier that the binding site of the lithium cation is at the carbonyl oxygen based on the interaction between amide complexes and metal ions by infrared 
(a)

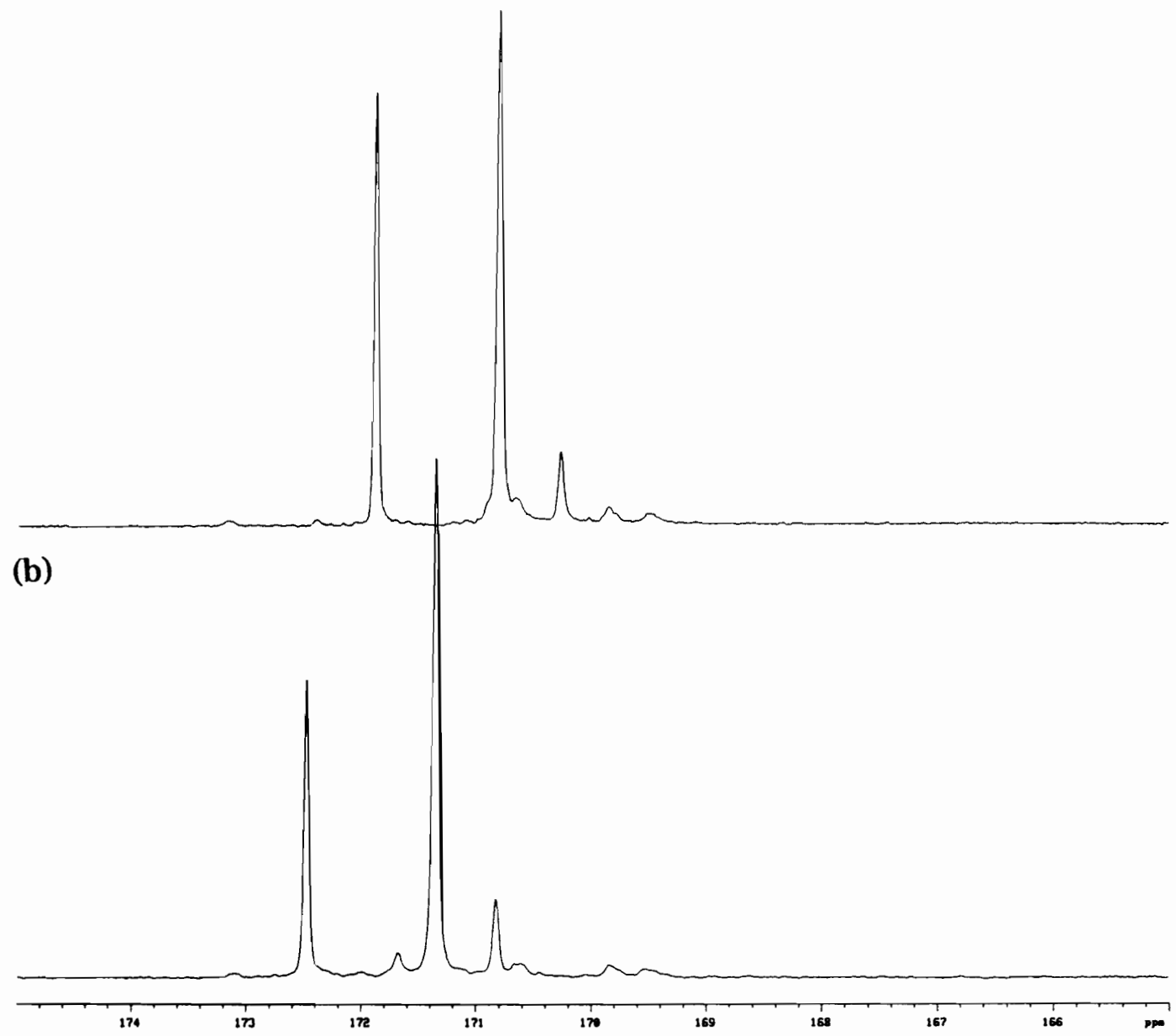

Figure 2. $\quad{ }^{13} \mathrm{C}$ NMR spectra of $\mathrm{CAB}$ in DMAc; (a) without $\mathrm{LiCl}$ (b) with $\mathrm{LiCl}$. 
spectroscopy (13) and X-ray structure analysis (14).

Therefore, from the results obtained by previous investigators and those in this study, it can be inferred that the downfield shift observed in the DMAC/LiCl/CAB solution is due to the ionic interactions between the $\mathrm{Li}$ cation and the carbonyl oxygens of DMAC and CAB.

2. Dynamic Viscoelastic Properties:

Figure 3 shows the relationship between dynamic shear viscosity and frequency of $35 \%(\mathrm{w} / \mathrm{w})$ and $50 \%(\mathrm{w} / \mathrm{w})$ CAB solutions without and with a varying amounts of Licl. since $\mathrm{CAB}$ solutions at $>40 \%(\mathrm{w} / \mathrm{w})$ concentration behave in a liquid crystalline manner, the viscosity of the $50 \%(\mathrm{w} / \mathrm{w})$ solution is expectedly lower than that at $35 \%(w / w)$. Such a decline in viscosity with increase in concentration is typical for liquid crystalline solutions. A detailed discussion of the viscoelastic behavior of the liquid crystalline cellulose ester solutions has been presented earlier (7).

A slight increase in viscosity of the $35 \%$ (w/w) (isotropic) solution is detected with the addition of $1 \%(\mathrm{w} / \mathrm{w})$ Licl. A similar viscosity increase was also observed by suto (15) in isotropic solutions of hydroxypropyl cellulose in water containing sodium chloride. Since DMAc is a good solvent for $C A B$, it is expected that $C A B$ molecules are well-surrounded by DMAC molecules, and that there is no intermolecular bonding. When Licl is added to the solution, there is a strong ionic interaction between the carbonyl oxygen of DMAc and Li 


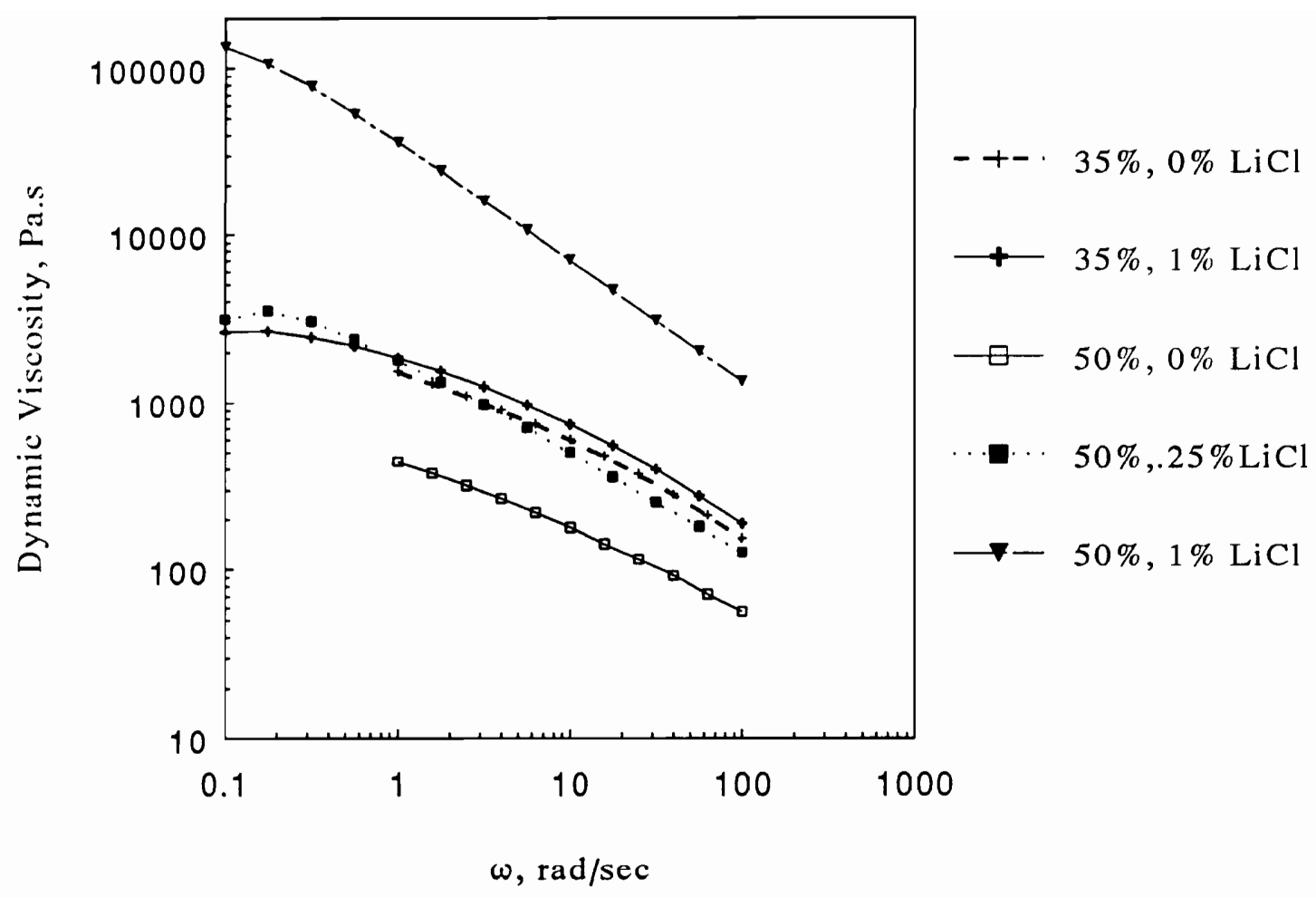

Figure 3. Dynamic viscosity versus frequency of $35 \%(w / w)$ and $50 \%(w / w) ~ C A B$ solutions without and with varying amount of $\mathrm{LiCl}$. 
cations which increases the degree of DMAC-DMAC interaction and reduces the DMAC-CAB intermolecular forces. Therefore, with a small amount of Licl, the intermolecular interaction of $\mathrm{CAB}$ molecules increases and solution viscosity rises. An increase in viscosity for cellulose solutions in DMAc/Licl has been reported previously with increasing in Licl content $(10,11)$

The viscosity of the $50 \%(w / w)$ anisotropic solution increased drastically with the increase in Licl content. Such viscosity behavior was also observed for the liquid crystalline solution of hydroxylpropyl cellulose in water with the addition of $\mathrm{NaCl}$ (15). For anisotropic solutions, there seems to be a competition between the alteration of the liquid crystalline structure and the conformational changes in the $C A B$ molecules caused by the ionic interaction between DMAC and Licl. Presence of Licl is causing the anisotropic phase to become isotropic and this change is proportional to the Licl concentration. Since the rise in viscosity is greater for anisotropic solutions than isotropic ones [at constant Licl concentration i.e. $1 \%(\mathrm{w} / \mathrm{w})]$, it clearly indicates that isotropization of the anisotropic phase had a greater influence on the viscosity behavior.

Figures 4 and 5 show the relationship between dynamic elastic modulus $\left(G^{\prime}\right)$ and dynamic loss modulus ( $\left.G^{\prime \prime}\right)$ at different frequencies for $35 \%(\mathrm{w} / \mathrm{w})$ and $50 \%(\mathrm{w} / \mathrm{w})$ concentration $C A B$ solutions with varying amounts of LiCl. The 


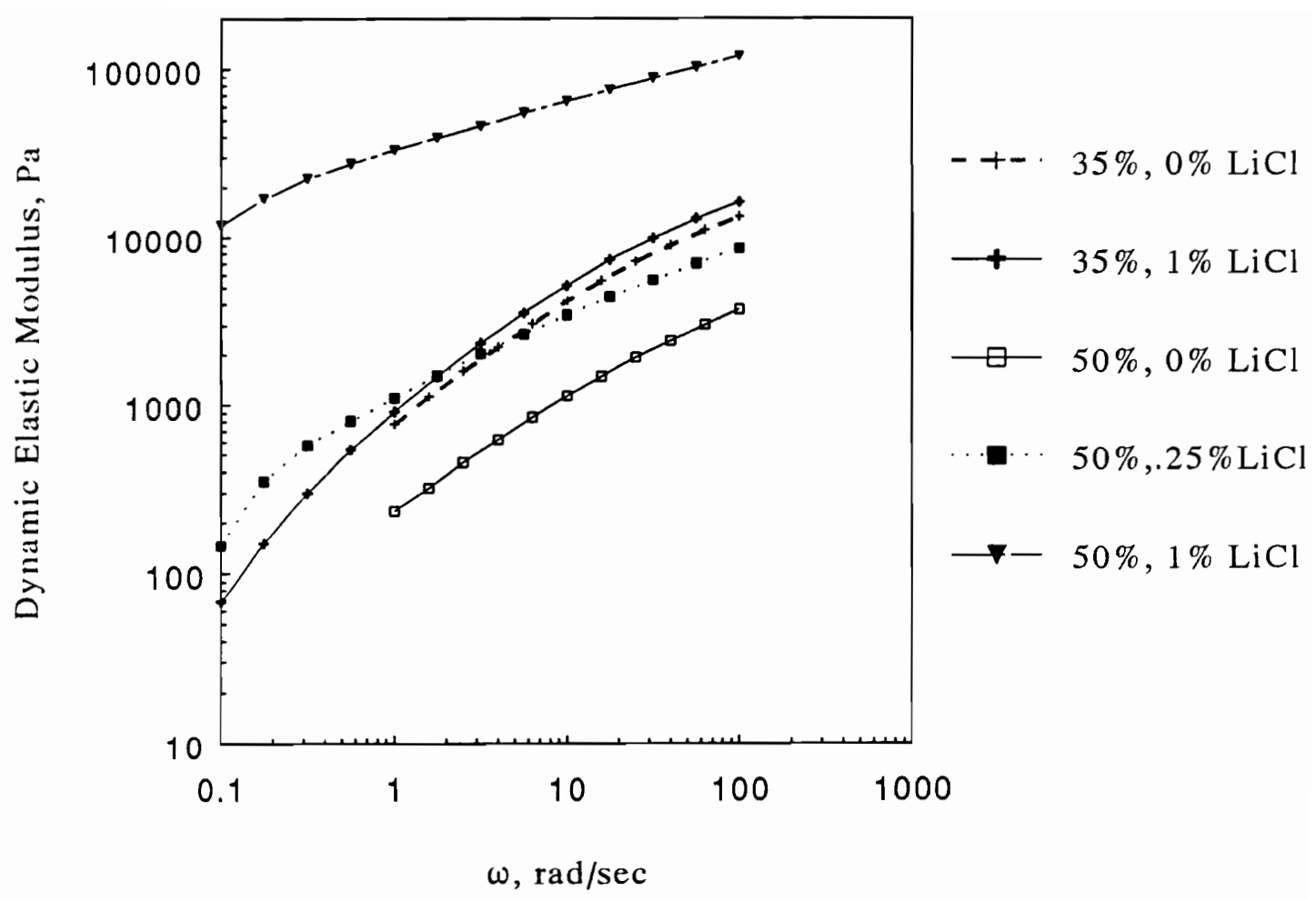

Figure 4. Dynamic elastic modulus versus frequency of 35\% (w/w) and $50 \%$ (w/w) CAB solutions without and with varying amount of $\mathrm{LiCl}$. 


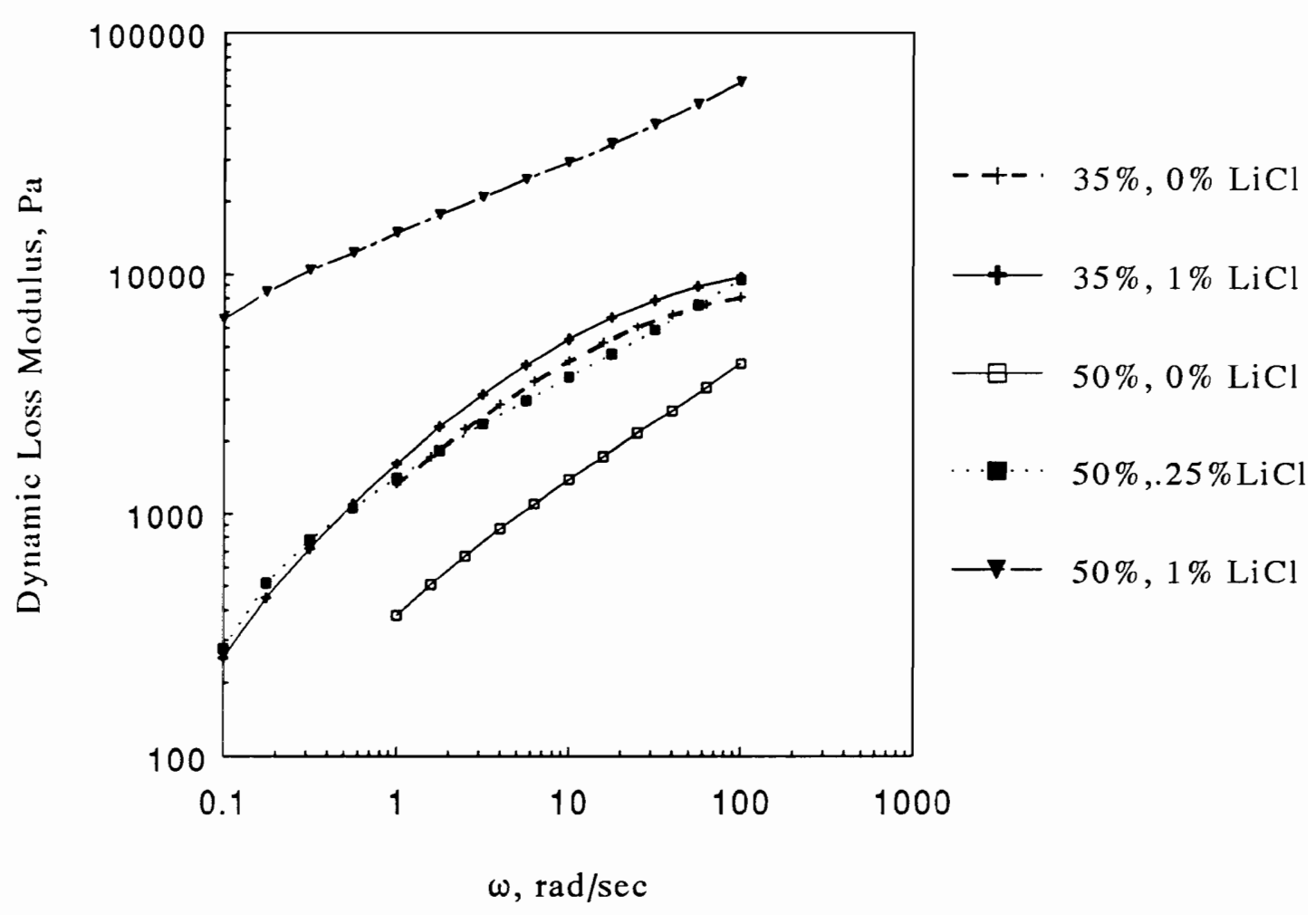

Figure 5. Dynamic loss modulus versus frequency of $35 \%(w / w)$ and $50 \%$ (w/w) CAB solutions without and with varying amount of $\mathrm{LiCl}$. 
behavior of the concentration dependence of $G^{\prime}$ and $G^{\prime \prime}$ is the same as that observed for dynamic viscosity, i.e. after the solution becomes anisotropic, $G^{\prime}$ and $G^{\prime \prime}$ values decline. As with any viscoelastic material, $G^{\prime}$ and $G^{\prime \prime}$ rises with frequency. In the presence of Licl, the solutions become more elastic and the damping characteristics increases. Similar effects on $G^{\prime}$ and $G^{\prime \prime}$ were observed for aqueous systems sodium carboxymethylcellulose in the presence of calcium and magnesium chloride salts (16).

3. Polarized Optical Microscopy:

Figure 6 (a) - (c) show the photomicrographs of $50 \%(w / w)$ $\mathrm{CAB}$ solutions with $0 \%, 0.25 \%$, and $1 \%(\mathrm{w} / \mathrm{w}) \mathrm{LiCl}$, respectively. All the solutions were birefringent and exhibited liquid crystalline behavior. However, it is observed that there is a transformation from anisotropic to isotropic phase with the increase in Licl concentration. Similar results were obtained for a cholesteric solutions of hydroxypropyl cellulose in DMAC containing Iicl (17). Fig. 6 (c) clearly shows the presence of isotropic regions which were not displayed in solutions with little or no Licl. These results support the observed rise in viscosity of anisotropic solutions.

4. Processing and Mechanical Properties:

Table I shows the spinning conditions and mechanical properties of the fibers with and without LiCl spun from anisotropic solutions. Since all the solutions were viscoelastic, there was release of stored energy which 
(a)

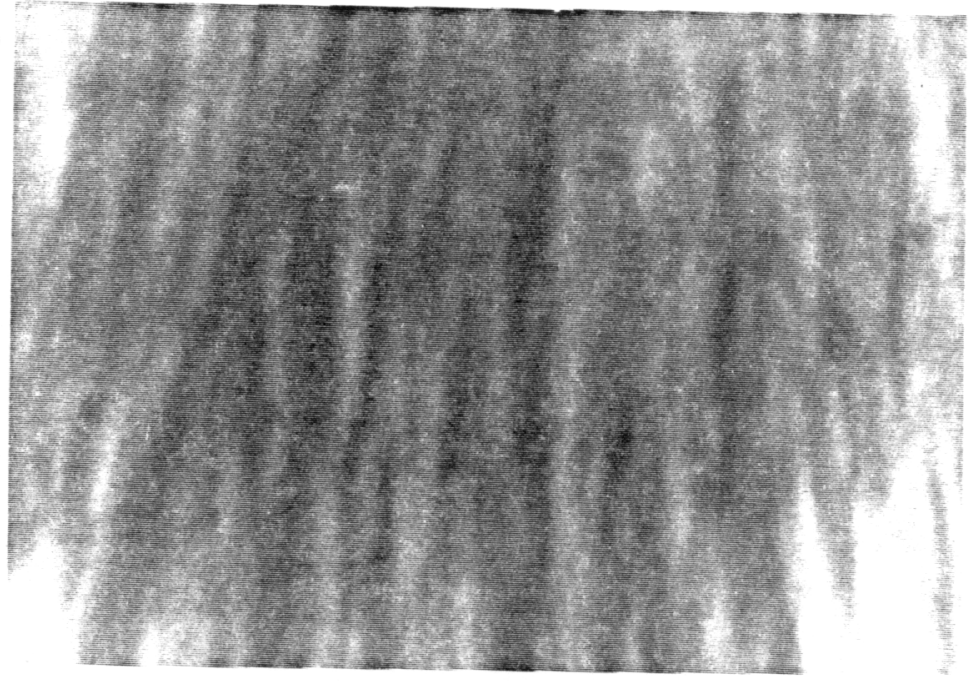

(b)

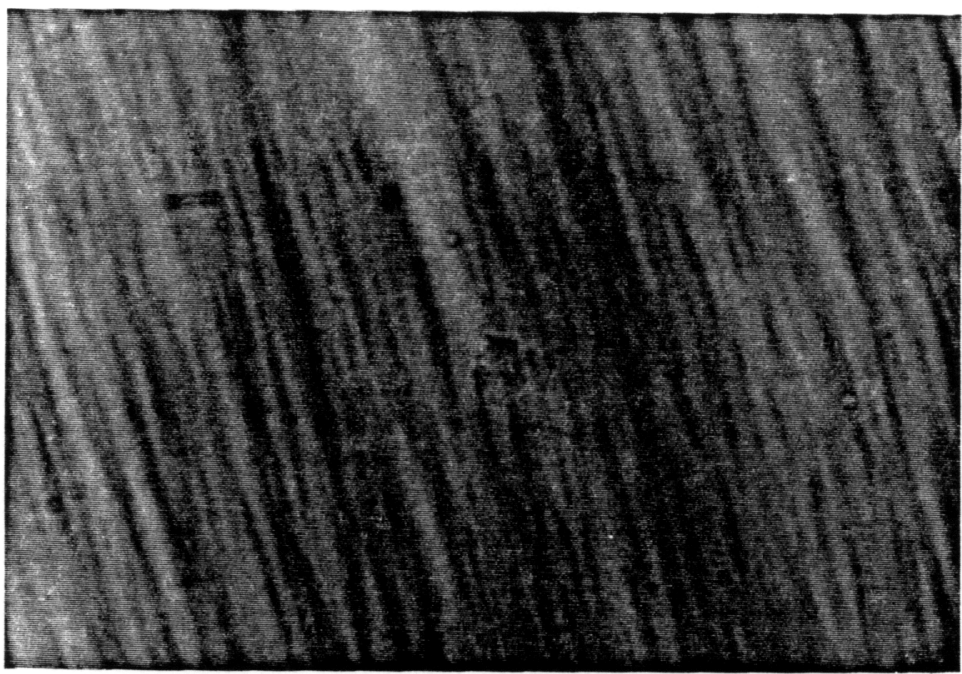

(c)

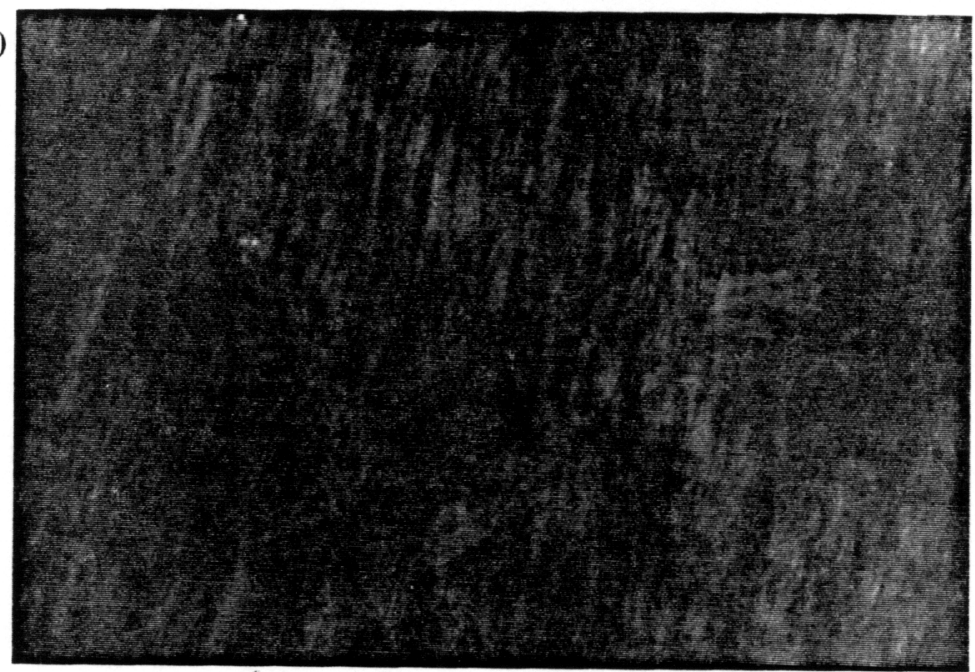

$50 \mu \mathrm{m}$

Figure 6. Polarized optical micrographs of 50\% (w/w) CAB solutions; (a) $0 \% \mathrm{LiCl}$ (b) $0.25 \% \mathrm{LiCl}$ (c) $1 \% \mathrm{LiCl}$. 
Table I. Spinning Conditions and Mechanical Properties of CAB Fibers containing Lithium Chloride.

\begin{tabular}{|c|c|c|c|c|c|c|}
\hline $\begin{array}{c}C_{p} \\
(w / w \&)\end{array}$ & $\begin{array}{c}\mathrm{V}_{\mathrm{L}}{ }^{1)} \\
\mathrm{m} / \mathrm{min}\end{array}$ & $\begin{array}{l}\text { Draw }^{2} \\
\text { Ratio }\end{array}$ & $\begin{array}{l}D \\
\mu \mathrm{m}\end{array}$ & $\begin{array}{l}\text { Modulus at } \\
50^{\circ} \mathrm{C} \text {, GPa }\end{array}$ & $\begin{array}{l}\text { strength at } \\
50^{\circ} \mathrm{C} \text {, MPa }\end{array}$ & $\begin{array}{l}\mathrm{LiCl} \\
\mu \mathrm{g} / \mathrm{g} \\
\end{array}$ \\
\hline$C A B-50$ & 11.0 & 0.80 & 178 & 8.2 & 137.5 & -- \\
\hline$C A B-50$ & 21.0 & 1.50 & 152 & 3.7 & 95.8 & -- \\
\hline $\begin{array}{l}C A B-50 \\
0.258 \\
\text { LiCl }\end{array}$ & 11.0 & 0.80 & 178 & 17.8 & 131.7 & 900 \\
\hline $\begin{array}{l}\text { CAB-50 } \\
0.25 \% \\
\text { LiCl }\end{array}$ & 21.0 & 1.50 & 127 & 7.5 & 170.6 & 900 \\
\hline
\end{tabular}


resulted in die-swelling of the solutions and slowed down the the filament velocity as it emerged from the die. Therefore, some of the draw ratios were less than unity as it was calculated based on $V_{0}$ instead of the reduced filament velocity. Modulus and strength (except for draw ratio of 0.8 ) values increased for the fibers containing Licl. This indicates the presence of interactions between $\mathrm{LiCl}$ and the polymer backbone.

Cellulose fibers spun from isotropic solutions of $\mathrm{N}-$ methyl morpholine N-oxide containing Licl did not show any change in mechanical properties (18). However, additions of ammonium chloride or calcium chloride to the solution strongly improved the mechanical properties of the cellulose fibers due to molecular association of the solvent with the salts (16). But there was no trace of the salts in the cellulose fibers. 5. Morphology:

Figures 7 (a) and (b) reveal the surface morphology of the fibers spun from an anisotropic solutions at constant take-up speed without and with Licl, respectively. Fibers without salt had a fibrillar ring structure, whereas the fibers with Licl were very smooth. This change in morphology could be due to the reduction in coagulation rate caused by the ionic interactions between DMAC and Licl. This has previously been shown to produce smooth fiber structures. 


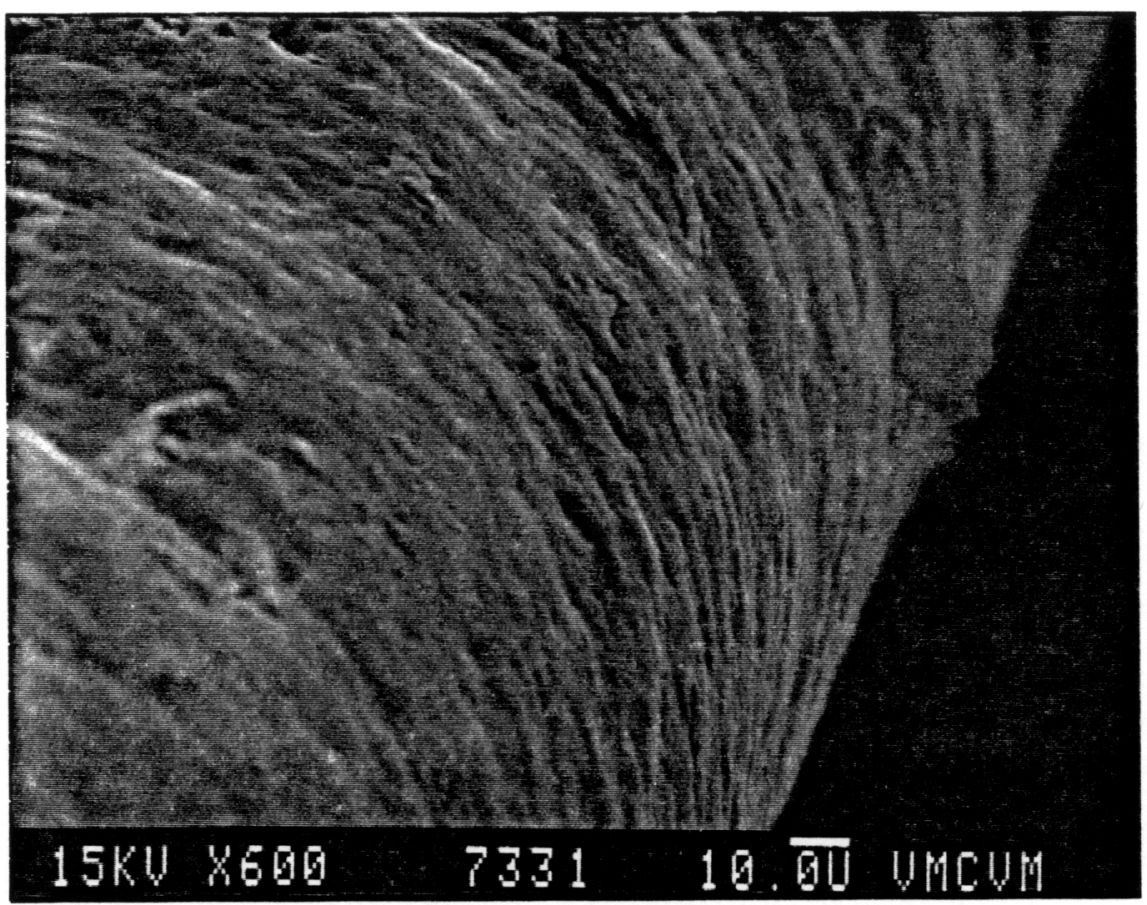

(a)

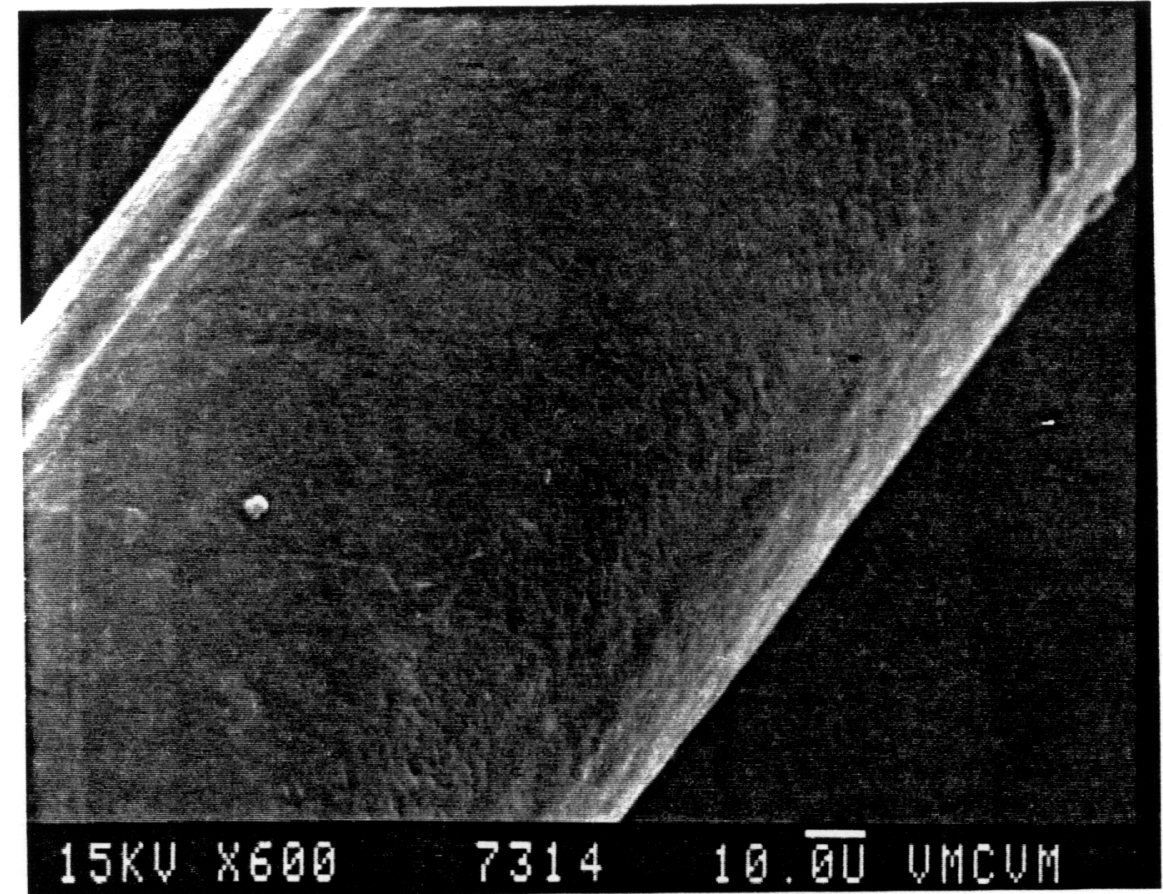

(b)

Figure 7. Scanning electron micrographs of the surface of CAB fibers spun at $11 \mathrm{~m} / \mathrm{min}$; (a) $0 \mu \mathrm{g} / \mathrm{g} \quad$ (b) $900 \mu \mathrm{g} / \mathrm{g}$. 


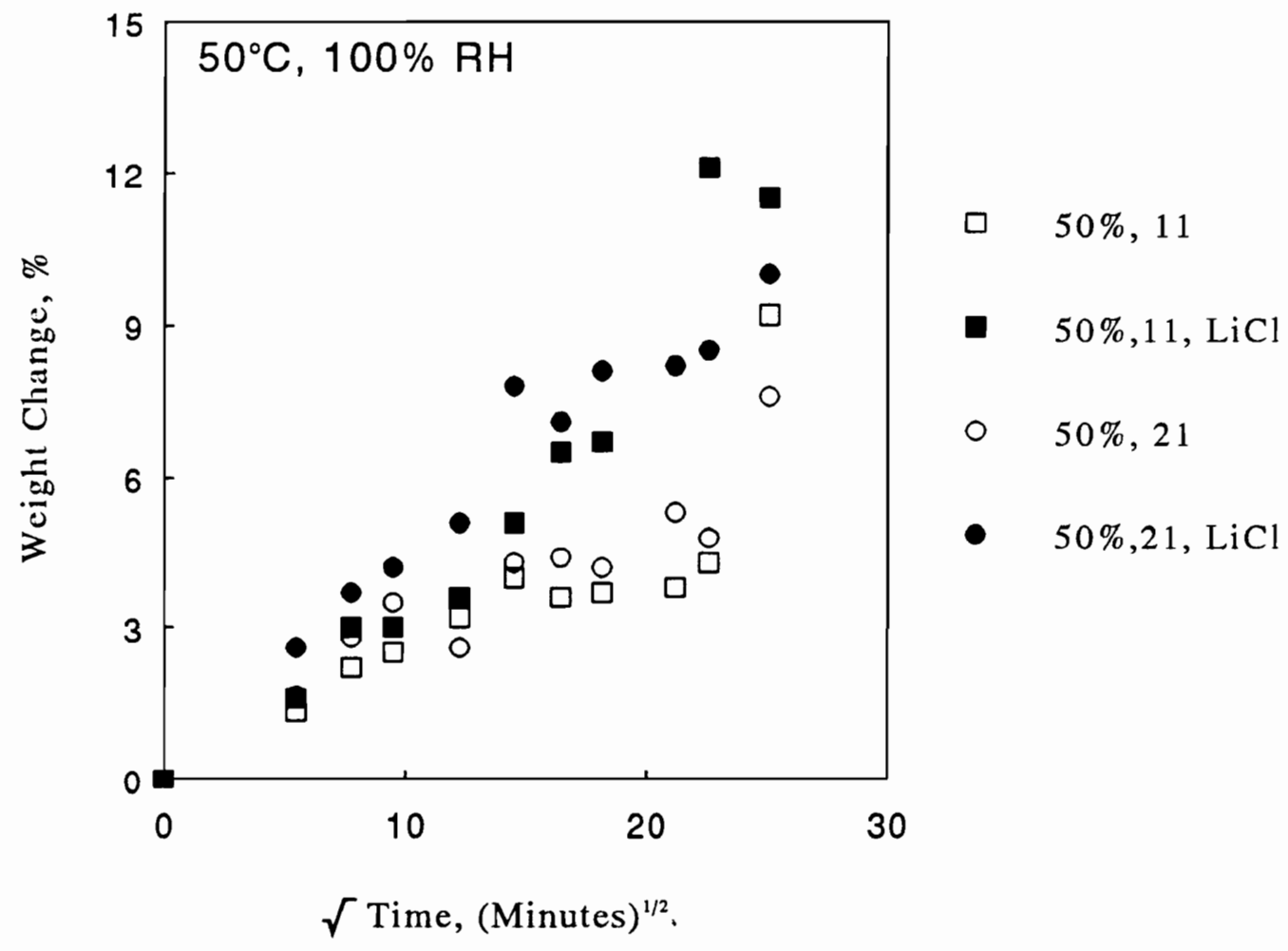

Figure 8. Weight change as a function of time of fibers exposed to air at $100 \%$ relative humidity. 
6. Moisture Absorption:

Figure 8 shows the moisture uptake for the fibers with and without LiCl at $50^{\circ} \mathrm{C}$ and approximately $100 \%$ relative humidity. Even though the data appear scattered, perhaps influenced by condensation problem, they do suggest that fibers containing Licl absorbed more moisture than those without Licl. This may be due to the presence of (hydroscopic) Licl molecules in the fibers.

7. Mechano-sorptive Creep Behavior:

Test conditions are listed in Table II. For identification purposes, we will denote the fibers spun at 11 $\mathrm{m} / \mathrm{min}$ as $\mathrm{CAB}-11$ and $\mathrm{CAB}-11-\mathrm{s}$ without and with Licl salt, respectively; and the fibers spun at $21 \mathrm{~m} / \mathrm{min}$ as $\mathrm{CAB}-21$ and CAB-21-S without and with LiCl salt, respectively.

The test results for $C A B$ fibers without salt are shown in Figures 9 and 10. From these results, it is seen that the creep data approximate straight lines when plotted on a loglog graph. Therefore, the creep obeys the power law. These results show that the fibers without salt ( $C A B-11$ and $C A B-21$ ) exhibit mechano-sorptive phenomenon, viz; the creep rates are higher under cyclic moisture conditions than those under constant moisture conditions. The test results are tabulated in Table III. To quantify the mechano-sorptive creep in these fibers, the ratio of the creep rate under transient moisture conditions to that under constant moisture conditions could be 
Table II. Test Conditions for CAB Fibers During creep Experiment.

\begin{tabular}{|c|c|c|c|}
\hline Type of Fiber & $\begin{array}{l}v_{L \prime} \\
m / m i n\end{array}$ & Temperature, ${ }^{\circ} \mathrm{C}$ & Applied stress, MPa \\
\hline $\mathrm{CAB}$ & 11 & 50 & 13.8 \\
\hline $\mathrm{CAB}$ & 21 & 50 & 9.6 \\
\hline CAB with Licl & 11 & 50 & 13.2 \\
\hline CAB with Licl & 21 & 50 & 17.1 \\
\hline
\end{tabular}




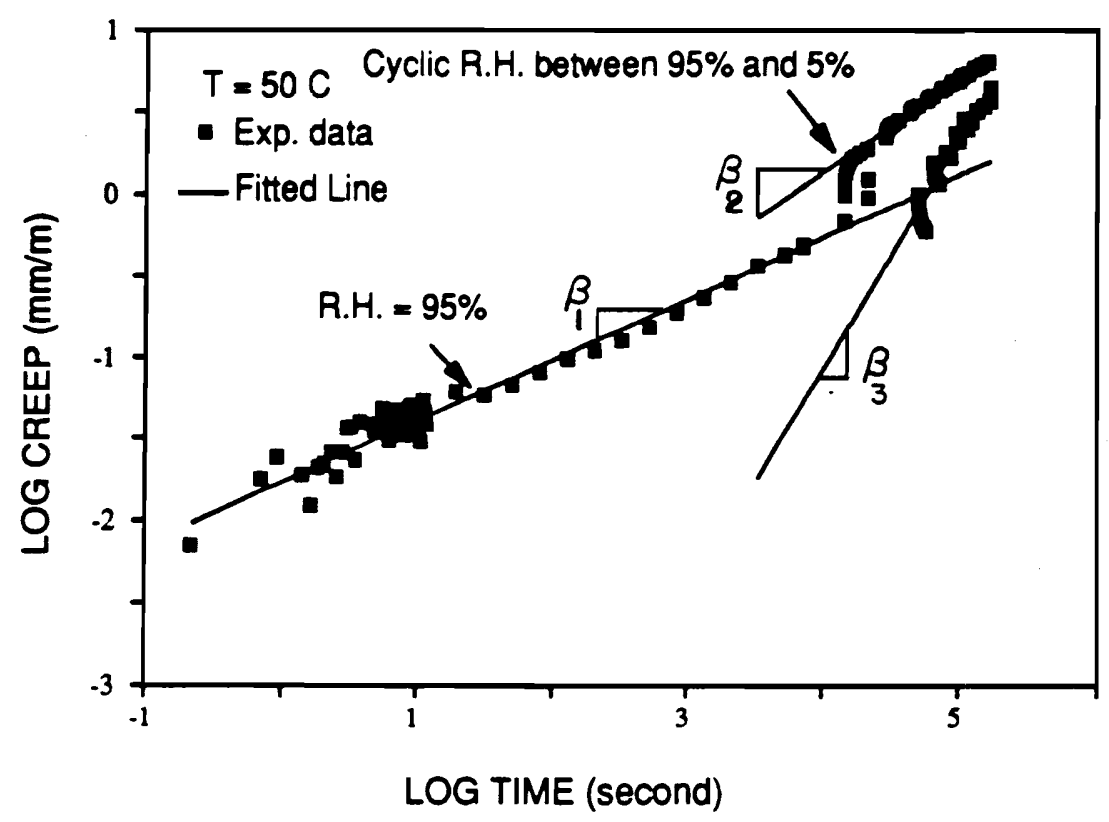

Figure 9. Creep of CAB-11 fiber. 


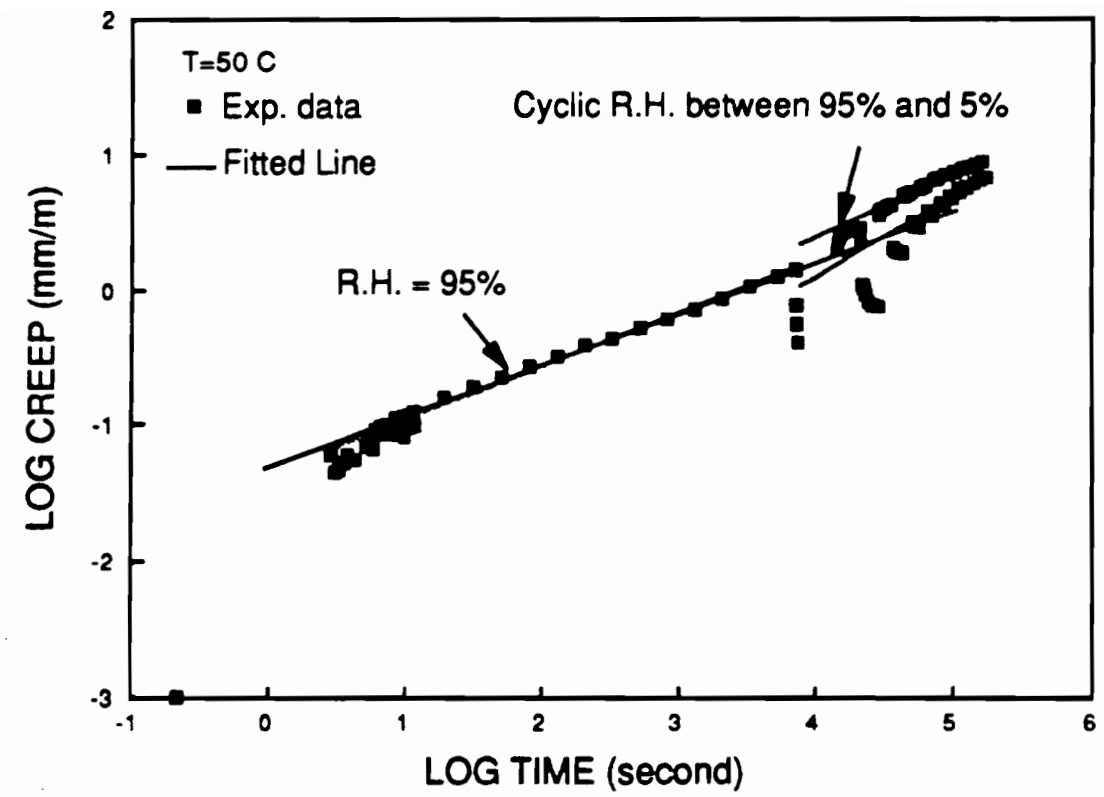

Figure 10. Creep of CAB-21 fiber. 


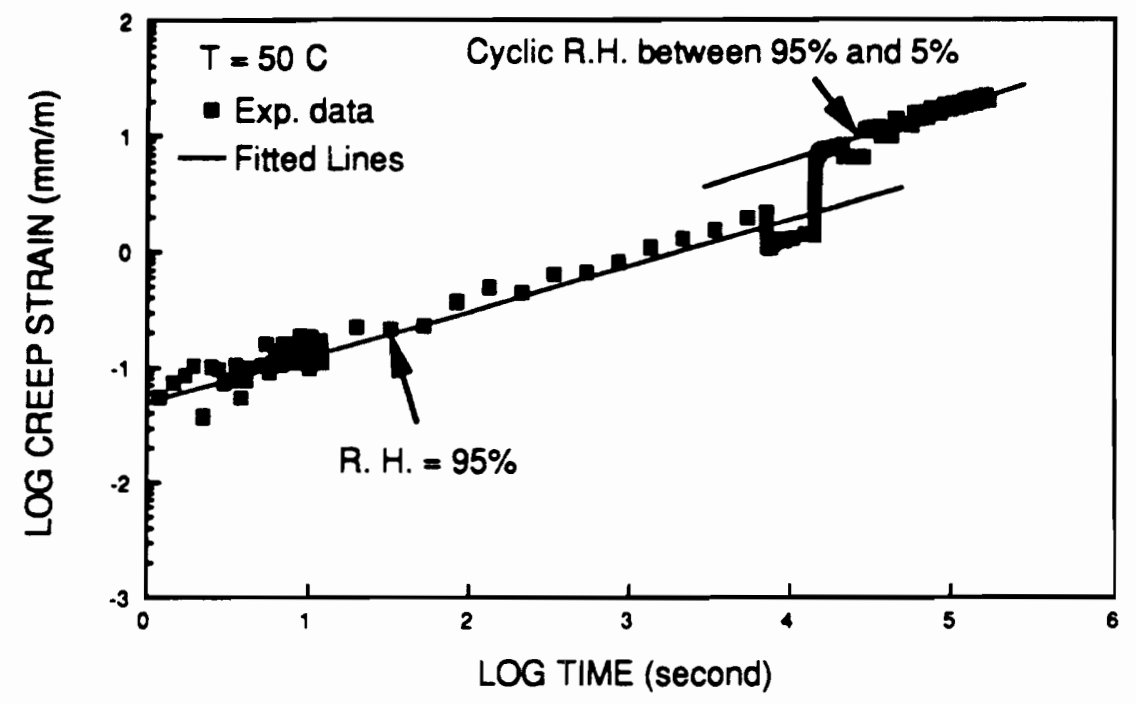

Figure 11. Creep of CAB-11-S fiber. 


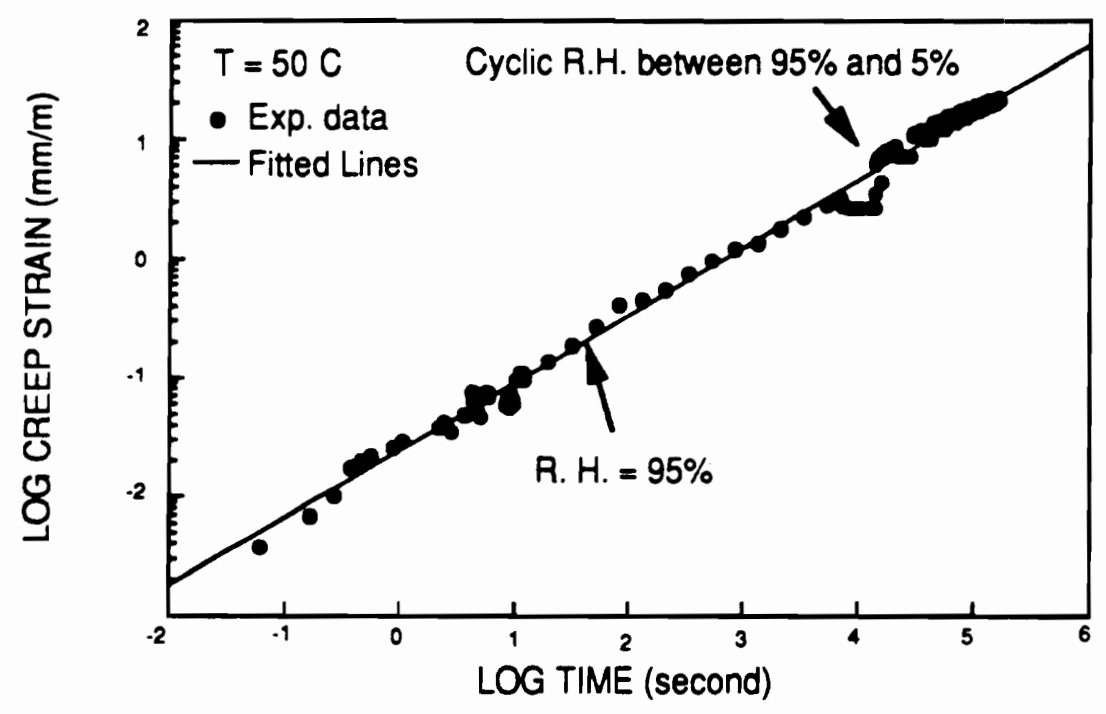

Figure 12. Creep of CAB-21-S fiber. 
used $(19,20)$. This ratio is termed the mechano-sorptive ratio. Creep rates under constant moisture condition $\left(\beta_{1}\right)$ and those under cyclic moisture conditions $\left(\beta_{2}\right)$, together with the mechano-sorptive ratio (r), are shown in Table III. Creep strain shows two distinct lines during the cyclic humidity conditions. The upper line corresponds to the creep strain during the wet portion of the cycle, and the lower line represents strain during the dry periods. This indicates that there is a substantial amount of axial strain which is associated with the different moisture contents. The increased slope, especially for the $C A B-11$ fibers, indicates substantial mechano-sorptive behavior.

Figures 11 and 12 show the creep results for the fibers containing salt $(\mathrm{CAB}-11-\mathrm{S}$ and $\mathrm{CAB}-21-5)$. The results are also tabulated in Table III. These fibers show little, if any, mechano-sorptive behavior. This is consistent with the observation that the LiCl enhances intermolecular interaction thereby preventing intermolecular slippage and thus creep. Hypothetically, the mechano-sorptive phenomenon in Kevlar fibers may be due to the osmotic pressure induced by the transport of moisture catalyzed by traces of residual sodium sulphate salt $(100 \mu \mathrm{g} / \mathrm{g})$ during the manufacturing process. However, mechano-sorptive effects are actually reduced in the $\mathrm{CAB}$ fibers with $900 \mu \mathrm{g} / \mathrm{g} \mathrm{LiCl}$ salt as compared to fibers without LiCl salt. This suggests that salt in fibers is not 
Table III. Mechano-Sorptive creep Results of CAB Fibers

\begin{tabular}{|c|c|c|c|c|}
\hline & $\boldsymbol{\beta}_{1}$ & $\beta_{2}$ & $\beta_{3}$ & $\left(\beta_{2} / \beta_{1}\right)$ \\
\hline & \multicolumn{4}{|c|}{$C A B-11$} \\
\hline Sample 1 & 0.38 & 0.57 & 1.41 & 1.50 \\
\hline Sample $\neq 2$ & 0.33 & 0.49 & 1.27 & 1.48 \\
\hline Average & 0.36 & 0.53 & 1.34 & 1.49 \\
\hline & \multicolumn{4}{|c|}{$C A B-21$} \\
\hline Sample 1 & 0.40 & 0.47 & 0.60 & 1.18 \\
\hline Sample 2 & $\underline{0.48}$ & $\underline{0.55}$ & $\underline{0.70}$ & 1.15 \\
\hline Average & 0.44 & 0.51 & 0.65 & 1.17 \\
\hline \multicolumn{5}{|c|}{$C A B-21-S$} \\
\hline Sample \#1 & 0.42 & 0.40 & -- & 0.95 \\
\hline Sample $\# 2$ & 0.49 & 0.52 & -- & 1.06 \\
\hline Sample 3 & $\underline{0.42}$ & $\underline{0.46}$ & -- & 1.10 \\
\hline Average & 0.44 & 0.46 & -- & 1.04 \\
\hline \multicolumn{5}{|c|}{$C A B-21-5$} \\
\hline Sample \#1 & 0.49 & 0.37 & -- & 0.76 \\
\hline Sample 2 & 0.50 & 0.48 & -- & 0.98 \\
\hline Sample 3 & $\underline{0.54}$ & $\underline{0.40}$ & -- & 0.94 \\
\hline Average & 0.51 & 0.42 & & 0.89 \\
\hline
\end{tabular}


the cause for mechano-sorptive behavior.

During the study of the mechano-sorptive behavior of Kevlar fibers, hydrogen bonding and crystallite rotation mechanisms have been proposed to explain the mechano-sorptive creep $(20,21)$. Kevlar fibers are composed of hydrogen bonded crystallites oriented at angles (about $10^{\circ}$ ) relative to the fiber axis. In an absorption process, small and polar water molecules may approach the hydrogen bonding sites and temporarily disrupt these hydrogen bonds. After the temporary breakage of the hydrogen bonds between the bonded crystallites, water molecules may bridge in between them to form new hydrogen bonds. In a desorption process, when water molecules leave the fiber, the hydrogen bonds, previously formed by the bridged water molecules, may break. These breakages in hydrogen bonds cause crystallite rotations toward the fiber axis and, therefore, increases in creep when the fiber is under load. This racheting mechanism was supported both by the measured mechano-sorptive creep activation energy $(\approx 20 \mathrm{~kJ} / \mathrm{mole}$ ) which is similar to the hydrogen bond dissociation energy and the increase in elastic modulus which indicates crystallite rotation $(20,21)$.

Therefore, in the above mentioned mechanism, two major points are important. First, the molecular structure must contain hydrogen bonds. Second, the deformation or the creep process must be controlled by the hydrogen bonded structure. 
For example, in the case of Kevlar fibers, it has been shown that the deformation process of Kevlar fibers is controlled by the hydrogen bonding between the oriented crystallites (22). Mechano-sorptive creep behavior is exhibited in CAB fibers due to the presence of hydrogen bonds. To examine the role of hydrogen bonds, two major groups of fibers were studied earlier in this continued research (4). The first group of fibers, Technora (produced by Teijin Ltd., is a copolyamide fiber prepared from 50/25/25 mole ratio of terephthaloyl chloride, p-phenylenediamine and 3,4'diaminodiphenyl ether monomers) and Twaron [produced by Akzo Co., is based on poly (p-phenylene terephthalamide)] which both contain hydrogen bonds. The second group of fibers, high density polyethylene [HDPE] and poly(methyl methacrylate) [PMMA] fibers, contain no hydrogen bonds. The creep test results had suggested that Technora and Twaron fibers exhibited mechano-sorptive behavior, while HDPE and PMMA did not show this behavior. These experimental results suggested that hydrogen bonds are responsible for the mechano-sorptive phenomenon observed.

Since the take-up speed of $C A B-11$ is lower than that of CAB-21, its crystallite angle should be greater (i.e., relatively low degree of orientation). Experimental results indicated that mechano-sorptive effect in CAB-11 is more pronounced than in $\mathrm{CAB}-21$ due to the greater crystallite angles in $\mathrm{CAB}-11$ fibers. Experimental results from Kevlar 
fibers have shown that the mechano-sorptive effects are more pronounced in Kevlar 29 fibers, least pronounced in Kevlar 149 fibers, and intermediate in Kevlar 49 fibers (19). This order of mechano-sorptive effects is the same as that of the crystallite angles of Kevlar.

It needs to be noted that the above mechanism is proposed mainly to explain the mechano-sorptive phenomenon in highly crystalline polymers. Aramid and $\mathrm{CAB}$ fibers are highly crystalline as they are spun from liquid crystalline solutions. For amorphous or semi-crystalline polymers with low to moderate crystallinity, the creep behavior is dominated by the amorphous regions rather than hydrogen bonding at the crystallite interfaces. For example, even though nylon-6,6 fibers contain hydrogen bonds, they do not exhibit mechanosorptive creep (4). [The crystallinity of nylon-6,6 fibers is generally about 50\% (23)]. Therefore, it is believed that its deformation behavior is controlled by the amorphous region rather than by the hydrogen bonds between crystallites.

It has been shown earlier by ${ }^{13} \mathrm{C}$ NMR measurements that Li cation interacts very strongly with the carbonyl oxygen of DMAC and CAB. However, DMAC is absent in the fibers and the fact that the added Licl salt actually reduces the mechanosorptive phenomenon in $C A B$ fibers could be explained with the electrostatic interactions between $\mathrm{Li}^{+}$and the $\mathrm{CAB}$ polymer chain. Carbonyl groups on the acetyl and butyryl substituents 
are sites available for ionic interactions. $\mathrm{Li}^{+}$cation can form strong electrostatic bonds with carbonyl oxygen and unsubstututed hydroxyl groups along the polymer chain, thus increasing the intermolecular forces between the polymer chains. These electrostatic bonds are stronger than hydrogen bonds (24). Therefore when water enters the fiber, water molecules may not be able to break these electrostatic bonds, hence reducing or eliminating mechano-sorptive behavior. Although, the moisture uptake is more for fibers with Licl as observed by moisture absorption experiment, the excess water uptake at $95 \%$ humudity cycle is unable to break the electrostatic bonds.

\section{CONCLUSIONS}

1. There is a strong ionic interaction between the carbonyl oxygen of DMAC and $C A B$ with the lithium cation. slight viscosity rise in the isotropic solutions is due to the change in the conformation of CAB molecules by LiCl. However, enormous rise in viscosity in anisotropic solutions is due to isotropization of the anisotropic phase.

2. Mechano-sorptive behavior is demonstrated by CAB fibers. This is attributed to the hydrogen bonding between the crystallite structure. It is believed that transient moisture conditions temporarily disrupt the hydrogens bonds, allowing crystallite rotation and a ratcheting of 
strain under load.

3. The presence of Licl reduces the mechano-sorptive behavior presumably due to the formation of electrostatic bonds between the lithium cation and the carbonyl oxygen on the substituents and unsubstituted hydroxyl groups on the $C A B$ backbone. This shows that the residual salt in Kevlar fibers is probably not responsible for the increased creep under cyclic moisture conditions. 


\section{REFERENCES}

1. V. Davé, W. G. Glasser and G. L. Wilkes (to be published).

2. A. F. Turbak, in Encyclopedia of Polym. sci. and Engr., H. F. Mark, N. M. Bikales, C. G. overberger and G. Menges, Eds., Wiley, New York, 14, 145, 1987.

3. J. Z. Wang, F. A. Kamke and D. A. Dillard, J. Mat. Sci., $26,5113,1991$.

4. J. Z. Wang, V. Davé, W. G. Glasser and D. A. Dillard, STP-ASTM Symp. on High Temp. and Environmental Effects on Polymeric Composites, San Diego, 1991.

5. C. M. Buchanan, J. A. Hyatt and D. W. Lowman, Macromolecules, $20,2750-2754,1987$.

6. C. M. Buchanan, J. A. Hyatt and D. W. Lowman, Carbohyd. Res., 177, 228-234, 1988 .

7. V. Davé and W. G. Glasser, in Dynamic viscoelasticity of Biomaterials, ACS Symp. Ser., W. G. Glasser and H. Hatakeyama, Eds., (In Press).

8. H. Blades, U.S. Patent 3767756,1972 .

9. J. Z. Wang and D. A. Dillard, Exptl. Tech., Sept/Oct., $47,1991$.

10. A. El-Kafrawy, J.Appl. Polym. Sci., 27, 2435-2443, 1982 .

11. C. L. McCormick, P. A. Callais and B. H. Hutchinson, Jr., Macromolecules, 18(12), 2394-2401, 1985. 
12. C. P. Rao, P. Balaram and C. N. R. Rao, J. Chem. Soc., Faraday Trans., I, 76, 1008-1013, 1980.

13. C. N. R. Rao, H. S. Randhawa, N. V. R. Reddy and D. Chakravorty, Spectrochim. Acta A, 31, 1283, 1975.

14. J. Bello, D. Haas and H. R. Bello, Biochemistry, 5, 2539, 1966.

15. S. Suto, W. Nishibori, K. Kudo and M. Karasawa, J. Appl. Polym. Sci., 37, 737-749, 1989.

16. T. Matsumoto and K. Mashiko, Polym. Engr. and Sci., $28(6), 393-402,1988$.

17. M. J. Seurin and P. Sixou, Eur. Polym. J., 23(1), 77-87, 1987.

18. H. Chanzy, M. Paillet and R. Hagege, Polymer, 31, 400405,1990 .

19. J. Z. Wang, D. A. Dillard, M. P. Wolcott, F. A. Kamke and G. L. Wilkes, J. of Comp. Mats., 24, 994-1009, 1990 .

20. J. Z. Wang, D. A. Dillard and T. C. Ward, J. Polym. Sci.: Polym. Phys. Ed., (In Press).

21. J. Z. Wang, Ph.D. Dissertation, Virginia Polytechnic Institute and state University, Blacksburg, VA 24061, 1990.

22. M. G. Norholt and R. v. d. Hout, Polymer, 26, 310-316, 1985.

23. H. W. Starkweather, P. Zoller and G. A. Jones, J. Polym. Sci.: Polym. Phys. Ed., 22, 1615-1621, 1984. 
24. T. L. Brown and H. E. LeMay, Chemistry, Prentice-Hall, New Jersey, 327-330, 1981. 
6.0 CELLULOSE-BASED FIBERS FROM LIQUID CRYSTALLINE SOLUTIONS. V. PROCESSING AND MORPHOLOGY OF BLENDS WITH LIGNIN. 


\title{
CELLULOSE-BASED FIBERS FROM LIQUID CRYSTALLINE SOLUTIONS.
}

V. Processing and Morphology of Blends with Lignin.

\begin{abstract}
Continuous fibers of blends of cellulose acetate butyrate (CAB) and of cellulose (C) with lignin (L) were spun from liquid crystalline solution. Morphological investigations implies that $C A B$ and $L$ formed blends with considerable phase mixing whereas $C$ and $L$ were partially phase mixed. The porosity of the surface structure of $\mathrm{CAB} / \mathrm{L}$ fibers increased with an increase in $\mathrm{L}$ content. The mechanical properties of the fibers with $L$ increased due to phase mixing of $C A B$ and $L$ molecules in the fiber matrix. This study shows that phase mixing can be enhanced by coagulation during wet spinning.
\end{abstract}




\section{INTRODUCTION}

The area of polymer blends and mixtures is rapidly growing. Certainly, blending is a simple way of obtaining a variety of properties in materials for a particular application at a reasonable price/performance ratio. Nonmesomorphic polymer blends have been extensively studied (1$3)$, and incompatibility is observed in many cases unless specific interactions exist between different constituents.

When one component is liquid crystalline, the polymer blend may produce exceptional properties leading to ultrahigh modulus composite materials (4-8). In case of mixtures of rigid-rod like components and random coils in a solvent, the latter component is predicted to be virtually excluded from the liquid crystalline phase (9). This is due to the entropy effect related to the interference of the random coils with the mutual orientation of rod-like molecules. Aharoni's experiment on ternary system of tetrachloroethane / polyisocyanate (POIC) / polystyrene supported the prediction, as polystyrene was not present in detectable quantities in the anisotropic POIC phase (10). Bianchi et al. (11) also showed that the flexible polymer, $x-500$ (polyterephthalamide of $p$ aminobenzhydrazide), was not observed in the anisotropic phase of poly (p-benzamide) (PBA) in a ternary system of $\mathrm{X}-500 / \mathrm{PBA}$ / dimethylacetamide. They also extended the study to two mesogenic polymers, cellulose acetate (CA) and hydroxypropyl 
cellulose (HPC), in dimethylacetamide and showed that CA and HPC were not compatible in isotropic and anisotropic solutions giving rise to three coexisting phases which still have not yet been theoretically predicted (12). similar incompatibility was observed between anisotropic solutions of HPC and ethyl cellulose (EC) in acetic acid (13). However, bulk morphology of these blend systems have not been investigated.

In the cell wall of wood, the carbohydrate component is closely associated with lignin - a three-dimensional crosslinked polymer. Lignin (L) is perceived to serve as the structural matrix imparting strength and rigidity to the cell wall. The interaction of $I$ and cellulose (C) in this natural composite is not well understood. Blend studies on meltextruded and solvent-cast films of HPC and $L$ have shown the presence of secondary interactions between the two components and represented a partially miscible system (14). However, blends of $I$ with EC and cellulose acetate butyrate (CAB) were determined to be immiscible (15) .

We have already studied the processing and morphological features of fibers from liquid crystalline solutions of $C A B$ (16), C (17) and cellulose hexanoate (17). Effects of 1ithium chloride on $C A B$ were studied by creep tests under cyclic moisture conditions (18). The objective of the present work is to blend $L$ with liquid crystalline solutions of $C A B$ and $C$ to understand the effects of $L$ (an amorphous non-mesogenic component) on the liquid crystallinity of the cellulosic 
solutions (semi-rigid component) and to evaluate the blend morphology of the fibers spun from these solutions.

\section{EXPERIMENTAL}

\section{Materials:}

Cellulose acetate butyrate ( $C A B$ 500-5) was obtained from Eastman Kodak, Kingsport, TN. The degree of substitution (DS) of acetyl (DS $\mathrm{AC})$ and butyryl $\left(\mathrm{DS}_{\mathrm{BU}}\right)$ groups was 0.29 and 2.57 , respectively. $\mathrm{DS}_{\mathrm{OH}}$, determined by difference, was 0.14 .

Whatman CF-11 cellulose powder was used as received. Tricarbanilate derivative of $C$ was prepared (19) to make solutions in HPLC-grade tetrahydrofuran for determination of molecular parameters using gel permeation chromatography.

Unmodified organosolv lignin was supplied by Aldrich (Cat. No. $37,101-7$ ). Reagent grade dimethylacetamide (DMAC) and lithium chloride (LiCl) were used as received.

II. Methods.

1. Preparation of Blended Solutions:

(a) $\mathrm{CAB}$ and $\mathrm{L}$.

Individual component solutions of dried $C A B$ and $L$ were prepared in DMAC. These were mechanically mixed for 12 hours at ambient temperature to prepare blended solutions. Since it has already been shown that this particular CAB behaves as a liquid crystalline solution in DMAc at $35 \%(\mathrm{w} / \mathrm{w})$ concentration (20), the total solids content in all the solutions was maintained at a constant value of $40 \%(w / W)$ and the $L$ content 
varied from 4 to $20 \%(w / w)$ of organic solutes.

(b) C and L.

Weighed amounts of DMAC and dried $L$ were added to $a$ weighed amount of $\mathrm{C}$ which was soaked in DMAc for 3 days. The mixture was heated to reflux temperature (approximately $165^{\circ} \mathrm{C}$ ) for 20-30 minutes while being mechanically stirred under nitrogen atmosphere. The mixture was allowed to cool to about $100^{\circ} \mathrm{C}$, and a weighed amount of $\mathrm{LiCl}$ was added under steady stirring. The mixture was stirred for about 30 minutes at $80^{\circ} \mathrm{C}$ before it was allowed to cool to room temperature. Stirring was continued for about 20 hours at room temperature. C and $\mathrm{L}$ concentration was $12 \%(\mathrm{w} / \mathrm{w})$ and $0.5 \%(\mathrm{w} / \mathrm{w})$ defined as grams of $C$ and L per 100 grams of (C, L, DMAC and LiCl), respectively. L concentration was $4 \%(\mathrm{w} / \mathrm{W})$ based on grams of I per 100 grams of binary mixture of dried $\mathrm{C}$ and L. LiCl concentration was $7.8 \%(w / w)$ and was defined as grams of LiCl per 100 grams of binary solution (DMAC and LiCl).

The blended solutions were allowed to equilibrate for 1 week prior to analysis and fiber spinning.

2. Viscosity Measurements:

A Rheometrics Mechanical Spectrometer (RMS 800) was used to determine the rheological properties of the concentrated blended solutions. The solutions were placed in a paralleldisk geometry. The dynamic mechanical properties were measured at $26^{\circ} \mathrm{C}$ using a strain amplitude of $25 \%$ of the value at which 
all the solutions showed viscoelasticity. The frequency ranged from 0.1 to $100 \mathrm{rad} / \mathrm{sec}$.

3. Polarized Optical Microscopy:

Polarized optical microscopy was performed with a zeiss Axioplan Microscope. Small portions of the solutions were placed between microscope slide and cover slip, and was inspected for birefringence between the cross polarizers of the microscope at room temperature.

4. Fiber Spinning:

Continuous blended fibers were spun by a dry jet/wet spinning method from a capillary with a diameter of $300 \mu \mathrm{m}$ as described in our earlier publication (16). The flow rate of the solutions varied between 1 and $3 \mathrm{~cm}^{3} / \mathrm{min}$. The fibers were taken-up on the spools at speeds varying from $11-25 \mathrm{~m} / \mathrm{min}$. The air-gap was about 1 inch. Water was used as the coagulant at about $30^{\circ} \mathrm{C}$. The fiber-wound spools were immersed in beakers containing water for 8 hours and were then allowed to air-dry for about 8-10 hours after which the fibers were vacuum-dried for 24 hours at approximately $50^{\circ} \mathrm{C}$. No post-spinning treatments were performed.

5. Fiber Diameter:

Fiber diameter (D) was determined by a Nikon UM-2 Measurescope equipped with a Quadra-Chek III attachment. The diameter was generally constant along an extended length of the fiber. The reported value is an average of three to four 
measurements along the fiber length.

6. Mechanical Properties:

The mechanical properties of the fibers were determined on an Instron 1130 Test Instruments following the procedure of ASTM standard D3822. Tests were conducted at room temperature and $67 \%$ relative humidity with 1 inch gauge length at a strain rate of 0.2 inches/min. Single blended fiber was used during testing. The linear density of the fibers varied between 90 and 150 denier for $C$ and L blends, and between 94 and 270 denier for $C A B$ and $L$ blends. The values of initial modulus (E), breaking tenacity $\left(\sigma_{b}\right)$, breaking toughness (BT) and elongation at break $\left(\varepsilon_{b}\right)$ represented averages of 6-10 measurements.

7. Thermal Analysis:

Thermal analysis data of the $C A B / L$ blended fibers were collected using a DuPont 912 DSC at a heating of $10^{\circ} \mathrm{C} / \mathrm{min}$ under nitrogen atmosphere from $30^{\circ} \mathrm{C}$ to $280^{\circ} \mathrm{C}$. After the first run, the samples were quenched to $30^{\circ} \mathrm{C}$ and re-heated to $280^{\circ} \mathrm{C}$ to observe the glass transition temperature of the material. During the sample preparation, the fibers were cut into small pieces. These were placed carefully into the DSC pans. The pans were sealed cautiously. The weight of the samples was low and varied between 3 to $6 \mathrm{mgs}$. as it was difficult to fill the pan completely with short light-weight fibers. 
Table I. Chemical and Molecular Characteristics of cellulose Tricarbanilate, Cellulose Acetate Butyrate and Lignin.

\begin{tabular}{||c|c|c|c||}
\hline \hline & $\begin{array}{c}\text { Cellulose } \\
\text { Tricarbanilate }\end{array}$ & $\begin{array}{c}\text { Cellulose } \\
\text { Acetate } \\
\text { Butyrate }\end{array}$ & $\begin{array}{c}\text { Organosolv } \\
\text { Lignin }\end{array}$ \\
\hline$\left\langle\mathrm{M}_{\mathrm{n}}\right\rangle\left(\times 10^{3}\right)$ & 110 & 53 & 0.8 \\
\hline$\left\langle\mathrm{M}_{\mathrm{w}}\right\rangle\left(\times 10^{3}\right)$ & 180 & 125 & 3.5 \\
\hline $\mathrm{DP}_{\mathrm{a}}$ & 212 & 150 & 4 \\
\hline $\mathrm{MHS}-$ Constant (a) & 0.83 & 0.95 & 0.18 \\
\hline $\begin{array}{c}\text { Intrinsic viscosity } \\
(\mathrm{dL} / \mathrm{g})\end{array}$ & 1.34 & 1.51 & 0.051 \\
\hline \hline
\end{tabular}




\section{Scanning Electron Microscopy:}

The fibers were observed on a JEOL JSM-35C scanning electron microscope with an accelerating voltage of $15 \mathrm{kV}$. Fracture surfaces were formed in liquid nitrogen. The fibers were mounted on aluminum specimen mounts (EMSL) with an angle of $45^{\circ}$. The fibers were coated at the bottom by Ladd silver Conducting Paint and were then sputter coated by pure gold for 1 minute (thickness $\sim 9 \mathrm{~nm}$ ) in a SPI sputter coater.

9. Transmission Electron Microscopy:

Blended fibers were embedded in Poly/Bed 812 (Polysciences, Inc.) cured at $60^{\circ} \mathrm{C}$ for 48 hours in flat molds. 80-90 $\mathrm{nm}$ thick sections were cut across the cross-section and longitudinal-section from the embedded fibers with a diamond knife mounted on a Reichert Ultracut E microtome. All the cut sections were mounted on copper grids. Staining was carried out for 30 minutes by exposing some of the copper grids to vapors of $0.5 \%$ stabilized aqueous solution of ruthenium tetroxide $\left(\mathrm{RuO}_{4}\right)$ (Polysciences, Inc.) in a small thin layer chromatography jar. The copper grids with $\mathrm{CAB}$ and $\mathrm{L}$ blend sections were observed on JEOL JEM-100CX-II and those with C and $L$ sections were observed on Philips STEM EM-420 electron microscopes at an accelerating voltage of $80 \mathrm{kV}$.

\section{RESULTS AND DISCUSSION}

Table I summarizes the molecular characteristics of $\mathrm{C}$ (tricarbanilate), $C A B$, and $L$. It should be noted that $L$ is a 
relatively small molecule in comparison to $C$ and $C A B$, and its MHS-constant, a, represents random coil conformation.

I. Dynamic Viscoelastic Properties:

(a) Blended Solutions of $\mathrm{CAB} / \mathrm{L}$.

Figure 1 (a) represents the relationship between dynamic shear viscosity and concentration of blended solutions with varying amount of $\mathrm{L}$ content, at different frequencies. In these solutions, $\mathrm{CAB}$ is gradually replaced by increasing amounts of $L$ to maintain the total solids content constant at $40 \%(w / w)$. The viscosity of the solution decreases with increasing $L$ content. All the solutions exhibit shear thinning behavior.

The relationship between dynamic elastic modulus (G') and dynamic loss modulus (G'') for the blended solutions at different frequency is summarized in Figs. 1 (b) and (c), respectively. Similar to any viscoelastic material, G' and G' increase with increasing frequency. But there is a gradual drop in $G^{\prime}$ and $G^{\prime}$ ' with increasing $L$ content. These results reveal that the blended solutions are viscoelastic in nature but the amount of energy stored and dissipated per cycle of deformation decreases with increasing I content.

(b) Blended solutions of $\mathrm{C} / \mathrm{L}$.

Figure 2 (a) represents the relationship between dynamic shear viscosity and frequency for a $12 \%(w / w)$ C solution and a $12 \%(w / w)$ C solution containing $0.5 \%(w / w)$ L concentration, i.e., this amounts to $4 \%$ I of organic solute solids. The 
(a)

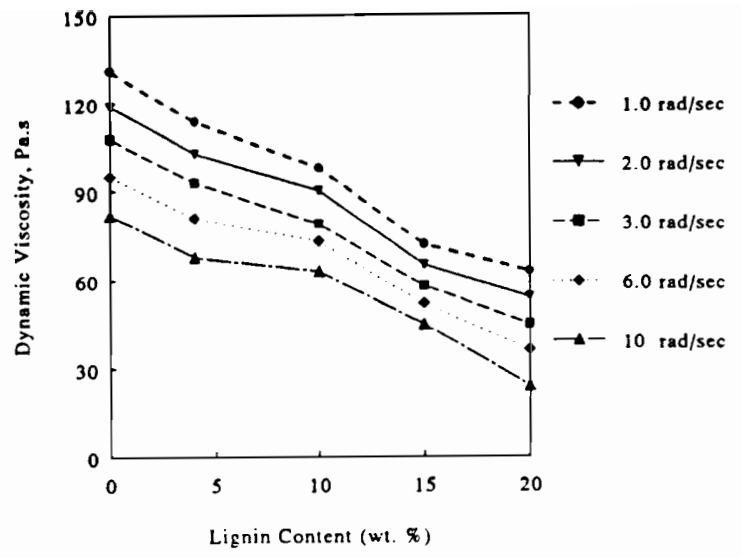

(b)

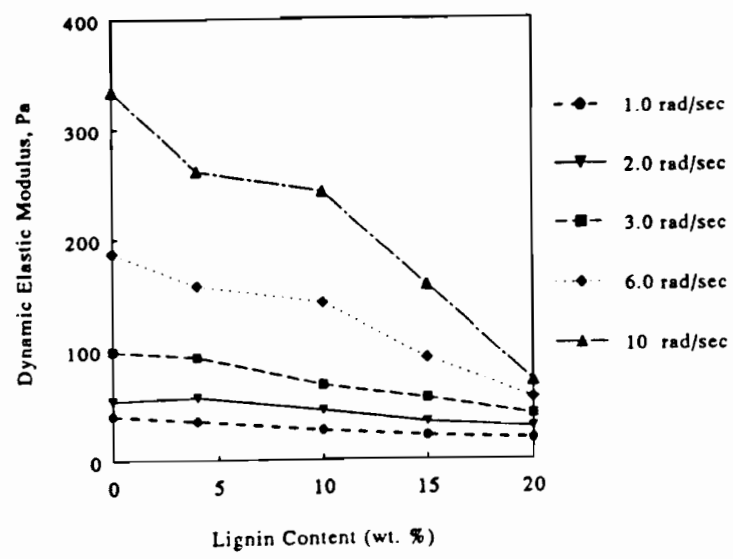

(c)

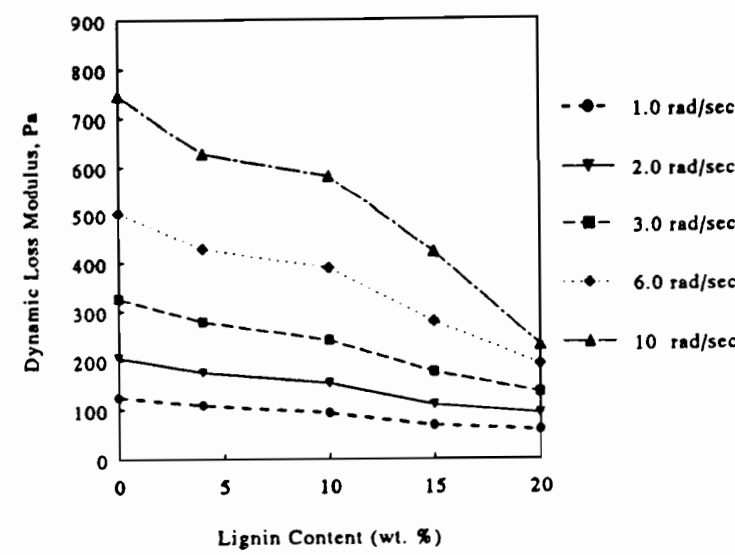

Figure 1. (a) Dynamic viscosity, (b) dynamic elastic modulus and (c) dynamic loss modulus versus concentration of $\mathrm{CAB} / \mathrm{L}$ solutions with varying amount of lignin content at different frequencies. 
(a)

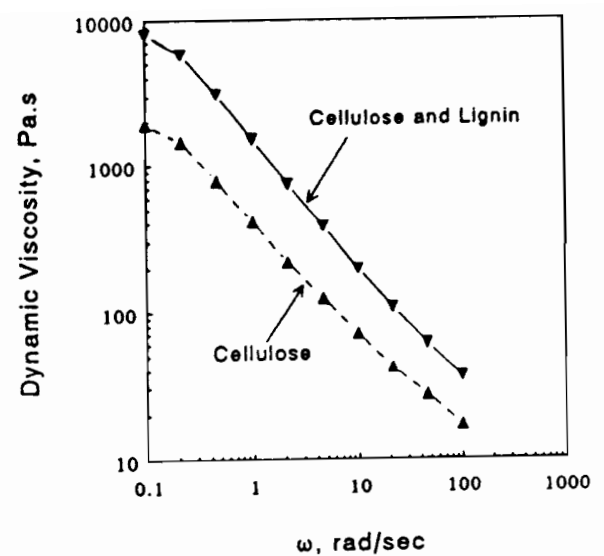

(b)

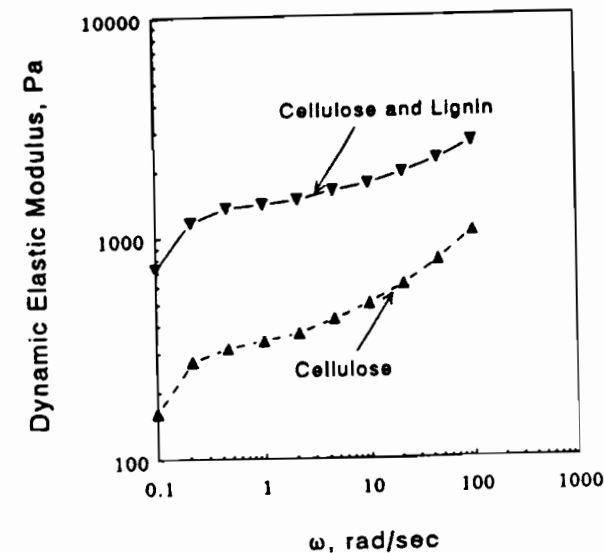

(c)

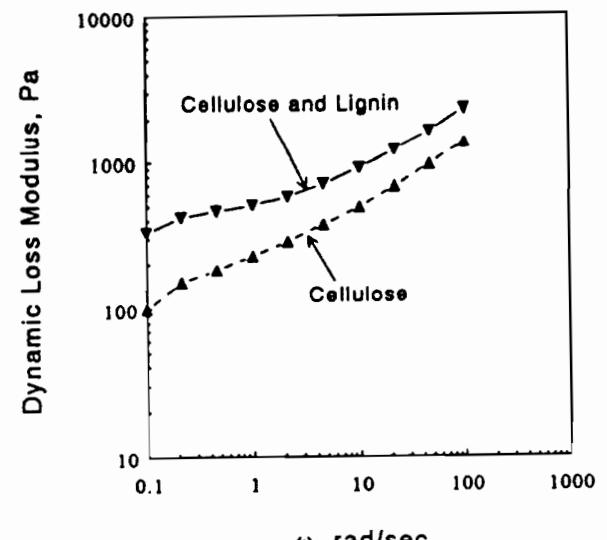

Figure 2. (a) Dynamic viscosity, (b) dynamic elastic modulus and (c) dynamic loss modulus versus frequency of $12 \%(\mathrm{w} / \mathrm{w})$ cellulose solution and $12 \%(w / w)$ cellulose solution containing $0.5 \%(w / w)$ lignin. 
viscosity of the $C$ solution containing $L$ increases unexpectedly at all frequencies.

Figures 2 (b) and (c) show the relationship between $G^{\prime}$ and $G^{\prime \prime}$ and frequency for the $C$ and $L$ solutions, respectively. The elastic and damping characteristics rise dramatically with only $0.5 \%$ (w/w) L concentration (i.e., $4 \%$ L of organic solids) in the solution. The increase in $G^{\prime}$ is greater than $G^{\prime \prime}$.

It should be noted that viscoelastic properties decreases for $C A B / L$ blends and increases for $C / L$ blends.

II. Polarized Optical Microscopy:

(a) Blended Solutions of $C A B / L$.

Figs. 3 (a) to (d) show the polarized optical micrographs of the blended solutions with $0 \%, 4 \%, 15 \%$ and $20 \%$ (w/w) L content, respectively. It is observed that the completely anisotropic solution at $40 \%(w / w)$ concentration without $L$ (Fig. 3a) gradually becomes biphasic in the presence of increasing L (Figs. 3b-d). Similar observations were reported for ternary solutions of cellulose triacetate/poly (methyl methacrylate) /trifluoroacetic acid-methylene chloride (21). Without phase diagrams, these observations remain purely qualitative. However, transmission electron microscopy of the films prepared from these $C A B / L$ solutions have shown direct evidence of cholesteric morphology (22).

(b) Blended Solutions of $\mathrm{C} / \mathrm{L}$.

Figures 4 (a) and (b) show the polarized optical micrographs of $12 \%(w / w)$ C solution and $12 \%(w / w)$ solution 


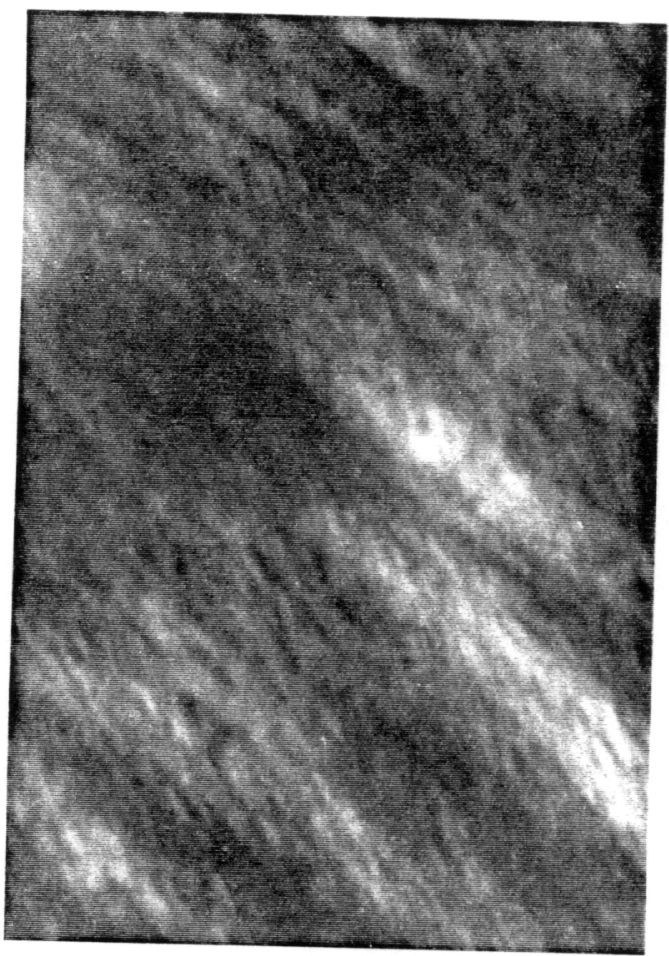

(a)

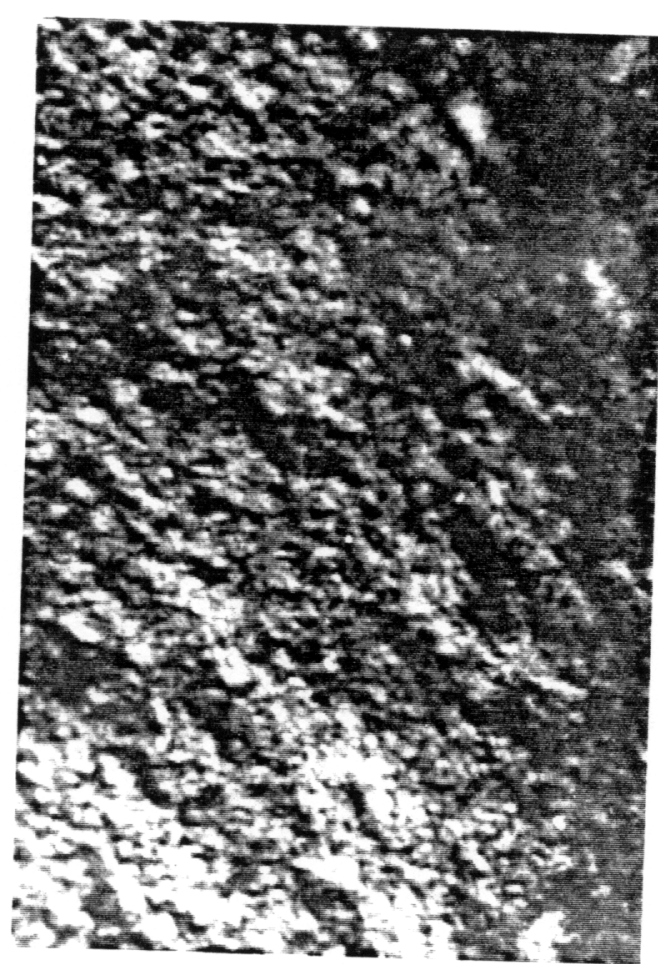

(b)

$50 \mu \mathrm{m}$

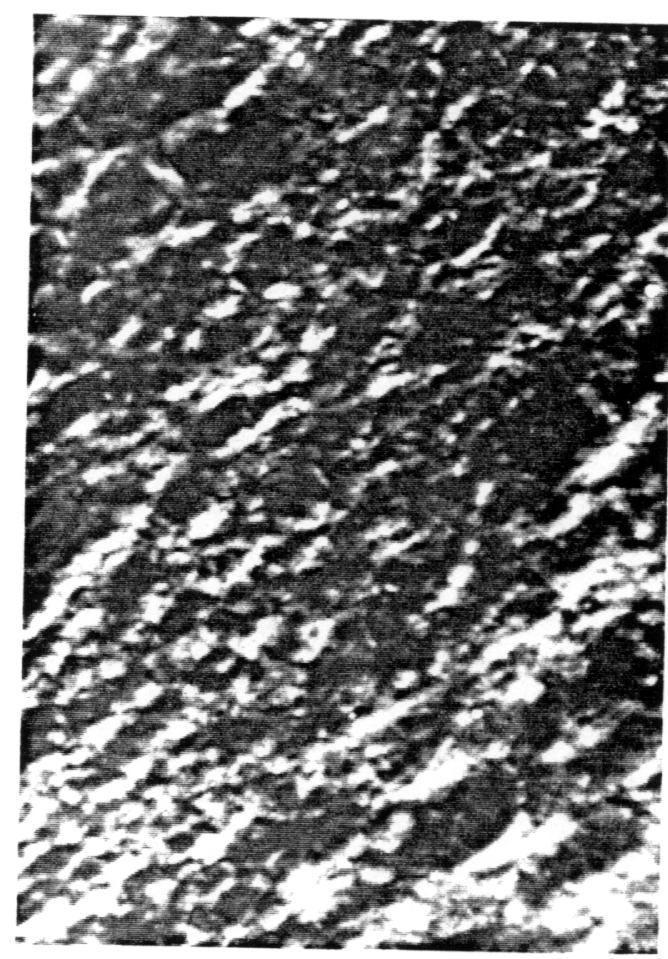

(c)

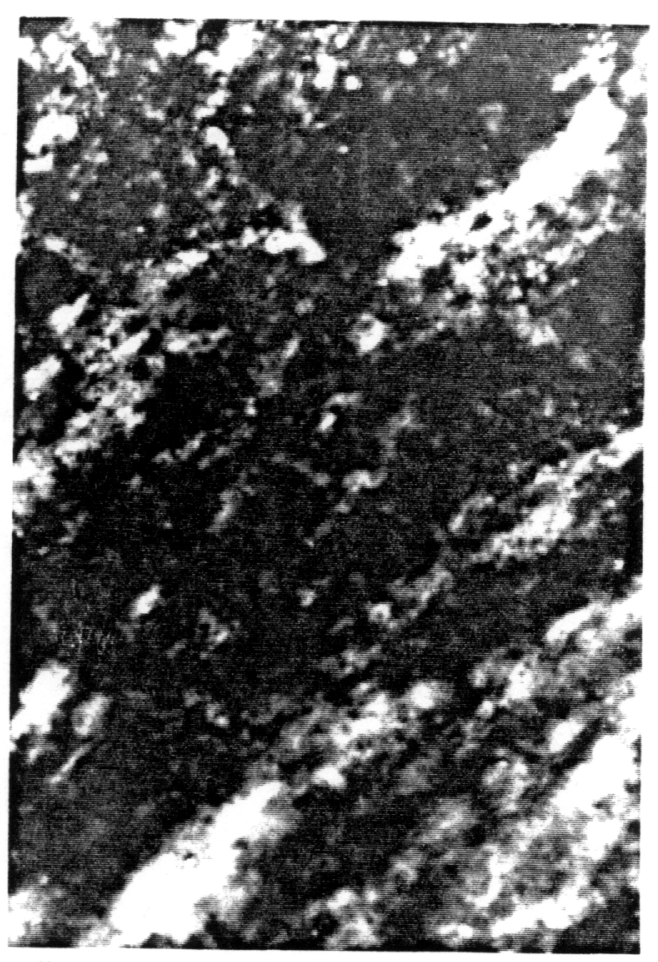

(d)

Figure 3. Polarized optical micrographs of $\mathrm{CAB} / \mathrm{L}$ solutions with varying amount of lignin content; (a) $0 \%(w / w)(b) 4 \%(w / w)$ (c) $15 \%(w / w)$ (d) $20 \%$ $(w / w)$. 
(a)

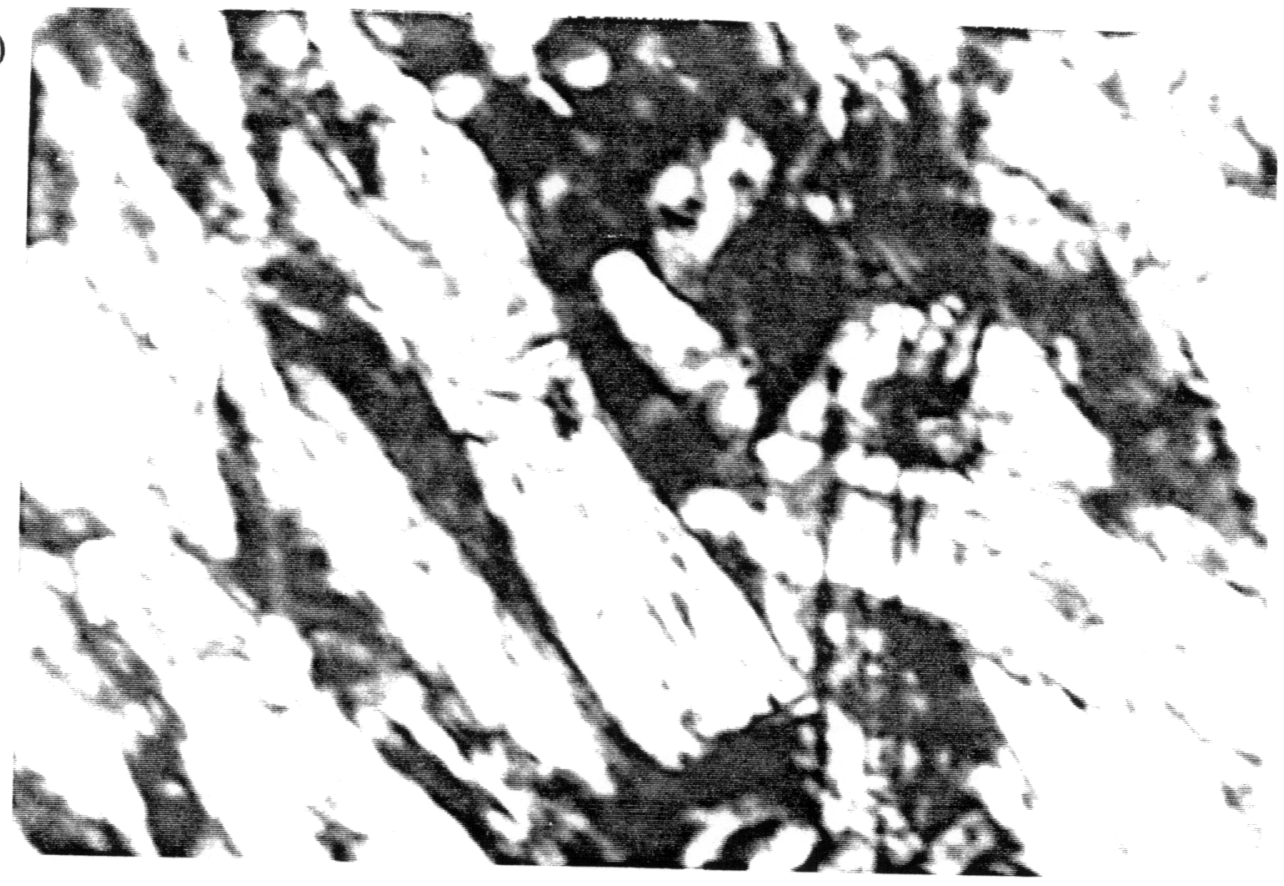

(b)

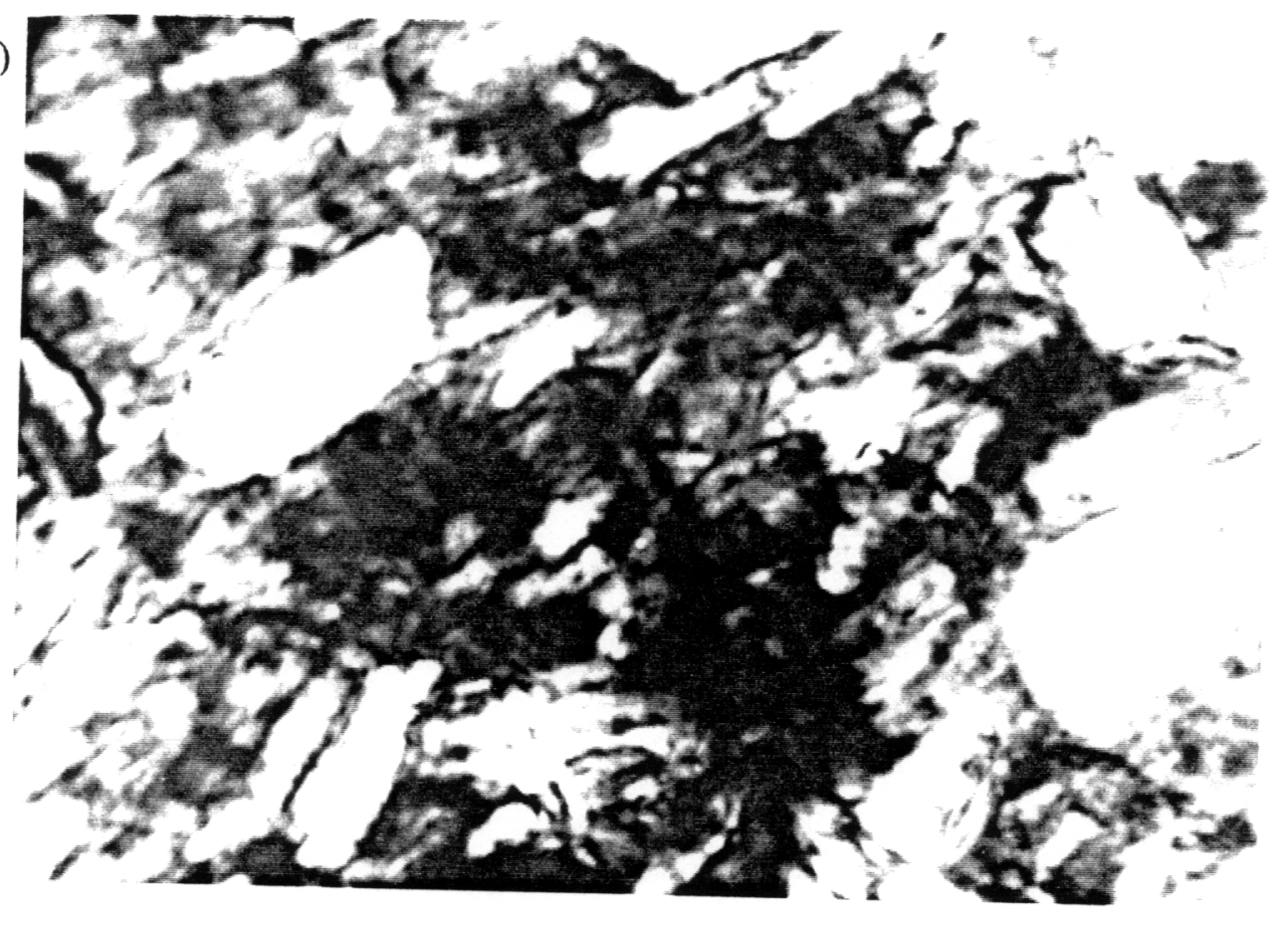

$50 \mu \mathrm{m}$

Figure 4. Polarized optical micrographs of (a) $12 \%(w / w)$ cellulose solution and (b) $12 \%(w / w)$ cellulose solution containing $0.5 \%(w / w)$ lignin. 
Table II. Spinning Conditions and Mechanical Properties of Fibers from blends of Cellulose Acetate Butyrate and Lignin's.

\begin{tabular}{|c|c|c|c|c|c|c|c|c||}
\hline $\begin{array}{l}\text { Lignin } \\
\text { Content } \\
\text { (wt.8) }\end{array}$ & Phase & $\begin{array}{c}\mathrm{V}_{\mathrm{L}}^{3)} \\
\mathrm{m} / \mathrm{min}\end{array}$ & $\begin{array}{l}\text { Draw } \\
\text { Ratio }\end{array}$ & $\begin{array}{l}\mathrm{D} \\
\mu \mathrm{m}\end{array}$ & $\begin{array}{c}\mathrm{E} \\
\mathrm{g} / \mathrm{d}^{3}\end{array}$ & $\begin{array}{l}\sigma_{\mathrm{b}} \\
\mathrm{g} / \mathrm{d}\end{array}$ & $\begin{array}{l}\varepsilon_{\mathrm{b}} \\
\text { \% }\end{array}$ & $\begin{array}{l}\mathrm{BT} \\
\mathrm{g} / \mathrm{d}\end{array}$ \\
\hline 0 & $\mathrm{~A}$ & 11 & 0.80 & 214 & 10.5 & 0.4 & 16.0 & 0.05 \\
\hline 0 & $\mathrm{~A}$ & 15 & 1.07 & 156 & 12.2 & 0.6 & 21.3 & 0.08 \\
\hline 0 & $\mathrm{~A}$ & 21 & 1.50 & 155 & 22.6 & 0.8 & 11.0 & 0.06 \\
\hline 4 & $\mathrm{~A}$ & 11 & 0.80 & 241 & 18.4 & 0.8 & 8.6 & 0.04 \\
\hline 4 & $\mathrm{~A}$ & 15 & 1.07 & 207 & 18.2 & 0.8 & 9.7 & 0.05 \\
\hline 4 & $\mathrm{~A}$ & 21 & 1.50 & 158 & 18.5 & 0.9 & 12.6 & 0.07 \\
\hline 10 & $\mathrm{~A}$ & 11 & 0.80 & 304 & 17.0 & 0.7 & 11.7 & 0.06 \\
\hline 10 & $\mathrm{~A}$ & 15 & 1.07 & 247 & 18.4 & 0.8 & 11.4 & 0.06 \\
\hline 10 & $\mathrm{~A}$ & 21 & 1.50 & 200 & 16.9 & 0.7 & 14.8 & 0.07 \\
\hline 15 & $\mathrm{~B}$ & 11 & 0.80 & 208 & 14.3 & 0.7 & 13.8 & 0.06 \\
\hline 15 & B & 15 & 1.07 & 196 & 15.2 & 0.7 & 14.6 & 0.07 \\
\hline 15 & B & 21 & 1.50 & 167 & 17.5 & 0.8 & 15.4 & 0.08 \\
\hline 20 & B & 11 & 0.26 & 190 & 20.6 & 0.6 & 9.5 & 0.05 \\
\hline 20 & B & 15 & 0.35 & 167 & 20.8 & 0.7 & 12.0 & 0.06 \\
\hline 20 & B & 21 & 0.50 & 161 & 16.6 & 0.6 & 10.5 & 0.04 \\
\hline \hline
\end{tabular}

1) The total solids content in all the solutions was maintained at a constant value of $40 \%(w / w)$ and lignin content varied from 4$20 q(w / w)$.

2) $B$ and $A$ designate biphasic and anisotropic liquid crystalline phases, respectively, by microscopy.

3) $V_{L}$ represents the velocity of the take-up roller. $V_{0}$ represents the velocity of the spinning solution in the spinnerette hole. It was kept constant at $14 \mathrm{~m} / \mathrm{min}$ except for the fibers with 20 (wt. of) lignin content where $V_{0}$ was $42.5 \mathrm{~m} / \mathrm{min}$.

4) Draw Ratio $=V_{L} / V_{0}$.

5) $1 \mathrm{GPa} \approx 8.5 \mathrm{~g} / \mathrm{d}$. 
Table III. Spinning Conditions and Mechanical properties of Fibers from blends of Cellulose and Lignin ${ }^{1)}$.

\begin{tabular}{|c|c|c|c|c|c|c|c|c|c|}
\hline $\begin{array}{l}\text { Lignin } \\
\text { Content } \\
\text { (wt. o) }\end{array}$ & $\mathrm{Ph}^{2}$ & $v_{0}$ & $V_{L}^{3)}$ & $\begin{array}{l}\text { Draw } \\
\text { Ratio }\end{array}$ & $\begin{array}{l}D \\
\mu \mathrm{m}\end{array}$ & $\underset{g / d^{g}}{E}$ & $\begin{array}{l}\sigma_{b} \\
g / d\end{array}$ & $\begin{array}{l}\varepsilon_{\mathrm{b}} \\
\xi\end{array}$ & $\begin{array}{l}\text { BT } \\
g / d\end{array}$ \\
\hline 0 & B & 21 & 15 & 0.72 & 144 & 105.1 & .61 & 1.5 & .01 \\
\hline 0 & B & 21 & 21 & 1.00 & 115 & 110.5 & .42 & 2.0 & .02 \\
\hline 0 & B & 21 & 30 & 1.43 & 103 & 144.1 & 1.1 & 2.0 & .02 \\
\hline 4 & B & 14 & 11 & 0.80 & 147 & 98.9 & .81 & 2.1 & .02 \\
\hline 4 & B & 14 & 18 & 1.30 & 100 & 91.6 & .82 & 1.3 & .02 \\
\hline 4 & B & 14 & 21 & 1.50 & 98 & 101 & .84 & 2.4 & .02 \\
\hline 4 & B & 21 & 15 & 0.72 & 110 & 81.6 & .72 & 2.9 & .02 \\
\hline
\end{tabular}

1) The fibers without lignin were spun from $12 \%$ (w/w) concentration cellulose solution. Lignin content is based on the weight of cellulose and lignin in the blended solution.

2) B designate biphasic liquid crystalline phase.

3) $V_{L}$ represents the velocity of the take-up roljer in $\mathrm{m} / \mathrm{min}$. $v_{0}$ represents the velocity in $\mathrm{m} / \mathrm{min}$ of the spinning solution in the spinnerette hole.

4) Draw Ratio $=V_{L} / V_{0}$.

5) $1 \mathrm{GPa} \approx 7.3 \mathrm{~g} / \mathrm{d}$. 
containing $0.5 \%(\mathrm{w} / \mathrm{w}) \quad \mathrm{L}$ concentration $(4 \% \quad \mathrm{~L}$ on total organics), respectively. The $\mathrm{C}$ solution is liquid crystalline in the presence of $\mathrm{L}$. However, there appears to be an increase in the isotropic region. This observation is similar to $\mathrm{CAB} / \mathrm{L}$ solutions.

\section{Processing:}

Tables II and III show spinning conditions and mechanical properties of fibers from blends of $L$ with $C A B$ and $C$, respectively. The first column represents the L content (wt. $\%$ ) in the spinning solution. The extrusion velocity, $V_{0}$, and the take-up velocity, $V_{L}$, varied from 14 to $21 \mathrm{~m} / \mathrm{min}$, and 11 to 30 $\mathrm{m} / \mathrm{min}$, respectively. The values of the shear rates corresponding to the $\mathrm{V}_{\mathrm{o}}$ values were 6220,9330 and $18880 \mathrm{~s}^{-1}$, respectively. A draw ratio of less than 1 is due to die swell. Liquid crystallinity and low viscosity improved the spinnability of $\mathrm{CAB} / \mathrm{L}$ solutions. However, fiber spinning was difficult for solutions with $20 \%(\mathrm{w} / \mathrm{w})$ lignin content due to the increase in the low-viscosity $L$ component. In spite of being liquid crystalline, high viscosity decreased the spinnability of the $C / L$ solutions.

IV. Mechanical Properties:

Figures 5 (a) and (b) show the modulus and tenacity variation with $L$ content (wt. $\%)$, respectively, for blended fibers from $C A B$ and $L$. There is an almost $80 \%$ improvement in the modulus of the fibers with only $4 \% \mathrm{~L}$ content, and this 
(a)

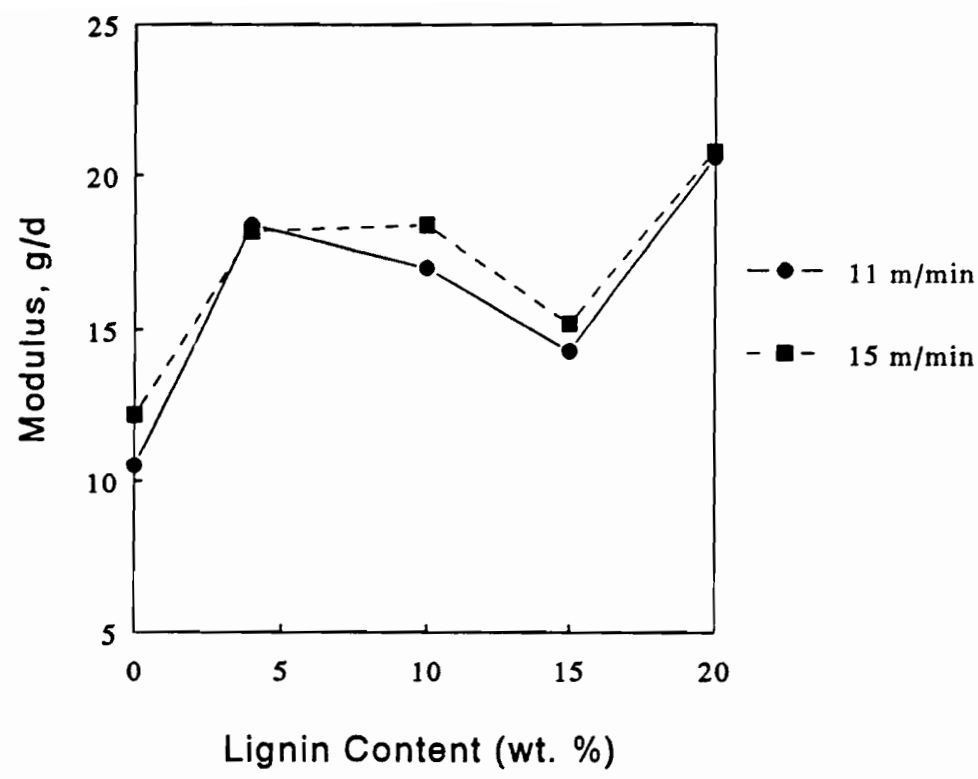

(b)

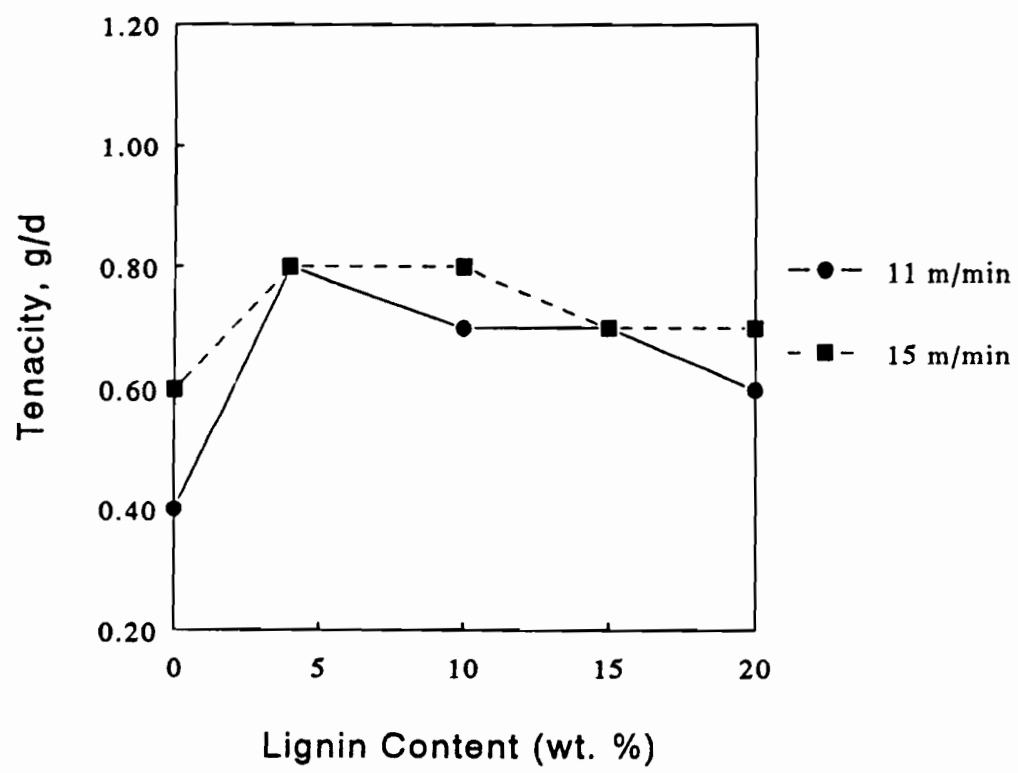

Figure 5. (a) Modulus and (b) tenacity versus lignin content (wt.\%) for $\mathrm{CAB} / \mathrm{L}$ fibers at different take-up speeds. 
remains unchanged at higher $\mathrm{L}$ contents. It is surprising to see the failure to change in fiber properties with increasing L content. L has been shown to be a "modulus-builder" in polymer blends (14). Take-up speed does not have any influence on the modulus, although the value decreases somewhat at 21 $\mathrm{m} / \mathrm{min}$ for the fibers with $\mathrm{L}$. The rise in tenacity values follows the same trend as modulus.

The modulus of blended fibers spun at nearly the same draw ratio from $C$ and $L$ declines by about $20 \%$ as compared to the fibers without $L$ (Table III). The orientation of the cellulose chains is possibly reduced due to the presence of the amorphous $I$ component. However, there is an improvement in tenacity for the blended fibers. Spinning speeds and mechanical properties of the fibers made from steam-exploded wood solutions in $\mathrm{N}$-methyl morpholine $\mathrm{N}$-oxide improved with a decrease in $\mathrm{L}$ content and a relative increase in degree of polymerization of $C(23)$.

It is surprising to note that $L$ causes the mechanical properties to increase for $\mathrm{CAB}$, and to decrease for $C$. v. Thermal Analysis:

Table IV represents the thermal analysis data of the fibers from blends of $C A B$ and $L$. Reduction in heat of fusion is observed for the blended fibers with increasing $L$ content. Therefore, the presence of an amorphous $\mathrm{L}$ component reduces the amount of crystallinity in the fibers. A glass transition temperature was not observed during the first DSC scan, and 
Table IV. Glass transition temperature $\left(T_{g}\right)$ and melting parameters $\left(T_{m}, \Delta H\right)$ of Fibers from blends of Cellulose Acetate Butyrate and Lignin determined by DSC.

\begin{tabular}{|c|c|c|c|l||}
\hline Lignin Content (wt. 8$)$ & $\mathrm{V}_{\mathrm{L}, \mathrm{m} / \mathrm{min}}$ & $\mathrm{T}_{\mathrm{f}}{ }^{\circ} \mathrm{C}$ & $\mathrm{T}_{\mathrm{m},}{ }^{\circ} \mathrm{C}$ & $\Delta \mathrm{H}, \mathrm{J} / \mathrm{g}$ \\
\hline 0 (CAB Powder) & ---- & 97 & 162 & 13.67 \\
\hline 0 & 11.0 & 103 & 148 & 13.23 \\
\hline 0 & 15.0 & 102 & 147 & 12.91 \\
\hline 0 & 21.0 & 102 & 147 & 13.09 \\
\hline 4 & 11.0 & 104 & 147 & 12.75 \\
\hline 4 & 15.0 & 106 & 147 & 12.63 \\
\hline 4 & 21.0 & 105 & 146 & 12.93 \\
\hline 10 & 11.0 & 102 & 146 & 12.38 \\
\hline 10 & 15.0 & 104 & 146 & 12.74 \\
\hline 10 & 21.0 & 104 & 146 & 12.43 \\
\hline 15 & 11.0 & 99 & 142 & 9.48 \\
\hline 15 & 15.0 & 105 & 143 & 11.27 \\
\hline 15 & 21.0 & 97 & 143 & 9.91 \\
\hline 20 & 11.0 & 100 & 143 & 10.88 \\
\hline 20 & 15.0 & 98 & 143 & 11.53 \\
\hline 20 & 21.0 & 97 & 143 & 11.28 \\
\hline 100 (Lignin Powder) & ---- & 100 & ----- & ------ \\
\hline \hline
\end{tabular}


the reported $\mathrm{T}_{\mathrm{g}}$ values are from the second scan of the melted fibers. Single glass transition temperatures and similar solubility parameters of 11.1 and $11.6\left(\mathrm{cal} . \mathrm{cm}^{3}\right)^{1 / 2}$ for $\mathrm{L}$ and $C A B$ (15), respectively, serve as evidence for miscibility.

These observations suggest that $\mathrm{L}$ and $\mathrm{CAB}$ are miscible at L contents of $\leq 20 \%$ in the fiber structure.

VI. Scanning Electron Microscopy (SEM):

Figures 6 (a) and (b) show the SEM micrographs of the surface of blended fibers of $\mathrm{CAB}$ with 15 and 20 (wt.\%) L content, respectively. Two observations can be made from these figures. First, the pore size is uniform and small ( $3-4 \mu \mathrm{m})$ for fibers with $15 \%$ L content, whereas the pore size with $20 \%$ $L$ is non-uniform and large $(-7-10 \mu \mathrm{m})$. At $0 \% \mathrm{~L}$, the fiber surface is relatively smooth with small pore size $(\sim 1-3 \mu \mathrm{m})$. The increase in porosity with $L$ content may be due to an increase in coagulation rate in the presence of $L$. Second, the roughness of the fiber surface has increased with increase in L content, but there are no indications of phase separation between $C A B$ and $L$. However, small particles of $\mathrm{L}$ are detected on the fiber surface with $20 \%$ L content [see arrow in Fig. 6 (b)]. The presence of $L$ did not show any different morphological features in blended fibers from $C$ and $L$. VII. Transmission Electron Microscopy (TEM):

Further evidence of phase mixing between $C A B$ and $C$ with $L$ is attained by TEM. Figures 7 (a) and (b) show the skin and 


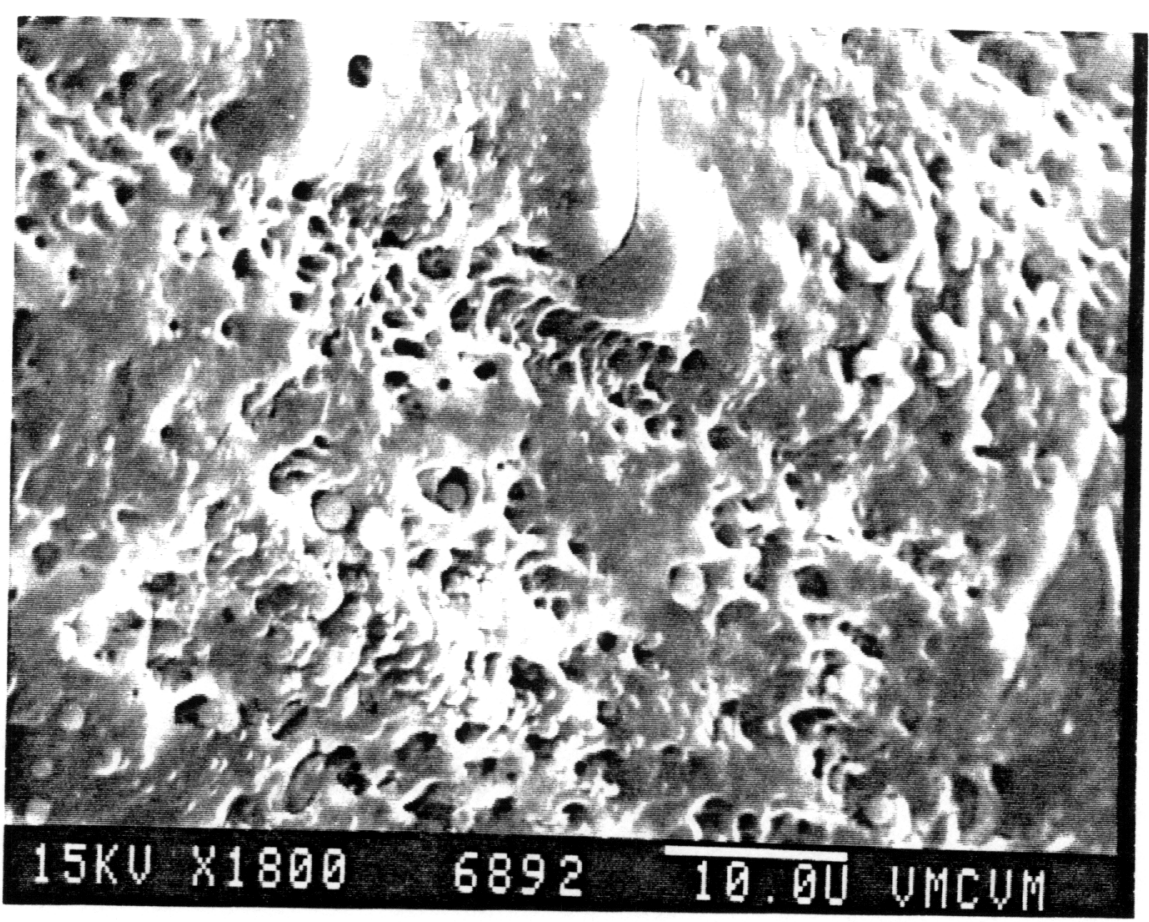

(a)

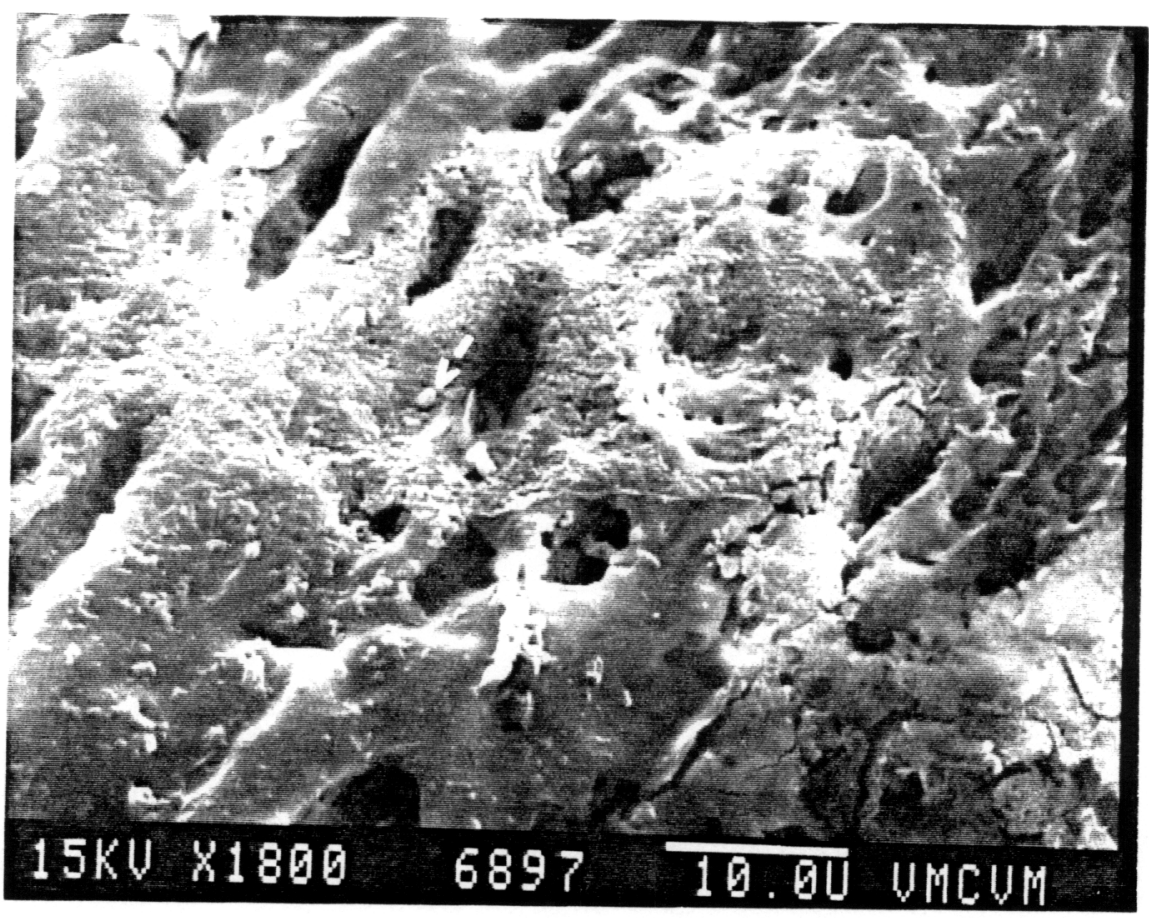

(b)

Figure 6. Scanning electron micrographs of the surface of CAB/L fibers with varying amount of lignin content; (a) $15 \%(w / w)$ (b) $20 \%(w / w)$. 


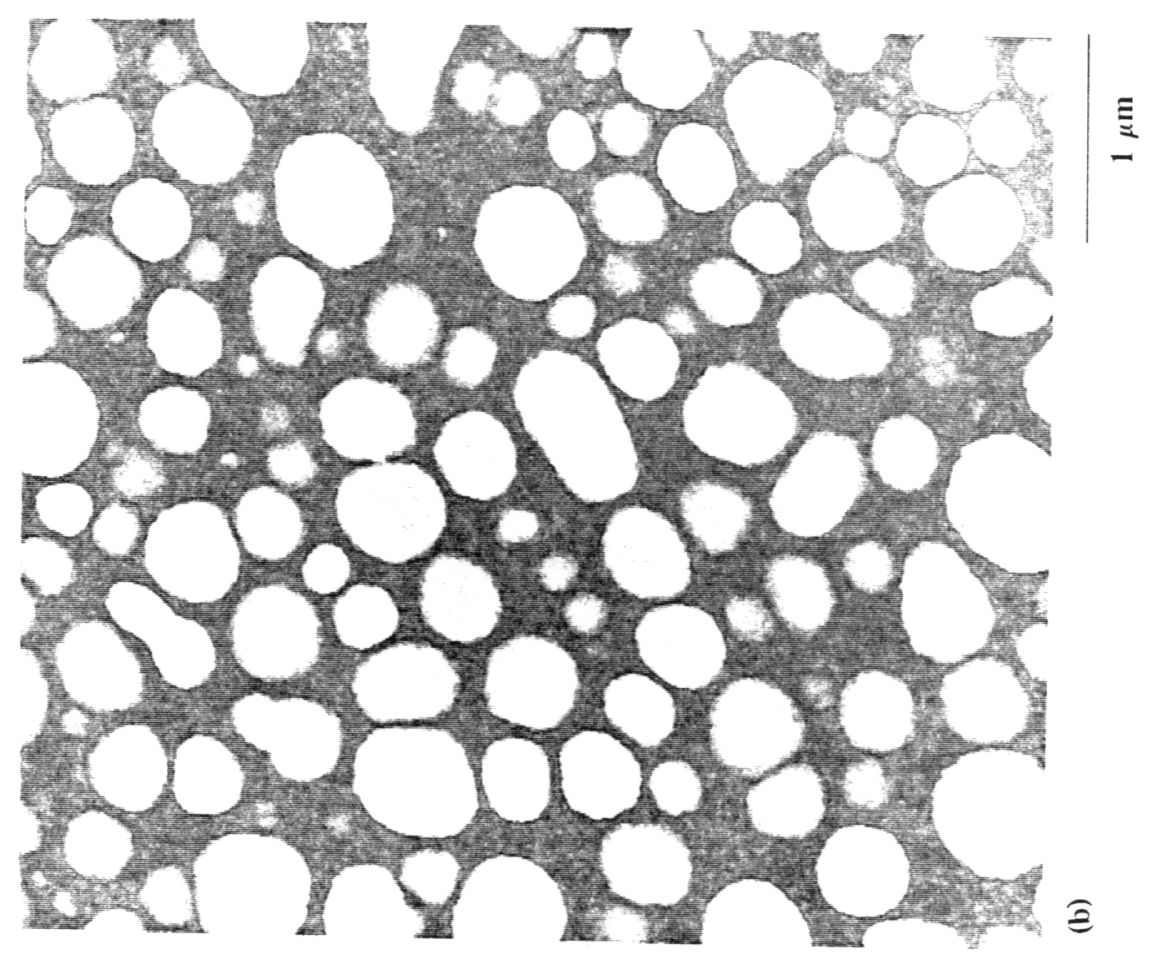

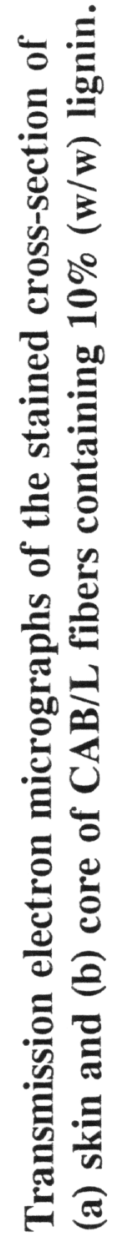

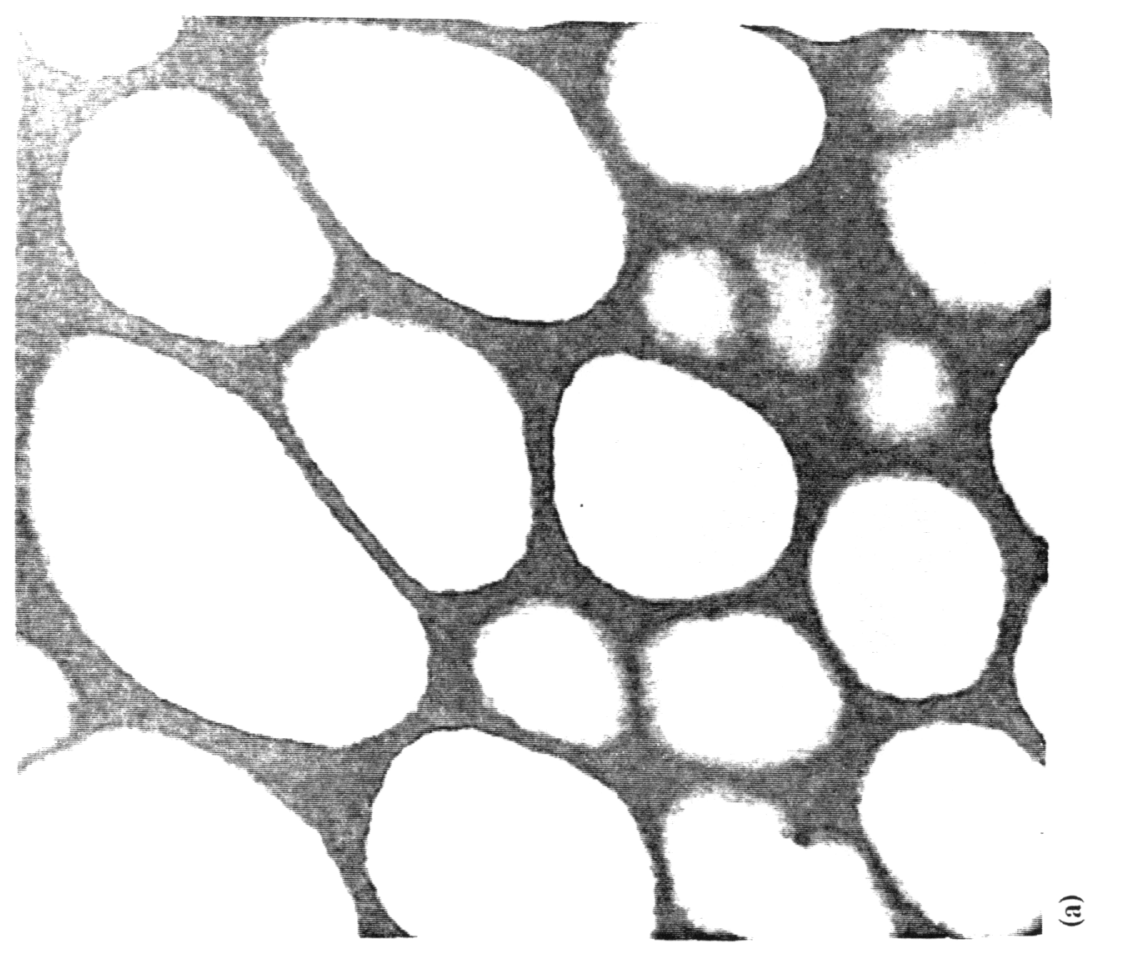


core of fiber cross-sections, respectively, of CAB with $10 \% \mathrm{~L}$ content stained by $\mathrm{RuO}_{4}$. The maximum pore size of the outer skin is $\sim 1-2 \mu \mathrm{m}$ and that of the core is less than $1 \mu \mathrm{m}$. This difference in skin and core morphology of the fibers is due to the solidification process during coagulation. SEM and TEM results indicate that the size of the pores on the fiber surface increases with increasing $L$ content. Total area of the pores in the core of all fibers was approximately $30 \%$ as determined by digital imaging analysis. The continuous (dark) matrix represents the blended $\mathrm{CAB}$ and $\mathrm{L}$ morphology, and implies considerable phase mixing. similar morphology was observed for all the fibers with varying amount of $L$ content.

Fig. 8 shows the TEM of the stained cross-section of $C$ fibers containing $4 \% \mathrm{~L}$. These fibers did not show skin-core effect and were devoid of pores. The figure shows that I domains $(\langle 1 \mu \mathrm{m})$ were well dispersed in the $\mathrm{C}$ matrix. However, some regions of the fibers had aggregates $(1-3 \mu \mathrm{m})$ of the $\mathrm{L}$ phase, as shown by the arrows. This demonstrates heterogenous distribution of $L$ in the $C$ matrix and represents a partially mixed system. TEM of the steam-exploded wood fibers had shown a homogenous structure with microdomains of $L$ and $C$ in the range of $10-20 \mathrm{~nm}(23)$.

Electron microscopy results suggest that the fibers from $C A B / L$ are significantly phase mixed and those from $C / L$ are partially phase mixed. 


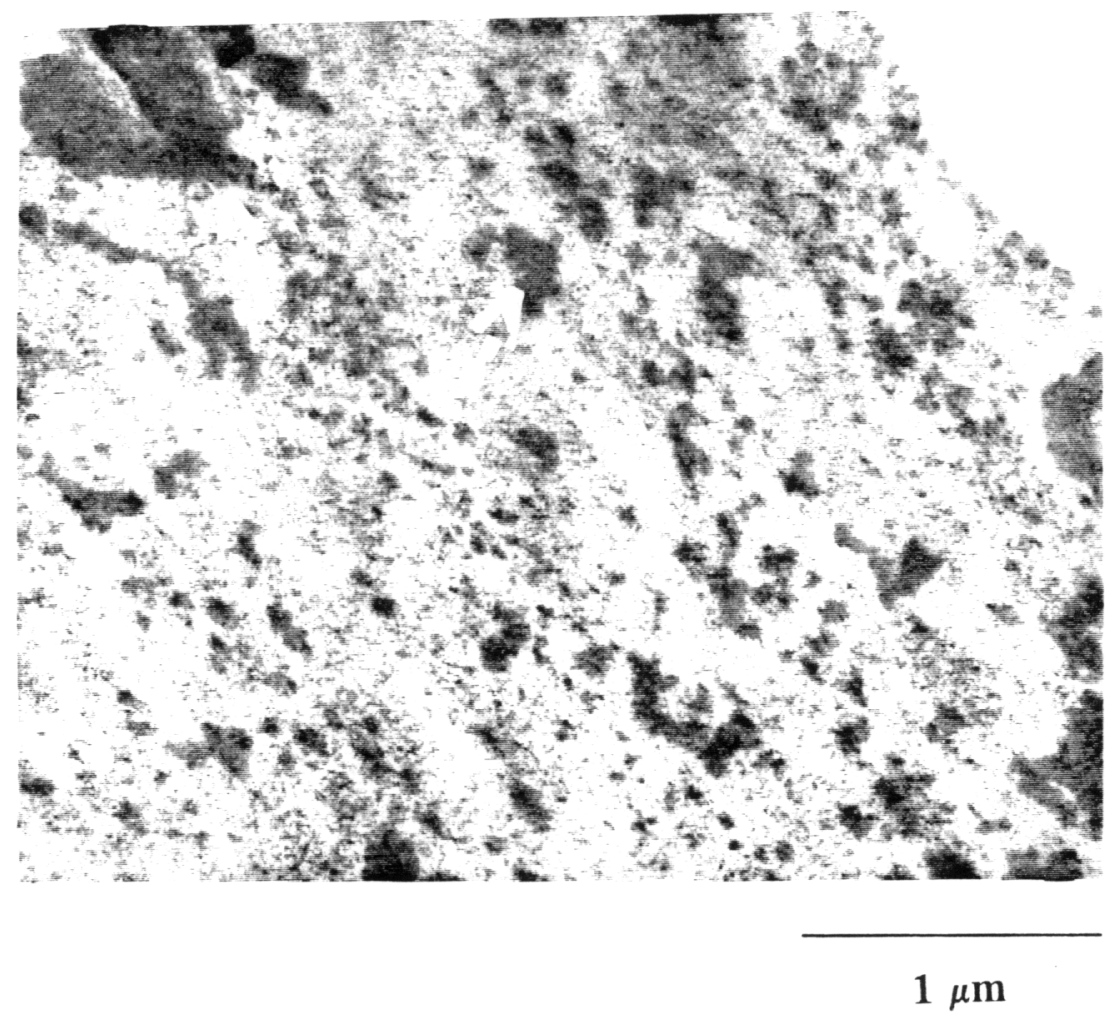

Figure 8. Transmission electron micrograph of the stained cross-section of $\mathrm{C} / \mathrm{L}$ fibers containing $4 \%$ (w/w) lignin. 
$L$ is serving as a plasticizer in the $C A B / L$ blends, as the viscosity drops with increasing $L$ content. In contrast, the viscosity increases in the $\mathrm{C} / \mathrm{L}$ blends. This increase can be attributed to either hydrogen bonding between $L$ and $C$, or to the presence of aggregates of $\mathrm{L}$ molecules in the $\mathrm{C}$ solution. There is no evidence at the moment for the former possibility. The aggregated L phase, as observed in TEM of $\mathrm{C} / \mathrm{L}$ fibers, can increase the viscosity of the solution. Therefore, the latter possibility seems to be a reasonable hypothesis.

The modulus and tenacity of the fibers from $C A B / L$ solutions improved with lignin content. This may be due to the orientation of $\mathrm{L}$ and $\mathrm{CAB}$ molecules during spinning. A single $\mathrm{T}_{\mathrm{g}}$ was observed for all the $\mathrm{CAB} / \mathrm{L}$ fibers. Earlier work on the films of $C A B / L$ blends had also demonstrated single $T_{g}$ transitions up to $\leq 20 \% \mathrm{~L}$ content by DSC (15). This is good indication of miscibility in $\mathrm{CAB} / \mathrm{L}$ fibers. There are no signs of phase separation from SEM and TEM results, and this suggests that $C A B / L$ fibers are miscible. The improvement in the mechanical properties of $\mathrm{CAB} / \mathrm{L}$ fibers can be attributed to high levels of mixing with good interfacial adhesion between the two components.

It can be inferred from the optical microscopy results of $\mathrm{CAB} / \mathrm{L}$ and $\mathrm{C} / \mathrm{L}$ that $\mathrm{L}$ forms the isotropic phase. Fibers spun from these solutions are expected to phase separate due to reduced interaction between the amorphous $\mathrm{L}$ phase and the 
liquid crystalline $C A B$ or $C$ phase. However, from electron microscopy results, $L$ is well distributed in the $C A B / L$ and $C / L$ blends even at low weight fractions, i.e. 4\%. Usually for capillary flow, like spinning, lower viscosity minor component (L) would tend to disperse and encapsulate the higher viscosity major component ( $\mathrm{CAB}$ or $\mathrm{C}$ ) (24). Rearrangement and phase separation of the blend components with different viscosities is possible during spinning. Since rearrangement is a kinetic process, it usually does not occur during wet spinning due to the immediate coagulation of the solution. This enhances the individual blend component to be well distributed as shown for $C A B / L$ fibers. Fiber spinning is, therefore, a good route to producing blends with significant phase mixing. 


\section{CONCLUSIONS}

1. Dynamic viscoelastic solution properties decreases for $\mathrm{CAB} / \mathrm{L}$ blends and increases for $\mathrm{C} / \mathrm{L}$ blends.

2. Polarized optical microscopy results demonstrate that the blended solutions of $\mathrm{CAB} / \mathrm{L}$ and $\mathrm{C} / \mathrm{L}$ are liquid crystalline.

3. SEM and TEM provide adequate evidence for concluding that the pore size on the fiber surface of $\mathrm{CAB} / \mathrm{L}$ blends increases with increase in $\mathrm{L}$ content.

4. Modulus and tenacity of the fibers from $C A B / L$ blends improve in the presence of $L$. This is due to the formation of blends with good phase mixing as observed by SEM and TEM. The high rate of the coagulation process during fiber spinning enhances the phase mixing.

5. Mechanical properties of $\mathrm{C} / \mathrm{L}$ fibers decline with $\mathrm{L}$ content; and they form blends with partial phase mixing. 


\section{REFERENCES}

1. D. J. Walsh, J. S. Higgins, and A. Maconnachie, Polymers, Blends and Mixtures, NATO ASI Series, Applied Sciences No. 89, Martnus Nijoff: Dordrech, 1985.

2. D. R. Paul and S. Newman, Polymer Blends, Academic Press, New York, Vol. 1, 1978.

3. O. Olabisi, L. M. Robeson and M. T. Shaw, Polymer-Polymer Miscibility, Academic Press, New York, 1979.

4. Z. Ophir and Y. Ide, Polym. Engr. Sci., 23 (14), 792, 1983 .

5. E. Joseph, G. L. Wilkes and D. G. Baird, Polym. Engr. Sci., 25 (7), 377, 1985.

6. E. Suokas, J. Sarlin and P. Tormala, Mol. Cryst. Liq. Cryst., 153, 515, 1987.

7. A. Pirnia and C. S. P. Sung, Macromolecules, 21, 2669, 1988 .

8. P. G. Hedmark, J. M. R. Lopez, M. Westdahl, P. -E. Werner, J. -F. Jansson and U. W. Gedde, Polym. Engr. Sci., 28 (19), 1248, 1988.

9. P. J. Flory, Adv. Polym. Sci., 59, 1, 1984 .

10. S. M. Aharoni, Polymer, 21, 21, 1980.

11. E. Bianchi, A. Ciferri and A. Tealdi, Macromolecules, 15, 1268,1982 .

12. E. Marsano, E. Bianchi and A. Ciferri, Macromolecules, $17,2886,1984$. 
13. S. Ambrosino, T. Khallala, M. J. Seurin, A. Ten Bosch, F. Fried, P. Maissa and P. Sixou, J. Polym. Sci.: Part C: Polym. Letts., 25, 351, 1987.

14. T. G. Rials and W. G. Glasser, J. Appl. Polym. Sci., 37 , $2399,1989$.

15. T. G. Rials and W. G. Glasser, wood and Fiber Sci., 21 (1), 80, 1989 .

16. V. Davé, W. G. Glasser and G. L. Wilkes (To Be Published)

17. V. Davé and W. G. Glasser (To Be Published).

18. V. Davé, J. Wang, W. G. Glasser and D. Dillard (To Be Published).

19. R. Evans and A. F. A. Wallis, Fourth Int. Symp. on Wood and Pulp. Chem., Paris, 1, 201, 1987.

20. V. Davé and W. G. Glasser, in viscoelasticity of Biomaterials, ACS Symp. Ser., W. G. Glasser and H. Hatakeyama, Eds., (In Press).

21. Y. K. Hong, D. E. Hawkinson, E. Kohout, A. Garrard, R. E. Fornes and R. D. Gilbert, in Polymer Association structures, ACS Symposium Series 384 , A. M. El-Nokaly, Ed. , 184, 1989 .

22. V. Davé, W. G. Glasser and G. L. Wilkes (To Be Published) 
23. H. Chanzy, M. Paillet, A. Peguy and R. Vuong, in wood and Cellulosics: Industrial Otilisation, Biotechnology, structure and Properties, J. F. Kennedy, G. O. Phillips and P. A. Williams, Eds., Ellis Horwood Ltd., Ch. 63, 573,1987 .

24. D. R. Paul, in Polymer Blends, Vol. 2, Ch. 16, 167, 1978. 
7.0 EVIDENCE OF CHOLESTERIC MORPHOLOGY IN FILMS OF CELLULOSE ACETATE BUTYRATE BY TRANSMISSION ELECTRON MICROSCOPY. 


\title{
EVIDENCE FOR CHOLESTERIC MORPHOLOGY IN FILMS OF
}

\author{
CELLULOSE ACETATE BUTYRATE BY
}

\section{TRANSMISSION ELECTRON MICROSCOPY.}

\begin{abstract}
Transmission electron microscopy provides direct evidence of helicoidal cholesteric morphology in films of cellulose acetate butyrate prepared by solvent casting from liquid crystalline solutions in dimethylacetamide. This observation of cholesteric structure reported here, we believe, is the first time for mixed esters of cellulose. Cholesteric morphology was also observed in solvent cast blended films of cellulose acetate butyrate and lignin from liquid crystalline solutions. There are indications that lignin particles may be serving as nucleating surfaces or sites of direct termination for some of the observed fingerprint pattern.
\end{abstract}




\section{INTRODUCTION}

Extensive attempts have been made to prepare polymeric films with cholesteric order by different techniques (1-3). since the first observation of cholesteric liquid crystalline textures in a solution of hydroxypropyl cellulose [HPC] (4), the field of cellulosic liquid crystals has expanded rapidly, and there have been numerous reports of lyotropic and thermotropic liquid crystalline systems with cellulose and its derivatives (5-7). The semi-rigid character of the cellulose backbone is responsible for the development of mesophases in these materials ( 8 ). Besides scientific pursuit, one primary motivation to study cellulosic liquid crystalline systems has been the potential of industrially preparing high strength/ high modulus regenerated fibers.

In this paper, we present the first evidence for the cholesteric structure in films of cellulose acetate butyrate $(C A B)$, and in blended films of $C A B$ and lignin prepared from liquid crystalline solutions by transmission electron microscopy (TEM) .

\section{EXPERIMENTAL}

\section{Materials.}

Cellulose acetate butyrate ( $C A B$ 500-5) was obtained from Eastman Kodak, Kingsport, TN. The degree of substitution (DS) of acetyl $\left(D_{\mathrm{AC}}\right)$ and butyryl (DS $\left.\mathrm{BU}\right)$ groups was 0.29 and 2.57, respectively. $\mathrm{DS}_{\mathrm{OH}}$ was determined by difference $\left[\mathrm{DS}_{\mathrm{OH}}=3\right.$ - 
$\{0.29+2.57\}=0.14\}$. Number average molecular weight $\left(\left\langle\mathrm{M}_{n}\right\rangle\right)$, polydispersity, and intrinsic viscosity (I.V.) were 52,600 $\mathrm{gm} / \mathrm{mole}, 2.4$, and $1.51 \mathrm{dL} / \mathrm{g}$, respectively.

Unmodified organosolv lignin was supplied by Aldrich (Cat. No. $37,101-7$ ). Its $\left\langle M_{n}\right\rangle$, polydispersity, and I.V. were $800 \mathrm{gm} / \mathrm{mole}, 4.4$, and $0.05 \mathrm{dL} / \mathrm{gm}$, respectively.

Reagent grade dimethylacetamide (DMAC) was used as received.

II. Methods:

1. Film Preparation:

Since we have found by rheological investigations that this particular $C A B$ behaves as a liquid crystalline solution in DMAC at $35 \%(\mathrm{w} / \mathrm{w})$ concentration $(9)$, the total solids content in all the solutions was maintained at a constant value of $40 \%(\mathrm{w} / \mathrm{w})$ and the lignin content varied from 0 to $20 \%$ (w/w) of organic solutes. Individual component solutions of dried $C A B$ and lignin were prepared in DMAC. These were mechanically mixed for 12 hours at ambient temperature to prepare blended liquid crystalline solutions. The solutions were allowed to equilibrate for 1 week.

Portions of the viscous solutions were poured on to Teflon molds and a glass rod was gently pushed over the solutions to form thin films. Films from sheared solutions were prepared by simply applying a unidirectional force using a glass slide. The molds were covered by glass plates and the 
solvent was allowed to evaporate at ambient conditions for 5 days. The resulting solid films were removed from the molds, and dried in a vacuum oven at $60^{\circ} \mathrm{C}$ for 48 hours to remove residual solvent. Amorphous films were prepared by heating the dried films in a hot press and quenching them from the melt state.

2. Polarized Optical Microscopy:

The films were observed between cross polarizers for birefringence with a Zeiss Axioskop Microscope at room temperature.

3. Transmission Electron Microscopy:

Films were embedded in Poly/Bed 812 (Polysciences, Inc.) cured at $60^{\circ} \mathrm{C}$ for 48 hours in flat molds. $80-90 \mathrm{~nm}$ thick sections were cut across the cross-section and longitudinalsection from the embedded films with a diamond knife mounted on a Reichert Ultracut E microtome. Care was taken to cut sections from different layers of the films. All the cut sections were mounted on copper grids. In some cases, staining was carried out for 30 minutes by exposing some of the copper grids to vapors of $0.5 \%$ stabilized aqueous solution of ruthenium tetroxide $\left(\mathrm{RuO}_{4}\right)$ (Polysciences, Inc.) in a small thin layer chromatography container. The copper grids were observed on a JEOL JEM-100CX-II electron microscope operated at an accelerating voltage of $80 \mathrm{kV}$. All the micrographs were bright field images of the cut sections. 


\section{RESULTS AND DISCUSSION}

Figure 1 shows the optical micrograph of the CAB film containing $20 \%(\mathrm{w} / \mathrm{w})$ lignin observed between cross polarizers. There are indications of molecular orientations similar to helicoidal arrangements characteristic of cholesteric liquid crystals. Since the cholesteric pitch is equal to or shorter than $1 \mu \mathrm{m}$, it is difficult to define the features of the pattern with visible light. Therefore, further evidence for cholesteric morphology was obtained by electron microscopy.

Freeze-fractured surfaces of the same film were observed by scanning electron microscopy (SEM), but there was no clear indication of any structural layering. Similar difficulty in obtaining useful structural detail by SEM was also encountered by Hara et al. (10) for cholesteric mesophases of thermotropic copolyesters.

Figure 2 (a) shows the TEM micrograph of the unstained cross-section of the same film. A well-ordered cholesteric arrangement is revealed with a periodicity ranging from 500$900 \mathrm{~nm}$ which is in good correspondence with the optical microscopy observations. Films cut in the longitudinal-section showed analogous patterns. Similar cholesteric morphology was also seen in the $C A B$ films without lignin. However, the cholesteric structure is more pronounced in presence of lignin. It is not surprising to observe the twisted cholesteric order in the CAB films as comparable observations 


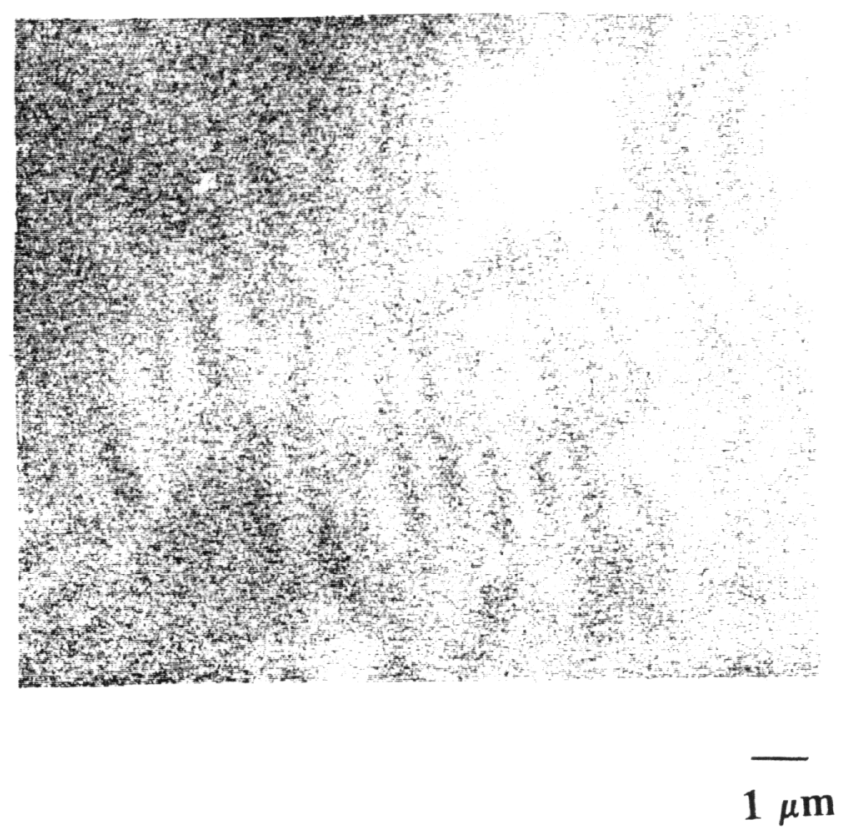

Figure 1. Polarized optical micrograph of the CAB film containing $20 \%(\mathrm{w} / \mathrm{w})$ lignin. 


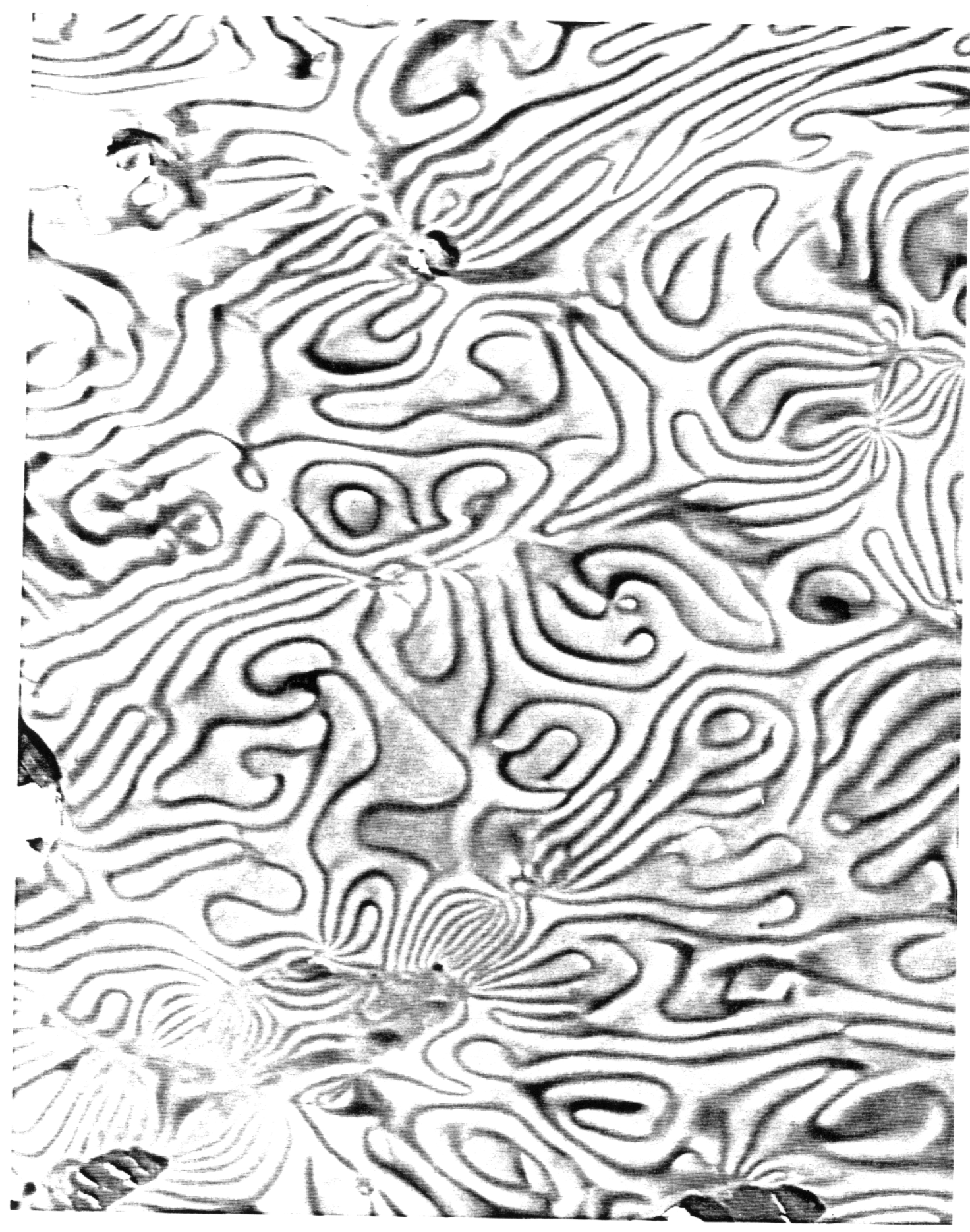

(a)

$1 \mu \mathrm{m}$

Figure 2. (a) Transmission electron micrograph of an unstained cross-section of the CAB film containing $20 \%$ (w/w) lignin. 
have been made by TEM for solvent cast films from nonlignin containing liquid crystalline solutions of cellulose acetate [CA] and cellulose triacetate [CTA] (11). However, their structures were more tightly arranged with periodicity of about 25-45 nm. The bulky butyryl side-chain substituent in $C A B$ may prevent the molecules to organize in a compact manner, and this may eventually lead to a wider periodicity between the striation lines. TEM observations of the sheared and amorphous lignin containing films did not exhibit the mesophase structure. This is due to the permanent disruption of the structure by shearing and thermal treatment.

The cholesteric structures of thermotropic copolyesters exhibited in the study of Hara et al. (10) are comparable to those reported in this paper. They determined the cholesteric pitches (200-500 nm) by circular dichroism (CD) and reflection spectra which correlated well with their TEM observations. CD measurements were not conducted in our study as these measurements were unable to indicate the pitch of the cholesteric structure in CA and CTA films (11).

Giasson et al. (11) had no explanation for the cholesteric structures observed in their study. Hara et al. (10) interpreted their structures to represent typical disclinations $(+\pi$ and $-\pi)$ and translation dislocations. In our study, $+\pi$ disclinations (mostly $\lambda^{+}$-type) were observed more frequently than $-\pi$ disclinations (mostly $\lambda$-type). Translation 
dislocations (mostly $\tau \cdot \lambda^{+}$) were also observed occasionally. The cholesteric liquid crystalline texture we observe is represented by well-defined twisted structures. This type of pattern exhibits certain regularity in electron density which corresponds to the helicoidal supramolecular organization typical of a cholesteric mesophase. In regions with highest contrast, a near regular spacing is observed as the lines are arranged perpendicular to the electron beam. However, in regions with lower contrast, a broad or irregular spacing is noticed as the lines are obliquely organized to the incident electron beam.

It should be noted that the distance (pitch) between the dark striation lines varies in some portions of the film. The variation in pitch does not appear to be influenced by induced strains during the cutting process of the films. Pitch is affected by inherent viscosity or molecular weight of the polymer (10). Higher inherent viscosity corresponds to larger pitch or a smaller twist angle. The intrinsic viscosity of $C A B$ used in our investigation is higher than the inherent viscosity of most of the cholesteric copolyesters utilized by Hara et al. (10). This may be the cause for wider pitch in the structures observed in our study.

Figure 2 (b) shows the TEM of an unstained longitudinalsection of the film containing $20 \%$ (w/w) lignin. Banded texture is seen to originate from the surface of the lignin 


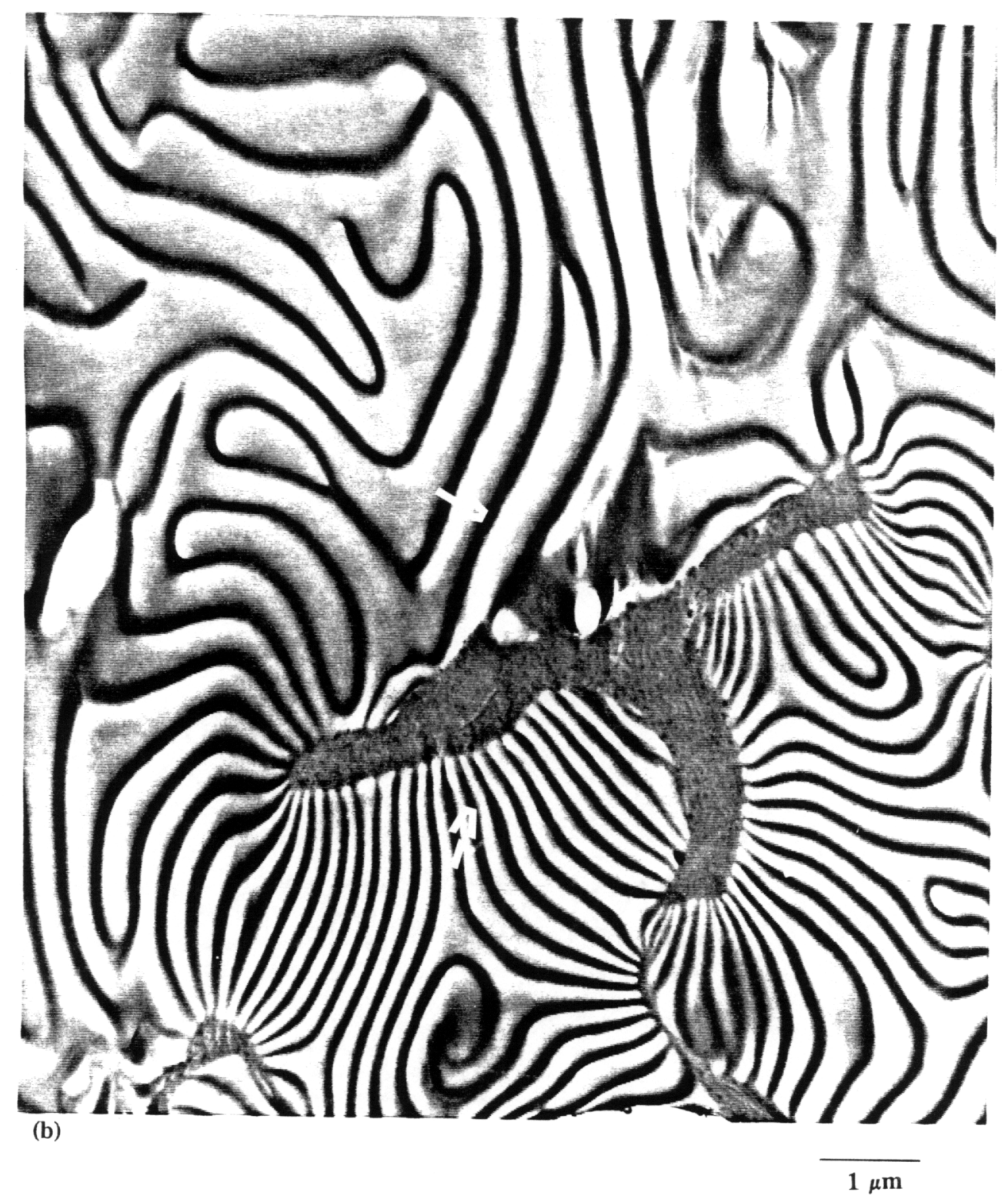

Figure 2. (b) Transmission electron micrograph of an unstained longitudinalsection of the CAB film containing $20 \%$ (w/w) lignin. 
particle which suggests that the occurrence of this particulate phase may serve as a nucleating or terminating surface for the observed ordered structure. We do not have direct evidence for this conjecture since there are other striation lines which do not commence from lignin. This ascertains that lignin is not responsible for the cholesteric morphology. It should be noted that the pitch between the dark lines is a function of closeness to the lignin particle (see arrows) .

The cholesteric structure is not consistently observed in all the regions as these $C A B / l i g n i n$ films are macrophase separated. This structure, due to possible disruption, is not present in regions where there is a high concentration of lignin particles. Therefore, this pattern occurs primarily due to the solidified liquid crystalline order from the $C A B$ solution. This is the first time a cholesteric morphology is observed for cellulose acetate butyrate.

Figure 3 shows the TEM of the stained cross-section of the film containing $20 \%(\mathrm{w} / \mathrm{w})$ lignin. While there is some increase in phase contrast due to staining by $\mathrm{RuO}_{4}$, it is apparent from the previous figure (Fig. 2) that staining is not needed to allow observance of the cholesteric texture. 


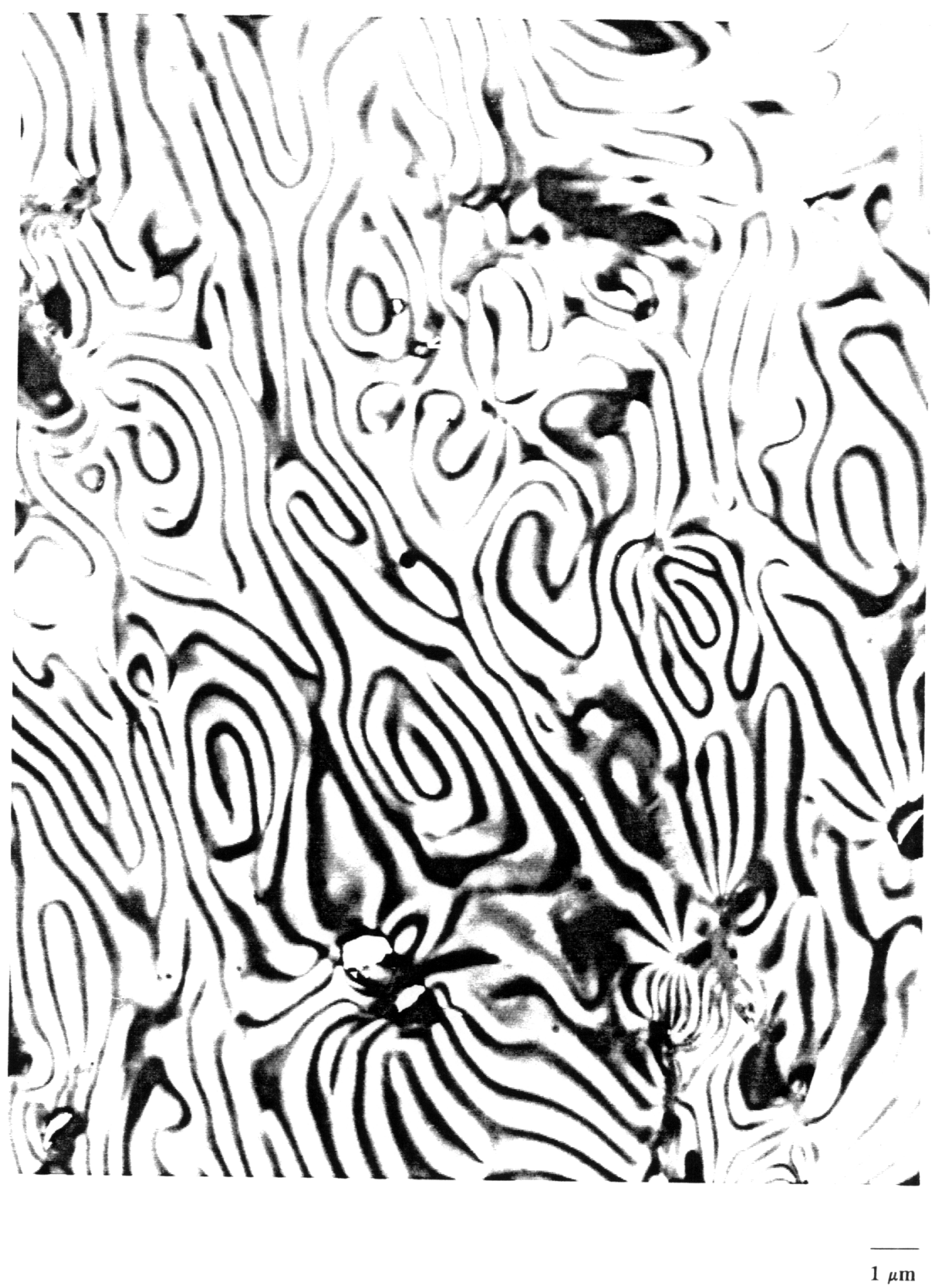

Figure 3. Transmission electron micrograph of a stained cross-section of the $C A B$ film containing $20 \%(w / w)$ lignin. 


\section{REFERENCES}

(1) W. R. Krigbaum, A. Ciferri, J. Asrar, and H. Toriumi, Mol. Cryst. Lia. Cryst., 76, 79, 1981.

(2) H. Finkelmann and G. Rehage, Makromol. Chem. Rapid Commun., 1, 733, 1980 .

(3) P. Shannon, Macromolecules, 17, 1873, 1984.

(4) R. S. Werbowyz and D. G. Gray, Mol. Cryst. Liq. Cryst., $34,97,1976$.

(5) D. G. Gray, J.Appl. Polym. Sci., Appl. Polym. Symp., 37 , $179,1983$.

(6) R. D. Gilbert and P. A. Patton, Progr. in Polym. Sci., 9 $(2 / 3), 115,1983$

(7) R. D. Gilbert, in ACS Symp. Ser. No. 433, 22, 259, 1990.

(8) D. G. Gray, Faraday Discus. Chem. Soc., 79, 257, 1985.

(9) V. Davé and W. G. Glasser, in viscoelasticity of Biomaterials, ACS Symp. Ser. (In Press).

(10) H. Hara, T. Satoh, T. Toya, S. Iida, and S. Orii, Macromolecules, 21, 14, 1988 .

(11) J. Giasson, J-F. Revol, A. M. Ritchey, and D. G. Gray, Biopolymers, $27,1999,1988$. 
8.0 MOLECULAR ORGANIZATION OF LIGNIN DURING CARBONIZATION. 


\title{
MOLECULAR ORGANIZATION OF LIGNIN DURING CARBONIZATION
}

\begin{abstract}
Thermotropic liquid crystalline behavior of lignin-like model compounds, lignin, (commercial) petroleum pitch and blends of lignin and pitch were studied using hot-stage optical microscopy. The results indicate that, while all dimeric lignin-like model compounds exhibit thermotropic liquid crystalline phenomena in some temperature range, the liquid crystalline behavior remains incomplete for lignin. The temperature at which a maximum amount of LC behavior is observed for lignin, large carbonized particles are observed as well. This prevents the formation of a molecularly organized fluid phase from ever developing. The inability to totally fluidize at elevated temperatures is likely to be held responsible for lignin's (lignin derivative's) inability to produce high strength, high modulus carbon fibers.
\end{abstract}




\section{INTRODUCTION}

Carbon fibers are the preferred reinforcing component of advanced composites due to high strength, high stiffness, light weight, fatigue resistance and vibration damping. High strength and high modulus in carbon fibers requires flawless structure and development of highly anisotropic graphitic crystallites oriented along the fiber axis during the process of production (1). During the production of carbon fibers, the fibrous precursor must be carbonized at $1200-1400^{\circ} \mathrm{C}$ in an inert atmosphere into a material of greater strength while maintaining its fibrous structure. For this reason, a stabilization stage is introduced in which the precursor fibers are heated under tension at $200^{\circ} \mathrm{C}-300^{\circ} \mathrm{C}$ in the presence of air. This will cause cross-linking on the surface of the fibers and will prevent that they shrink, melt, or fuse together.

Carbon fibers are manufactured by thermally treating fibers composed of polyacrylonitrile (PAN), (petroleum and coal) pitch, or rayon (1-3). Carbon fibers from rayon suffer from low carbon yield (20-25\%) due to formation of tarry substances like levoglucosan during the stabilization stage. Chain fragmentation eliminates orientation from the precursor and the product is an amorphous char. Properties can be improved by very expensive processing like stretch graphitization. PAN-based carbon fibers are by far the most 
important due to their high carbon yield (50\%) and good mechanical properties. However, the cost of these carbon fibers is very high and extensive work is being carried out to find new precursors.

Pitch is a complex mixture of thousands of mainly aromatic organic compounds with an average molecular weight of several hundreds. Pitch is isotropic in both liquid and solid states. Brooks and Taylor (4) found that as pitch is heated above $400^{\circ} \mathrm{C}$, small spheres appeared and gradually increase in size with time and temperature. There is no change in sphere structure when pitch is cooled to room temperature as the high viscosity permits mesophase to cool with little disruption of the microstructure (5). Therefore the structure of hightemperature pitch materials can be studied at room temperature. The process of coalescence of mesophase spheres continues with simultaneous thermal dehydrogenative condensation reactions leading to larger aromatic molecules (5). When these molecules are sufficiently large (molecular weight ca. 1,000) and flat, they favor the formation of a carbonaceous mesophase which is a discotic nematic liquid crystal with parallel stacking (6). There is no contact between adjacent molecules. Structurally, mesophase pitch is similar to conventional nematic liquid crystals, but unlike nematic phases, they are formed through chemical processes. Fibers spun from mesophase pitch are highly oriented, with the 
molecular planes aligned along the fiber axis. These fibers are excellent precursors for manufacturing carbon fibers (7). But pitch is a complex material, and certainly cannot be regarded as a single-component liquid crystalline system. The emphasis now is on the synthesis of pure, chemically stable single-component systems of relatively simple disc-like molecules which exhibit striking analogies with carbonaceous mesophase pitch $(8-11)$.

Carbon fibers have also been manufactured from lignin (7). Lignin-based carbon fibers, Kayacarbon, were first developed and made commercially available by Nippon Kayaku Co. on a pilot scale. The process involve carbonization of dryspun fibers from lignin dissolved in an alkaline solution with poly (vinyl alcohol) added as a plasticizer $(12,13)$. The lignin-based carbon fibers have the advantage of lower temperature and shorter period of stabilization stage since the cross-linking reactions are facilitated by oxygencontaining functional radicals $(12,13)$. A short cross-linking stage and high carbonization yield contribute to reducing the production cost of carbon fibers from lignin. This makes lignin an attractive precursor as high yield is a dominant factor in the processing and the economics of carbon fiber technology.

However, electron microscopy studies on the Kayacarbon showed poorly developed fibrillar structures and lack of homogeneity, orientation and interlinking between the 
crystallized layer planes. Large number of continuous and discontinuous graphitized inclusions were found due to catalytic graphitization by impurities (e.g., sodium and sodium compounds). These inclusions were effectively decoupled from the basic structure and thus weaken the fibers $(14,15)$. Microvoids of complicated shapes and sizes were also observed $(16,17)$. These flaws, and the lack of orientation gives rise to carbon fibers with low modulus and low tensile strength. This could be the reason for commercial abandonment of ligninbased carbon fibers by Nippon Kayaku Co. in 1973.

Sudo and his associates from the Forest Products Research Institute in Tsukuba, Japan, have demonstrated that carbon fibers can be produced from lignin by converting it into a pitch-like material using multi-step chemical modification processes $(18,19)$. In the first process $(18)$, lignin was successfully modified to a molten viscous material with suitable properties for melt-spinning by hydrocracking followed by heat-treatment in vacuum. This process eliminates hydroxymethyl groups and aliphatic hydroxyl groups. This degradative process seems to induce flow in lignin by the removal of active functional groups. This method requires operating costs of hydrocracking and high initial investments. In the second process, the lignin pitch was prepared by phenolation of lignin followed by heat-treatment in vacuum (19). This process may remove active functional groups by the condensation of phenol to the lignin molecule which reduces 
molecular interactions and thereby increases fluidity in lignin. The resultant carbon fibers from both processes were better than Kayacarbon. However, due to relatively low modulus and low tensile strength, these fibers fall into the category of general-purpose carbon fibers.

High modulus, high strength carbon fibers can be prepared from lignin if the molecules are organized in their melt state in an anisotropic, liquid crystalline form prior to solidification. This process resembles the formation of fibers from carbonaceous mesophase pitch. This molecular orientation, or the lack thereof, appears to be the primary limitation for the formation of high modulus carbon fibers from lignin.

The main features that favor liquid crystal formation are long, narrow, rod-shaped molecules with high aspect ratio (L/D). Presence of rigidity imparted by double bonds and ring structures along the long axis of molecules further enhances liquid crystal formation. The majority of the liquid crystalline structures possess two or more aromatic or heteroaromatic rings, one or more bridging groups that binds the rings together and two terminal groups usually on the long axis of the molecule $(20)$.

Lignin is one of the major components of the plant kingdom, and its basic structural units are 4-hydroxyphenyl, 3-methoxy-4-hydroxyphenyl (guaiacyl), and 3,5-dimethoxy-4hydroxyphenyl (syringyl) groups. The degradation products of lignin are 4-hydroxybenzaldehyde, vanillin and syringaldehyde. 
Liquid crystalline properties were not observed in ligninderived compounds which were synthesized based on a homologous series of 4-n-alkoxybenzoic acid derivatives from vanillin and syringaldehyde (21). This may have been due to hindrances caused by the presence of large methoxyl groups attached to the aromatic ring to form molecular stacking which leads to stable mesophase. This work was based on the knowledge that the homologous series of 4-n-alkoxybenzoic acids formed thermotropic liquid crystalline compounds. In order to reduce the effects of methoxyl groups, benzoyloxybenzoic acid derivatives, cholesteryl benzoate derivatives and benzylidenep-aminobenzoic acid derivatives having the guaiacyl structure were synthesized from vanillin (21-23). All three acid derivatives exhibited thermotropic liquid crystalline behavior.

The goals of the present study are to investigate thermotropic liquid crystalline behavior of lignin-like model compounds, lignin and lignin blends with pitch; and properties of carbon fibers made from hydroxypropyl lignin pitch.

\section{EXPERIMENTAL}

I. Materials.

1. Lignin-like Model Compounds: The four compounds used in this study were two biphenyls $(5-5)$, a $\beta$-ether model $(\beta-0-4)$, and phenyl coumaran $(\beta-5)$.

2. Lignin: The organosolv lignin used was obtained from Kodak 
as an experimental product.

3. Hydroxypropyl Lignin (HPL): Organosolv Red Oak HPL was obtained in accordance to earlier reports (24).

4. Petroleum Pitch: The pitch was obtained from Ashland Oil Company under the designation Ashland A-240.

\section{Methods.}

1. Acetylation of Lignin:

Lignin was acetylated according to the procedure of Gierer and Lindberg (25) with some minor changes. It was freeze-dried for 24 hours before acetylation. Lignin was first dissolved in distilled pyridine before an equal amount of distilled acetic anhydride was added to the solution with continuous stirring under flowing dry nitrogen $(40 \mathrm{~mL}$ of pyridine and acetic anhydride per gram of lignin). The reaction was carried out at $0^{\circ} \mathrm{C}$ for 24 hours. The acetylated lignin was recovered by precipitating the reaction mixture in $0.1 \mathrm{~N} \mathrm{HCl}$ (approximately ten times the volume of the reaction mixture). The acetylated lignin was filtered and washed several times with $0.1 \mathrm{~N} \mathrm{HCl}$ to remove the pyridine. This was followed by washing with distilled water to remove $\mathrm{HCl}$ from the lignin derivative. The derivative was collected and freeze-dried for 24 hours and stored in a desiccator. Lignin acetate was soluble in tetrahydrofuran (THF) which is required for many molecular weight determinations. 
2. Preparation of Mesophase Pitch from A-240:

Mesophase pitch was prepared by following the procedure of previous investigators (26-28) with some changes. A-240 was heated under nitrogen flow in a pyrex jar to $425^{\circ} \mathrm{C}$. The pitch was continuously stirred throughout the heating process to keep the heating process homogenous. The temperature was maintained at $425^{\circ} \mathrm{C}$ for three hours (residence time). After one hour of residence time, a drop of pitch was placed on a microscope slide which was covered by a cover slip. A similar sample was prepared after two hours. It was difficult to prepare such samples after three hours as the pitch became too viscous. These slides were examined for mesophase formation on the optical microscope stage with cross-polarizers.

3. Preparation of Pitch and Carbon Fibers from HPL:

This work was done at the Forest Products Research Institute in Tsukuba, Japan, on the HPL prepared in our laboratory. About 20 gms. of HPL were hydrocracked in the presence of $1 \mathrm{gm}$ (based on lignin) of Raney-Nickel catalyst in $200 \mathrm{~mL}$ of $5 \%$ aqueous sodium hydroxide. The initial hydrogen pressure was $5 \times 10^{-3} \mathrm{GPa}$, and the reaction time and temperature were 1 hour and $250^{\circ} \mathrm{C}$. After cooling the reaction mixture, the solution was filtered to remove the catalyst and the residue. The yield of hydrocracked lignin was $61 \%$ based on starting material. The hydrocracked HPL was heat treated at $250^{\circ} \mathrm{C}$ for 70 minutes in a stream of nitrogen to remove 
volatile and/or thermally unstable compounds and prepare HPL pitch. Total yield of lignin-pitch was $50.3 \%$. The pitch was spun into fibers by melt spinning through a single hole spinnerette under nitrogen pressure at $153^{\circ} \mathrm{C}-165^{\circ} \mathrm{C}$. The fibers were wound on to a bobbin at a winding speed of about 100 $\mathrm{m} / \mathrm{min}$. These as-spun fibers (also called Green Fibers) were thermostabilized in air by heating up to $210^{\circ} \mathrm{C}$ at a rate of $1-$ $2^{\circ} \mathrm{C} / \mathrm{min}$. After the heat treatment, the fibers were carbonized in nitrogen by heating at a rate of $5^{\circ} \mathrm{C} / \mathrm{min}$. up to $1,000^{\circ} \mathrm{C}$. The yield of the carbon fibers based on the starting material was $26 \%$

4. Preparation of Blends:

Blends of lignin and A-240 pitch were prepared by mixing weighed amounts of lignin and A-240 in a mortar by pestle. 5. Gel Permeation Chromatography:

Narrow distribution polystyrene standards were obtained from Polymer Laboratories and American Polymer Standards Corporation. These standards were dissolved in HPLC-grade THF, and they were used for constructing a universal calibration curve $(\log [\eta] \mathrm{M}$ versus retention volume) (29).

Molecular weights were determined using the viscotek Differential Viscometer, Model 100, in series with the Waters 410 Refractive Index monitor, three waters Ultrastyragel columns with pore diameters of $10^{3} \AA, 10^{4} \AA$ and $10^{6} \AA$, and a waters 510 HPLC pump. Samples were introduced via the waters 
U6K injection port. Data collection was performed with a personal computer interfaced to the detection and injection system. Data manipulations were handled with the Unical software package 2.71 provided by Viscotek. Solutions of the samples were carefully prepared in HPLC-grade THF to ensure accurate concentrations which ranged from $3-6 \mathrm{mg} / \mathrm{mL}$. Concentrations of polystyrene standards were molecular weight dependent, ranging from 0.01 to $5 \mathrm{mg} / \mathrm{mL}$.

6. Hot-stage Optical Microscopy:

Lignin-like model compounds, lignin, HPL, A-240 pitch and blends of lignin and A-240 samples (few milligrams) were placed between microscope slide and cover slip. This set was heated in the hot-stage of a Zeiss Axioplan Universal optical Microscope before it was examined for birefringence between cross polarizers. The heating and cooling rate was $5^{\circ} \mathrm{C} / \mathrm{min}$ and $2.5^{\circ} \mathrm{C} / \mathrm{min}$, respectively. The hot-stage compartment containing the sample was continuously flushed with dry nitrogen in order to maintain an inert atmosphere and continuously remove pyrolysis gases.

\section{Differential Scanning Calorimetry (DSC):}

The thermal analysis of the lignin-like model compounds was determined on a Perkin-Elmer DSC-2C interfaced to a Thermal Analysis Data Station (TADS). All the compounds were analyzed at a heating and cooling rate of $5^{\circ} \mathrm{C} / \mathrm{min}$ and $2.5^{\circ} \mathrm{C} / \mathrm{min}$, respectively, under a purge of dry nitrogen. 
8. ${ }^{13}$ C NMR Spectroscopy:

The ${ }^{13} \mathrm{C}$ NMR spectra of HPL, hydrocracked HPL and heattreated HPL (concentration: $150 \mathrm{mg} / 0.75 \mathrm{ml}$ deuterated dimethyl sulfoxide) solutions were recorded on a Varian 400 FT-NMR spectrometer operated at $100 \mathrm{MHz}$ and spectral width of 25,000 Hz. The observed spectra for HPL, hydrocracked HPL and heattreated HPL solutions were obtained after 3500,1550 , and 1550 repetitions, respectively at $25^{\circ} \mathrm{C}$ in $5 \mathrm{~mm}$ NMR tubes using the polarization transfer standard Distortionless Enhancement Polarization Transfer (DEPT) technique.

9. Scanning Electron Microscopy:

The carbon fibers from HPL were observed on a JEOL JSM35C Scanning Electron Microscope with an accelerating voltage of $15 \mathrm{kV}$. The carbon fibers were mounted on aluminum specimen mounts (EMSL) at $45^{\circ}$ angle. The fibers were coated at the bottom by Ladd silver conducting Paint, and they were then sputter-coated with pure gold for 1 minute ( $9 \mathrm{~nm}$ thickness) in a SPI sputter coater.

10. Transmission Electron Microscopy:

The carbon fibers were embedded in Poly/Bed 812 (Polysciences, Inc.) cured at $60^{\circ} \mathrm{C}$ for 48 hours in flat molds. 80-90 nm sections were microtomed across the cross-section from the embedded fibers. All sections were mounted on copper grids, and they were observed on Philips STEM EM-420 electron microscope. 
Table I. Chemical and Molecular Characteristics of Lignin, HPL, Hydrocracked HPL and Heat-treated HPL.

\begin{tabular}{||l|l|l|l|l||}
\hline \hline & Lignin & HPL & $\begin{array}{c}\text { Hydrocracked } \\
\text { HPL }\end{array}$ & $\begin{array}{c}\text { Heat } \\
\text { Treated HPL }\end{array}$ \\
\hline$\left\langle M_{a}\right\rangle$ & 2,900 & 1,900 & 400 & 780 \\
\hline$\left\langle M_{w}\right\rangle$ & 12,000 & 5,600 & 600 & 2000 \\
\hline$\left\langle M_{w}\right\rangle /\left\langle M_{n}\right\rangle$ & 4.1 & 2.9 & 1.5 & 2.5 \\
\hline MHS-Constant (a) & 0.41 & 0.24 & 0.17 & 0.21 \\
\hline Log K & -2.81 & -2.21 & -1.9 & -2.1 \\
\hline $\begin{array}{l}\text { Intrinsic } \\
\text { Viscosity } \\
{[\eta], \text { dL/g }}\end{array}$ & 0.056 & 0.044 & 0.034 & 0.036 \\
\hline
\end{tabular}


TABLE II. Typical Properties of Commercial Ashland Petroleum Pitch

\begin{tabular}{||c|c|c||}
\hline & Test Method & $\begin{array}{c}\text { A-240 } \\
\text { Pitch }\end{array}$ \\
\hline Glass Transition Temperature, ${ }^{\circ} \mathrm{C}$ & ASTM D-3104 & 120 \\
\hline Density, g/CC, $25^{\circ} \mathrm{C}$ & Beckman Pycnometer & 1.23 \\
\hline Benzene Insolubles, \& & ASTM D-2317 & 5.0 \\
\hline Quinone Insolubles, \& & ASTM D-2318 & Nil \\
\hline Carbon, Weight 8 & ASTM D-2416 & 51.8 \\
\hline Ash, Weight 8 & ASTM D-2415 & 0.10 \\
\hline Sulfur, Weight 8 & ASTM D-1552 & 2.2 \\
\hline Viscosity, Pa.s, $163^{\circ} \mathrm{C}$ & Brookfield & 0.70 \\
\hline Viscosity, Pa.s, $177^{\circ} \mathrm{C}$ & Brookfield & 0.27 \\
\hline Viscosity, Pa.s, $188^{\circ} \mathrm{C}$ & Brookfield & 0.14 \\
\hline
\end{tabular}




\section{RESULTS AND DISCUSSION}

Table I summarizes molecular characteristics of lignin, HPL, hydrocracked HPL, and heat-treated HPL. Relevant data describing commercial petroleum pitch are given in Table II. 1. Lignin-like Model Compounds:

Figure 1 shows the model compounds used in this study. (a) The biphenyl model compound (Fig. 1a) was heated on the hot-stage until it became isotropic at $130^{\circ} \mathrm{C}$. On cooling from the isotropic melt, spherulites were observed to appear at about $95^{\circ} \mathrm{C}$, which grew with time (Fig. 2a). These spherulites are typically 1,500 to $2,000 \mu \mathrm{m}$ in size. The DSC scan on as received sample shows two melting endotherms; a smaller one at $104^{\circ} \mathrm{C}$ and a sharp peak at $119^{\circ} \mathrm{C}$ (Fig. 3a). On cooling this sample in the DSC, a crystallization exotherm from $115^{\circ} \mathrm{C}$ to $80^{\circ} \mathrm{C}$ is observed (Fig. 3b) which agrees with our observation using the hot-stage polarized optical microscope. After the crystallization was complete, the sample was heated again very slowly, in the DSC. The DSC scan for such a sample again shows two sharp endotherms at $116.5^{\circ} \mathrm{C}$ and $122^{\circ} \mathrm{C}$ (Fig. 3C). The sample in Fig. 2 a was heated slowly on the hot-stage. On heating, the spherulites begin to melt at about $105^{\circ} \mathrm{C}$ (Fig. $2 \mathrm{~b}$ ). On further heating, the compound seemed to transform into a nematic liquid crystalline phase at $-113^{\circ} \mathrm{C}$ (Fig. 2C) as the texture of the crystals changed and disclination lines characteristic of nematic liquid crystalline phase appeared. At $120^{\circ} \mathrm{C}$, a 


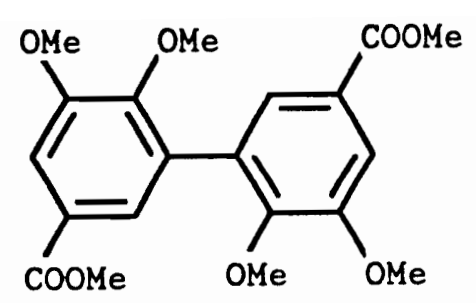

(a)

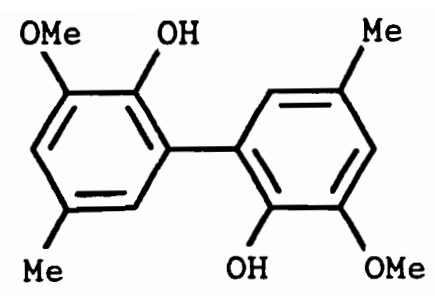

(b)

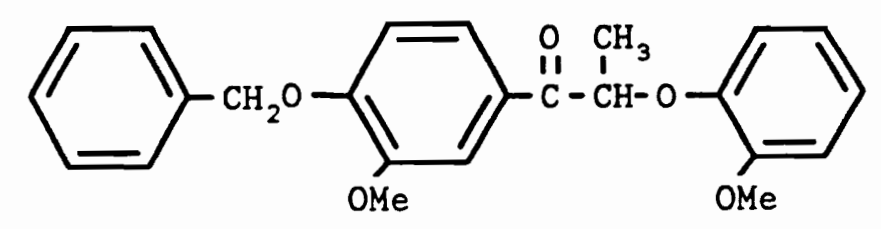

(c)

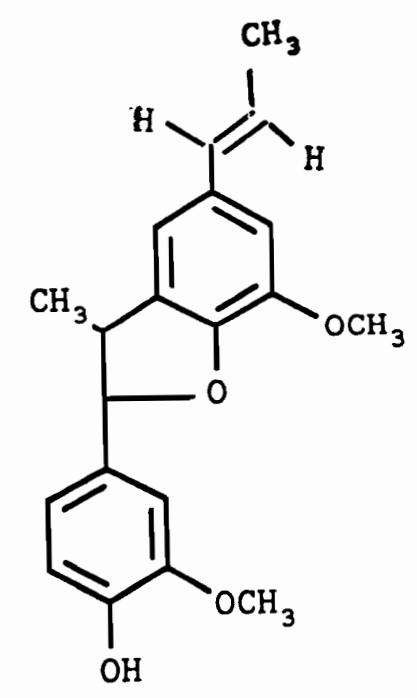

(d)

Figure 1. Chemical structures of lignin-like model compounds;

(a) Biphenyl [5-5] (b) Biphenyl [5-5] (c) $\beta$-ether model (d) Phenyl coumaran $(\beta-5)$. 

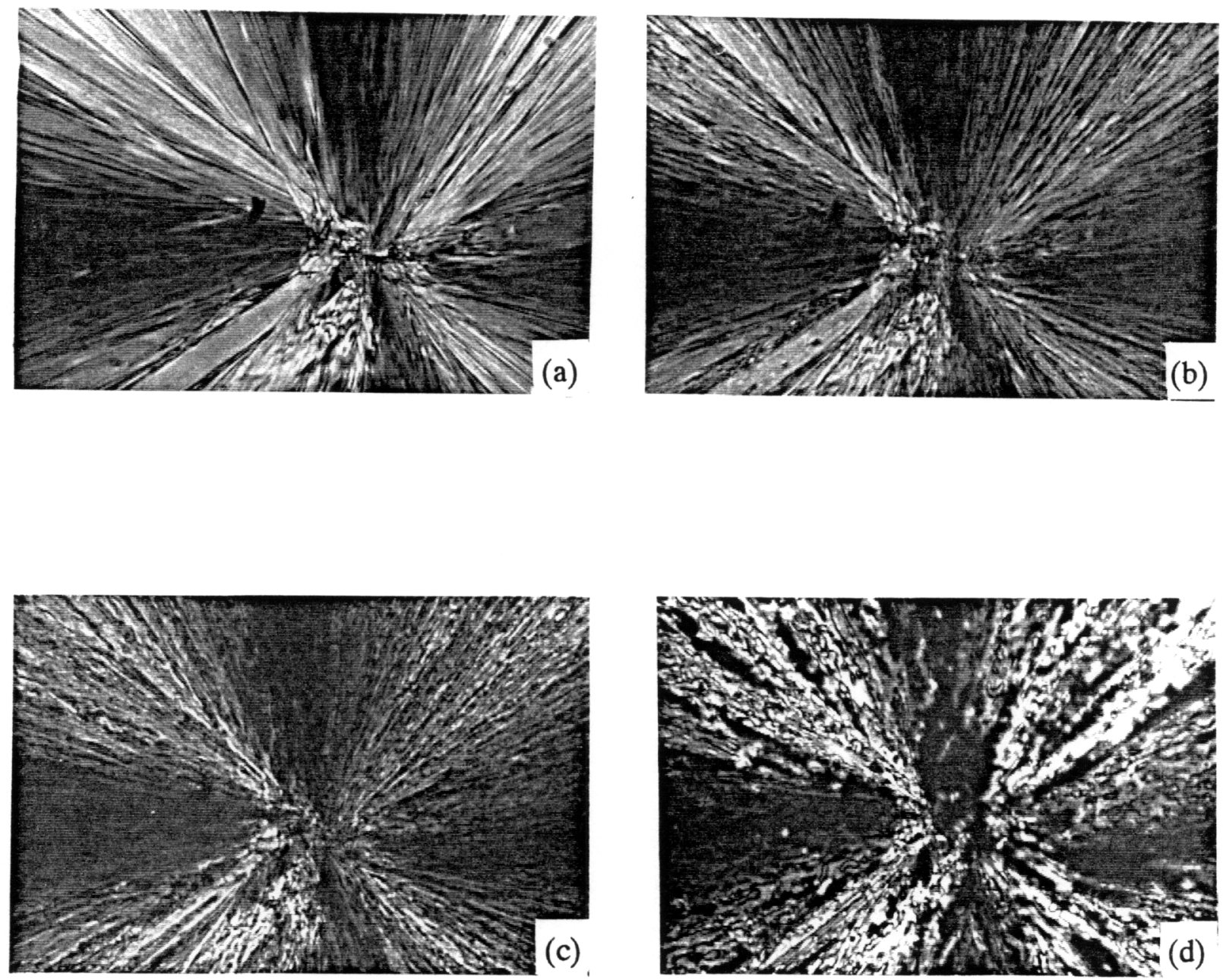

$50 \mu$

Figure 2. Polarized optical micrographs of biphenyl [5-5] (Fig. 1a) crystallized isothermally at $95^{\circ} \mathrm{C}$ and heated on hot-stage as a function of temperature; (a) $95^{\circ} \mathrm{C}$ (b) $105^{\circ} \mathrm{C}$ (c) $113^{\circ} \mathrm{C}$ (d) $120^{\circ} \mathrm{C}$. 


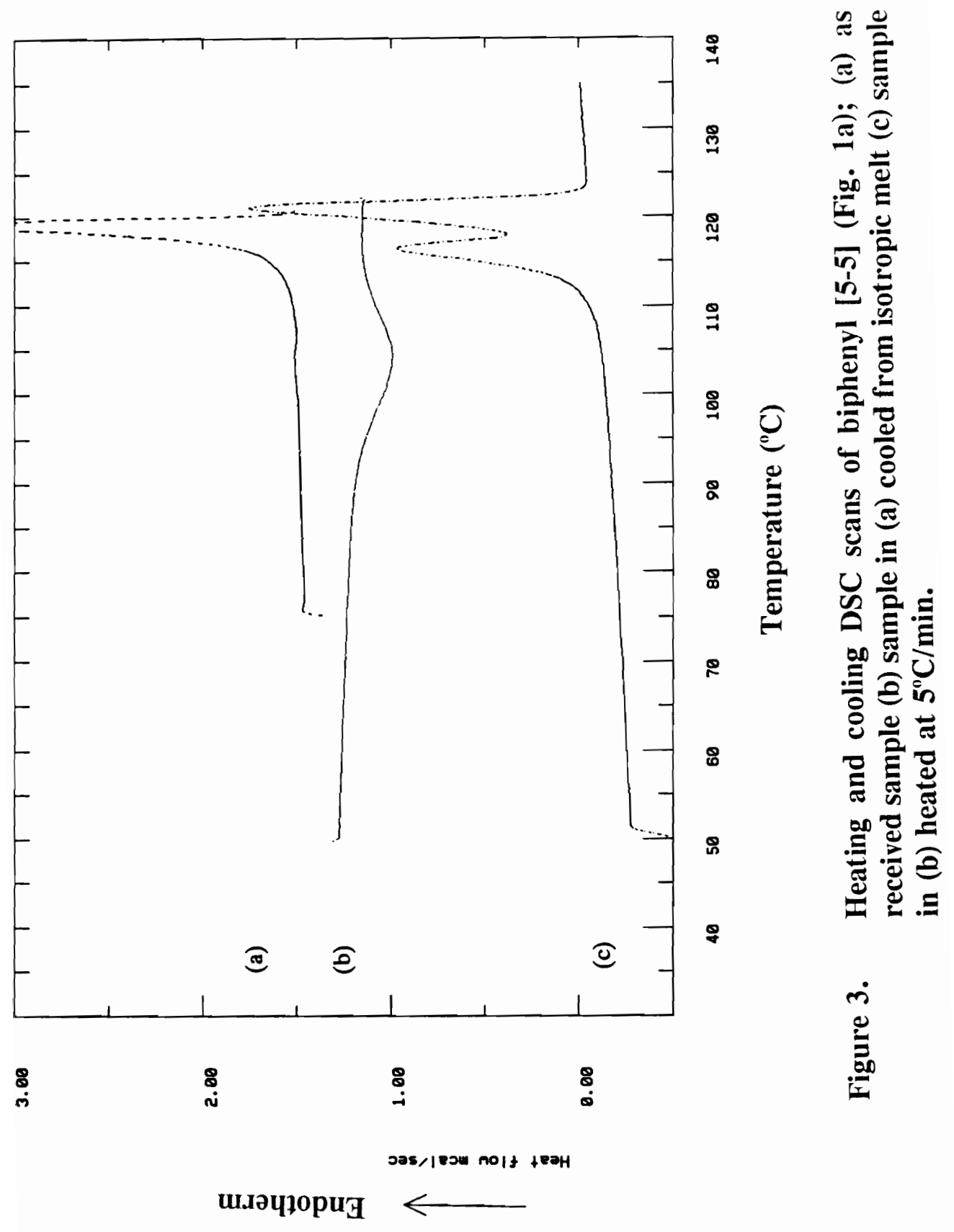


complete transformation of $\mathrm{K} \rightarrow \mathrm{N}$ [crystal (K) to nematic (N)] phase occurs (Fig. 2d). This nematic phase was stable up to $126^{\circ} \mathrm{C}$ before it became isotropic at $128^{\circ} \mathrm{C}$. The endotherm transition in Fig. $3 \mathrm{C}$ at $116.5^{\circ} \mathrm{C}$ agrees with polarized optical microscopic observations, and it suggests that this transition is due to a $\mathrm{K} \rightarrow \mathrm{N}$ transition; and that the endothermic transition at $122^{\circ} \mathrm{C}$ is due to an $\mathrm{N} \rightarrow \mathrm{I}$ transition [nematic (N) to isotropic (I)]. In the case of an as received sample (Fig. $3 a)$, the $\mathrm{K} \rightarrow \mathrm{N}$ transition is at $104^{\circ} \mathrm{C}$, which is lower by $12.5^{\circ} \mathrm{C}$ as compared to the isothermally crystallized sample. The reason for this lower transition temperature may be attributed to smaller or less perfect crystals in as received sample compared to the isothermally crystallized sample. This would also explain the reason for the much lower heat of transition in the as received sample at $104^{\circ} \mathrm{C}$. On the other hand, the higher endothermic peak in the as received sample shows very close resemblance to that of the isothermally crystallized sample for temperature and heat of fusion. This conclusion is further supported by DSC observations on quenched samples. When this compound is quenched and reheated, the phase transitions observed by polarized optical microscopy and DSC were similar to those observed for the as received sample in Fig. 3a. From all these observations, one can conclude that the biphenyl model compound $1 \mathrm{a}$ behaves as a thermotropic liquid-crystalline material. 
(b) Biphenyl model compound (Fig. 1b) was heated on the hotstage until it became isotropic at $135^{\circ} \mathrm{C}$. On cooling from the isotropic melt, spherulites appear and grow in size at $98^{\circ} \mathrm{C}$ (Fig. 4a). Similar experiments by DSC reveal an exothermic transition between $95^{\circ} \mathrm{C}$ and $50^{\circ} \mathrm{C}$ (Fig. 5a), and this agrees with polarized optical microscopic observations. On re-heating the sample (Fig. 4a), spherulitic textures did not change until $115^{\circ} \mathrm{C}$. At $118^{\circ} \mathrm{C}$, the spherulites seemed to have melted and the texture changes. Disclination lines characteristic of a nematic liquid crystalline phase were clearly observed (Fig. 4b). The $\mathrm{K} \rightarrow \mathrm{N}$ transition is completed by further heating to $120^{\circ} \mathrm{C}$ (Fig. 4C). This nematic phase was stable up to $128^{\circ} \mathrm{C}$. It became isotropic at $129^{\circ} \mathrm{C}$. This observation was further supported by DSC. The sample in Fig. 5a was heated slowly in the DSC. The DSC scan in Fig. 5b shows a broad exothermic peak at $\sim 75^{\circ} \mathrm{C}$, a small endothermic peak at $113^{\circ} \mathrm{C}$ followed by a sharp endothermic peak at $123^{\circ} \mathrm{C}$. Due to slower rates of crystallization of this compound, a complete crystallization does not occur at a cooling rate of $2.5^{\circ} \mathrm{C} / \mathrm{min}$. On slow heating, further crystallization occur and is observed in the form of an exotherm at $\sim 75^{\circ} \mathrm{C}$. Based on polarized optical microscopy, the lower endothermic peak at $-113^{\circ} \mathrm{C}$ corresponds to an $\mathrm{K} \rightarrow \mathrm{N}$ transition and the higher one to an $\mathrm{N} \rightarrow \mathrm{I}$ transition. When this compound is quenched from the melt, the DSC heating scan (Fig. 5c) shows a similar behavior as that observed for 

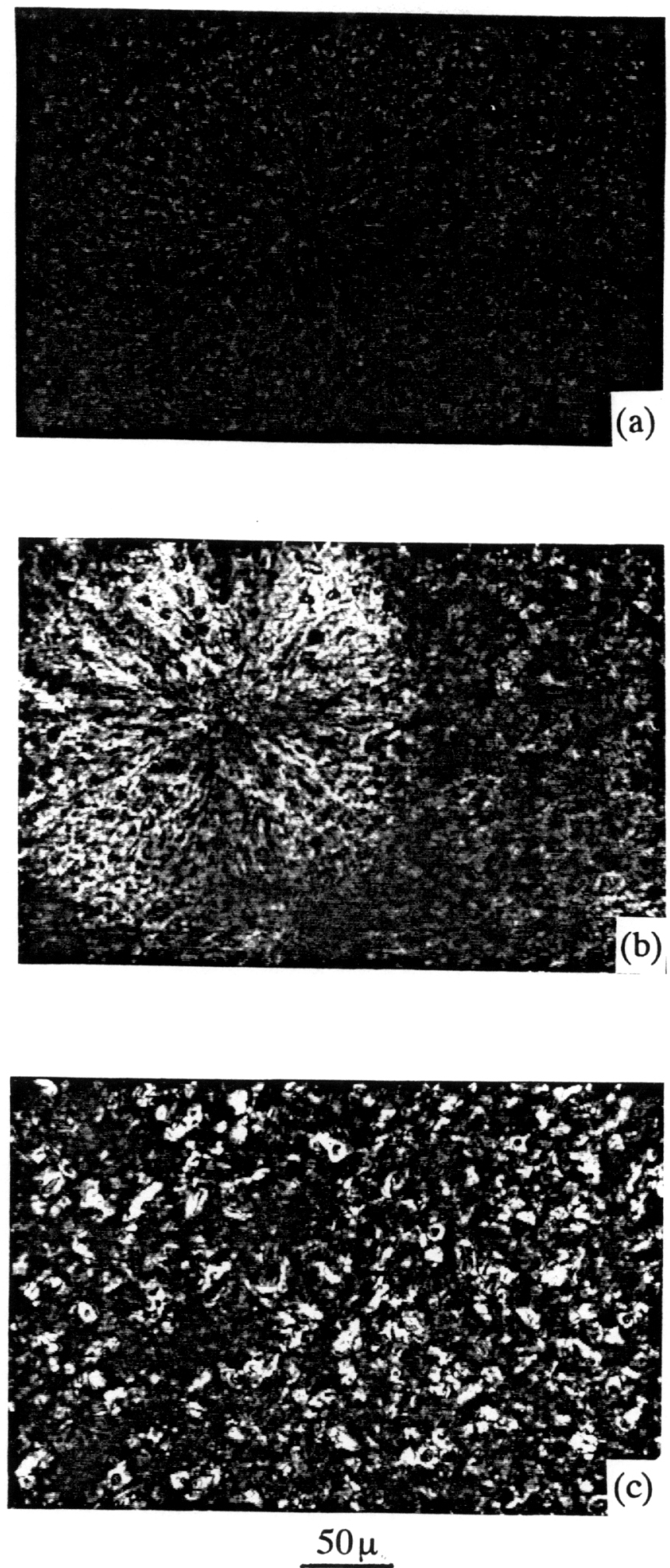

Figure 4. Polarized optical micrographs of biphenyl [5-5] (Fig. 1b) crystallized isothermally at $98^{\circ} \mathrm{C}$ and heated on hot-stage as a function of temperature; (a) $98^{\circ} \mathrm{C}$ (b) $118^{\circ} \mathrm{C}$ (c) $120^{\circ} \mathrm{C}$. 


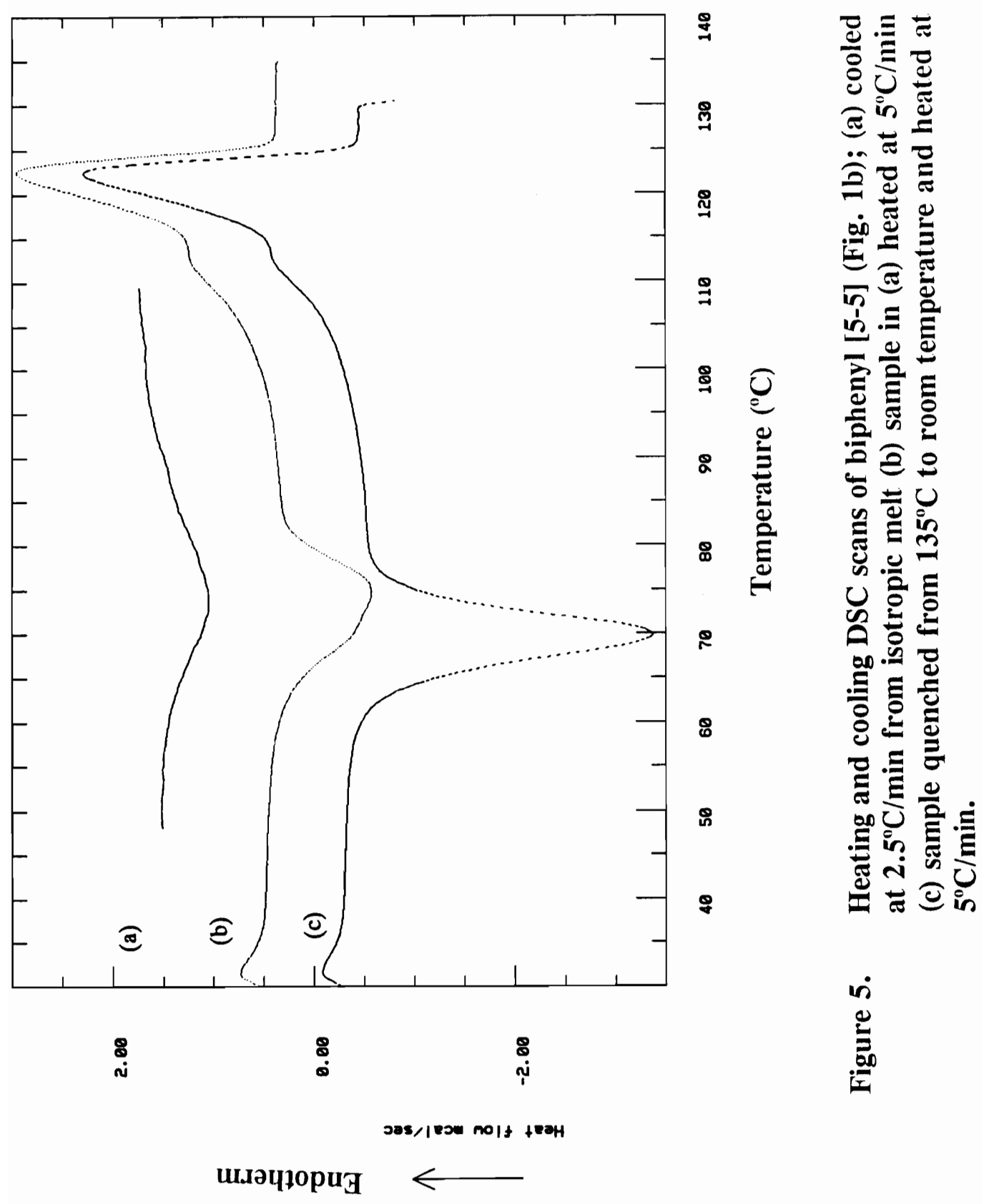


the slow-cooled sample 5b. However, the exothermic peak is sharper and at lower temperature as one would expect from a quenched sample when heated in DSC. Once again, the polarized optical microscopy results were similar to that observed for slow-cooled samples. In this case, very small size spherulites were observed to appear at $\sim 55^{\circ} \mathrm{C}$ on heating a quenched sample, which transformed into a nematic phase at $112^{\circ} \mathrm{C}$ followed by an $\mathrm{N} \rightarrow \mathrm{I}$ phase at $127^{\circ} \mathrm{C}$. These observations clearly indicate the thermotropic mesophase transition of this model compound. (c) As received $\beta$-ether model (Fig. 1c) was heated on the hotstage, and an $\mathrm{K} \rightarrow \mathrm{N}$ transition was observed. The crystals melted at $102.5^{\circ} \mathrm{C}$ (Fig. $6 \mathrm{a}$ ) and a nematic phase formed as disclination lines were observed at $103.5^{\circ} \mathrm{C}(\mathrm{K} \rightarrow \mathrm{N})$ (Fig. 6b) . Fig. 7 gives the DSC scan of the as received sample. This scan is characterized by a small endotherm at $\sim 93^{\circ} \mathrm{C}$ and a broad endotherm at $105^{\circ} \mathrm{C}$. Based on polarized optical microscopy results, the lower endotherm could correspond to an $\mathrm{K} \rightarrow \mathrm{N}$ transition and the higher one to an $\mathrm{N} \rightarrow \mathrm{I}$ transition. There were no transitions observed during the cooling run, second heating run or on quenched samples. Even isothermal annealing in the suitable temperature range did not show any development of crystallinity. These DSC observations are supported by polarized optical microscopy. Upon cooling from above the isotropic transition, the sample remained non-birefringent even when annealed isothermally between $50^{\circ} \mathrm{C}$ and $85^{\circ} \mathrm{C}$ for 24 

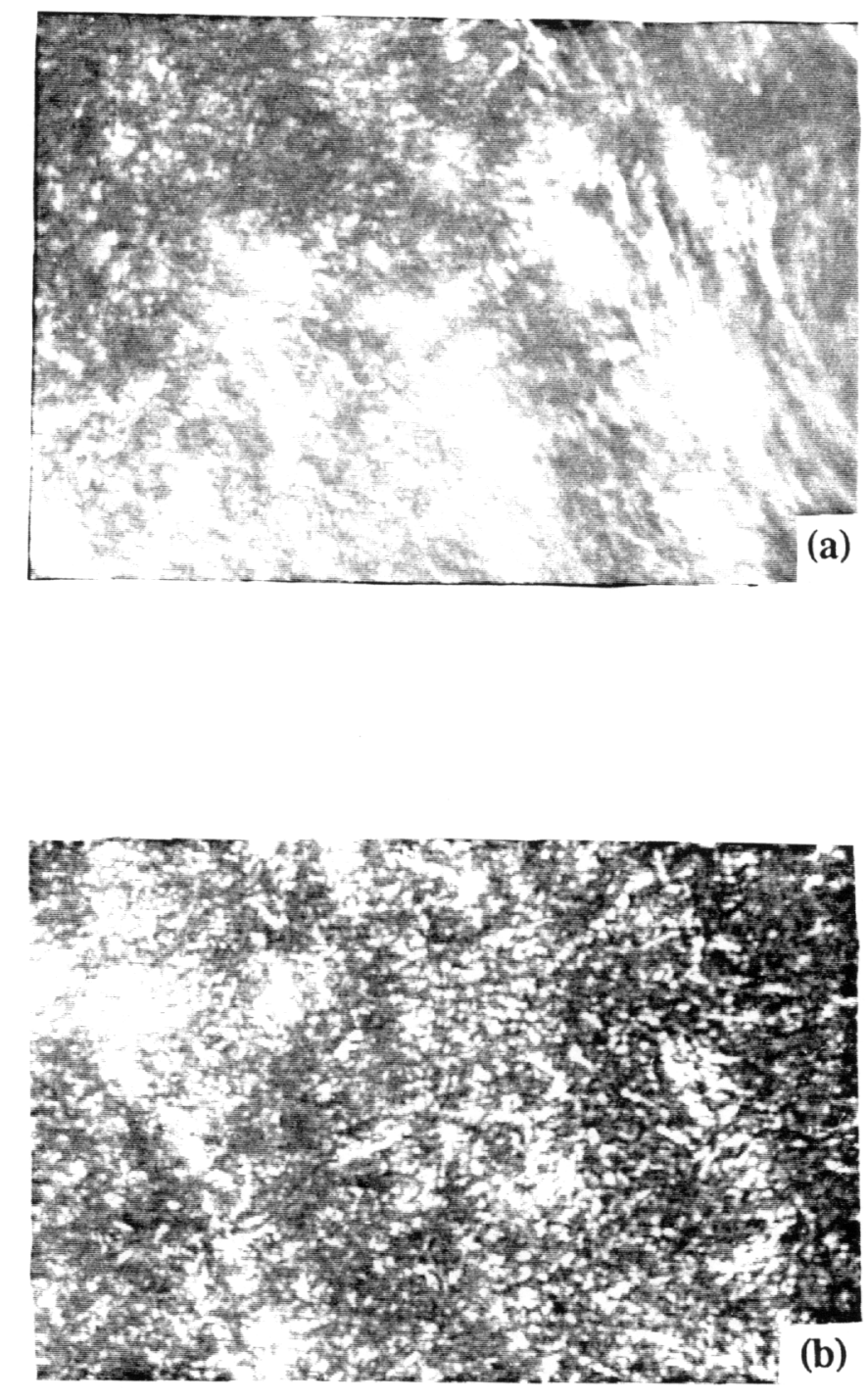

$50 \mu \mathrm{m}$

Figure 6. Polarized optical micrographs of $\beta$-ether model (Fig. 1c) heated on hotstage as a function of temperature; (a) $102.5^{\circ} \mathrm{C}$ (b) $103.5^{\circ} \mathrm{C}$. 


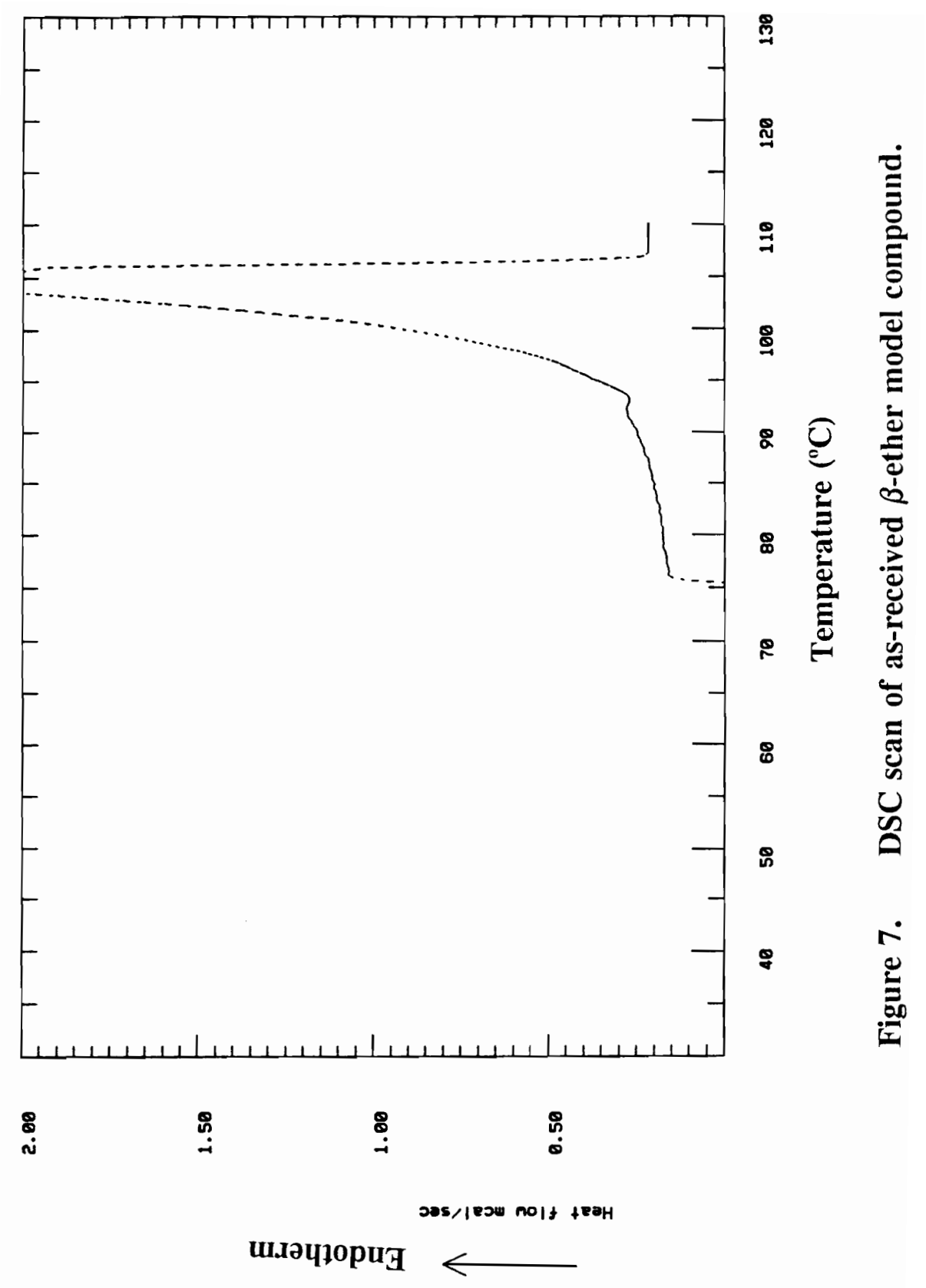


hours. These results suggest that this compound exhibits thermotropic liquid-crystalline behavior only during the first heating run, and this indicates that the nematic mesophase is not very stable, unlike the biphenyls compounds described earlier.

(d) As received phenyl coumaran model compound (Fig. 1d) was heated on the hot stage. At $131.2^{\circ} \mathrm{C}$ the crystals melted and characteristic disclination lines of a nematic phase appeared at $131.8^{\circ} \mathrm{C}$. This phase began to grow. It was completely nematic at $132.7^{\circ} \mathrm{C}(\mathrm{K} \rightarrow \mathrm{N})$ and isotropic at $133.8^{\circ} \mathrm{C}(\mathrm{N} \rightarrow \mathrm{I})$. Fig. $8 a$ gives the DSC scan obtained on heating the as received sample. A broad endotherm between $122-135^{\circ} \mathrm{C}$ was observed. The temperature range over which $\mathrm{K} \rightarrow \mathrm{N}$ and $\mathrm{N} \rightarrow \mathrm{I}$ transitions were observed (by polarized optical microscopy) covers only $2.6^{\circ} \mathrm{C}$. Therefore, it is likely that the two transitions may be buried inside the broad melting endotherm observed in Fig. 8a. When this sample was annealed isothermally, it was possible to separate the two transitions to the extent that they were detectable by DSC. Fig. 8b gives one typical DSC scan obtained on slow heating of a quenched sample (from $135^{\circ} \mathrm{C}$ to $25^{\circ} \mathrm{C}$ ). This scan is characterized by a small exotherm at $85^{\circ} \mathrm{C}$ (this indicates crystallization of the compound) followed by two sharp endotherms at $113^{\circ} \mathrm{C}$ and $131^{\circ} \mathrm{C}$. When the same sample was crystallized from the melt isothermally at $85^{\circ} \mathrm{C}$ on the hotstage, large spherulites were observed which suggest that the 


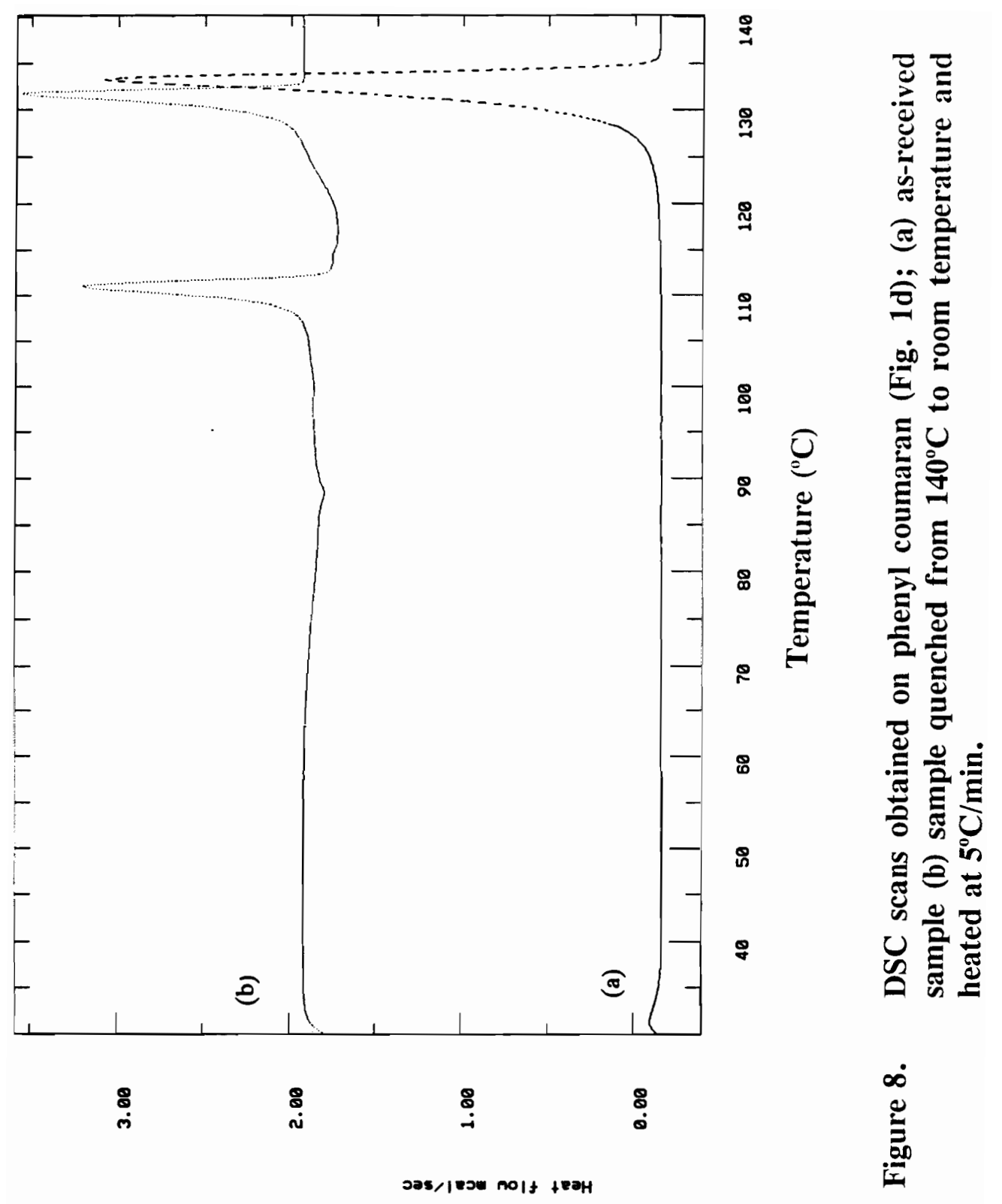

ш.ләчрория $\longleftarrow$ 

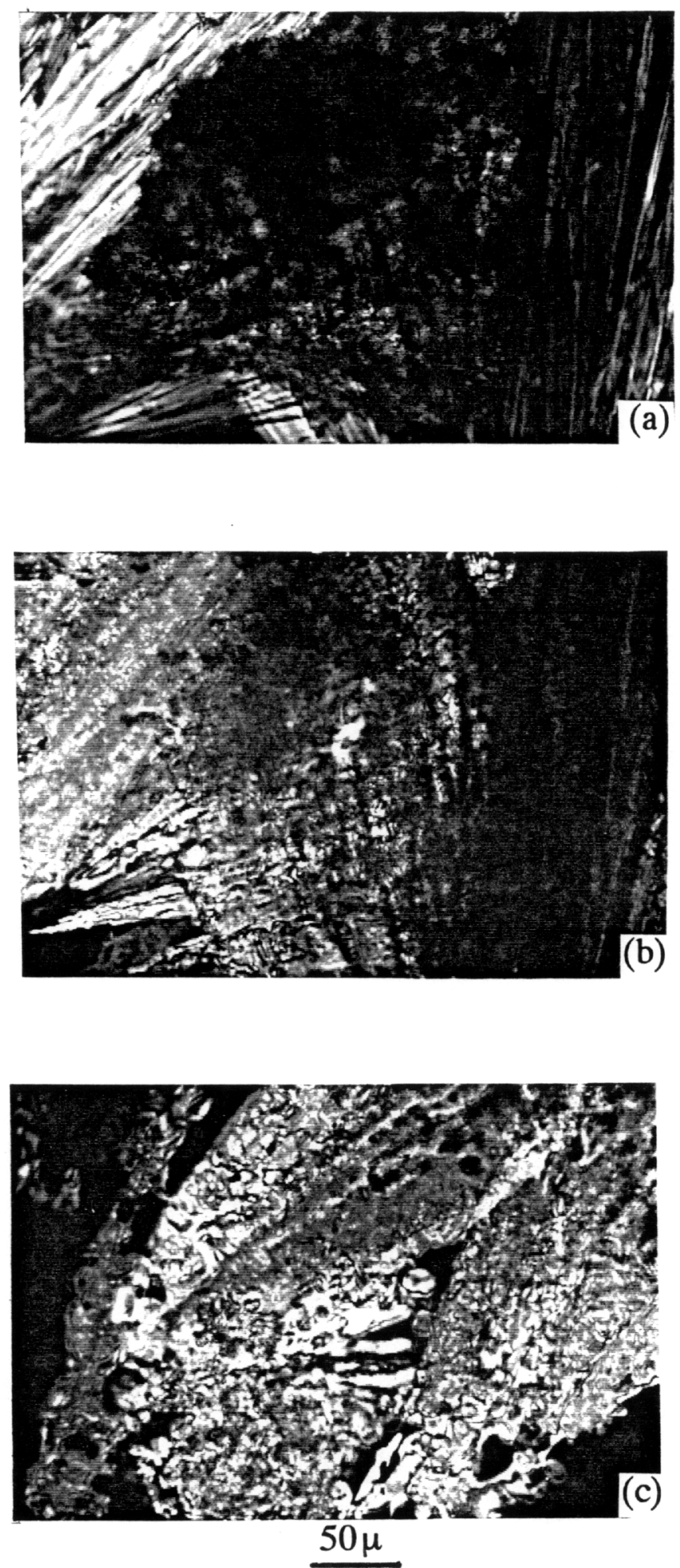

Figure 9. Polarized optical micrographs of phenyl coumaran crystallized isothermally at $85^{\circ} \mathrm{C}$ and heated on hot-stage as a function of temperature; (a) $105^{\circ} \mathrm{C}$ (b) $110^{\circ} \mathrm{C}$ (c) $130^{\circ} \mathrm{C}$. 
exotherm in Fig. $8 \mathrm{~b}$ was due to crystallization. On heating this sample on the hot-stage to $105^{\circ} \mathrm{C}$, the spherulitic texture seemed to melt, and the formation of a nematic phase was observed (Fig. 9a). Complete nematic phase transition occurs by further heating to $110^{\circ} \mathrm{C}-115^{\circ} \mathrm{C}$ (Fig. 9b). This nematic phase was stable up to $130^{\circ} \mathrm{C}$ (Fig. 9C), and it becomes isotropic at $131.8^{\circ} \mathrm{C}$. These temperatures agree well with the two endotherms observed in Fig. $8 \mathrm{~b}$, and they suggest that the lower endotherm represents a $\mathrm{K} \rightarrow \mathrm{N}$ transition and the higher one represents an $\mathrm{N} \rightarrow \mathrm{I}$ transition. Polarized optical microscopy results similar to isothermally crystallized samples were also observed for the quenched samples (not shown here). Once again, these polarized optical microscopy and DSC results indicate that the phenyl coumaran model compound forms thermotropic liquid crystals.

Table III summarizes all major thermal transitions in the model compounds as observed by optical microscopy and DSC.

No one has reported on the thermotropic liquidcrystalline behavior of the lignin-like model compounds studied here. The phenyl groups in the biphenyl (5-5) compounds impart the necessary rigidity and unidimensionality to the structures which promote the formation of liquid crystalline structure. The hydroxyl hydrogens are substituted by methyl groups, and it is therefore hypothesized that intramolecular hydrogen bonding may not exist in the first 
TABLE III. Summary of Liquid-crystalline Behavior of Lignin-like Model Compounds.

\begin{tabular}{|c|c|c|c|c|}
\hline $\begin{array}{l}\text { Model } \\
\text { Compounds }\end{array}$ & $\begin{array}{l}\text { POM') } \\
\text { (Heating) }\end{array}$ & $\begin{array}{c}\text { DSC } \\
\text { (Heating) }\end{array}$ & $\begin{array}{l}\text { POM } \\
\text { (Cooling) }\end{array}$ & $\begin{array}{c}\text { DSC } \\
\text { (Cooling) }\end{array}$ \\
\hline $\begin{array}{c}\text { (a) Biphenyl } \\
(5-5)\end{array}$ & $\begin{array}{ll}\text { b) } & \left(\mathrm{T}_{\mathrm{m}}\right)_{105} \\
\text { d) } & (\mathrm{K} \rightarrow \mathrm{N})_{120} \\
(\mathrm{~N} \rightarrow \mathrm{I})_{128} & \end{array}$ & 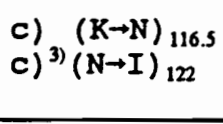 & a) $\left(T_{c}\right)_{95}$ & b) $\left(T_{c}\right)_{115-80}$ \\
\hline $\begin{array}{c}\text { (b) Biphenyl } \\
(5-5)\end{array}$ & $\begin{array}{ll}\text { b) } & \left(\mathrm{T}_{\mathrm{m}}\right)_{118} \\
\text { c) } & (\mathrm{K} \rightarrow \mathrm{N})_{120} \\
& (\mathrm{~N} \rightarrow \mathrm{I})_{129} \\
\end{array}$ & $\begin{array}{l}\text { b) }\left(T_{c}\right)_{75} \\
\text { b) }(K \rightarrow N)_{113} \\
\text { b) }(N \rightarrow I)_{123}\end{array}$ & a) $\left(T_{c}\right)_{98}$ & a) $\left(T_{c}\right)_{95-50}$ \\
\hline $\begin{array}{l}\text { (c) } \beta \text {-ether } \\
(\beta-0-4)\end{array}$ & $\begin{array}{ll}\text { a) } & \left(T_{\mathrm{m}}\right)_{102.5} \\
\text { b) } & (\mathrm{K} \rightarrow \mathrm{N})_{103.5} \\
& (\mathrm{~N} \rightarrow \mathrm{I})_{104}\end{array}$ & 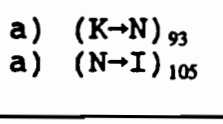 & & \\
\hline $\begin{array}{c}\text { (d) Phenyl } \\
\text { Coumaran } \\
(\beta-5)\end{array}$ & $\begin{array}{ll}\text { a) } & \left(T_{m}\right)_{105} \\
\text { b) } & (\mathrm{K} \rightarrow \mathrm{N})_{115} \\
& (\mathrm{~N} \rightarrow I)_{131.8}\end{array}$ & $\begin{array}{l}\text { b) }\left(T_{c}\right)_{85} \\
\text { b) }(K \rightarrow N)_{113} \\
\text { b) }(N \rightarrow I)_{131}\end{array}$ & & \\
\hline
\end{tabular}

1) Polarized Optical Microscopy.

2) Corresponds to the figure no. of polarized optical micrograph for the transition.

3) Corresponds to the figure no. of DSC scan for the transition.

4) $T_{m}$ and $T_{c}$ are the melting and crystallization temperature.

5) $(\mathrm{K} \rightarrow \mathrm{N})_{x}$ and $(\mathrm{N} \rightarrow \mathrm{I})_{y}$ refers to crystal to nematic transition and nematic to isotropic transition at $x$ and $y$ temperature, respectively. 
compound (Fig. 1a). In the second biphenyl (5-5) compound (Fig. 1b), intramolecular hydrogen bonds are likely to be present due to the interaction of unsubstituted hydroxyl groups. There are also intermolecular and intramolecular hydrogen bonds due to interaction between the hydroxyl and the methoxy oxygen groups (30). These interactions will make the second biphenyl compound stiffer than the first one.

The 1,4-disubstituted benzene rings usually form the standard building-block from which the majority of the liquidcrystal molecular structures are derived (20). The $\beta$-ether model compound is also based on a similar building-block. It consists of 3 benzene rings which make the molecule long, narrow and rigid. But the alkyl ether bridging units impart some molecular flexibility. This is the most likely reason why this compound exhibits an unstable liquid crystalline behavior as the nematic phase was observed only during the heating run.

The phenyl coumaran compound consists of 2 phenyl rings and a five membered ring as the bridging group. This makes the structure very rigid, and the compound very clearly exhibits nematic phase transition.

These observations demonstrate that all dimeric ligninlike model compounds are capable of forming thermotropic liquid crystalline materials as all exhibit nematic phase transitions within a narrow temperature range. An enrichment of a fluid phase with such structures may therefore be expected to yield highly organized systems. Fiber spinning 
from such systems may produce oriented fibers, which can be carbonized to yield high modulus carbon fibers.

2. Lignin:

Table I represents the chemical and molecular characteristics of lignin. Lignin, when heated, flows at about $110^{\circ} \mathrm{C}$. At $200^{\circ} \mathrm{C}$, clear anisotropic droplets are observed (Fig. 10) which persist for ca. 1 hour. As the temperature is raised to $300^{\circ} \mathrm{C}$, these droplets gradually fade and disappear at $400^{\circ} \mathrm{C}$. Lignin gets completely carbonized above $440^{\circ} \mathrm{C}$.

Lignin is an amorphous, aromatic biopolymer consisting of phenylpropane units $\left(\mathrm{C}_{6}-\mathrm{C}_{3}\right)$ which are linked together by ether and carbon-carbon bonds (31). Many of the units are trifunctional which makes lignin a 3-dimensional non-meltable polymer (Fig. 11). Experimental evidence on lignin monolayers suggests that the structure is moderately flexible, possibly elongated and cross-linked (32). There is no change in monolayer film thickness with molecular weight which advocates two-dimensional, planar conformation.

During the hot-stage experiments, anisotropic droplets observed at approximately $200^{\circ} \mathrm{C}$ in the lignin molecule suggest that planar conformation of the lignin structure favors formation of anisotropy to a certain extent, but it remains incomplete possibly due to 3-dimensional molecular diversity and a partially cross-linked structure. Molecular breadth is considered an important feature in the correlation between 


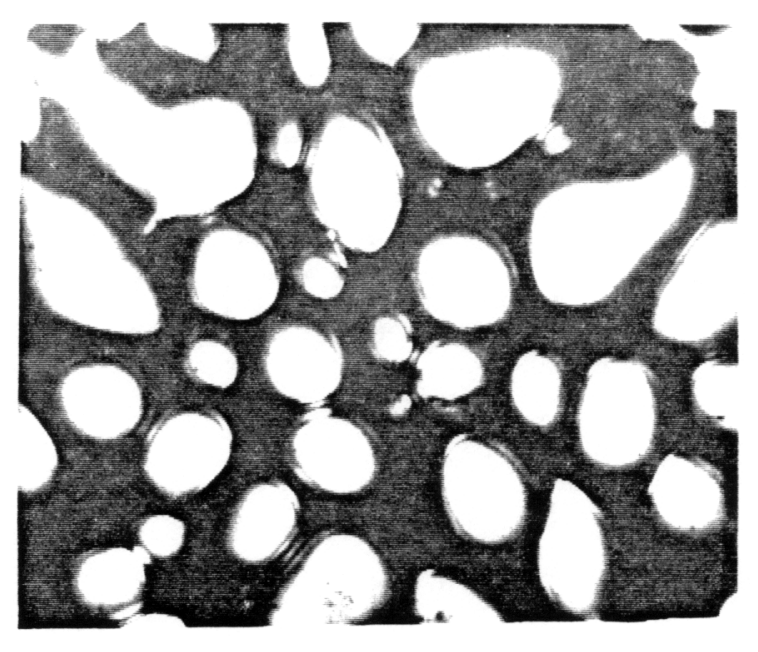

$50 \mu \mathrm{m}$

Figure 10. Polarized optical micrograph of lignin at $200^{\circ} \mathrm{C}$ when heated on hotstage. 


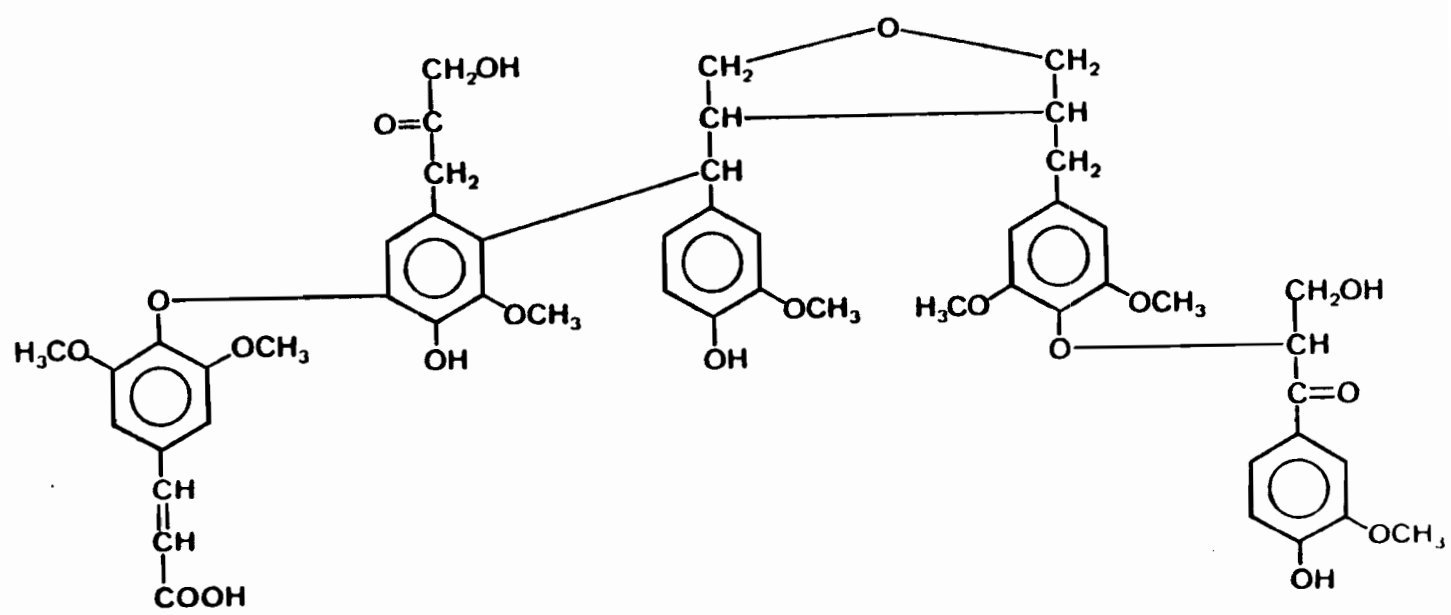

Figure 11. Chemical structure of organosolv lignin. 
thermal stability and chemical constitution of mesophases (33). An increase in molecular breadth would normally reduce the anisotropy of molecular shape, thus depressing the stability of both nematics and smectics. Lignin is a "broad" molecule (large diameter) which reduces the anisotropy (i.e., L/D ratio) of the structure. Low molecular weight lignin fractions should form liquid crystalline systems as the structures will be more well-defined and narrow. The observation of liquid crystalline behavior in the dimeric lignin-model compounds supports this hypothesis.

3. Pitch $(A-240)$ :

Table II shows typical properties of A-240 pitch. When pitch is heated on the hot-stage, optically anisotropic spheres are observed which begin to develop at $120^{\circ} \mathrm{C}$, and their formation gradually increases by coalescence as the temperature rises to $425^{\circ} \mathrm{C}$. Figs. 12 (a) and (b) show the micrographs of the pitch sample that was kept at $425^{\circ} \mathrm{C}$ for 1 hour and 2 hours, respectively. A clear indication of carbonaceous mesophase formation and molecular organization is indicated which becomes uniform with time. Textures of this nature for $A-240$ have been reported earlier when in situ observations were made by optical microscopy $(34,35)$.

Figs. 13 (a) and (b) show the micrographs of the pitch which was heated in a pyrex jar and then quenched to room temperature from $425^{\circ} \mathrm{C}$ after a residence time of 1 and 2 
(a)

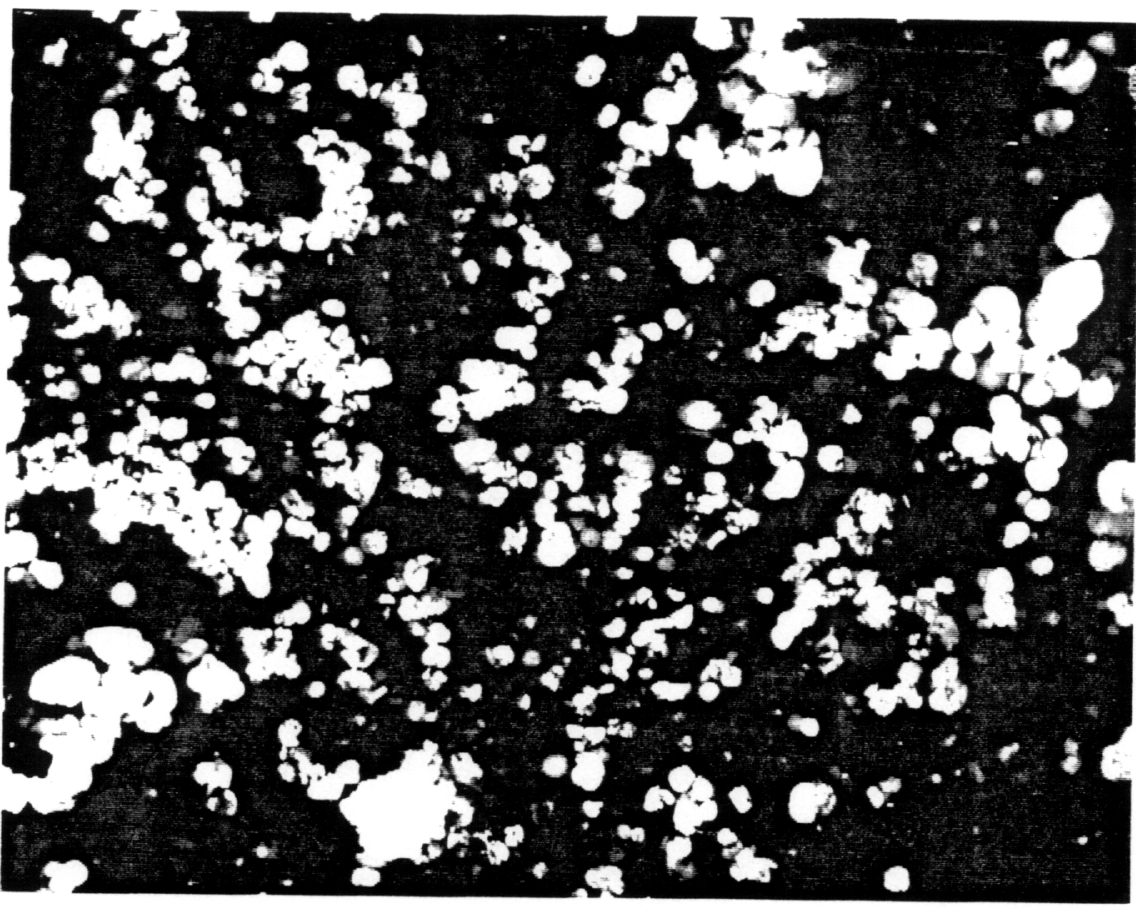

(b)

$$
50 \mu \mathrm{m}
$$

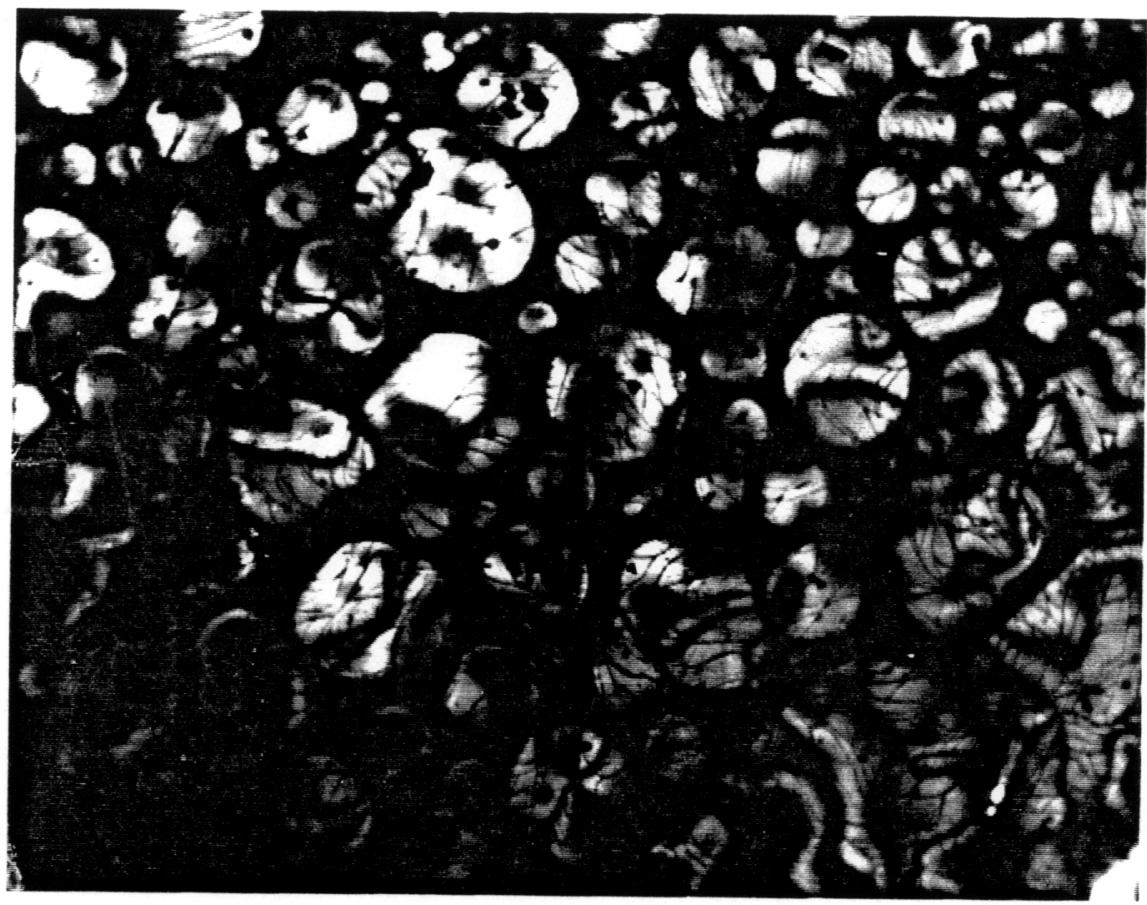

Figure 12. Polarized optical micrographs of A-240 pitch when heated isothermally at $425^{\circ} \mathrm{C}$ on hot-stage as a function of time; (a) 1 hour (b) 2 hours. 
(a)

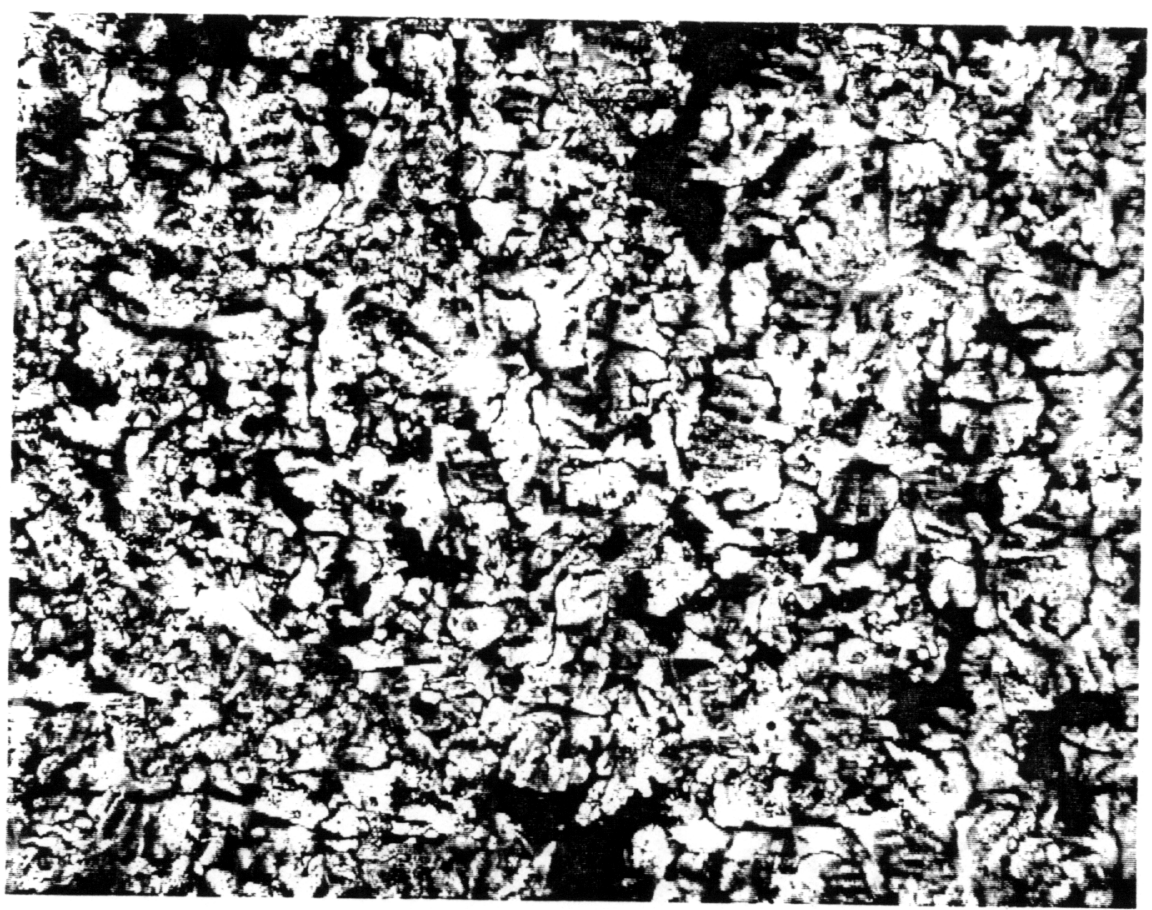

(b)

$50 \mu \mathrm{m}$

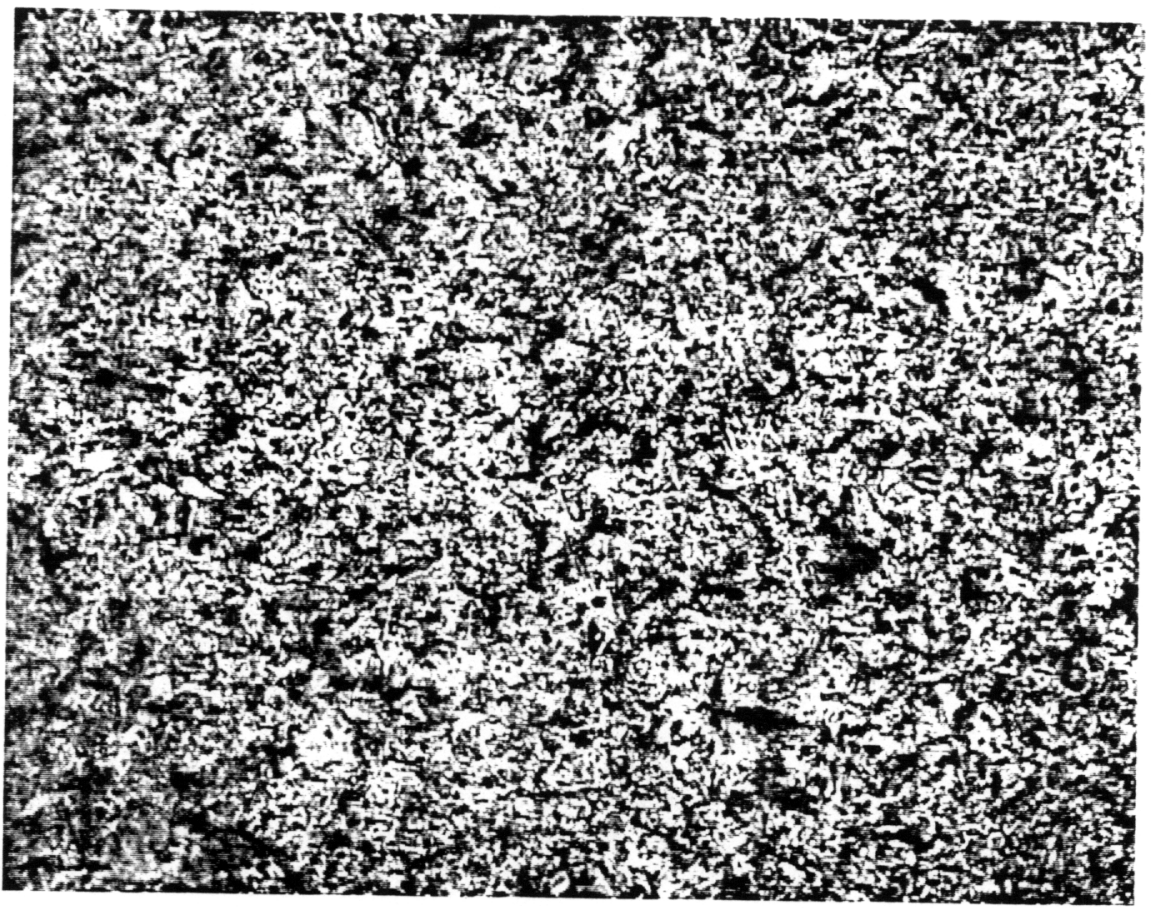

Figure 13. Polarized optical micrographs of quenched A-240 pitch at room temperature after isothermal heat-treatment at $425^{\circ} \mathrm{C}$ with varying residence time (a) 1 hour (b) 2 hours. 
hours, respectively. After 2 hours, the mesophase structure is fibrous and wedge disclinations are apparent (Fig. 13b). This type of morphology was observed when the structure of carbonaceous mesophase was investigated by conventional metallographic methods $(6,36,37)$. There is a difference in the morphologies between Figs. 12 and 13. This maybe due to continuous stirring of the pitch while being heated which makes the system into a homogenous carbonaceous mesophase. 4. Blends of Pitch $(A-240)$ and Lignin:

The blend of pitch and lignin containing $25 \%(w / w)$ of lignin was heated on the hot-stage. The lignin portion of the blend begins to flow at $110^{\circ} \mathrm{C}$, and spheres are seen to develop at $120^{\circ} \mathrm{C}$ as pitch molecules are undergoing molecular organization. Pitch and lignin seem to form a compatible blend at lower temperature. At $200^{\circ} \mathrm{C}$, droplets of lignin are seen to develop, and there is a simultaneous growth of mesophase formation from pitch. Fig. 14 is the micrograph of the blend which was kept at $425^{\circ} \mathrm{C}$ for 2 hours. The figure clearly shows that there is mesophase formation due to $75 \%$ pitch, but the development of a homogenous, anisotropic fluid is prevented by the premature carbonization of lignin as, at higher temperature, lignin and pitch remain as incompatible blends. Similar observations were seen for the blend containing $50 \%$ $(w / w)$ and $75 \%(w / w)$ lignin, respectively.

From these observations of blends of pitch and lignin 


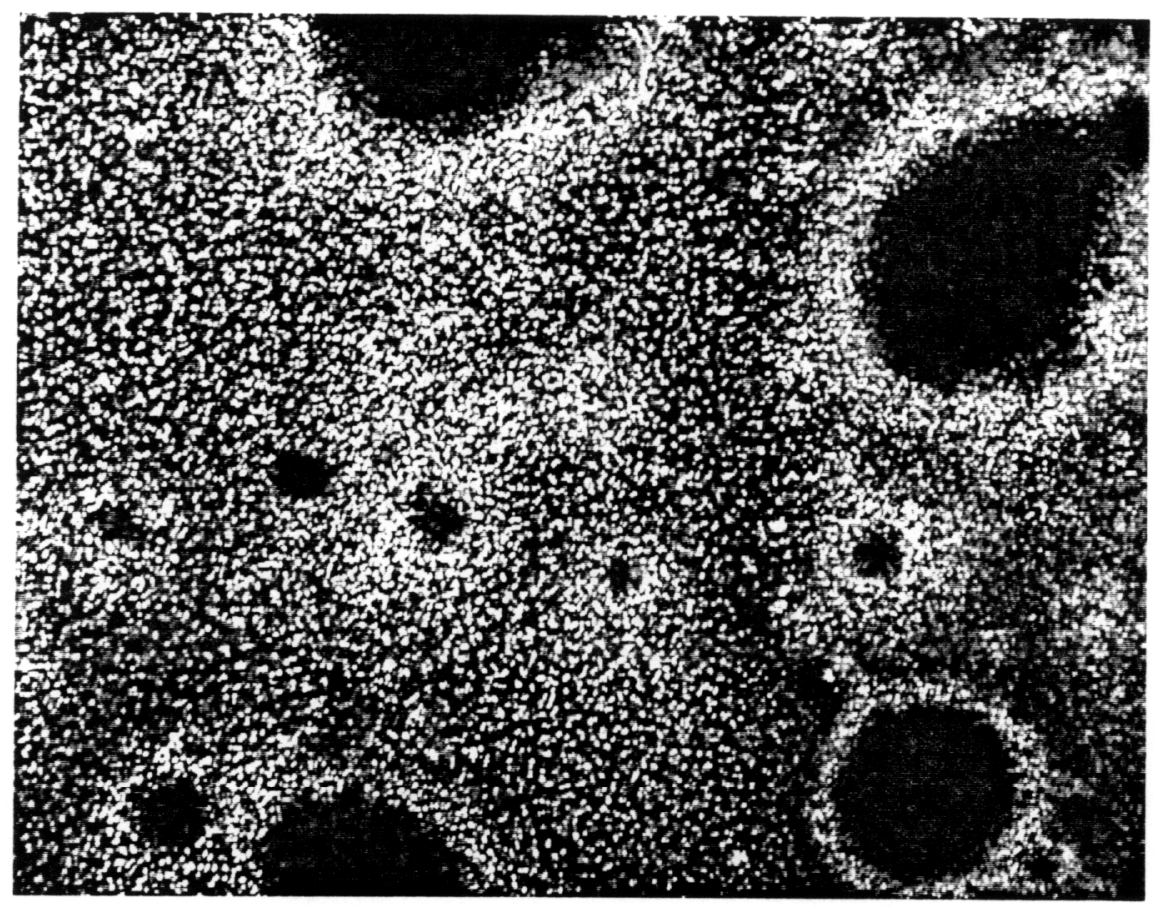

$50 \mu \mathrm{m}$

Figure 14. Polarized optical micrograph of the blend of A-240 pitch and lignin containing $25 \%(\mathrm{w} / \mathrm{w})$ lignin after isothermal heating at $425^{\circ} \mathrm{C}$ for 2 hours on hot-stage. 
containing varying amounts of lignin, it is clear that lignin interferes with the development of a homogenous carbonaceous mesophase of pitch due to premature carbonization. These observations are supported by the fact that carbonaceous mesophase sphere growth and coalescence is hindered by the presence of insoluble carbon particles (38). Flow can be induced in lignin by the process of hydrocracking $(18,19)$. This route produces lignin pitch which flows and is processable at high temperature. However, lignin needs to be degraded (just as coal) before this can happen.

5. Hydroxypropyl Lignin (HPL):

The chemical and molecular characteristics of HPL are shown in Table I. HPL is obtained by reacting propylene oxide with the hydroxyl groups of lignin (24) (Fig. 15). This chemical modification improves the flow property of lignin without degradation. On heating, HPL flows into a homogenous liquid at about $80^{\circ} \mathrm{C}$ which ultimately gets carbonized at higher temperature $\left(>400^{\circ} \mathrm{C}\right)$ without any signs of mesophase formation. Propoxylation may not increase the molecular flexibility as the possibility of intramolecular hydrogen bonding, and subsequent conformational stability would be retained. The flow properties of lignin improve by propoxylation, but the molecular breadth (diameter) will most likely hinder mesophase formation in HPL. 


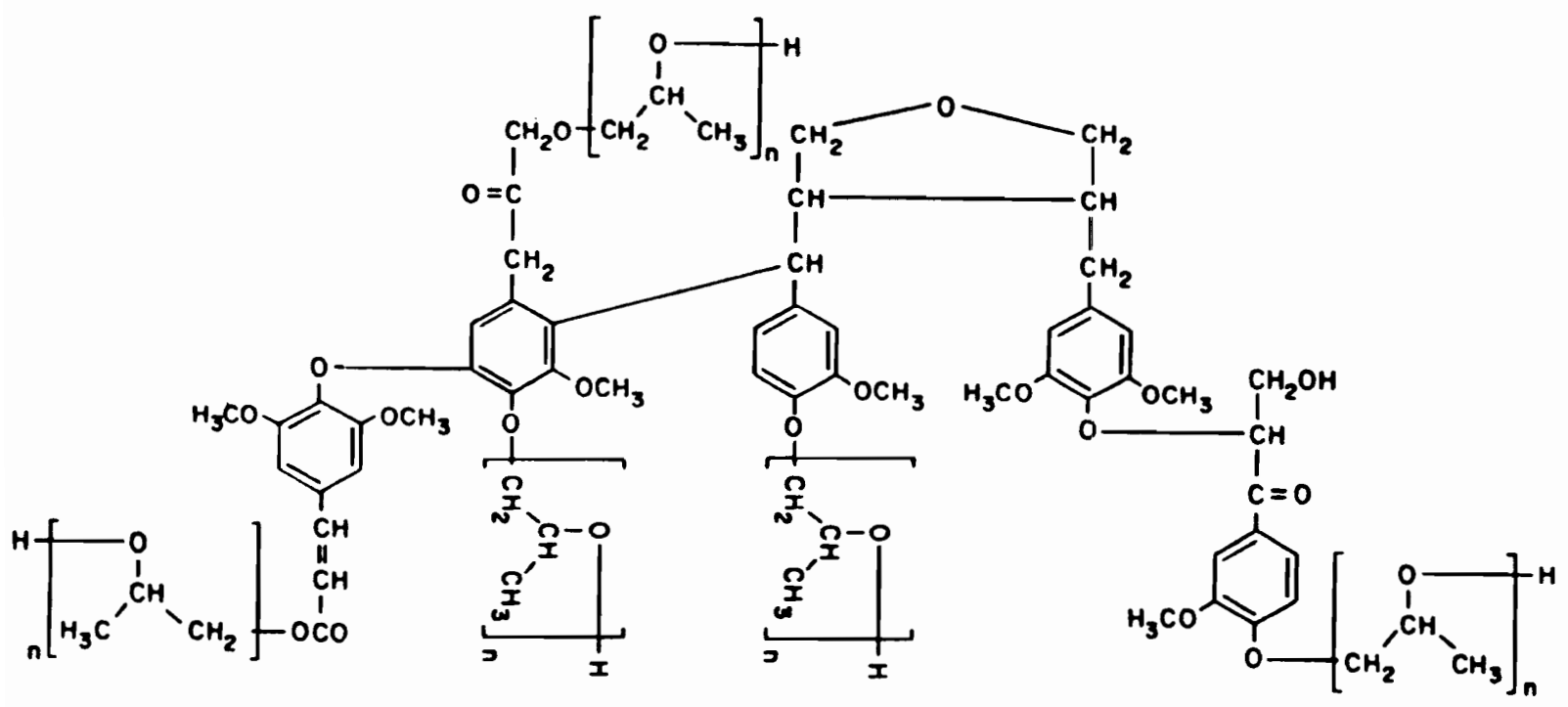

Figure 15. Schematic representation of hydroxypropyl (organosolv) lignin derivative $(n=1)$. 
6. Hydroxypropyl Lignin Pitch:

Attempts were made to improve the flow behavior of HPL by hydrocracking and heat-treatment. Table I shows the molecular characteristics of hydrocraked and heat-treated HPL. It is observed that, on comparing with HPL, $\left\langle\mathrm{M}_{\mathrm{p}}\right\rangle$ and $\left\langle\mathrm{M}_{\mathrm{w}}\right\rangle$ values for hydro-cracked lignin decrease due to degradation whereas the values for heat-treated HPL increase due to removal of lowmolecular thermally unstable compounds and condensation (Fig. 16) .

${ }^{13} \mathrm{C}$ NMR scans were carried out to verify the chemical changes taking place during hydrocracking and heat-treatment. Figs. 17 (a) - (c) shows the NMR of HPL, hydrocracked HPL and heat-treated HPL, respectively. In Fig. 17a, the peaks at $\sim 20$ $\mathrm{ppm}$, 64-68 ppm and 72-80 ppm are from the $\mathrm{CH}_{3}, \mathrm{CH}$, and $\mathrm{CH}_{2}$ groups, respectively, of propylene oxide. These peaks almost disappear in Figs. $17 \mathrm{~b}$ and 17c. However, the methoxyl peak ( $\sim 56 \mathrm{ppm})$ from lignin exists even after heat-treatment. The peaks from $100-120 \mathrm{ppm}$ are assigned to $\mathrm{c}-2, \mathrm{c}-5$ and $\mathrm{c}-6$ in the aromatic nucleus of lignin. These peaks are present with strong intensity in hydrocracked HPL (Fig. 17b), and they decline somewhat in heat-treated HPL. These NMR scans indicate that upon hydrocracking and heat-treatment, there is cleavage of propylene oxide from HPL, and HPL pitch contains heavily condensed structures with an abundance of aromatic moieties. The molecular weight data supports this hypothesis. 


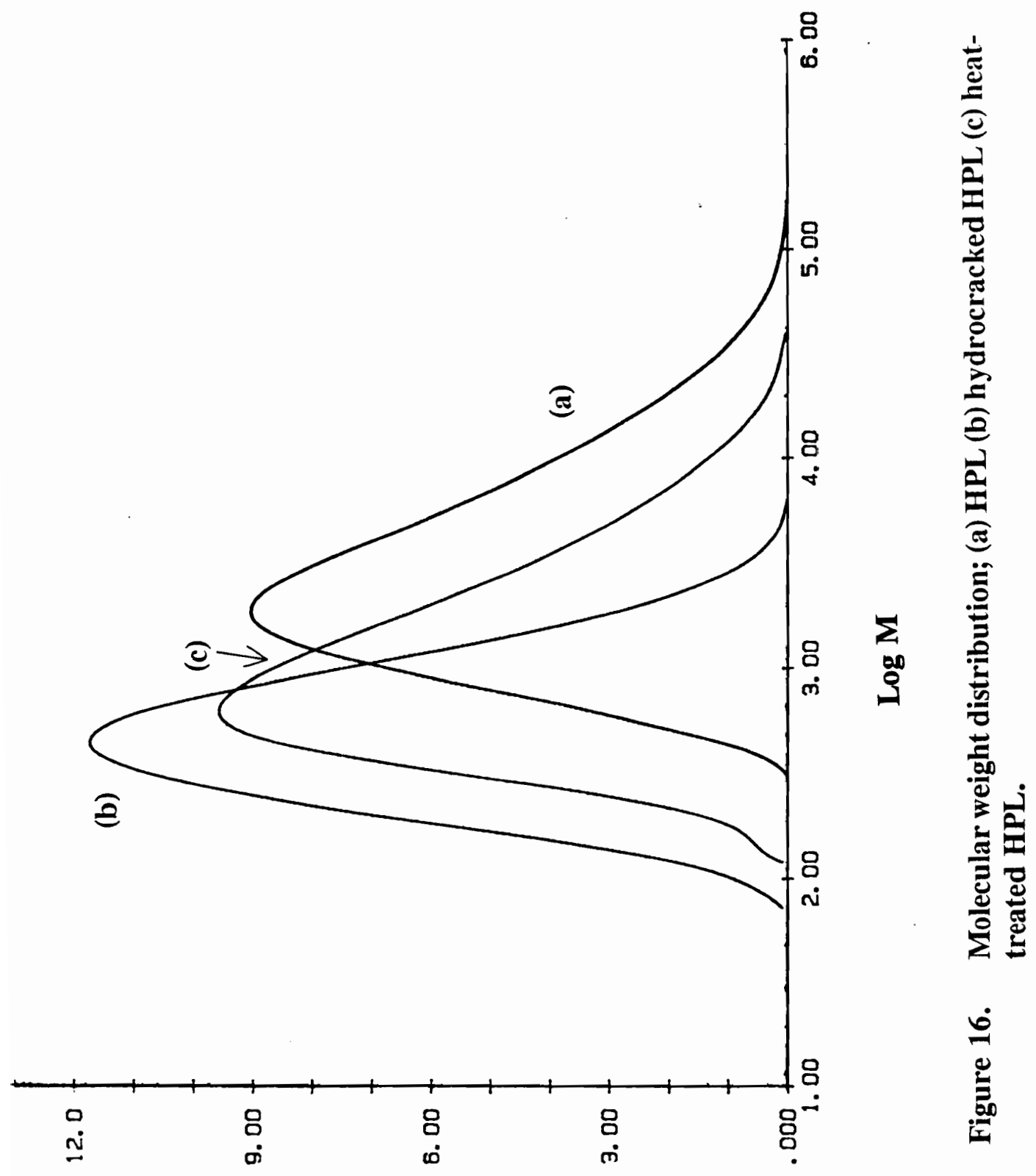

I-0I $\times(\text { (W 80I) })^{\mathbb{a}} M$ 


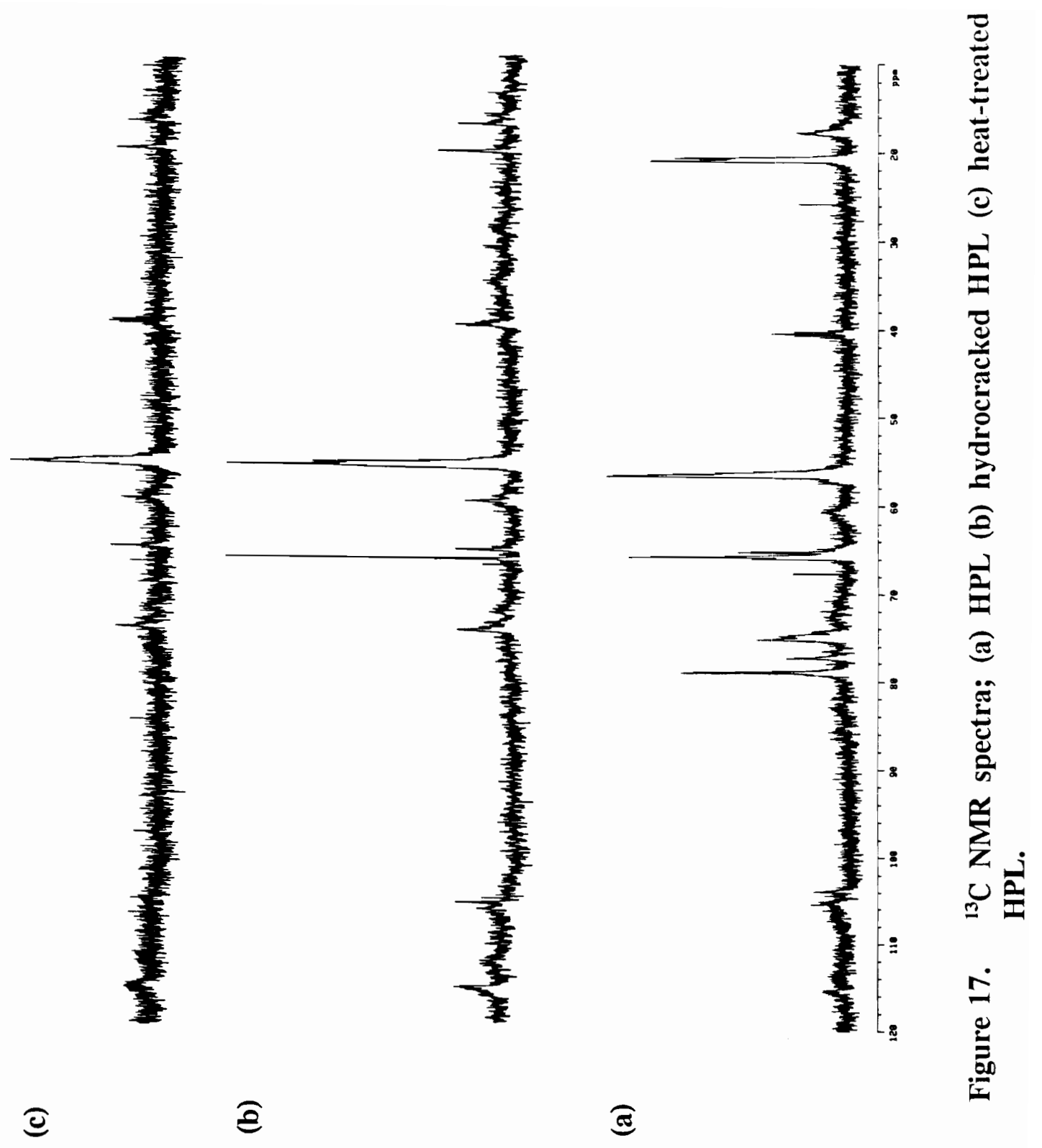


7. Mechanical Properties:

Table IV compares the mechanical properties of carbon fibers from PAN, mesophase-pitch, rayon, Kayacarbon (lignin/PVA), lignin pitch and HPL pitch. The fibers from PAN and mesophase pitch are comparable, but the fibers from rayon (without hot-stretching) were lower than the commercial fibers and are comparable to lignin pitch carbon fibers. HPL pitch fibers were, however, inferior to lignin pitch fibers.

8. Scanning Electron Microscopy:

The surface morphologies of green fibers and carbon fibers from heat-treated HPL pitch are shown in Figs. 18 (a) and 18 (b), respectively. Green fibers are quite smooth except for some particulate depositions on the surface. Carbon fibers from HPL pitch are extremely smooth, and the absence of any structural defects on the surface produces fibers which have a higher tensile strength than Kayacarbon (Table IV). 9. Transmission Electron Microscopy:

The carbon fibers from HPL were extremely brittle as they shattered during the microtoming process. The cross-section of the fibers did not show any layered arrangement of carbon lattice fringes along the fiber axis at high magnifications. This indicates that the fibers are amorphous. Fig. 19 is the TEM of the cross-section of the carbon fiber at a lower magnification. The lack of orientation, primarily, is responsible for the inferior mechanical properties. 
Table IV. Physical Properties of Carbon Fibers (CF) from Different Precursors.

\begin{tabular}{|c|c|c|c|c|}
\hline CARBON FIBER TYPE & $\begin{array}{l}\text { Tensile } \\
\text { Strength } \\
\text { (GPa) }\end{array}$ & $\begin{array}{l}\text { Tensile } \\
\text { Modulus } \\
\text { (GPa) }\end{array}$ & $\begin{array}{l}\text { Diameter } \\
(\mu \mathrm{m})\end{array}$ & $\begin{array}{c}\varepsilon_{\mathrm{b}} \\
(q)\end{array}$ \\
\hline $\begin{array}{l}\text { Polyacrylonitrile } \\
(\text { PAN })^{1}\end{array}$ & 3.65 & 231 & $5-7$ & $1.3-1.8$ \\
\hline Mesophase Pitch & 2.1 & 520 & 10 & 0.4 \\
\hline $\begin{array}{l}\text { Rayon } \\
\text { After Hot-stretching }\end{array}$ & $\begin{array}{l}0.4-0.7 \\
3.1 \\
\end{array}$ & $\begin{array}{l}20-55 \\
620 \\
\end{array}$ & & \\
\hline $\begin{array}{l}\text { Kayacarbon } \\
\text { (i) } 1500^{\circ} \mathrm{C}^{4)} \\
\text { (ii) } 2000^{\circ} \mathrm{C} \\
\end{array}$ & $\begin{array}{l}0.25 \\
0.29\end{array}$ & $\begin{array}{l}27.0 \\
24.0\end{array}$ & $\begin{array}{l}12-14 \\
12-14\end{array}$ & $\begin{array}{l}1.0 \\
1.0\end{array}$ \\
\hline $\begin{array}{l}\text { Lignin-Pitch } \\
\text { (i) Process I } \\
\text { (ii) Process II }\end{array}$ & $\begin{array}{l}0.66 \\
0.52\end{array}$ & $\begin{array}{l}40.7 \\
48.9\end{array}$ & $\begin{array}{l}7-9 \\
21-26\end{array}$ & $\begin{array}{l}1.63 \\
1.06\end{array}$ \\
\hline HPL-Pitch & 0.33 & ----- & $6-15$ & ------ \\
\hline
\end{tabular}

1) T-300, Thornel Product Information, Amoco Performance Products.

2) P-75S, Thornel Product Information, Amoco Performance Products.

3) From Reference No. 1 .

4) Carbonization Temperatures. 
(a)

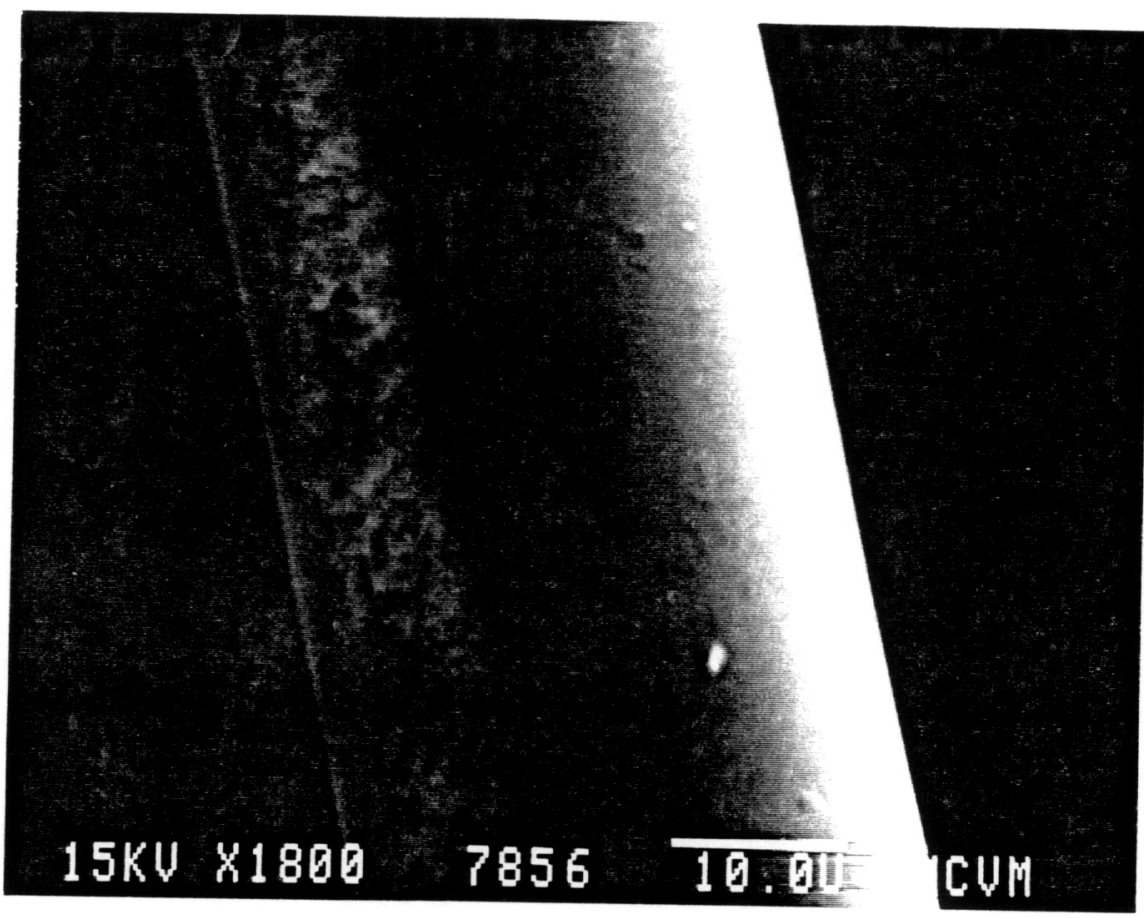

(b)

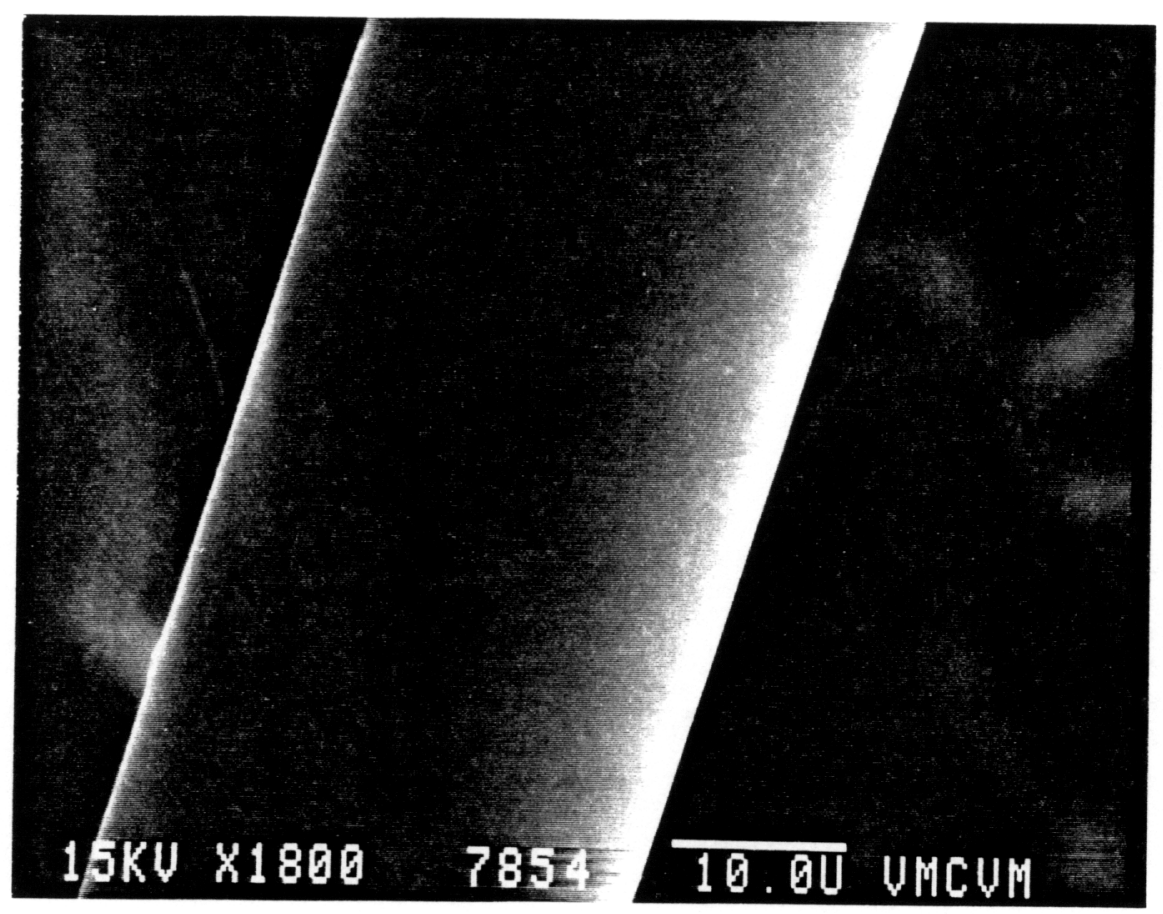

Figure 18. Scanning electron micrographs of the surface of fibers spun from heattreated HPL; (a) green fibers (b) carbon fibers. 


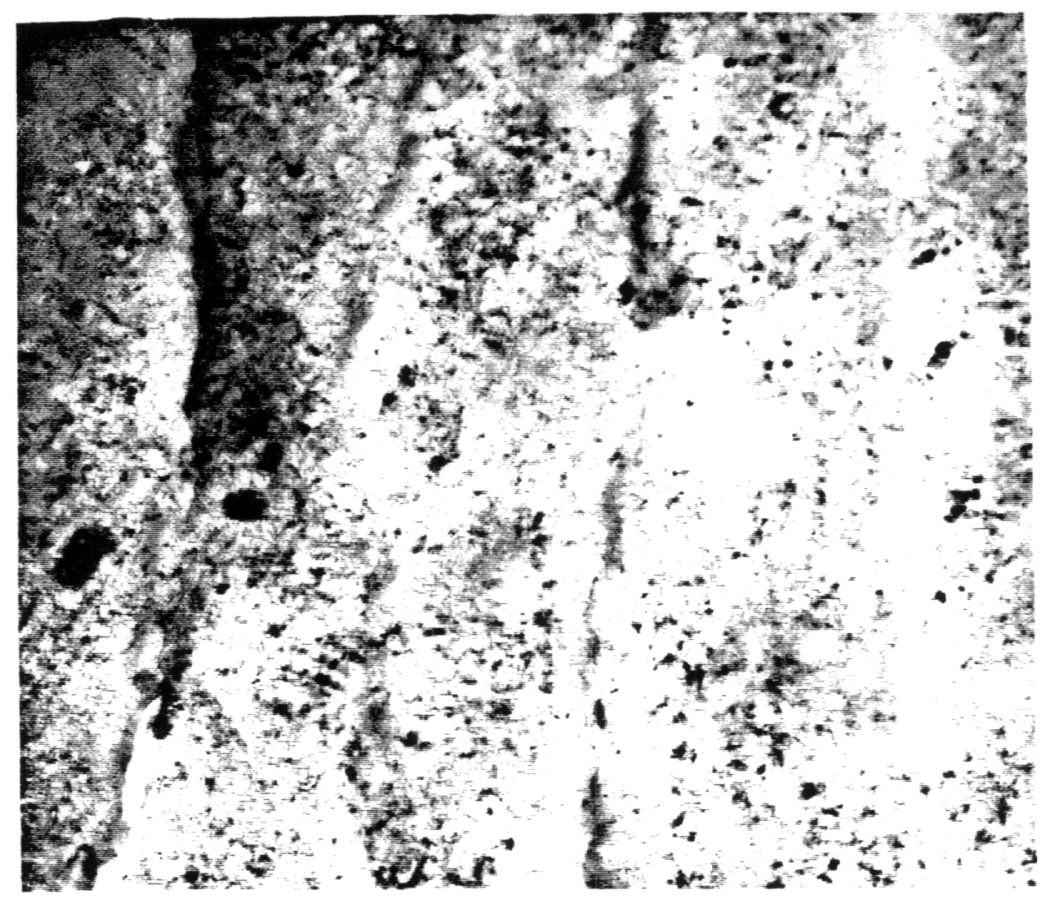

$100 \mathrm{~nm}$

Figure 19. Transmission electron micrograph of the cross-section of carbon fibers from heat-treated HPL. 


\section{CONCLUSIONS}

1. Dimeric lignin-like model compounds studied here are capable of developing thermotropic liquid-crystalline materials as observed by hot-stage optical microscopy and differential scanning calorimetry.

2. Lignin, having chemistry similar to that of pitch, appears to posses the necessary ability for molecular organization during heating while in fluid phase. Restraints are recognized for the formation of organized fluids which are related to significant non-uniformity in terms of responding to heating (i.e., the presence of solid particulates which never undergo fluidization, and which carbonize and char while surrounded by an anisotropic solution). If it were possible to eliminate those substances that resist fluidization during heating and have structures similar to the lignin-like model compounds, it is possible that lignin could then a display pitch-like carbonaceous mesophase.

3. The degradative process of hydrocracking and heattreatment eliminates the active functional groups which reduces molecular interactions and increases fluidity in lignin and hydroxypropyl lignin. Carbon fibers from hydroxylpropyl lignin pitch have a defect-free surface morphology, but due to the lack of molecular orientation, the properties are inferior to those of other carbon 
fibers. It maybe possible to improve the molecular orientation by fiber spinning from blends with mesophase pitch. The carbon fibers from lignin and HPL are classified as general purpose carbon fibers (GPCF) due to their inferior mechanical properties. 


\section{REFERENCES}

1. J. Riggs, "Carbon Fibers", in Encyclopedia of Polymer Sci. Engr., Vol. 2, John Wiley \& Sons, NY, 640-685, 1985.

2. P. J. Goodhew, A. J. Clarke and J. E. Bailey, "A Review of Fabrication and Properties of Carbon Fibers", J. Mat. Sci.\& Engr., 17, 3-30, 1975.

3. J. B. Donnet and O. P. Bahl, "Carbon Fibers", in Encyclopedia of Phy. sci. and Tech., Vol. 2, Academic Press, 515-529, 1987.

4. J. D. Brooks and G. H. Taylor, "The Formation of some Graphitizable Carbons", Chemistry and Physics of Carbon, Vol. 4, 243-286, 1968.

5. L. S. Singer, "The Mesophase in Carbonaceous Pitches, Faraday Discuss. Chem. Soc., 79, 265-272, 1985.

6. J. E. Zimmer and J. L. White, "Disclination structures in the Carbonaceous Mesophase", Advances in Liquid Crystals, Vol. 5, 157-213, 1982 .

7. S. Otani, "Carbonaceous Mesophase and Carbon Fibers", Mol. Cryst. Liq. Cryst., 63, 249-264, 1981.

8. S. Chandrasekhar, B. K. Sadashiva and K. A. Suresh, "Liquid Crystals of Disc-like Molecules", Pramāna, 9(5), $471-480,1977$. 
9. H. Gasparoux, "Carbonaceous Mesophase and Disc-like Molecules", Liquid Crystals of one and Two Dimensional order, springer Series in Chemical Physics, Eds. W. Helfrich and G. Hoppke, 373-395, 1980.

10. J. Billard, "Discotic Mesophases: A Review", Iiquid Crystals of One and Two Dimensional order, Springer Verlag Series in Chemical Physics, Eds. W. Helfrich and G. Hoppke, 373-382, 1980 .

11. H. Gasparoux, C. Destrade and N. H. Tinh, "Nematic Disclike Liquid Crystal: a model for the carbonaceous mesophase", 15th Biennial Conference on Carbon (Extended Abstracts), $176-177,1981$.

12. Y. Fukuoka, "Carbon Fiber Made from Lignin (Kayacarbon)", Jap. Chem. Quart., 5(3), 63-66, 1969.

13. "Kayacarbon", Manufacturer's Brochure, Nippon Kayaku Co. Ltd. , 1970.

14. D. J. Johnson, I. Tomizuka and O. Watanabe, "Fine Structures of Lignin-Based Carbon Fibers", Carbon, 13 (4), $321-325,1975$.

15. D. J. Johnson and I. Tomizuka, "The Fine structure of Lignin- and Pitch-Based Carbon Fibers", Plastics \& Polymers Conference Supplement No. 6, 20-24, 1974.

16. I. Tomizuka, T. Kurita, Y. Tanaka and O. Watanabe, "Voids in the Carbon Fibers Produced from Lignin and PVA", Yogyo-Kyokai-Shi, $79(12), 460-469,1971$. 
17. I. Tomizuka and D. J. Johnson, "Microvoids in Pitch-Based and Lignin-Based Carbon Fibers as Observed by X-Ray Small-Angle Scattering", Yogyo-Kyokai-Shi, 86(4), 186192,1978 .

18. K. Sudo and K. Shimizu, "Carbon Fibers From Lignin", Fourth Int. Symp. on wood and Pulp Chem., 1, 101-104, 1987.

19. K. Sudo, "Carbon Fibers From Lignin", Presentation at the ACS Conference, Cellulose Division, Toronto, 1988.

20. Hans Kelker and Rolf Hatz, Handbook of Iiquid crystals, Verlag Chemie, 34-41, 1980 .

21. K. Kasuga and H. Hatakeyama, "Synthesis and Liquid Crystalline Properties of Homologous Series Having Guaiacyl Structure as Central Linkage: 4, 4'-n-Alkoxy-3'Oxybenzoic Acid", Cellulose Chem. Technol., 18, 469-477, 1984 .

22. K. Kasuga and H. Hatakeyama, "Synthesis and Thermal Properties of Cholesteryl 4-n-Alkoxy-3-methoxybenzoates", Mol. Cryst. Liq. Cryst., 147, 1-14, 1987.

23. K. Kasuga, H. Hatakeyama and T. Hatakeyama, "Liquid Crystals Having Lignin-Related structures", Chapter 50, Cellulose, structural and Functional Aspects, Eds., J. F. Kennedy, G. O. Phillips and P. A. Williams, Ellis Horwood Series in Poly. Sci. and Tech., John Wiley \& Sons, 385$390,1989$. 
24. L. C.-F. Wu and W. G. Glasser, "Engineering Plastics from Lignin. I. Synthesis of Hydroxypropyl Lignin", J. Appl. Polym. Sci., 29(4), 1111-1123, 1984.

25. J. Gierer and O. Lindberg, Acta Chem. Scand. Series B, $34,161-170,1980$.

26. Y. D. Park, Y. Korai and I. Mochida, "Roles of Extraction in the Preparation of Mesophase Pitch", 17th Biennial Conference on Carbon (Extended Abstracts), 151-152, 1985.

27. Y. D. Park, Y. Korai and I. Mochida, "Preparation of Anisotropic Pitch by Carbonization Under Vacuum", 17th Biennial Conference on Carbon (Extended Abstracts), 153154,1985 .

28. W. Kowbel and J. Don, "The Microstructure Study of Mesophase by Microscopic Techniques", 18th Biennial Conference on Carbon (Extended Abstracts), 286-287, 1987.

29. W. G. Glasser, V. Davé and C. E. Frazier, "Molecular Weight Distribution of (Semi-) Commercial Lignin Derivatives", J. Wood Chem. Technol. (In Press).

30. M. Remko and J. Polčin, "MO Investigations on Lignin Model Compounds. XII. PCILO Calculations of Conformational structure and Hydrogen Bonds in 2,2'Dihydroxybiphenyl, 2,2'-Dimethoxybiphenyl and 2,2'Dihydroxy-3'-methoxybiphenyl", Int. J. of Res. in Phy. Chem. and Chem. Phys. (Z. Phy. Chem. Neue Folge), 120, 18,1980 . 
31. W. G. Glasser and S. S. Kelley, "Lignin", in Encyclopedia of Polymer Sci. and Engr., Vol. 8, 2nd Edition, John Wiley \& Sons, 795-852, 1987.

32. P. Luner and U. Kempf, "Properties of Lignin Monolayers at the Air-Water Interface", Tappi, 53(11), 1970.

33. G. W. Gray, B. Jones and F. Marson, "Effect of halogen substitution on the mesomorphism of trans-p-nalkoxycinnamic acids", J. Chem. Soc., 1417, 1956.

34. F. Kfoury, H. Gasparoux, P. Delhaes, F. Albugues and Y. Grenie, "Mesophase Formation from Different Pitches: Texture Characterization, Magnetic Anisotropy and Rheological Properties", 16th Biennial Conference on Carbon (Extended Abstracts), 80-81, 1983.

35. K. J. Chen and R. J. Diefendorf, "Preferred Orientation of Mesophase Pitch", 18th Biennial Conference on Carbon (Extended Abstracts), 292-293, 1987.

36. J. Dubois, C. Agache and J. L. White, "The Carbonaceous Mesophase Formed in the Pyrolysis of Graphitizable Organic Materials", Metallography, 3, 337-369, 1970.

37. J. L. White and J. E. Zimmer, "Disclination Structures in Carbonaceous Mesophase and Graphite", Surface and Defect Properties of Solids", 5, 16-35, 1976.

38. H. Honda, H. Kimura, Y. Sanada, S. Sugawara and T. Furuta, "Optical Mesophase Texture and X-ray Diffraction Pattern of the Early-stage Carbonization of Pitches", Carbon, 8, 181-189, 1970. 
9.0 LIQUID CRYSTALLINE PHASE FORMATION IN CONCENTRATED TRI-O- $\alpha$-NAPHTHYLMETHYL CELLULOSE SOLUTIONS. 


\title{
LIQUID CRYSTALLINE PHASE FORMATION IN CONCENTRATED
}

TRI-O- $\alpha$-NAPHTHYLMETHYL CELLULOSE SOLUTIONS.

\begin{abstract}
Tri-0- $\alpha$-naphthylmethyl cellulose was prepared by homogenous phase reaction using $\mathrm{SO}_{2}$-diethylamine (DEA)dimethyl sulfoxide (DMSO) as the solvent system. Observations made by dynamic mechanical spectrometry and by cross polarized optical microscopy revealed liquid crystalline behavior for the concentrated solutions of tri-0- $\alpha$-naphthylmethyl cellulose in dimethylacetamide (DMAc). Experimental and calculated (predicted) critical volume fraction of the derivative, $V_{p}^{c}$, did not show agreement. It is determined that $v_{p}^{c}$ is not greatly affected by bulky substituents on the cellulose backbone.
\end{abstract}




\section{INTRODUCTION}

In recent years, numerous publications have appeared pertaining to liquid crystalline behavior of concentrated solutions of cellulose and cellulose derivatives in different solvent systems. Our research efforts are directed towards understanding the effects of different types of side chain substituents on the onset of liquid crystallinity in cellulose derivative solutions. We have established in our earlier investigations $(1,2)$ that solution anisotropy can be reached at lower concentration in cellulose esters with large and bulky substituents as compared to smaller ones. The general trend was that the experimental critical volume fraction $\left(V_{p}^{c}\right)$ values were lower than the calculated (predicted) values from Flory's theory. We would like to gain further insights on the effects of side chain substituents on liquid crystalline behavior by extending our investigations to homogeneously prepared cellulose ether derivatives.

The objective of the present study is to examine the lyotropic liquid crystalline behavior of tri-0- $\alpha-$ naphthylmethyl cellulose in DMAc in relation to the bulky naphthyl groups on the cellulose backbone.

\section{EXPERIMENTAL}

1. Preparation of Tri-0- $\alpha$-Naphthylmethyl Cellulose:

Tri-0- $\alpha$-Naphthylmethyl cellulose was prepared according to the method described by Isogai et al. $(3,4), 10$ gms of 
Whatman cellulose was dissolved in a nonaqueous $\mathrm{SO}_{2}-$ diethylamine (DEA)-dimethyl sulfoxide (DMSO) solvent mixture, and powdered sodium hydroxide was added to the solution. Chloromethylnaphthylene was added drop-wise to the cellulose solution with heating up to $85^{\circ} \mathrm{C}$. Total reaction time was approximately 14 hours. The following conditions were different from Isogai's methodology. All reactions were carried out under nitrogen, and the solution was poured into 4 liters of distilled water after the reaction ended. The polymer formed a stable emulsion which settled nicely to the bottom of the beaker. The liquid layer was decanted off, and more distilled water was added with vigorous stirring. This procedure was repeated until the liquid layer was colorless. Then the water was decanted off again, and 3 liters of methanol was added to cause precipitation of the polymer. The product was filtered and extracted with methanol in a soxlet for at least 72 hours. The color of the polymer remained bright orange even after extracting the product by acetone for 48 hours. The polymer was dried, dissolved in tetrahydrofuran (THF), and centrifuged to remove any insoluble material. The supernatant was concentrated and then precipitated into methanol. The polymer was finally dried under high vacuum for 24 hours. Yields were typically 70 to $80 \%$ of the theoretical value. The product was soluble in THF, chloroform, dioxane and dimethylacetamide; and insoluble in methanol, ethanol and 
acetone.

2. Determination of Molecular Parameters:

Solution of tri-0- $\alpha$-naphthylmethyl cellulose was prepared in HPLC-grade THF. The molecular parameters were determined using high pressure gel permeation chromatography with a differential viscosity detector (Viscotek Model No. 100) and a differential refractive index (concentration) detector (Waters 410) in sequence. All calculations were based on a universal calibration curve.

3. NMR Spectroscopy:

All NMR spectra were collected on a Varian Unity 400 instrument at 400 and $100 \mathrm{MHz}$ for proton and carbon nuclei, respectively. Samples for proton NMR were prepared in $5 \mathrm{~mm}$ NMR tubes by dissolving 25 to $40 \mathrm{mill}$ igrams of sample in about 0.5 to $0.7 \mathrm{mls}$ of deuterated chloroform. Samples for carbon NMR were prepared in $10 \mathrm{~mm}$ NMR tubes by dissolving 150 to 200 milligrams of sample in about 2.5 to $3 \mathrm{mls}$ of deuterated chloroform. The two-dimensional spectra is a standard Double Quantum Filtered CosY (DQCOSY) run at $40^{\circ} \mathrm{C}$.

4. Preparation of Concentrated Solutions:

A complete range of tri-o- $\alpha$-naphthylmethyl cellulose solutions $[5-50 \%(w / w)]$ were prepared by mechanically mixing known weights of naphthyl cellulose with DMAc at ambient temperatures. The solutions were allowed to equilibrate for 2 weeks prior to analysis. 
Table I. Molecular, Chemical and Thermal Characteristics of Tri-0- $\alpha$-Naphthylmethy 1 Cellulose.

\begin{tabular}{||l|l|}
\hline$\left\langle M_{\mathrm{p}}\right\rangle, \mathrm{gm} /$ mole $\left(10^{-3}\right)$ & 43 \\
\hline$\left\langle\mathrm{M}_{\mathrm{w}}\right\rangle, \mathrm{gm} /$ mole $\left(10^{-3}\right)$ & 150 \\
\hline$\left\langle\mathrm{M}_{\mathrm{w}}\right\rangle /\left\langle\mathrm{M}_{\mathrm{n}}\right\rangle$ & 3.5 \\
\hline $\mathrm{DP}$ & 74 \\
\hline Degree of Substitution & 3.0 \\
\hline MHS-Constant, a & 0.64 \\
\hline Log $\mathrm{K}$ & -3.4 \\
\hline Intrinsic Viscosity $[\eta], \mathrm{dL} / \mathrm{g}$ & 0.64 \\
\hline Melting Temperature $\left({ }^{\circ} \mathrm{C}\right)$ & 94 \\
\hline Heat Capacity, J/gm & 64.7 \\
\hline
\end{tabular}


5. Viscosity Measurements:

These measurements of the solutions below $30 \%$ (w/w) concentration were made at $25^{\circ} \mathrm{C}$ using a Wells-Brookfield Cone/Plate Viscometer at different rotation speeds. Rheometrics Mechanical Spectrometer (RMS 800) was used to determine the rheological properties of $30 \%(\mathrm{w} / \mathrm{w})$ and higher concentrated solutions. The solutions were placed in a parallel-disk geometry. The dynamic mechanical properties were measured at $26^{\circ} \mathrm{C}$ using strain amplitude of $25 \%$ of the value at which the respective sample showed viscoelasticity. The frequency ranged from 0.1 to $100 \mathrm{rad} / \mathrm{sec}$.

6. Polarized Optical Microscopy:

Microscopy experiments were performed on a Zeiss Axioplan Universal Microscope. Small portions of solution were placed between microscope slide and cover slip, and this was examined for birefringence between the cross polarizers of the microscope at room temperature.

\section{RESULTS AND DISCUSSION}

The molecular, chemical and thermal characteristics of tri-o- $\alpha$-naphthylmethyl cellulose are summarized in Table I. The $\mathrm{DP}_{\mathrm{n}}$ of unmodified whatman cellulose (parent polymer) was about 220, but that of the cellulose ether derivative was reduced to 74 . This is due to the degradative nature of the reaction.

Figures 1 (a) and (b) show the proton and two-dimensional 


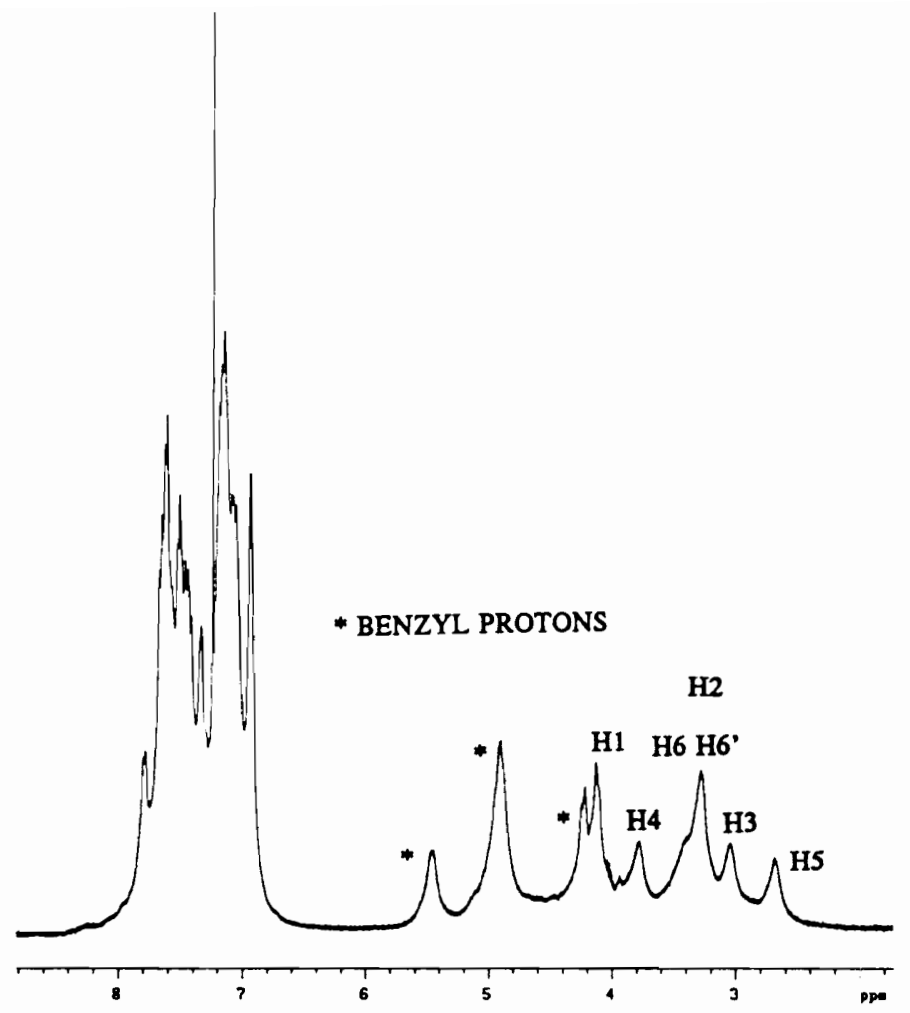

Figure 1. (a) Proton NMR spectra of Tri-O- $\alpha$-naphthylmethyl Cellulose. 


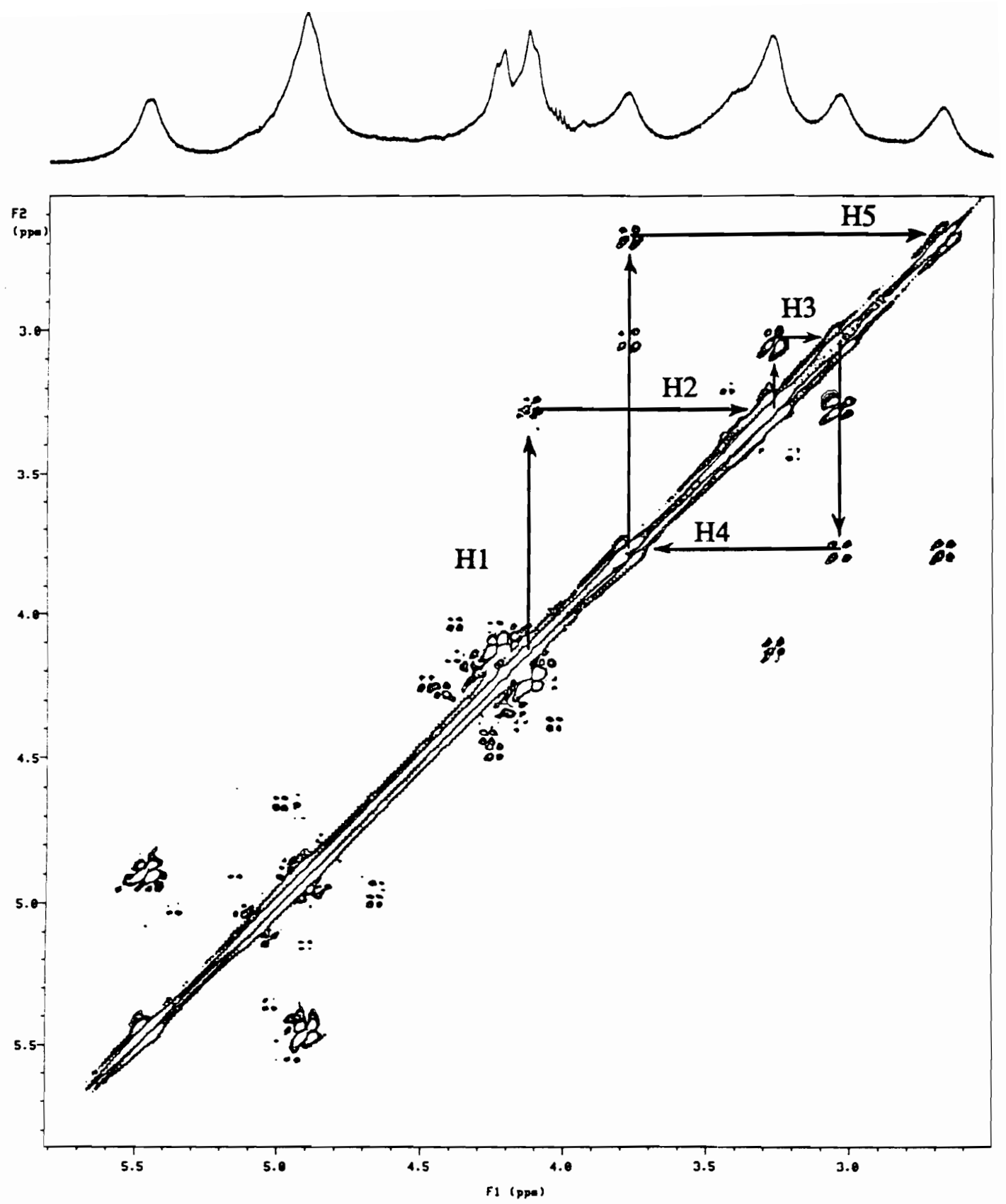

Figure 1. (b) Two dimensional COSY (DQCOSY) spectra of Tri-O- $\alpha$ naphthylmethyl Cellulose. 


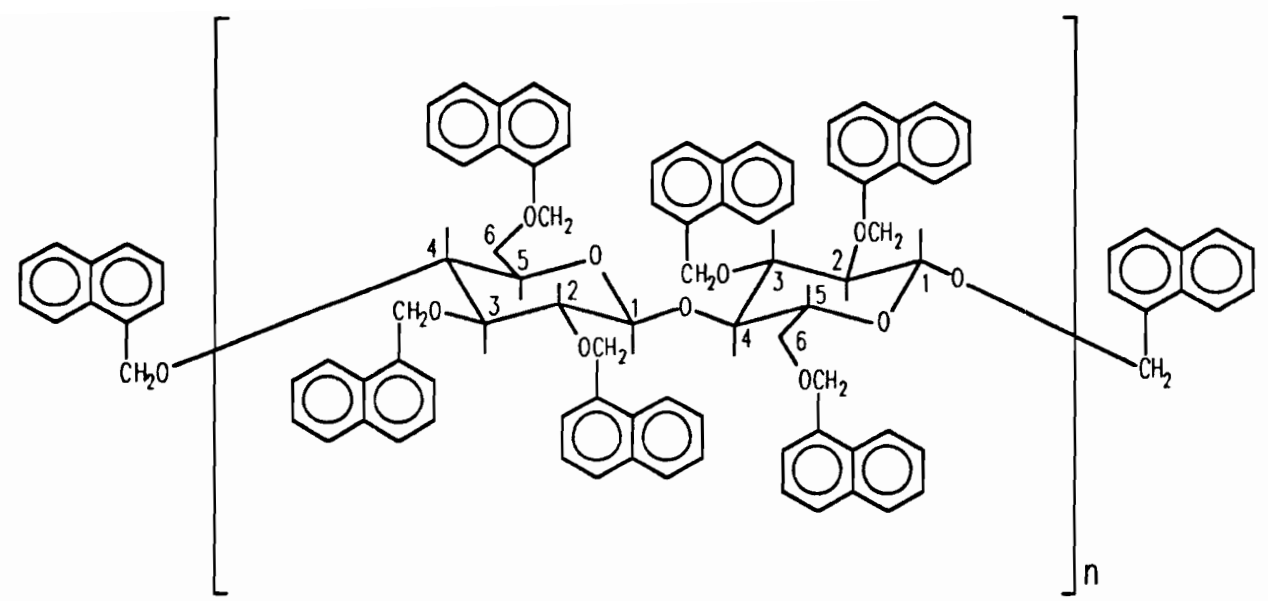

Figure 1. (c) Chemical Structure of Tri-O- $\alpha$-naphthylmethyl Cellulose. 
COSY (DQCOSY) spectra of tri-0- $\alpha$-naphthylmethyl cellulose. Figure 1 (c) show the chemical structure of the derivative. Table II lists the peak assignments of proton signals as indicated on the spectra. Proton assignments are based upon the DQCOSY experiment shown in Figure 1 (b). The point of reference in the DQCOSY experiment is $\mathrm{H} 1$, located at $\delta=4.12$ ppm. The identity of the $\mathrm{H} 1$ proton signal was confirmed by heteronuclear correlation (HETCOR) experiments performed on fluorobenzyl cellulose (5). The HETCOR experiment allows us to correlate the H1 proton signal with the carbon-13 signal of $\mathrm{Cl}$ of the anhydroglucose residue. The carbon-13 signal at position $\mathrm{Cl}$ of cellulose derivatives is known to appear around $101 \mathrm{ppm}$. Therefore a clear correlation between $\mathrm{Cl}$ and $\mathrm{H} 1 \mathrm{gives}$ us the precise starting point for the interpretation of the DQCOSY experiment. Furthermore, the $\mathrm{H} 1$ proton signals of arylmethyl cellulose derivatives have similar chemical shifts. Therefore the HETCOR experiment on fluorobenzyl cellulose provides the identification of the $\mathrm{H} 1$ proton signal for most arylmethyl cellulose derivatives, such as naphthyl cellulose. Consequently, by analogy to fluorobenzyl cellulose, the reference point, $\mathrm{H} 1$, is established for naphthyl cellulose. The remaining backbone proton signals are then easily identified from Figure $1(b)$. On the other hand, the benzyl signals of the naphthyl derivative, which range from $\delta=4.2$ to $5.5 \mathrm{ppm}$, are identified by their characteristic chemical shift. The DQCOSY experiment used here is not able to identify 


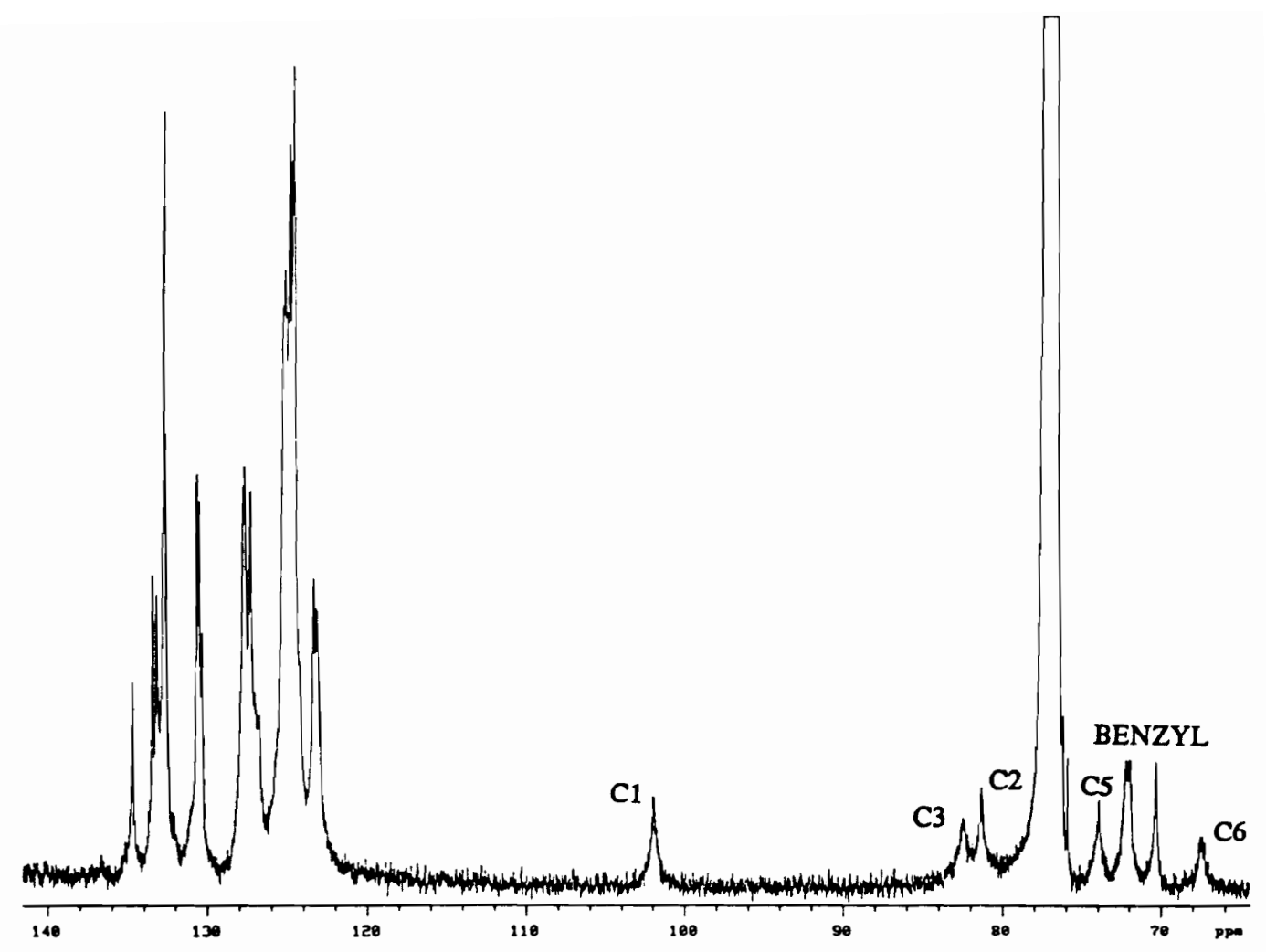

Figure 2. $\quad{ }^{13} \mathrm{C}$ NMR spectra of Tri-O- $\alpha$-naphthylmethyl Cellulose. 
Table II. Peak Assignments of Proton and Carbon Signals of Tri-0- $\alpha$-Naphthylmethyl Cellulose.

\begin{tabular}{|c|c|c||}
\hline & $\begin{array}{c}\text { Anhydroglucose Unit } \\
\text { (ppm) }\end{array}$ & $\begin{array}{c}\text { Benzy } \\
\text { (ppm) }\end{array}$ \\
\hline H1 & 4.12 & \\
\hline H2 & 3.28 & \\
\hline H3 & 3.04 & \\
\hline H4 & 3.78 & \\
\hline H5 & 2.68 & \\
\hline H6 & 3.40 & \\
\hline H6' & 3.20 & $5.19,4.91,4.2$ \\
\hline Benzy1 Protons & & \\
\hline C1 & 101.9 & \\
\hline C2 & 81.2 & \\
\hline C3 & 82.4 & $72.3,72.2,71.4$ \\
\hline C4 & 77 & \\
\hline C5 & 74 & \\
\hline C6 & 67.4 & \\
\hline Benzy1 Carbons & 77 & \\
\hline
\end{tabular}


the location of the benzyl signals relative to positions 2,3 , and 6. Note that the benzyl proton signals of Figure 1 (a) appear as two sets of doublets, and one singlet. The benzyl signal at $4.91 \mathrm{ppm}$ overlaps one half of the doublet which is centered at $5.19 \mathrm{ppm}$. Also, the signal for $\mathrm{H} 1$, at $3.28 \mathrm{ppm}$ overlaps one half of the doublet which is centered at $4.2 \mathrm{ppm}$.

Figure 2 shows the ${ }^{13} \mathrm{C}$ spectra of tri-o- $\alpha$-naphthylmethyl cellulose. Table II lists the peak assignments of carbon signals as indicated on the spectra. These assignments are based upon distortionless enhancement by polarization transfer (DEPT) and HETCOR experiments performed on tri-O-pfluorobenzylcellulose (5). The carbon NMR of such arylmethyl cellulose derivatives are nearly identical. Therefore the carbon assignments for naphthyl cellulose are from direct analogy to fluorobenzyl cellulose.

Figure 3 (a) show the relationship between the dynamic shear viscosity and concentration of tri-0- $\alpha$-naphthylmethyl cellulose at different frequencies. The viscosity increases with concentration up to a maximum before decreasing with further increase in concentration. The figure reveals very explicitly that tri-o- $\alpha$-naphthylmethyl cellulose becomes liquid crystalline at $41 \%(\mathrm{w} / \mathrm{w})$. Such viscosity behavior is typical for liquid crystalline solutions, and has been shown earlier for cellulose acetate $(6,7)$, ethyl cellulose $(8)$, hydroxypropyl cellulose $(7,9)$ and cellulose triacetate $(10)$. 
(a)

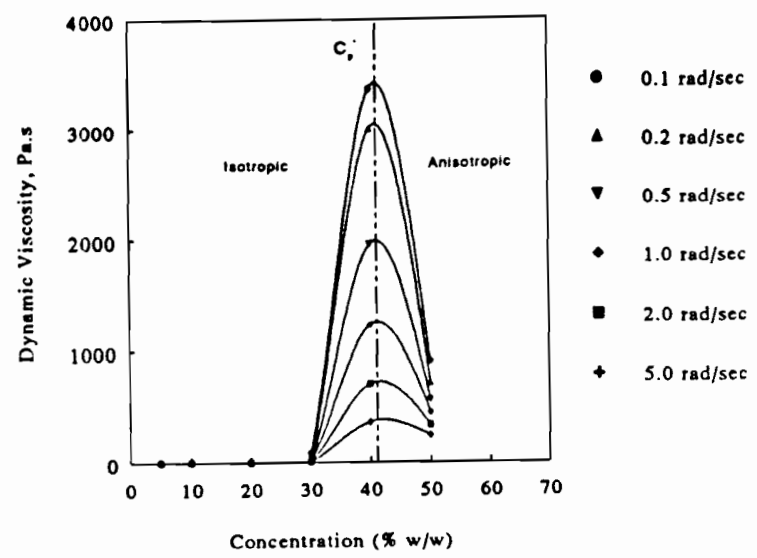

(b)

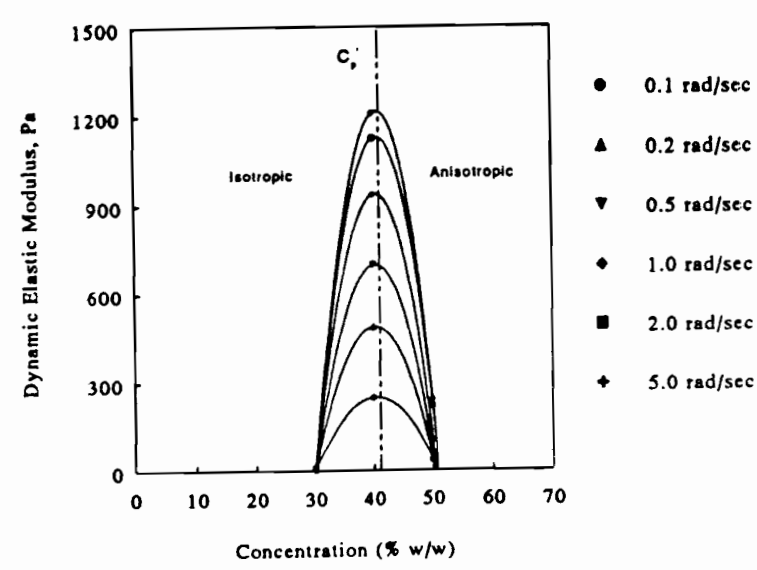

(c)

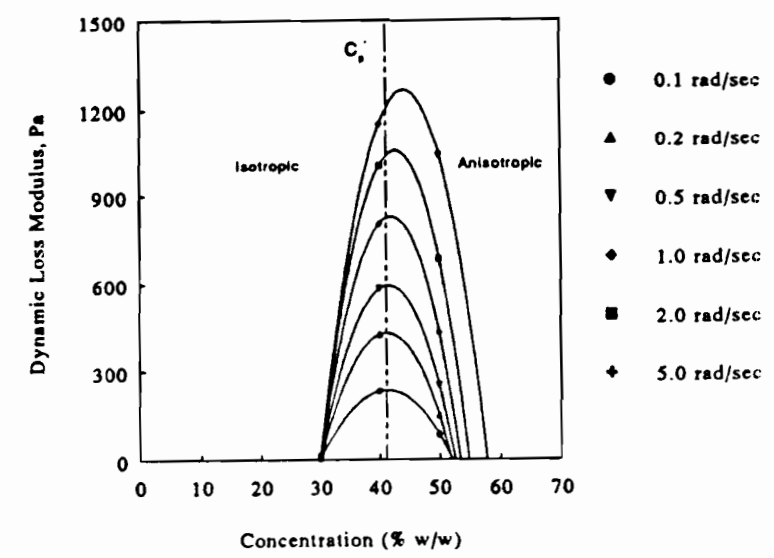

Figure 3. (a) Dynamic viscosity (b) dynamic elastic modulus and (c) dynamic loss modulus versus concentration of tri-0- $\alpha$-naphthylmethyl cellulose solutions at different frequencies. 
This change in viscosity is due to a change in the structure of the solution. As the concentration rises, the size and density of the ordered regions increases and a suspension of ordered clusters in an isotropic matrix is created (11). The energy dissipated by these clusters during flow is less than the total sum of the energy dissipated by each individual molecule; hence the viscosity decreases with concentration.

Shear thinning of the anisotropic solution begins several decades of shear rate lower for an anisotropic solution than for an isotropic solution of the same viscosity (12). In this case, shear thinning of the biphasic solution (40\%) is more than that of the anisotropic solution (50\%) and the effect is more pronounced at higher shear rate. The reason for this behavior is unclear.

Figs. 3 (b) and (c) show the relationship between dynamic elastic modulus $\left(G^{\prime}\right)$ and dynamic loss modulus $\left(G^{\prime \prime}\right)$ and concentration of tri-0- $\alpha$-naphthylmethyl cellulose at different frequencies. The behavior of the concentration dependence of $G^{\prime}$ is the same as that observed for dynamic viscosity, i.e., after the solution becomes anisotropic $G^{\prime}$ value decline. But the $G^{\prime \prime}$ value of the anisotropic solution is almost comparable to the biphasic solution, especially at high frequencies. As with any viscoelastic material, $G^{\prime}$ and $G^{\prime \prime}$ rise with frequency.

Figures 4 (a) and (b) show the polarized optical micrographs of $40 \%(\mathrm{w} / \mathrm{w})$ and $50 \%(\mathrm{w} / \mathrm{w})$ concentrations of the derivative, respectively. The micrographs show the transition 

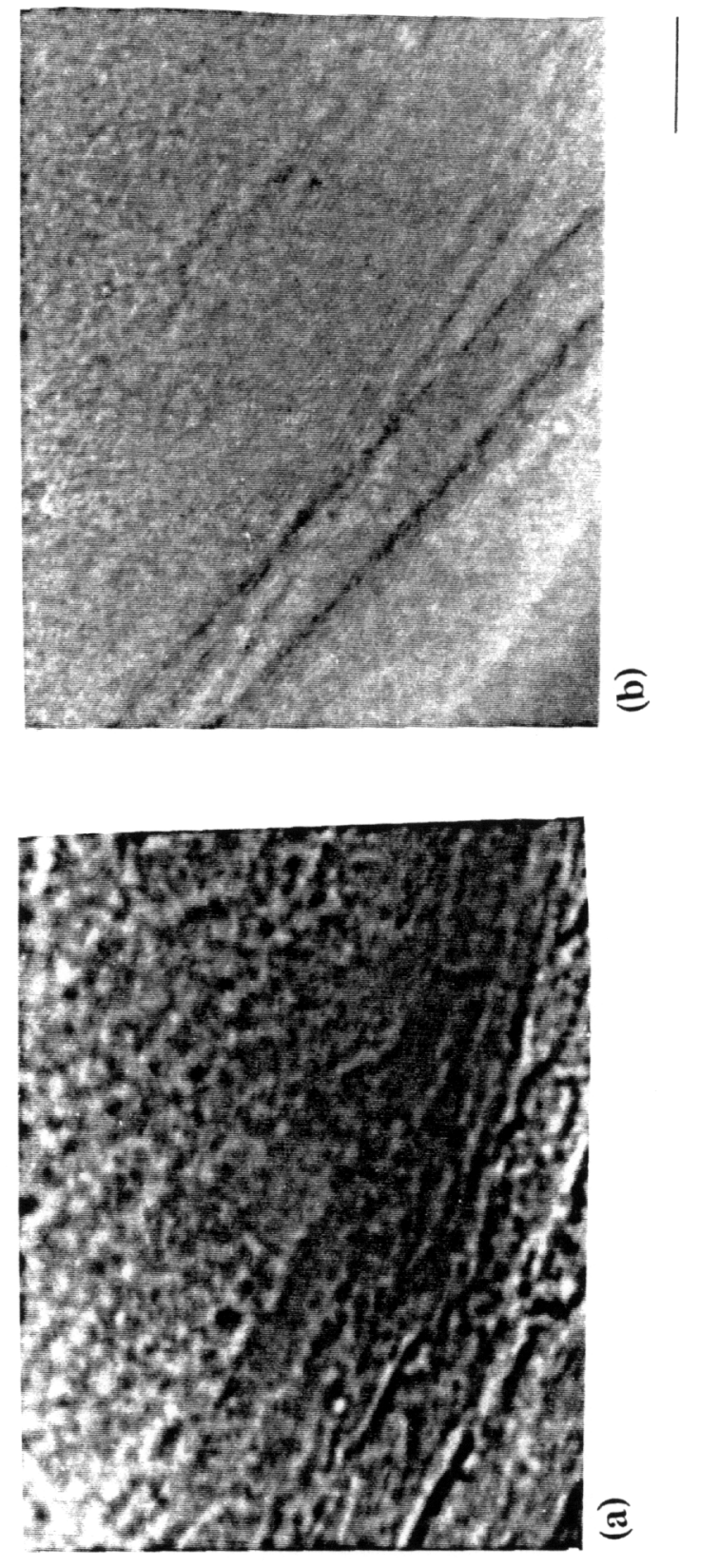

$\sum_{\text {in }}^{\Xi}$

递

战

을

造

을

茎

⿹ํㅇ

ํํㅇ

范

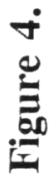


from biphasic (Fig. 4a) to anisotropic solution (Fig. 4b). There is a good correlation between dynamic viscosity measurements and polarized optical microscopy results which confirm that the onset of liquid crystalline behavior in tri$0-\alpha$-naphthylmethyl cellulose in DMAc is at $41 \%(w / w)$. No one has yet reported the lyotropic mesophase formation of tri-0- $\alpha-$ naphthylmethyl cellulose.

Tri-o- $\alpha$-naphthylmethyl cellulose melted at $94^{\circ} \mathrm{C}$, but did not exhibit thermotropic liquid crystalline behavior. However, thermotropic liquid crystallinity was previously reported for this particular cellulose ether derivative with crystal to smectic, smectic to nematic, and nematic to isotropic transitions at $140^{\circ} \mathrm{C}, 160^{\circ} \mathrm{C}$ and $200^{\circ} \mathrm{C}$, respectively (13).

The MHS-constant, $a$, of tri-o- $\alpha$-naphthylmethyl cellulose was 0.64 (Table I), and represents a flexible polymer chain. Values of ' $a$ ' for cellulose and cellulose derivatives are in the range of 0.9 to 1.0 and indicates that they are semi-rigid polymers. In spite of low ' $a$ ' value, the derivative in our study formed lyotropic solutions at high concentrations. This means that stiffness of the chain is much higher than predicted by MHS equation, and liquid crystal formation is due to intramolecular and intermolecular interactions.

Experimental value $(0.35)$ of $V_{p}^{c}$ is lower than the calculated value (0.46) [see Table III and Appendix A for calculations] for tri-0- $\alpha$-naphthylmethyl cellulose. 
Table III. Theoretical Values of Molecular Parameters for Cellulose Derivatives.

\begin{tabular}{||c|c|c|c|c|c|c|c|}
\hline $\begin{array}{c}\text { Cell. } \\
\text { Der. }\end{array}$ & $\begin{array}{c}\mathrm{M}_{\mathrm{u}}{ }_{\mathrm{g}} / \mathrm{mole} \\
\mathrm{CA}^{1)}\end{array}$ & $\mathrm{d}, \AA$ & $\mathrm{I}_{\mathrm{k}}, \AA$ & $\mathrm{q}, \AA$ & $\mathrm{x}_{\mathrm{k}}$ & $\begin{array}{l}\mathrm{V}_{\mathrm{p}}^{\mathrm{c}}{ }^{\prime} \\
\mathrm{Th} .\end{array}$ & $\begin{array}{l}\mathrm{v}_{\mathrm{p}}^{\mathrm{c}}{ }^{\prime} \\
\text { Expt }\end{array}$ \\
\hline $\mathrm{CAB}-1$ & 303.3 & 8.06 & 207.7 & 103.85 & 25.77 & 0.29 & 0.38 \\
\hline $\mathrm{CAB}-2$ & 326.2 & 9.32 & 207.7 & 103.85 & 22.29 & 0.33 & 0.32 \\
\hline $\mathrm{CAB}-3$ & 354.1 & 9.71 & 207.7 & 103.85 & 21.40 & 0.34 & 0.29 \\
\hline $\mathrm{NC}^{2)}$ & 582 & 12.68 & 192.3 & 96.15 & 15.16 & 0.46 & 0.35 \\
\hline
\end{tabular}

1) Cellulose acetate and cellulose acetate butyrates (CA, CAB-1, CAB-2, $(A B-3)$ : values obtained from reference no. 1 .

2) Tri-o- $\alpha$-naphthylmethyl cellulose: values from Appendix A. 
Critical volume fraction was not greatly affected by molecular weight for hydroxypropyl cellulose (HPC) (16) and (acetoxypropyl) cellulose (APC) (17) in DMAc. So, we can compare the experimental results obtained in this study with our previous work on cellulose esters with different types of substituents (1), even though the molecular weights differ to some extent. Cellulose hexanoate values are not compared as it is not highly substituted as all the other derivatives considered here (2). Figure 5 show the relationship between the experimental and predicted values of $\mathrm{V}_{\mathrm{p}}^{\mathrm{c}}$ and molecular weight of repeat unit, $M_{u}$, for cellulose esters (see Table III) and tri-o- $\alpha$-naphthylmethyl cellulose. The experimental values of $V_{p}^{c}$, in general, are lower than the theoretical values for all the cellulose derivatives (Figure 5) . $\mathrm{V}_{\mathrm{p}}^{\mathrm{c}}$ decreases with $M_{u}$ for cellulose esters but the trend does not follow our prediction that large and bulky substituents $\left(M_{u}\right)$ will enhance the formation of liquid crystalline solutions at lower concentration (1).

It seems that Flory's theory overestimates the $v_{p}^{c}$, and the difference is greater at higher $M_{u}$. This difference may be due to intermolecular interactions and molecular association between side chains which lowers $V_{p}^{c}$. It is possible that cellulose ethers have different type of interactions than cellulose esters. During the study of liquid crystalline solutions of HPC in DMAC, in order to obtain complete 


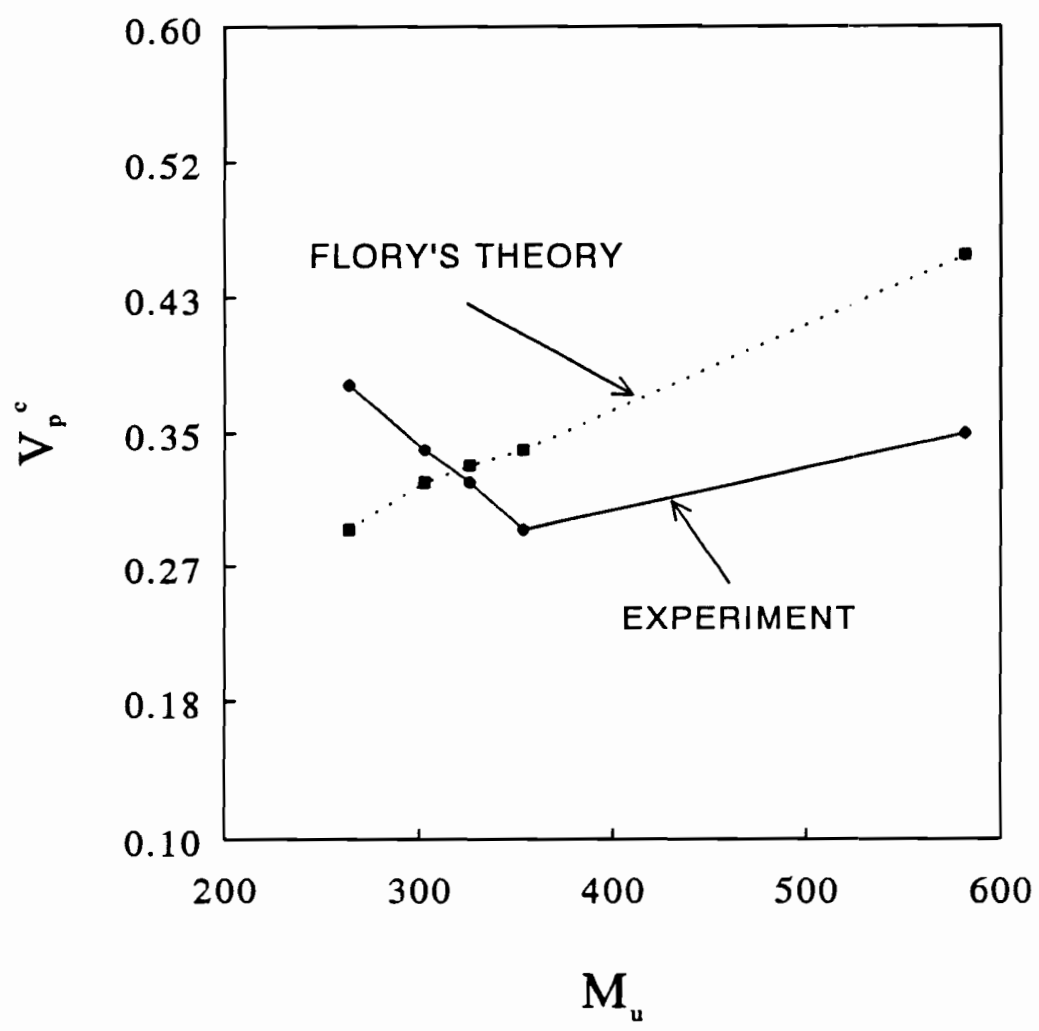

Figure 5. Experimental and theoretical variation of critical volume fraction of cellulose derivatives with molecular weight of the repeating unit. 
agreement between the experimental and calculated values of $\mathrm{V}_{\mathrm{p}}^{\mathrm{c}}$, presence of small degree of soft interactions was speculated $(16,18)$.

$\mathrm{V}_{\mathrm{p}}^{\mathrm{c}}$ is definitely related to molecular parameters associated with chain rigidity. Aspect ratio, $\mathrm{x}_{\mathbf{k}}$, is affected by persistence length (q) and diameter of the chain. $q$ for cellulose and cellulose derivatives range from 70 to $110 \AA$ (19). In our study, we assumed $q$ to be $103.85 \AA$ and $96.15 \AA$ for cellulose esters and tri-0- $\alpha$-naphthylmethyl cellulose, respectively. It is necessary to experimentally determine $q$ values for all our derivatives to get accurate theoretical results. However, experimentally determined q values too give predicted $V_{p}^{c}$ values higher than experimental results (19). Therefore, our assumption of $q$ values seems to be within acceptable range. It is complicated to solve the discrepancy between the predicted and experimental $V_{p}^{c}$ values as there is no successful theory to explain the universal behavior of semi-rigid liquid crystalline polymers like cellulose and its derivatives. Our contribution in this area of active research is that molecular weight of the repeat unit of cellulose derivatives does not seem to influence the critical volume fraction, $V_{p}{ }^{c}$, to a great extent. 


\section{CONCLUSIONS}

1. Tri-o- $\alpha$-naphthylmethyl cellulose was successfully prepared by employing a nonaqueous cellulose solvent, $\mathrm{SO}_{2}-\mathrm{DEA}-\mathrm{DMSO}$.

2. Tri-o- $\alpha$-naphthylmethyl cellulose solution in DMAC exhibited liquid crystalline behavior as observed by viscosity measurements and cross polarized optical microscopy results.

3. Flory's theory overestimates $V_{p}{ }^{c}$. Molecular weight of repeat unit of cellulose derivatives does not seem to influence $v_{p}^{c}$.

\section{APPENDIX A}

Equations used for theoretical calculations are taken from reference no. 14 .

1. Calculation of molecular weight of repeat unit, $\mathbf{M}_{u}$ :

$$
\mathrm{M}_{\mathrm{v}}=162+3(11 \times 12+9)-3=582 \mathrm{gm} / \mathrm{mole}
$$

2. Calculation of chain diameter, $d$ :

$$
\begin{aligned}
d & =\left(M_{u} / N_{A} \rho l_{u}\right)^{1 / 2} \\
d & =\left(582 / 6.02 \times 10^{23} \times 1.17 \times 10^{-24} \times 5.14\right)^{1 / 2} \\
& =12.68 \AA
\end{aligned}
$$

3. Calculation of Kuhn segment length, $I_{k}: l_{k}=\left\langle r_{2}\right\rangle_{0} / n l_{u}$ From light scattering [Wirick and Waldman, (15)] $\left\langle\mathrm{r}^{2}\right\rangle_{0} / \mathrm{n}=1000 \AA^{2}$ for cellulose ethers. $I_{k}=1000 / 5.14=192.30 \AA$ 
4. Calculation of Persistence Length, q:

$$
\begin{aligned}
& q=l_{k} / 2 \\
& q=192.30 / 2=96.15 \AA
\end{aligned}
$$

5. Calculation of Aspect Ratio, $\mathrm{X}_{\mathbf{k}}$ :

$$
\begin{aligned}
& x_{k}=2 q / d \\
& x_{k}=2 \times 96.15 / 12.68=15.16
\end{aligned}
$$

6. Calculation of Critical Volume Fraction, $V_{p}^{c}$ :

$$
\begin{aligned}
& \mathrm{V}_{\mathrm{p}}^{\mathrm{c}}=8 / \mathrm{X}_{\mathrm{k}}\left(1-2 / \mathrm{X}_{\mathrm{k}}\right) \\
& \mathrm{V}_{\mathrm{p}}^{\mathrm{c}}=8 / 15.16(1-2 / 15.16)=0.46
\end{aligned}
$$




\section{REFERENCES}

1. V. Davé and W. G. Glasser, in viscoelacticity of Biomaterials, ACS Symp. Ser., W. G. Glasser and H. Hatakeyama, Eds. (In Press).

2. V. Davé and W. G. Glasser (In Preparation).

3. A. Isogai, A. Ishizu and J. Nakano, J.Appl. Polym. Sci., 29, 2097-2109, 1984 .

4. A. Isogai, A. Ishizu and J. Nakano, J. Appl. Polym. Sci., $29,3873-3882,1984$.

5. C. E. Frazier and W. G. Glasser (In Preparation).

6. S. Dayan, P. Maissa, M. J. Vellutini and P. Sixou, J. Polym. Sci.: Polym. Lett. Ed., 20, 33-43, 1982.

7. S. Dayan, J. M. Gilli and P. Sixou, J. Appl. Polym. Sci., $28,1527-1534,1983$.

8. S. Suto, J. Polym. Sci.: Polym. Phys. Ed., 22, 637-646, 1984 .

9. P. Navard and J. M. Haudin, J. Polym. Sci.: Polym. Phys. Ed., 35, 189-201, 1986.

10. Y. K. Hong, D. E. Hawkinson, E. Kohout, A. Garrard, R. E. Fornes and R. D. Gilbert in Polymer Association structure, M. A. El-Nokaly, Ed., ACS Symp. Ser. \# 384, Ch. 12, 184-203, 1989 .

11. D. G. Baird, J. Rheology, 24 (4), 465-482, 1980.

12. K. F. Wissburn, J. Rheology, 25 (6), 619-662, 1981. 
13. A. Isogai, A. Ishizu and J. Nakano, J. Appl. Polym. Sci., $30,345-353,1985$.

14. P. J. Flory, Adv. in Polym. Sci., 59, 2-36, 1984.

15. M. G. Wirick and M. H. Waldman, J. Appl. Polym. Sci., 14, 579,1970 .

16. G. Conio, E. Bianchi, A. Ciferri, A. Tealdi and M. A. Aden, Macromolecules, 16 (8), 1264-1270, 1983.

17. G. V. Laivins, P. Sixou and D. G. Gray, J. Polym. Sci.: Part B: Polym. Phys., 24, 2779-2792, 1986.

18. M. A. Aden, E. Bianchi, A. Ciferri, G. Conio and A. Tealdi, Macromolecules, 17 (10), 2010-2015, 1984.

19. E. Bianchi, A. Ciferri, G. Conio, L. Lanzavecchia and M. Terbojevich, Macromolecules, 19 (3), 630, 1986. 
10.0 THERMAL TRANSITIONS OF CELLULOSE ACETATE. 


\title{
THERMAL TRANSITIONS OF CELLULOSE ACETATE.
}

\begin{abstract}
Thermal transitions of cellulose acetate (CA) with degree of substitution 2.44 were determined by differential scanning calorimetry. Transition temperatures were observed at $40^{\circ} \mathrm{C}$ and $190^{\circ} \mathrm{C}$, respectively. Detailed investigations by thermogravimetric analysis and infrared spectroscopy concluded that the former transition was due to loss of absorbed moisture. The latter transition was attributed to the glass transition temperature of $C A$. Melting point of $C A$ was at $231^{\circ} \mathrm{C}$.
\end{abstract}




\section{INTRODUCTION}

Multiple thermal transitions are observed in all cellulose derivatives. Mandelkern and Flory (1) have reported transitions at $30^{\circ} \mathrm{C}$ and $105^{\circ} \mathrm{C}$ in dilatometric studies of cellulose triacetate $\left(44.8 \%\right.$ acetyl) and at $60^{\circ} \mathrm{C}$ and $120^{\circ} \mathrm{C}$ for cellulose acetate $(38.2 \%$ acetyl). There are some publications that describe various transition temperatures of cellulose acetate by differential scanning calorimetry (DSC) that do not agree $(2-4)$. All polysaccharide ester derivatives show considerable affinity for water due to the presence of ether oxygen, of unesterified hydroxyls and of ester groups. Scandola et al. (5-8) have studied the viscoelastic relaxations of cellulose acetate by dynamic mechanical spectroscopy (DMTA).

The main objective of this investigation is to identify the thermal transitions of cellulose acetate by DSC.

\section{EXPERIMENTAL}

I. Materials.

Cellulose acetate (CA 394-60) was obtained from Eastman Kodak, Kingsport, TN. The average acetyl content was 39.4 weight $\%$ and the degree of substitution $\left(D_{\mathrm{AC}}\right)$ was 2.44 . II. Methods.

1. Differential Scanning Calorimetry (DSC):

The thermal analysis of cellulose acetate (CA) was determined on a DuPont $912 \mathrm{DSC}$ at a heating rate of $10^{\circ} \mathrm{C} / \mathrm{min}$ 
under nitrogen atmosphere.

2. Fourier Transform-Infrared Spectroscopy (FT-IR):

FT-IR spectroscopy was carried out on a Nicolet 5SXC Spectrometer for a very thin solvent-cast film of CA before and after drying to detect shift in carbonyl or hydroxyl peaks.

3. Thermogravimetric Analysis (TGA):

TGA was determined on a Perkin-Elmer TGS-2 for un-dried and dried CA powder under nitrogen atmosphere at $10^{\circ} \mathrm{C} / \mathrm{min}$.

\section{RESULTS AND DISCUSSION}

Figure 1 shows the DSC scan up to $125^{\circ} \mathrm{C}$ heated at $10^{\circ} \mathrm{C} / \mathrm{min}$ of cellulose acetate (CA) which was dried at $125^{\circ} \mathrm{C}$ for 8 hours. A broad endotherm is observed at $\sim 71^{\circ} \mathrm{C}$. Figure 2 is the DSC scan of the same sample re-run immediately after quenching from $125^{\circ} \mathrm{C}$. Fig. 2 shows that the broad endotherm peak at about $71^{\circ} \mathrm{C}$ (Fig. 1) has almost disappeared and the melting transition of $\mathrm{CA}$ is at $231^{\circ} \mathrm{C}$. Similar observations were made for samples dried at different temperatures. The broad endotherm peak re-appeared, as in Fig. 1, after the sample was stored at $30^{\circ} \mathrm{C}$. Therefore, the process was reversible; it could be due to absorbed moisture or physical aging.

Fig. 3 represents the FT-IR of un-dried and dried film of CA. There are almost no changes observed in the spectra, except for a slight reduction in the hydroxyl peak probably due to drying. Similar IR spectra are observed with the dried 


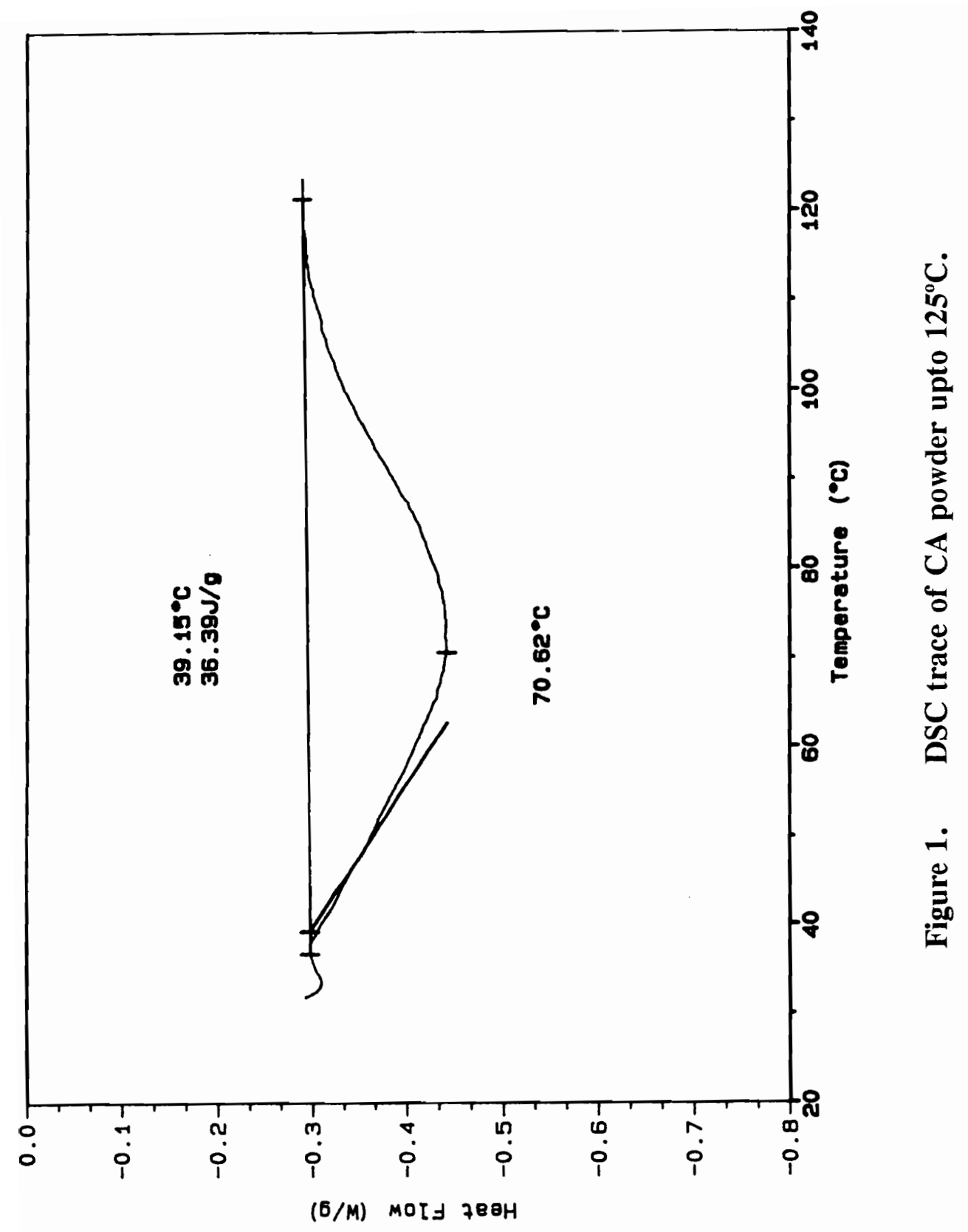

шләчрория 


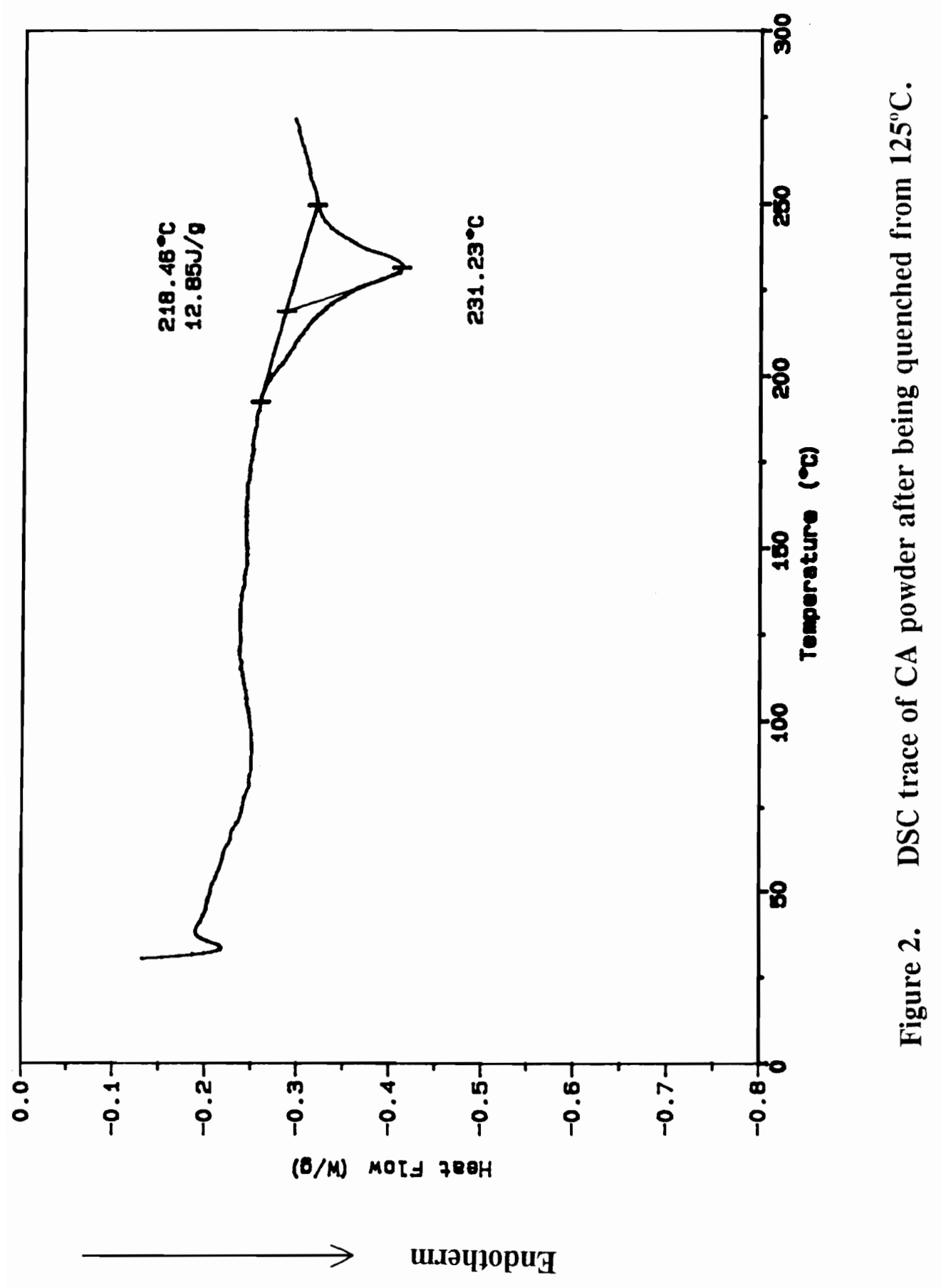




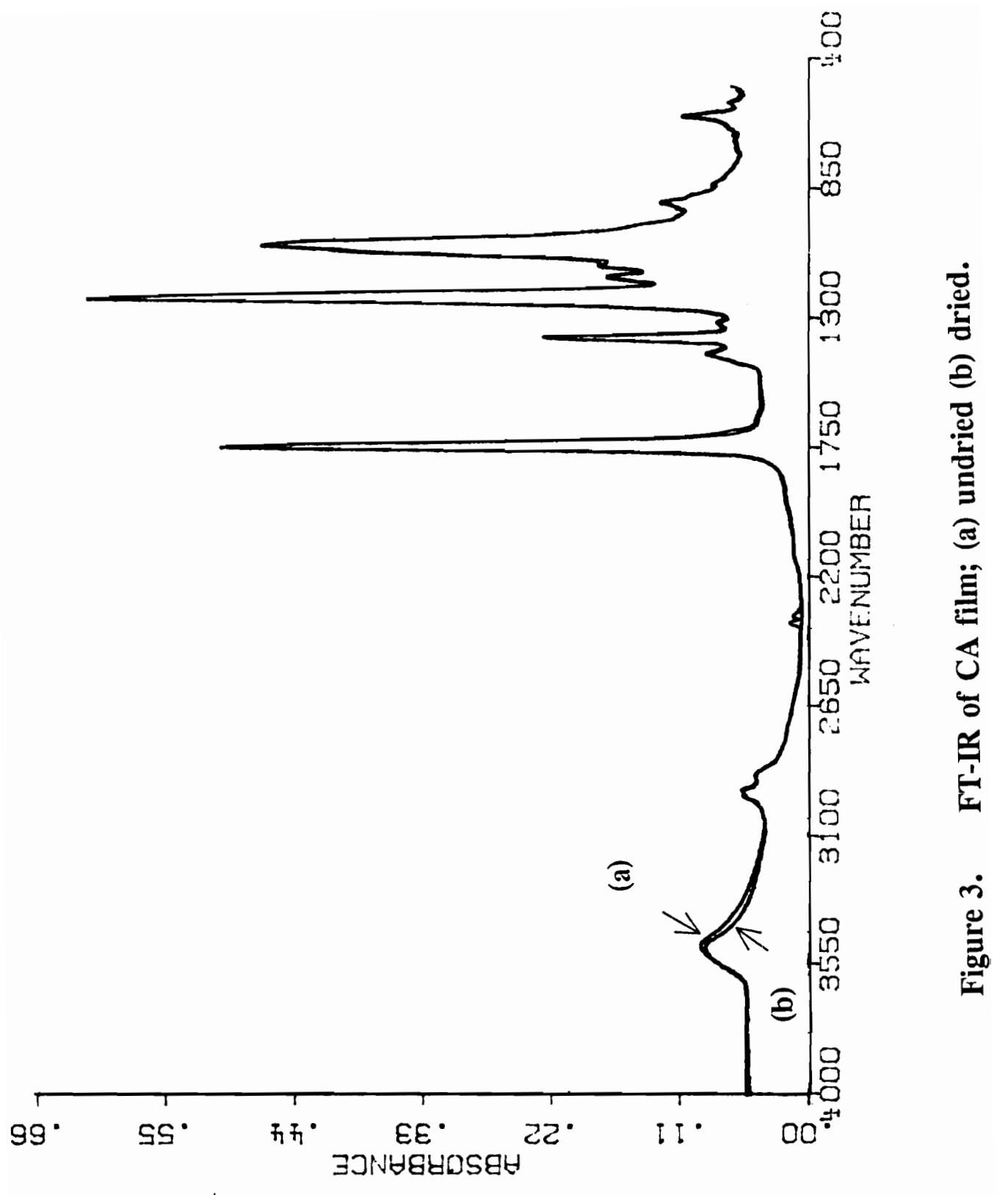


film after 5 days. This shows no sign of physical aging in cellulose acetate.

Fig. 4 shows the DSC scan of a CA sample run after being quenched from $270^{\circ} \mathrm{C}$ to $-60^{\circ} \mathrm{C}$. Two transitions were observed at about $40^{\circ} \mathrm{C}$ and $190^{\circ} \mathrm{C}$, respectively. The former transition at $40^{\circ} \mathrm{C}$ is suspected to be for cellulose acetate plasticized by moisture and the latter at $190^{\circ} \mathrm{C}$ is glass transition of cellulose acetate.

Fig. 5 shows the TGA for the un-dried and dried CA samples, and weight loss is clearly indicated at about $50^{\circ} \mathrm{C}$, and this suggests loss of moisture.

Scandola et al. (5-8) made similar observations after detailed investigations on the effects of moisture on viscoelastic properties of cellulose acetate by DMTA, DSC, and TGA. The reversible endotherm at about $70^{\circ} \mathrm{C}$ (by DSC), reversible $\beta^{*}$ transition at about $50^{\circ} \mathrm{C}$ (by DMTA) and weight loss (by TGA) were related to the presence of absorbed moisture. They also observed $\alpha, \beta$ and $\gamma$ relaxations at $197^{\circ} \mathrm{C}$, $-38^{\circ} \mathrm{C}$ and $-88^{\circ} \mathrm{C}$ for the main chain, local motions of the main chain, and absorbed moisture respectively. The latter two transitions were not observed in our study. These transitions are shown in Fig. 6 (reproduced from reference 8 ).

Since the transitions observed in our study correlate well with those obtained by scandola et al. (5-8), this paper confirms the thermal transitions of $C A$. 


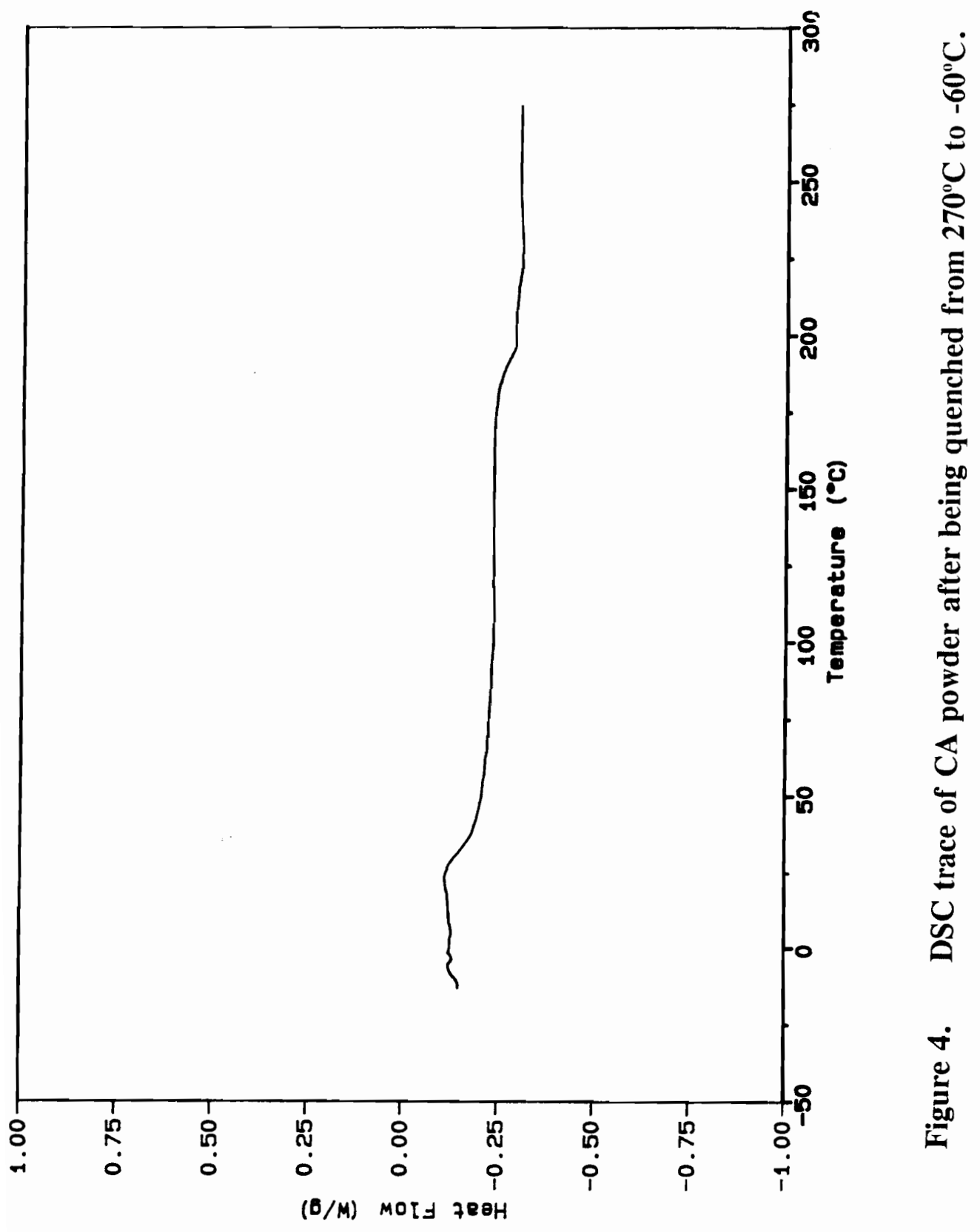

ш.љурория 


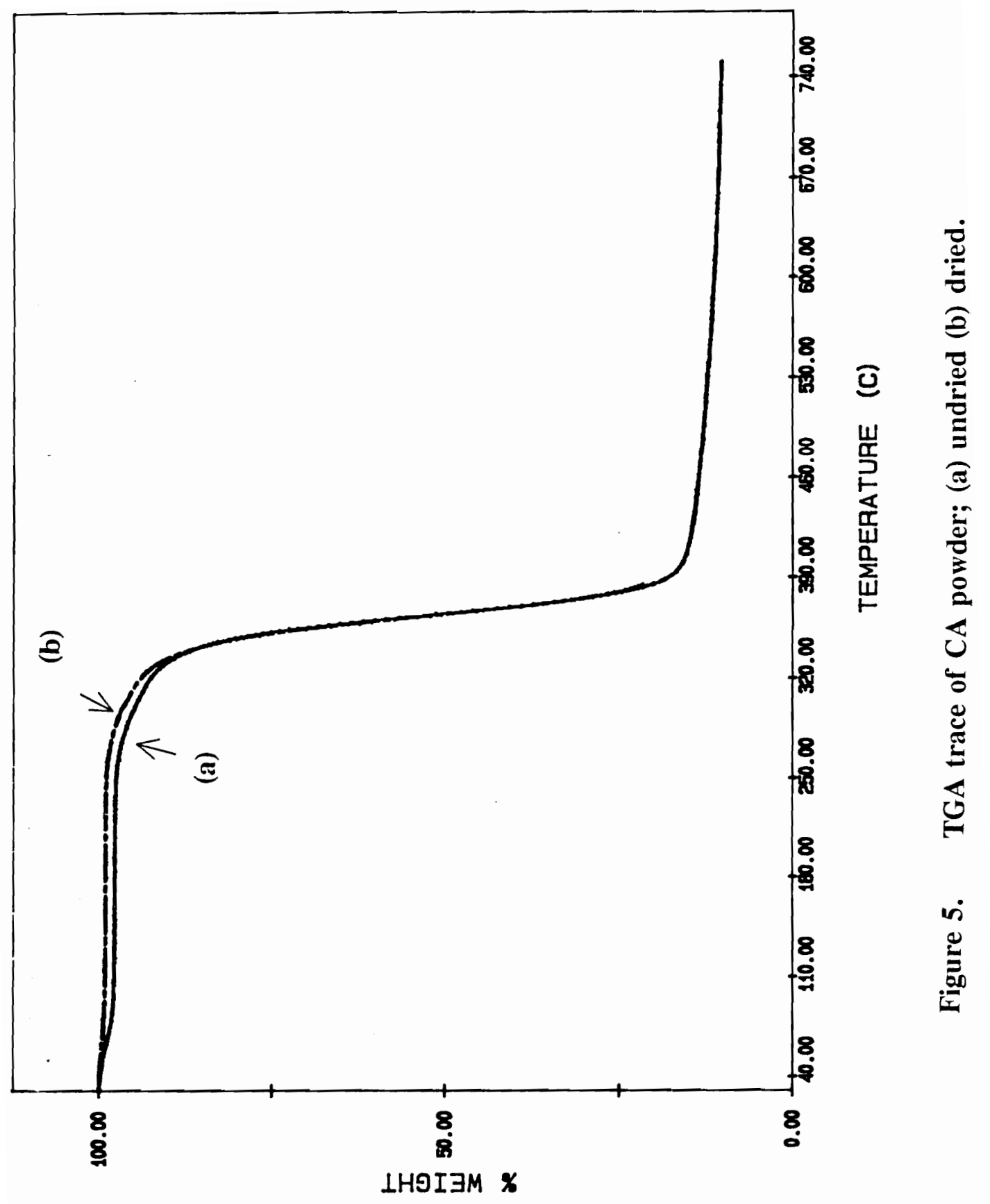




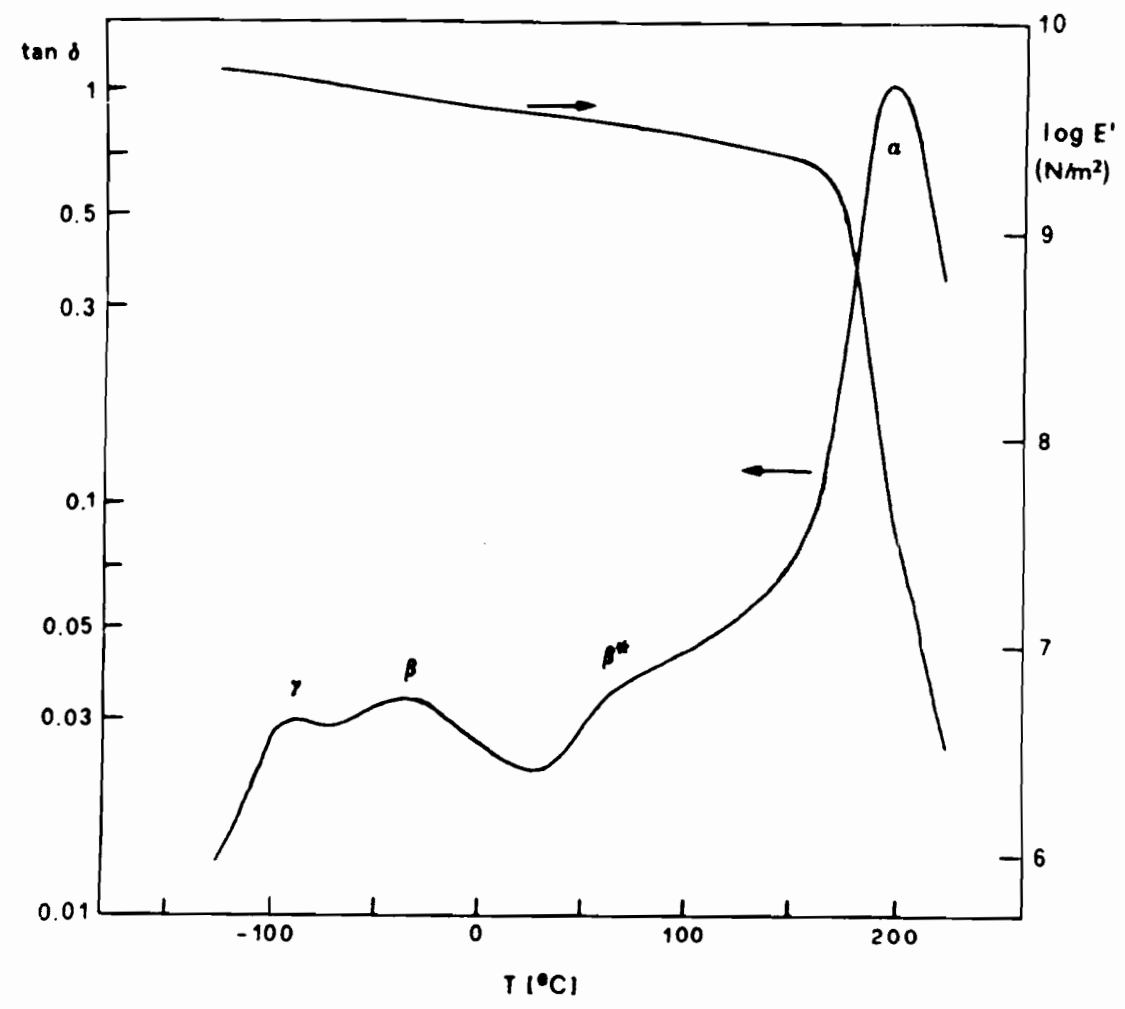

Figure 6. Dynamic Mechanical Properties of Cellulose Acetate (DS 2.4) at $3 \mathrm{~Hz}$. (Reproduced from reference no. 8) 


\section{CONCLUSIONS}

Thermal transitions of cellulose acetate were determined by DSC. The broad endotherm peak and the transition temperature at $-40^{\circ} \mathrm{C}$ are due to absorbed moisture. $T_{g}$ and $T_{m}$ of $\mathrm{CA}$ are at $190^{\circ} \mathrm{C}$ and $231^{\circ} \mathrm{C}$, respectively. 


\section{REFERENCES}

1. L. Mandelkern and P. J. Flory, J. Am. Chem. Soc., 73, $3206,1951$.

2. S. Nakamura, S. Shindo and K. Matsuzak, J. Polym. Sci.. Part B, 9, 591, 1971.

3. J. M. G. Cowie and R. J. Ranson, Makromol. Chem., 143, $110,1971$.

4. R. B. Seymour and E. L. Johnson, J. Polym. Sci.: Polym. Chem. Ed., 16, 1, 1978 .

5. M. Scandola and G. Ceccorulli, Polymer, 26, 1953, 1985.

6. M. Scandola and G. Ceccorulli, Polymer, 26, 1958, 1985.

7. G. Ceccorulli, M. Pizzoli and M. Scandola, Polym. Comm., $27,229,1986$.

8. M. Pizzoli, M. Scandola and G. Ceccorulli, in wood and Cellulosics: Industrial Utilisation, Biotechnology, structure and Properties, J. F. Kennedy, G. O. Phillips and P. A. Williams, Eds., Ellis Horwood Ltd., Ch. 11, $105,1987$. 
11.0 RECOMMENDATIONS. 


\section{RECOMMENDATIONS}

Some of the questions in the area of fiber spinning and liquid crystalline solutions of cellulose and cellulose derivatives were answered in this research. However, few questions specific to these materials requires additional study.

Recommendations are provided below for further work in this field.

1. Experimental determination of the persistence length of all the cellulose derivatives used in this study.

2. Thermal analysis of mixed esters of cellulose which are prepared heterogeneously and homogeneously.

3. Conduct mechano-sorptive creep experiments on cellulose acetate butyrate fibers before and after soaking them in solutions of lithium chloride.

4. Construct phase diagram for the ternary system of lignin/cellulose/dimethylacetamide to understand the effects of lignin on the liquid crystalline solutions of cellulose and cellulose derivatives. 
12.0 APPENDIX. 


\section{APPENDIX}

\section{A. Fiber Spinning Apparatus.}

The spinning apparatus consisted of (i) Solution Delivery System, (ii) spinning Pack Assembly, (iii) Capillary Spinnerette, (iv) Coagulation Bath, and (v) Take-up Device. Each segment of the apparatus is described in details. I. DELIVERY SYSTEM.

It comprised of a programmable infusion and withdrawal syringe pump (Harvard Apparatus Model No. 44). The unique features of the Pump 44 resulted from the use of microcontroller technology. The pump was capable of delivering the solutions at a constant steady flow rate.

II. SPINNING PACK ASSEMBLY.

The pack assembly is illustrated in Figure 1 (a) and (b). It consisted of a top and bottom assembly and both mated to a 5.45 inches long and 1.24 inches internal diameter seamless tube which functioned as the solution reservoir. The volume of the reservoir was $\sim 100 \mathrm{~cm}^{3}$. All parts were manufactured from 304 stainless steel. The seals employed on the pack assembly were constructed from sintered aluminum and were provided by Mott Metallurgical Corporation, Farmington, Connecticut. This is the state-of-the-art pack design as it completely disassembles for easy cleaning. A 12 inches long plunger was constructed from stainless steel which could easily slide in and out of the cylindrical reservoir. A thick stainless steel 
(a)

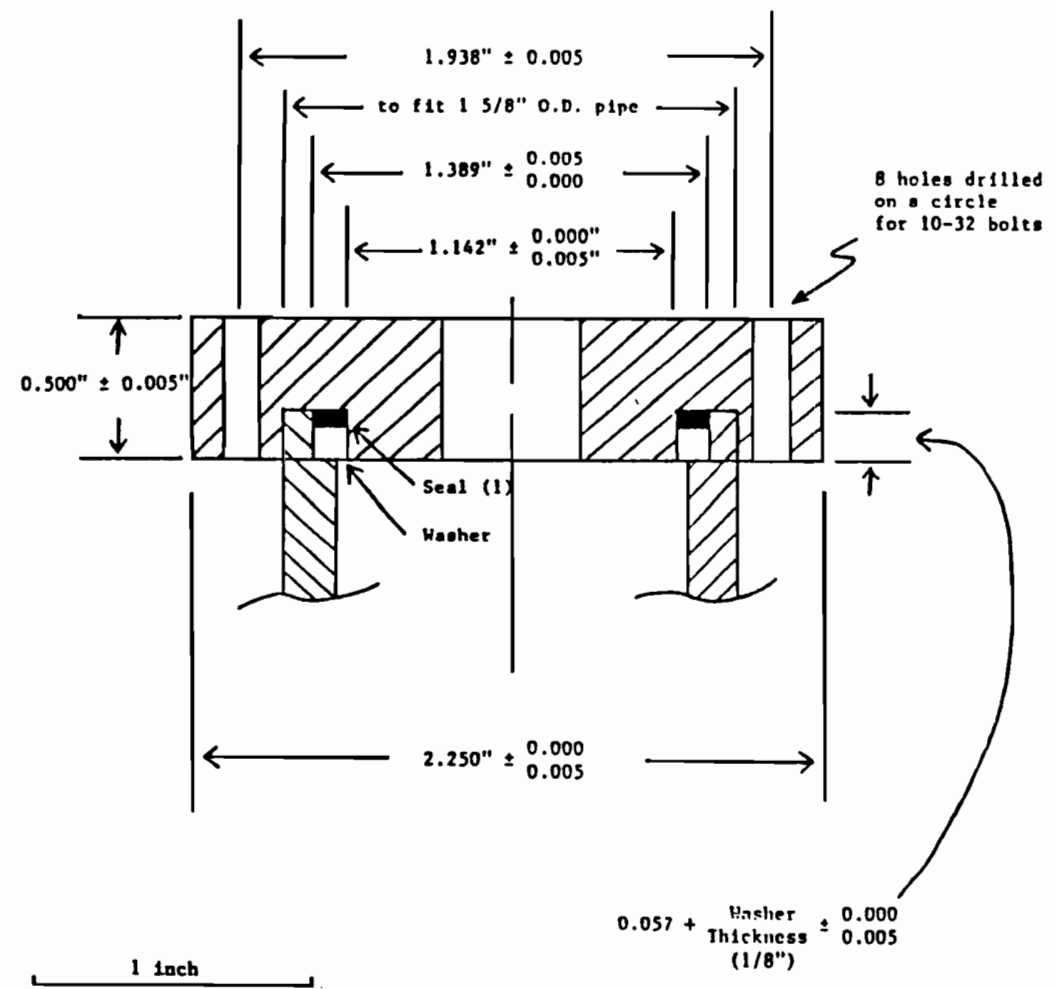

(b)

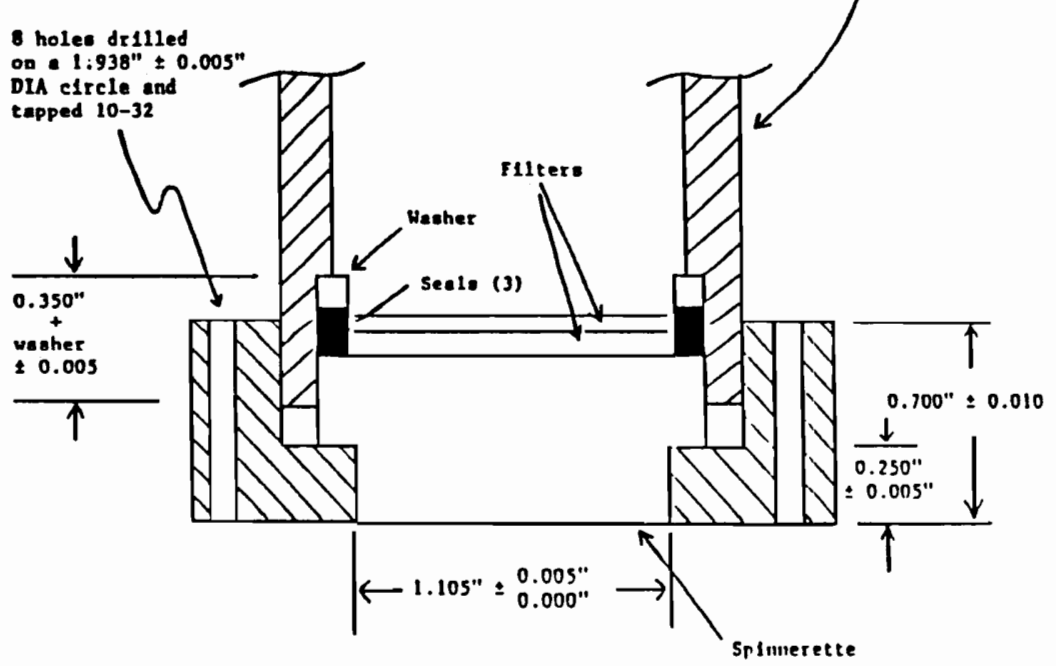

Note: Total S.S. plpe length 1s $5.45^{\prime \prime} \pm 0.010^{\prime \prime}$

1 1nch

Figure 1. Design of spinning pack; (a) top assembly (b) bottom assembly. 
square plate was fastened on the top of the plunger which in turn was held by the retaining brackets to the pusher block of the pump.

\section{CAPILLARY SPINNERETTE.}

The spinnerette was custom designed from 316 stainless steel by Nippon Nozzle Co., Ltd., Osaka, Japan. Their agent in the U.S.A. is Frankl and Thomas Inc., Greenville, south Carolina. Figure 2 shows the complete design of the single hole spinnerette with capillary diameter of $0.3 \mathrm{~mm}$, capillary length of $1.2 \mathrm{~mm}$ (i.e., L/D Ratio =4) and entrance angle of $60^{\circ}$. Fiber extrusion was stable due to high L/D ratio. The spinnerette was fitted in the bottom pack assembly [Fig. 1 (b) ] .

IV. COAGULATION BATH.

It was fabricated from poly(methyl methacrylate) [plexiglass]. The dimensions of the bath were $1 \mathrm{~m}$. (length) $\mathrm{x}$ $0.15 \mathrm{~m}$. (breadth) $\times 0.11 \mathrm{~m}$. (depth). slots were built in the bath to hold glass rods which served as guide rollers for the fibers during the coagulation process. Water was used as the coagulant to remove dimethylacetamide from the fibers.

V. TAKE-UP DEVICE.

Custom Scientific Instruments, Inc. Take-up System (Model CS-194T-053) was used as the take-up device for the wet-spun fibers (Figure 3 ). The fibers were wound on the interchangeable spool tubes as they emerged from the coagulation 


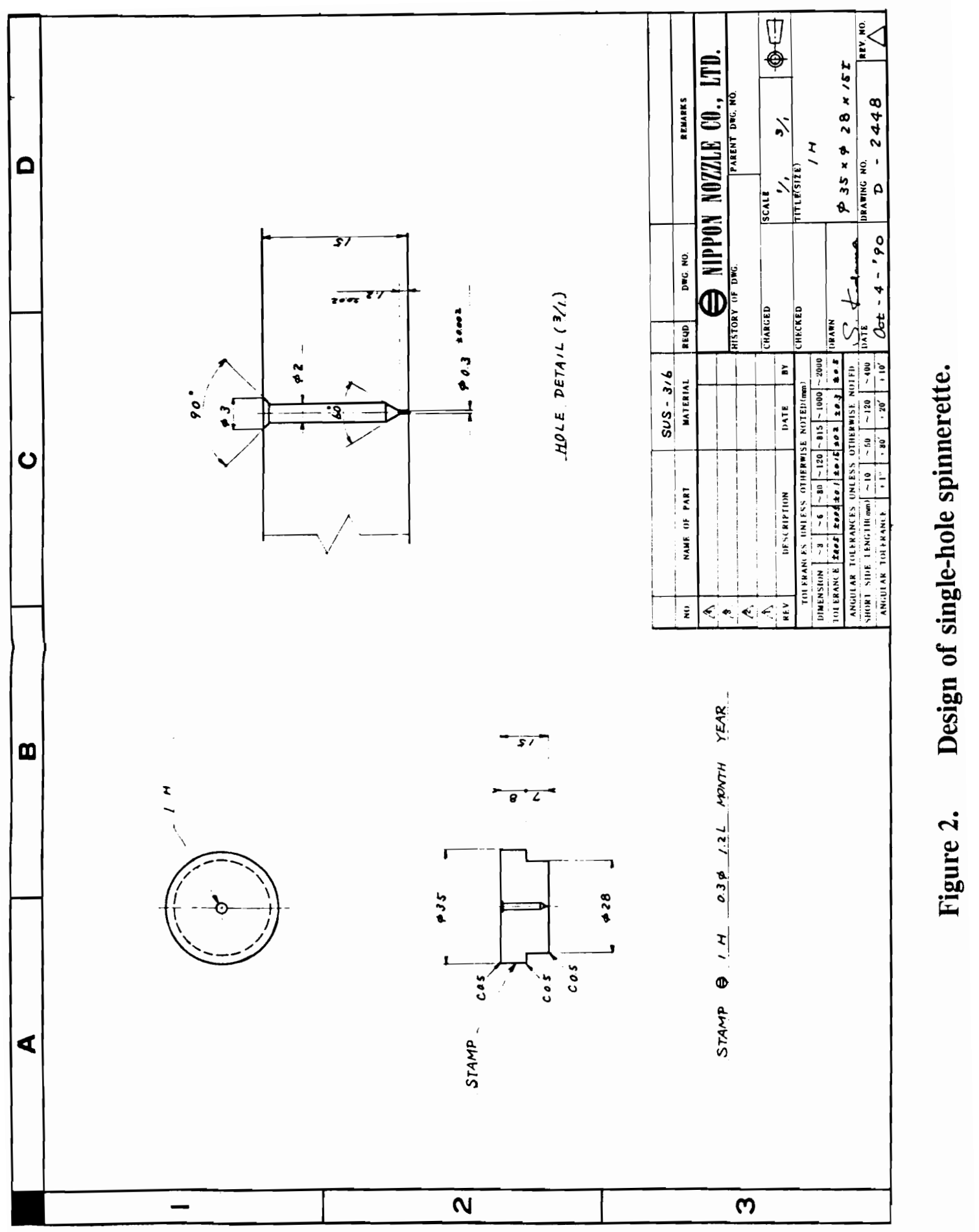




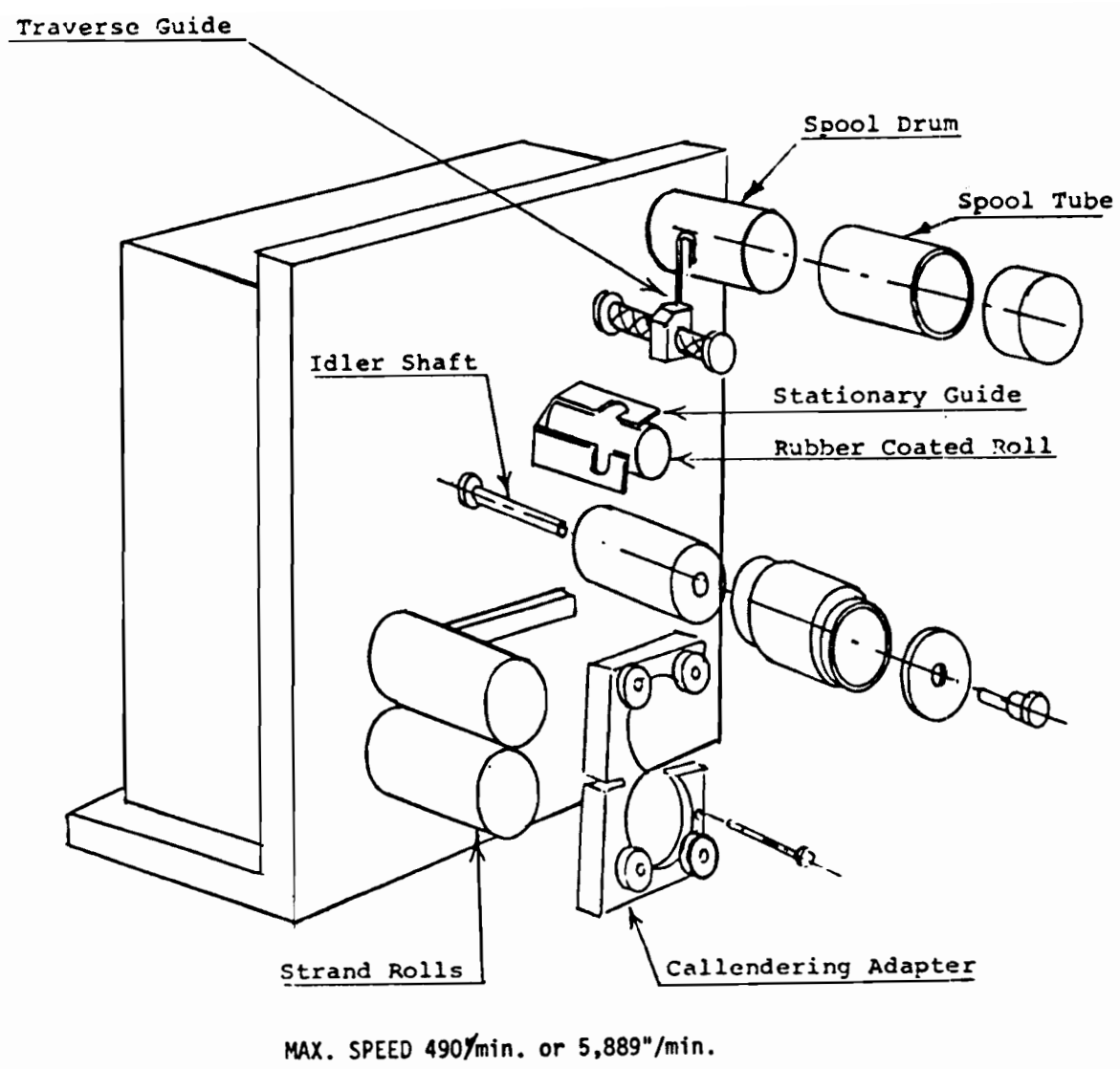

Figure 3. Schematic representation of the take-up device. 


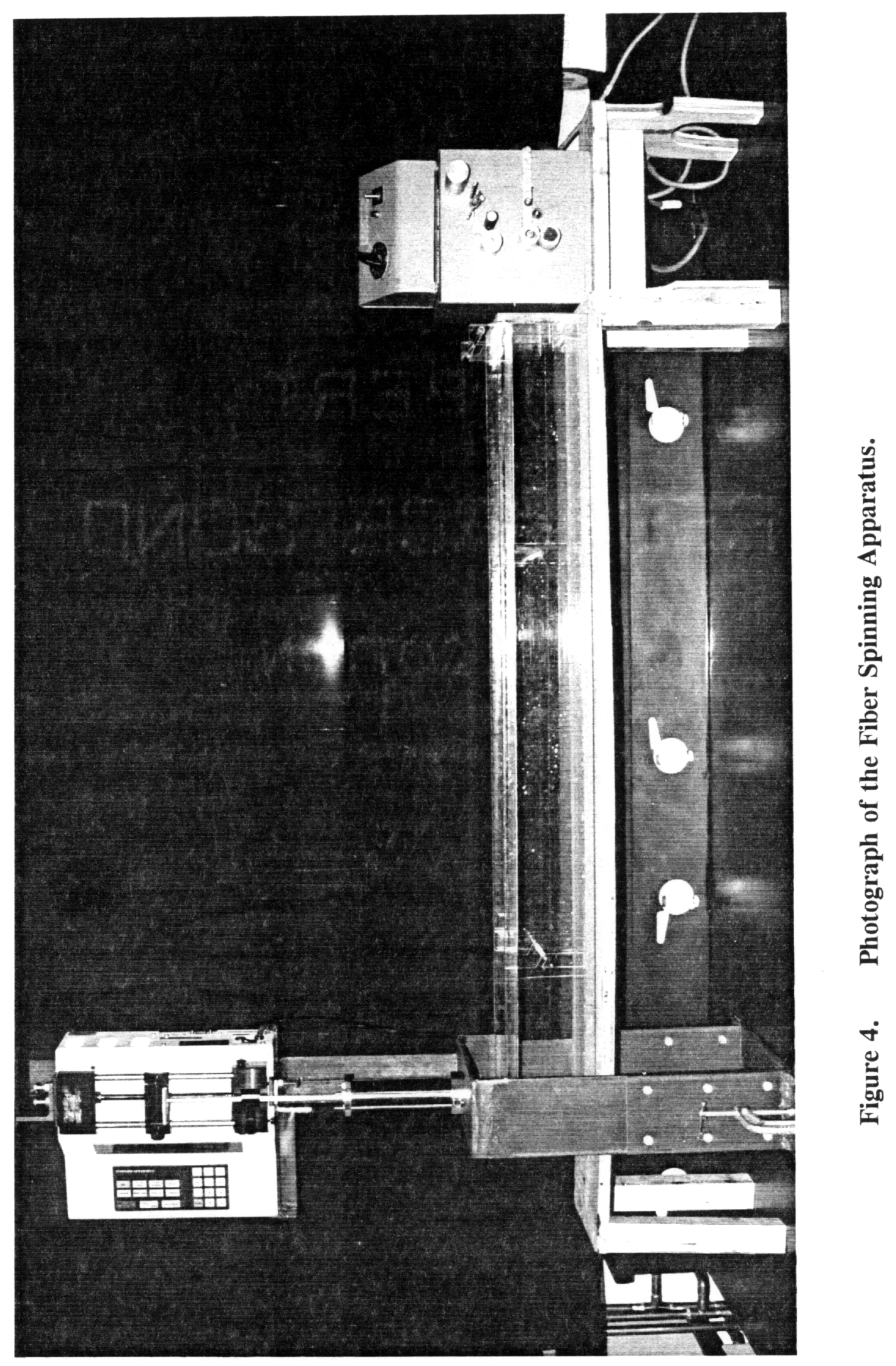


bath. A small piece of double stick scotch tape was placed on the spool surface to hold the fibers during the initial startup process. Spool tube was held in place by an adapter which was screwed into the spool drum. The take-up speed of the spool tube could be varied to match the rate of extrusion to produce the desired fiber diameter. AMETEK Model 1726 Digital Tachometer was used to measure the revolutions per minute (RPM) of the spool tube. The measurements were made optically (non-contact) by scanning a strip of retroreflective tape (target) attached to the rotating spool tube adapter. Figure 4 shows the photograph of the spinning apparatus. B. Processing of Fibers.

Concentrated solutions (viscosity range 500 - $5000 \mathrm{~Pa} . \mathrm{s}$ ) were transferred into the cylindrical reservoir and were extruded by the pump via the plunger through the single-hole spinnerette into the coagulation bath at a steady flow rate. The pump automatically adjusted the pressure required to maintain steady flow when the diameter of the reservoir was entered into the microcontroller. Since the shear rate at the capillary wall in the spinnerette at a particular flow rate was constant, the fiber properties from different concentrated solutions with varying viscosities could be compared.

The air gap between the spinnerette and the water surface was about 1 inch. The solution extrudate formed into fiber by the coagulation process. At start-up, single fiber was picked 
up by a long tweezers and was manually dragged slowly towards the take-up device. The fiber was stuck on the tape on the spool surface. The spool was powered gradually to the desired winding speed. Fiber was slipped into the hook of the traverse guide when steady fiber production was achieved. The hook traversed the fiber across the spool and made the winding process very uniform. The glass rods were gradually lowered into their respective slots to secure the fiber below the water surface. This removed the solvent from the fibers very efficiently. The fiber was cut after the desired amount of fiber was wound on the spool at a particular winding speed. To assure complete removal of solvent from the fibers, the fiberwound spool was removed from the spool drum and was immersed in a beaker containing water for 8-10 hours. The spool was allowed to air-dry for 48 hours, and then vacuum oven-dried for 24 hours at $\sim 50^{\circ} \mathrm{C}$. 
VITA

Vipul Davé, son of Mr. and Mrs. B. M. Davé, was born on June 3, 1962 in Rajkot, India. He attended secondary school at st. Joseph's College, Calcutta, India, from where he graduated in May, 1981. He then received his B.S. in Textile Technology from the University of Baroda, Baroda, India in December, 1985.

Immediately after receiving his B.S., vipul entered the Master's program in Polymer science at the University of Massachusetts at Lowell in January, 1986 under the supervision of Dr. Stanley C. Israel. He then transferred to Michigan State University in September, 1987 for pursuing his $\mathrm{Ph} . \mathrm{D}$ in Materials Science and Engineering. Attracted by the outstanding polymer program, he transferred to Virginia Tech in January 1988, where he earned his Ph.D. in Materials Engineering Science in February, 1992 under the wing of Dr. Wolfgang G. Glaser.

He married Shruti (daughter of Mr. and Mrs. J. K. Kusumgar) on May 26, 1991.

369 